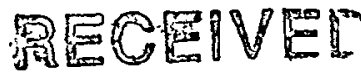 OCT 312800 OSTI
}

\section{In Situ Redox Manipulation Proof-of-Principle Test at the Fort Lewis Logistics Center: Final Report}

\author{
V. R. Vermeul \\ J.E. Szecsody \\ M. D. Williams \\ B. N. Bjornstad \\ J. C. Evans \\ T. L. Liikala
}

September 2000

Prepared for the U.S. Army Forces Command

Through a Related Services Agreement

with the U.S. Department of Energy

under Contract DE-AC06-76RL01830

Pacific Northwest National Laboratory

Richland, Washington 99352 


\section{DISCLAIMER}

This report was prepared as an account of work sponsored by an agency of the United States Government. Neither the United States Government nor any agency thereof, nor any of their employees, make any warranty, express or implied, or assumes any legal liability or responsibility for the accuracy, completeness, or usefulness of any information, apparatus, product, or process disclosed, or represents that its use would not infringe privately owned rights. Reference herein to any specific commercial product, process, or service by trade name, trademark, manufacturer, or otherwise does not necessarily constitute or imply its endorsement, recommendation, or favoring by the United States Government or any agency thereof. The views and opinions of authors expressed herein do not necessarily state or reflect those of the United States Government or any agency thereof. 


\section{DISCLAIMER}

Portions of this document may be illegible in electronic image products. Images are produced from the best available original document. 


\section{Summary}

Pacific Northwest National Laboratory (PNNL) conducted a proof-of-principle test at the Fort Lewis Logistics Center to determine the feasibility of using the In Situ Redox Manipulation (ISRM) technology for remediating groundwater contaminated with dissolved trichloroethylene (TCE). ISRM creates a permeable treatment zone in the subsurface to remediate redox-sensitive contaminants in groundwater. The permeable treatment zone is formed by injecting a chemical reducing agent (sodium dithionite with $\mathrm{pH}$ buffers) into the aquifer through a well to reduce the naturally occurring ferric iron in the sediments to ferrous iron. Once the reducing agent is injected and given sufficient time to react with aquifer sediments, residual chemicals and reaction products are withdrawn from the aquifer through the same well used for the injection. Redox-sensitive contaminants such as TCE, moving through the treatment zone under natural groundwater flow conditions, are destroyed. TCE is degraded via reductive dechlorination within the ISRM treatment zone to benign degradation products (i.e., acetylene, ethylene). Prior to the proof-of-principle field test, the ISRM technology was successfully demonstrated in laboratory experiments for the reductive dechlorination of dissolved TCE using sediments from the Fort Lewis site.

The Logistics Center was placed on the National Priorities List in December 1989 because of TCE contamination in groundwater beneath the site. A Federal Facilities Agreement between the Army, the U.S. Environmental Protection Agency, and the Washington State Department of Ecology became effective in January 1990, and a Record of Decision (ROD) was signed in September 1990. The major components of the ROD included installation of two pump-and-treat systems for the upper aquifer and further investigation of the lower aquifer and other potential sources of contamination. The pump-andtreat systems became operational in August 1995. Fort Lewis asked PNNL to provide technical support in accelerating Installation Restoration Program site remediation and significantly reducing site life-cycle costs at the Logistics Center. In support of this program, ISRM was selected as an innovative technology for bench and field-scale demonstration.

Emplacement of the ISRM treatment zone was accomplished through a series of four separate dithionite injection tests conducted between November 10, 1998 and March 29, 2000. An extensive program of chemical monitoring was also performed before, during, and after each injection to evaluate the performance of ISRM. Prior to emplacement of the ISRM treatment zone, the site was extensively characterized with respect to geologic, hydrologic, and geochemical properties. Sediment core samples collected for the characterization studies were analyzed in bench-scale column tests at PNNL to determine reducible iron content. These site-specific hydrogeologic and geochemical data were used to develop the emplacement design of the pilot-scale (i.e., single injection well) ISRM treatment zone.

Performance data obtained from the proof-of-principle test indicate that field-scale reductive dechlorination of TCE using the ISRM technology is feasible. A treatment zone was created in the subsurface that reduced TCE concentrations as much as $92 \%$ on the downgradient side of the reduced zone, from a background concentration of approximately $140 \mathrm{ppb}$ to approximately $11 \mathrm{ppb}$. The appearance of the principal degradation product, acetylene, also confirmed that TCE destruction was occurring. Analysis of sediment samples collected from post-test boreholes showed a high degree of iron reduction, which helped to confirm the effectiveness of the treatment zone emplacement. 
Another important goal of the testing program was to provide assurances that chemical treatment of the subsurface did not result in undesirable secondary effects, including formation of toxic TCE degradation products, mobilization of trace elements, and degradation of hydraulic performance. Results obtained from the Fort Lewis ISRM proof-of-principle test, which are consistent with results from previous ISRM studies (both bench- and field-scale), indicate that no significant secondary effects were identified that could limit full-scale application of this technology. 


\section{Acknowledgments}

The authors wish to acknowledge the important roles of J. S. Fruchter as ISRM Principal Investigator and J. G. Bush as Technical Group Manager in this project. We also thank K. B. Olsen and D. S. Sklarew for their helpful technical reviews, and S. Q. Bennett for her editorial review and document assembly. 



\section{Acronyms and Abbreviations}

\begin{tabular}{|c|c|}
\hline AFB & Air Force Base \\
\hline ALG & American Lake Gardens (Tract) \\
\hline ASTM & American Society for Testing and Materials \\
\hline BTC & breakthrough curve \\
\hline CERCLA & Comprehensive Environmental Response, Compensation, and Liability Act \\
\hline CFR & Code of Federal Regulations \\
\hline DCE & dichloroethylene \\
\hline DNAPL & dense nonaqueous phase liquid \\
\hline DO & dissolved oxygen \\
\hline DoD & U.S. Department of Defense \\
\hline DOE & U.S. Department of Energy \\
\hline DRMO & Defense Reutilization and Marketing Office \\
\hline DWS & federal drinking water standards \\
\hline $\mathrm{EC}$ & electrical conductivity \\
\hline Ecology & Washington State Department of Ecology \\
\hline EGDY & East Gate Disposal Yard \\
\hline $\mathrm{Eh}$ & oxidation reduction potential \\
\hline EM-50 & DOE Office of Science and Technology \\
\hline EPA & U.S. Environmental Protection Agency \\
\hline ESD & explanation of significant difference \\
\hline $\mathrm{Fe}(\mathrm{III})$ & ferric iron \\
\hline $\mathrm{Fe}(\mathrm{II})$ & ferrous iron \\
\hline FFA & Federal Facilities Agreement \\
\hline FORSCOM & U.S. Army Forces Command \\
\hline GC/MS & gas chromatography/mass spectrometry \\
\hline HRS & hazard ranking score \\
\hline IC & ion chromatography \\
\hline ICP-MS & inductively coupled plasma-mass spectrometry \\
\hline ICP-OES & induction coupled plasma-optical emission spectrometry \\
\hline IP & integrated program \\
\hline IRP & installation restoration program \\
\hline ISRM & In Situ Redox Manipulation \\
\hline
\end{tabular}




$\begin{array}{ll}\text { MCL } & \text { maximum contaminant level } \\ \text { MROD } & \text { Mount Rainier Ordnance Depot } \\ \text { NCP } & \begin{array}{l}\text { National Contingency Plan } \\ \text { NPL }\end{array} \\ \text { National Priorities List } \\ \text { ORP } & \begin{array}{l}\text { oxidation reduction potential } \\ \text { OST }\end{array} \\ \text { PNNL } & \text { Pacific Northwest National Laboratory } \\ \text { POL } & \text { petroleum, oil, and lubricants } \\ \text { POPT } & \text { proof-of-principle test } \\ \text { Ppb } & \text { parts per billion } \\ \text { RA } & \text { remedial action } \\ \text { RCRA } & \text { Resource Conservation and Recovery Act } \\ \text { RFA } & \text { RCRA Facility Assessment } \\ \text { RI/FS } & \text { Remedial Investigation/Feasibility Study } \\ \text { ROD } & \text { record of decision } \\ \text { SPH } & \text { six-phase heating } \\ \text { TCA } & \text { trichloroethane } \\ \text { TCE } & \text { trichloroethylene } \\ \text { USACE } & \text { U.S. Army Corps of Engineers } \\ \text { USGS } & \text { U.S. Geological Survey } \\ \text { VOA } & \begin{array}{l}\text { volatile organic analysis } \\ \text { VoClatile organic compound }\end{array} \\ & \end{array}$




\section{Contents}

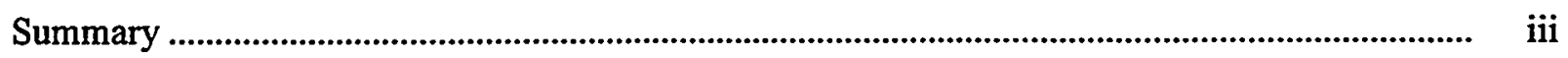

Acknowledgments...................................................................................................................

Acronyms and Abbreviations........................................................................................................ vii

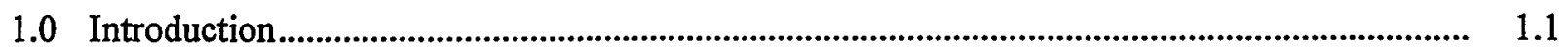

1.1 Background...........................................................................................................

1.1.1 Contamination Assessment ................................................................................. 1.5

1.1.2 Technology History ........................................................................................ 1.7

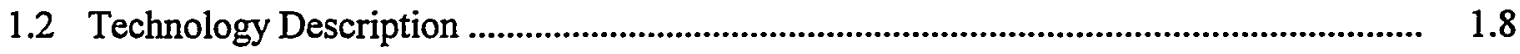

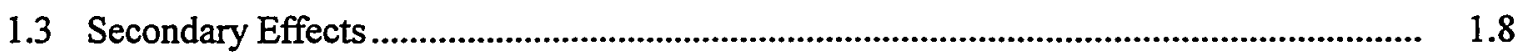

1.4 Field Test Objectives .............................................................................................. 1.10

1.5 Organization of the Report ............................................................................................... 1.10

2.0 Summary of Proof-of-Principle Test Activities.......................................................................... 2.1

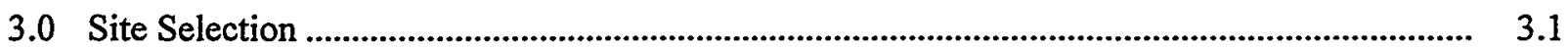

3.1 Regulatory Criteria …………................................................................................... 3.1

3.2 Well Installation Costs ...........................................................................................

3.3 Hydrogeologic Criteria .............................................................................................. 3.2

3.4 Geochemical Criteria ............................................................................................. 3.2

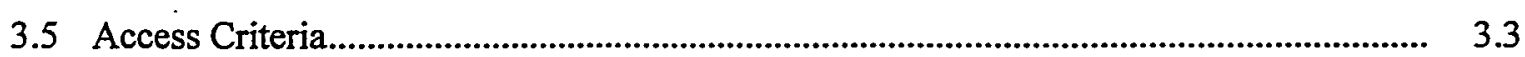

4.0 Site Characterization ............................................................................................................

4.1 Hydrogeology of the ISRM Site .............................................................................

4.1.1 Steilacoom Gravel ............................................................................................. 4.3 
4.1.2 Upper Till (Qvt1) ....................................................................................

4.1.3 Upper Outwash Gravel (Gg-u) and Sand (Gs-u) ........................................... 4.4

4.1.4 Lower Till (Qvt2) ......................................................................................... 4.4

4.1.5 Lower Outwash Gravel (Gg-l) and Sand (Gs-l) .............................................. 4.4

4.1.6 “Older" Till (tu) .................................................................................................. 4.5

4.1.7 Nonglacial Deposits (ng) ................................................................................ $\quad 4.5$

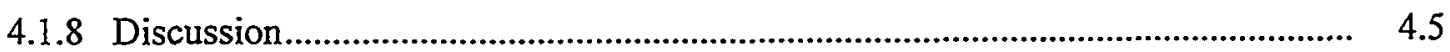

4.1.9 Hydrology of the ISRM Site.......................................................................... 4.6

4.2 Well Installation ......................................................................................................... 4.6

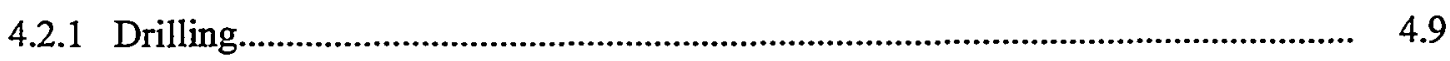

4.2.2 Sediment Sampling ...................................................................................... 4.11

4.2.3 Well Development............................................................................................ 4.11

4.3 Geologic Characterization .......................................................................................... 4.12

4.3.1 Geologic Logging ....................................................................................... 4.12

4.3.2 Particle-Size Analyses ................................................................................... 4.12

4.3.3 Bulk Density ................................................................................................... 4.12

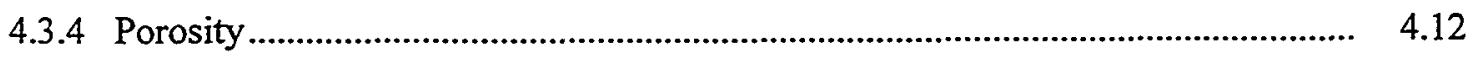

4.3.5 Geophysical Logging................................................................................ 4.17

4.4 Geochemical Characterization.................................................................................... 4.19

4.5 Vertical Contaminant Distribution .............................................................................. 4.19

4.6 Baseline Groundwater Chemistry ................................................................................ 4.20

4.7 Hydrologic Characterization ..................................................................................... 4.24

4.8 Groundwater Flow Rate and Direction...................................................................... 4.25 
5.0 Site Setup for Proof-of-Principle Test ....................................................................................

5.1 Component Description ...........................................................................................

5.1.1 Site Utilities ................................................................................................

5.1.2 Monitoring Equipment …............................................................................... 5.2

5.1.3 Analytical Measurements ............................................................................... 5.4

5.1.4 Injection and Withdrawal Equipment................................................................. 5.5

5.2 Description of Equipment Integration/Operation............................................................. 5.7

5.2.1 Tracer and Dithionite Injection ....................................................................... 5.7

5.2.2 Groundwater Sample Collection ......................................................................... 5.7

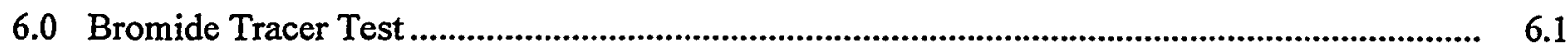

6.1 Tracer Test Objective ................................................................................................ 6.1

6.2 Tracer Test Description .............................................................................................

6.3 Results and Discussion ..............................................................................................

7.0 Treatment Zone Emplacement................................................................................................

7.1 Emplacement Strategy ...................................................................................................

7.2 Emplacement Description .............................................................................................

7.2.1 Dithionite Injection/Withdrawal 1...................................................................... 7.4

7.2.2 Dithionite Injection/Withdrawal 2a, 2b, 3, and 4 ............................................. 7.8

7.2.3 Injection/Withdrawal Tests 2a/2b........................................................................ 7.9

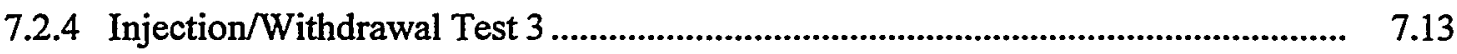

7.2.5 Injection/Withdrawal Test 4 ............................................................................

7.3 Estimation of Percent Reduction from Dithionite Injection Data ....................................... 7.13

7.4 Mobilization of Trace Elements ...................................................................................... 
8.0 Performance Assessment of Emplaced ISRM Treatment Zone

8.1 Performance Ássessment Groundwater Monitoring .

8.1.1 TCE Monitoring

8.1.2 TCE Degradation Products

8.1.3 Field Parameter Monitoring

8.1.4 Trace Metals Monitoring

8.1.5 Common Anions Monitoring

8.2 Relationship Between Field-Scale Iron Reduction and TCE Degradation Rates

8.3 Treatment Zone Reductive Capacity

8.4 Hydraulic Performance

9.0 Summary and Conclusions

10.0 References

Appendix A - Geochemical Reactions for Remediation of TCE

Appendix B - Analytical Methods.

Appendix C - Hydrogeologic Setting. Well Construction Summaries, and As-Built Diagrams .......

Appendix E-Baseline Chemistry

E.1

Appendix F - Tracer Test.

F.1

Appendix G - Dithionite Injection/Withdrawal.

G.1

Appendix $\mathrm{H}$ - Post-Injection Performance Assessment Monitoring

H.1 


\section{Figures}

$1.1 \quad$ Map of Fort Lewis .............................................................................................................

1.2 Detailed Map of Study Area Showing TCE Plume Beneath Fort Lewis Logistics Center in September 1999 ..................................................................................................................... 1.3

1.3 In Situ Redox Manipulation Conceptual Diagram .................................................... 1.9

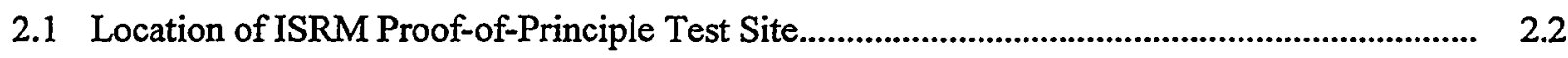

4.1 Borehole Locations ..................................................................................................................

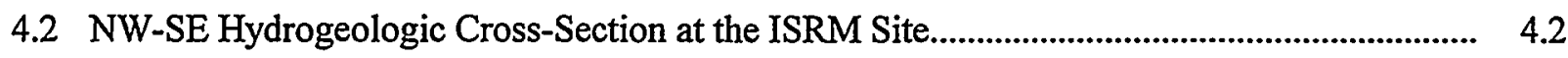

4.3 NE-SW Hydrogeologic Cross-Section at the ISRM Site................................................. 4.2

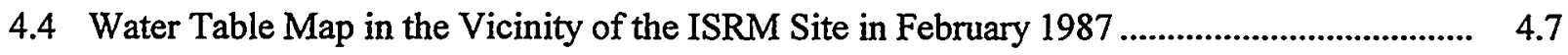

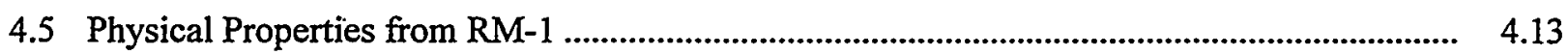

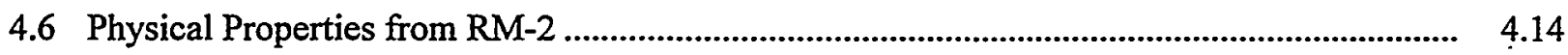

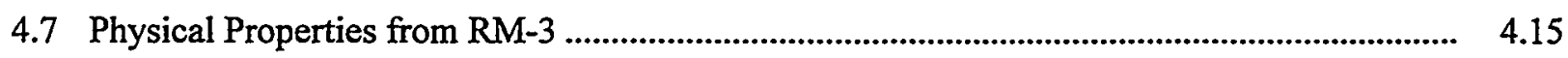

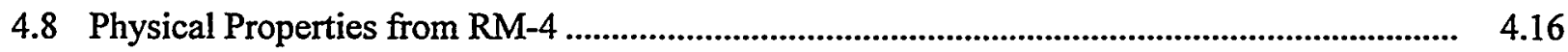

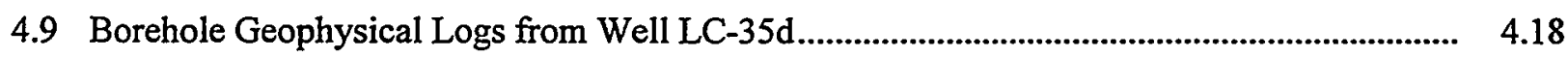

4.10 Potentiometric Surface Contour Maps Representing a) Low Water Level Conditions on October 19, 1999, b) Medium Water Level conditions on January 1, 2000, and

3) High Water Level conditions on February 2, 1999

4.11 Groundwater Velocity and Direction Observed at the ISRM Proof-of-Principle Test Site During Field Testing.

4.12 Comparison of Monthly Precipitation and Water Level Elevation at RM-9.

4.13 Comparison of Hydrographs for Wells Within and Outside the DRMO Complex.

5.1 Schematic Drawing of the Tracer and Dithionite Injection System Used at the Fort Lewis ISRM POPT Field Demonstration 
5.2 Schematic Drawing of the Groundwater Sample Acquisition System Used at the Fort Lewis ISRM POPT Field Demonstration

6.1 Fort Lewis ISRM Proof-of-Principle Site Bromide Tracer Test Showing the Duration of the Test Phases and Bromide Concentrations from the Injection/Withdrawal Streams.

6.2 Mass Balance of Injected and Withdrawn Bromide to Calculate the Percent Bromide Mass Recovery Achieved During the Withdrawal

7.1 Dithionite Injection/Withdrawal Test 1

7.2 Comparison of Breakthrough Curves Between the Bromide Tracer Test (Br-) and EC from Injection 1 for Selected Wells.

7.3 Temperatures and EC BTC Measured During the Preheat Phase for Dithionite Injection/Withdrawal 2

7.4 Dithionite Injection/Withdrawal Test 2a

7.5 Contour Plot of Percent Fe(II) Reduction Within the Targeted Portion of the Aquifer After Each Dithionite Injection/Withdrawal Test.

7.6 Observed Trace Metals Concentrations in Withdrawal Water During the 64-Hour Withdrawal Phase

8.1 TCE Concentration Trends at ISRM Site for Wells RM-1A, RM-2, RM-5, RM-8, and RM-9

8.2 Normalized TCE Concentration Trends at ISRM Site for Wells RM-7, RM-10, RM-11, and RM-13 Expressed as a Percentage of Influent Concentration at Well RM-3

8.3 TCE Destruction Trend Plot for Well RM-2

8.4 TCE Destruction Trend Plot for Well RM-5

8.5 TCE Destruction Trend Plot for Well RM-1A

8.6 TCE Concentration Contours Following Each Injection

8.7 TCE Contours in Groundwater at ISRM Site for Last Sampling Period Including New Wells, RM-14 and RM-15

8.8 Trend Plots for Acetylene in Groundwater for all Four Injections

8.9 Trend Plot for $\mathrm{pH}$ and Electrical Conductivity in Well RM-5. 
$8.11 \mathrm{pH}$ Distribution on $6 / 21 / 00$

8.12 (a) Basline Eh Distribution Injection 4, (b) Eh Distribution Pre-Injection 4, (c) Eh After Injection 4 Withdrawal, (d) Eh Injection 4 Plus One Month, (e) Eh Injection 4 Plus Two Months, and (f) Injection 4 Plus Three Months.

8.13 Sulfate Concentration Trends for Wells RM-5 and RM-6

8.14 Estimated Field-Scale TCE Degradation Rates Versus Average Percentage Iron Reduction ..

8.15 Pathline Showing Groundwater Path Through the Injection 4 Fe(II) Percent Reduction Estimates and Latest TCE Monitoring Results.

8.16 Sediment Oxidation of a Mixture of all RM Borehole Sediments (a) That was $100 \%$ Reduced by Dithionite in Laboratory Experiments, and (b) Sediment from FM-4, 61.8-ft Depth That was Field Reduced

8.17 Comparison of Pressure Response Data for (a) RM-5, (b) RM-7, and (c) RM-8 Under Changing Test Conditions

8.18 Pressure Response at $\mathrm{t}=100 \mathrm{~min}$ for Injection Well RM-9 Relative to Pressure Responses in Selected Monitoring Wells.

8.19 Pressure Response at $t=100 \mathrm{~min}$ for Selected Monitoring Wells

8.20 Average Pressure Response at $t=100 \mathrm{~min}$ for Selected Monitoring Wells.

\section{Tables}

4.1 ISRM Well Completion Summary Information

4.2 Bulk Density and Total Porosity Statistics for the Stratigraphic Units

4.3 Vertical TCE Distribution in the Groundwater Beneath the ISRM Test Site.

4.4 Summary of Pre-Injection Groundwater Geochemical Parameters

4.5 Summary of Pre-Injection TCE, DCE, and Acetylene Concentrations in Groundwater at POPT Site. 
4.6 Summary of Pre-Injection Common Anion Concentrations in Groundwater at POPT Site ..... $\quad 4.23$

4.7 Summary of Pre-Injection ICP-OES Trace Metals Concentrations in Groundwater at POPT Site

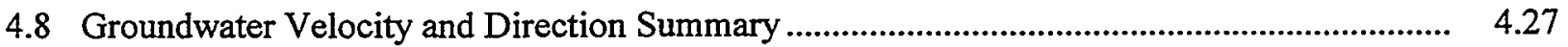

5.1 Field Parameter Monitoring Electrode Specifications...................................................... 5.3

6.1 Bromide Tracer Test Injection Concentration Summary ......................................................

7.1 Summary of Fort Lewis ISRM POPT Field Injection Tests..........................................

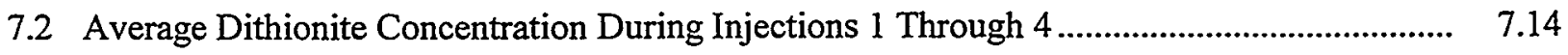

7.3 Dithionite-Sediment Contact Time During Injections 1 Through 4 .................................... 7.15

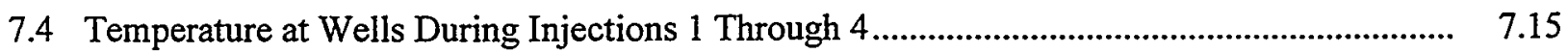

7.5 Predicted Cumulative Iron Reduction at Well Locations .......................................................... 7.16

7.6 Species Showing Elevated Concentrations in Injection 2 Withdrawal Water......................... 7.19

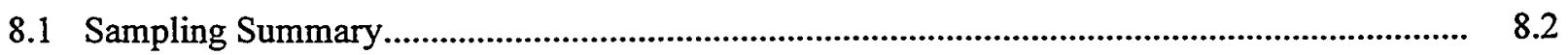

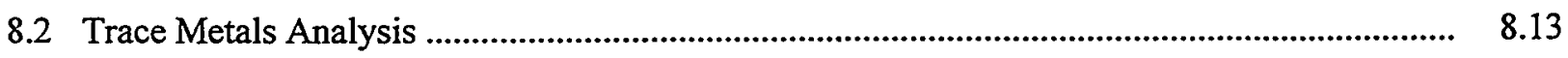

8.3 Qualitative Reducing Conditions Observed in Sediment Cores ........................................... 8.19

8.4 Results of Sediment Reduction and Oxidation Experiments.............................................. $\quad 8.20$ 


\subsection{Introduction}

Fort Lewis is a U.S. Army Forces Command (FORSCOM) installation in western Washington, on the southeastern shore of Puget Sound, approximately 10 miles southwest of Tacoma (Figure 1.1). Its boundaries encompass 86,176 acres within Pierce and Thurston counties. Fort Lewis is an active military training facility for both weapons qualification and field training, whose mission is to maintain the combat readiness of assigned units. The working population is approximately 22,100 personnel, including military, civilian, and contractor employees.

The installation Logistics Center, formerly known as the Fort Lewis Quartermaster Motor Base and the Mount Rainier Ordnance Depot (MROD), was built in 1941. It was activated in 1942 and consisted of shops, warehouses, and barracks. In mid-1942, the facility was transferred to ordnance jurisdiction and renamed MROD. MROD was responsible for furnishing ordnance supplies and rebuild services to military installations within the states of Idaho, Montana, Oregon, and Washington, and to overseas and Alaskan installations through Pacific Coast Army terminals. Items serviced by MROD included artillery, combat and transport vehicles and assemblies, fire control material, guided missile material, and small arms. In 1963, the facility was turned over to the Logistics Center (Figure 1.2) to serve as the primary non-aircraft maintenance facility for the post.

Trichloroethylene (TCE) was used at the Logistics Center in large quantities as a cleaning and degreasing solvent until the mid-1970s, when it was replaced by trichloroethane (TCA). The waste TCE was commonly mixed with petroleum, oil, and lubricants (POL) and stored in drums for disposal. The volume of waste produced is uncertain but may have been as much as six to eight drums per month (Shannon \& Wilson 1986). Some of this material was used to assist in the burning of other wastes. Disposal occurred at several locations, including the East Gate Disposal Yard (EGDY), located upgradient of the Logistics Center (see Figure 1.2). The EGDY was used as an uncontrolled disposal site between 1946 and 1960. Disposal trenches were excavated in the yard and on adjacent land to the southwest.

The Logistics Center was placed on the National Priorities List (NPL) in December 1989 as a result of TCE contamination in groundwater beneath the site (see Figure 1.2). A Federal Facilities Agreement (FFA) between the U.S. Environmental Protection Agency (EPA), the Washington State Department of Ecology (Ecology), and the Army was formalized in January 1990. This FFA established the procedural framework and schedule for developing, implementing, and monitoring appropriate response actions at the Logistics Center. A Record of Decision (ROD) was signed by EPA, Ecology, and the Army in September 1990. The selected remedial action consisted of two pump-and-treat systems to treat groundwater in the upper aquifer and further investigation of the lower aquifer and remaining potential sources of soil contamination. The Interstate 5 (I-5) pump-and-treat system was designed for plume containment and consists of a series of extraction wells, a treatment facility, and recharge galleries near the northwestern edge of the site. The East Gate system was designed to contain and remediate the source area (EGDY) and facilitate the flushing of secondary groundwater sources within the high-concentration zones beneath the EGDY. 


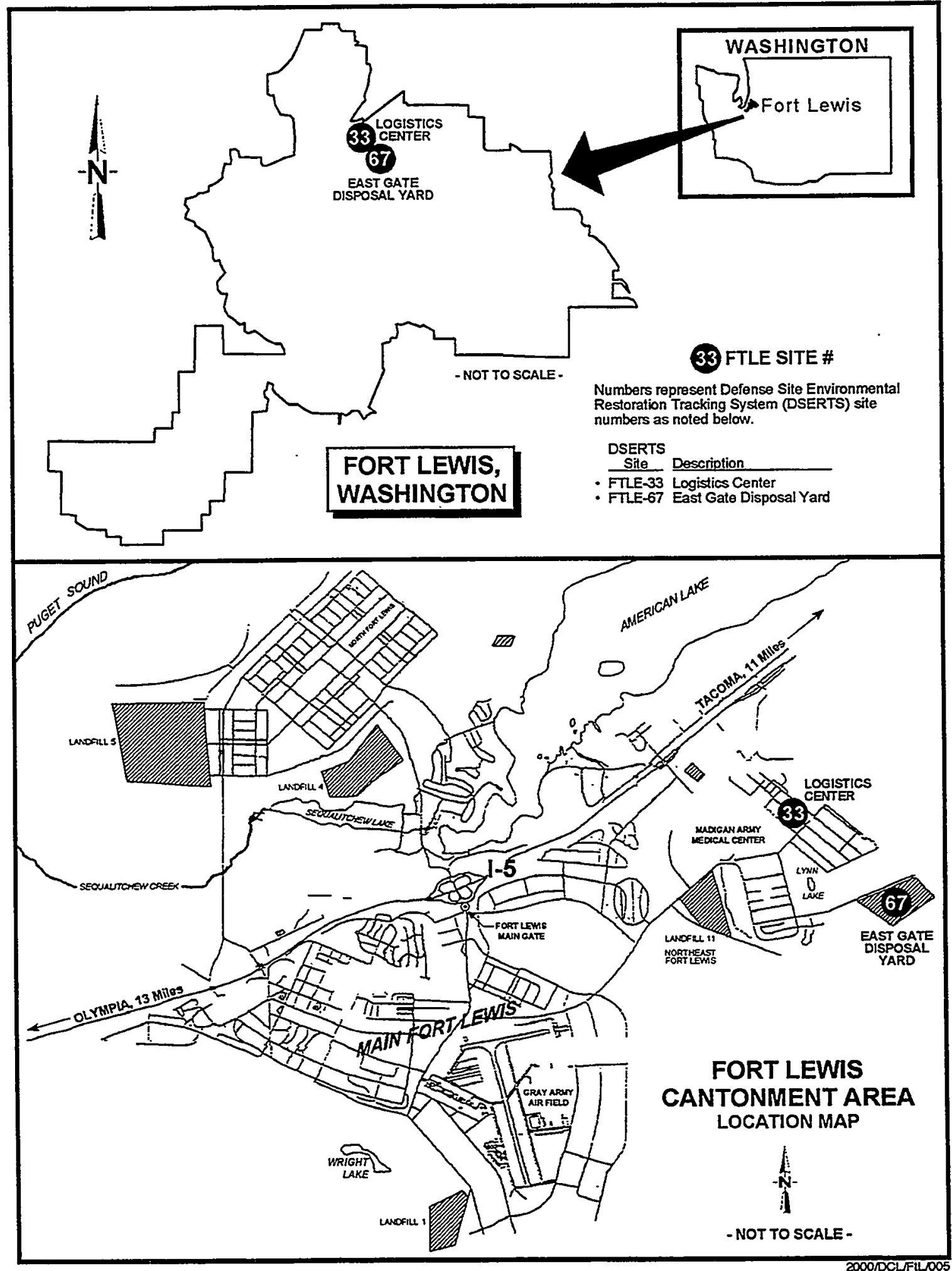

Figure 1.1. Map of Fort Lewis 


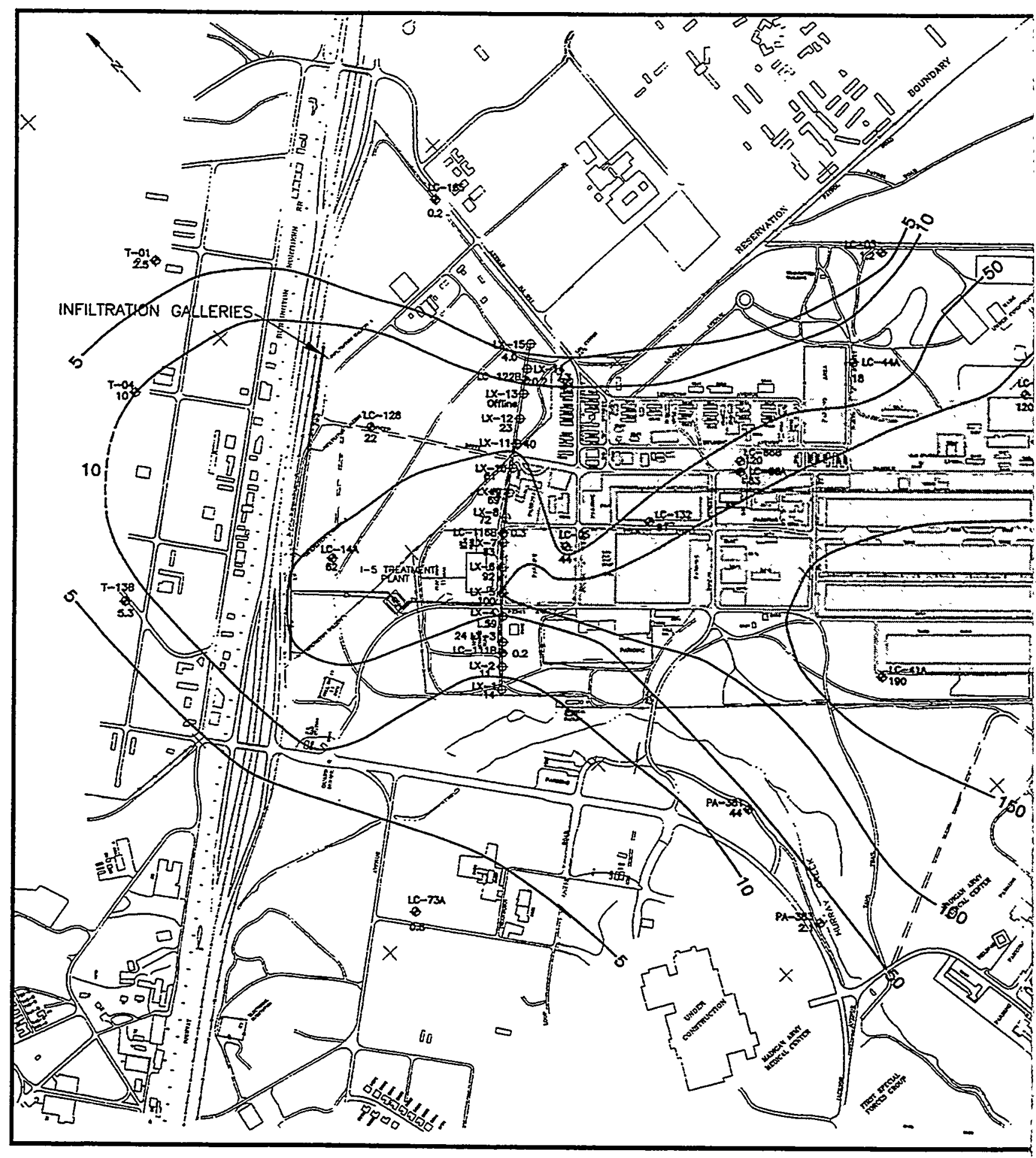

Figure 1.2. Detailed Map of Study Area Showing TCE PI 


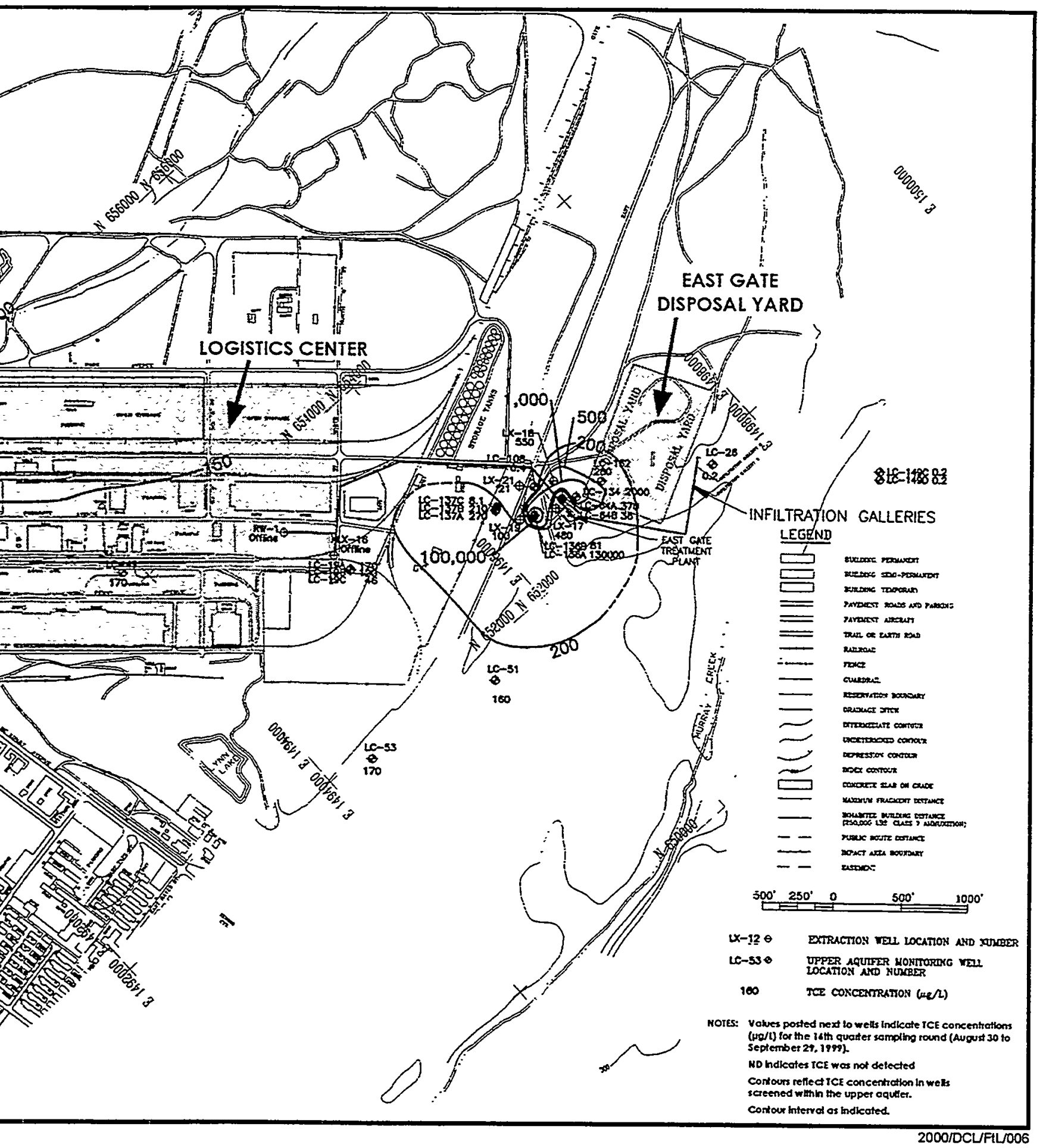

e Beneath Fort Lewis Logistics Center in September 1999 
In 1997, Fort Lewis asked Pacific Northwest National Laboratory (PNNL) to visit the installation and assess the waste treatment operations at the Logistics Center. From this visit and a review of supporting documentation provided by Fort Lewis, PNNL identified enhancement opportunities in four areas relative to the current treatment operations (Cantrell et al. 1998). Fort Lewis then asked PNNL to provide technical support in accelerating Installation Restoration Program (IRP) site remediation and significantly reducing site life-cycle costs at the Logistics Center. PNNL proposed a program to evaluate and test new and innovative (non-standard) remedial technologies. This program was endorsed by Fort Lewis and FORSCOM. The specific objectives of the program are to:

1. Apply state-of-the-art/innovative remedial technologies to identify, isolate/contain, or treat in an accelerated fashion contaminants of concern to regulatory acceptable levels.

2. Evaluate, optimize, and enhance the effectiveness of existing groundwater remediation systems in reducing contaminants to regulatory acceptable levels, leading to closure of these systems significantly earlier than current estimates.

3. Complete associated remedial and administrative tasks to remove the Logistics Center from the NPL.

PNNL is now in the third year of this program at Fort Lewis and has made significant progress with laboratory and field-testing of the innovative remedial technology called In Situ Redox Manipulation (ISRM). ISRM involves creating a permeable treatment barrier in the subsurface to remediate redoxsensitive contaminants in groundwater. The permeable treatment barrier is created within or downstream of a contaminant plume, and redox-sensitive contaminants moving through this barrier are destroyed or immobilized. The initial feasibility of ISRM for treating dissolved TCE contamination in the groundwater beneath the Logistics Center in situ was demonstrated in the proof-of-principle test (POPT) reported in this document. Activities include site selection, characterization, and setup; baseline sampling and analysis; tracer testing; treatment zone emplacement; and performance assessment. The laboratory work has been reported in detail in Szecsody et al. (2000).

\subsection{Background}

Historical information on the regulatory status of and contamination assessment for the Logistics Center and ISRM technology are provided in this section.

\subsubsection{Contamination Assessment}

An Installation Assessment Report was completed for Fort Lewis in September 1983. This report identified sites, including the Logistics Center, requiring further inspection. The Logistics Center, a landfill, and 14 other sites from the Resource Conservation and Recovery Act (RCRA) Facility Assessment (RFA) requiring further investigation make up the 16 sites in the Fort Lewis FFA.

Several studies have been conducted on the groundwater contamination beneath the Logistics Center since the Installation Assessment Report. In 1983, EPA and the Tacoma-Pierce County Health Department initiated studies of potential groundwater contamination in the American Lake Gardens Tract 
(ALG), an area north of the Logistics Center, in response to public concern. These studies confirmed the presence of TCE and cis 1,2-dichloroethylene (DCE) in several domestic water-supply wells in ALG, apparently originating from the north at McChord Air Force Base (AFB), and revealed indications of contamination beneath the Logistics Center. As a result, a groundwater investigation began in November 1984 to determine whether TCE and DCE contamination at ALG was coming from the Logistics Center. This investigation defined the northern and eastern boundaries of a large area of contaminated groundwater consisting primarily of TCE with lesser concentrations of DCE beneath the Logistics Center. The plume of groundwater contamination was shown to be moving along the centerline of the Logistics Center (see Figure 1.2).

Knowledge of contamination beneath the Logistics Center prompted an EPA directed groundwater study of the Tillicum area (a small town immediately downgradient of the Logistics Center) in November 1985 to specifically address the potential impact on a nearby public water supply well. The study concluded that widespread, low-level TCE contamination was present beneath Tillicum, apparently originating from the Logistics Center. The contamination was also found to extend into the next lower aquifer. In December 1985, a second groundwater investigation of the Logistics Center began to identify sources of the TCE contamination, determine the areal extent of the contamination (particularly at the southern and western edges), and develop a conceptual remedial action to remove the contamination. The study defined a large zone of contaminated groundwater beneath the Logistics Center that contained significant concentrations of TCE and DCE. The contaminated zone was found to be at least 10,000 feet in length, 2,500 feet in width, and to extend at least 80 feet below the water table. The primary source of the contamination was identified as the EGDY. The results of this investigation warranted a remedial investigation/feasibility study (RI/FS) for the Logistics Center starting in October 1986. EPA's investigation of Tillicum resulted in adding it to the Logistics Center RI/FS study area in February 1988. The RI was finalized in November 1988.

The Logistics Center was nominated for the NPL in July 1989 and placed on the NPL in December 1989 with a hazard ranking score (HRS) of 35.48. Soil, groundwater, surface water, and sediment analyses indicated that groundwater contamination was the principal threat at the site. The FS was finalized in May 1990, and the ROD was signed in September 1990. The selected remedial action (RA) consisted of pumping and treating groundwater in the upper aquifer and further investigation of the lower aquifer and remaining potential sources of soil contamination. This investigation found that the complex hydrogeology beneath the Logistics Center might allow contaminated groundwater from the upper aquifer to migrate to the lower aquifer through permeable soil lenses (see Appendix C).

The ROD for the Logistics Center specified that if the lower aquifer were found to be contaminated, it would be pumped and treated using the existing onsite treatment facilities. The Fort Lewis IRP Technical Working Group, consisting of representatives from Fort Lewis, EPA, Ecology, PNNL, and the U.S. Geological Survey (USGS), determined that this action may not be beneficial to the treatment of this aquifer because it may pull additional contaminated groundwater down from the upper aquifer. They determined that innovative remedial technologies should be employed to contain and remediate the existing contaminated groundwater. An explanation of significant difference (ESD) from the ROD for the Logistics Center was signed by the Army and EPA in September 1998. This ESD, prepared in accordance with the Comprehensive Environmental Response, Compensation, and Liability Act 
(CERCLA) and the Code of Federal Regulations (CFR), was necessary to document changes to the selected remedy outlined in the ROD. The ESD addresses enhancements being implemented in the overall strategy for remediating the site and has become part of the Administrative Record pursuant to the National Oil and Hazardous Substance Pollution Contingency Plan (NCP).

A Master Environmental Restoration Action Plan was prepared in March 2000 to completely integrate available resources and technologies to accelerate cleanup of the Logistics Center, significantly reduce IRP life-cycle costs, and delist this site from the NPL. It summarizes the strategic and tactical planning and decision rationale on enhanced contaminant source and groundwater plume cleanup plans for the site, of which ISRM is an integral part. The plan is consistent with the Department of Defense's (DoD) desire to find an exit strategy from pump and treat and will be updated as developments warrant.

Pursuant to the initial assessment of existing treatment operations in 1997 and in conjunction with the Master Environmental Restoration Action Plan, viable remedial technologies were subjected to further screening and evaluation. Three innovative technologies, ISRM, Six-Phase Heating (SPH), and one technology to be determined, were selected in this process for laboratory and field-testing. However, funding constraints limited immediate testing to one technology. ISRM was nominated because of its potential to isolate the source area from the remainder of the plume.

\subsubsection{Technology History}

Development of the ISRM technology was initiated at PNNL in 1991 with funding from U.S. Department of Energy (DOE) Office of Health and Environmental Research Subsurface Science Program. As part of this project, laboratory proof-of-principle studies were conducted and conceptual design and preliminary planning documents were prepared. However, attempts to control redox potential in an aquifer must overcome various scale-up complications arising from the interaction between contaminants, reducing agents, groundwater, and the natural variability of the subsurface. Therefore, in 1993, the project was transferred to DOE's Office of Science and Technology (OST). A site for the initial field test of the ISRM technology was selected in 1994. Bench-scale, intermediate-scale, and field-scale tests of the technology were funded through an OST EM-50 Integrated Program (IP) from 1993 through 1995 and through the Plumes and Subsurface Contaminant Focus Areas in 1996. This laboratory- and field-scale approach provided the means to evaluate the scale-up and extrapolation of the results from controlled laboratory-scale studies to the less certain conditions encountered in the subsurface.

Before going to the field, a series of experiments was performed at the bench and intermediate scales to test different reagents for their efficiency in manipulating redox conditions. Reagents tested included sulfite, thiosulfate, hydroxylamine, and dithionite. These tests were used to determine the nature of the reactions that could occur and the efficiency with which they would be induced by the reagent. The factors assessed include the kinetics of dithionite disproportionation, $\mathrm{Fe}(\mathrm{III})$ reduction, and the subsequent reoxidation of the reduced phases.

Comparative bench-scale batch studies under anoxic conditions established that dithionite was the most effective reductant for the structural ferric iron found in the silt and clay fractions of sediment samples collected from the Hanford Site in southeastern Washington State (see Section 4.4 for results 
from Fort Lewis sediment). These tests also confirmed that dithionite has a limited lifetime in these sediments due to 1) oxidation by $\mathrm{Fe}$ (III) present in the layer-silicate mineral structures or as oxyhydroxides and 2) a disproportionation reaction that was catalyzed by contact with mineral surfaces. Further experiments established that the longevity of the dithionite was prolonged by buffering the solution at neutral or higher $\mathrm{pH}$. Using buffered solutions at room temperature, these experiments indicated that the half-life of the dithionite ion would be approximately two or three days under the conditions in the Hanford unconfined aquifer. This half-life allows enough time for reduction of structural iron in the aquifer solids, while ensuring that dithionite does not remain in the groundwater for extended periods.

\subsection{Technology Description}

The ISRM technology (Figure 1.3) creates a permeable reactive barrier using a chemical treatment to reduce existing $\mathrm{Fe}$ (III) (ferric iron) to $\mathrm{Fe}(\mathrm{II})$ (ferrous iron) in aquifer sediments. A chlorinated solvent such as TCE is dechlorinated as the contaminated groundwater passes through the barrier under natural gradient conditions. The iron reduction is accomplished through the injection and withdrawal of a reducing agent, sodium dithionite, with potassium carbonate $\mathrm{pH}$ buffer, using standard groundwater wells. Sodium dithionite is a strong reducing agent that has many desirable characteristics for this type of application, including instability in the natural environment (on the order of days for complete breakdown) and reaction and degradation products that ultimately oxidize to sulfate.

This reduction of sediments in the aquifer requires sufficient contact time with the reductant and, in a homogeneous system, results in a cylindrical treatment zone centered around the injection/withdrawal well. This reaction or contact time is predetermined in laboratory experiments and depends on factors such as groundwater temperature, mass of iron, type of iron oxides, and sediment texture (Szecsody et al. 2000; Istok et al. 1999). The permeable reactive barrier is designed to dechlorinate TCE for decades, but it will eventually be oxidized (mainly by dissolved oxygen in groundwater) and lose its effectiveness. If additional reductive capacity is needed, the barrier can be regenerated at a reduced cost using the previously installed wells. The scientific basis of iron reduction mechanisms, sediment oxidation mechanisms, and TCE degradation are discussed in detail in Appendix A and Szecsody et al. (2000).

\subsection{Secondary Effects}

Potential secondary effects associated with the ISRM technology include metals mobilization, residuals concentrations, hydraulic performance (i.e., aquifer plugging), and dissolved oxygen depletion. In previous bench- and field-scale demonstrations of ISRM, none of these effects were shown to exceed technical or regulatory limits. This section presents a brief discussion of the secondary effects and includes references to more detailed information.

During development of the ISRM technology, one of the primary regulatory and stakeholder concerns was the potential for releasing unwanted constituents as the chemical treatment zone is formed. For example, as the reductive environment is formed, otherwise stable minerals or hydroxides can be broken down to release metals such as arsenic and manganese. The ISRM technology has been field-tested at several sites, including a proof-of-principle test at the Hanford 100-H Area for removing chromium from groundwater (Fruchter et al. 2000), a treatability test at the Hanford 100-D Area (Williams et al. 1999a), 


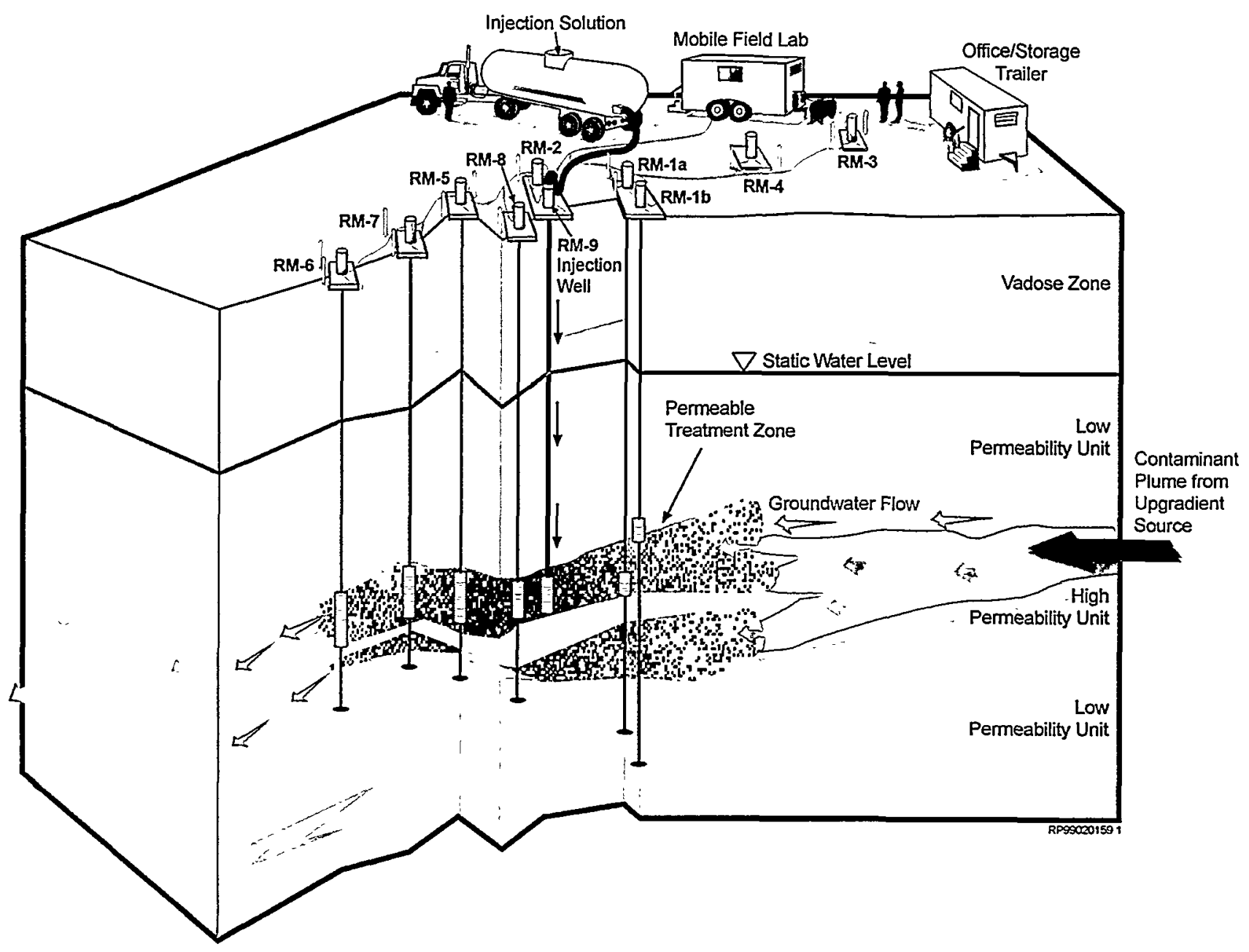

Figure 1.3. In Situ Redox Manipulation Conceptual Diagram. Schematic hydrology shown in diagram is based on the ISRM proof-of-principle test site at the Fort Lewis Logistics Center.

and a 2,000-foot-long ISRM barrier currently under construction at the same 100-D Area location. In addition, for each of these sites, batch and column tests were conducted to investigate the release of trace metals and gain regulatory approval for the field-scale injection. Laboratory tests have also been run on sediments from the Frontier Hard Chrome site (EPA) and Moffett Federal Airfield. Results from these field- and laboratory-scale tests indicate that, although trace metals are mobilized and exceed regulatory limits during the injection and withdrawal phases of the barrier emplacement, most are removed during the withdrawal, and following the emplacement do not migrate outside the reduced zone in significant enough quantities to create a regulatory concern.

Another concern associated with the ISRM technology was the potential to leave reaction products from the dithionite treatment (i.e., residual chemicals) in the aquifer. The primary reaction product of the dithionite injection is sulfate, which is regulated under a secondary drinking water standard (see Section 8). In the field tests cited above, the injection and withdrawal phases of the emplacement were 
designed to meet regulatory and stakeholder-mandated residual sulfate concentration requirements. Increasing the duration of the withdrawal phase to recover additional residuals does impact the costs of emplacement and wastewater disposal.

Analysis of hydraulic performance data from all ISRM field demonstrations to date (Fruchter et al. 2000; Williams et al. 1999a) has not indicated a significant reduction in formation permeability from deployment of the ISRM technology. The hydraulic test analysis did indicate a near-well decrease in permeability at the injection/withdrawal well following the injection. This small zone of reduced permeability (i.e., skin effect) is attributed to entrapment of suspended or colloidal material, or mineralization associated with the carbonate buffer, in the sandpack zone and well screen during the withdrawal phase. This near-well reduction in permeability caused no adverse effects during the injection or withdrawal phases of the demonstrations and did not result in any significant degradation in the overall hydraulic performance of the treatment zone.

Another secondary effect associated with the ISRM technology that may be of concern at some sites is oxygen depletion. At the ISRM treatability test site at the Hanford 100-D Area, proximity to the Columbia River $(\sim 500 \mathrm{ft})$ and potential salmon-spawning habitat resulted in regulatory and stakeholder sensitivity. To address regulatory concerns, a modeling study simulated this near-river system and investigated mechanisms important to attenuation of the anoxic plume. The model predicted how far downgradient from the ISRM barrier acceptable dissolved oxygen concentrations were achieved (Williams et al. 1999b; Williams and Oostrom 2000). At the 100-D site, the numerical model predicted 75 to $95 \%$ oxygen saturation at the river and determined that air entrapment caused by water table fluctuations (associated with diurnal fluctuations in river stage) had the greatest impact on attenuation of the anoxic plume.

As discussed, none of the potential secondary effects associated with the ISRM technology has been shown to exceed technical or regulatory limits. However, because the secondary effects of concern are highly dependent on site hydrogeology and geochemistry, the required data were collected to quantify these effects during the ISRM proof-of-principle demonstration at Fort Lewis.

\subsection{Field Test Objectives}

The objective of the ISRM proof-of-principle test was to determine the field-scale feasibility of this innovative remediation technology for treating dissolved TCE contamination in the groundwater at the Fort Lewis Logistics Center. Bench-scale studies were conducted in parallel with these field activities, but a field-scale test was required to determine the feasibility at a larger scale in the complex hydrogeologic and geochemical conditions of the subsurface.

\subsection{Organization of the Report}

A summary of the POPT activities is provided in Section 2. Site selection and site characterization of the POPT site are presented in Sections 3 and 4, respectively. Site setup is summarized in Section 5.

Details of the tracer test and treatment zone emplacement are contained in Sections 6 and 7, respectively. 
Section 8 assesses the performance of the emplaced treatment zone. Conclusions are provided in Section 9 and references cited in the text are listed in Section 10. Supporting documentation can be found in the appendixes. 


\subsection{Summary of Proof-of-Principle Test Activities}

This section contains a brief description of activities associated with the ISRM proof-of-principle test (POPT) and the objective of each of these activities. Detailed descriptions and results from these activities are contained in this report. The following list contains a brief description of the main activities associated with the ISRM POPT:

- Site Selection. Site selection criteria were developed and used to compare potential demonstration sites. All potential demonstration site locations were subjected to the criteria, which resulted in the final selection of the Fort Lewis ISRM POPT test site (Figure 2.1).

- Test Plan Preparation. A treatability test plan was prepared and submitted to the Fort Lewis IRP Technical Working Group prior to initiation of field activities.

- Hydraulic Gradient/Groundwater Flow Determination. Local-scale hydraulic gradient and groundwater flow direction was investigated using existing site wells. This information was used to align the ISRM well network with the natural groundwater flow direction.

- Well Installation and Geologic/Geochemical Characterization. Sonic drilling was used to install a central injection/withdrawal well and monitoring wells. As initial characterization boreholes were advanced, vertically discrete sediment and groundwater samples were collected. Sediment samples were analyzed for physical and chemical properties; groundwater samples were analyzed for standard field parameters and TCE concentration to determine their distribution with depth.

- Hydrologic characterization. Hydraulic tests (step drawdown, constant-rate discharge) were conducted in the injection well to evaluate its hydraulic performance and provide estimates of formation hydraulic conductivity and storativity. Water-level data from ISRM monitoring wells were incorporated into the analysis of local-scale hydrologic gradient and groundwater flow direction.

- Baseline Groundwater Monitoring. Three rounds of groundwater samples were collected from all site monitoring wells prior to any injection activities to determine baseline conditions. Samples were analyzed in the field for standard field parameters (dissolved oxygen [DO], electrical conductivity [EC], $\mathrm{pH}$, oxidation-reduction potential [ORP], temperature) and submitted for laboratory analysis of volatile organic compounds (VOCs), trace metals, and major ions).

- Tracer Test. A conservative tracer was injected into the central injection well and monitored in surrounding monitoring wells to estimate extent of the injected plume and the volumes/rates of injected reagent required to create an ISRM treatment zone. The tracer arrival data were also used to design the sampling strategy for the dithionite injection.

- Dithionite Injection/Withdrawal. A sodium dithionite solution was injected through the central injection well; after a reaction period, unreacted dithionite and reaction products were withdrawn 


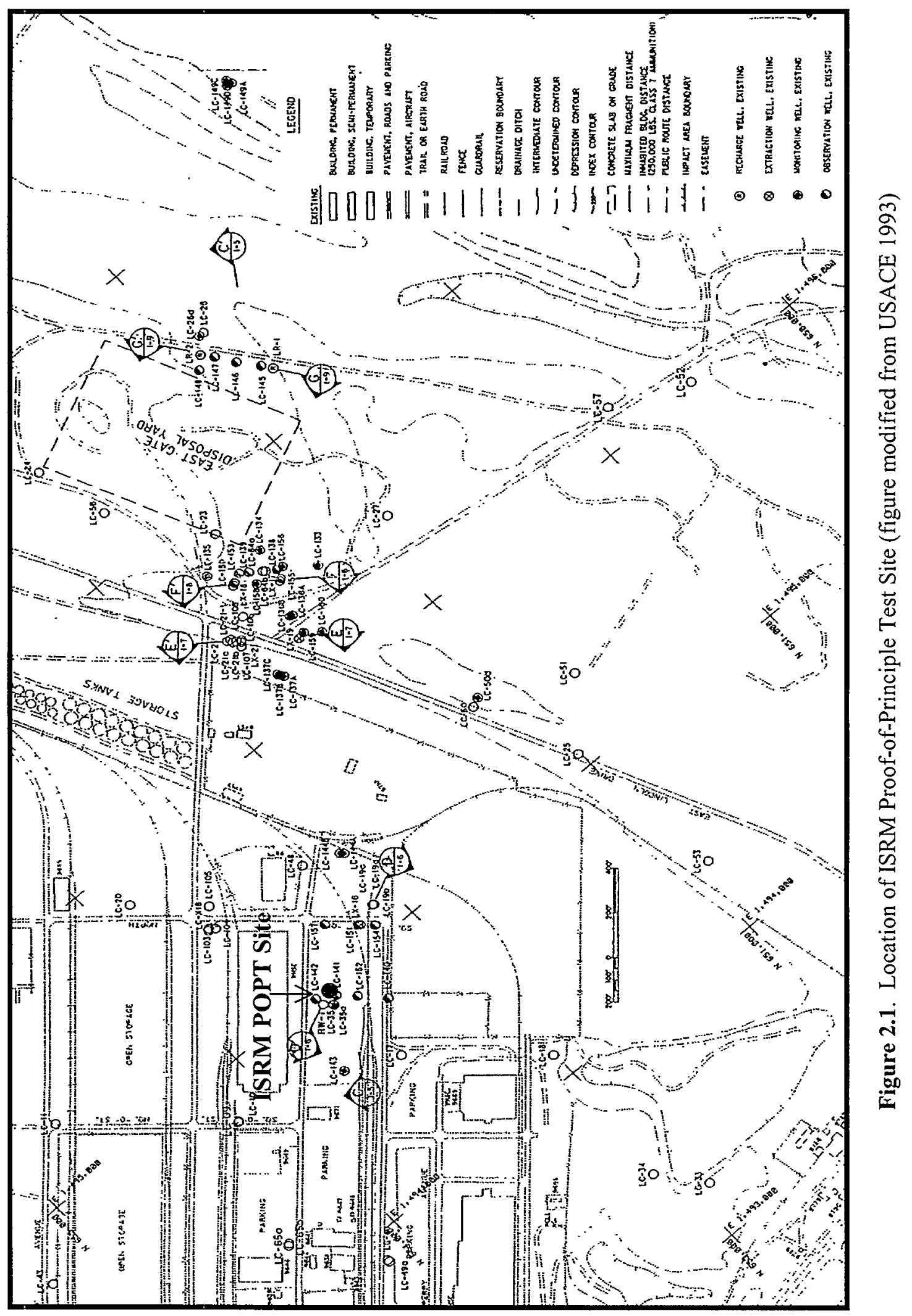


through the injection well. During the injection, dithionite arrival was monitored at all wells to determine the radial extent of the injection. During the withdrawal phase, concentrations were monitored to determine residuals, reaction products, and the percentage of injected mass recovered.

- Sediment Core Sample. Following creation of the ISRM treatment zone, core holes were drilled and sediment samples collected from within the reduced zone. Samples were collected and transported to the laboratory under an anoxic environment where they were analyzed for reductive capacity and percentage of iron reduced. This information was used to estimate the reduction efficiency achieved with the injection and the anticipated life expectancy of the treatment zone.

- Performance Assessment Groundwater Monitoring. After the ISRM treatment zone was created, groundwater samples were collected from all monitoring wells at the POPT site and compared with baseline levels to assess the performance of the ISRM treatment zone. 


\subsection{Site Selection}

Several locations within the Fort Lewis Logistics Center were considered as potential sites for the ISRM POPT. During the site selection phase, various regulatory, cost, hydrogeologic, geochemical, and site access criteria were developed to provide a framework for selecting a suitable demonstration site. Based on these criteria and input from the Fort Lewis IRP Technical Working Group, a site near the East Gate secondary extraction well RW-1 (see Figure 2.1) was selected to demonstrate the technology.

The following sections contain a brief description of the site selection criteria and a discussion of their application to siting the ISRM POPT at Fort Lewis.

\subsection{Regulatory Criteria}

The participation of representatives from regulatory and stakeholder groups during the planning and design phases of a technology field demonstration will help guide the selection of a demonstration site that has administrative support from the agencies that control activities on the site. This would be the case for sites with contamination problems that might benefit from the technology being developed. In turn, those managing the site must be convinced that there will be minimal adverse impact to the site, especially if it could preclude future remediation options.

To encourage the development of cleanup alternatives, EPA established treatability studies in the CERCLA legislation that provide performance and cost data to aid in remedy selection and design. Treatability studies are performed to provide site-specific testing of innovative technologies for which general data are not readily available. Formal permitting of a cleanup activity is unnecessary for a treatability study, although the responsible administrative parties for the site must be agreeable to the designation. Treatability studies are a desirable alternative to formal permits because of the simplified approval procedure. For this reason, a site where a CERCLA treatability study is appropriate and desirable is required. Regulatory acceptance for the ISRM POPT at Fort Lewis was gained through participation in the IRP technical working group.

\subsection{Well Installation Costs}

A significant cost associated with a field-scale ISRM demonstration is well installation. The selected site should have features that minimize both the number of wells that must be installed and the installation cost per well/core hole. Sites with existing wells that can be used as monitoring wells are especially desirable, not only for the savings in installation costs but also for the geologic and hydrologic characterization information accompanying these wells.

The selected ISRM POPT site was near a large number of existing wells. Although none-of these wells could be used to monitor the test due either to their location or screen interval depth, they did provide information on the hydrogeology and groundwater chemistry of the site. Based on various cross-sections presented in Shannon \& Wilson (1986), Ebasco (1988), and USACE $(1992,1993)$, the 
transmissive portion of the upper aquifer in this area was expected to be thinner and shallower than at other proposed locations. These hydrogeologic conditions would minimize the drilling and sampling costs associated with well installation.

\subsection{Hydrogeologic Criteria}

The primary objective of the ISRM POPT was to demonstrate that the ISRM technology could be used to treat dissolved TCE at the Fort Lewis site. Because there are many factors that can complicate interpretation of data obtained from the field test, it is not desirable to site the POPT in an area that is hydrogeologically complex. Hydrogeologic conditions at the selected test site affect the ability to control and monitor the reagent injection and assess the performance of the emplaced ISRM treatment zone. Sites with relatively slow groundwater velocities (less than $2 \mathrm{ft} /$ day) in relatively thin aquifers (less than $30 \mathrm{ft}$ thick) afford a higher degree of control over the extent of transport.

As discussed in Section 3.2, previous hydrogeologic characterization near the selected ISRM POPT site indicated that the transmissive portion of the upper aquifer in this area was relatively thin and well defined, which minimized the costs associated with treatment zone emplacement (e.g., well installation, sampling complexity, chemical costs).

Groundwater velocity requirements can be estimated from the design width of the emplaced treatment zone and the TCE reduction reaction half-life. Velocity requirements must be met to ensure that the migrating TCE plume will not move through the reduced zone too quickly for completion of the TCE degradation process. Higher-velocity zones can be accommodated by making the treatment zone wider or more reduced.

For a POPT, however, it is desirable to select a region with velocities low enough that a smaller treatment zone can be emplaced, thus minimizing cost. Based on a treatment zone design width of $50 \mathrm{ft}$ and a maximum TCE concentration of $200 \mathrm{ppb}$ near the demonstration site, the maximum contaminant velocity that would still result in the desired 5-ppb output concentration was calculated to be approximately $4 \mathrm{ft} /$ day. A review of existing hydrogeologic data from the selected site indicated that groundwater and contaminant velocity did not exceed this limit.

\subsection{Geochemical Criteria}

The permeable treatment zone is created by reducing Fe(III) to Fe(II) within the aquifer sediments. Naturally occurring Fe(III) content should be at least $0.05 \%$ of the total soil fraction. The selected site must meet this criterion. No sediment core samples were available for analysis from any of the proposed ISRM POPT locations; however, analysis of outwash gravel samples obtained by the USGS during the installation of monitoring wells at other locations within the Logistics Center indicated that adequate iron was present within the formation to support the ISRM technology (see Section 4.4 for detailed information on iron content at the POPT site).

Because of the costs associated with drilling, characterizing, and conducting a technology demonstration in a highly contaminated aquifer, it is desirable to locate the test site in an area with moderate levels 
of contamination. Levels should be sufficient to assess the performance of the emplaced treatment zone adequately. In addition, efforts should be made to ensure that the presence of constituents other than the contaminants of concern (e.g., TCE/DCE) do not negatively impact emplacement or performance assessment of the treatment zone. The site that was selected for the ISRM POPT is located within an area of sufficient TCE contamination $(\sim 140 \mathrm{ppb})$ to assess the performance of the emplaced treatment zone while limiting the cost and health and safety issues associated with working at a highly contaminated site.

\subsection{Access Criteria}

These criteria include not only unencumbered access for personnel, vehicles, and heavy equipment but also access to site utilities (e.g., electrical power, water, wastewater disposal) and verification that ISRM POPT activities would not interfere with any ongoing or planned Logistics Center activities.

Because the selected ISRM POPT site was located within a Defense Reutilization and Marketing Office (DRMO) property storage yard, initially the unencumbered access for personnel and vehicles criterion was not met. However, negotiations with DRMO management resulted in an acceptable solution: an interior portion of the storage yard was fenced off, and a fenced corridor with locking gate provided access to the site. Site utilities, including electrical power from a nearby extraction well, water from a nearby fire hydrant, and wastewater disposal to the Fort Lewis sanitary sewer, were all readily available at the selected ISRM POPT site, and no significant impact to Logistics Center activities was identified. However, to accommodate the test, a modification was made to existing pump-and-treat operations. The East Gate secondary extraction wells (RW-1 and LX-16) were deactivated and placed on standby on July 23, 1998. PNNL analysis indicated that the East Gate secondary well field had fulfilled its design function as outlined in the Phase II Design Report (USACE 1993). Therefore, the shutdown would not impact cleanup design goals. 


\subsection{Site Characterization}

This section contains a description of the hydrogeology of the Fort Lewis ISRM POPT site, a description of the wells installed at the site, and a discussion of the geologic, geochemical, and hydrologic characterization activities conducted at the site. For further details of the geological setting, see Appendix C.

\subsection{Hydrogeology of the ISRM Site}

Twenty-one recently drilled boreholes and two pre-existing wells (see Figure 4.1) were used to define the late-Pleistocene stratigraphy of the site (Bjornstad and Vermeul 2000). The high-density borehole network encompasses a small area (about 30 by $120 \mathrm{ft}$ ), elongated in a northwest-southeast direction. Two hydrogeologic cross-sections, showing the subsurface stratigraphic relationships between units, are presented in Figures 4.2 and 4.3.

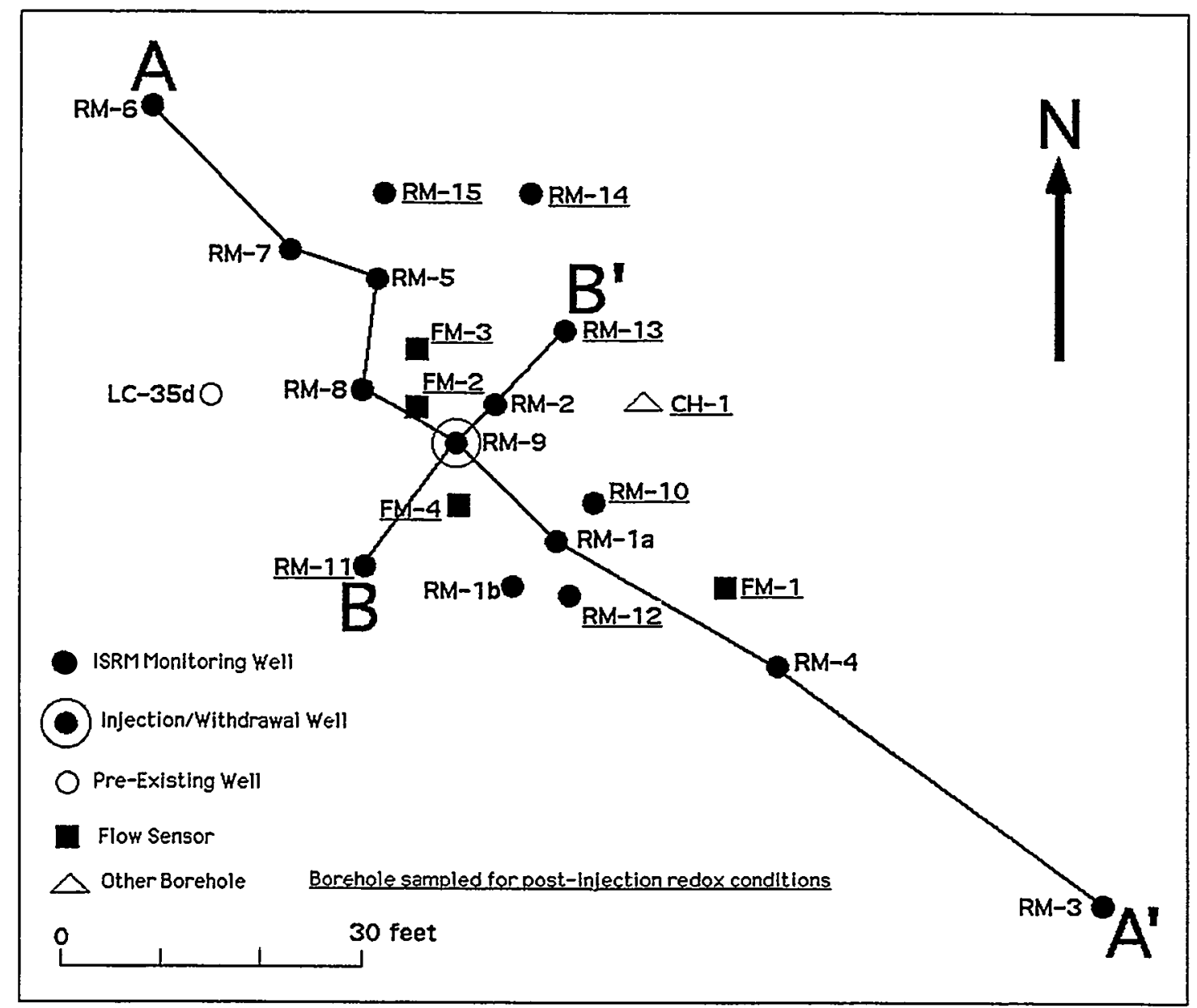

Figure 4.1. Borehole Locations 


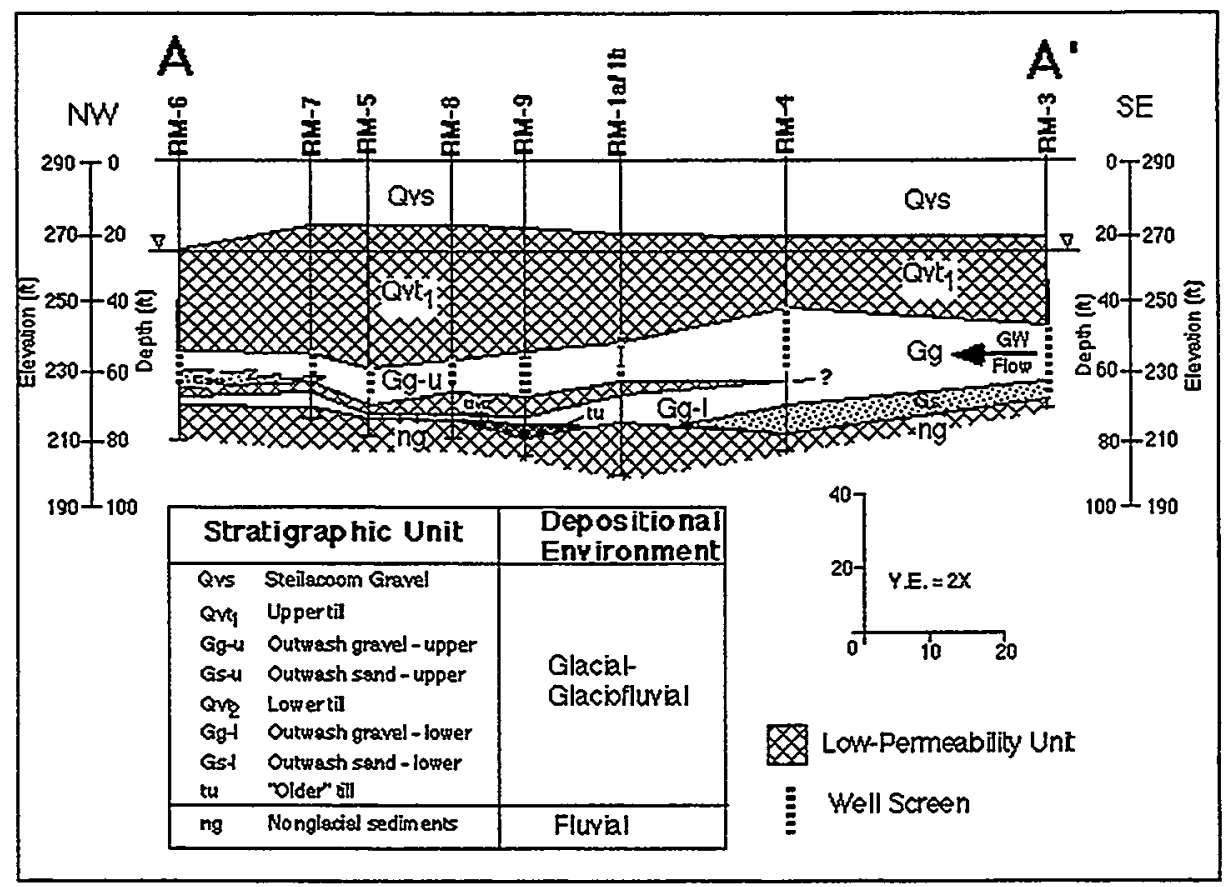

Figure 4.2. NW-SE Hydrogeologic Cross-Section at the ISRM Site (see Figure 4.1 for location of cross-section)

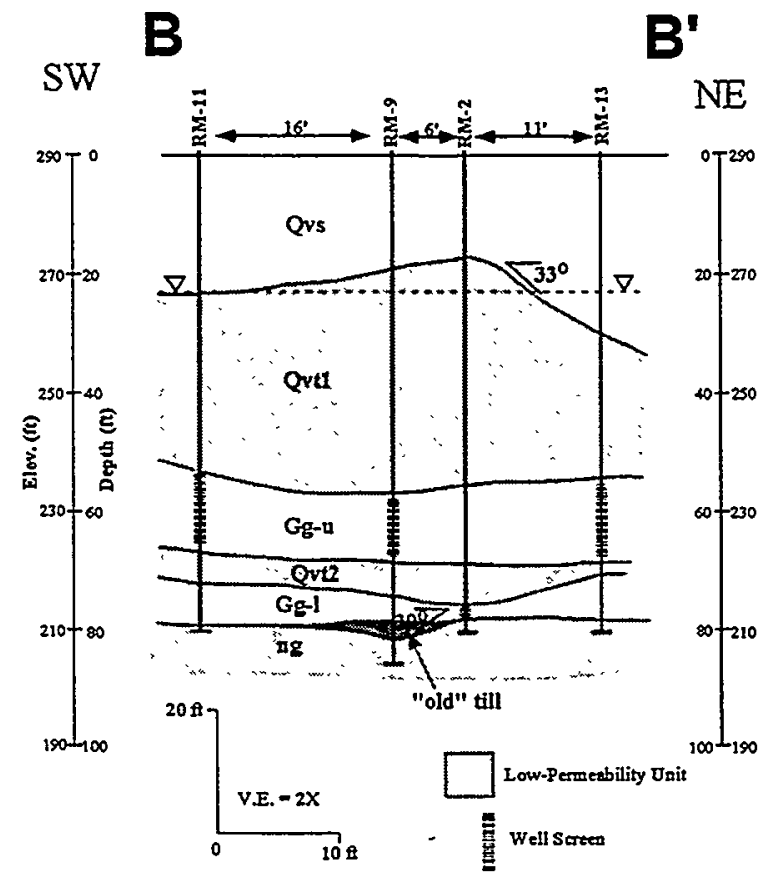

Figure 4.3. NE-SW Hydrogeologic Cross-Section at the ISRM Site (see Figure 4.1 for location of cross-section) 
The surface of the ISRM site is essentially flat and $290 \mathrm{ft}$ above mean sea level. Glacial till and outwash, deposited during the last glaciation (Vashon), extend to a depth of approximately 70-80 feet below the surface; below this is a distinctive sequence of nonglacial fluvial silt and sand. Surface facilities on the site rest on a thick bed (several tens of feet) of outburst flood gravels (Steilacoom Gravel) left behind during the last deglaciation (Figure C.1).

The stratigraphy and lithology of the ISRM site (Figures 4.1 to 4.3 ) are based on logging and sampling of the 21 new boreholes (Figure 4.1) drilled since 1998. Well completion summaries and asbuilt diagrams for these wells, which show the detailed stratigraphy and lithology of the late-Pleistocene sediments, are presented in Appendix C. Most of the boreholes were advanced through the Vashon Drift and into the underlying non-glacial deposits (ng); this contact lies at a depth of 69 to $81 \mathrm{ft}$ below ground surface. Vashon Drift deposits within the study area include the Steilcoom Gravel (Qvs), an upper till (Qvt1), an upper outwash gravel (Gg-u), a lower till (Qvt2), a lower outwash gravel (Gg-1) and/or sand (Gs-1), and an "older" till.

\subsubsection{Steilacoom Gravel}

The Steilacoom gravel blankets the ISRM site and is characterized by consistently coarse, clastsupported gravel containing well-rounded pebbles and cobbles in a sand matrix. Wet colors range from dark gray to dark grayish brown. The upper 1 to $2 \mathrm{ft}$ is stained dark brown to black due to near-surface weathering and high concentrations of decomposed organic matter. Locally, boulders up to 1.5 feet in diameter occur; however, most clasts do not exceed 3 inches in diameter (Woodward-Clyde 1997).

The gently sloping outburst flood plain formed by the Steilacoom Gravel is broken by irregularities produced by the intersections of braided stream channels and depressions of irregular shape (see Figure 4.2). The lowermost portion of Steilacoom Gravel may actually be equivalent to recessional outwash associated with the retreat of the last glaciation, as described in Woodward-Clyde (1997). However, because the texture of the recessional outwash is so much like that of the Steilacoom Gravel and because no difference was detectable in the highly disturbed sonic drill cuttings, no attempt was made to distinguish recessional outwash unit from the Steilacoom Gravel. For this reason, any recessional outwash present is combined with the Steilacoom Gravel unit in this report.

The Steilacoom Gravel mantles the underlying upper till unit. Under the ISRM site the Steilacoom Gravel thins to as little as 17 feet over a northwest trending buried ridge of till. The gravels rapidly thicken radially away from this ridge, where they are up to 30 feet thick.

\subsubsection{Upper Till $\left(Q^{2} t_{1}\right)$}

The upper till is typically a gray, well-graded gravel in a matrix of sand, silt, and clay. The contact with the overlying Steilacoom Gravels was identified primarily through a color change (brown to gray) accompanied by an apparent increase in silt/clay content and decrease in sorting (i.e., increase in grading). While the till contains abundant gravel-size clasts similar to the Steilacoom Gravels, the matrix, as observed in core samples, is predominantly silt. Often the till contains lenses of sandy gravel with little or no silt present. These probably represent deposition within meltwater channels beneath the glacier. 
The upper till is not very dense and therefore probably represents an ablation till. Apparently, due to the higher concentration of fines and relatively low density, the matrix material in core samples from the upper till had a "runny" consistency. This is attributed to the sonic drilling method, which vibrates the saturated sediments intensely, leading to liquefaction of the fine-grained matrix. The top of the till forms a northwest-trending ridge, centered over Wells RM-7 and -8, with up to 13 feet of vertical relief beneath the ISRM site and a range in thickness from 20 to 41 feet.

\subsubsection{Upper Outwash Gravel (Gg-u) and Sand (Gs-u)}

Below the upper till is the upper outwash gravel (Gg-u) unit, which consists of a clast-supported, loose, grayish-brown, pebble-cobble gravel within a sandy matrix. The mean grain size ranges from granule to medium pebble. The upper outwash gravel is differentiated from the overlying and underlying till layers by a matrix that is predominantly sand and by its unconsolidated nature. A well-sorted, grayish-brown, medium-grained sand (Gs-u) occurs at the base of the upper outwash gravel in the northern portion of the ISRM site, at wells RM-6 and RM-7 (Figure 4.2). The upper outwash gravel/sand unit was chosen as the targeted treatment interval for the ISRM POPT.

The top of the outwash gravel and sand unit dips steeply to the west-southwest in the vicinity of the ISRM site. It is up to 15 feet lower on the west end than the east. The base of the outwash gravel and sand unit forms another ridge-like feature similar to that seen between the Steilacoom Gravel and upper till units. The thickness of the unit thins to less than 10 feet along this buried ridge and thickens away from it.

\subsubsection{Lower Till $\left(\mathrm{Qvt}_{2}\right)$}

The lower till unit is thinner and discontinuous across the site area. Where the lower till pinches out to the southeast, the upper and lower outwash units are in direct contact with each other and therefore cannot be differentiated. In general, the lower till is much more dense than the upper till and probably represents a lodgement till. A distinctive brown sandy silt layer, only a few inches thick, caps the lower till in two of the boreholes (RM-2 and RM-4).

The lower till unit also appears to form a northwest-trending ridge up to $7 \mathrm{ft}$ thick at well $\mathrm{RM}-2$. The unit thins away from this point and is missing in the area southeast of well RM-4. Where present, the base of the lower till unit dips radially inward toward Well RM-8.

\subsubsection{Lower Outwash Gravel (Gg-l) and Sand (Gs-l)}

Where the lower till is present, a lower sequence of outwash gravel and/or sand can be differentiated. In core samples, the lower outwash gravel is similar to the upper outwash gravel. The Gg-l unit consists of clast-supported, loose, grayish-brown, pebble-cobble gravel within a sandy matrix; the Gs-1 unit consists of well-sorted, grayish-brown, medium-grained sand. This sand unit would often heave up inside the casing during drilling, indicating that the lower outwash sand is very loose and unconsolidated. 
The lower outwash gravel and sand unit thickens to the east and south. The unit ranges from 1.2 to $15 \mathrm{ft}$ thick where the upper defining unit (Qvt2) is present. The base of the lower outwash gravel and sand unit, which lies in contact with the nonglacial deposits, dips steeply (up to $40^{\circ}$ ) to the southwest beneath the ISRM site (see Figure 4.3).

\subsection{6 “Older" Till (tu)}

An "older" lodgement till was encountered in two adjacent borings (RM-9 and FM-4) along the upper contact with the non-glacial sediments (see Figures 4.2 and 4.3) at an elevation of 210 to $220 \mathrm{ft}$. In RM-9 it consists of four feet of weathered, brown, massive, extremely compact and dense matrix-supported gravelly sand, silt, and clay. The density and color suggest it is much older and has undergone much more weathering and compaction than the younger, overlying tills. This unit may be equivalent to the "undifferentiated till" (tu) identified in previous publications (USACE 1993; Ebasco 1988, 1993; Woodward-Clyde 1997), where it has been reported at elevations below $240 \mathrm{ft}$.

\subsubsection{Nonglacial Deposits (ng)}

Nonglacial deposits underlying outwash gravel, outwash sand, or "older" till are characterized by stratified alluvial sand and silt; the upper contact lies at an elevation of 210 to $220 \mathrm{ft}$. The nonglacial deposits at the ISRM site are equivalent to the Olympia Beds of Troost (2000) and the Qpv unit of Logan et al. (2000). This unit is easily differentiated from the overlying glacial deposits primarily by its color, texture, and grading. Specifically, nonglacial deposits are distinguished by a predominance of volcanic sand grains, paleosols, purplish to pink hues, and presence of peat, tephra, wood, and mudflows (Troost et al. 1998). Unlike the overlying deposits, no gravel-sized clasts ( $>2 \mathrm{~mm}$ ) were encountered in the nonglacial sediments. At the ISRM site, nonglacial deposits are typically more compact and display internal sedimentary structures (e.g., grading, cross bedding, etc.) and evidence for weak soil development, including bioturbation and horizonation. Colors in the core often have a distinctive blue or olive cast, indicating a chemically reduced state. Exceptions occurred near the contacts with more transmissive units (e.g., overlying outwash), where the nonglacial deposits were often oxidized brown to yellowish brown. Sedimentary textures consist of mixtures of poorly graded sand and silt, which often fine upward. The mean grain size is very fine sand.

The upper surface of the nonglacial deposits dips steeply (up to $40^{\circ}$ ) to the southwest in the immediate vicinity of the ISRM site. The base of the nonglacial deposits was not encountered in any of the new boreholes but may be up to $60 \mathrm{ft}$ thick, based on a previous study (USACE 1993).

\subsubsection{Discussion}

Because of the distinctive nature of the nonglacial sediments, this stratigraphic unit is easy to identify, is continuous beneath the study area, and forms a good control bed from which to perform stratigraphic correlation. However, considerable uncertainty remains about the exact relationship between the complex assemblage of Vashon-stage sediments (interstratified glacial tills and outwash gravels/sands) overlying the nonglacial strata despite the high borehole density. Criteria used to differentiate glacial tills from outwash gravel include the amount of silt in the matrix, matrix color, and sorting. Generally, tills have a 
higher silt content, are gray in color, and more poorly sorted than outwash gravel, which is interpreted to have a higher percentage of sand, appears in shades of brown, and is moderately sorted. Perhaps the percentage of fines and sorting are an artifact related to different vibrations or some other variable related to sonic drilling. The alternative explanation is that the heterogeneity is inherent to the formation, which would require high-relief erosional "scour and fill" features and/or rapid lateral facies changes to account for the variability observed in these glacial deposits.

If the variations in particle size, color, and sorting are real and not drilling-induced, at least two cycles of Vashon-stage glacial advance and retreat are represented; however, there could be more. This is demonstrated in the summary geologic logs (Appendix C) where glacial outwash and till are repeatedly interstratified (e.g., RM-14). Correlation of the individual subunits within the Vashon sequence is often difficult, and choosing contacts between subunits appears to be somewhat arbitrary. Based on our interpretation, there appear to be at least three till units. Two (Qvt2 and "older" till) out of three till units are discontinuous across the study area, which is only $120 \mathrm{ft}$ long.

\subsubsection{Hydrology of the ISRM Site}

The leaky confined aquifer beneath the ISRM site lies at an elevation of approximately $235 \mathrm{ft}$, about 55 feet below ground surface (see Figures 4.2 and 4.3). Most of the vadose zone is composed of Steilacoom Gravel. The regional (logistics center scale) groundwater flow direction is to the northwest (Figure 4.4). A discussion of efforts to quantify the local-scale groundwater flow rate and direction is contained in Section 4.8. The potentiometric surface at the ISRM site generally lies within the upper till unit. As discussed in the previous sections, the hydrostatigraphy is complex due to the diverse nature of the sediments and highly variable lateral and vertical extent of lithologic units. Groundwater flow is concentrated in the higher permeability, coarse-grained facies. Beneath the ISRM site, these facies exist below the upper till within the underlying glacial outwash sand and gravel (Figures 4.2 and 4.3). Hydrologic characterization of the outwash gravel unit is discussed in Section 4.6. The outwash sand (Gs) and gravel (Gg) are separated into a lower and upper unit by an intervening lower till (Qvt2). The lower till pinches out to the southeast where the upper and lower outwash units are undifferentiated. Aquifer tests and responses observed during the tracer and dithionite injection tests suggest hydraulic communication exists between the upper and lower outwash units (see Sections 4.6 and 7.3). The upper outwash gravel unit was chosen as the targeted treatment interval for the ISRM POPT.

\subsection{Well Installation}

This section describes the field activities associated with installation and sampling of one injection well and 15 monitoring wells (Figure 4.1). Monitoring wells installed at the site included 12 wells completed in the upper outwash gravel and sand unit (Gg/Gs-u) that comprises the targeted treatment zone, two wells completed in the lower outwash gravel and sand unit (Gg/Gs-l), and one well completed across the bottom of the upper till unit (Qvt1). A well completion summary is included in Table 4.1. These wells were used to support hydrogeologic characterization, design, emplacement, and performance assessment activities associated with the ISRM POPT field demonstration. Wells were installed in three separate drilling campaigns, one before injection testing activities began, one after the first dithionite 


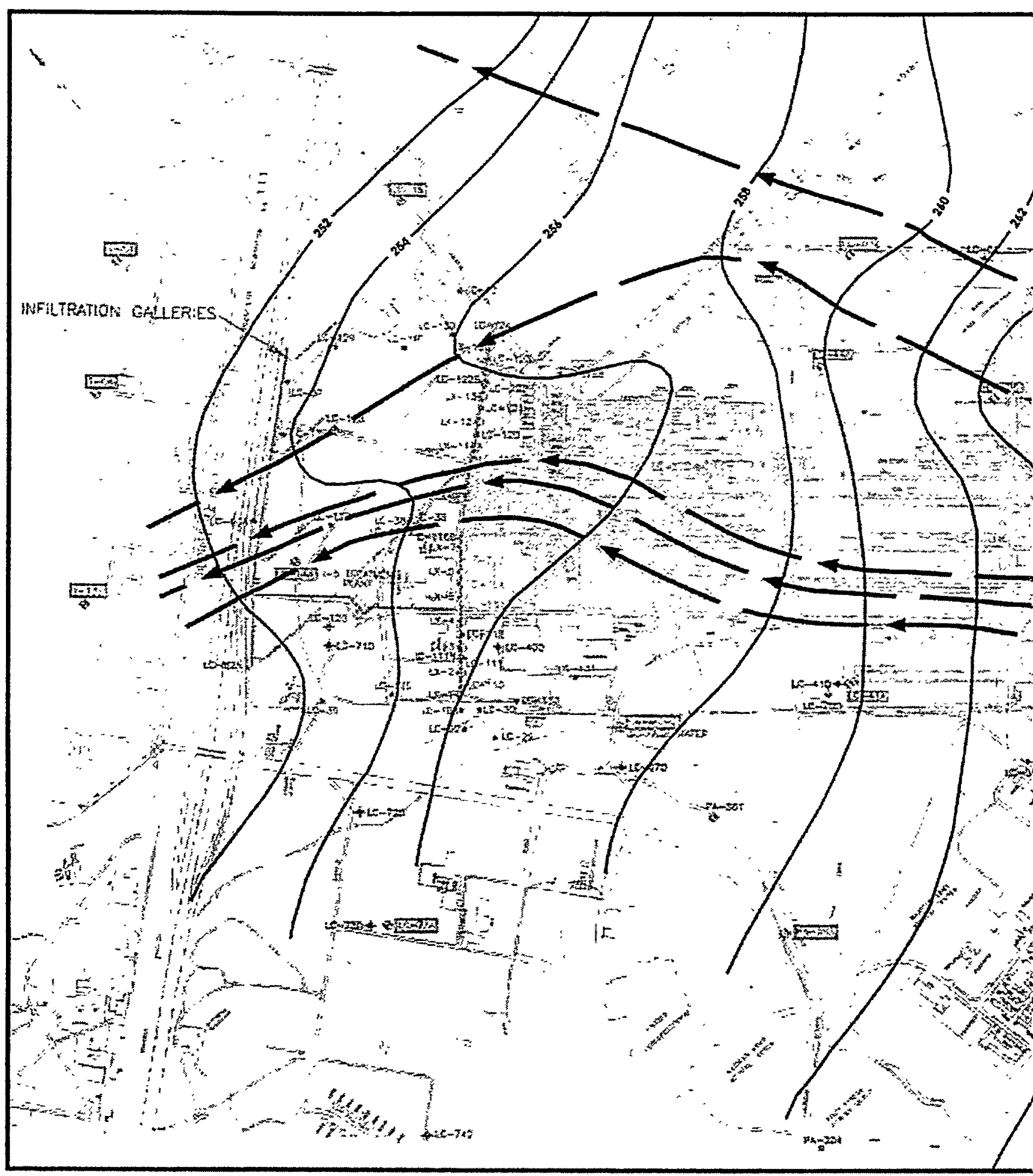

Figure 4.4. Water Table Map in the Vicinity of the ISRM Site in Febru 
Table 4.1. ISRM Well Completion Summary Information

\begin{tabular}{|l|c|c|c|c|}
\hline \multicolumn{1}{|c|}{ Well ID } & $\begin{array}{c}\text { Radial Dist. from } \\
\text { Injection Well (ft) }\end{array}$ & $\begin{array}{c}\text { Screened } \\
\text { Interval (ft bgs) }\end{array}$ & $\begin{array}{c}\text { Stratigraphic } \\
\text { Unit Screened }\end{array}$ & Well Diameter \\
\hline RM-1a & 14.8 & $60.2-64.2$ & Gg-u & 4 \\
\hline RM-1b & 15.5 & $49.2-53.2$ & Qvt1/Gg-u & 4 \\
\hline RM-2 & 5.5 & $75.5-77.0$ & Gg-l & 4 \\
\hline RM-3 & 80.2 & $49.6-68.6$ & Gg/Gs & 4 \\
\hline RM-4 & 40.2 & $43.5-62.5$ & Gg-u & 4 \\
\hline RM-5 & 17.5 & $61.3-70.3$ & Gg-u & 4 \\
\hline RM-6 & 44.2 & $56.3-65.3$ & Gg-u & 4 \\
\hline RM-7 & 24.2 & $53.6-62.6$ & Gg-u & 4 \\
\hline RM-8 & 10.2 & $58.6-65.1$ & Gg-u & 4 \\
\hline RM-9 & $--\cdots-----$ & $57.6-66.9$ & Gg-u & 8 \\
\hline RM-10 & 15.2 & $54.4-58.8$ & Gg-u & 4 \\
\hline RM-11 & 15.1 & $53.0-64.9$ & Gg-u & 4 \\
\hline RM-12 & 20.3 & $70.2-79.6$ & Gg-1 & 4 \\
\hline RM-13 & 15.0 & $55.5-67.4$ & Gg-u & 4 \\
\hline RM-14 & 18.6 & $62.0-71.5$ & Gg-u & 2 \\
\hline RM-15 & 25.7 & $57.1-69.2$ & Gg-u & 2 \\
\hline
\end{tabular}

injection, and one after the final dithionite injection at the site. In addition to the 16 wells installed at the site, five additional boreholes were drilled to collect sediment core samples and install four in situ groundwater velocity sensors.

\subsubsection{Drilling}

As discussed above, 15 monitoring wells and one injection well were installed. For 13 of the monitoring well installations, an 8-in. borehole was advanced to total depth and completed with 4-in. PVC casing and screen. For two of the monitoring well installations, a 6-in. borehole was advanced to total depth and completed with 2-in. PVC casing and screen; and for the injection well installation, a 12-in. borehole was advanced to total depth and completed with 8-in. PVC casing and screen. All screen material consisted of 20-slot continuous wire wrap ( $v$-wire) screen. Table 4.1 contains well construction information for the 16 wells installed at the site, including well diameter, radial distance from the injection well, stratigraphic unit screened, and screen interval depth. Detailed well construction information is contained in the well construction summaries and as-built diagrams contained in Appendix $\mathrm{C}$. Installation and completion of these wells was conducted in accordance with Washington Administrative Code Standards ("Minimum Standards for Construction and Maintenance of Wells," Chapter 173-160). 
Sonic drilling was selected for hydrogeologic characterization and well installation at the ISRM field test site due to the following several requirements of the demonstrated technology:

- The selected method must be capable of drilling a straight borehole. Wells and/or coreholes were drilled close to previously drilled wells.

- The selected method must not introduce large volumes of air into the formation (e.g., air rotary) that could significantly alter the redox condition of collected samples. Because the ISRM technology involves redox processes, measurement of the aquifer's baseline and manipulated redox condition is an important objective.

- The selected method must be capable of a relatively fast penetration rate to minimize costs associated with PNNL field monitoring, analysis, and oversight.

In accordance with the Site-Specific Health and Safety Plan, organic vapors were monitored with a photoionization detector as a precautionary measure. No results obtained from subsurface drill cuttings, water samples, or sampling equipment were above background levels. Drill cuttings were contained in 55-gallon drums, as directed by the Fort Lewis Environmental Point of Contact. All derived waste was disposed of in accordance with the Investigation Derived Waste Management Plan. Drilling was conducted in three separate campaigns, discussed below.

\section{Campaign \#1}

During the initial drilling campaign (August 1998), designed to provide site-specific characterization information and the initial well network needed to monitor the ISRM injection tests, one injection well (RM-9) and nine monitoring wells (RM-1a/b through RM-8) were installed at the site. Both sediment and aqueous samples were collected as the boreholes were advanced. Sediment samples were collected in a split-spoon sampler (Section 4.2.2). Aqueous samples were collected within a temporary screen using a portable small-diameter, variable-speed submersible pump (Grundfos RediFlo 2). Aqueous samples were analyzed in an onsite mobile laboratory as they were collected to determine the vertical distribution of contamination and provide the information required to correctly locate the screen interval (Section 4.5).

\section{Campaign \#2}

During the second drilling campaign (May 1999), four additional monitoring wells (RM-10 through RM-13) and two in situ groundwater velocity sensors (HydroTechnics, Inc.) were installed. Sediment samples were collected as the boreholes were advanced. The monitoring wells and flow meters were installed following the first dithionite injection to meet additional geohydrologic characterization and injection monitoring needs identified during the tracer test and first dithionite injection test. 


\section{Campaign \#3}

The primary objective of the final drilling campaign (June 2000) was to collect sediment core samples for measuring reductive capacity. This information was needed to assess the field-scale efficiency of the dithionite injections and to estimate the longevity of the emplaced treatment zone. Samples were collected using anoxic preservation methods and shipped to the laboratory for analysis. Additional information on sample collection and reductive capacity measurements is contained in Section 8.3. To leverage the investment in these post-injection coreholes, two were completed as additional monitoring wells (2-in. screen and casing was used to minimize cost), and two were used to install in situ groundwater velocity sensors. Additional information on groundwater flow velocity and direction is contained in Section 4.8.

\subsubsection{Sediment Sampling}

All 21 new boreholes were drilled and sampled using the resonant sonic drill method. Core samples were obtained by applying a resonating harmonic vibration to a core barrel or split-spoon sampler ahead of the sonically advanced casing. Between core runs, the casing was advanced and cleaned out with a vibrating core barrel. A multitude of sediment samples were collected during drilling of the boreholes prior to well installation. These included over 180 grab samples collected from core-barrel cuttings and retained in clear plastic bags as each hole was advanced. The cuttings were subsampled and placed into labeled Zip-Loc bags approximately every $5 \mathrm{ft}$ or change in lithology. Grab samples were collected from all new boreholes except of RM-1b. RM-1b was only a few feet away from RM-la and considered to be geologically the same as RM-1a.

Intervals of particular interest (e.g., key contacts, injection zone lithology, etc.) were cored with a 5-ft.-long, 4-in.-diameter split-spoon sampler lined with plastic lexan precut into 6-in. lengths. A total of 6016 -in. core segments were geologically logged, capped, and archived. Of these, 21 were submitted for physical property analysis. Analyses performed included particle size, bulk density, and total porosity analysis (see Section 4.3). Another 209 core segments were collected anoxically in a glove box and submitted for geochemical analysis. The results of these analyses are reported in Section 4.4. A complete list of samples, including their location, lithology, and relative stratigraphic position, can be found in Appendix I.

\subsubsection{Well Development}

All site monitoring wells were developed before groundwater sampling began. Well development was conducted in two phases. The first phase consisted of bailing and/or surging, as required, during well completion (i.e., after placing the filter pack but before placing the annular seal) to settle the sandpack and remove fine-grained material generated during drilling. Immediately following well completion, an appropriately sized submersible pump was installed, and the wells were pumped and surged until any remaining fine-grained material was removed from the installation and the well had achieved an acceptable yield and turbidity level. 


\subsection{Geologic Characterization}

Geologic characterization at the ISRM site included geologic logging of all wells and laboratory measurement of particle-size distribution, bulk density, and porosity of core samples from four wells (RM-1, -2, -3, and -4). Most of the analyzed samples were from the upper and lower glacial outwash units, although three samples were from till and two from non-glacial sediments. Changes in the bulk density, porosity, and particle size $(\%<2 \mathrm{~mm})$ with depth are shown, graphically, in Figures 4.5 through 4.8. Limited borehole geophysical logging was also performed as part of geologic characterization.

\subsubsection{Geologic Logging}

A geologist was on site at all times during drilling to supervise and document drilling and sampling activities as well as compile a geologic log for each of the boreholes. Sediments were described in the field using the standard criteria [e.g., Unified Soil Classification System (ASTM 1986) and Last and Liikala (1987)]. Sediment characteristics logged included lithology, particle-size distribution, grading (sorting), mineralogy, wet color, degree of consolidation, cementation, roundness, odor, and structure. A single-page summary log, showing results of the laboratory analytical data along with the field geologic $\log$, is presented in Figures 4.5 through 4.8. A summary geologic log is presented for each of the 21 new boreholes in Appendix C.

\subsubsection{Particle-Size Analyses}

Particle-size analysis via the dry-sieve method was performed on 21 sediment core samples submitted for analysis. Two of the samples that contained $>25 \%$ coarse silt ( 45 micron diameter), were separated further using the hydrometer method. Particle-size statistics, including the $\%<2 \mathrm{~mm}$, median, mean, standard deviation, and skew are arranged by stratigraphic unit in Table 4.2. The results of particle-size analysis indicate most samples are a well-graded (i.e., poorly sorted) mixture of mud, sand, and gravelsize particles, with the exception of the non-glacial unit, which is less well-graded and consists primarily of sand and/or silt.

\subsubsection{Bulk Density}

Dry bulk density was also measured on portions of the 21 sediment core samples submitted for analysis. Bulk density ranged from 1.65 to $2.32 \mathrm{~g} / \mathrm{cm}^{3}$ (Table 4.2). Among the different stratigraphic units, the Qvtl unit shows the most internal variation in bulk density, ranging from 1.68 to $2.29 \mathrm{~g} / \mathrm{cm}^{3}$. The upper outwash gravel unit (Gg-u) has the highest density (average $2.24 \mathrm{~g} / \mathrm{cm}^{3}$ ), while the ng layer is the least dense (average $1.73 \mathrm{~g} / \mathrm{cm}^{3}$ ). Even though tills are often more compacted, a higher bulk density for the outwash gravel is attributed to its higher concentration of pebble and cobble clasts.

\subsubsection{Porosity}

Total porosity was calculated using particle density and dry bulk density measured for each of the core samples. Because porosity is a function of bulk density, there is a direct inverse relationship 


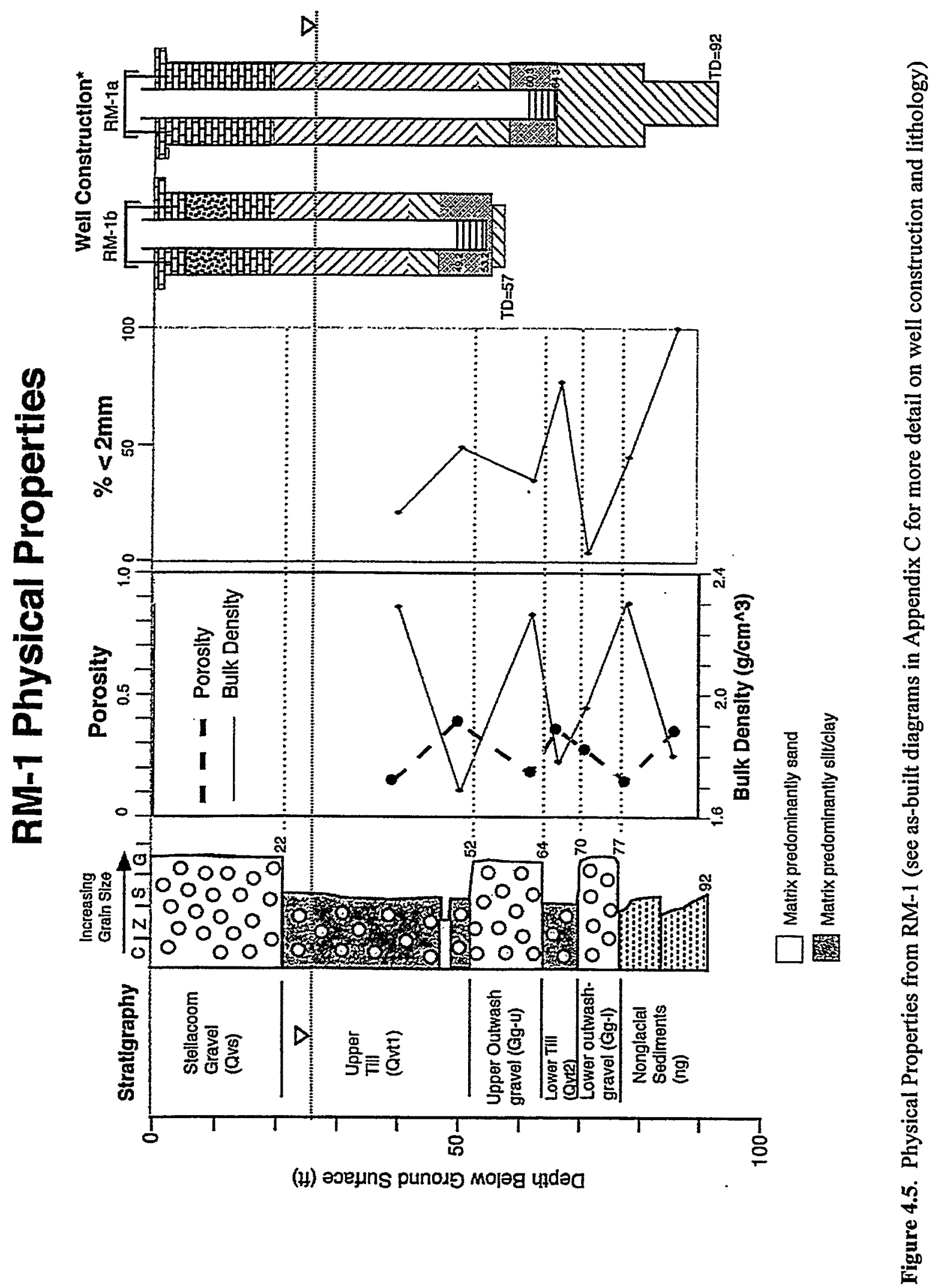


This page cannot be converted.

Please view the native document

for the original page. 


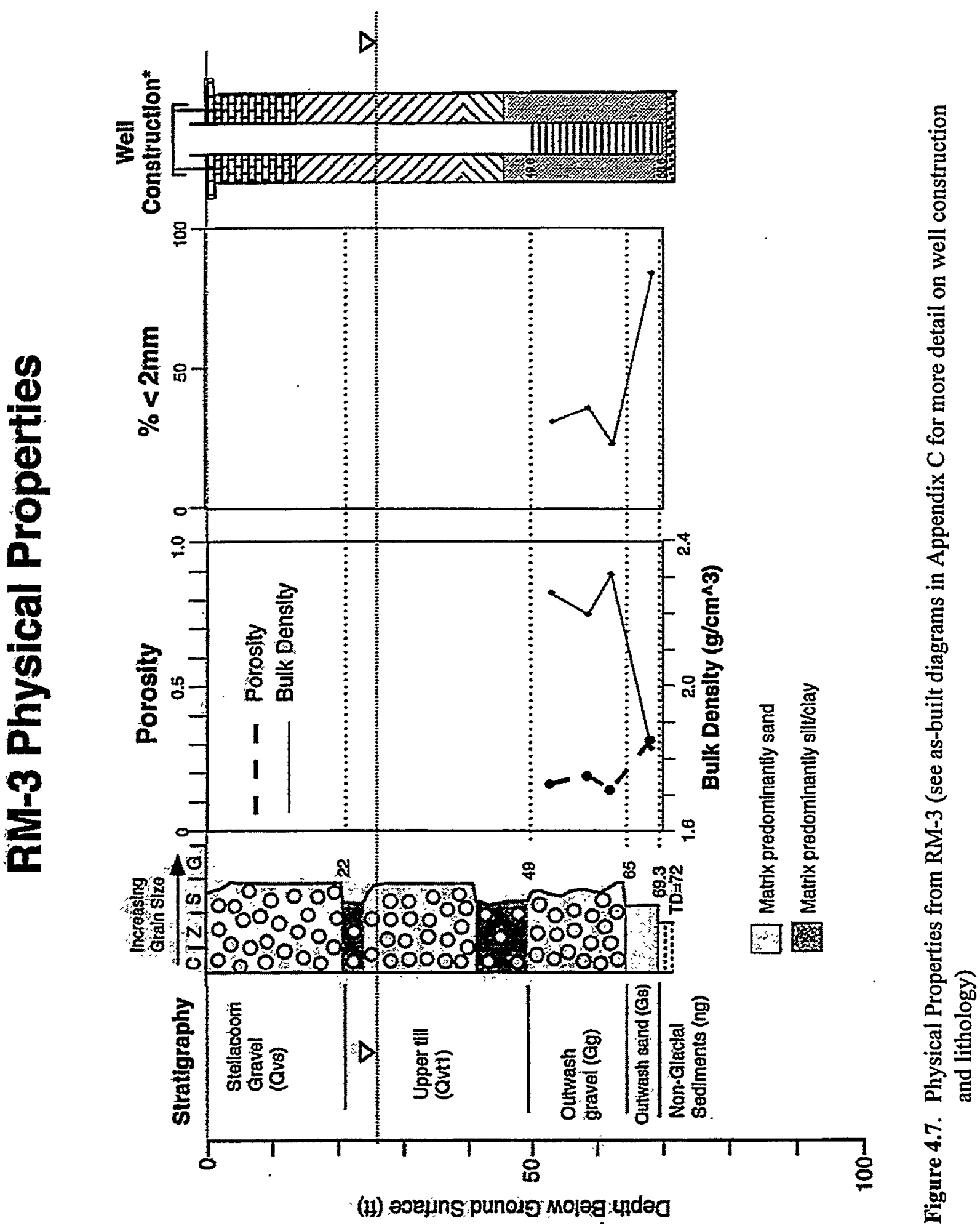




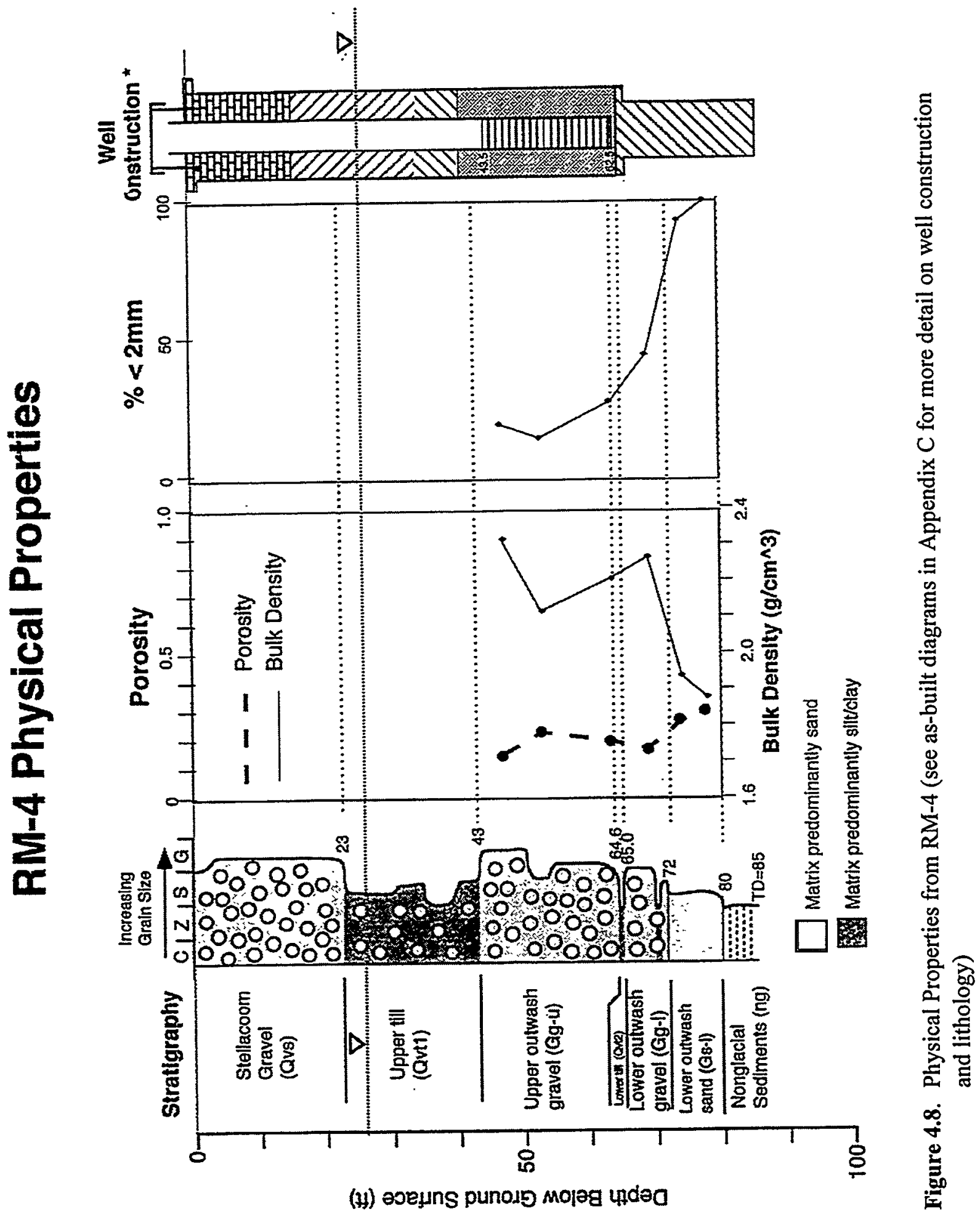


Table 4.2. Bulk Density and Total Porosity Statistics for the Stratigraphic Units

\begin{tabular}{|c|l|c|c|c|c|c|c|}
\hline \multicolumn{9}{|c|}{ Stratigraphic Unit } & & \\
\hline & & Qvt1 & Gg-u & Qvt2 & Gg-l & Gs-1 & ng \\
\hline Bulk Density & \# Samples & 3 & 9 & 1 & 2 & 3 & 2 \\
\hline$\left(\mathrm{g} / \mathrm{cm}^{3}\right)$ & Range & $1.68-2.29$ & $2.12-2.32$ & 1.77 & $1.95-2.27$ & $1.83-1.94$ & $1.65-1.80$ \\
\hline & Average & 2.05 & 2.24 & 1.77 & 2.11 & 2.02 & 1.73 \\
\hline & Std Dev. & 0.33 & 0.07 & NA & 0.23 & 0.06 & 0.11 \\
\hline \multirow{3}{*}{$\begin{array}{c}\text { Total Porosity } \\
(\%)\end{array}$} & \# Samples & 3 & 9 & 1 & 2 & 3 & 2 \\
\cline { 2 - 8 } & Range & $15-39$ & $15-23$ & 36 & $17-28$ & $27-32$ & $35-40$ \\
\cline { 2 - 8 } & Average & 24.7 & 18.4 & 36 & 22.5 & 29.7 & 37.5 \\
\cline { 2 - 8 } & Std Dev. & 12.7 & 2.6 & NA & 7.7 & 2.5 & 3.5 \\
\hline
\end{tabular}

between these two parameters (see Figures 4.5 through 4.8 ). Total porosity ranges from 15 to $40 \%$. The ng layer displays the highest porosity (average $37.5 \%$ ), while the upper outwash gravel unit (Gg-u) displays the lowest porosity (average 18.4\%). The porosity of the upper till unit (Qvt1) is especially variable compared with other stratigraphic units.

\subsubsection{Geophysical Logging}

To assess whether downhole geophysical logs could be used to assist in stratigraphic interpretations and correlations at the ISRM site, geophysical logging was performed in two of the new wells (RM-1 and RM-2) and one of the older wells (LC-35D) (Figure 4.9). Three types of geophysical tools, natural gamma, resistivity, and temperature, were used in one or more of the boreholes.

The natural gamma logs were obtained from RM-1 before well completion within the 8-in. steel casing and after well completion within the 4-in. PVC casing. A natural gamma log was also obtained from two other PVC-cased and completed wells (RM-2 and LC-35D). Electrical resistivity logs were obtained from three PVC-cased and completed wells (RM-1, RM-2 and LC-35D), while a temperature log was obtained from LC-35D only.

The results do not show any consistent trends in the natural gamma or resistivity signals in the glacial drift ( 0 to $80 \mathrm{ft}$ depth). A decrease in the amount of gamma rays detected between 25 to $30 \mathrm{ft}$ is probably related to attenuation associated with the water table. Distinct boundaries observed in the resistivity log appear to conform to changes in the type of annular material used to complete the well rather than stratigraphic or lithologic boundaries.

The temperature log did provide useful information. The water temperature in LC-35D decreases steadily from $\sim 54.5^{\circ} \mathrm{F}$ at the water table ( $26 \mathrm{ft}$ depth) to a low of $\sim 52.7^{\circ} \mathrm{F}$ around the 90 - $\mathrm{ft}$ depth (Figure 4.9). Below $90 \mathrm{ft}$ the water temperature slowly increases. The temperature profile in Figure 4.9 indicates the colder water below $\sim 58 \mathrm{ft}$ has a different recharge source, perhaps from colder meltwater streams coming off the Cascades, than the warmer water above. The water temperature in the injection zone (58 to $72 \mathrm{ft} \mathrm{depth}$ ) at well LC-35d appears to be a near-constant $52.8^{\circ} \mathrm{F}\left(11.5^{\circ} \mathrm{C}\right)$. 


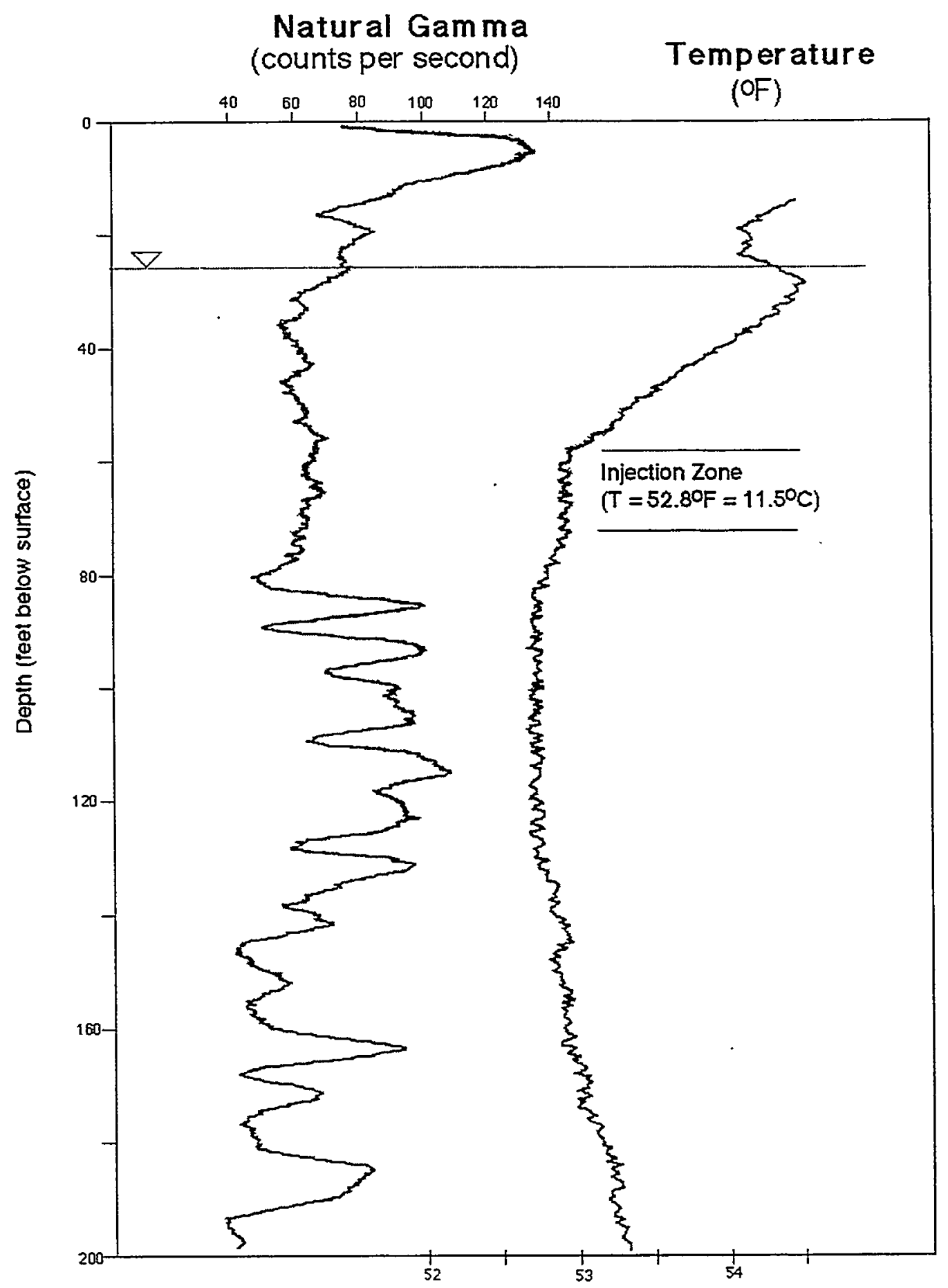

Figure 4.9. Borehole Geophysical Logs from Well LC-35d 


\subsection{Geochemical Characterization}

Laboratory experiments were conducted with Fort Lewis sediment collected from POPT boreholes to determine the spatial variability of the reducible iron mass and reduction/oxidation rates (detailed in Szecsody et al. 2000). Results from 27 column experiments showed that the sediments from the POPT site averaged $62.8 \pm 39.7 \mu \mathrm{mol} / \mathrm{g}(0.351 \pm 0.222 \%)$ of reducible iron. Sediment samples from well RM-9 (POPT site injection well) averaged $49.1 \pm 3.25 \mu \mathrm{mol} / \mathrm{g}(0.274 \%)$ of reducible iron (three experiments). Column experiments were also conducted with sediment samples from six U.S. Geological Survey (USGS) wells located near (but not at) the POPT site. Sediments from the USGS wells averaged $45.6 \pm 33.3 \mu \mathrm{mol} / \mathrm{g}(0.255 \pm 0.186 \%)$ of reducible iron, so the POPT site appeared to average $30 \%$ greater reducible iron than the larger-scale average of aquifer sediments.

The rate of iron reduction was used to design the dithionite injection strategy at the POPT site. This iron reduction rate was determined from laboratory dithionite reduction experiments in batch and column systems. The column reduction experiments averaged $6.82 \pm 2.46$ hours for the reduction half-life (27 experiments) as opposed to 3.1 hours for six batch experiments $\left(25^{\circ} \mathrm{C}\right)$. Reduction in columns is likely slower due to physical access limitations to sites, which does not occur in batch systems.

The applicability of batch and column studies to the field scale depends on accounting for large-scale chemical and physical variability: While column experiments do incorporate some aspects of the field scale such as the advective flow of mobile solutes through the reactive immobile surfaces, these small systems are not representative of all aspects of the field scale. Groundwater systems contain 1) natural physical and chemical heterogeneities, 2) unique flow fields, 3) different temperature, and 4) a wide range of porous media sizes that are not represented in column experiments. Because of the small (1-cmdiameter $\times 10-\mathrm{cm}$-length; up to 10-cm-diameter $\times 50-\mathrm{cm}$-length) size of columns, natural sediment from cores is sieved and repacked so does not incorporate natural heterogeneity patterns (but rather point samples). The chemical spatial variability of the sediment was addressed in this study by statistical variability in experiments with sediments from different boreholes and of different depths. Larger-scale glacial or fluvial features that result in zones of differing hydraulic conductivity and iron content are not incorporated in small-scale laboratory experiments but are integrated into the field-scale POPT test. Therefore, the comparison of the mass of iron reduction with the resulting TCE dechlorination rate (Section 8 ) provides some measure of the influence of heterogeneity.

\subsection{Vertical Contaminant Distribution}

As discussed in Section 4.2, during well installation activities at the ISRM field test site, depth discrete aqueous samples were collected as the boreholes were advanced. Aqueous samples were analyzed as they were collected in an onsite mobile laboratory facility using a Viking SpectraTrak fieldportable gas chromatograph/mass spectrometer (GC/MS). Samples were submitted for analysis immediately after collection so that no preservation was required. Samples were analyzed by a heated headspace method using commercially certified external and internal standards for quantitation. 
Because of limitations inherent in the headspace method used in these analyses, a high level of confidence is not placed in the accuracy of the reported values. However, the method does have acceptable precision and provides a good relative measure of the variability in TCE concentration with depth.

A summary of the vertical TCE concentration data obtained during this investigation is contained in Table 4.3. In general, the data indicate that TCE is relatively constant within the targeted treatment zone (i.e., the upper outwash gravel and sand unit $[\mathrm{Gg} / \mathrm{Gs}-\mathrm{u}]$ ). The data also indicate that the lower outwash gravel and sand unit has an approximately $60 \%$ higher TCE concentration than the targeted treatment zone. Also, the one sample collected from a sand lens within the upper till (Qvtl) indicates concentrations above the targeted zone are approximately $30 \%$ lower than in the targeted treatment zone.

Table 4.3. Vertical TCE Distribution in the Groundwater Beneath the ISRM Test Site

\begin{tabular}{|l|c|c|c|}
\hline Well W & $\begin{array}{c}\text { Depth Interval } \\
(\mathbf{f t})\end{array}$ & $\begin{array}{c}\text { TCE Concentration } \\
(\mu \mathrm{g} / \mathrm{L})\end{array}$ & Stratigraphic Unit \\
\hline RM-1a & $50-55$ & 80 & Gg-u \\
\hline RM-1a & $55-60$ & 81 & Gg-u \\
\hline RM-1a & $60-65$ & 81 & Lg-u \\
\hline RM-1a & $65-70$ & 75 & Qvt2 \\
\hline RM-1a & $70-75$ & 103 & Gg-1 \\
\hline RM-1a & $75-80$ & 135 & Gg-1 \\
\hline RM-2 & $40-45$ & 56 & Qvt1 \\
\hline RM-2 & $55-60$ & 80 & Gg-u \\
\hline RM-2 & $60-65$ & 93 & Gg-u \\
\hline RM-2 & $65-70$ & 82 & Gg-u \\
\hline RM-2 & $75-80$ & 130 & Gg-1 \\
\hline RM-3 & $50-55$ & 78 & Gg \\
\hline RM-3 & $55-60$ & 64 & Gg \\
\hline RM-3 & $60-65$ & 75 & Gg \\
\hline RM-3 & $67-72$ & 93 & \\
\hline
\end{tabular}

The observed vertical concentration profile is consistent with the conceptual model of the site and the physical characteristics of the TCE. The lower concentrations observed in the upper till would be expected at a location distant from the primary source area when natural recharge to the groundwater system is significant. The increasing TCE concentration with depth is also consistent with the physical properties of the contaminant (i.e., TCE is a dense nonaqueous phase liquid [DNAPL]).

\subsection{Baseline Groundwater Chemistry}

Before the ISRM injection tests began at the POPT site, three rounds of groundwater samples were collected from the 10 initially installed wells. Samples were collected on September 30, 1998, October 12, 1998, and November 9, 1998. Field parameters measured at that time included electrical conductivity (EC), temperature, $\mathrm{pH}$, and dissolved oxygen (DO). Laboratory measurements were also 
performed for volatile organics, acetylene, common anions, and trace metals. All three sets of samples were analyzed by headspace GC/MS for volatile organics while only the September 9, 1998, and October 10, 1998 samples were analyzed by the GC purge and trap method. Because there was some disagreement between the two methods, apparently associated with the headspace sample introduction technique, only the purge and trap results are reported here. All samples collected were analyzed for trace metals by induction coupled plasma-optical emission spectrometry (ICP-OES) Method 6010. Ion chromatography measurements were performed on all three sets of samples for fluoride, chloride, nitrate, phosphate, and sulfate. Only the first sweep taken on September 30, 1998 was analyzed for acetylene because, as expected, all measurements were reported as nondetects at a reasonably high level of sensitivity $(0.06 \mathrm{ppb})$. A detailed discussion of analytical methods is contained in Section 5.1.3.

A summary of the field parameter measurements is given in Table 4.4. Groundwater samples taken from all nine wells show a relatively uniform composition with respect to $\mathrm{EC}, \mathrm{DO}$, and $\mathrm{pH}$. $\mathrm{EC}$ measurements were relatively low with a range of at most a factor of 2. DO measurements spanned a somewhat larger range but tended to cluster around approximately $50 \%$ of saturation, while the $\mathrm{pH}$ distribution was relatively narrow with an average value just slightly below neutrality. Table 4.5 summarizes the results of the GC analysis for TCE, DCE, and acetylene prior to the first dithionite injection.

TCE measurements in the two sweeps averaged $136.6 \mathrm{ppb}$ for the 10 wells prior to injection. The DCE measurements average $13.9 \mathrm{ppb}$, approximately 10 times lower. DCE concentrations exhibited greater variability, which is probably related to analytical limitations associated with the lower levels. All samples required a 10-fold dilution to bring the TCE concentration within the linear range for the photoionization detector. The dilution tended to adversely impact the analytical precision for the initially more dilute DCE. All acetylene measurements were recorded as non-detects. Those samples also showed no detectable quantities of ethene, ethane, or chloroacetylene.

Table 4.4. Summary of Pre-Injection Groundwater Geochemical Parameters

\begin{tabular}{|l|c|c|c|c|c|c|}
\hline Well & $\begin{array}{c}\text { Average EC } \\
(\mu \mathbf{S} / \mathbf{c m})\end{array}$ & $\begin{array}{c}\text { Range EC } \\
(\mu \mathbf{S} / \mathbf{c m})\end{array}$ & $\begin{array}{c}\text { Average DO } \\
(\mathbf{m g} / \mathbf{L})\end{array}$ & $\begin{array}{c}\text { Range DO } \\
(\mathbf{m g} / \mathbf{L})\end{array}$ & Average $\mathbf{p H}$ & Range $\mathbf{p H}$ \\
\hline $\mathrm{RM}-1 \mathrm{~A}$ & 131 & 115 to 159 & 4.32 & 4.13 to 4.50 & 6.68 & 6.53 to 6.76 \\
\hline $\mathrm{RM}-1 \mathrm{~B}$ & 163 & 128 to 189 & 2.92 & 1.57 to 4.00 & 6.94 & 6.81 to 7.06 \\
\hline $\mathrm{RM}-2$ & 133 & 106 to 169 & 4.67 & 4.29 to 5.00 & 6.82 & 6.69 to 6.89 \\
\hline $\mathrm{RM}-3$ & 126 & 99 to 161 & 6.77 & 6.01 to 8.00 & 6.61 & 6.33 to 6.76 \\
\hline $\mathrm{RM}-4$ & 126 & 107 to 154 & 5.23 & 4.98 to 5.60 & 6.71 & 6.63 to 6.76 \\
\hline $\mathrm{RM}-5$ & 132 & 109 to 160 & 4.25 & 3.79 to 4.50 & 6.87 & 6.78 to 6.93 \\
\hline $\mathrm{RM}-6$ & 143 & 129 to 135 & 2.51 & 1.39 to 4.00 & 7.16 & 7.11 to 7.14 \\
\hline $\mathrm{RM}-7$ & 128 & 113 to 125 & 5.74 & 5.32 to 5.50 & 6.82 & 6.86 to 6.87 \\
\hline $\mathrm{RM}-8$ & 128 & 114 to 148 & 6.25 & 5.66 to 7.20 & 6.76 & 6.60 to 6.86 \\
\hline $\mathrm{RM}-9$ & 121 & 102 to 146 & 6.02 & 5.63 to 6.90 & 6.77 & 6.61 to 6.82 \\
\hline All Wells & 134 & 99 to 189 & 4.8 & 1.34 to 8.00 & 6.8 & 6.53 to 7.14 \\
\hline
\end{tabular}


Table 4.5. Summary of Pre-Injection TCE, DCE, and Acetylene Concentrations in Groundwater at POPT Site

\begin{tabular}{|l|c|l|c|c|c|}
\hline Well & $\begin{array}{c}\text { Average } \\
\text { TCE } \\
\text { (ppb) }\end{array}$ & $\begin{array}{c}\text { Range TCE } \\
\text { (ppb) }\end{array}$ & $\begin{array}{c}\text { Average } \\
\text { DCE } \\
\text { (ppb) }\end{array}$ & $\begin{array}{c}\text { Range DCE } \\
\text { (ppb) }\end{array}$ & $\begin{array}{c}\text { Acetylene } \\
\text { (ppb) }\end{array}$ \\
\hline $\mathrm{RM}-1 \mathrm{~A}$ & 128 & 121 to 134 & 9.8 & 8.9 to 10.2 & $<0.06$ \\
\hline $\mathrm{RM}-1 \mathrm{~B}$ & 124 & 104 to 142 & 12.3 & 9.2 to 15.3 & $<0.06$ \\
\hline $\mathrm{RM}-2$ & 143 & 133 to 153 & 17.4 & 15.6 to 19.1 & $<0.06$ \\
\hline $\mathrm{RM}-3$ & 120 & 117 to 123 & 11.5 & 8.9 to 14.0 & $<0.06$ \\
\hline $\mathrm{RM}-4$ & 133 & 124 to 142 & 11.9 & 10.4 to 13.3 & $<0.06$ \\
\hline $\mathrm{RM}-5$ & 154 & 148 to 159 & 15.0 & 14.4 to 15.5 & $<0.06$ \\
\hline $\mathrm{RM}-6$ & 129 & 103 to 155 & 19.2 & 15.3 to 23.1 & $<0.06$ \\
\hline $\mathrm{RM}-7$ & 155 & 144 to 165 & 16.1 & 12.9 to 19.2 & $<0.06$ \\
\hline $\mathrm{RM}-8$ & 153 & 141 to 164 & 14.2 & 11.4 to 17.0 & $<0.06$ \\
\hline $\mathrm{RM}-9$ & 124 & 122 to 124 & 9.4 & 9.8 to 10.2 & $<0.06$ \\
\hline All Wells & 136.6 & & 13.9 & & $<0.06$ \\
\hline
\end{tabular}

Table 4.6 summarizes the ion chromatography (IC) measurements made on all three rounds of samples collected from the 10 wells. Common anions were present at relatively low levels in groundwater at the POPT site before the first injection. Chloride and sulfate levels were relatively low $(2.53 \mathrm{mg} / \mathrm{L}$ and $6.56 \mathrm{mg} / \mathrm{L}$, respectively), while fluoride and phosphate were below detection. Nitrate was, however, detectable at nearly uniform levels throughout all 10 wells, averaging $2.16 \mathrm{mg} / \mathrm{L}$. While this is well below the primary federal regulatory limit of $45 \mathrm{mg} / \mathrm{L}$ for nitrate, it may represent a distant contamination source worthy of further investigation. Nitrate contamination of groundwater does not appear to have been addressed by previous environmental investigations at Fort Lewis. They have concentrated on organics (primarily TCE) and, to a lesser extent, trace metals as known or potential contaminants. Nitrate contamination can result from many potential sources, including fertilizers, septic drainage fields discharge, chemicals, and degradation of certain munitions such as TNT. Nitrate levels have remained constant or decreased throughout the project, ensuring that the source is unrelated to the dithionite injections. The differences observed in anion concentrations for well RM- $1 \mathrm{~b}$ relative to other wells sampled are most likely associated with this wells screened internal, which was completed almost entirely in the upper till unit (Qvt1).

Table 4.7 contains summary information for trace element analysis by ICP-OES on all three rounds of samples collected from the 10 wells. The samples were filtered in the field and thus represent a dissolved component only. The trace metals data are generally unremarkable, with only a handful of major and minor cations such as sodium, potassium, calcium, magnesium, strontium, and barium present at detectible levels. Very minor amounts of manganese and iron were also detectable in some samples. 


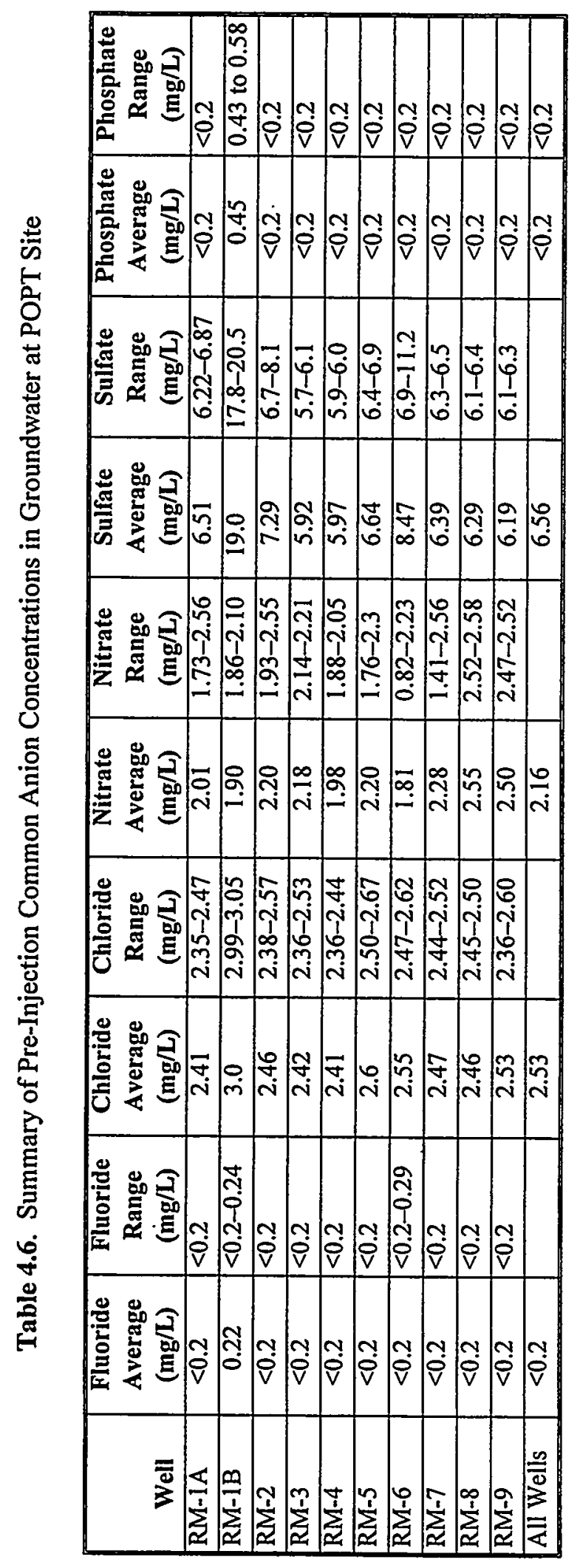


Table 4.7. Summary of Pre-Injection ICP-OES Trace Metals Concentrations in Groundwater at POPT Site

\begin{tabular}{|l|c|c|}
\hline Constituent & $\begin{array}{c}\text { Average } \\
\text { (mg/L) }\end{array}$ & \multicolumn{1}{|c|}{$\begin{array}{c}\text { Range } \\
\text { (mg/L) }\end{array}$} \\
\hline Aluminum & $<0.1$ & $<0.1$ \\
\hline Arsenic & $<0.06$ & $<0.06$ \\
\hline Barium & 0.0047 & 0.004 to 0.009 \\
\hline Beryllium & $<0.002$ & $<0.002$ \\
\hline Boron & $<0.005$ & $<0.005$ \\
\hline Cadmium & $<0.02$ & $<0.02$ \\
\hline Calcium & 13.9 & 13 to 15 \\
\hline Chromium & $<0.04$ & $<0.04$ \\
\hline Cobalt & $<0.004$ & $<0.004$ \\
\hline Copper & $<0.006$ & $<0.006$ \\
\hline Iron & 0.013 & $<0.005$ to 0.044 \\
\hline Lead & $<0.06$ & $<0.06$ \\
\hline Lithium & $<0.02$ & $<0.02$ \\
\hline Magnesium & 6.11 & 6.7 to 6.9 \\
\hline Manganese & 0.015 & U to 0.18 \\
\hline Molybdenum & $<0.06$ & $<0.06$ \\
\hline Nickel & 0.075 & U to 0.27 \\
\hline Potassium & 1.76 & 1.6 to 2.2 \\
\hline Selenium & $<0.06$ & $<0.06$ \\
\hline Silicon & $<0.1$ & $<0.1$ to 12 \\
\hline Silver & $<0.02$ & $<0.02$ \\
\hline Sodium & 5.70 & 5.5 to 12 \\
\hline Strontium & 0.073 & 0.068 to 0.081 \\
\hline Vanadium & $<0.02$ & $<0.02$ \\
\hline Zinc & $<0.006$ & $<0.006$ \\
\hline
\end{tabular}

\subsection{Hydrologic Characterization}

Following installation and development of the ISRM well network, a constant-rate discharge test was conducted in the injection well (RM-9) to determine the local-scale hydraulic properties of the aquifer and the specific capacity of the injection well prior to emplacement of the ISRM treatment zone. These test data were representative of baseline (pre-injection) aquifer conditions and were incorporated into the design analysis of the proof-of-principle technology demonstration. Hydrologic test data were analyzed using peer-reviewed analytical methods that were applicable to the given test conditions. Because one of the primary objectives of the hydraulic testing program was to assess any adverse effects (e.g., aquifer plugging) associated with the ISRM technology, emphasis was placed on the analysis of test results from monitoring wells within the targeted treatment zone (RM-1a, RM-5, RM-7, and RM-8). 
A 720-minute constant-rate discharge test was conducted on September 20, 1998. The discharge rate was regulated and maintained at a relatively constant rate $( \pm 0.5 \%)$, averaging $80.3 \mathrm{gpm}$ for the duration of the test. The flow rate was monitored with turbine flow meters and pressure transducers were installed in the stress well (RM-9) and selected monitoring wells to monitor pressure response during the test (see Section 5 for details).

Based on the hydrogeologic information collected during installation of site monitoring wells (Śection 4.3), a leaky confined aquifer model was selected as the best representation of observed aquifer conditions. Thickness of the upper outwash gravel within the targeted treatment zone ranged from 9 to $16 \mathrm{ft}$ with an average of $12 \mathrm{ft}$. Interpretation of the tracer injection test and the first dithionite injection test indicated that, in the vicinity of the injection well (RM-9), the lower-till aquitard unit (Qvt2) was noncontinuous and the upper and lower outwash gravel units were hydraulically connected. This connectivity is evidenced by the relatively quick pressure response and tracer/dithionite arrival responses observed at RM-2.

Early and intermediate time-pressure response data (i.e., $\mathrm{t}<=100 \mathrm{~min}$ ) were used in the analysis. Selection of this data segment for analysis was based on a consistently observed late-time deflection from the theoretical leaky confined well response for both drawdown and pressure recovery data from the constant-rate discharge test and subsequent testing activities (i.e., tracer test and dithionite injections). This late time deflection was most likely caused by a local reduced flow boundary, which is not unexpected given the variability in formation thickness and relatively high level of heterogeneity observed at the site. Another possible model that would account for this S-shaped well response (a steep early-time segment, a flat intermediate-time segment, and a steeper late-time segment) is the delayed yield response associated with unconfined aquifers (Neuman 1972). However, because the geology observed at the site indicates a locally leaky-confined system, a leaky confined model was used and analysis targeted the early and intermediate time data segments.

Plots of the observed pressure response and type curve matches for selected monitoring wells are shown in Appendix D. Hydraulic test data were analyzed with a leaky confined aquifer model (Hantush 1960) using type curves generated with the Waterloo Hydrogeologic, Inc. software package AquiferTest. Data analysis resulted in estimates for transmissivity and storativity from 7,000 to $9,600 \mathrm{ft}^{2} /$ day and 0.0009 and 0.002 , respectively. Based on an average transmissivity of $8700 \mathrm{ft}^{2} /$ day and an average thickness of $12 \mathrm{ft}$, the average hydraulic conductivity of the upper outwash gravel unit beneath the ISRM test site is approximately $700 \mathrm{ft} /$ day.

\subsection{Groundwater Flow Rate and Direction}

Two approaches were used to quantify the local-scale groundwater flow rate and direction at the Fort Lewis ISRM proof-of-principle test site. The primary approach involved standard hydrologic analysis techniques based on measurements of hydraulic gradient, hydraulic conductivity, and porosity (i.e., application of Darcy's Law). Because measuring the local hydraulic gradient was complicated by seasonal variability in recharge and limitations in the number and location of available, appropriately screened monitoring wells, an innovative approach was applied at the site that provides a direct 
measurement of groundwater velocity. In situ groundwater flow sensors (HydroTechnics, Inc.) were installed, two sensors during the second drilling campaign and two more during the third drilling campaign.

The failure of the first two sensor installations, which allowed the natural formation sediments to collapse around the sensors, resulted in the formation of a hypothesis that the outwash gravels beneath Fort Lewis were too poorly sorted, contained too many large cobbles, and in some cases contained too large a silt fraction for the in situ flow sensors to be effective. During a subsequent drilling campaign, HydroTechnics, Inc. provided two additional sensors to demonstrate their applicability in heterogeneous sediments like those encountered at the POPT site. During the second installation, a sandpack was placed around the flow sensors to provide a more integrated measurement of flow velocity over the scale of the sensor array. Interpretation of these data indicated that the second installation also failed to provide representative measurements of in situ groundwater flow velocity and direction.

Due to the failure of the in situ flow sensors, estimates of groundwater velocity and direction at the site were based solely on local-scale hydraulic gradient measurements. The relatively high permeability aquifer materials composing the targeted treatment zone made measuring the hydraulic gradient difficult using the network of wells installed at the ISRM POPT site. Most monitoring wells were installed close together to meet injection and performance assessment monitoring objectives, and the high-permeability aquifer materials result in a relatively flat gradient, making differences in water level between the wells small and difficult to measure precisely. To provide an estimate of the local-scale (i.e., DRMO complex) hydraulic gradient, local area monitoring wells were identified that were completed over the appropriate depth interval and could be used to construct a potentiometric surface map.

As indicated in Figure 4.10, the medium and low water level conditions, which represent the typical groundwater flow regime observed over the duration of the POPT, are characterized by a general northerly flow with a northeasterly deflection in the flow in the vicinity of the ISRM test site (near RM-3 and RM-6). Although there are insufficient data to fully characterize the cause and extent of this deflection, available data indicate there may be a significant change in flow direction near the test site under the typical flow regime. This deflection in groundwater flow direction may be associated with thinning of the outwash gravel aquifer in the vicinity of the ISRM test site. As discussed in Section 3, one of the site selection criteria, which were developed to meet the POPT objectives, was to target a relatively thin outwash gravel unit to minimize drilling and chemical costs associated with demonstration of the ISRM technology. However, if the targeted geologic feature is not aerially extensive, local groundwater flow may be affected by the localized thinning of the unit and associated reduction in transmissivity, causing the observed deflection in groundwater flow. There are not enough data to characterize the aerial extent of this targeted feature.

Based on water level contour maps generated from various data sets collected over the duration of the POPT, four wells were selected, two ISRM monitoring wells (RM-3 and RM-6) and two of the closest pre-existing monitoring wells (LC-143 and LC-152), for calculating groundwater velocity and direction using a least-squares-fit algorithm (WATER-VELTM, In-Situ, Inc. Software Series). These data are presented in Table 4.8 and Figure 4.11. 


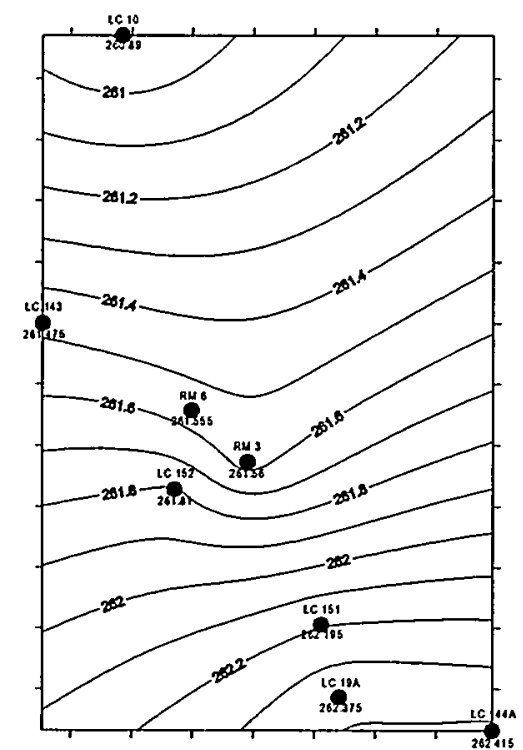

(a)

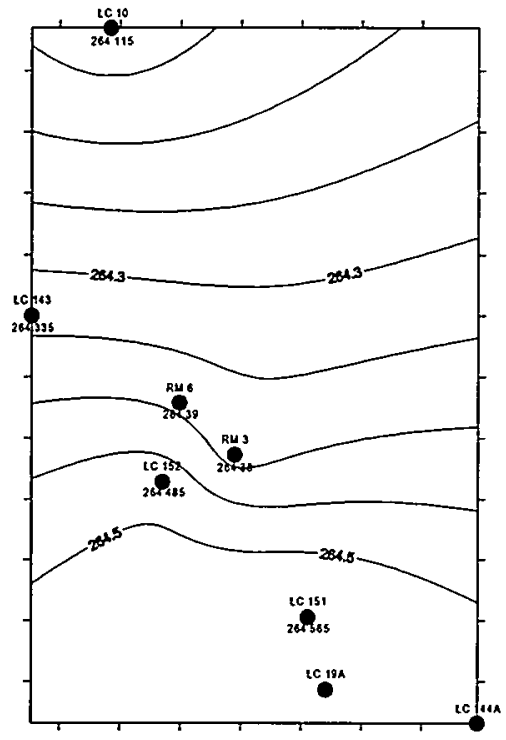

(b)

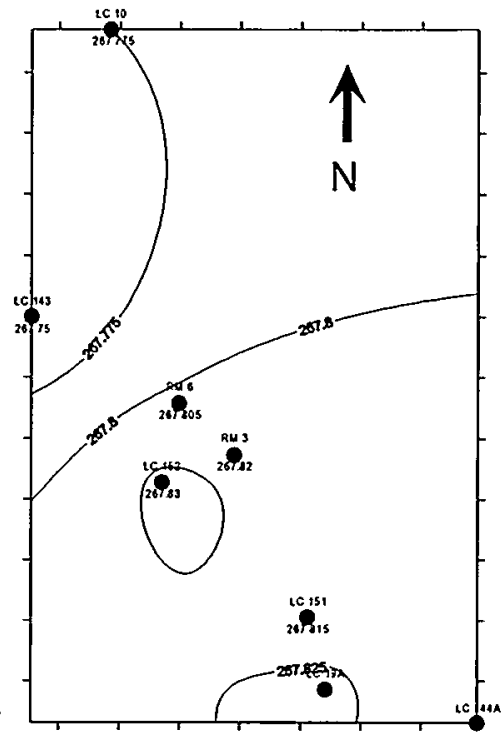

(c)

Figure 4.10. Potentiometric Surface Contour Maps Representing a) Low Water Level Conditions on October 19, 1999, b) Medium Water Level Conditions on January 1, 2000, and c) High Water Level Conditions on February 2, 1999

Table 4.8. Groundwater Velocity and Direction Summary

\begin{tabular}{|c|c|c|}
\hline Date Measured & V (ft/d) & Azimuth (deg) \\
\hline $2 / 8 / 99$ & 1.4 & 243 \\
\hline $4 / 1 / 99$ & 0.7 & 259 \\
\hline $10 / 19 / 99$ & 6.9 & 26 \\
\hline $11 / 30 / 99$ & 5.9 & 28 \\
\hline $1 / 6 / 00$ & 3.5 & 25 \\
\hline $2 / 8 / 00$ & 3.4 & 19 \\
\hline $3 / 8 / 00$ & 2.4 & 18 \\
\hline $3 / 20 / 00$ & 2.7 & 19 \\
\hline $4 / 25 / 00$ & 3.6 & 24 \\
\hline $5 / 23 / 00$ & 4.5 & 25 \\
\hline $6 / 12 / 00$ & 5.0 & 30 \\
\hline $6 / 20 / 00$ & 5.1 & 28 \\
\hline
\end{tabular}

As indicated, there have been two separate flow regimes observed at the POPT site: the typical regime characterized by a relatively consistent northeasterly direction and a low flow regime observed during the winter of 1998-1999. Groundwater velocities under the typical regime have ranged from 2.4 to $6.9 \mathrm{ft} /$ day with a time weighted average velocity of approximately $4 \mathrm{ft} /$ day. Under this regime, there is a strong correlation between water level elevation and groundwater velocity, with the highest velocity associated with low water level conditions and velocity decreasing as the water level rises. During extremely high water level conditions, the local gradient flattens (Figure 4.10c) and groundwater velocity 


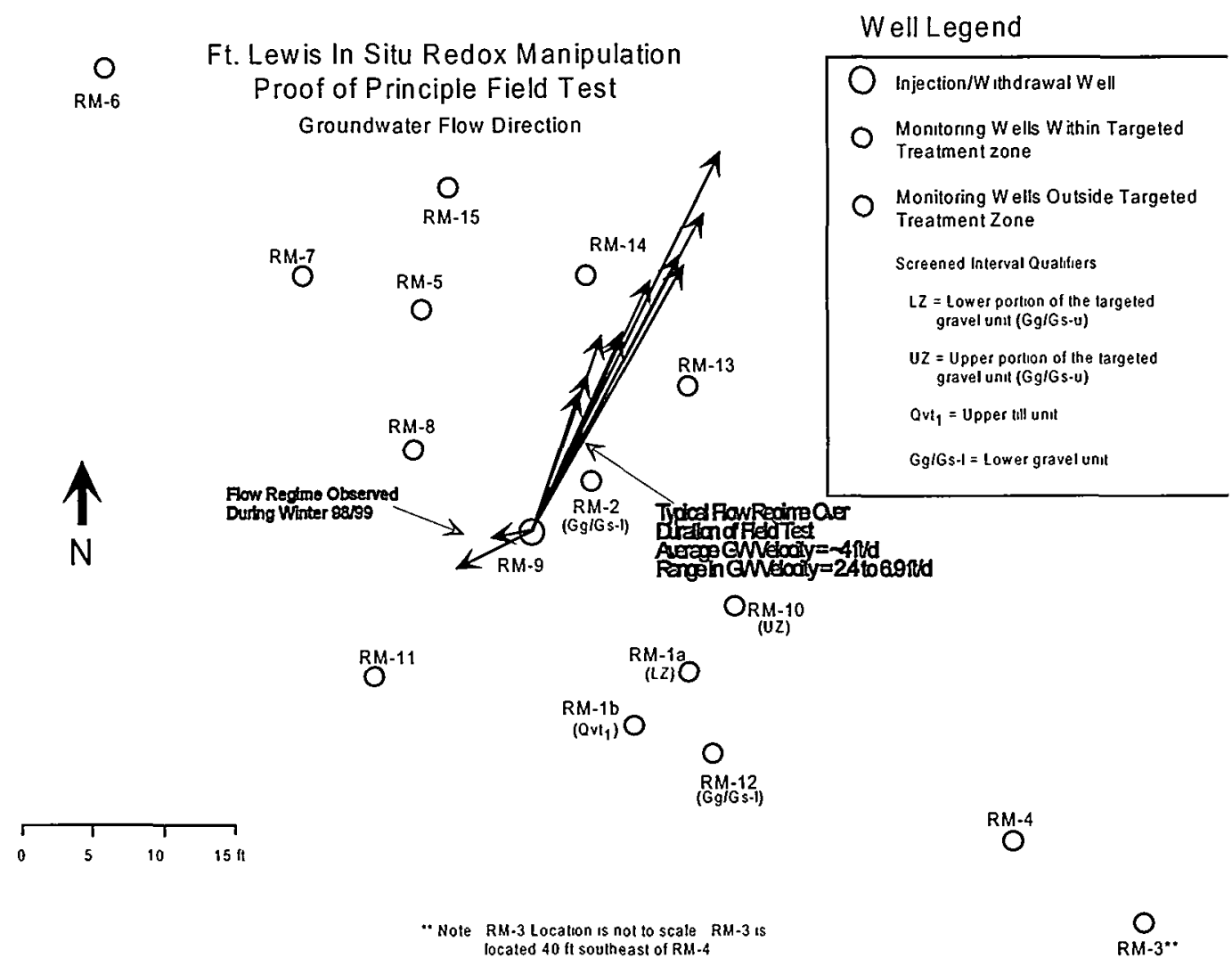

Figure 4.11. Groundwater Velocity and Direction Observed at the ISRM Proof-of-Principle Test Site During Field Testing

is significantly reduced. Fluctuations in site water levels result from seasonal variations in precipitation and the associated groundwater recharge (Figure 4.12).

This flattening of the local groundwater gradient is most likely associated with differences in recharge characteristics between the industrially zoned DRMO complex where the test site is situated and the naturally forested area just outside the DRMO complex. Although sufficient data to quantitatively substantiate this theory are not available, the comparison of hydrographs for wells within the DRMO complex with wells in the forested area (Figure 4.13) indicate significant differences in recharge characteristics. For the period inspected, more than twice the water level change was observed in monitoring wells located within the DRMO complex during a seasonal high recharge period (winter 1997-1998 data segment) than in monitoring wells outside the complex in natural vegetation.

These data indicate that surface conditions and possibly storm water drainage systems may be increasing local recharge to the groundwater system near the DRMO complex. This difference in recharge condition is not unexpected given the relatively high precipitation levels at Fort Lewis and the lack of vegetation within the complex to enhance evapotranspiration. The localized increase in water level elevations created by this spatial and temporal variability in recharge distribution acts to decrease the gradient near the ISRM test site and subsequently lower the groundwater velocity. 


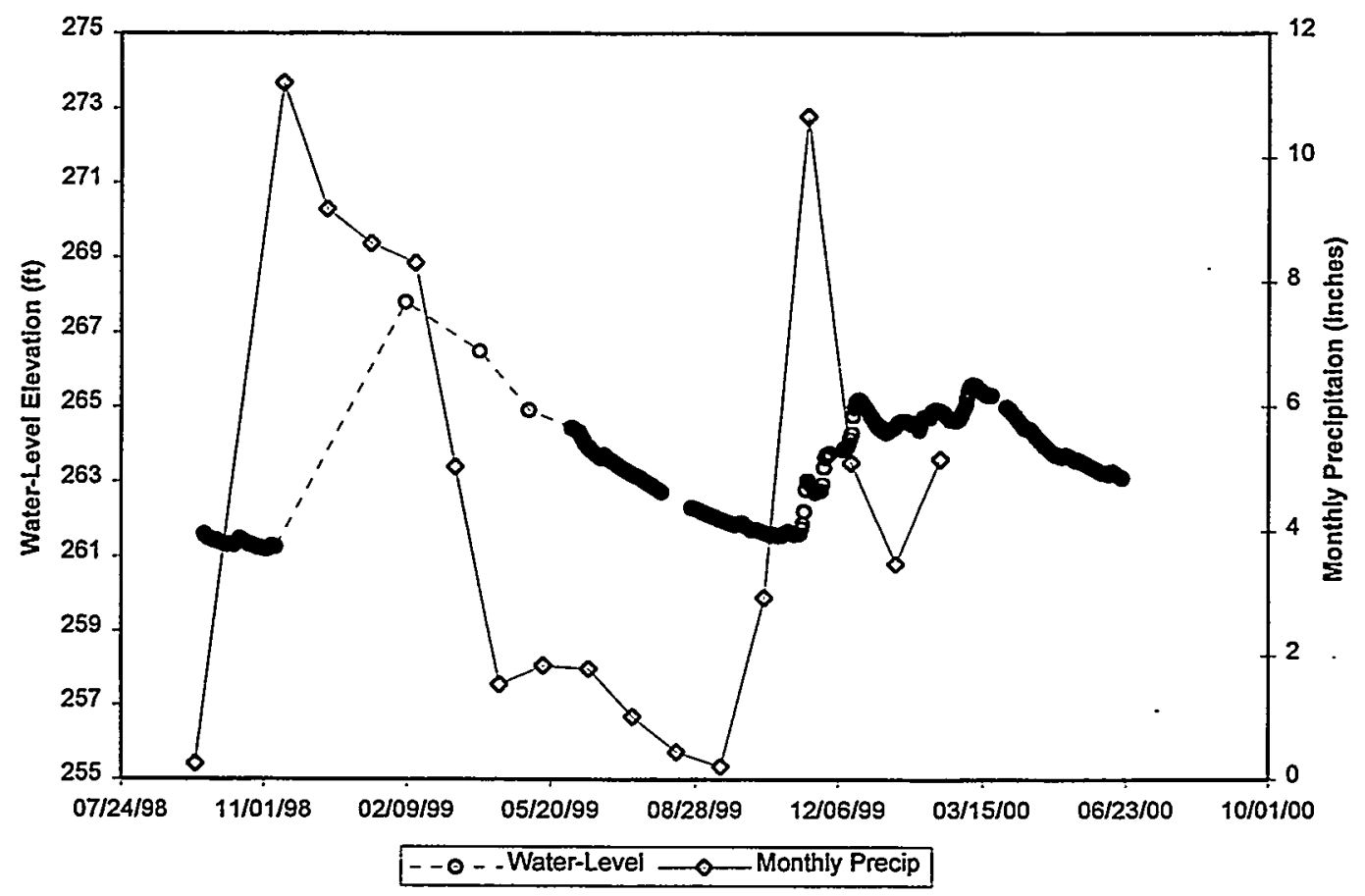

Figure 4.12. Comparison of Monthly Precipitation and Water Level Elevation at RM-9

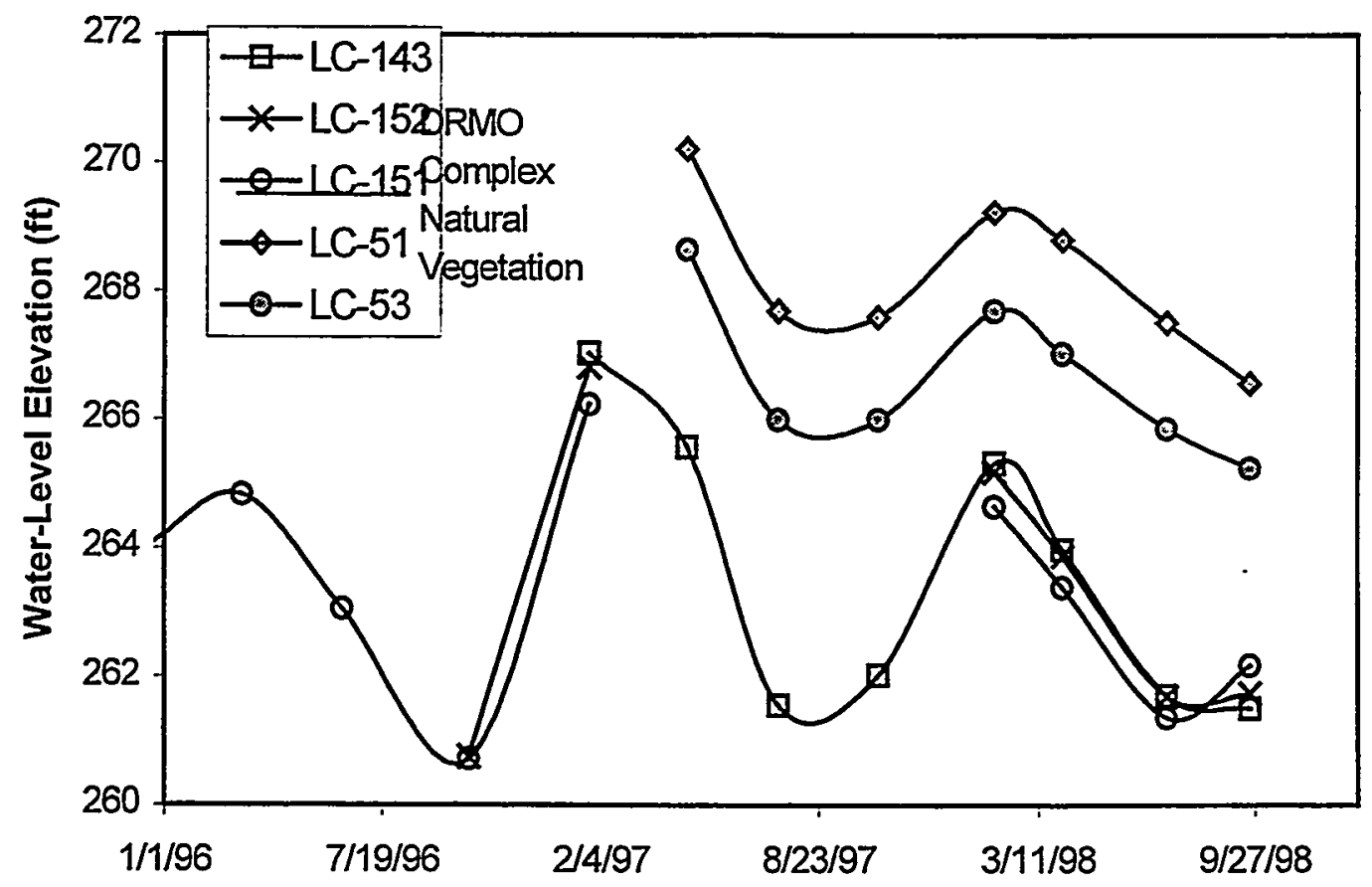

Figure 4.13. Comparison of Hydrographs for Wells Within and Outside the DRMO Complex 
As indicated in Figure 4.11, the up- and downgradient monitoring wells included in the POPT well network are not aligned with the typical groundwater flow direction, which would be the desired configuration. Because limited test site scale information on the groundwater flow direction was available prior to installation of the ISRM well network, orientation of the well network was based on the historic logistics center scale water table map (Figure 4.4).

Due to the lack of local-scale, spatially distributed water level data for the upper outwash gravel unit, a high level of confidence cannot be placed in the accuracy of the resulting groundwater velocity and direction estimates. In addition, performance assessment groundwater monitoring data contained in Section 8.1 indicates a more northerly or north-northwesterly flow direction may predominate. Although the small scale of the ISRM treatment zone and lack of water-level data limit our ability to predict groundwater flow direction accurately, the available data are sufficient to provide a semi-quantitative estimate of groundwater velocity and a measure of the seasonal variability in groundwater flow. Water table contour maps for three measurement dates representing high, medium and low water level conditions are shown in Figure 4.10. 


\subsection{Site Setup for Proof-of-Principle Test}

This section includes a description of the site utilities, monitoring equipment, analytical equipment, injection equipment, and the integration of these components into the operational systems required to conduct the ISRM POPT.

\subsection{Component Description}

The following is a description of the various components required to conduct a field-scale demonstration of the ISRM technology.

\subsubsection{Site Utilities}

Site utility requirements for a field-scale demonstration of the ISRM technology include access to electrical power, water supply, and wastewater disposal.

\section{Water Supply}

To conduct the tracer and dithionite injection tests, a substantial source of water is needed to make up the injection solutions. If a water supply is not available on site, groundwater can be withdrawn from wells prior to the test and stored in onsite storage tanks until the injection tests are conducted. At the Fort Lewis POPT site, a nearby fire hydrant supplied the water needed for dilution of the concentrated tracer and dithionite solutions; each test used over 40,000 gallons of water at rates as high as 90 gpm. Static pressure at the hydrant was $60 \mathrm{psi}$, and the temperature of the water ranged from 12 to $16^{\circ} \mathrm{C}$. Prior to the injection tests, water samples from the hydrant were submitted for VOC, major ions, and trace metals analysis; no constituents were present at levels that prevented use of the water from the hydrant.

\section{Electrical Service}

Electrical power is required to operate site facilities, including a mobile laboratory and associated analytical equipment, office/storage trailer, and injection/monitoring equipment. Site power can be supplied by appropriately sized generators; however, line power is preferable because, once installed, it is virtually maintenance free and more reliable than generators. At the Fort Lewis POPT site, an existing power service for the turbine extraction pump in RW-1 was modified to meet the requirements of the ISRM field demonstration.

\section{Boiler}

Because dithionite reaction rates are significantly reduced under the relatively cold groundwater temperature conditions at Fort Lewis, a boiler was used to preheat the aquifer prior to dithionite injection and to warm the injection solution during the various injection phases. The water supply source (hydrant) was warmed from an ambient temperature that ranged from 12 to $16^{\circ} \mathrm{C}$ to an injection temperature of as 
high as $32^{\circ} \mathrm{C}$ using an oil-fired steam boiler. Design calculations indicated a 25 hp steam boiler would be capable of supplying the heat required to meet injection temperature specifications. The boiler unit was rated at $862 \mathrm{lb}$ steam/hour and fired on \#2 fuel oil. The boiler was rated at 150-psi design pressure and was equipped with flame safeguard control, pressure operating controls, and low-water cutoff controls. Supply water was heated using a Bell and Gossett shell and tube heat exchanger. At high fire, which was required to raise the injection stream at $50 \mathrm{gpm}$ from ambient to approximately $30^{\circ} \mathrm{C}$, the boiler consumed approximately 7.5 gallons of fuel per hour.

\section{Wastewater Disposal}

Due to the selected emplacement method of the ISRM treatment zone at the Fort Lewis POPT field site (injection/withdrawal or push/pull), relatively large volumes of wastewater were generated (see Section 7, Table 7.1). Several options were considered for treatment/disposal of the generated wastewater. Fort Lewis environmental and sanitary sewer operations personnel were notified and given information regarding the volume and expected constituent concentrations in the wastewater. Based on that information, it was determined that the ISRM withdrawal water could be discharged directly to the Fort Lewis sanitary sewer. Fort Lewis utilities personnel were contacted, and suitable access to the sanitary sewer was identified approximately $700 \mathrm{ft}$ from POPT site. Temporary piping was installed to provide continuous access for wastewater disposal during ISRM field-testing activities.

\subsubsection{Monitoring Equipment}

\section{Sampling Pumps}

Dedicated Grundfos RediFlo2 sampling pumps with integral well seals were installed in all site monitoring wells. The sample tubing from each of these sampling pumps was routed inside an onsite mobile laboratory and connected directly to a sampling manifold. Sample pumps were operated using a manufacturer-supplied variable-speed control box (converts standard 110-V single-phase power into three-phase power to meet the requirements of Rediflo2 sampling pumps) and a project-developed multichannel interface (pump switch box) that allowed multiple sample pumps to be operated using a single control box.

\section{Sampling Manifold}

A project-developed sampling manifold allowed all sampling streams to be routed into a central manifold for monitoring field parameters (in a flow-through monitoring assembly) and collecting groundwater samples. The advantage of this type of system is that all field parameter measurements are made using a single set of electrodes, which improves data quality and comparability of spatially distributed measurements. Consistent labeling between the sampling manifold and pump switch box simplified selection of the well to be sampled and reduced the chance of operator error during the frequent sampling associated with the injection tests. 


\section{Field Parameter Measurements}

Field parameters were monitored using $\mathrm{pH}$, temperature, electrical conductivity, and dissolved oxygen electrodes installed in a flow-through monitoring assembly. The flow-through assembly was designed to minimize the amount of "dead space" within the monitoring chamber and resulted in flowthrough residence times of less than three seconds under standard monitoring conditions. Purge volumes pumped prior to sample collection were determined by monitoring stabilization of field parameters. The field parameter monitoring electrodes used during this field test met the specifications shown in Table 5.1.

Table 5.1. Field Parameter Monitoring Electrode Specifications

\begin{tabular}{|l|l|l|l|}
\hline \multicolumn{1}{|c|}{ Parameter } & \multicolumn{1}{c|}{ Manufacturer/Model \# } & \multicolumn{1}{c|}{ Range } & Accuracy/Reproducibility \\
\hline $\mathrm{pH}$ & Oakton/WD-35615 & $\mathrm{pH} \mathrm{2-16}$ & $\pm 0.05 \mathrm{pH}$ \\
\hline Eh & Metron/10-565-3116 & & \\
\hline Temperature & Oakton/WD-35607 & $0.0-100^{\circ} \mathrm{C}$ & $\pm 0.5^{\circ} \mathrm{C}$ \\
\hline $\begin{array}{l}\text { Electrical } \\
\text { conductivity }\end{array}$ & Oakton/WD-35607 & $0.0-199.9 \mathrm{mS}$ & $\pm 50 \mu \mathrm{S}$ \\
\hline Dissolved oxygen & Orion/810 & $0-20 \mathrm{ppm}$ & $\pm 0.1 \mathrm{ppm}$ \\
\hline Bromide (tracer test) & Cole-Parmer/P-27502-05 & $0.4-79,900 \mathrm{ppm}$ & $\pm 2 \%$ full scale \\
\hline
\end{tabular}

\section{Field Parameter Verification}

Field parameters were also monitored on a separate verification station consisting of a system using micro-flow-through electrodes and a syringe pump.

\section{Water-Level/Pressure Response Measurements}

Pressure transducers ( 10 and $20 \mathrm{psi}, 0.1 \%$ of full scale accuracy) were installed in selected wells to monitor pressure response during hydraulic and dithionite/tracer injection tests and continuously recorded using a Campball Scientific CR10 datalogger. Transducer readings were validated periodically with water level measurements during all phases of testing to check for transducer drift.

Water levels were measured using a high-accuracy, National Institute of Standards and Technology traceable, non-stretch, metal-taped, water level meter marked in $0.01 \mathrm{ft}$ gradations. When collecting water level measurements for gradient determination, measurements were collected over as short a time period as possible. Initial measurements were rechecked throughout the measurement period to quantify any water level changes due to external stress (e.g., pump-and-treat operation, barometric pressure change). 


\subsubsection{Analytical Measurements}

A comprehensive series of analytical measurements were made throughout the project in support of the field objectives. These included measurements made in the field in an onsite trailer-based mobile laboratory during the injection/withdrawal tests or during sample collection for groundwater monitoring. More extensive measurements were performed later under more rigorous laboratory conditions in a number of different PNNL in-house laboratories. A complete description of all the methodologies used as well as the associated QA/QC controls can be found in Appendix B, "Analytical Methods."

During the injection/withdrawal activities, dithionite measurements were performed in the mobile laboratory using an ultraviolet absorption system with an on-line automatic dilution capability. Field measurement of dithionite was needed because of the inherent instability of that reagent, rendering return to a remote laboratory impractical. Dithionite calibration standards were freshly prepared in the field from pure reagent materials.

Samples were collected from each well for analysis of VOCs, acetylene, trace metals, and common anions during groundwater monitoring. Samples were returned to PNNL, usually within 24 hours, and either analyzed immediately or archived for subsequent analysis depending upon analytical requirements.

Samples collected for volatile organic analysis (VOA) (e.g., TCE and DCE) were collected in headspace-free, brown glass VOA vials, preserved in the field with hydrochloric acid to retard bacterial degradation, and stored at reduced temperature $\left(4^{\circ} \mathrm{C}\right.$ nominal $)$ prior to analysis. An attempt was made to analyze the laboratory samples as promptly as possible. In most cases, analytical determinations were made in well under the EPA specified 14-day holding time. Two determinant methods were used. For much of the initial work, a GC/MS method using headspace sample introduction was used. The GC/MS method provided a powerful identification capability that permitted a sensitive search for all volatile species, providing assurance that TCE and DCE were the only significant species of concern found at the site both before and after dithionite treatment. Calibration problems associated with the headspace method resulted in a switch to a more conventional gas chromatography method (PNL-VOA3) that follows essentially the same protocols as EPA SW-846 Method 502.2. Commercially prepared calibration standards were used for quantitation. Cross-calibration checks between the commercial standards and several in-house standards prepared by weighing neat reagents provided very good assurance of accurate results.

Separate acid preserved samples were collected for acetylene assay in headspace-free, brown glass 40-mL VOA vials. The acetylene samples were analyzed by a GC system employing a special HP-PLOT-type column with exceptional separation capabilities for ethane, ethene, and acetylene. The GC employed a flame ionization detector for detection and quantitation. Sample introduction was by the headspace method with commercially prepared gas mixtures used for calibration.

Trace metal samples were collected in 20-mL acid washed plastic vials. Concentrated Ultrex nitric acid was included in each vial as a preservative. Samples were analyzed initially by induction coupled plasma-optical emission spectroscopy (ICP-OES). Because ICP-OES detection sensitivity proved 
inadequate to reach required maximum concentration levels (MCL) limits for several key elements (As and $\mathrm{Sb}$ ), some samples were also submitted for analysis by induction coupled plasma-mass spectrometry (ICP-MS). The ICP-OES protocol used is internally documented as PNL-ALO-211.2. It generally followed EPA SW-846 Method 6010 protocols, while the ICP-MS protocols are based on EPA 6020.

Ion chromatography was performed on unpreserved samples collected in 20 -mL plastic vials. Two different laboratories were used for this work. Both used variants on EPA Method 300.0 modified to include sulfite and thiosulfate. The PNNL methods are internally documented as PNL-IC-1 and PNL-ALO-212, respectively.

All field and laboratory data were entered into a Microsoft Access database. The database included individual tables for Analytical Data, Field Sampling Information, and Constituents (CAS numbers). The Analytical Data table contained over 7600 records representing individual analytical determinations. The Field Sampling Information table contained 463 records, corresponding to that number of individual sampling events, with each event usually generating multiple archived samples for additional analytical work.

\subsubsection{Injection and Withdrawal Equipment}

\section{Injection Manifold}

The injection manifold (Figure 5.1) consists of an injection pump and appropriately routed piping, valving, and flow rate monitoring equipment. The manifold is used to control (both rate and concentration), monitor, and sample the injection solutions. The manifold was constructed of 316 stainless steel and used stainless steel ball valves for both diversion/shutoff and flow control valves.

\section{Injection Pump}

A 0.75 hp Grundfos stainless steel multi-stage centrifugal pump (Model \# CRN2-30) was used for injecting the concentrated solution. The injection tubing that extended from the well-head to the center of the injection interval was constructed of 1.5-in.-diameter stainless steel.

\section{Fluid Metering Pump}

A fluid metering pump made by FMI Inc. (QD-2) was used to meter a small amount of dithionite $(\sim 150 \mathrm{~mL} / \mathrm{min})$ into the injection stream during the preheat phase of Injections 2 through 4 . The metered dithionite was required to remove dissolved oxygen from the supply water.

\section{Turbine Flow-Meters}

Omega turbine flow meters were installed to measure the flow rate of the various streams and the total injection flow rate. A 2-in.-diameter flow meter (Model \# FTB109) was used to monitor the dilution 


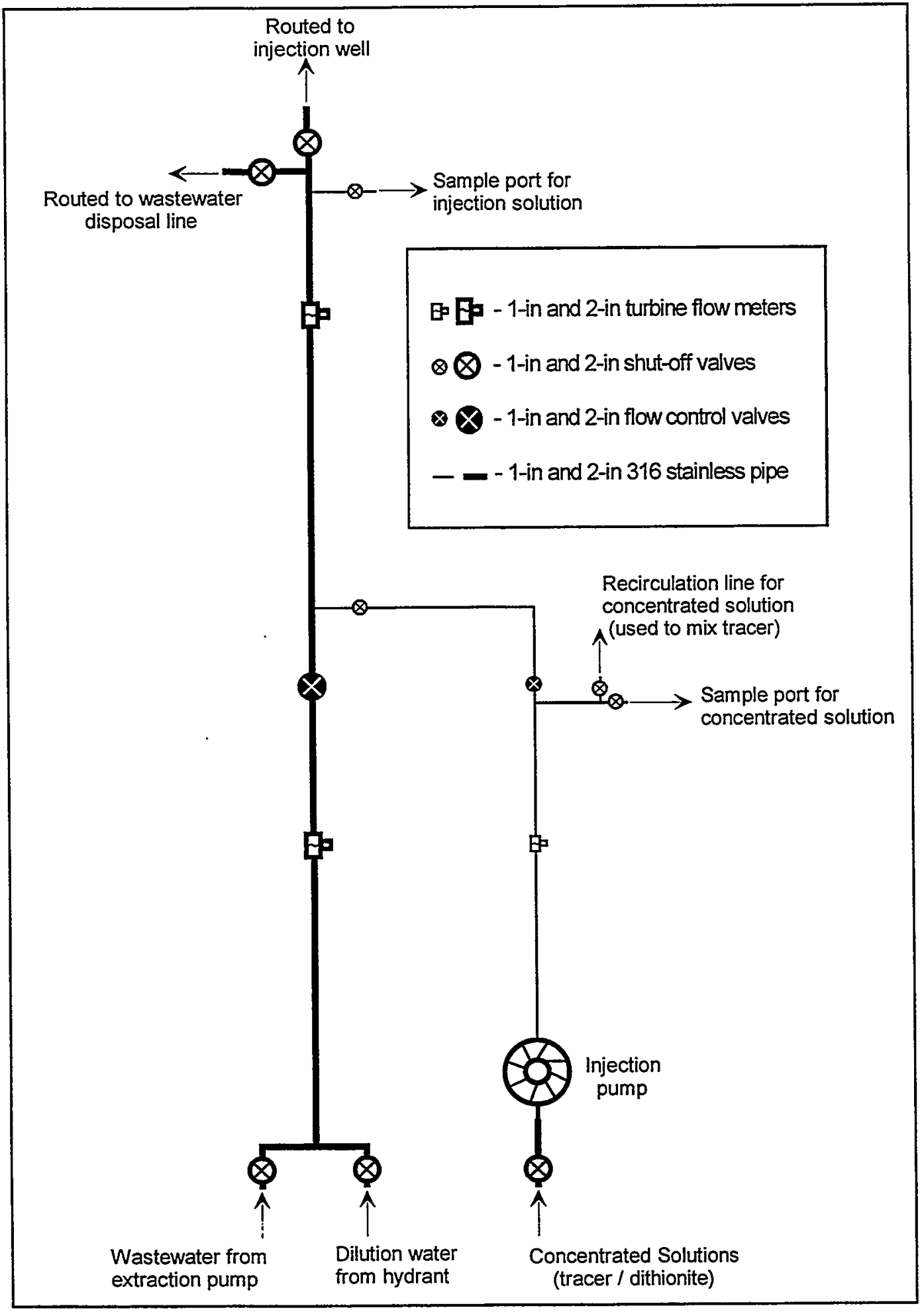

Figure 5.1. Schematic Drawing of the Tracer and Dithionite Injection System Used at the Fort Lewis ISRM POPT Field Demonstration 
water and total injection rate; a 1-in. diameter flow meter (Model \# FTB106) was used to monitor the injection rate of the concentrated tracer/dithionite solutions. These flow meters were continually logged with a Campbell Scientific CR10 datalogger.

\section{Submersible Extraction Pump}

A $3.0 \mathrm{hp}$, 6-in. diameter Grundfos stainless steel submersible pump (Model \# 80S30-3) was used as the extraction pump during the withdrawal phase of the test. The extraction pump was installed on a 2-in.-diameter stainless steel riser.

\subsection{Description of Equipment Integration/Operation}

The following is a description of how the various equipment components were integrated into the systems required to conduct an ISRM POPT.

\subsubsection{Tracer and Dithionite Injection}

The tracer and dithionite injection tests were conducted using the equipment described above and illustrated in Figure 5.1. The desired injection concentration was achieved by mixing the concentrated tracer (tracer test) or dithionite (dithionite injection test) solutions with dilution water from the fire hydrant. Injection pressure for the concentrated solutions and dilution water was provided by the stainless steel injection pump and the 60 -psi static pressure of the hydrant, respectively. The two injection streams were mixed within the injection manifold before the solution arrived at the point of injection (i.e., the center of the injection well's screen interval). Supply water from the hydrant was routed through the heat exchanger and raised to the desired temperature before entering the injection manifold.

All injection flow rates (concentrated solution, dilution water, total) were monitored with turbine flow meters and controlled by manually adjusting flow control valves. Sample ports were located on the manifold so that samples of the concentrated and injection solutions could be collected throughout the injection test.

Following the injection and residence phases of the test, remaining dithionite and reaction products were extracted through the central injection/withdrawal well using a submersible extraction pump. Wastewater generated during the withdrawal phase was routed back through the injection manifold to a wastewater disposal line that discharged to the Fort Lewis sanitary sewer. Withdrawal water was routed back through the injection manifold so that the same flow monitoring and control equipment used to monitor/control the injection could be used to monitor/control the withdrawal.

\subsubsection{Groundwater Sample Collection}

Groundwater sample collection during the ISRM POPT was conducted using the equipment described in Section 5.1.2 and illustrated in Figure 5.2. The groundwater sampling equipment consisted of dedicated variable-speed submersible sampling pumps installed in all site monitoring wells with sample tubing 


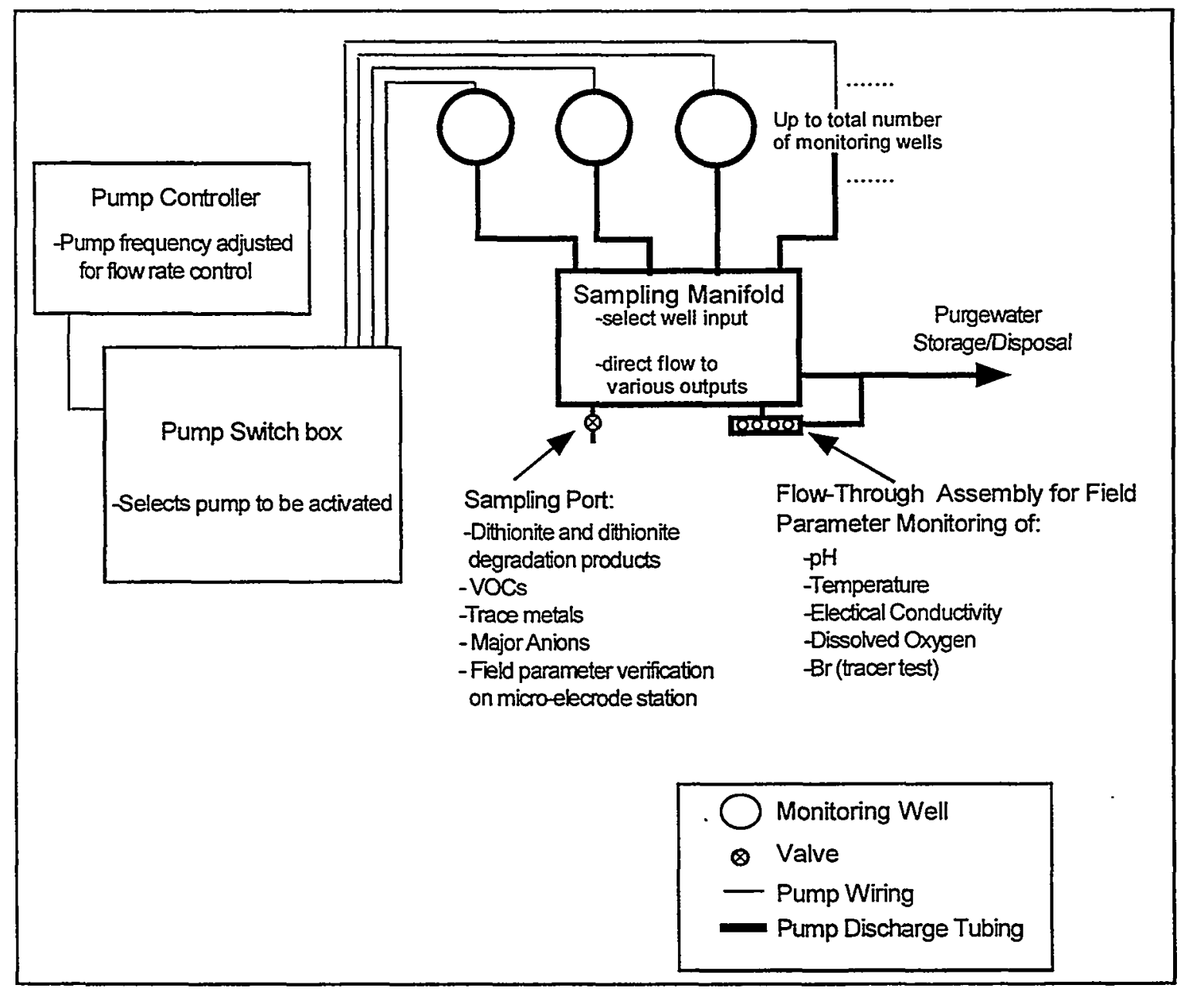

Figure 5.2. Schematic Drawing of the Groundwater Sample Acquisition System Used at the Fort Lewis ISRM POPT Field Demonstration

and control wiring routed to a central location inside the onsite mobile laboratory where groundwater field parameters were monitored (in a flow-through monitoring assembly) and groundwater samples were collected. The advantage of this type of system is that all field parameter measurements were made using a single set of electrodes, which improves data quality and comparability of spatially distributed measurements.

The procedure for monitoring field parameters and collecting groundwater samples using this equipment is described below:

1. Select well to be sampled on pump switch box and sampling manifold.

2. Move starter switch on variable speed control box to the start position. Pump frequency was preset to provide a purge rate of approximately $3 \mathrm{gpm}$. 
3. Following displacement of any air bubbles trapped in the sample tubing (generally within the first 20 to 30 seconds), divert approximately $1 \mathrm{gpm}$ of sample stream to flow-through monitoring assembly.

4. Monitor field parameters until they have stabilized. In general, field parameters stabilized after two to three minutes of purging. Wells were purged for approximately three minutes $(9 \mathrm{gal})$ and five minutes (15 gal) during the tracer/dithionite injection tests and pre- and post-experiment monitoring, respectively.

5. Record field parameter measurements and collect required groundwater samples.

6. Collect sample in $10 \mathrm{~mL}$ syringe for field parameter verification on the micro-electrode station.

7. Select next well to be sampled and repeat process. 


\subsection{Bromide Tracer Test}

\subsection{Tracer Test Objective}

A conservative tracer test was conducted at the ISRM proof-of-principle site in October 1998 using a potassium bromide solution. This test was similar to the planned dithionite injection/withdrawal tests for emplacement of the reduced zone. The objectives of the tracer test, which were developed to aid in designing the dithionite injection/withdrawal tests, included estimating the radial extent of injected solution, assessing spatial variability (heterogeneities), calculating recovery of the tracer, testing field equipment, refining field operations, and determining sampling protocols. A description of the test and its results is provided below.

\subsection{Tracer Test Description}

The tracer test was conducted using an injection/withdrawal (or push-pull) technique that consisted of three phases (see Figure 6.1): injection, residence, and withdrawal. In the injection phase, the solution is injected into a central injection/withdrawal well (RM-9, as shown in Figure 4.1). The residence phase provides time for the solution to react with the aquifer sediments (in the case of sodium dithionite) and potentially drift in the aquifer. In the withdrawal phase, the solution is extracted from the aquifer by pumping from the same well used for injection. Aqueous samples were collected from the injection and withdrawal stream and from the surrounding monitoring wells. Bromide ion-selective electrodes were used for bromide measurements in the field trailer at the time of sample collection to monitor the performance of the test. Archive samples were also collected and measured for bromide using ion chromatography (IC) in the laboratory following the test. More than 300 aqueous samples were collected during the tracer test and analyzed by both ion-selective electrodes and IC.

During the tracer test, a potassium bromide $(\mathrm{KBr})$ solution $(80 \mathrm{mg} / \mathrm{L} \mathrm{Br})$ was injected into the aquifer through the injection/withdrawal well (RM-9) at 100 gpm for 7.4 hours, for a total volume of 44,500 gallons. A concentrated $\mathrm{KBr}$ solution was prepared in a 7,000-gallon mixing tank and diluted in-line during the injection to the required concentration using the local water supply (fire hydrant). The injection phase was followed by a 16-hour residence phase to determine the impact of groundwater drift on the injected plume during this period. The residence phase was followed by the withdrawal phase in which 140,400 gallons of groundwater were pumped from the injection/ withdrawal well at a rate of $90 \mathrm{gpm}$ for 26 hours. This withdrawal volume represented 3.2 injection volumes.

\subsection{Results and Discussion}

A mass balance calculation of the injected bromide mass and the withdrawal mass resulted in a $72 \%$ recovery after extracting 3.2 injection volumes (Figure 6.2). The withdrawal mass was calculated by integrating the bromide concentration (by IC) of samples collected from the withdrawal stream. 
Ft. Lewis Bromide Tracer Test - Injection/Withdrawal Well (RM-9)

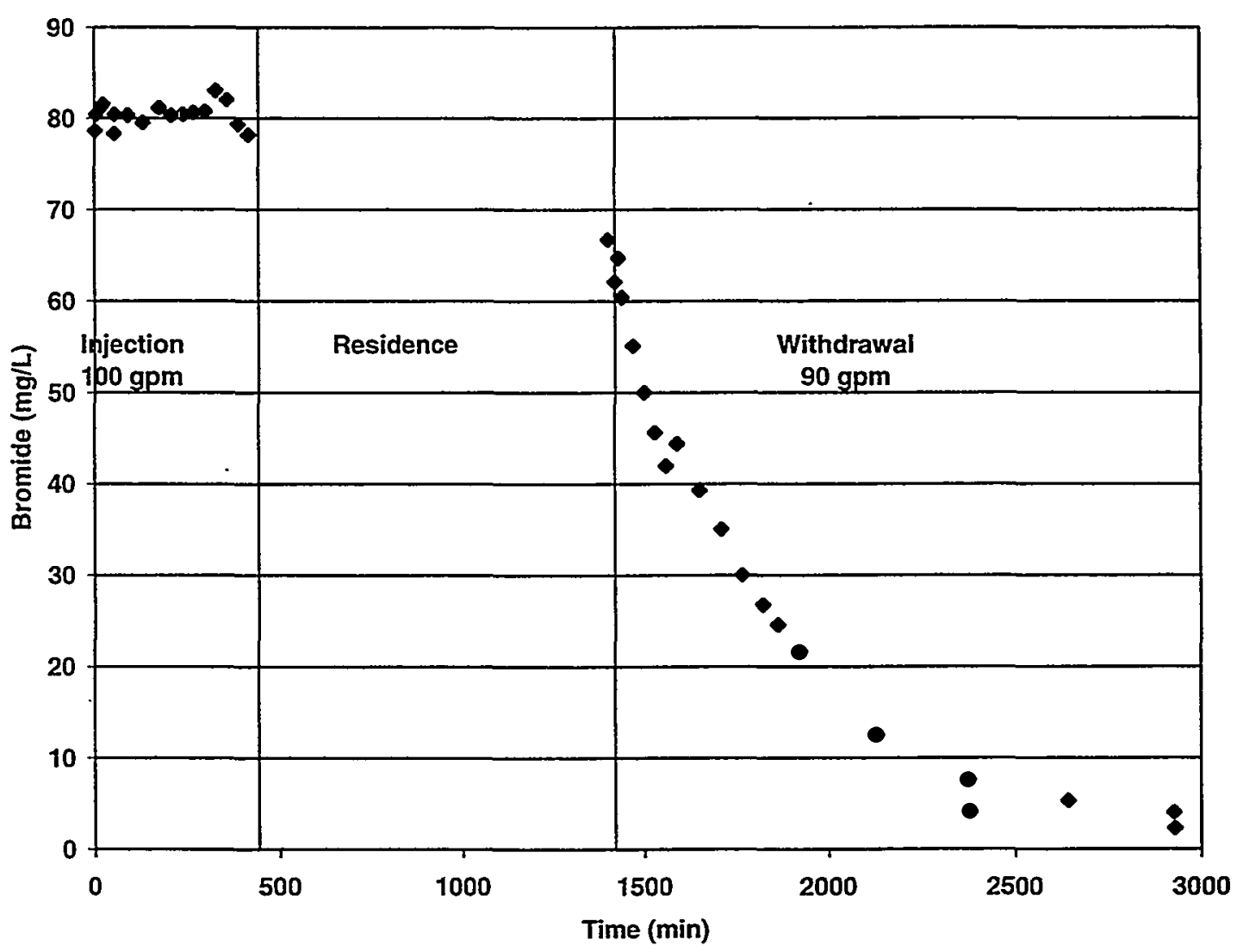

Figure 6.1. Fort Lewis ISRM Proof-of-Principle Site Bromide Tracer Test Showing the Duration of the Test Phases (injection/residence/withdrawal) and Bromide Concentrations from the Injection/Withdrawal Streams (Well RM-9)

Bromide breakthrough curves (BTCs) were constructed for all of the monitoring wells during the test and are included in Appendix F. A summary of the concentrations midway through ( $\sim 3.7 \mathrm{hr})$ and at the end of the injection phase $(\sim 7.4 \mathrm{hr})$ is provided in Table 6.1. At the time of the tracer test, only wells numbered up to RM-9 were installed (RM-10 to RM-15 were installed later). The BTCs and summary table show a significant variability in arrival times measured at the site that were not correlated with radial distance. One example of this variability can be seen in well $\mathrm{RM}-4$, which is located at a $40-\mathrm{ft}$ radial distance southeast of the injection well. Well RM-4 measured $68 \%$ of the injection concentration by the midpoint of the injection phase and had higher concentrations than three wells that were significantly closer to the injection well (RM-1A, RM-5, and RM-7). Bromide was not detected during the entire test in well RM-6, which is at a similar distance but on the opposite side of the injection well from RM-4. A radially symmetric analytic model (Hoopes and Harleman 1967) used to fit the BTC for RM-4 (using a 0.20 porosity and average thickness of the upper portion of the aquifer), showed that 4.6 times more flux than was used for the tracer test would be required to fit these measurements. 


\section{Ft. Lewis Br-Tracer Mass Balance}

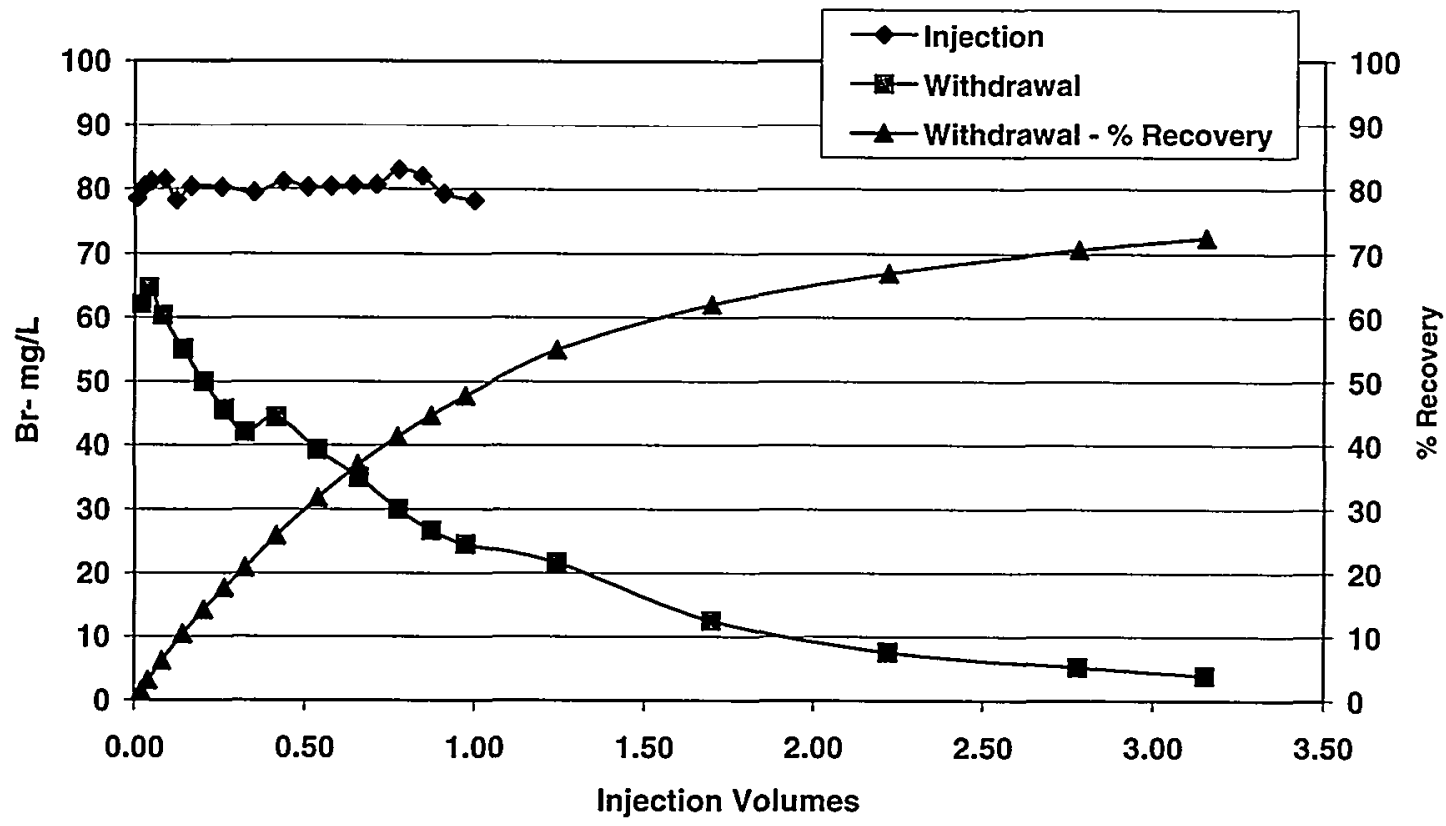

Figure 6.2. Mass Balance of Injected and Withdrawn Bromide to Calculate the Percent Bromide Mass Recovery Achieved During the Withdrawal (IC measurements for bromide from the injection and withdrawal streams [RM-9] are shown)

Table 6.1. Bromide Tracer Test Injection Concentration Summary

\begin{tabular}{|l|c|c|c|c|c|}
\hline Well Name & $\begin{array}{c}\text { Radial } \\
\text { Distance } \\
(\mathbf{f t})\end{array}$ & $\begin{array}{c}\text { Mid-Injection } \\
\text { Br- Conc. }^{-}\end{array}$ & $\begin{array}{c}\text { Mid-Injection } \\
\mathbf{B r}^{-} \text {\% }\end{array}$ & $\begin{array}{c}\text { End Injection } \\
\text { Br- Conc. }^{-} \\
(\mathbf{m g} / \mathbf{L})\end{array}$ & $\begin{array}{c}\text { End Injection } \\
\mathbf{B r}^{-} \text {\% }\end{array}$ \\
\hline RM-1A & 14.8 & 4.1 & 51 & 52 & 65 \\
\hline RM-1B & 15.5 & 1.1 & 1.4 & 3.1 & 3.9 \\
\hline RM-2 & 5.5 & 67 & 84 & 76 & 95 \\
\hline RM-3 & 80.2 & 0.7 & 0.9 & 3.3 & 4.1 \\
\hline RM-4 & 40.2 & 54 & 68 & 63 & 79 \\
\hline RM-5 & 17.5 & 52 & 65 & 66 & 82 \\
\hline RM-6 & 44.2 & 0 & 0.0 & 0 & 0.0 \\
\hline RM-7 & 24.2 & 2.1 & 2.6 & 13 & 16 \\
\hline RM-8 & 10.2 & 79 & 99 & 81 & 101 \\
\hline RM-9 & 0.0 & 80 & 100 & 79 & 99 \\
\hline
\end{tabular}


Additionally, well RM-3, which is $80 \mathrm{ft}$ southeast of the injection well (in the same direction as $\mathrm{RM}-4$ ), had detectable bromide by the second half of the injection phase. Although a three-dimensional heterogeneous model is needed to assess these BTCs in the context of the entire system (because the total flux injected will be apportioned based on the vertical and horizontal spatial variability in permeabilities), the use of a simple analytic model on individual BTCs can be useful for interpreting test results and helping guide future data collection.

While wells RM-3 and RM-4 on the southeastern side of the injection well would indicate overall preferential flow in this direction, wells RM-1a and RM-1b, which are closer to the injection well on this side (15 ft), had lower concentrations than RM-4. However, well RM-1a is partially screened across the lower portion of the Gg-u unit, while well RM-1b is screened at the very top of the unit, primarily in the upper till (Qvt1) unit. Well RM-10, which was installed at a later date for use in subsequent injection/ withdrawal tests (see discussion in Section 7), was screened over the depth interval between the RM$\mathrm{la} / \mathrm{lb}$ screen intervals to determine whether higher fluxes were occurring in the central/upper portion of the aquifer, which would help explain the BTCs measured at RM-4 and RM-3.

The BTCs for wells on the northwest side of the injection/withdrawal well (RM-8, RM-5, RM-7, and RM-6) seemed to exhibit a response more consistent with that expected for a radial injection. These BTCs all behaved monotonically, showing decreasing concentrations with increasing radial distance. However, analysis of these BTCs using the radially symmetric analytic model mentioned above showed a trend of decreasing flux required with increasing distance. Fluxes 3.5 times greater than used in the test (i.e., $350 \mathrm{gpm}$ in the model versus $100 \mathrm{gpm}$ in the test) were needed to fit the BTC for RM-8. For well RM-5, a flux 1.4 times greater than the actual flux used was needed to fit the BTC; and for RM-7, a flux of one-half that used for the test was needed. While the overall fluid mass balance was not conducted, the lower fluxes inferred near well RM-7 should help balance the higher fluxes estimated for other areas.

Well RM-2 was screened below the thin Qvt2 till layer to provide information on leakage through this unit associated with stresses applied in the injection/withdrawal well, which is screened above the Qvt2 layer. Bromide concentrations were monitored in well RM-2 during the tracer test. While significant bromide concentrations were measured in well RM-2 during the injection phase, indicating leakage through this zone, bromide concentrations in this lower portion of the aquifer recovered as rapidly as those within the upper portion of the aquifer during the withdrawal phase (see Appendix F).

Testing of groundwater sampling protocols before the tracer test and during the injection phase showed that a three-minute purge at $2 \mathrm{gpm}$ (six gallons total) was sufficient prior to sample collection. This was based on the stability of measured parameters (temperature, $\mathrm{pH}, \mathrm{EC}$, and DO) when switching between wells. The resulting purge volumes used were applicable only during forced gradient conditions at the site. 


\subsection{Treatment Zone Emplacement}

\subsection{Emplacement Strategy}

The ISRM treatment zone was created by injecting and withdrawing a sodium dithionite solution with a potassium carbonate/ bicarbonate $\mathrm{pH}$ buffer to reduce the naturally occurring $\mathrm{Fe}(\mathrm{III})$ in the aquifer sediments to $\mathrm{Fe}(\mathrm{II})$. Reagent injection and withdrawal were performed through a central well at the site (well RM-9, Figure 4.1) using a push-pull technique, as described previously. Bench-scale testing with aquifer sediments from the site (as detailed in Section 4.4 and Szecsody et al. 2000) determined that these sediments contain a large amount of reducible Fe(III) (mean of $0.35 \mathrm{wt} \%$ ). These laboratory studies also demonstrated that the TCE degradation rates are a function of the percentage of reduction of Fe(III) to $\mathrm{Fe}(\mathrm{II})$ in the sediments from the site. A significant percentage of reduction ( $>30$ to $40 \%$ ) is required to achieve the TCE degradation rates needed for adequate ISRM treatment zone performance at the Fort Lewis site with its low aquifer temperature $\left(11^{\circ} \mathrm{C}\right.$ ) and high groundwater velocity (up to $7 \mathrm{ft} /$ day). Multiple dithionite injection/withdrawal operations were required to meet these specifications. To gain an understanding of the relationship between iron reduction by dithionite and TCE degradation at the fieldscale, four dithionite injection/withdrawal tests were conducted with a time interval (several months) between each injection/withdrawal test for monitoring TCE and acetylene. These data were used for evaluating the interim ISRM performance at successively increasing percentages of iron reduction at the site.

The objective of the dithionite injection/withdrawal tests was to create a reducing zone in the targeted portion of the aquifer (unit Gg-u, as shown in Figure 4.2) to significantly lower dissolved TCE concentrations in groundwater migrating through the treatment zone. While the bench-scale studies had demonstrated the feasibility of the ISRM concept at a small scale, the field test incorporated all additional complexities of full-scale remediation (hydraulic conductivity/physical heterogeneity, iron oxide spatial heterogeneity, groundwater velocity, and direction variability). The injection/withdrawal well (RM-9) and most of the monitoring wells at the site are screened in the Gg-u unit. A thin, semi-continuous layer of glacial till (Qvt2) constitutes the base of the targeted treatment zone. Monitoring wells were installed below this lower permeability till layer to determine the amount of leakage that occurred during the injection/withdrawal tests.

The dithionite injection/withdrawal tests conducted at the site are summarized in Table 7.1. Analysis of the emplacement monitoring data and performance of Injection 1 resulted in changes in the emplacement design for the subsequent injections to increase emplacement efficiency. Changes in the emplacement design were related to minimizing density sinking effects caused by the reagent density, high aquifer permeability, and enhanced leakage from the targeted portion of the aquifer through the Qvt2 aquitard to the Gg-l unit in the lower portion of the aquifer. Another design change for tests following Injection 1 was heating the aquifer from an ambient temperature of 11 to 25 to $30^{\circ} \mathrm{C}$ to increase the dithionite/Fe(III) reduction reaction rate. Increasing the reduction reaction rate was of particular importance because density effects were limiting the dithionite/ sediment contact time in the upper Gg-u portion of the aquifer. Additional monitoring wells (RM-10, RM-11, RM-12, and RM-13) were installed at the 
Table 7.1. Summary of Fort Lewis ISRM POPT Field Injection Tests

\begin{tabular}{|c|c|c|c|c|c|}
\hline Test/Date & $\begin{array}{c}\text { Injection } \\
\text { Volume/Rate/ } \\
\text { Duration }\end{array}$ & $\begin{array}{c}\text { Injection } \\
\text { Concentration }^{(a) /} \\
\text { Temperature }\end{array}$ & $\begin{array}{l}\text { Residence } \\
\text { Duration }\end{array}$ & $\begin{array}{c}\text { Withdrawal } \\
\text { Volume/Rate/ Duration }\end{array}$ & $\begin{array}{c}\text { Recovery } \\
\%\end{array}$ \\
\hline $\begin{array}{l}\text { Bromide tracer } \\
10 / 13 / 98 \text { to } \\
10 / 15 / 98\end{array}$ & $\begin{array}{l}44,500 \mathrm{gal} / \\
100 \mathrm{gpm} / 7.4 \mathrm{hr}\end{array}$ & $\begin{array}{l}80 \mathrm{mg} / \mathrm{L} \mathrm{Br}- \\
15^{\circ} \mathrm{C}\end{array}$ & $16.1 \mathrm{hr}$ & $140,400 \mathrm{gal} / 90 \mathrm{gpm} / 26 \mathrm{hr}$ & $\begin{array}{c}72 \% \\
(3.2 \mathrm{IV})\end{array}$ \\
\hline $\begin{array}{l}\text { Dithionite } 1 \\
11 / 10 / 98 \text { to } \\
11 / 14 / 98\end{array}$ & $\begin{array}{l}50,600 \mathrm{gal} \\
(6,500 \mathrm{gal} \\
\text { reagent }) / \\
100 \mathrm{gpm} / 8.4 \mathrm{hr}\end{array}$ & $\begin{array}{l}0.07 \mathrm{M} \text { dithionite } \\
13^{\circ} \mathrm{C}\end{array}$ & $38.8 \mathrm{hr}$ & $\begin{array}{l}\mathrm{RM}-9: 246,850 \mathrm{gal} / \\
90 \mathrm{gpm} / 45.5 \mathrm{hr} \\
\mathrm{RM}-2: 11,500 \mathrm{gal} / 3 \mathrm{gpm} \\
\text { to } 4.8 \mathrm{gpm}\end{array}$ & $\begin{array}{l}\sim 50 \% \\
(5.1 \mathrm{IV})\end{array}$ \\
\hline $\begin{array}{l}\text { Injection } 2 \\
\text { preheat } \\
8 / 5 / 99 \text { to } 8 / 6 / 99\end{array}$ & $\begin{array}{l}129,000 \mathrm{gal} / \\
100 \mathrm{gpm} / 21.5 \mathrm{hr}\end{array}$ & $\begin{array}{l}\text { No Dithionite } \\
20 \text { to } 22^{\circ} \mathrm{C}\end{array}$ & NA & NA & NA \\
\hline $\begin{array}{l}\text { Injection } 2 \mathrm{a} \\
\text { preheat } \\
8 / 9 / 99 \text { to } \\
8 / 10 / 99\end{array}$ & $\begin{array}{l}44,400 \mathrm{ga} / \\
50.5 \mathrm{gpm} / \\
14.7 \mathrm{hr}\end{array}$ & $\begin{array}{l}\text { No Dithionite } \\
22^{\circ} \mathrm{C}\end{array}$ & NA & NA & NA \\
\hline $\begin{array}{l}\text { Dithionite } 2 a \\
8 / 10 / 99 \text { to } \\
8 / 14 / 99\end{array}$ & $\begin{array}{l}123,500 \mathrm{gal} \\
(5,569 \mathrm{gal} \\
\text { reagent }) / 50 \mathrm{gpm} \\
/ 40.9 \mathrm{hr}\end{array}$ & $\begin{array}{l}0.027 \mathrm{M} \text { dithionite } \\
22 \text { to } 28^{\circ} \mathrm{C}\end{array}$ & $32 \mathrm{hr}$ & $\begin{array}{l}\text { RM-9: } 124,500 \mathrm{gal} / \\
90 \mathrm{gpm} / 23 \mathrm{hr}\end{array}$ & $\begin{array}{c}27 \% \\
(1.0 \mathrm{IV})\end{array}$ \\
\hline $\begin{array}{l}\text { Dithionite } 2 b \\
8 / 16 / 99 \text { to } \\
8 / 22 / 99\end{array}$ & $\begin{array}{l}148,300 \mathrm{gal} \\
(5,922 \mathrm{gal} \\
\text { reagent }) / 50 \mathrm{gpm} \\
/ 49.4 \mathrm{hr}\end{array}$ & $\begin{array}{l}0.025 \mathrm{M} \text { dithionite } \\
24 \text { to } 30^{\circ} \mathrm{C}\end{array}$ & $30.8 \mathrm{hr}$ & $\begin{array}{l}\text { RM-9: } 344,319 \mathrm{gal} / \\
89.7 \mathrm{gpm} / 64 \mathrm{hr} \\
\mathrm{RM}-2: 79,145 \mathrm{gal} / \\
20.6 \mathrm{gpm} / 64 \mathrm{hr}\end{array}$ & $\begin{array}{c}71 \% \\
(2.9 \mathrm{IV})\end{array}$ \\
\hline $\begin{array}{l}\text { Injection } 3 \\
\text { preheat } \\
12 / 1 / 99 \text { to } \\
12 / 2 / 99\end{array}$ & $\begin{array}{l}51,000 \\
\mathrm{gal} / 50 \mathrm{gpm} / \\
17 \mathrm{hr}\end{array}$ & $\begin{array}{l}5 \times 10^{-4} \mathrm{M} \\
\text { dithionite } \\
28^{\circ} \mathrm{C}\end{array}$ & NA & NA & NA \\
\hline $\begin{array}{l}\text { Dithionite } 3 \\
12 / 2 / 99 \text { to } \\
12 / 9 / 99\end{array}$ & $\begin{array}{l}142,000 \text { gal } \\
(5,225 \text { gal } \\
\text { reagent }) / \\
50 \mathrm{gpm} / 47.2 \mathrm{hr}\end{array}$ & $\begin{array}{l}0.02 \mathrm{M} \text { dithionite } \\
29^{\circ} \mathrm{C}\end{array}$ & $47.85 \mathrm{hr}$ & $\begin{array}{l}\text { RM-9: } 374,000 \mathrm{gal} / 90 \\
\mathrm{gpm} / 71 \mathrm{hr} \\
\mathrm{RM}-2: 93,500 \mathrm{gal} / \\
20 \mathrm{gpm} / 71 \mathrm{hr}\end{array}$ & $\begin{array}{c}61 \% \\
(3.3 \mathrm{IV})\end{array}$ \\
\hline $\begin{array}{l}\text { Injection } 4 \\
\text { preheat } \\
3 / 21 / 00\end{array}$ & $\begin{array}{l}35,000 \mathrm{gal} / \\
50.4 \mathrm{gpm} / \\
11.5 \mathrm{hr}\end{array}$ & $\begin{array}{l}5 \times 10^{-4} \mathrm{M} \\
\text { dithionite } \\
26.5^{\circ} \mathrm{C}\end{array}$ & NA & NA & NA \\
\hline $\begin{array}{l}\text { Dithionite } 4 \\
3 / 21 / 00 \text { to } \\
3 / 29 / 00\end{array}$ & $\begin{array}{l}137,000 \mathrm{gal} \\
(6,069 \mathrm{gal} \\
\text { reagent }) / \\
40.6 \mathrm{gpm} / \\
55.9 \mathrm{hr}\end{array}$ & $\begin{array}{l}0.0275 \mathrm{M} \\
\text { dithionite } \\
31^{\circ} \mathrm{C}\end{array}$ & $76.5 \mathrm{hr}$ & $\begin{array}{l}\text { RM-9: } 333,000 \mathrm{gal} / 88 \\
\mathrm{gpm} / 63 \mathrm{hr} \\
\mathrm{RM}-2: 82,000 \mathrm{gal} / \\
22 \mathrm{gpm} / 63 \mathrm{hr}\end{array}$ & $\begin{array}{c}58 \% \\
(3.0 \mathrm{IV})\end{array}$ \\
\hline
\end{tabular}


site between Injection 1 and Injection 2 to better understand the spatial variability seen in the breakthrough curves during the tracer test (see Section 6) and dithionite Injection 1 . The specific details of the emplacement process and results are provided in Section 7.2.

\subsection{Emplacement Description}

The basic emplacement operation consisted of three phases; injection, residence, and withdrawal, as described in Section 6. The emplacement process is described below with specific details provided in the subsections related to each injection/withdrawal test. Table 7.1 summarizes the operations of each test. The main differences in the operation of the injection/withdrawal tests were between Injection 1 and the remaining injections $(2 a, 2 b, 3$, and 4$)$.

Approximately 6,000 gallons of concentrated sodium dithionite solution with a potassium carbonate/ bicarbonate $\mathrm{pH}$ buffer was delivered to the site in a tanker truck for each injection/withdrawal test. Prior to shipment to the ISRM site, the solution was chilled (during the dissolving process), and the headspace of the tank was blanketed with nitrogen gas to prevent oxidation with atmospheric oxygen. The molar concentration of potassium carbonate was four times that of the sodium dithionite to maintain a high $\mathrm{pH}$ for enhanced stability of dithionite. This results in a pH of 11 in the injection solution. The mass of chemicals used for each injection/withdrawal test was as follows:

$\begin{array}{ll}\text { Sodium dithionite (90\% purity, low trace metals) } & 5,300 \mathrm{lb}(2400 \mathrm{~kg}) \\ \text { Potassium carbonate } & 15,000 \mathrm{lb}(6800 \mathrm{~kg}) \\ \text { Potassium bicarbonate } & 1,000 \mathrm{lb}(450 \mathrm{~kg})\end{array}$

The concentrated reagent was pumped directly from the tanker truck and diluted inline using a local water supply from a fire hydrant near the site. The volume of concentrated reagent in the tanker truck was calculated onsite from tank level measurements and was used to determine the injection rate of the concentrated reagent. The dithionite concentration was also measured from the tanker truck solution to verify the mass and purity delivered. A complete description of the process and analytical equipment used at the site is provided in Section 5. Dithionite was measured at the field site in the field trailer using two automated high-performance liquid chromatography (HPLC) systems connected to syringe pumps for sample dilution with anoxic water. Because of its instability, dithionite must be measured at the site shortly after sample collection. A positive pressure of argon gas was maintained within the tanker of concentrated dithionite solution throughout the injection.

Injection into well RM-9 began after the concentrated solution and dilution water flow rates had been determined and set. During all phases of these tests, aqueous samples were collected from the sampling manifold inside the field trailer. In-line probes were connected to the sampling manifold for direct measurement of temperature, $\mathrm{EC}, \mathrm{pH}, \mathrm{DO}$, and $\mathrm{Eh}$. Aqueous samples for dithionite measurement were collected directly into $10-\mathrm{mL}$ syringes via a luer-lock fitting on the manifold to prevent oxidizing the samples with atmospheric oxygen. These samples were measured immediately after collection for dithionite concentration. A second station in the field trailer was used to verify the field parameter measurements $(\mathrm{DO}, \mathrm{EC}, \mathrm{pH})$ made with the in-line probes at the sampling manifold. This system used a syringe pump to inject a sample collected into a $10-\mathrm{mL}$ syringe at the sampling manifold into a series of 
microelectrodes. Archive samples were also collected at the sampling manifold for laboratory analysis of sulfur, major anions, and trace metals. The samples collected for trace metals were filtered using a disposable $0.45 \mu \mathrm{m}$ filter and preserved with ultra-pure nitric acid. Chemical data collected during the test were recorded on datasheets, entered into electronic spreadsheets, and plotted to monitor the progress of the tests in the field trailer.

Following the injection phase, the residence phase provided additional time for the dithionite to react with the aquifer sediments. Aqueous samples were collected during the residence phase and measured for dithionite and field parameters. The duration of the residence phase was determined by the dithionite concentrations measured at the site. Because the reagent plume drifts with the groundwater flow and is denser than the groundwater, an extended-duration residence phase can reduce the recovery of unreacted reagent and reaction products during the withdrawal phase (Williams et al. 1994).

During the withdrawal phase, unreacted reagent and reaction products were extracted by pumping from the central injection/withdrawal well (RM-9). The withdrawal water was disposed of in a sanitary sewer located on the western side of the main DRMO building. Permission was obtained from Fort Lewis for disposal of the withdrawal water based on the water quality and volume of the withdrawal stream. Samples collected from the injection and withdrawal streams were analyzed for total sulfur, which accounts for the dithionite reaction products (sulfate, sulfite, and thiosulfate), and used to calculate a mass balance (i.e., percent of injected reagent recovered). Monitoring during the withdrawal phase of Injection/Withdrawal 1 showed that the concentrations measured in well RM-2 (located below the targeted portion of the aquifer) were not decreasing as rapidly as measured during the tracer test, which was due to sinking of the dense reagent. To improve the recovery percentage, withdrawal from well RM-2 was added to the total withdrawal stream using the sampling pump. During subsequent injection tests (i.e., Tests 2 through 4), a higher capacity submersible pump was installed to further improve recovery and reduce residual chemicals in this lower portion of the aquifer. The total volume of water withdrawn during the withdrawal phases ranged from 3 to 5 injection volumes (IV). The percentage of recovery was calculated for each dithionite injection/withdrawal test (see Table 7.1).

\subsubsection{Dithionite Injection/Withdrawal 1}

The reagent concentration for Injection/Withdrawal 1 was $0.07 \mathrm{M}$ sodium dithionite solution, with $0.28 \mathrm{M}$ potassium carbonate, and 0.028 potassium bicarbonate, resulting in a solution density of $1.051 \mathrm{~g} / \mathrm{cm}^{3}$. A total volume of 50,600 gallons of reagent was injected into well RM-9 at a rate of $100 \mathrm{gpm}$ for 8.4 hours. Aqueous samples were collected at roughly five-minute intervals with samples collected from the injection stream and all the monitoring wells every hour. BTCs for dithionite and EC are provided for the injection/withdrawal well and the monitoring wells in Appendix F. Figure 7.1 shows the dithionite and EC measurements for well RM-9 and selected monitoring wells during the test. Comparisons of BTCs from previous ISRM field demonstrations (Fruchter et al. 1996) have shown that the EC measurements behave much like a conservative tracer (e.g., bromide). EC and dithionite measurements relative to the injection concentration can be used to determine the amount of dithionite that has reacted along the flow path from the injection/withdrawal well to the specific monitoring well. This can be seen in the separation between the dithionite and EC breakthrough curves in Figure 7.1b for well RM-8. The dithionite concentration for RM- 8 was $85 \%$ of the injection concentration by the end of 

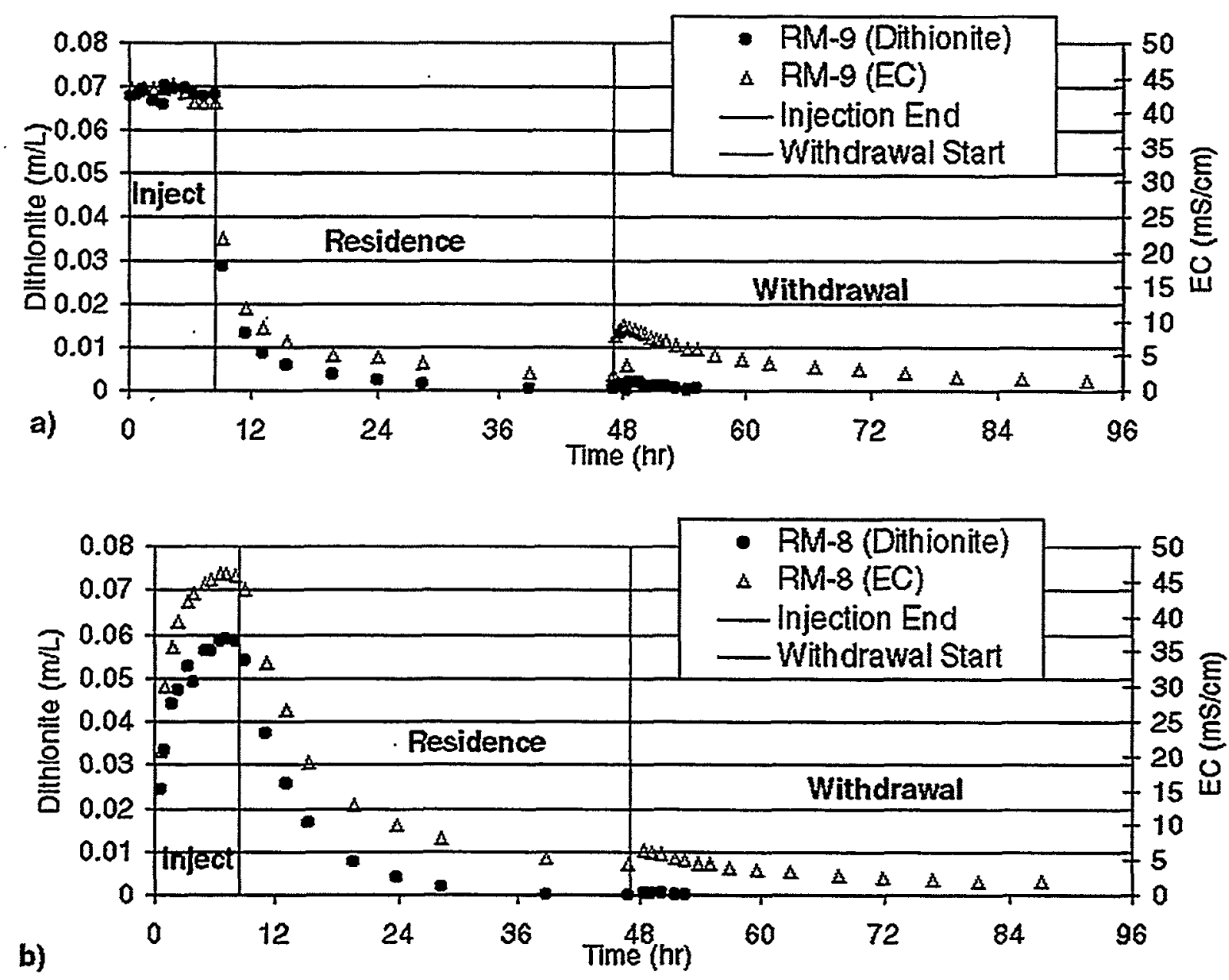

b)

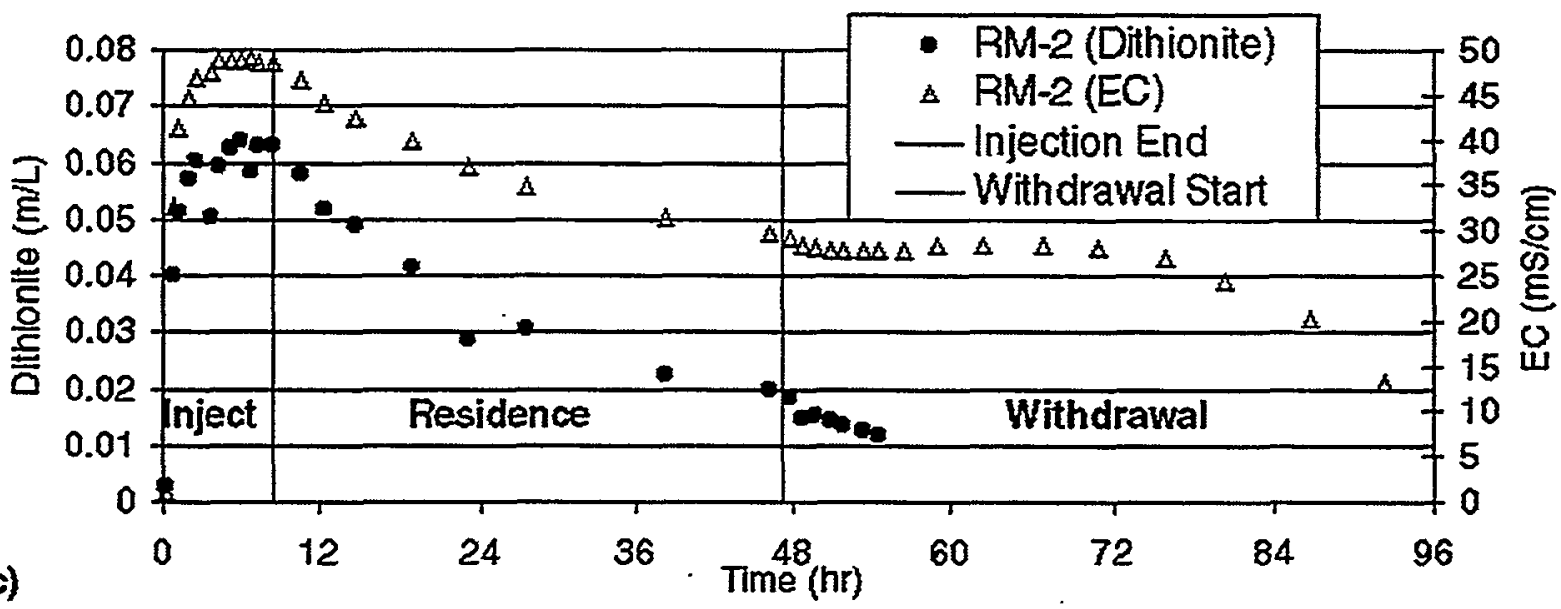

Figure 7.1. Dithionite Injection/Withdrawal Test 1. Dithionite and EC BTC for selected wells: RM-9 (injection/withdrawal stream), well RM-8 (at a radial distance of $10 \mathrm{ft}$ ), and well RM-2 (at a radial distance of $5 \mathrm{ft}$ but completed in the lower portion of the aquifer). BTCs for all wells are in Appendix F. Details on the test are provided in Table 7.1. 
the injection, even though the EC at RM-8 indicated full injection EC concentration. This difference is due to consumption of dithionite by both the disproportionation and iron reduction reactions along the flow path from the injection well to $\mathrm{RM}-8$ (at a 10-ft radial distance).

The overall extent of the injected dithionite plume was similar to the extent of the bromide plume in the tracer test (see Section 6), with notable differences during the residence phase due to the higher density of the dithionite solution. The extent of the dithionite plume on the southeast side of the injection/ withdrawal well was greater than measured on the northwest side. Well RM-4, to the southeast of well RM-9 at a radial distance of $40 \mathrm{ft}$, had a concentration and arrival time response similar to well RM-5, which is only $17 \mathrm{ft}$ to the northwest of the injection/withdrawal well. Well RM-4 also saw a higher concentration than well RM-7, which is $25 \mathrm{ft}$ northeast of the injection/withdrawal well. Trace amounts of dithionite and increases in EC were measured in wells farthest from the injection/withdrawal well (RM-3, $80 \mathrm{ft}$ to the southeast and RM-6, $44 \mathrm{ft}$ to the northwest). An analysis of the dithionite concentrations and iron reduction estimates for each well during the injection tests is provided in Section 7.3.

The duration of the residence phase for Injection 1 was 38.8 hours. The most pronounced effect from density sinking can be seen during the residence phase in Figures 7.1a and $\mathrm{b}$. This caused the rapid decrease in EC measurements for wells screened in the targeted portion of the aquifer (Gg-u). EC measurements during the residence phase decreased by $94 \%$ for well RM-9 (7.1a) and $90 \%$ for well RM8 (Figure 71.b). The decrease in EC during the residence phase was much less for RM-2, which was screened below the thin Qvt2 aquitard layer (shown in Figure 7.1c). Effects of the increased density of the dithionite solution on the BTCs can be seen in Figure 7.2, which compares the EC BTCs from the dithionite test and bromide BTCs from the tracer test.

Minimal density effects are expected from the bromide solution during the tracer test. In the tracer test, only a $14 \%$ decrease in tracer concentration was measured for well RM-9 (Figure 7.2a) and a 19\% decrease for well RM-8 (Figure 7.2b) during the residence phase. While the residence for the tracer test was much shorter than it was in Injection 1, most of the decrease occurred in the first 12 hours, as shown in Figures 7.2 $\mathrm{a}$ and 7.2b. For well RM-2, the decrease in tracer concentration during the residence phase of the tracer test was greater than the $\mathrm{EC}$ decrease for this well during Injection 1 (Figure 7.2c). As shown in the example of well RM-8 in Figure 7.2b, the EC arrivals for wells screened in the targeted portion of the aquifer were slower than the bromide arrival time during the injection phase. Both were conducted at the same injection rate (100 gpm). Well RM-2, which is screened in the lower portion of the aquifer, had a faster EC arrival during the dithionite injection test than the bromide did during the tracer test. The relative peak concentration was also greater for EC than for bromide for well RM-2. Losses of reagent due to sinking to the lower portion of the aquifer slowed down the advection of reagent in the upper portion of the aquifer. Density effects were enhanced at the Fort Lewis site over other ISRM field test sites due to the high permeability of the aquifer and the leaky Qvt2 aquitard at the base of the targeted aquifer. Comparison of EC BTCs with bromide tracer BTCs at sites with lower permeability aquifers and more continuous aquitards bounding the bottom showed much less pronounced density effects during all phases of testing. 

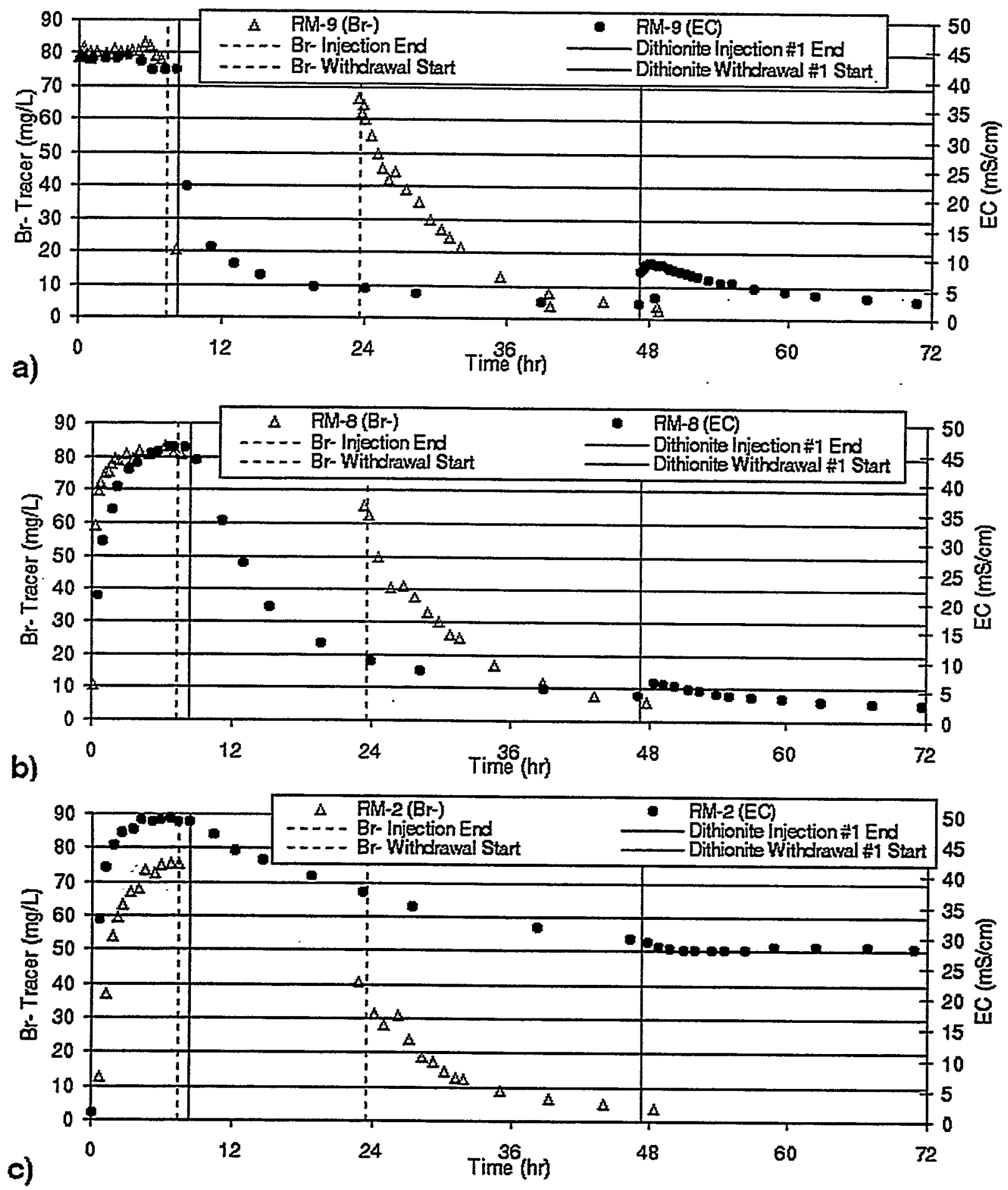

Figure 7.2. Comparison of Breakthrough Curves Between the Bromide Tracer Test $\left(\mathrm{Br}^{-}\right)$and EC from Injection 1 for Selected Wells. Details of the tests are provided in Table 7.1. 
During the withdrawal phase, 258,000 gallons were pumped from wells RM-9 and RM-2; this was 5.1 times the injection volume. The majority of the withdrawal water was pumped from well RM-9 at $90 \mathrm{gpm}$ for $45.5 \mathrm{hr}$ (246,850 gallons from RM-9 versus 11,500 gallons from RM-2). Extraction from well RM-2 was conducted during the withdrawal phase using the submersible sampling pump after it was observed that the concentrations were not decreasing as rapidly as expected based on the tracer results (see Figure 7.2c). The volume extracted from RM-2 is estimated because the discharge was not measured with a flow meter. The percent recovery for the withdrawal phase of this test, based on the total sulfur mass balance of the injection and withdrawal stream, was approximately $50 \%$. The recovery calculated from well RM-9 was $43 \%$ with an estimate of $7 \%$ for RM-2. Density effects were evident in well RM-2 from observations of concentration response to pumping. Pumping from well RM-2 was started and stopped throughout the withdrawal phase. Concentrations measured in this well decreased during the short intervals of pumping but rebounded once pumping had ceased.

For Injection 1, density sinking limited the contact time of dithionite with the sediments in the targeted portion of the aquifer ( $\mathrm{Gg}-\mathrm{u}$ ). Additionally, the low aquifer temperature yielded slow reaction rates for the dithionite/Fe(III) reduction reaction. These combined effects resulted in a low efficiency of iron reduction. Reduced iron estimates based on average dithionite concentrations and temperature for all the tests are provided in Section 7.3. Changes in the design and operation for the remaining injection/ withdrawal tests to increase their efficiency are described in the following section.

\subsubsection{Dithionite Injection/Withdrawal $2 \mathrm{a}, 2 \mathrm{~b}, 3$, and 4}

Factors negatively impacting the efficiency of Injection/Withdrawal 1 were aquifer temperature, density effects, and heterogeneities. The design and operation of subsequent dithionite injection/ withdrawal tests were changed after the analysis of the results from Injection 1 . The changes that were implemented more than doubled the percent iron reduction during the remaining injection/withdrawal tests using the same mass of dithionite based on iron reduction estimates from dithionite BTCs (see Section 7.3). Changes made for Injection/Withdrawal tests $2 a, 2 b, 3$, and 4 are described below.

Ambient aquifer temperatures at the site are approximately $11^{\circ} \mathrm{C}$. The temperature of the local water source used for diluting the concentrated reagent was only about $13^{\circ} \mathrm{C}$. These low temperatures resulted in slow kinetics of the dithionite/iron reduction reaction (see Szecsody et al. 2000). A steam boiler was used at the site to heat the local water to $20^{\circ}$ to $30^{\circ} \mathrm{C}$ during injection. The thermal storage of the aquifer sediments necessitated the addition of a pre-heat phase prior to the injection of dithionite. Approximately three pore volumes of water are needed to raise the temperature of a unit volume of saturated sediments to the input water temperature based on estimates of porosity and specific heat of the sediments at the site (assuming sufficient time for thermal equilibrium).

To minimize the impact of density effects, the injection concentration was lowered from the $0.07 \mathrm{M}$ dithionite used in Injection 1 to an average of $0.025 \mathrm{M}$ for subsequent injections. The potassium carbonate buffer concentration was also lowered to maintain the $4 \mathrm{x}$ concentration ratio. This lowered the reagent solution density to $\sim 1.017 \mathrm{~g} / \mathrm{cm}^{3}$, which reduced the fluid density contrast between the reagent and groundwater in the aquifer and lowered the vertical buoyancy force. The reduction in injection concentration resulted in an increase in the injection volume for the same mass of dithionite. The 
injection rate was reduced from the $100 \mathrm{gpm}$ used in Injection/Withdrawal 1 to about $50 \mathrm{gpm}$ for two reasons. First, to limit the radial extent of the dithionite injection to create a smaller zone of higherpercentage iron reduction near the injection/withdrawal well rather than creating a very large area of low iron reduction. Szecsody et al. (2000) has shown in laboratory experiments that there is a nonlinear relationship between the percentage of iron reduction and TCE degradation rates. In general, the benefits of a smaller reduced zone with greater iron reduction outweigh the benefits of a larger reduced zone with lower iron reduction. The lower injection rates provide more contact time for the dithionite solution with sediments closer to the injection well. The second reason for using a lower injection rate is that it provides for a longer injection phase where the advective field created by the injection dominates the density sinking forces. In this case, the majority of iron reduction occurs during the injection phase. For an alternative injection strategy of high concentration and rapid injection (as used in Injection 1), the majority of the iron reduction would occur in the residence phase.

Four additional wells were installed at the site following dithionite Injection/Withdrawal 1 (RM-10, RM-11, RM-12, and RM-13) to address heterogeneities and increase our understanding of the spatial distribution of reagent during the injections. Wells RM-11 and RM-13 were installed to determine the extent of the reagent plume in the north and south directions, which were not covered by previously installed ISRM monitoring wells. Given the spatial variability at the site and the completion design of existing monitoring wells, information from non-ISRM wells could not be used to monitor reagent extent in these directions. Well RM-10 was installed in the center of the targeted treatment zone near well RM-1A to determine whether higher flow rates were occurring in the center of the aquifer (as indicated by the relatively rapid BTC in well RM-4 to the southeast). The last new monitoring well installed during this phase of drilling, RM-12, was installed to determine the extent of the reagent transport below the Qvt2 layer (the same unit monitored by well RM-2 but at a greater radial distance).

Details on each of the dithionite injection/withdrawal tests are provided in Table 7.1. EC, dithionite, and temperature BTCs for all the wells in these tests are provided in Appendix F. Because of the reduction in flow rate, longer injection period, and increased number of monitoring wells, the sampling frequency was decreased to three hours for all the wells in Tests 2,3, and 4. An hourly frequency for monitoring was used for Test 1 . There was a nine-month period between Injection/Withdrawal 1 and the remaining tests that was used for additional laboratory bench-scale tests, installation of additional monitoring wells, analysis of the results of the bromide tracer test and dithionite Injection/Withdrawal 1, and to revise the design for the remaining emplacement operations. The interval between Injection/ Withdrawal 2, 3, and 4 was about three months to provide time for three groundwater monitoring events to assess the interim TCE degradation performance at successively increased percentages of iron reduction. The following sections provide additional information on Injection Tests 2 through 4.

\subsubsection{Injection/Withdrawal Tests $2 a / 2 b$}

Two dithionite injection/withdrawal operations, each involving a single tanker-truck delivery to the site, were conducted in succession as part of Injection/Withdrawal 2 (2a and $2 b$ ). The two injection/ withdrawal tests were combined to increase the percentage of reduction in the reduced zone rapidly to levels closer to that needed for significant TCE degradation rates, as shown by the bench-scale tests. The low percentage of reduction created by these early injections was well below the threshold determined by 
bench-scale tests, so the tests could be combined without losing significant performance data. Since these tests were conducted sequentially, only one preheat phase was required for both, and the withdrawal phase for Test $2 \mathrm{a}$ was shortened. This reduced the overall effort required for the two tests.

The preheat phase of Injection 2 involved the injection of 129,000 gallons of water from the fire hydrant that was routed through the boiler and heated to $20^{\circ}$ to $22^{\circ} \mathrm{C}$ prior to injection at $100 \mathrm{gpm}$ for $21.5 \mathrm{hr}$. Aquifer temperatures increased from the ambient value of $11^{\circ}$ or $12^{\circ} \mathrm{C}$ to $17^{\circ}$ to $22^{\circ} \mathrm{C}$ in the targeted portion of the aquifer during the initial $21.5 \mathrm{hr}$ preheat, as shown for selected wells in Figure 7.3. Because the fire hydrant water had a lower EC (i.e., TDS) than the groundwater, the arrival of the injected water can also be seen in decreasing EC BTCs. Injection of the warm water ceased for three days and was restarted for another $14.7 \mathrm{hr}$ at a lower injection rate, $50.5 \mathrm{gpm}$, for $14.7 \mathrm{hr}$, resulting in the injection of an additional 44,400 gallons of water at $22^{\circ} \mathrm{C}$. The decrease in temperature during the three-day hiatus between aquifer preheating events was from $1^{\circ}$ to $3^{\circ} \mathrm{C}$.

Dithionite injection began after the second preheat operation. Dithionite Injection/Withdrawal Test 2a consisted of injecting 123,500 gallons of $0.027 \mathrm{M}$ dithionite solution at a rate of $50 \mathrm{gpm}$ for $40.9 \mathrm{hr}$. The injection phase was followed by a 32-hr residence phase. Density effects during the residence phase were less than the sinking observed in Injection 1 as shown for selected wells screened in the upper portion of the aquifer in Figure 7.4a and $\mathrm{b}$. The EC concentrations during $2 \mathrm{a}$ for RM-9 (Figure 7.4a) and RM-8 (Figure 7.4b) decreased by $67 \%$ and $54 \%$, respectively. For comparison, during the residence phase of Injection 1, EC measurements decreased by $94 \%$ for RM-9 (Figure 7.1a) and $90 \%$ for RM-8 (Figure 7.1b).

The volume of withdrawal water extracted relative to the injection volume for Test $2 \mathrm{a}$ was less than that in other tests because a second injection test (2b) was planned to directly follow Injection $2 a$. One injection volume (IV) of groundwater was extracted during the withdrawal phase from well RM-9 only, at a pumping rate of $90 \mathrm{gpm}$ for $23 \mathrm{hr}$. The lower-volume withdrawal for this test was done to prevent cooling the heated aquifer by pulling the surrounding lower-temperature groundwater toward the injection/withdrawal well. In addition, higher-density residuals in the lower portion of the aquifer would lower the density contrast during injection into the upper portion of the aquifer and reduce density sinking for Injection $2 \mathrm{~b}$. The mass balance calculated from the sulfur measurements for withdrawal stream samples resulted in only a $27 \%$ recovery after the one IV withdrawal for Test $2 \mathrm{a}$. The $2.9 \mathrm{IV}$ withdrawal for Test $2 b$ resulted in $71 \%$ recovery. The higher recovery for Test $2 b$ was due in part to the low recovery of residuals from Test $2 \mathrm{a}$. The withdrawal phase for Test $2 \mathrm{~b}$ involved pumping from both wells RM-9 (89.7 gpm) and RM-2 (20.6 gpm).

With the changes in design, it was estimated that more than twice the iron reduction took place in the targeted portion of the aquifer during Injection 2, than in Injection 1. These reduction estimates were made from the average dithionite concentration, temperature, and duration of the injection phases for each of the tests (see Section 7.3). Monitoring results from the new wells installed after Injection 1 showed that arrival times for the wells installed in the targeted portion of the aquifer at $15 \mathrm{ft}$ radial distances to the northeast (RM-13) and southwest (RM-11) were faster than those measured toward the northwest (RM-5). The arrival time in RM-10, located toward the southeast and screened over the central portion of the 

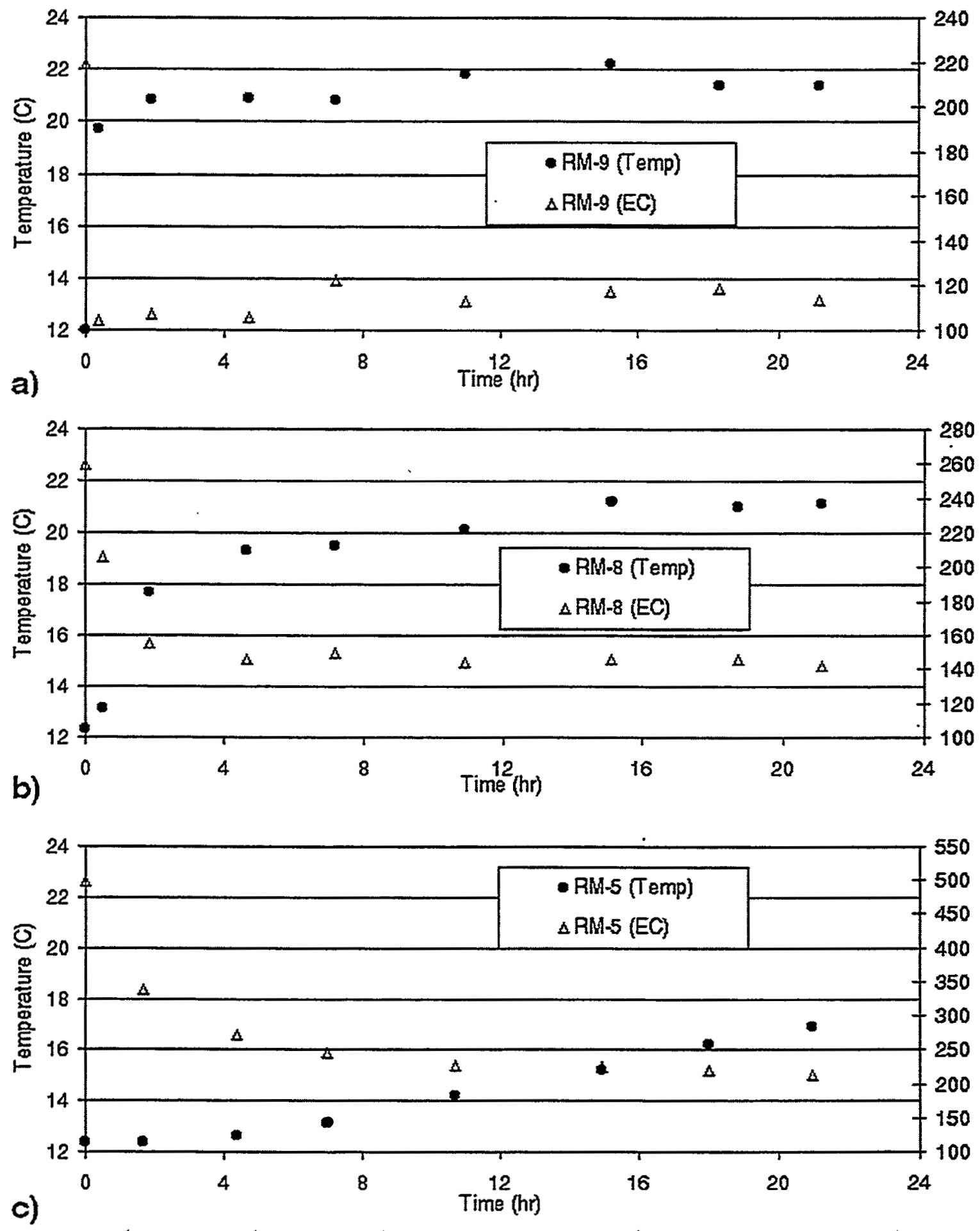

Figure 7.3. Temperatures and EC BTC Measured During the Preheat Phase for Dithionite Injection/ Withdrawal 2. The EC of the heated injection water was lower than the initial EC in the aquifer, resulting in decreasing BTCs for EC. 

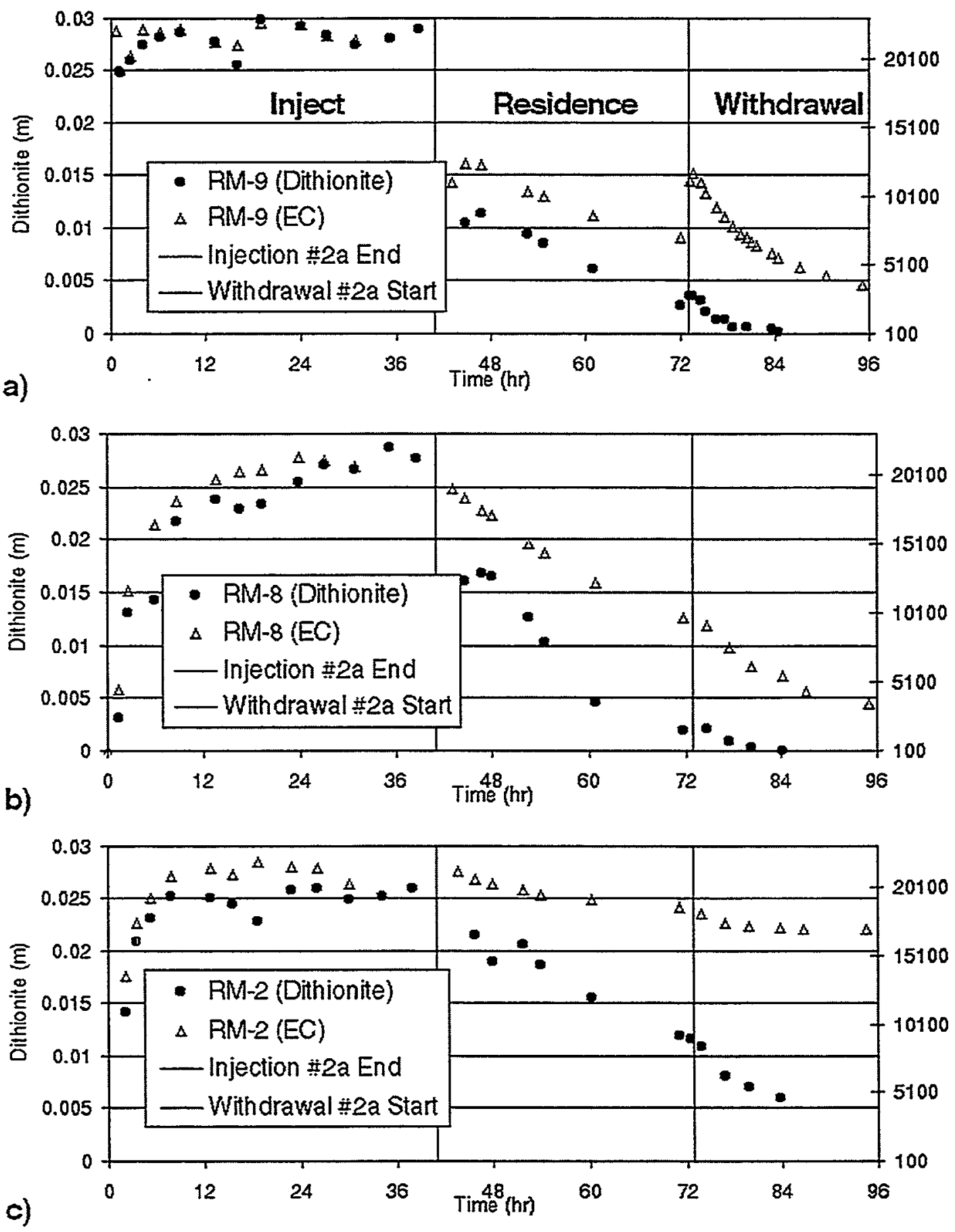

Figure 7.4. Dithionite Injection/Withdrawal Test 2a. Dithionite and EC BTC for Selected Wells: RM-9 (injection/withdrawal stream), well RM-8 (at a radial distance of $10 \mathrm{ft}$ ), and well RM-2 (at a radial distance of $5 \mathrm{ft}$ but completed in the lower portion of the aquifer). The design of Test $2 \mathrm{a}$ and subsequent tests was changed to use lower reagent concentrations to minimize density effects (compare with Figure 7.1). 
targeted zone (i.e., the depth interval between the RM-1a/lb screen interval depth) was faster than that measured for the RM-1a/1b cluster, indicating greater flux in the central/upper portion of the Gg-u unit. As discussed in Section 6.3, these data help explain the discrepancy in breakthrough response between the $\mathrm{RM}-1 \mathrm{a} / 1 \mathrm{~b}$ well cluster and wells RM-3 and RM-4, all to the southeast of the injection well. Lastly, the well installed below the Qvt2 layer in the Gg-1 unit (RM-12) showed substantial EC and dithionite concentrations, but they were lower and more delayed than RM-2, which is closer to the injection/ withdrawal well. Concentrations also decreased relatively rapidly in RM-12 during the withdrawal phase. A submersible pump was installed in well RM-12 to enhance recovery if required. Based on the EC concentration decrease observed in RM-12 during the withdrawal phase using wells RM-9 and RM-2 for extraction, withdrawal from RM-12 was not required.

\subsubsection{Injection/Withdrawal Test 3}

Dithionite Injection/Withdrawal Test 3 , which was conducted approximately three months after Injection 2, used a design similar to Injection 2. The modifications from Injection 2 included the addition of low concentrations of dithionite to the water used during the preheat phase to strip dissolved oxygen from the injected water. The concentrated dithionite solution was added to the $50 \mathrm{gpm}$ preheat injection stream using an FMI pump at about $150 \mathrm{~mL} / \mathrm{min}$. Slightly higher temperatures were used both during the aquifer preheat and during the dithionite injection to further enhance the dithionite/iron reaction rates. The lower dithionite concentration in Test $3,0.02 \mathrm{M}$ (compared with 0.025 in Test $2 \mathrm{~b}$ ) was due to a reduction in dithionite purity in the chemical shipment received for Injection 3 . The purity of the batch of dithionite used in this test was $20 \%$ lower than in previous tests. The total sulfur measurements from injection stream samples in Test 3 accounted for the total mass of chemical expected, but the sulfur was in a less reactive form than dithionite (e.g., sulfites). The recovery from the withdrawal phase of Test 3 was calculated at $61 \%$ of the injected mass after extracting 3.3 injection volumes.

\subsubsection{Injection/Withdrawal Test 4}

Dithionite Injection/Withdrawal Test 4 was the final reagent injection planned for treatment zone emplacement at the ISRM POPT site. This test also added low concentrations of dithionite to the injection stream during the preheat phase to remove dissolved oxygen from the water (similar to Test 3 ). The injection rate was slightly lower than that used in Injections 2 and 3, and the temperatures were slightly higher to further increase the amount of reduction near the injection/withdrawal well. A smaller withdrawal volume was also used in Test 4 than in Test $3(3.0 \mathrm{IV}$ versus $3.3 \mathrm{IV}$ ), which resulted in a $3 \%$ decrease in the estimated percent recovery during the withdrawal phase.

\subsection{Estimation of Percent Reduction from Dithionite Injection Data}

TCE is abiotically degraded by interaction with reduced Fe(II) species in the zone of the aquifer that has been treated with dithionite (see Section 1.2). To determine the efficiency of the field-scale injection strategy, the fraction of reduced iron resulting from the four dithionite injections was estimated. Data summarized from field injections includes dithionite concentration (Table 7.2), dithionite-sediment contact time (Table 7.3), and temperature (Tale 7.4). These data were used to estimate the fraction of iron oxides in the reduced sediments. Controlled laboratory experiments were used to establish rates of TCE 
degradation with Fort Lewis sediments under differing contact times, total mass of iron oxides, fraction of sediment reduced, temperature, and ionic strength (Szecsody et al. 2000). The effect of groundwater flow on iron reduction is minimal during the brief $(<100 \mathrm{hr})$ sediment-dithionite contact time required for iron reduction. The actual dithionite concentration observed at a well was more a complex function than a single concentration, but for estimating iron reduction, the integrated average concentration during dithionite breakthrough was used.

Injections 1 through 4 resulted in stepped increases in sediment reduction. For a homogeneous aquifer, locations nearest the injection well should be more highly reduced, given greater contact time with a higher dithionite concentration (and likely a higher temperature). The aquifer at Fort Lewis is very heterogeneous and anisotropic, as shown in the dithionite concentration and contact time data, where each well received a unique dithionite breakthrough curve that resulted in sediment reduction.

Based on the observed dithionite contact times and concentrations at the available monitoring wells, it was estimated that the series of injection sequences at the POPT site achieved a roughly cylindrical reduced iron zone in which the iron oxides in the sediments near the injection/withdrawal well were $50 \%$ to $60 \%$ reduced, with a much larger area surrounding it containing a lower percentage of iron reduction (Table 7.5). These data are also shown contoured for wells within the targeted portion of the aquifer

Table 7.2. Average Dithionite Concentration During Injections 1 Through 4

\begin{tabular}{|c|c|c|c|c|c|}
\hline Well & $\begin{array}{c}\text { Injection 1 } \\
\text { Dithionite } \\
(\mathbf{m o l} / \mathbf{L})\end{array}$ & $\begin{array}{c}\text { Injection 2a } \\
\text { Dithionite } \\
(\mathbf{m o l} / \mathbf{L})\end{array}$ & $\begin{array}{c}\text { Injection 2b } \\
\text { Dithionite } \\
(\mathbf{m o l} / \mathbf{L})\end{array}$ & $\begin{array}{c}\text { Injection 3 } \\
\text { Dithionite } \\
(\mathbf{m o l} / \mathbf{L})\end{array}$ & $\begin{array}{c}\text { Injection 4 } \\
\text { Dithionite } \\
(\mathbf{m o l} / \mathbf{L})\end{array}$ \\
\hline la & 0.015 & 0.01 & 0.012 & 0.006 & 0.01 \\
\hline Ib & Trace & Trace & Trace & Trace & Trace \\
\hline 2 & 0.04 & 0.025 & 0.0 .02 & 0.018 & 0.025 \\
\hline 3 & 0.01 & 0.02 & 0.005 & 0.005 & 0.006 \\
\hline 4 & 0.01 & 0.01 & 0.012 & 0.010 & 0.015 \\
\hline 5 & 0.01 & 0.01 & 0.015 & 0.012 & 0.014 \\
\hline 6 & None & Trace & Trace & Trace & Trace \\
\hline 7 & None & Trace & Trace & Trace & Trace \\
\hline 8 & 0.035 & 0.022 & 0.025 & 0.02 & 0.025 \\
\hline 9 & 0.07 & 0.03 & 0.025 & 0.02 & 0.028 \\
\hline 10 & -- & 0.01 & 0.014 & 0.014 & 0.017 \\
\hline 11 & -- & 0.012 & 0.01 & 0.010 & 0.014 \\
\hline 12 & -- & 0.01 & 0.012 & 0.008 & 0.013 \\
\hline 13 & -- & 0.02 & 0.022 & 0.02 & 0.025 \\
\hline
\end{tabular}


Table 7.3. Dithionite-Sediment Contact Time During Injections 1 Through 4

\begin{tabular}{|l|c|c|c|c|c|}
\hline Well & $\begin{array}{c}\text { Injection \#1 } \\
\text { Contact } \\
\text { Time (hr) }\end{array}$ & $\begin{array}{c}\text { Injection } \\
\text { \#2a Contact } \\
\text { Time (hr) }\end{array}$ & $\begin{array}{c}\text { Injection } \\
\text { \#2b Contact } \\
\text { Time (hr) }\end{array}$ & $\begin{array}{c}\text { Injection \#3 } \\
\text { Contact } \\
\text { Time (hr) }\end{array}$ & $\begin{array}{c}\text { Injection \#4 } \\
\text { Contact } \\
\text { Time (hr) }\end{array}$ \\
\hline $1 \mathrm{a}$ & 30 & 58 & 70 & 80 & 70 \\
\hline $1 \mathrm{~b}$ & & & 60 & 80 & 60 \\
\hline 2 & 55 & 62 & 72 & 76 & 60 \\
\hline 3 & $<1$ & 16 & 40 & 30 & 30 \\
\hline 4 & 19 & 50 & 63 & 56 & 50 \\
\hline 5 & 38 & 60 & 66 & 55 & 50 \\
\hline 6 & & & 70 & 60 & 60 \\
\hline 7 & & & 66 & 60 & 60 \\
\hline 8 & 18 & 57 & 64 & 63 & 60 \\
\hline 9 & 10 & 53 & 60 & 66 & 66 \\
\hline 10 & & 50 & 52 & 50 & 45 \\
\hline 11 & & 52 & 64 & 60 & 50 \\
\hline 12 & & 35 & 54 & 50 & 40 \\
\hline 13 & & 47 & 60 & 67 & 60 \\
\hline
\end{tabular}

Table 7.4. Temperature at Wells During Injections 1 Through 4

\begin{tabular}{|l|c|c|c|c|c|}
\hline Well & $\begin{array}{c}\text { Injection 1 } \\
\text { Temp. } \\
{ }^{\circ} \mathbf{C}\end{array}$ & $\begin{array}{c}\text { Injection 2a } \\
\text { Temp. } \\
{ }^{\circ} \mathbf{C}\end{array}$ & $\begin{array}{c}\text { Injection 2b } \\
\text { Temp. } \\
{ }^{\circ} \mathbf{C}\end{array}$ & $\begin{array}{c}\text { Injection 3 } \\
\text { Temp. } \\
{ }^{\circ} \mathbf{C}\end{array}$ & $\begin{array}{c}\text { Injection 4 } \\
\text { Temp. } \\
{ }^{\circ} \mathbf{C}\end{array}$ \\
\hline Ia & 11 & 16 & 18 & 17 & 19 \\
\hline $1 \mathrm{~b}$ & 11 & 13 & 14 & 14 & 14 \\
\hline 2 & 11 & 20 & 22 & 22 & 24 \\
\hline 3 & 11 & 12 & 12 & 12 & 14 \\
\hline 4 & 11 & 15 & 15 & 17 & 19 \\
\hline 5 & 11 & 20 & 21 & 25 & 27 \\
\hline 6 & & 13 & 13 & 14 & 15 \\
\hline 7 & & 13 & 14 & 14 & 14 \\
\hline 8 & 11 & 23 & 26 & 27 & 28 \\
\hline 9 & 11 & 25 & 27 & 28 & 29 \\
\hline 10 & & 20 & 21 & 23 & 24 \\
\hline 11 & & 19 & 18 & 20 & 21 \\
\hline 12 & & 13 & 14 & 15 & 18 \\
\hline 13 & & 24 & 26 & 27 & 28 \\
\hline
\end{tabular}


Table 7.5. Predicted Cumulative Iron Reduction at Well Locations

\begin{tabular}{|c|c|c|c|c|c|}
\hline Well & $\begin{array}{l}\text { Injection 1 } \\
\text { Percent Fe } \\
\text { Reduction }\end{array}$ & $\begin{array}{l}\text { Injection } 2 \mathbf{a} \\
\text { Percent Fe } \\
\text { Reduction }\end{array}$ & $\begin{array}{l}\text { Injection } 2 \mathbf{b} \\
\text { Percent Fe. } \\
\text { Reduction }\end{array}$ & $\begin{array}{l}\text { Injection } 3 \\
\text { Percent Fe } \\
\text { Reduction }\end{array}$ & $\begin{array}{l}\text { Injection } 4 \\
\text { Percent Fe } \\
\text { Reduction }\end{array}$ \\
\hline $1 \mathrm{a}$ & 3 & 9 & 16 & 21 & 26 \\
\hline $1 b$ & 0 & 0 & 0 & 0 & 0 \\
\hline 2 & 10 & 20 & 33 & 44 & 54 \\
\hline 3 & 0 & 0 & 0 & 0 & 0 \\
\hline 4 & 4 & 9 & 14 & 20 & 28 \\
\hline 5 & 4 & 10 & 18 & 25 & 33 \\
\hline 6 & 0 & 0 & 0 & $\overline{0}$ & 0 \\
\hline 7 & 0 & 0 & 0 & 0 & 0 \\
\hline 8 & 8 & 19 & 31 & 45 & 57 \\
\hline 9 & 6 & 18 & 31 & 46 & 59 \\
\hline 10 & $4^{(a)}$ & 9 & 16 & 24 & 31 \\
\hline 11 & $4^{(a)}$ & 9 & 14 & 20 & 26 \\
\hline 12 & $3^{(a)}$ & 7 & 11 & 15 & 19 \\
\hline 13 & $8^{(a)}$ & 19 & 32 & 47 & 60 \\
\hline Mean $^{(0)}$ & 5 & 13 & 23 & 33 & 42 \\
\hline \multicolumn{6}{|c|}{$\begin{array}{l}\text { (a) Well was installed after Injection } 1 \text {. Iron reduction estimates for Injection } 1 \text { for these } \\
\text { wells were based on similar wells. } \\
\text { (b) Treatment zone mean calculated from main treatment zone wells in the targeted Gg-u } \\
\text { portion of the aquifer (RM-1A, RM-5, RM-8, RM-9, RM-10, RM-11, and RM-13). }\end{array}$} \\
\hline
\end{tabular}

(Gg-u) in Figure 7.5. The size of the $>50 \%$ treatment zone is roughly $15 \mathrm{ft}$ in diameter (biased to the northeast of the injection/withdrawal well), and the $>25 \%$ treatment zone is roughly $30 \mathrm{ft}$ in diameter. The actual percentage of iron reduction, based on analysis of core samples collected after all injections were completed, is presented in Section 8.3.2. Eight cores from four boreholes were analyzed for the percentage of iron that was reduced by the field dithionite injections.

While the fraction of reduced iron can be estimated from the dithionite injection data, the resulting TCE reactivity (and measured TCE concentration in wells) in the reduced zone is strongly influenced by groundwater dynamics. In a static (non-flowing) system, the TCE degradation rate can be generally predicted, given the fraction of iron reduction, temperature, and contact time. Based on bench-scale test results conducted at typical Fort Lewis groundwater temperatures $\left(11^{\circ} \mathrm{C}\right)$, the TCE degradation half-life for $100 \%$ reduced sediment is 24 hours; for $50 \%$ reduced sediment it is 160 hours; and for $25 \%$ reduced sediment it is $\sim 600$ hours. Because a flowing system allows only limited contact time between the TCE and reduced sediment, the resulting TCE concentration measured at a well is a function of the TCEsediment reactivity upgradient of that location (i.e., path length and residence time in the reduced zone). The flow rate in most groundwater aquifers is relatively simple, slow, and constant. In contrast, the Fort Lewis POPT site has a relatively rapid groundwater flow rate (time-weighted average of $4 \mathrm{ft} /$ day) that changes direction and magnitude seasonally. 


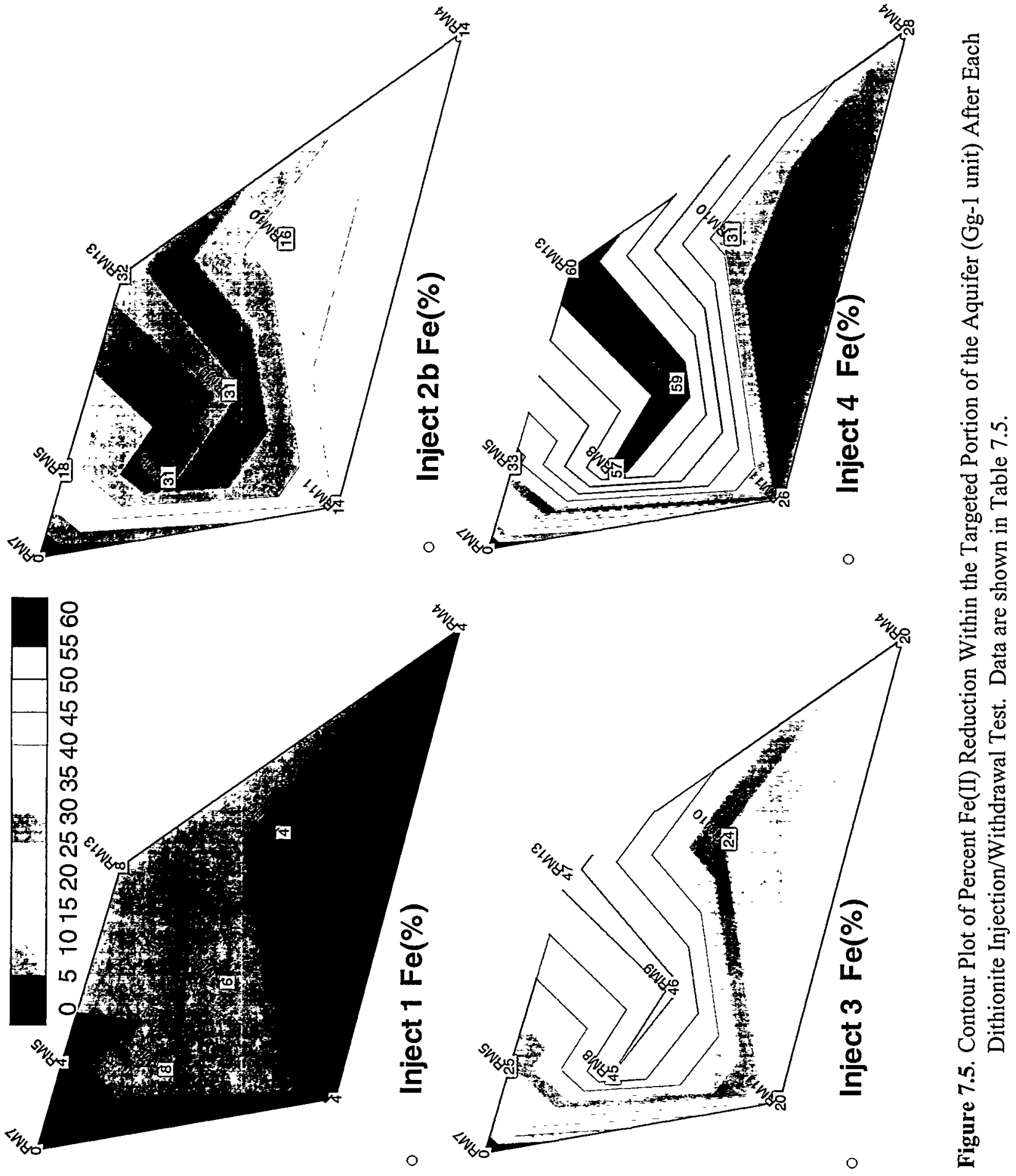


Observed TCE concentrations following barrier emplacement and assessment of barrier performance are discussed in Section 8.1.1. Estimates of field-scale TCE degradation rates at the various reduction percentages are provided in Section 8.2.

\subsection{Mobilization of Trace Elements}

This section discusses the results of the trace element analyses of the withdrawal stream during the dithionite injection/withdrawal tests. Baseline and post-emplacement trace element monitoring at the site is discussed in Section 8.1. The injected dithionite solution is a high $\mathrm{pH}$, high ionic strength, reducing medium. The injection solution thus has the potential to mobilize some trace elements through several processes, including reduction, amphoteric dissolution, and cation exchange. Samples of withdrawal water were analyzed to verify that metals mobilized during the dithionite injection, and subsequently withdrawn from the aquifer, resulted in wastewater concentrations that were within acceptable limits. Figure 7.6 shows the behavior of two key elements, iron and arsenic, during the withdrawal phase following the second injection series.

Iron is mobilized to a limited extent from the reduction of immobile ferric iron to the more mobile ferrous form. Following the withdrawal phase, iron has been reduced to acceptable levels. Arsenic shows similar behavior but at much lower levels because it is much less abundant. Even at its maximum, arsenic does not exceed the $50 \mathrm{ppb}$ EPA Drinking Water Standard. Other elements appearing at elevated levels in the withdrawal water include fluoride, aluminum, and manganese. Table 7.6 lists the peak concentrations of the affected species compared with their baseline concentrations.

Trace Metals Concentrations in Withdrawal Water Following Injection 2B

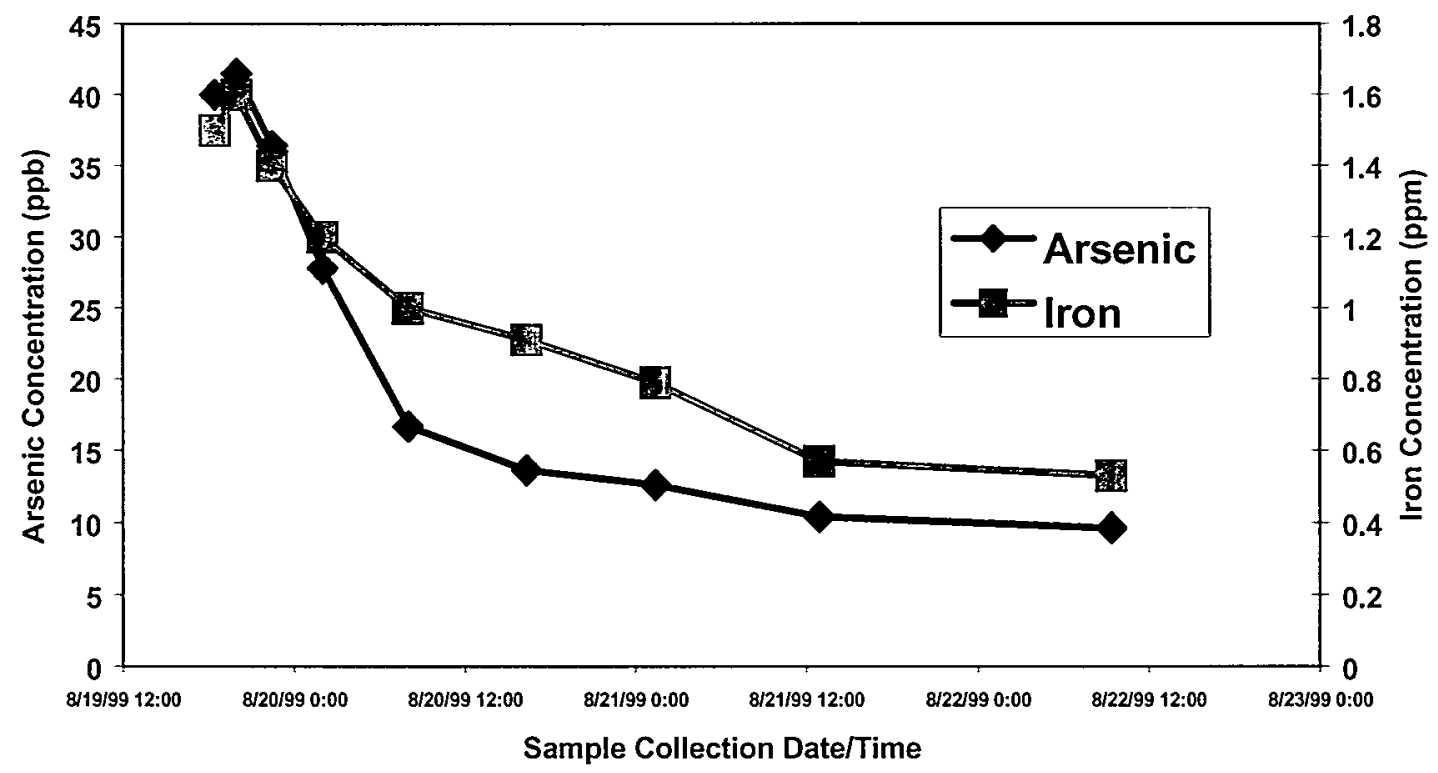

Figure 7.6. Observed Trace Metals Concentrations in Withdrawal Water During the 64-Hour Withdrawal Phase 
Table 7.6. Species Showing Elevated Concentrations in Injection 2 Withdrawal Water

\begin{tabular}{|l|c|c|}
\hline Species & Peak Concentration (ppb) & Baseline Concentration (ppb) \\
\hline Aluminum & 347 & $<50$ \\
\hline Arsenic & 42 & $<5$ \\
\hline Fluoride & 39,000 & $<200$ \\
\hline Iron & 1,600 & 0.005 to 44 \\
\hline Manganese & 431 & $<10$ to 180 \\
\hline
\end{tabular}




\subsection{Performance Assessment of Emplaced ISRM Treatment Zone}

This section addresses the measurements used to assess the effectiveness of the ISRM barrier placement. Section 8.1 considers the chemical monitoring results, including TCE, DCE, acetylene, electrical conductivity, $\mathrm{pH}$, dissolved oxygen, $\mathrm{Eh}$, trace metals, and common anions. Section 8.2 deals with the relationship between field-scale reduction and TCE degradation rates. Section 8.3 discusses methods for estimating treatment zone reductive capacity, including sediment core collection, laboratory studies of reductive capacity, and results of laboratory oxidation experiments on post-injection sediment core samples collected from the POPT site. Section 8.4 covers the hydraulic performance of the zone following the injections.

\subsection{Performance Assessment Groundwater Monitoring (post-injection)}

A comprehensive series of groundwater samples was collected from all available wells after each injection. Collection periods were approximately one month apart. Samples were collected after the first injection test from the original 10 wells, RM-1A through RM-9. Prior to the second injection test, four new wells, RM-10, RM-11, RM-12, and RM-13, were installed and added to the sampling list. Most subsequent data analysis used this same 14-well set. Two additional wells, RM-14 and RM-15, were installed three months after the final (fourth) injection. Because only one sample collection sweep was performed involving these wells, these data are generally not used in the subsequent analyses. However, data from RM-14 and RM-15 are useful for providing the most accurate representation of the spatial extent of the affected region of TCE degradation. These two additional wells will also be useful for future long-term monitoring efforts because both were installed near the region of maximum effect. Table 8.1 summarizes the sample collection program. Each collection sweep typically included samples for VOCs, acetylene, common anions, and trace metals. However, due to cost constraints, not all samples collected were analyzed for trace metals.

\subsubsection{TCE Monitoring}

As discussed in Section 7, the design of dithionite Injection 1 resulted in minimal iron reduction and subsequently very little TCE destruction. After Injection 1, TCE and DCE were monitored using an analytical method based on GC/MS with headspace sample introduction. Calibration problems associated with the method necessitated a change back to the more conventional purge-and-trap-based gas chromatography method (SW-846 8021) used for the earlier baseline measurements. Because of concerns over data comparability, however, only purge-and-trap data were used in the tabulations in this report or plotted on the figures. Consequently, most of these plots start with the monitoring measurements performed just before Injection 2. A complete discussion of the analytical methods used is presented in Appendix B, and the full data tabulations are included in Appendix H.

TCE measurements after Injection $2 a$ as well as the two subsequent injection tests (Injections 3 and 4) exhibited a pattern of initial decrease in concentration followed in most cases by some degree of return 
Table 8.1. Sampling Summary

\begin{tabular}{|c|c|c|}
\hline Activity & Date(s) & Wells Sampled \\
\hline Injectionl & $11 / 10 \% 14798$ & 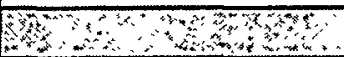 \\
\hline Post Inj. 1 & $12 / 1 / 98$ & RM-1A thru RM-9 \\
\hline Post Inj. 1 & $12 / 15 / 98$ & RM-1A thru RM-9 \\
\hline Post Inj. 1 & $1 / 5 / 99$ & RM-1A thru RM-9 \\
\hline Post Inj. 1 & $2 / 9 / 99$ & RM-1A thru RM-9 \\
\hline Post Inj. 1 & $4 / 1 / 99$ & $\overline{\mathrm{RM}}-1 \mathrm{~A}$ thru RM-9 \\
\hline Post Inj. 1 & $8 / 5 / 99$ & RM-1A thru RM-13 \\
\hline Injection $2 \mathrm{~A}^{\prime}:$ & $8 / 10=14 / 99$ & and \\
\hline Injection $2 \mathrm{~B}$ & $8 / 1632799$ & whot \\
\hline Post Inj. 2A/2B & $9 / 21 / 99$ & RM-1A thru RM-13 \\
\hline Post Inj. 2A/2B & $10 / 19 / 99$ & RM-1A thru RM-13 \\
\hline Post Inj. 2A/2B & $12 / 1 / 99$ & RM-1A thru RM-13 \\
\hline Injection 3 & $12 / 229 / 99$ & phe \\
\hline Post Inj. 3 & $1 / 6 / 00$ & RM-1A thru RM-13 \\
\hline Post Inj. 3 & $2 / 8 / 00$ & RM-1A thru RM-13 \\
\hline Post Inj. 3 & $3 / 20 / 00$ & RM-1A thru RM-13 \\
\hline Injection 4 & $3 / 2129100$ & 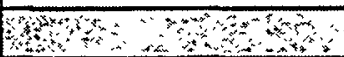 \\
\hline Post Inj. 4 & $4 / 26 / 00$ & RM-1A thru RM-13 \\
\hline Post Inj. 4 & $5 / 24 / 00$ & RM-1A thru RM-13 \\
\hline Post Inj. 4 & $6 / 21 / 00$ & RM-1A thru RM-15 \\
\hline
\end{tabular}

to higher levels. Figure 8.1 includes concentration trend plots for the five wells most profoundly affected by the creation of a reductive zone. Figure 8.2 shows the equivalent data for several other wells less profoundly impacted by the dithionite injections. In most cases, the increase in TCE concentration is most likely attributable to a combination of zone recovery from the treatment (see Section 8.1.3) and changes in groundwater velocity between sampling episodes. It is difficult to separate these effects because of the long periods involved and the need to proceed with additional injections during that same time frame. The change in concentration is most pronounced in wells monitoring portions of the treatment zone with the least TCE destruction (i.e., the shortest pathways through the barrier over which TCE destruction occurs). For wells monitoring portions of the treatment zone with a significant treatment pathway resulting in significant TCE destruction (e.g., RM-5), the change in concentration was much less prominent.

Three of the wells most profoundly impacted by the creation of a reduced zone (RM-2, RM-5, and RM-1a) are plotted in Figures 8.3 through 8.5 , which contain only data collected after a minimum threemonth recovery time following dithionite treatment. The plots are normalized to the background TCE concentration (140 ppb) and expressed in terms of percent of TCE remaining. This means that increasing barrier effectiveness would be expressed in terms of trends decreasing toward $0 \%$ residual TCE. The largest effect is seen for well RM-2, which was completed in the lower outwash gravel unit (Gg-1). A substantial amount of TCE destruction was observed in RM-2 after only one injection because density 


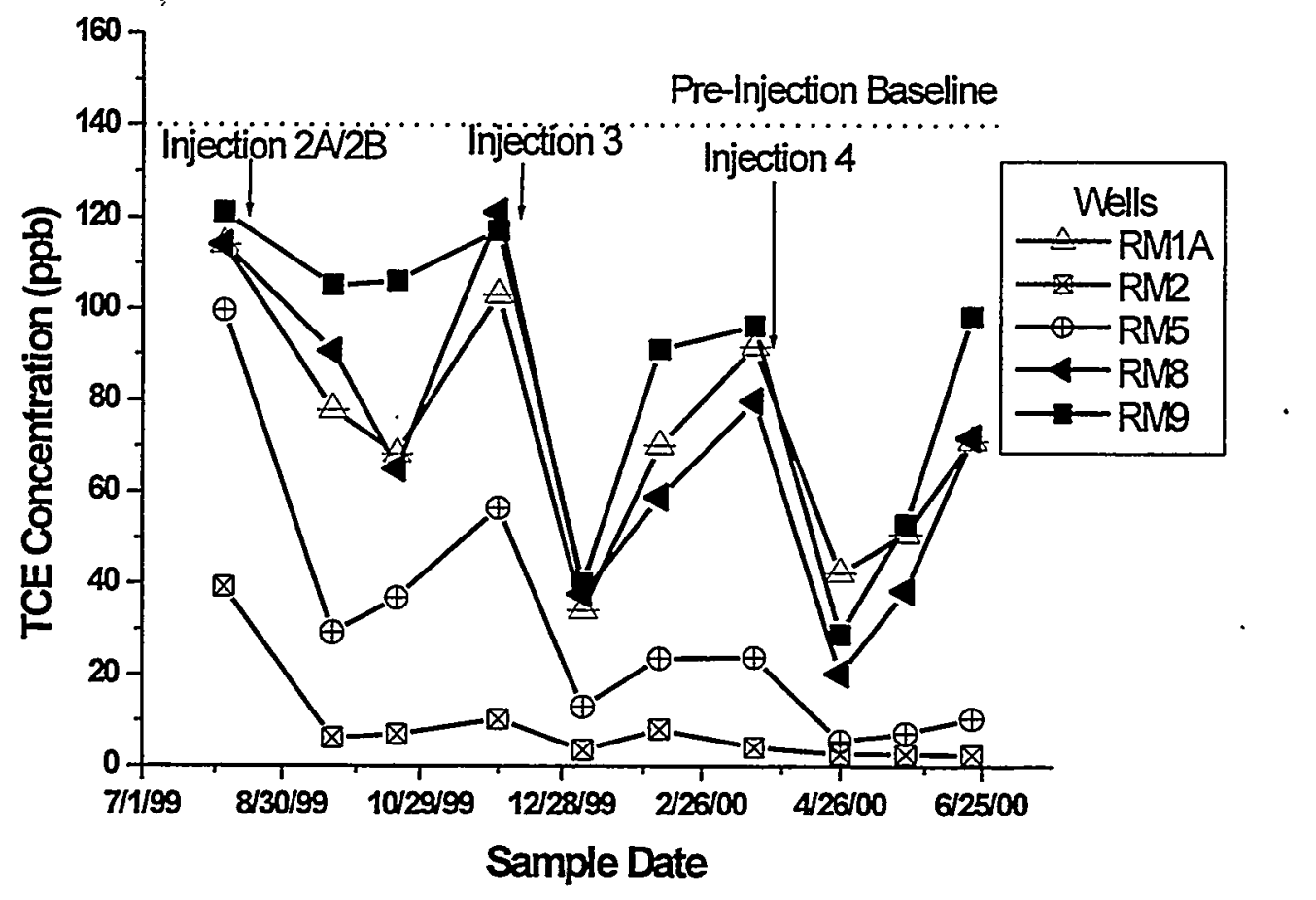

Figure 8.1. TCE Concentration Trends at ISRM Site for Wells RM-1A, RM-2, RM-5, RM-8, and RM-9

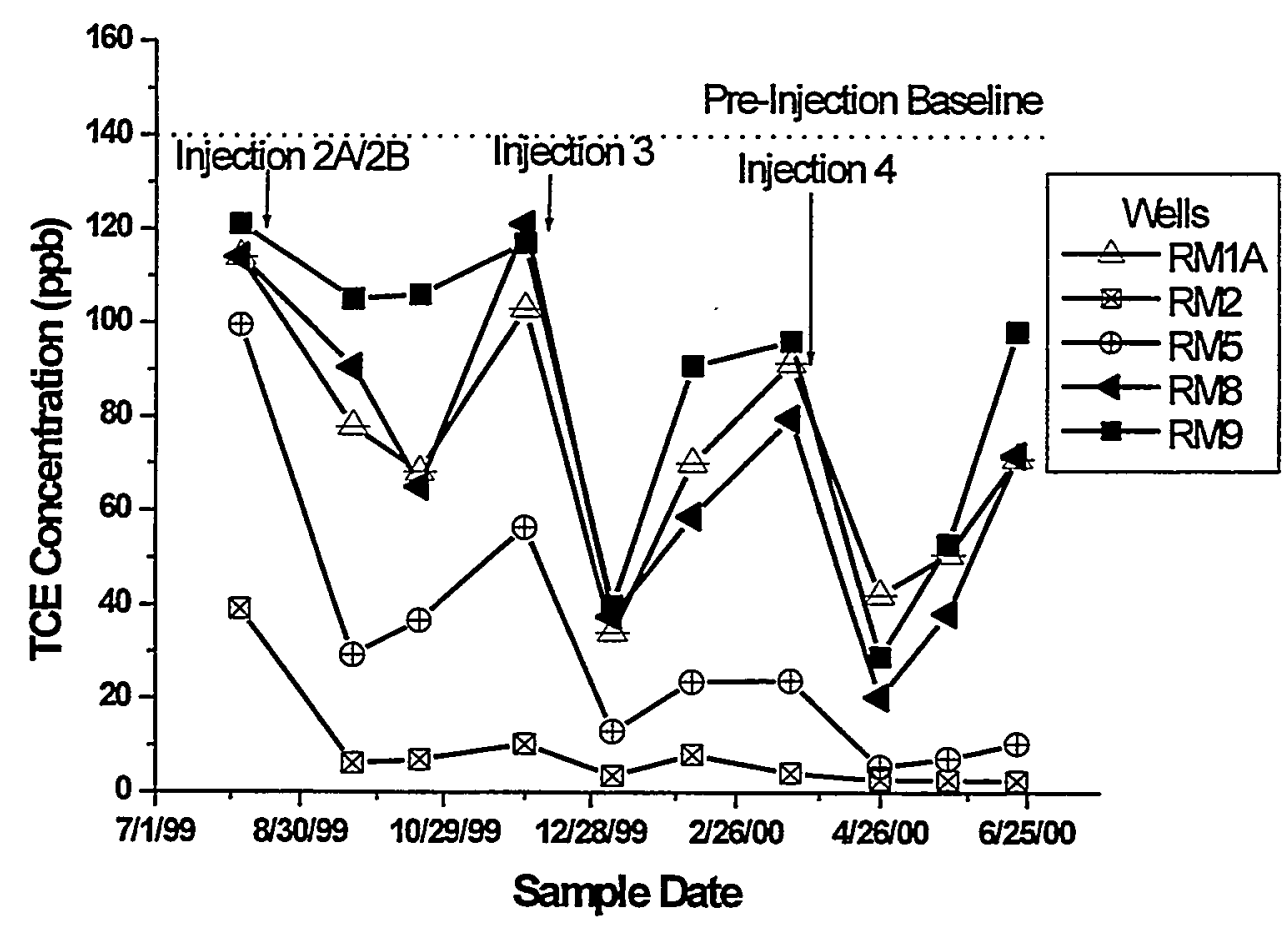

Figure 8.2. Normalized TCE Concentration Trends at ISRM Site for Wells RM-7, RM-10, RM-11, and RM-13 Expressed as a Percentage of Influent Concentration at Well RM-3 


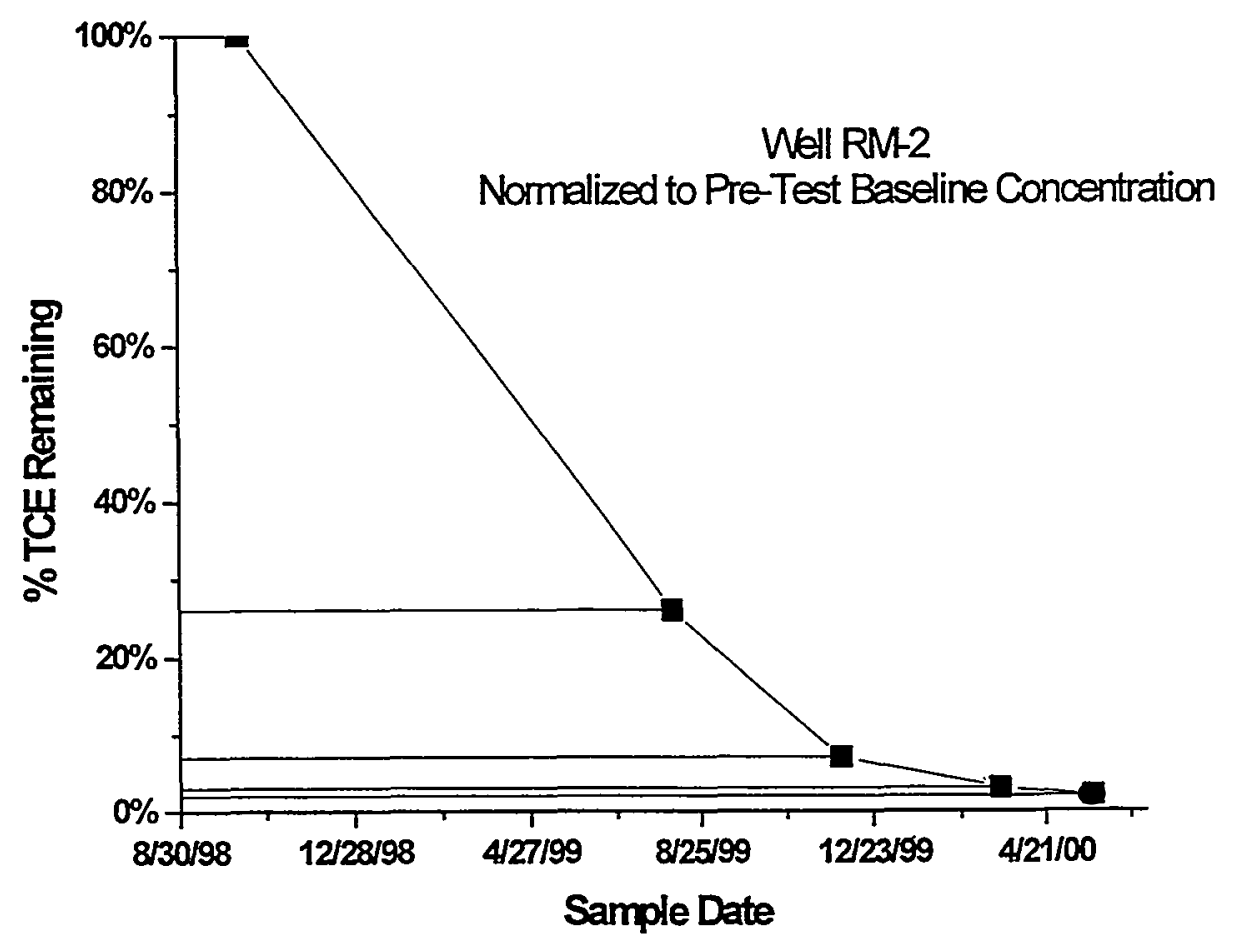

Figure 8.3. TCE Destruction Trend Plot for Well RM-2

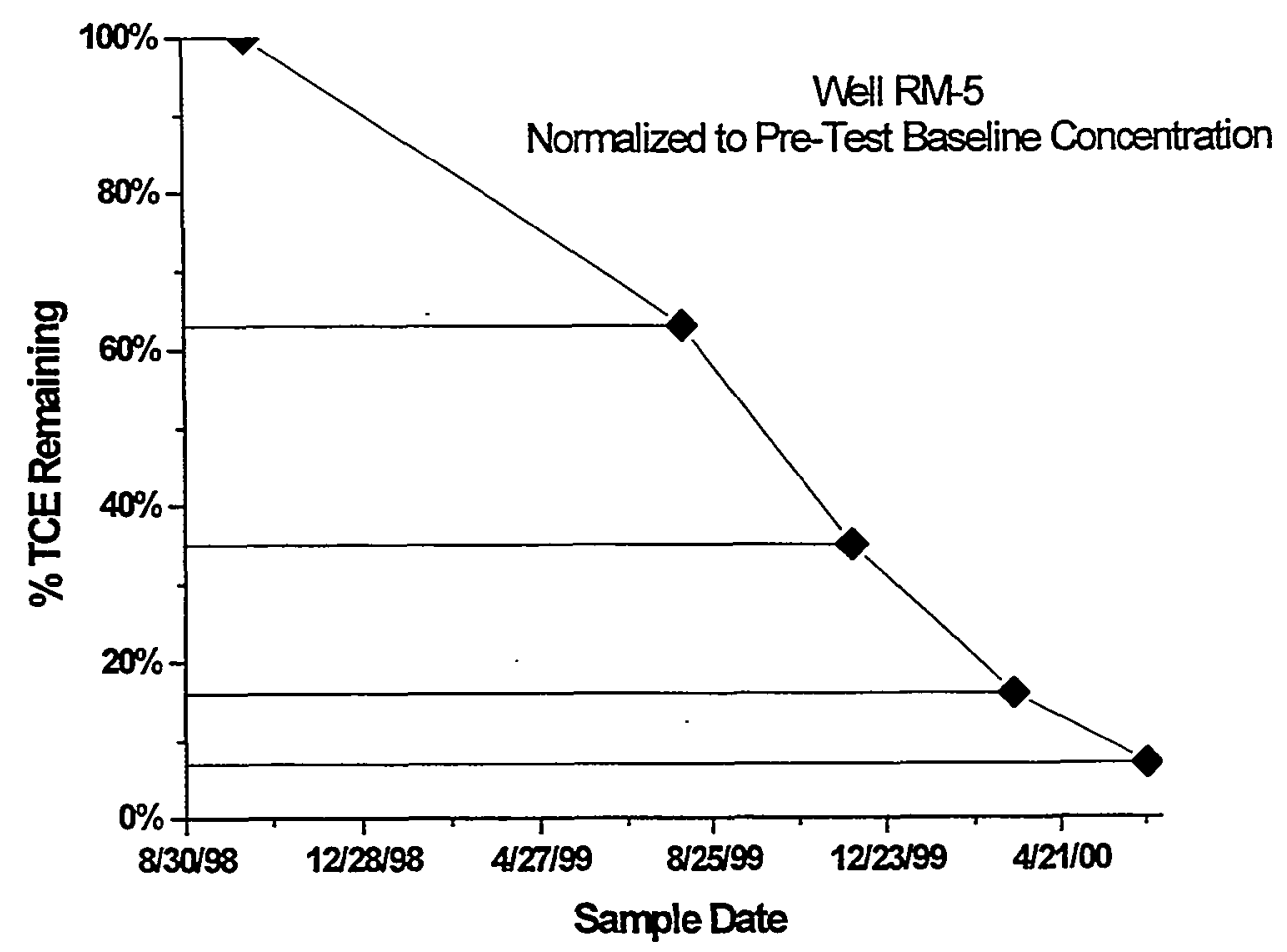

Figure 8.4. TCE Destruction Trend Plot for Well RM-5 


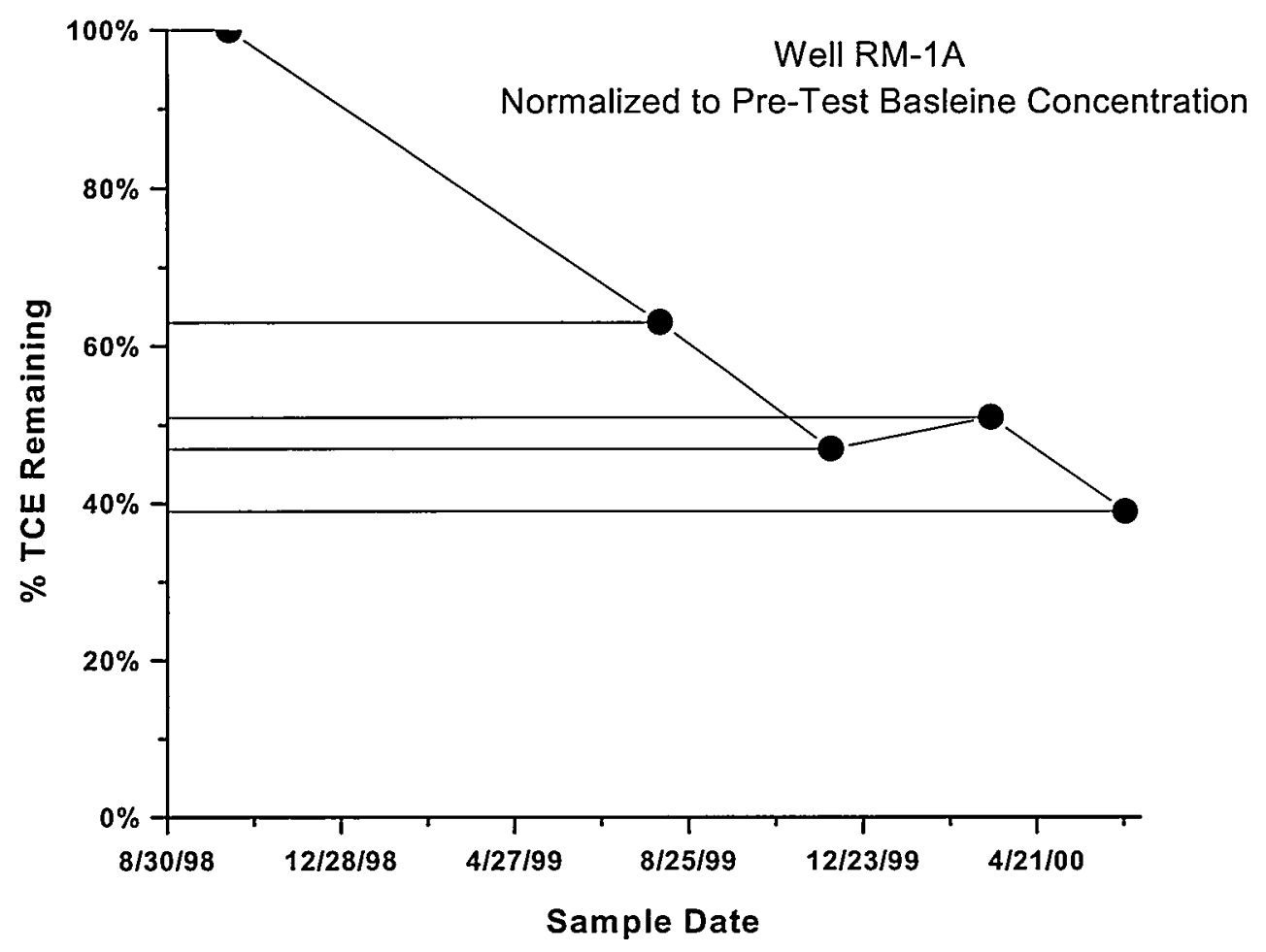

Figure 8.5. TCE Destruction Trend Plot for Well RM-1A

sinking of the reagent resulted in significantly more dithionite contact time for the sediments near RM-2. After four injections, residual TCE levels were very low ( $98 \%$ destruction). Because of the relatively atypical hydrogeologic conditions for this particular well (see Section 4.1), this result cannot be considered representative of conditions established within the treatment zone.

Comparable results are shown for well RM-5 in Figure 8.4. RM-5 was completed on the downgradient side of the targeted treatment zone and can be regarded as representative of the maximum degradation effect for the treated region. During the last sampling event following Injection 4 (June 21, 2000), TCE measurements showed $92 \%$ destruction of TCE relative to baseline measurements. RM-5 also showed an approximately linear improvement in barrier effectiveness with each successive injection. After four injections, barrier effectiveness appears headed for an asymptote, with additional injections likely to represent diminishing returns.

Other wells within the treatment zone showed significant but somewhat less profound effects for each injection, as indicated in Well RM-1A (Figure 8.5). It should be noted that, unlike RM-5, RM-1A is not located on the downgradient side of the treatment zone and subsequently monitors a shorter pathline through the treatment zone (i.e., less residence time for TCE destruction).

TCE concentration data collected during the last sampling event associated with each injection were contoured using a kriging algorithm and are presented in Figure 8.6. It should be noted that contour lines extending outside the region controlled by monitoring wells (i.e., TCE data) are extrapolated and may not 
represent actual conditions. For simplicity, the central injection well, RM-9, was used as the 0,0 reference point for the grids. Eleven wells, including RM-1a through RM-13, were used to construct the grid. RM-2 and RM-1b were not included because they were not within the targeted treatment zone. Two additional wells completed in June 2000, RM-14 and RM-15, were not included because data for earlier sampling periods were not available, and it was best to compare the four injection sequences on an equivalent basis. The four plots show increased TCE destruction with each additional injection. The plots also show the computed local groundwater flow vectors based on measured water levels at the time of sampling (see Section 4.8 for details). Groundwater velocity was not measured during the sampling period for the post-Injection 1 plot; however, as indicated in Figure 8.6, the effect of the first injection was minimal.

Figure 8.7 shows the post-Injection 4 data including the two additional wells, RM-14 and RM-15. Because these additional wells were completed on the downgradient side of the treatment zone, these data help to constrain the dimensions of the most affected region. Some manual adjustment was made to the contour plot to provide better agreement between the computed contour lines and individual data points. Because these measurements were made in mid-summer during a period of low precipitation and correspondingly high groundwater flow (see Section 4.8 for details on seasonal variability in flow direction), it represents less favorable conditions for TCE destruction performance. Performance monitoring at the POPT site will continue through March 2001.
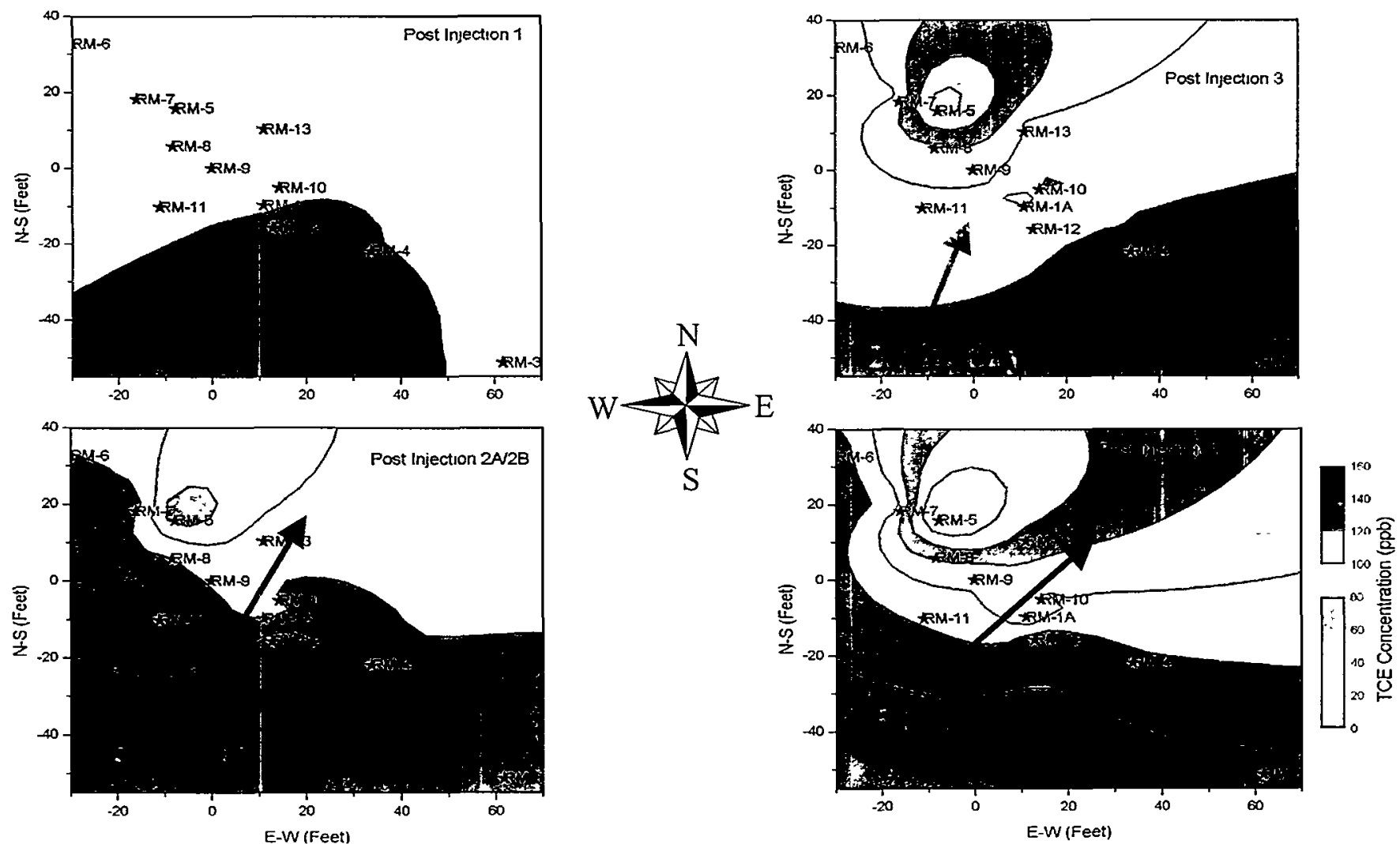

Figure 8.6. TCE Concentration Contours Following Each Injection 
TCE Contours from 6/21/00 sampling

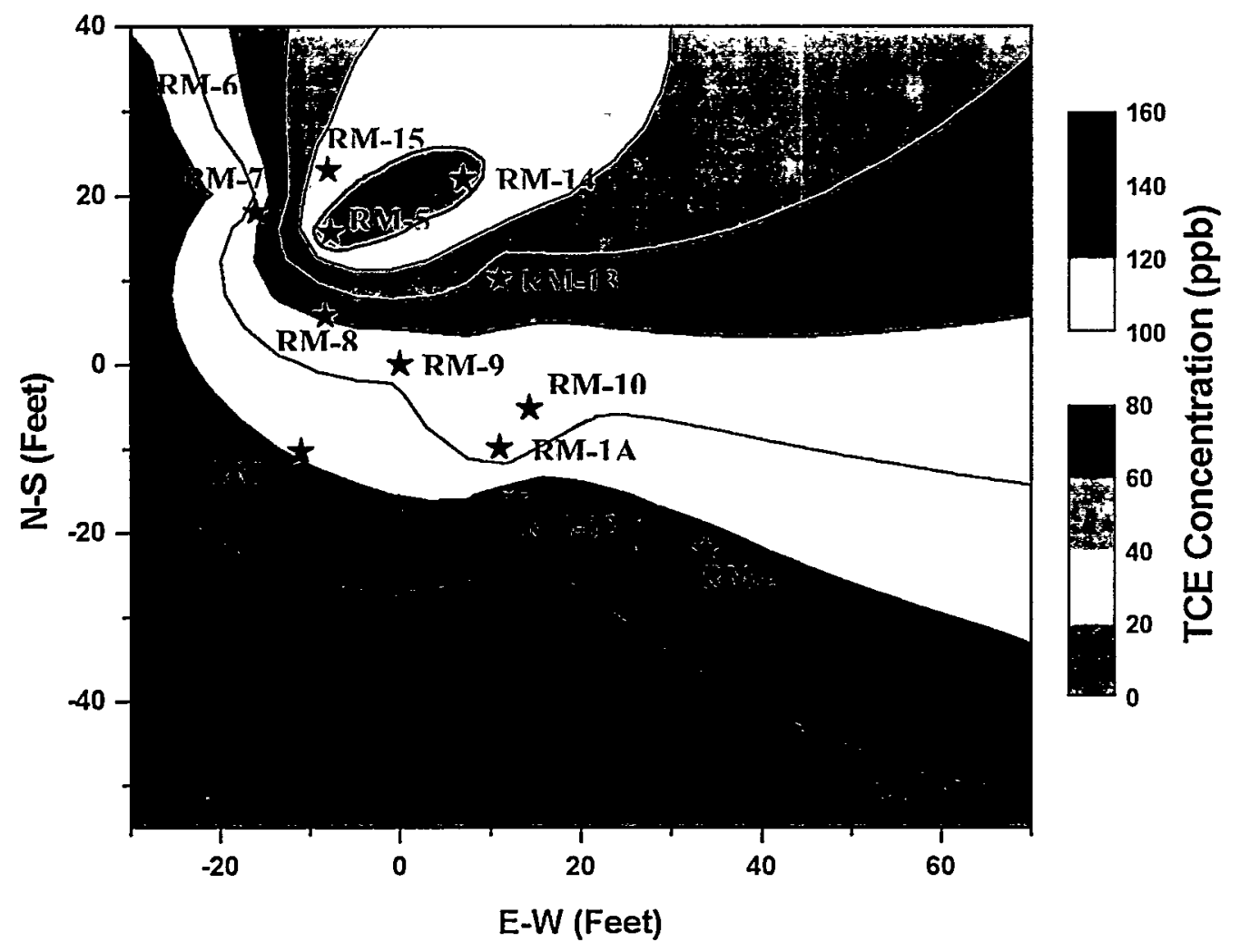

Figure 8.7. TCE Contours in Groundwater at ISRM Site for Last Sampling Period Including New Wells RM-14 and RM-15

\subsubsection{TCE Degradation Products}

Reductive dechlorination of TCE by ferrous iron generally proceeds through a process of reductive elimination, leading directly to production of chloroacetylene without significant production of the intermediate products associated with sequential dehydrohalogenation (see Appendix A). While some chloroacetylene production was observed in the early stages of laboratory column studies, the compound is known to be unstable and was thus not expected to be present in field-samples. No chloroacatylene was found in post-injection performance assessment groundwater samples. While some CIS-DCE was present in all cases, it was not observed to undergo any significant change in concentration during the test period. The presence of cis-DCE is likely to be associated with naturally occurring biodegradation processes in the extended plume. No vinyl chloride was found. As discussed in Section 4.6, no background acetylene was detectable at the site prior to dithionite treatment. Figure 8.8 shows the trend behavior of acetylene with time for the full injection series in wells exhibiting the largest effect. The two wells showing the largest acetylene production, RM-2 and RM-5, also exhibit the greatest decrease in TCE. Other wells show the same temporal trends in acetylene production but at lesser magnitude. Only the two wells most distant from the point of injection, RM-3 and RM-6, show no measurable acetylene production, which is consistent with the lack of TCE destruction at these wells. Production of acetylene exhibited a transient 
behavior in each case. The explanation for this effect is unclear but may be related to biodegradation of acetylene or its transport behavior. The maximum acetylene values observed fall far short of the amount required to provide a mass balance with the degraded TCE. However, due to acetylene's relative instability in the subsurface environment, a mass balance between TCE destruction and acetylene production was not an objective of this test. The primary objective of monitoring acetylene production was to provide a qualitative confirmation of TCE destruction.

\subsubsection{Field Parameter Monitoring}

Groundwater chemistry parameters ( $\mathrm{pH}, \mathrm{EC}, \mathrm{Eh}$, and $\mathrm{DO})$ were measured routinely during each sampling event. DO values were typically below detection in all wells after the first injection except for $\mathrm{RM}-3$. Trend behavior for $\mathrm{pH}$ and $\mathrm{EC}$ is shown in Figure 8.9 for well RM-5. Other wells within the treatment zone showed similar behavior with a large conductivity spike primarily associated with residual potassium carbonate buffer following each injection. The residual conductivity then dropped off markedly with time over the next few months after each injection as natural groundwater flow removed the material from the zone. The $\mathrm{pH}$ showed an increase of 1.6 after Injection 2 and more than an additional 0.6-pH units after the second injection series. The $\mathrm{pH}$ in RM-5 has continued to remain high in spite of the relatively large dropoff in conductivity following each injection test. This effect probably reflects to some extent the buffering capacity of the residual injection solution but may also be tied to the additional buffering capacity associated with precipitated carbonate minerals. The presence of the reduced zone itself also provides significantly high $\mathrm{pH}$ in situ buffering capacity. The spatial distribution of $\mathrm{EC}$ and $\mathrm{pH}$ residuals measured during the last post-Injection 4 sampling event (June 21,2000) are shown in Figures 8.10 and 8.11, respectively.

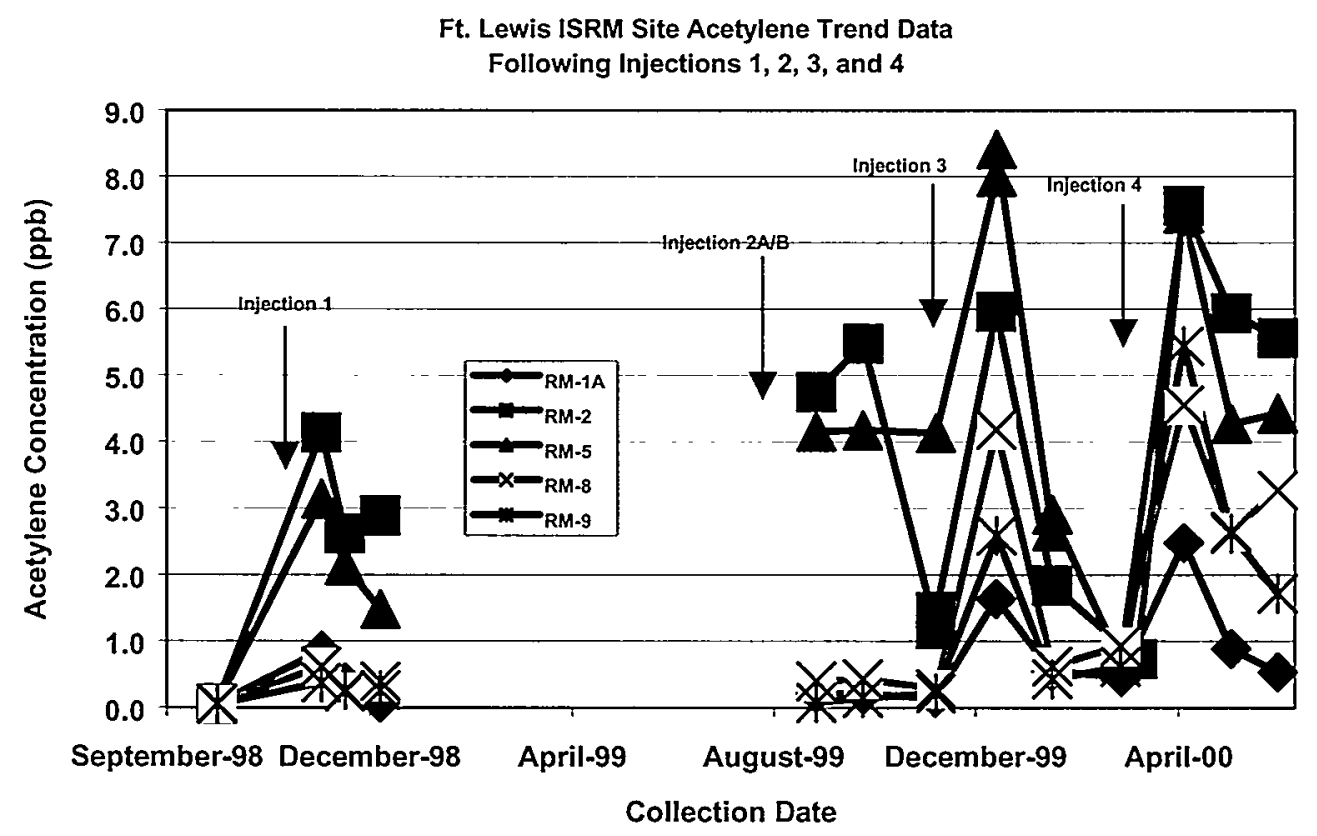

Figure 8.8. Trend Plots for Acetylene in Groundwater for all Four Injections 


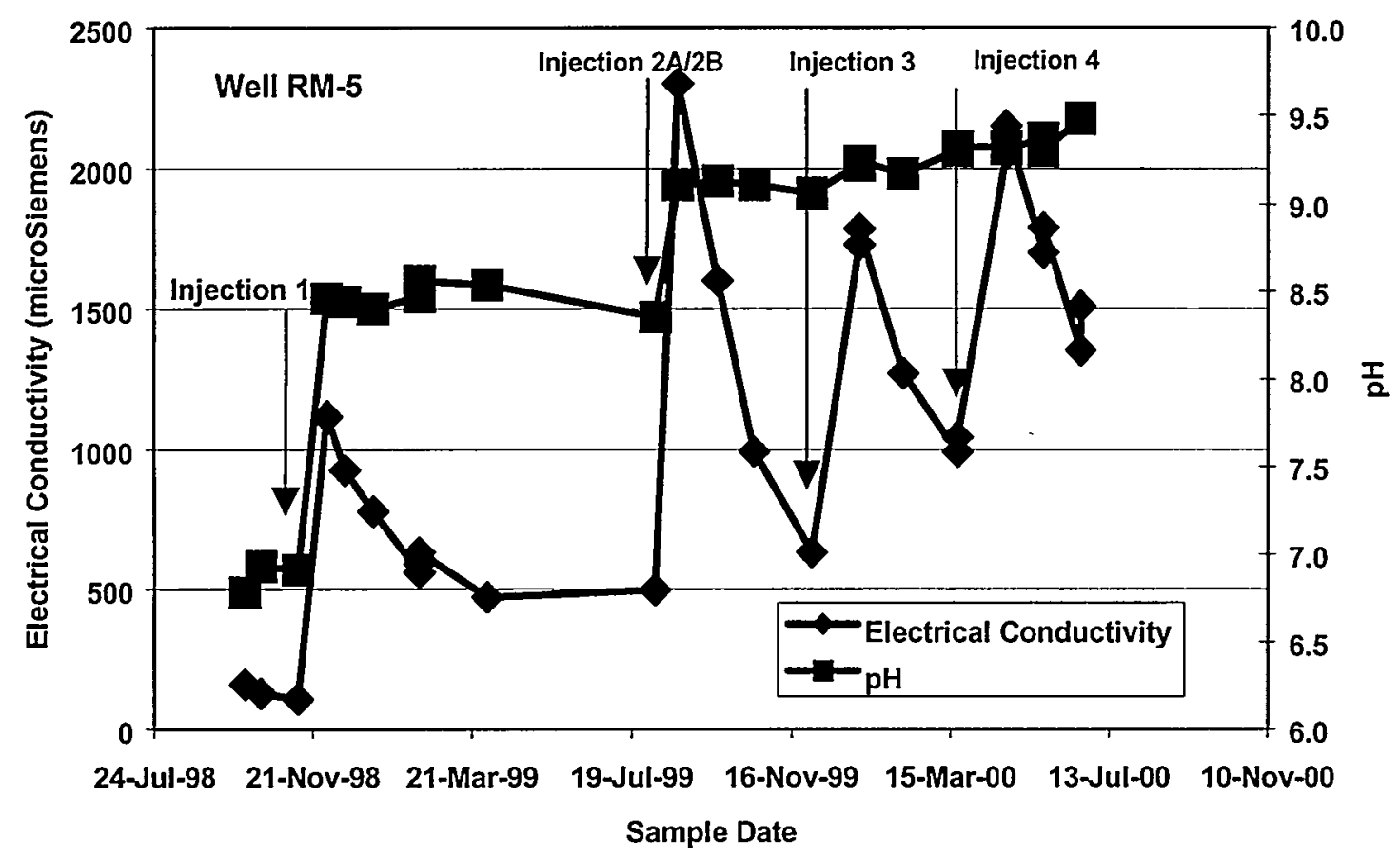

Figure 8.9. Trend Plot for $\mathrm{pH}$ and Electrical Conductivity in Well RM-5

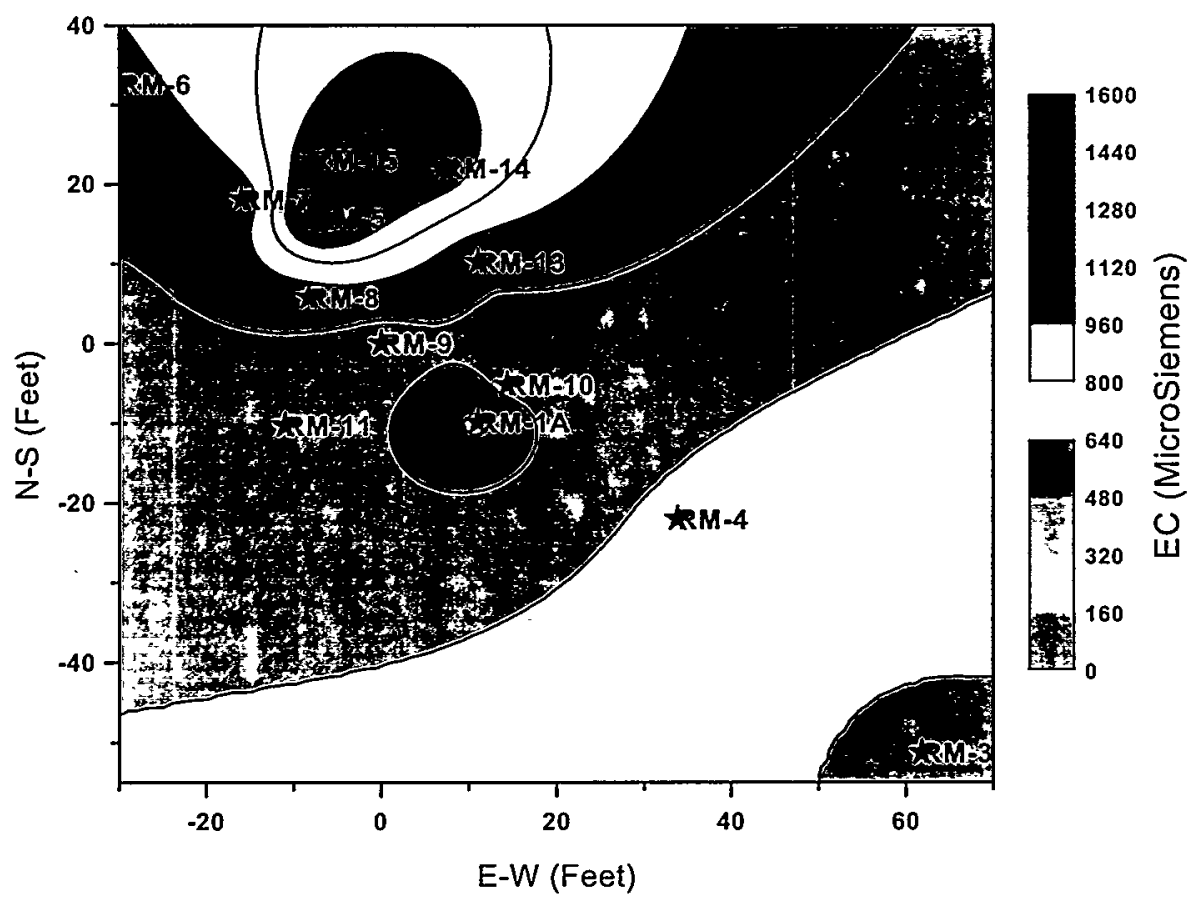

Figure 8.10. Electrical Conductivity Distribution on $6 / 21 / 00$ 


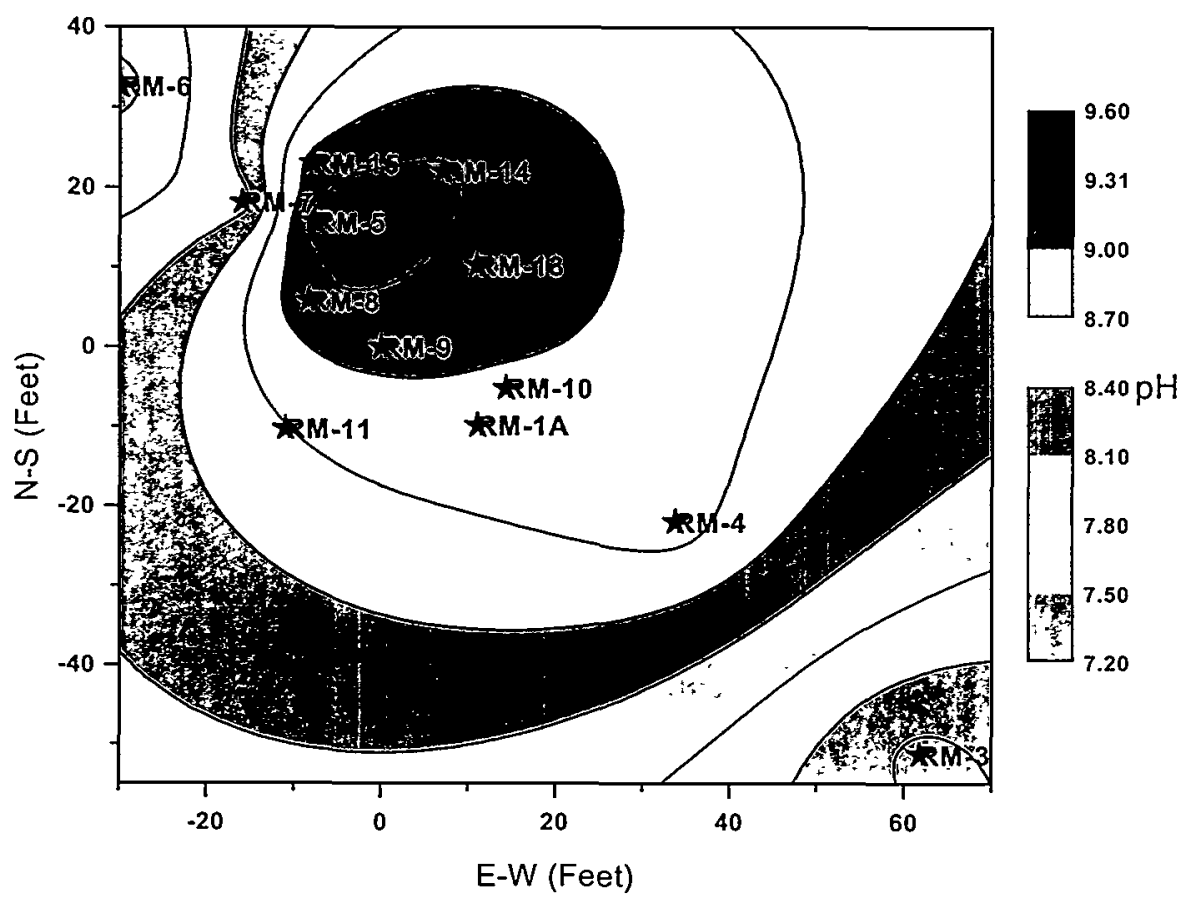

Figure 8.11. pH Distribution on $6 / 21 / 00$

As indicated in Figures 8.9 and 8.10, residual chemicals decreased in the treatment zone over the three-month monitoring period following each injection and the center of mass of the residuals plume was transported downgradient. Monitoring wells on the downgradient side of the treatment zone (RM-5, RM-14, and RM-15) remained at elevated levels relative to the surrounding well as of the June 2000 sampling event. These data indicate residuals remaining within the treatment zone following each injection are being transported downgradient, changing the geochemical conditions within the treatment zone. These results are consistent with the observed trend in TCE concentrations following each injection (see Section 8.1.1 and Figure 8.1)....

Oxidation-reduction potential (ORP or Eh) was also measured routinely during each sample collection series. The Eh distribution can be used to measure the development of the ISRM barrier formation and changes with time. Figures 8.12a-f shows measured Eh distributions on the site at various points in time. The first figure, 8.12a, shows the Eh for the pre-injection baseline measured on October 12, 1998. Groundwater taken from all wells was uniformly oxidizing as indicated by positive Eh values ranging from +63 to $+173 \mathrm{mV}$. Oxidizing conditions are shown graphically in red. Those data are also consistent with the observation of dissolved oxygen values ranging from $40 \%$ to $60 \%$ of saturation. Figure $8.12 \mathrm{~b}$, by contrast, shows the condition of the site after three dithionite injection tests. The green contours indicate reducing conditions toward the northwest corner of the site with oxidizing conditions toward the southeast corner of the study area. The dark green area thus represents the most highly reduced zone formed by the first three injection tests. Eh values at that time ranged down to $-299 \mathrm{mV}$. Immediately after the conclusion of operations associated with the fourth and final injection test, the entire study area shows uniformly reducing conditions. The blue color of the plot indicates Eh values as low as $-854 \mathrm{mV}$. 
a
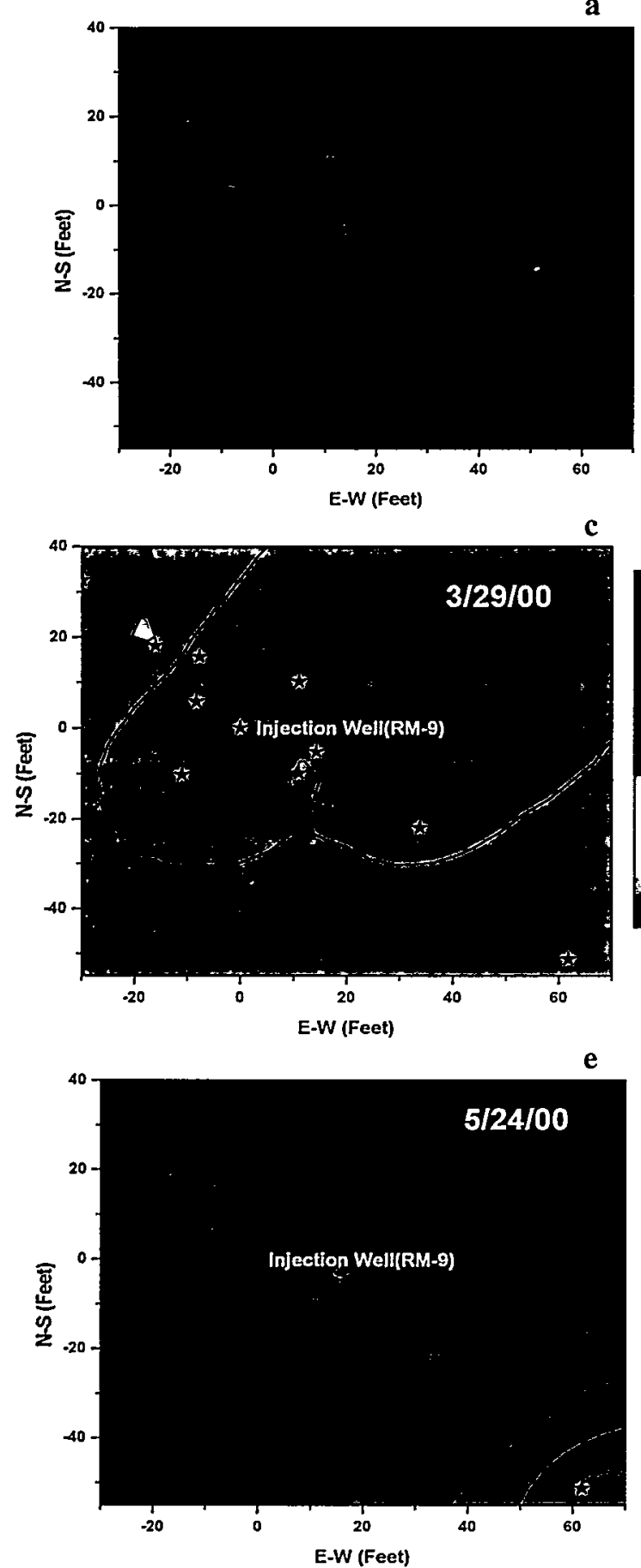

b
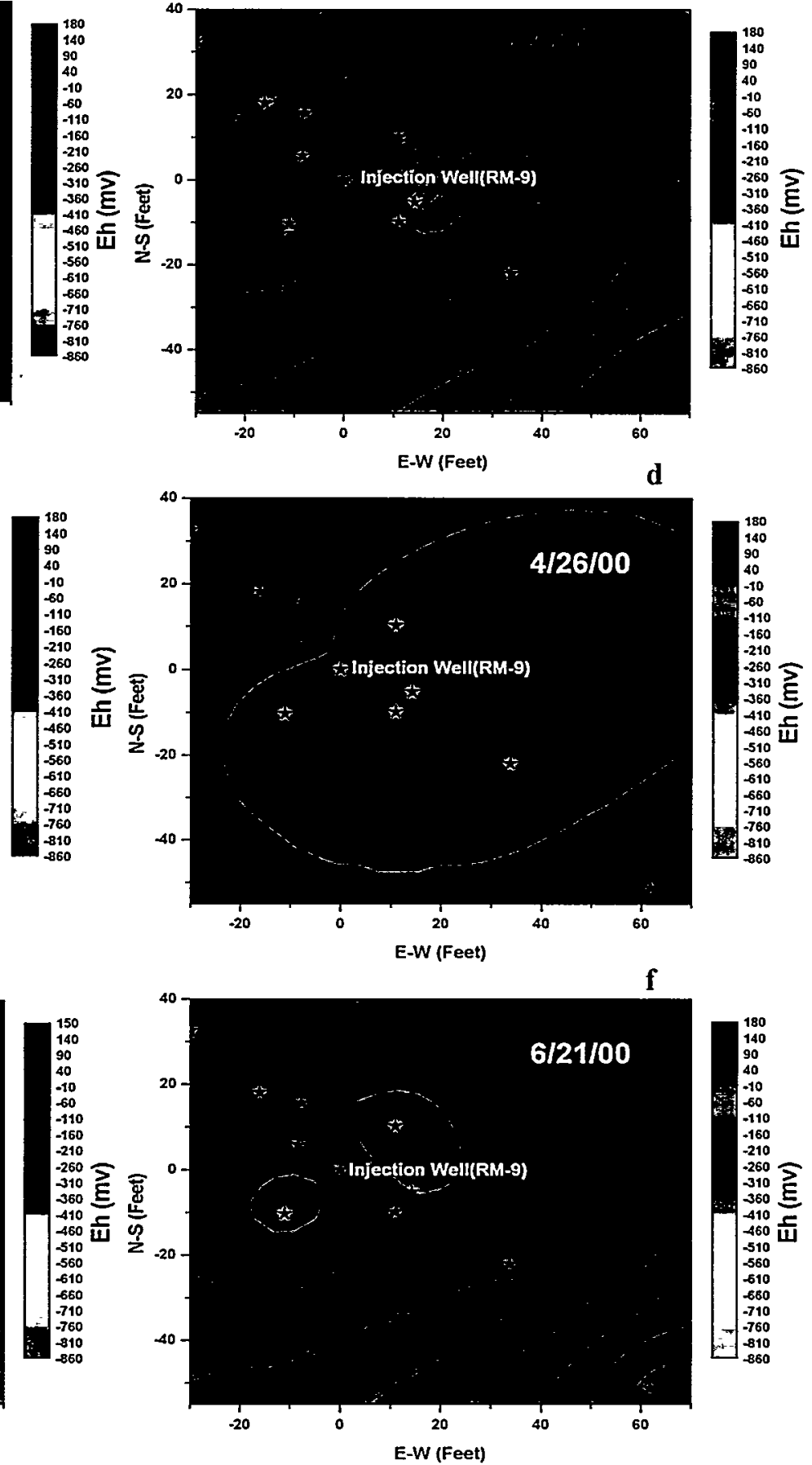

Figure 8.12. (a) Baseline Eh Distribution, (b) Eh Distribution Pre-Injection 4, (c) Eh After Injection 4 Withdrawal, (d) Eh Injection 4 Plus One Month, (e) Eh Injection 4 Plus Two Months, and (f) Injection 4 Plus Three Months 
However, as natural groundwater flow resumed, the Eh values at the site rose rapidly to more intermediate levels. The plot made from data taken one month later is now uniformly green, indicating a somewhat less strongly reducing environment with Eh values typically in the range of -300 to $-400 \mathrm{mV}$.

\subsubsection{Trace Metals Monitoring}

During the course of the project, trace metals samples were collected routinely. Trace metals monitoring had several goals:

1. Samples collected during the injection/withdrawal phase provided a measure of temporary mobilization for some species through reductive chemical transformations. Species of special concern included iron, manganese, and arsenic. Amphoteric species such as aluminum, beryllium, and zinc also have some potential for short-term mobilization under the high $\mathrm{pH}$ conditions associated with each injection. Metals mobilization results during the injection/withdrawal tests are discussed in Section 7.4 .

2. Discharge of withdrawal water to the sewer system required knowledge of trace element content for the waste stream (Section 7.4).

3. Post-test monitoring was intended to confirm that no long-term mobilization of trace elements had occurred because of the dithionite/buffer injections.

Two sets of post-test analyses were run using inductively coupled plasma mass spectrometry (ICP-MS), which was chosen because it is extremely sensitive (down to and typically well below Primary Drinking Water Standards [DWS]) for a wide range of elements. The first samples were taken October 19, 1999, two months after Injection 2; the second was collected June 21, 2000, three months after Injection 4.

Table 8.2 summarizes the trace metals analysis results. Baseline chemistry results along with primary and secondary DWS are included for comparison. The measurements presented in the table are given as ranges for all the wells studied; results from individual wells are included in Appendix $H$. None of the measurements exceeded the primary DWS for any of the species studied. A single measurement of antimony in RM-2 after the second injection series came close to the DWS set for that element, but measurements made after Injection 4 showed that, while antimony was some $\overline{\bar{w}} \bar{h}$ at elevated, it was still a factor of 3 below the DWS and of negligible importance in the other wells. The secondary DWS was exceeded in most wells for manganese, which is mobilized by reductive treatment. Manganese was particularly high in wells on the periphery of the zone of high reduction (RM-6, RM-7, RM-11, and RM-15. Arsenic can exhibit short-term enhanced mobility with reductive treatment but was not found at levels of concern. Significant elevation of iron to above the secondary DWS was seen in RM-1A, RM-2, RM-5, RM-6, and RM-15. 
Table 8.2. Trace Metals Analysis (all numbers in $\mathrm{mg} / \mathrm{L}$ )

\begin{tabular}{|c|c|c|c|c|c|}
\hline Constituent & $\begin{array}{l}\text { Pre-Test } \\
\text { Baseline }\end{array}$ & $\begin{array}{c}\text { Post-Injection } \\
\text { 2A/2B }\end{array}$ & $\begin{array}{c}\text { Post-Injection } \\
4\end{array}$ & $\begin{array}{c}\text { Primary } \\
\text { DWS }\end{array}$ & $\begin{array}{c}\text { Secondary } \\
\text { DWS }\end{array}$ \\
\hline Aluminum & $<\overline{0.1}$ & $<0.06$ & 0.03 to 0.08 & & 0.05 to 0.2 \\
\hline Antimony & $\mathrm{NA}$ & 0.00066 to 0.0055 & 0.0009 to 0.002 & 0.006 & \\
\hline Arsenic & $<0.06$ & $<0.005$ & 0.0005 to 0.004 & 0.05 & \\
\hline Barium & 0.004 to 0.009 & 0.0017 to 0.13 & 0.0012 to 0.19 & 2 & \\
\hline Beryllium & $<0.002$ & $\mathrm{NA}$ & $<0.0002$ & 0.004 & \\
\hline Bismuth & $\mathrm{NA}$ & $<0.00005$ & 0.0001 to 0.008 & & \\
\hline Boron & $<0.005$ & NA & $\mathrm{NA}$ & & \\
\hline Cadmium & $<0.02$ & $<0.003$ & $<0.0003$ & 0.005 & \\
\hline Calcium & 13 to 15 & 11 to 24 & 6.8 to 78 & & \\
\hline Chromium & $<0.04$ & $<0.0015$ & $<0.001$ & 0.1 & \\
\hline Cobalt & $<0.004$ & $\mathrm{NA}$ & 0.0014 to 0.06 & & \\
\hline Copper & $<0.006$ & $<0.005$ & 0.0009 to 0.012 & & 1 \\
\hline Iron & $<0.005$ to 0.044 & 0.014 to 1.4 & 0.06 to 1.59 & & 0.3 \\
\hline Lead & $<0.06$ & $<0.009$ & $<0.0001$ & 0.015 & \\
\hline Lithium & $<0.02$ & NA & $<0.004$ & & \\
\hline Magnesium & 6.7 to 6.9 & 4.1 to 44 & 4 to 32 & & \\
\hline Manganese & $\mathrm{U}$ to 0.18 & 0.041 to 0.81 & 0.03 to 1.35 & & 0.05 \\
\hline Mercury & NA & $\mathrm{NA}$ & $<0.0003$ & 0.002 & \\
\hline Molybdenum & $<0.06$ & $<0.37$ to 22.4 & 0.0005 to 0.011 & & \\
\hline Nickel & U to 0.27 & $<0.01$ to 0.275 & 0.0027 to 0.23 & & \\
\hline Potassium & 1.6 to 2.2 & 2.7 to 1550 & $\mathrm{NA}$ & & \\
\hline Selenium & $<0.06$ & $<0.025$ & $<0.0003$ & 0.05 & \\
\hline Silicon & $<0.1$ to 12 & $\mathrm{NM}$ & $<0.09$ to 15.6 & & \\
\hline Silver & $<0.02$ & $<.0003$ to 0.014 & 0.0008 to 0.0043 & & 0.1 \\
\hline Sodium & 5.5 to 12 & 4.8 to 440 & NA & & \\
\hline Strontium & 0.068 to 0.081 & NA & 0.04 to 0.54 & & \\
\hline Thallium & $\mathrm{NA}$ & $\mathrm{NA}$ & $<0.00002$ & 0.0005 & \\
\hline Thorium & $\mathrm{NA}$ & $<0.0002$ & $<0.002$ & & \\
\hline Tin & $\overline{\mathrm{NA}}$ & 0.000046 to 0.0001 & 0.0002 to 0.0017 & & \\
\hline Uranium & $\mathrm{NA}$ & 0.000072 to 0.00056 & $<0.000002$ to 0.0010 & & \\
\hline Vanadium & $<0.02$ & $\mathrm{NM}$ & $<0001$ to 0.0025 & & \\
\hline Zinc & $<0.006$ & $<0.05$ & $<0008$ to 0.038 & & 5 \\
\hline
\end{tabular}




\subsubsection{Common Anions Monitoring}

Performance assessment monitoring samples were subjected to IC analysis for common anions, including fluoride, chloride, nitrate, phosphate, sulfate, and sulfite (see Appendix $\mathrm{H}$ for analysis results). In general, the fluoride, chloride, and phosphate data proved to be of little interest. Fluoride levels remained very low except for short periods during injection and withdrawal. Similarly, chloride tended to stay at low baseline levels, and phosphate was consistently below the nondetect level. Nitrate was found routinely in all samples from the site at low levels, typically around $2 \mathrm{mg} / \mathrm{L}$ (as $\mathrm{NO}_{3}{ }^{-}$). The nitrate was observed to decrease following the dithionite injections. The decrease is probably associated with the reductive conditions because nitrate has been found in past work to be more readily reduced than TCE. The nitrate appeared to be widely distributed. Sulfite was observed in some samples following dithionite injection, primarily associated with well RM-2. In some cases, the sulfite levels were comparable to or greater than the sulfate levels in that well; however, sulfite was absent, or in very minor amounts in all other wells. Sulfite is relatively unstable under oxidizing conditions and no attempt was made to preserve sulfite prior to analysis; however, because the samples were typically free of DO as collected, sulfite proved to be persistent in the RM-2 samples.

Figure 8.13 shows the temporal behavior of sulfate concentrations in two wells, RM-5 and RM-6 (located within and outside the treatment zone, respectively). The RM-5 sulfate trend is comparable with that of EC (Figure 8.9) with large increases associated with each injection followed by decay in concentration as the sulfate is transported downgradient. The red line on the plot at $250 \mathrm{mg} / \mathrm{L}$ is the secondary DWS for sulfate. RM-5 has exceeded that standard following each injection, and sulfate has accumulated to some extent following the latter more closely spaced injections. The last measurement, taken in June 2000 , was $330 \mathrm{mg} / \mathrm{L}$, still above the standard; however, as natural groundwater flow continues to clear out the system, the sulfate concentration in RM-5 should drop below $250 \mathrm{mg} / \mathrm{L}$. The trend for RM- 6 was interesting because that well is some distance from the injection point (outside the targeted treatment zone). However, it should be noted that RM- 6 was influenced by low reagent concentrations during the injection. The observed sulfate pulse in RM- 6 suggests there may be a component of local groundwater flow during some portion of the monitoring period transporting the residual sulfate in that direction. Sulfate levels have been consistently high in well RM-2. The last three measurements taken one month apart were all around $1,500 \mathrm{mg} / \mathrm{L}$ with no apparent temporal trend, indicating lower groundwater velocities, and/or continued seepage of higher density residuals from the overlying Qvt2 unit, into the Gg-1 unit near RM-2. As indicated in the tabular sulfate results contained in Appendix $\mathrm{H}$, sulfate concentrations in wells monitoring the treatment zone have generally decreased to below the secondary standard of $250 \mathrm{mg} / \mathrm{L}$, except for wells on the downgradient side of the treatment zone (RM-5, RM-14, and RM-15) which continue to exceed the standard by 40 to $80 \mathrm{mg} / \mathrm{L}$ as of the June 2000 sampling event. Sulfate concentrations are not expected to exceed these levels as the residuals plume is transported downgradient and should eventually decline to below this level as the plume is dispersed and diluted by recharge. An example of previously observed rtes of sulfate decline over the three-month periods between injections can be seen in Figure 8.13 and Appendix H. 


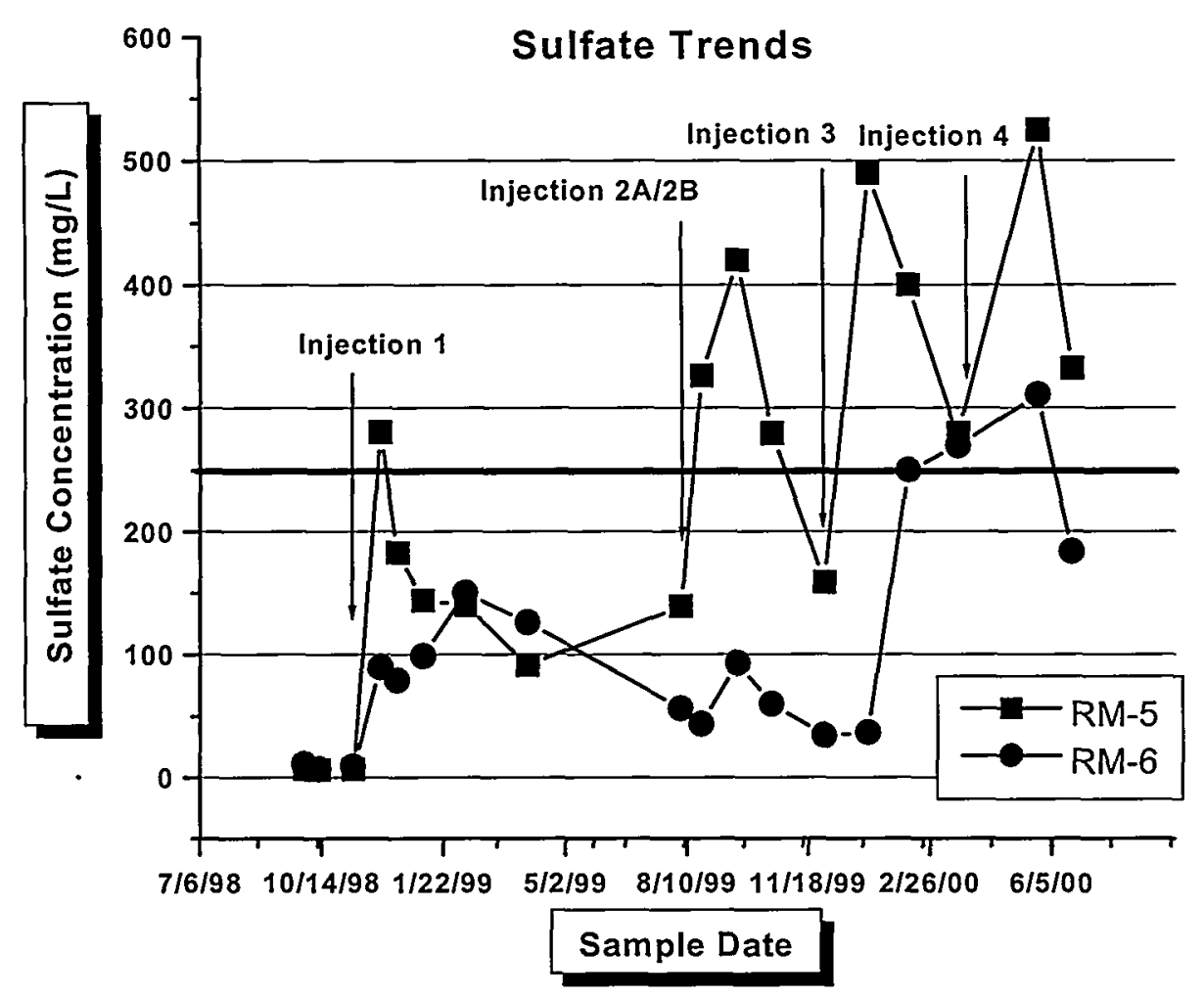

Figure 8.13. Sulfate Concentration Trends for Wells RM-5 and RM-6

\subsection{Relationship Between Field-Scale Iron Reduction and TCE Degradation Rates}

Two simple approaches are described below for quantifying the TCE degradation rates at the ISRM POPT test under conditions of varying iron reduction and groundwater flow velocity. Previous laboratory studies demonstrated that the TCE degradation rate is a function of the percentage of total reducible iron reduction of the sediment (Szecsody et al. 2000). The series of dithionite injection/withdrawal tests resulted in a spatial and temporal (based on monitoring periods between dithionite injection/withdrawal tests) distribution of iron reduction at the site. Estimates of iron reduction used in these analyses are based on the dithionite BTCs described in Section 7.3 and summarized in Table 7.5.

The first approach for determining field-scale TCE degradation rates is based on the overall performance of the reduced zone after each dithionite injection/withdrawal test. The following assumptions/ parameters were used: average iron reduction estimates for the targeted portion of the aquifer after each dithionite/injection withdrawal test (Table 7.5 and Figure 7.5), a constant input TCE concentration of $135 \mu \mathrm{g} / \mathrm{L}$, an output TCE concentration based on measurements from well RM-5, a constant pathlength through the reduced zone, and the groundwater velocity estimated at the time of TCE sampling from water level measurements (Section 4.8). This analysis uses only the latest sampling event between each injection/withdrawal test and following Injection 4 to minimize the residual effects of the dithionite injection/withdrawal tests on the TCE concentrations. A retardation factor of 2 , based on estimates from 
the USGS for the Fort Lewis site, was used to calculate the TCE residence time from the groundwater velocity. The results of this analysis are shown in Figure 8.14. Method 1 shows slow TCE degradation rates with a 24-day half-life at the low percentage iron reduction after Injection 1. The highest TCE degradation rate with a four-day half-life occurred as expected, after the last dithionite injection/ withdrawal test, which resulted in the highest estimated percent iron reduction. The estimated TCE degradation rate for Injections $2[23 \% \mathrm{Fe}(\mathrm{II})]$ and $3[33 \% \mathrm{Fe}(\mathrm{II})]$ did not follow the expected trend. While the TCE concentrations for well RM-5 following Injection 3 were lower than those following Injection 2, the groundwater flow velocity calculated during the TCE sampling was significantly lower during post-Injection 3 monitoring, which offset the TCE decrease.

The second approach examined only the latest Fe(II) and TCE data but accounts for the spatial distribution along the groundwater flow paths to derive the TCE degradation rates (see Figure 8.14, Method 2). The flow path was at a bearing of 28 degrees (NNE) based on the groundwater flow direction from water level measurements (Section 4.8). The pathline was divided into nine segments for each of the $5 \% \mathrm{Fe}$ (II) contour intervals in Figure 8.15a. Starting and ending TCE concentrations for each of these segments were determined from Figure 8.15b. The extent of the contour plots shown in Figure 8.15 was clipped to the existing wells at the ISRM proof-of-principle test site. The residence time for each segment

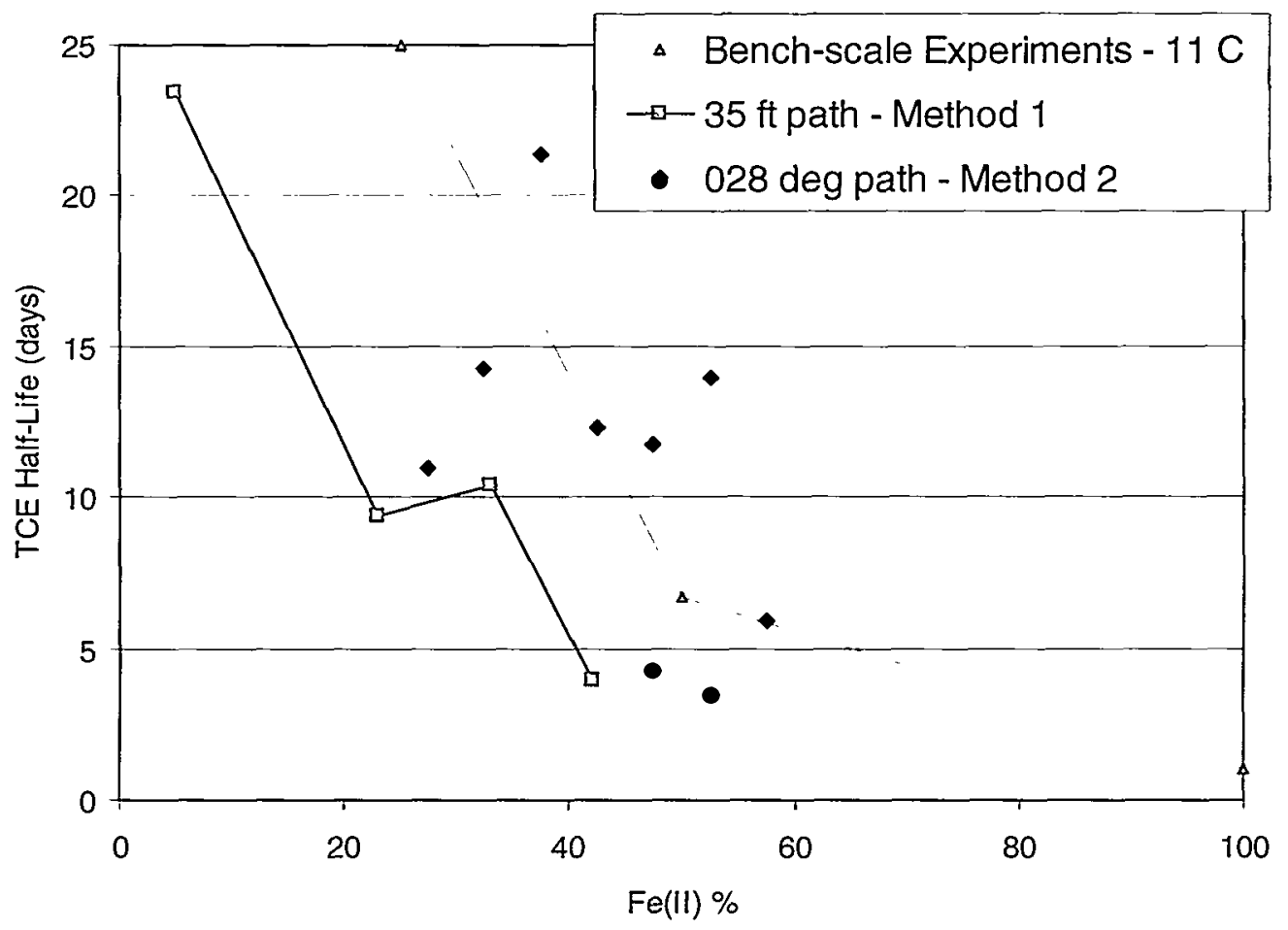

Figure 8.14. Estimated Field-Scale TCE Degradation Rates (expressed as half-life) Versus Average Percentage Iron Reduction. Degradation rates were calculated using two simplified methods (see text for details). Estimates of degradation rates from bench-scale data under field conditions are also shown. 


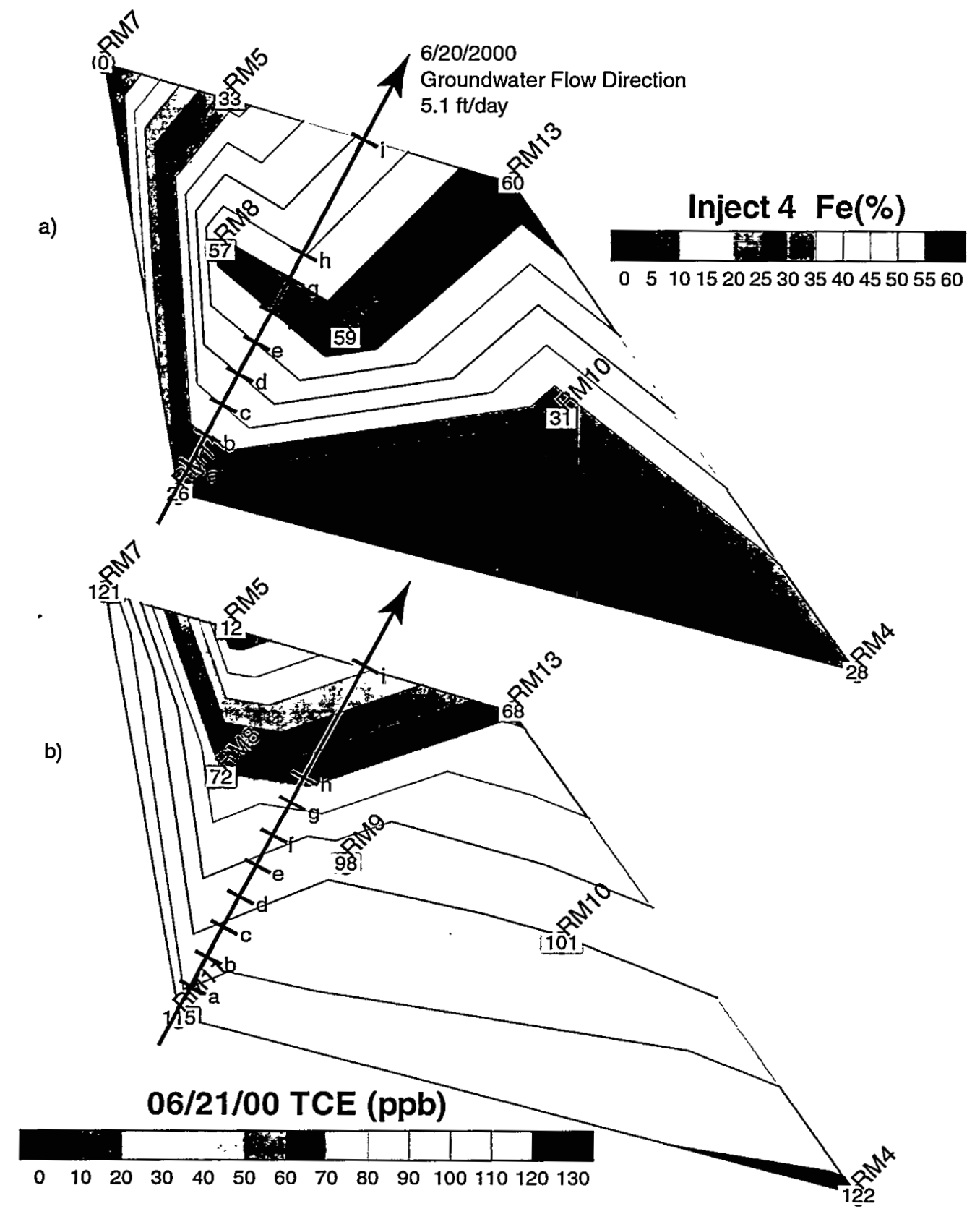

Figure 8.15. Pathline Showing Groundwater Path Through the Injection 4 Fe(II) Percent Reduction Estimates and Latest TCE Monitoring Results. The pathline was divided into nine segments (a to i) based on the $5 \% \mathrm{Fe}$ (II) isopleths. TCE degradation rates were estimated for each segment using the starting and ending TCE concentrations and residence time calculated in each segment. Results of this analysis are shown in Figure 8.14 (Method 2). The groundwater flow direction and velocity were based on water levels measured in wells around the DRMO facility. These contour plots were clipped to the extent of wells at the ISRM POPT site. 
was calculated based on the segment length, groundwater flow velocity of $5.1 \mathrm{ft} /$ day, and a retardation factor of 2 for TCE. The TCE degradation rate was then calculated for each of the segments and plotted with the percentage $\mathrm{Fe}(\mathrm{II})$ reduction for the segment on Figure 8.14. This figure shows considerable scatter in the results. Most of the scatter can be explained by the distinct difference in TCE degradation rates calculated in segments south of RM-9, which are relatively sluggish compared with the rates calculated for segments north of RM-9 (i.e., closer to the low TCE concentration well RM-5).

Local-scale variations in groundwater flow direction and velocity may explain some of the variability in the results. While the groundwater flow direction as determined by the methods used in this study is appropriate for larger-scale areas, differences in the local groundwater flow direction and rate can be expected at the smaller scale of the ISRM POPT site, particularly with the complex hydrostratigraphy at the site.

\subsection{Treatment Zone Reductive Capacity}

Sediment core samples collected during drilling in June 2000 were analyzed for the actual percent of iron oxides reduced by the field-scale dithionite injections (see Table 8.3). Cores collected for reductive capacity were retrieved with a 5 - $\mathrm{ft}$ long split-spoon sampler. Clean lexan liners, precut to $0.5 \mathrm{ft}$, were used to line the inside of the split spoon. Once the casing was advanced and the hole cleaned out, the split-spoon sampler was driven (vibrated) 5 -ft into the underlying undisturbed material. Upon bringing the split-spoon assembly to the surface, the head, shoe, and top half of the split spoon were removed. The lower half of the split spoon containing the sediment-filled 0.5 -lexan liners was immediately placed inside an argon-filled glovebox located onsite. Within the glovebox, the outsides of the individual liners were cleaned off and labeled with borehole number, depth interval, and an "up" arrow. Next, samples for reductive-capacity analysis were chosen; usually every other 0.5 - $\mathrm{ft}$ segment was selected. Preference was given to those cores that were from the interior portion of the 5 - $\mathrm{ft}$ core or appeared to be more reduced, avoiding the ends, which have a higher probability of containing disturbed sloughed material, or only partially full liners. Normally, three to four core segments were chosen for reductive capacity analysis from each 5-ft split-spoon interval. Before opening the glovebox, the reductive-capacity core liners were capped with plastic endcaps and secured with duct tape. After the glovebox was opened, core samples were immediately transferred to sealed schedule $40 \mathrm{PVC}$ tubes filled with anoxic water. The remaining core segments, not chosen for reductive capacity analysis, were used to collect geologic samples and construct a geologic log of the interval. Remaining liners filled with intact material at the end of sampling and geologic description were capped and stored as archive samples.

Qualitatively, a reduced zone was observed from the sediment cores by the change in sediment color to grey/black, which corresponds to siderite and iron sulfide (Table 8.3). While a grey or black color in sediment cores indicates a reducing zone, the intensity of the color cannot be used to quantify redox activity. Reduced sediment was observed in all cores. In all boreholes, the reduced zone extended from the bottom of the targeted treatment zone upwards through part to most of the unit. The topographic relief of the aquifer bottom coupled with the slightly higher density of the dithionite injection fluid (relative to groundwater) lead to the injection solution sinking and generally treating the lower portion of the aquifer, and likely sinking into and through the underlying aquitard (Qvt2). 
Table 8.3. Qualitative Reducing Conditions Observed in Sediment Cores

\begin{tabular}{||l|c|c|c||}
\hline \multicolumn{1}{|c|}{ Borehole } & $\begin{array}{c}\text { Aquifer Depth } \\
\text { (Ft) }\end{array}$ & $\begin{array}{c}\text { Reduced Zone } \\
\text { (Ft) }\end{array}$ & $\begin{array}{c}\text { Stratigraphic } \\
\text { Unit }\end{array}$ \\
\hline FM-3 & $56-68$ & $58-68$ & Gg-u \\
\hline FM-4 & $45-65$ & $61-65$ & Gg-u \\
\hline CH-1 & $50-73.5$ & $63.5-73.5$ & Gg-u \\
\hline RM-14 & $51-74.5$ & $60-74.5$ & Gg-u \\
\hline RM-15 & $58-70$ & $69-70$ & Gg-u \\
\hline
\end{tabular}

Eight sediment samples from the reduced interval of the boreholes were analyzed in laboratory experiments for the percent of iron that was reduced by the field-scale dithionite injection experiments. Sediment oxidation by dissolved oxygen-saturated water in columns was used to determine the mass of field-reduced iron (i.e., same procedure used in Section 4.4 for determining the amount of reducible iron). These experiments consisted of injecting oxygen-saturated water into sediment columns at a constant rate until oxygen was no longer consumed by the reduced iron. The mass of reduced iron was calculated from the oxygen loss throughout the experiment. To account for the spatial variability of the total mass of iron in sediment, three of the eight samples were re-reduced in the laboratory (with dithionite) and oxidized a second time to determine the maximum amount of reducible iron. This provides data to calculate the percent of reducible iron in sediment cores that was treated by the field-scale dithionite injections (i.e., a measure of the injection efficiency).

Results of the laboratory oxidation experiments on sediment cores from the POPT site (Table 8.4) indicate that the sediment was substantially reduced. The fraction of iron that was reduced by the field dithionite injections averaged $68 \%$ and varied from $23 \%$ to $100 \%$ (relative to the maximum reducible iron). The samples showed a distribution of reduction percentage decreasing with distance from the injection well (as expected), as well as skewed toward deeper samples being more highly reduced. The total mass of reducible iron (averaging $60.9 \pm 21.0 \mu \mathrm{mol} \mathrm{Fe}(\mathrm{II}) / \mathrm{g}$ [0.34\%] for all POPT sediments) indicates that the iron content is highly spatially variable. This average of 12 separate experiments provided a similar value for reducible iron to experiments with a mixture of all of the RM sediments $[60.3 \mu \mathrm{mol} \mathrm{Fe}(\mathrm{II}) / \mathrm{g}]$. The mass of reducible iron in the aquifer sediments (i.e., gravel unit) varied from $19.0 \mu \mathrm{mol} F(\mathrm{II}) / \mathrm{g}$ [RM-2, 62'] to $126 \mu \mathrm{mol} \mathrm{Fe}(\mathrm{II}) / \mathrm{g}$ [RM-1, 62'], although the underlying till had even more reducible iron [RM-14, 73', a sandy silt had $168 \mu \mathrm{mol} F($ (II)/g]. Due to the large amount of spatial variability of the reducible iron mass, a small number of points may not accurately assess the overall performance of the reduced zone. The amount of TCE degradation provides a larger-scale average of the overall performance because the degradation reaction is essentially averaged through sediments with differing amount of reduction.

Oxidation experiments show that the field-reduced sediment (Figure 8.16b) had considerable reductive capacity but less than the maximum amount that could be reduced in laboratory experiments (Figure 8.16a). Both samples had approximately the same mass of total reducible iron [about $63 \mu \mathrm{mol}$ $\mathrm{Fe}(\mathrm{II}) / \mathrm{g}]$, but the field-reduced sediment from FM-4 at a depth of $61.8 \mathrm{ft}$ indicated that the iron in this location was about 50\% reduced. At this level, the TCE degradation half-life is approximately 160 hours. 


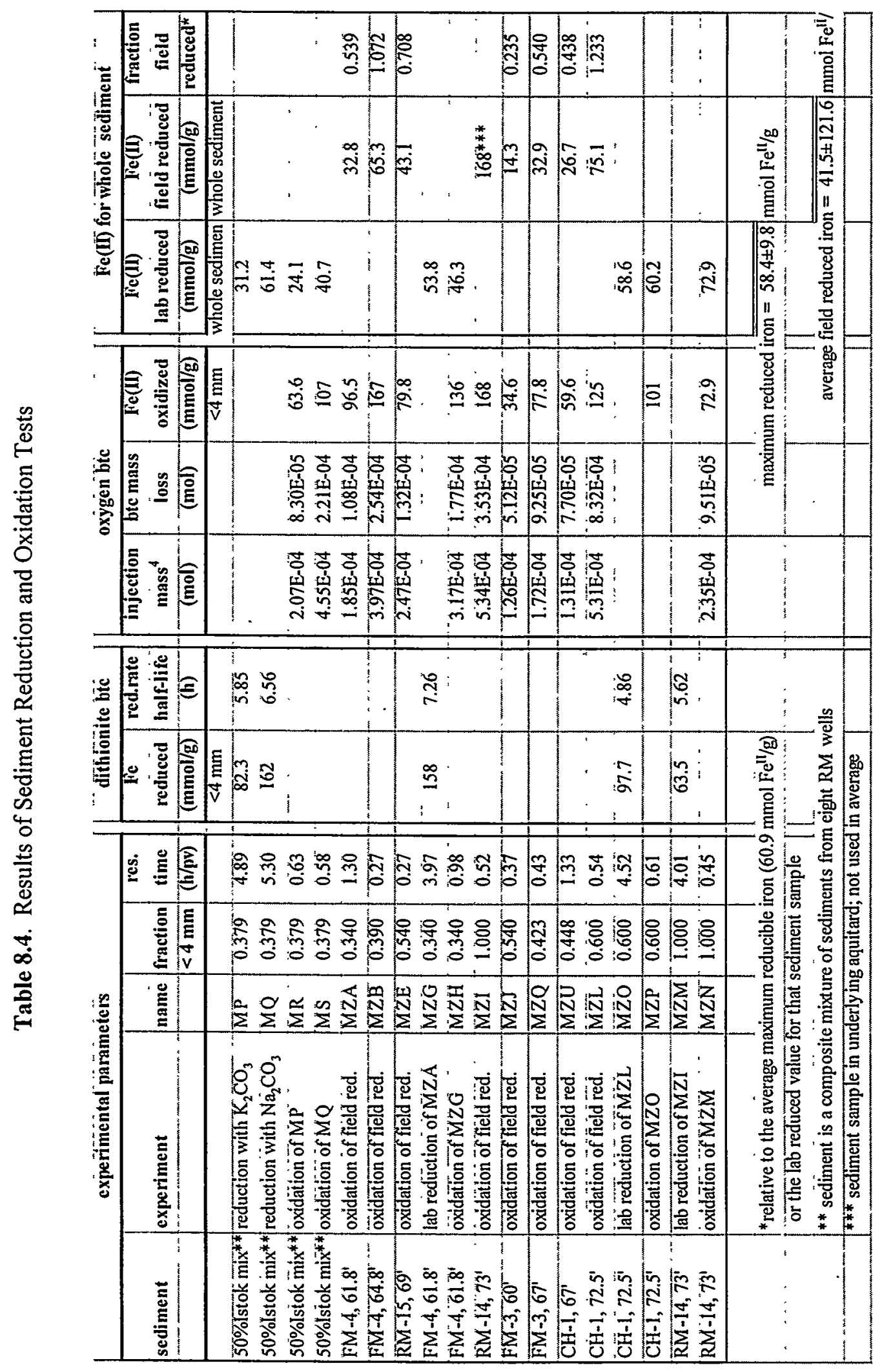



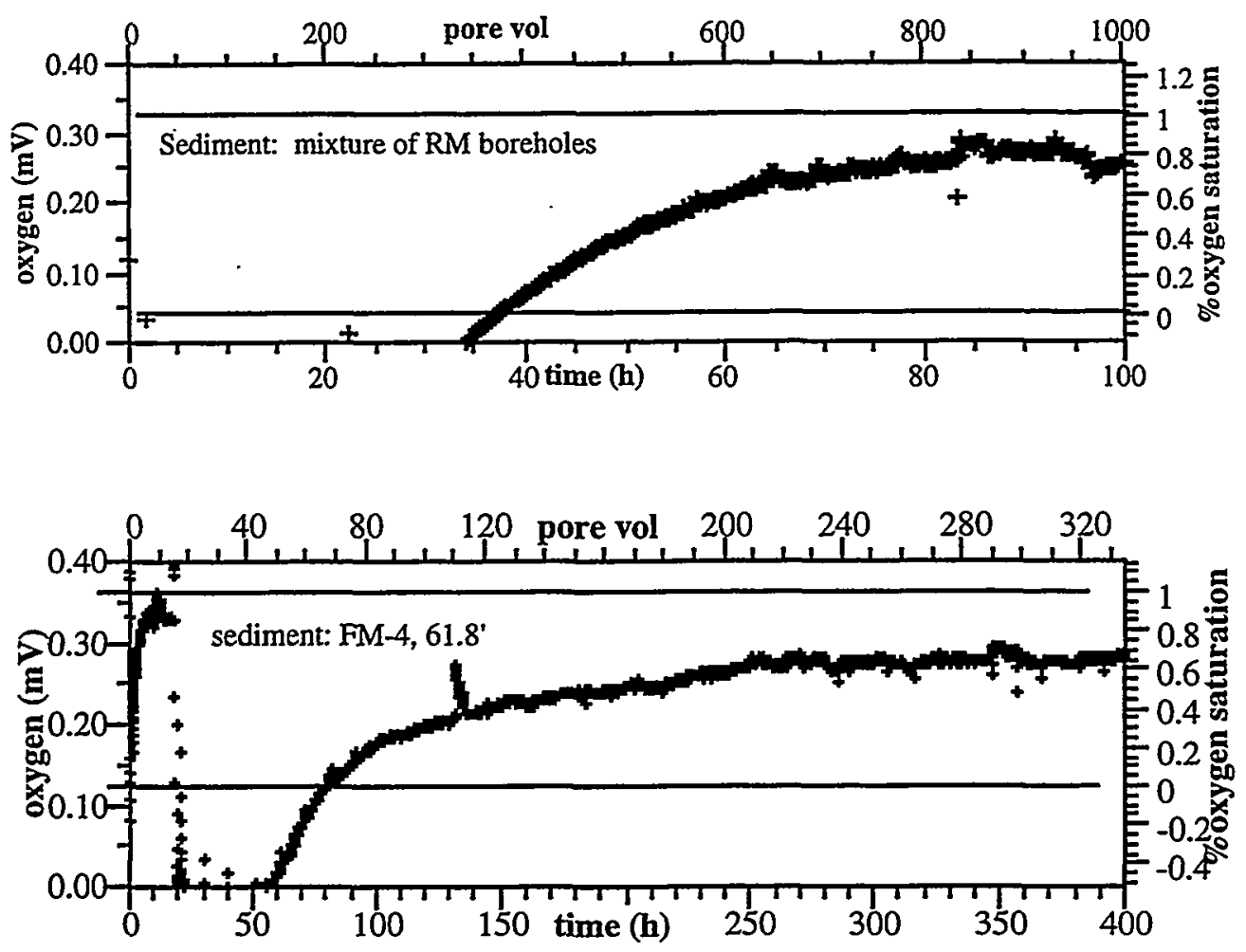

Figure 8.16. Sediment Oxidation of a Mixture of all RM Borehole Sediments (a) That was $100 \%$ Reduced by Dithionite in Laboratory Experiments, and (b) Sediment from FM-4, 61.8-ft Depth That was Field Reduced

This capacity represents approximately 320 pore volumes of treatment. A second sediment sample from borehole FM-4 (65 ft) indicated that the iron was $100 \%$ reduced, which would degrade TCE at a faster rate. The TCE degradation performance of the treatment zone can be estimated from the spatial resolution of this iron reduction data as well as the estimated iron reduction at well locations and the residence time of TCE in the reduced zone (Section 8.2).

\subsection{Hydraulic Performance}

As discussed in Section 4.6, pre-injection hydraulic testing was conducted to determine the localscale hydraulic properties of the aquifer and to provide a baseline for comparison with post-injection hydraulic responses. This information was used to assess any changes in formation permeability associated with the ISRM treatment. Because ISRM is a permeable barrier technology that relies on the flow of contaminated groundwater through the treatment zone under natural gradient conditions, it is important to determine whether the physical and chemical processes associated with the technology (i.e., injection of a chemical reducing agent) cause any significant reduction in the permeability of the formation. 
Data used to assess the degradation of hydraulic performance associated with implementation of ISRM included pressure buildup (injection phase), drawdown (withdrawal phase), and recovery (following withdrawal phase) responses collected during the various phases of the ISRM proof-ofprinciple demonstration. Pressure response and flow rate data were collected from the pre-injection (baseline) constant-rate discharge test, the tracer injection test, and the five individual dithionite injection tests. Selected data were analyzed using the analytical techniques discussed in Section 4.6.

Hydraulic performance analysis was focused on fully screened monitoring wells within the targeted treatment zone that were installed and instrumented before the first dithionite injection test at the site (RM-5, RM-7, and RM-8). Other monitoring wells within the treatment zone (RM-11 and RM-13) were not included in the analysis because they were installed after the first dithionite injection, so no baseline information was available.

Comparison of pre- and post-injection hydraulic test responses was complicated by changing test conditions throughout the various phases of field testing at the site. Test conditions were similar for the baseline constant-rate discharge test through the first dithionite injection test. However, following the first dithionite injection test, it was determined that significant quantities of dithionite were being fluxed into the lower outwash gravel unit during injection and that recovery of this mass by pumping only from the injection well was ineffective. As discussed in Section 4.6, the Qvt2 aquitard unit is noncontinuous near the injection well (RM-9), and the upper and lower outwash gravel units are hydraulically connected. To facilitate mass recovery from the lower outwash gravel unit and improve the overall percentage of residuals recovery, withdrawal phases for Injections $2 \mathrm{~b}$ through 4 were conducted with extraction pumps installed in RM-9 and RM-2, with a withdrawal rate division of $80 \%$ and $20 \%$, respectively.

Although tests run prior to dithionite Injection $2 \mathrm{~b}$ were run at flow rates that varied as much as $20 \%$, comparing these tests is easy because theoretical well response scales linearly with discharge rate. Scaling of pressure response data based on the test flow rate provides a valid comparison method as long as the same test well configuration is used for all tests. Comparing tests conducted with combined discharge from RM-9 and RM-2 with other tests conducted with discharge from RM-9 only is not strictly valid. However, because of the strong hydraulic connection between the upper and lower outwash gravel unit near the injection well and the small relative withdrawal rate in RM-2 (20\%), it was hypothesized that the pressure responses observed from combined RM-9/RM-2 withdrawal could be used to provide a qualitative or semi-quantitative assessment of changes in formation permeability.

To test this hypothesis, pressure response data from the withdrawal phase of dithionite Injection 2a (pumping from RM-9 only at $90 \mathrm{gpm}$ ) were compared with scaled pressure response data from the withdrawal phase of dithionite Injection $2 b$ (pumping from $R M-9$ at 90 gpm and $R M-2$ at 20 gpm). Data from these comparisons are shown in Figure 8.17. As indicated, comparison of scaled pressure response data under these varying test conditions show no consistent bias between the two data sets for the three monitoring wells analyzed. This analysis indicates that comparison of the scaled pressure responses from all of the various test phases, although not theoretically accurate under the changing test conditions discussed above, should provide an acceptable measure of the effects of the multiple injection/withdrawal tests conducted during the ISRM proof-of-principle test. 

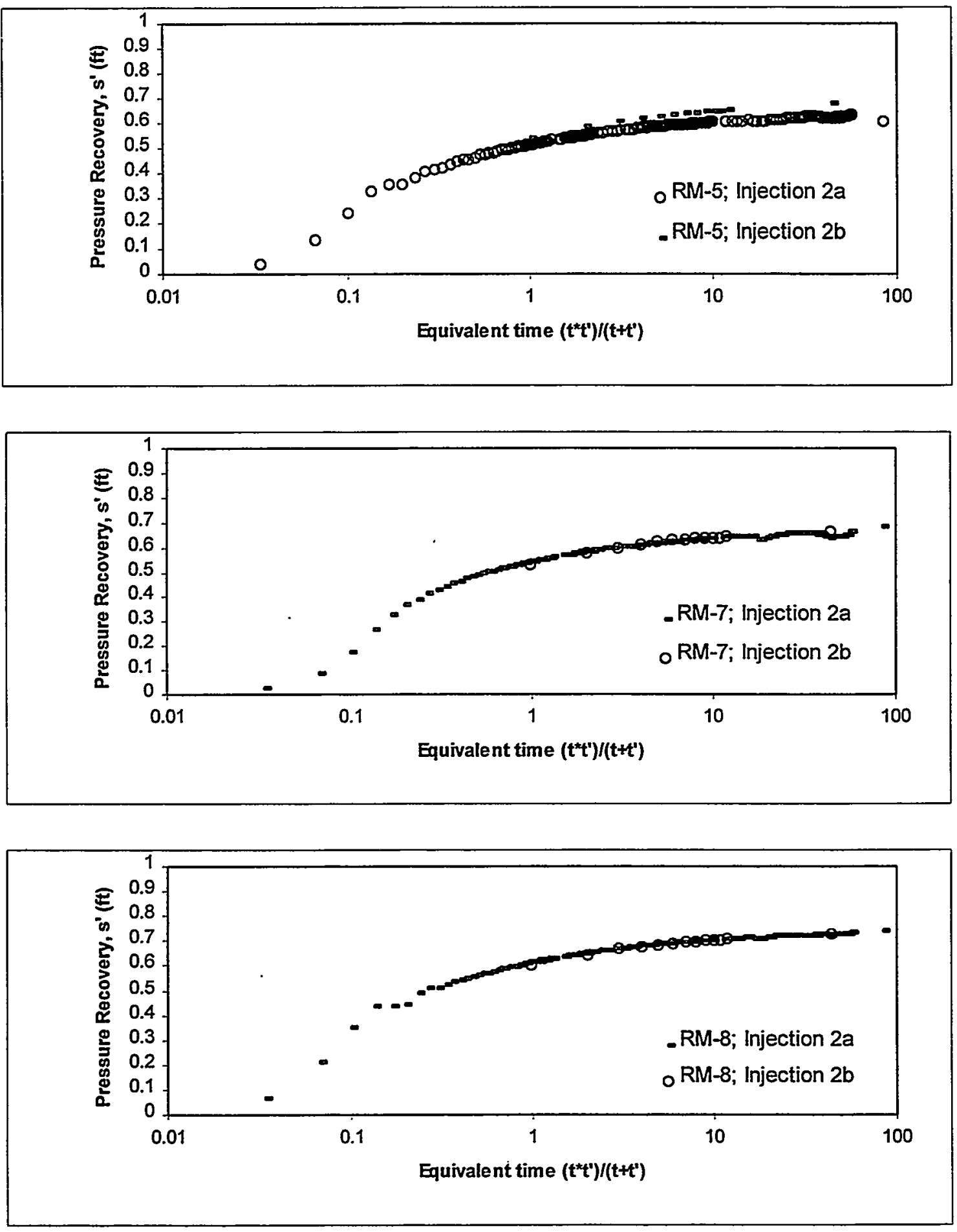

Figure 8.17. Comparison of Pressure Response Data for (a) RM-5, (b) RM-7, and (c) RM-8 Under Changing Test Conditions (i.e., pumping from RM-9 only [Injection 2a] versus combined pumping of RM-9 and RM-2 [Injection 2b]) 
Plots showing the observed pressure response at the injection well and selected monitoring wells for the various test phases are contained in Appendix E. Based on analysis of drawdown data from the withdrawal phases and pressure recovery data collected at termination of the withdrawal phases, it was determined that early- and intermediate-time pressure responses $(t<=100 \mathrm{~min})$ provided the most representative data set for determination of test site scale hydrologic properties. Late time data showed a consistent deflection from the theoretical well response, indicating that some type of reduced flow boundary had been encountered. This type of late-time response is not unexpected given the variability in formation thickness and relatively high level of heterogeneity observed at the site.

To assess changes in formation permeability over the duration of ISRM proof-of-principle injection/ withdrawal testing, the pressure response at a test time of 100 minutes (scaled to a flow rate of $90 \mathrm{gpm}$ ) was tabulated for the various test phases. As discussed previously, pressure response data were available from three phases of each injection/withdrawal test; the injection (pressure buildup), the start of the withdrawal (early-time drawdown data), and the end of the withdrawal (recovery following termination of pumping). These data are presented in Figures 8.18 through 8.20 .

Figure 8.18 shows pressure response in the injection well (RM-9) relative to pressure responses in the selected monitoring wells. These data indicate that plugging and unplugging of the injection well screen occurred over the multiple injections, as evidenced by the magnitude of variability in pressure response in the injection well relative to that in the observation wells. In Figure 8.19, the pressure responses observed in the monitoring wells are plotted without the injection well data, providing a better scale for interpretation of the data. Although there are anomalies, pressure response data from the selected monitoring wells do follow the same general trends. Figure 8.20 shows a composite response, generated by computing the mean pressure response of the three monitoring wells for each test phase. The composite response indicates there is a decreasing trend in formation permeability (i.e., increasing trend in pressure response) over the multiple injection/withdrawal tests.

To quantify the magnitude of this change, type curve analysis results for the three monitoring wells, both pre-injection and following Injection 4, were compared (Appendix F). Pre-injection transmissivities at the three monitoring locations ranged from 7,000 to $9,600 \mathrm{ft}^{2} /$ day compared with a post-injection range of 6,300 to $8,800 \mathrm{ft}^{2} /$ day. Based on these analysis results and an average aquifer thickness of $12 \mathrm{ft}$, the average hydraulic conductivity within the targeted treatment zone before the first dithionite injection and after the final injection is $700 \mathrm{ft} /$ day and $630 \mathrm{ft} /$ day, respectively. These data indicate a reduction in hydraulic conductivity of approximately $10 \%$ over the multiple test phases, or less than $1 \%$ per injection/ withdrawal operation.

The physical and/or chemical processes responsible for this reduction in permeability are not well understood. Likely physical processes include redistribution of fine-grained materials, either naturally occurring or generated during sonic drilling, by the relatively high pore water velocities associated with the injection/withdrawal tests. Chemical processes associated with the injection of a buffered chemical reducing agent (i.e., sodium dithionite) include dissolution and redistribution of metals throughout the treatment zone, clay flocculation, and precipitate formation. Although there are insufficient data to rule out either of these processes, the consistent trend from the initial baseline hydraulic testing through the final dithionite injection indicates that physical processes may dominate. If the observed changes in 


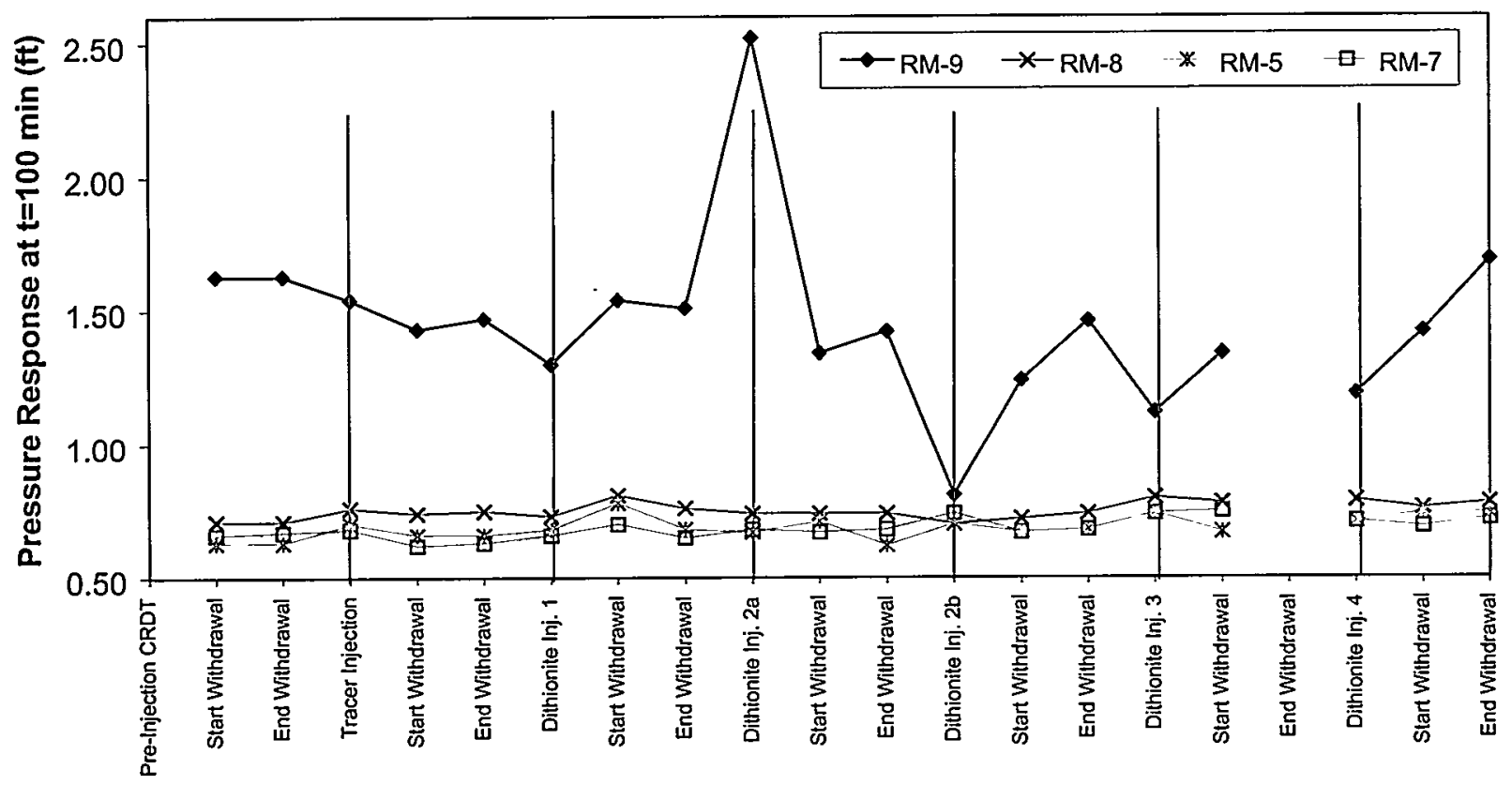

Figure 8.18. Pressure Response at $t=100 \mathrm{~min}$ for Injection Well RM-9 Relative to Pressure Responses in Selected Monitoring Wells

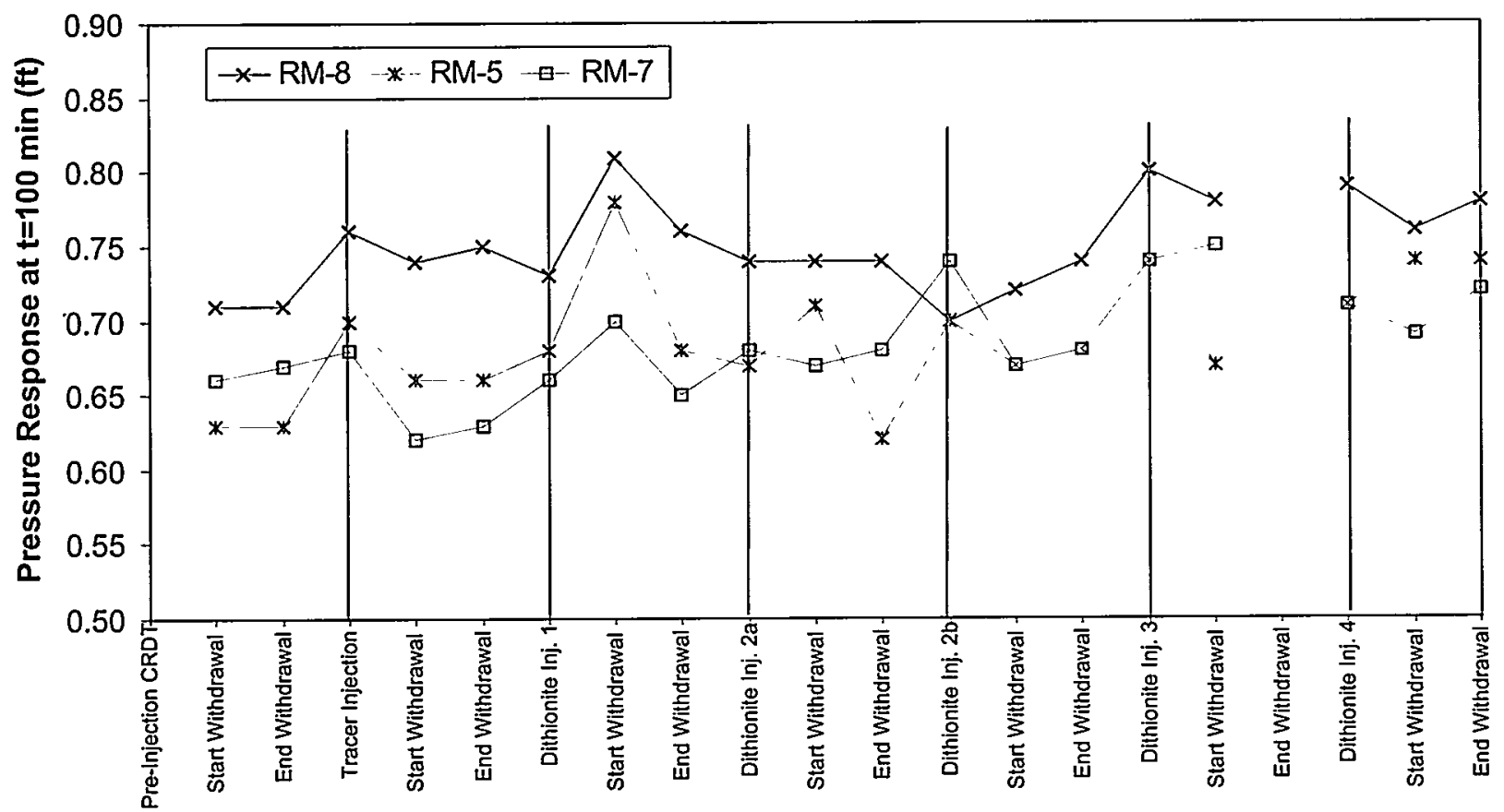

Figure 8.19. Pressure Response at $t=100 \mathrm{~min}$ for Selected Monitoring Wells 


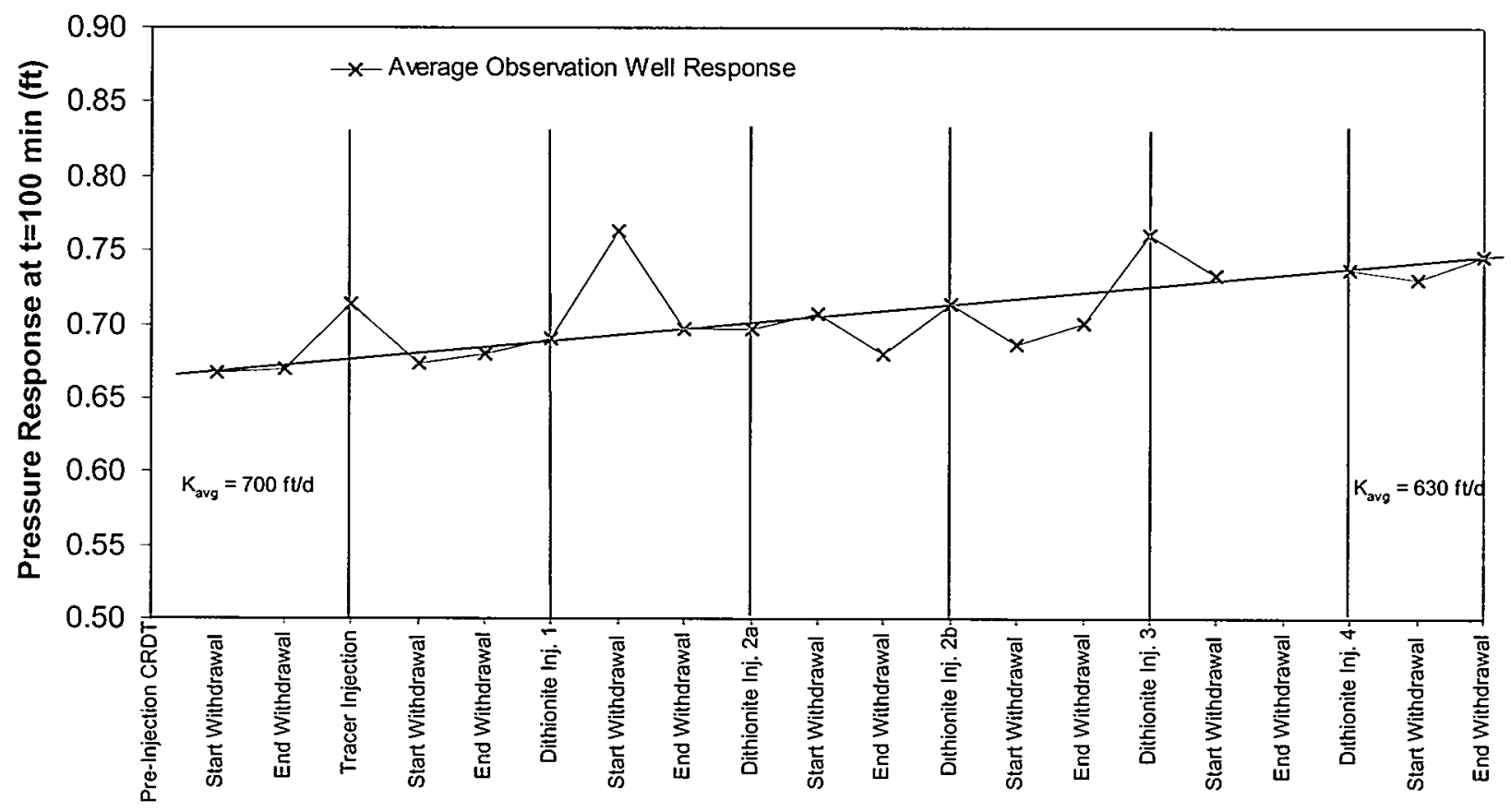

Figure 8.20. Average Pressure Response at $\mathrm{t}=100 \mathrm{~min}$ for Selected Monitoring Wells

permeability were associated primarily with injection of the chemical reducing agent, the trend in decreasing permeability should not have been observed until after the first dithionite injection. However, to investigate the aspects of sodium on permeability degradation in Fort Lewis sediments, a column experiment was conducted in which sediment was reduced with $0.09 \mathrm{~mol} / \mathrm{L}$ sodium dithionite and $0.36 \mathrm{~mol} / \mathrm{L}$ potassium carbonate (used in the field injections). The hydraulic head data indicated no apparent degradation in the hydraulic conductivity (pressure response in the column test actually indicated a $19 \%$ increase in conductivity) caused by sediment reduction or the potassium carbonate buffer. 


\subsection{Summary and Conclusions}

The objective of the ISRM proof-of-principle test was to determine the field-scale feasibility of the ISRM technology for the treatment of dissolved TCE contamination in the groundwater at the Fort Lewis Logistics Center. While the ISRM technology for TCE destruction was demonstrated in laboratory experiments using sediment from the Fort Lewis site, a field-scale test was required to determine the feasibility of applying the technology at a large scale in the complex hydrogeologic and geochemical conditions of the subsurface. Emplacement of the ISRM treatment zone was accomplished through a series of four separate dithionite injection tests conducted between November 10, 1998 and March 29, 2000. An extensive program of chemical monitoring was also performed before, during, and after each injection to evaluate ISRM's performance. Prior to emplacement of the ISRM treatment zone, the geologic, hydrologic, and geochemical properties of the site were characterized. Sediment core samples collected in connection with the characterization studies were analyzed in bench-scale column tests at PNNL to determine reducible iron content. These site-specific hydrogeologic and geochemical data were used to develop the emplacement design of the pilot-scale (i.e., single injection well) ISRM treatment zone.

Performance data obtained from the proof-of-principle test indicate that field-scale reductive dechlorination of TCE is feasible using the ISRM technology. A treatment zone was created in the subsurface that reduced TCE concentrations as much as $92 \%$ on the downgradient side of the reduced zone, from a background concentration of approximately $140 \mathrm{ppb}$ to approximately $11 \mathrm{ppb}$. The appearance of the principal degradation product, acetylene, also demonstrated that TCE destruction was occurring via reductive dechlorination. Preliminary analysis of sediment samples collected from post-test boreholes showed a high degree of iron reduction, which helped confirm the effectiveness of the treatment zone.

Laboratory analysis of sediment from the Fort Lewis site showed that it contains a relatively large amount of reducible iron $(0.25 \mathrm{wt} \%)$ and that a significant percentage $(>30$ to $40 \%)$ of iron reduction was required to achieve the necessary TCE degradation rates at the site given the low aquifer temperature $\left(11^{\circ} \mathrm{C}\right.$ ) and high groundwater velocities (time-weighted average value of $\sim 4 \mathrm{ft} / \mathrm{d}$ ). The technical objectives of this test and reagent requirements to achieve this reduction led to a series of four dithionite injection/withdrawal tests with monitoring periods between them to evaluate the interim TCE degradation performance. After the first injection, which had very low iron reduction efficiency, a number of design modifications were incorporated into the injections. These modifications included diluting of the reagent to minimize density sinking effects and preheating the treatment zone by injecting warm, anoxic water to increase the $\mathrm{Fe}(\mathrm{III})$ to $\mathrm{Fe}$ (II) reduction reaction rate. The dithionite/sediment contact time during the injection and residence phases was also increased. Including these design modifications as a standard procedure for all subsequent injections substantially improved iron reduction efficiency during the remaining injection tests (dithionite Injections $2 a, 2 b, 3$, and 4). Improved TCE degradation performance within the ISRM treatment zone was also observed after each dithionite injection/withdrawal test as the percentage of iron reduction at the site increased. 
Another important goal of the testing program was to provide assurances that chemical treatment of the subsurface did not result in undesirable secondary effects in Fort Lewis sediments, including formation of toxic TCE degradation products, mobilization of trace elements, and degradation of hydraulic performance. The field-scale study confirmed the extensive experience in the laboratory, which showed that TCE is degraded by reductive dechlorinatation, yielding benign products (acetylene, ethylene). While some pre-existing cis-DCE already was present in the Fort Lewis groundwater (associated with past biodegradation effects in the ambient plume), no significant change in the cis-DCE concentration was observed and no vinyl chloride was detected. Degradation of the dithionite itself does result in a substantial burden of sulfate being added to the groundwater. Most of the sulfate was withdrawn after each injection; however, reagent density and hydrogeologic conditions have resulted in consistently high sulfate levels in well RM-2. Residual levels of sulfate within the treatment zone did exceed secondary drinking water standards for a short time after each injection test and continue to exceed the $250 \mathrm{mg} / \mathrm{L}$ standard by 40 to $80 \mathrm{mg} / \mathrm{L}$ in three monitoring wells on the downgradient side of the treatment zone by June 2000. Sulfate concentrations are not expected exceed these levels as the residuals plume is transported downgradient and should eventually decline to below this level as the plume is dispersed and diluted by recharge.

As anticipated from previous field and laboratory ISRM tests, several trace metals were mobilized during emplacement of the ISRM treatment zone, the majority of which were recovered during the withdrawal phase of the process. Although concentrations in the withdrawal water did exceed drinking water standards, the withdrawal water met the criteria for wastewater disposal. After the treatment zone had been emplaced, none of the trace element concentrations exceeded primary drinking water standards; the secondary drinking water standards were exceeded for iron and manganese.

Hydraulic performance was found to degrade by a small amount over the 13 injection/withdrawal operations conducted at the test site over the duration of field activities. Although there are insufficient data to determine definitively whether the degradation was caused by physical or chemical processes, the consistent trend from the initial baseline hydraulic testing through the final dithionite injection indicates that physical processes may dominate. Interpretation of the available performance assessment data provided no indication that this small reduction in hydraulic conductivity ( $\sim 10 \%$ over the multiple test phases or less than $1 \%$ per injection/withdrawal operation) caused any significant degradation to the overall performance of the treatment zone. In summary, there do not appear to be any significant secondary effects that could limit full-scale application of this technology.

The primary objective of the ISRM proof-of-principle test was met. A single-well treatment zone was created and it was demonstrated that TCE could be reductively dechlorinated at the field scale. However, to quantify the performance and economic viability of a full-scale barrier deployment, a treatability test-scale demonstration is needed in which multiple injection wells are used to form an adequately sized linear barrier (e.g., 100 to $200 \mathrm{ft}$ long). It is not practical to attempt to obtain this information from a single-well proof-of-principle test. Uncertainties in determining groundwater flow direction at the accuracy required for a small-scale single-well test and associated local-scale hydrogeologic heterogeneities make detailed interpretation of the downgradient performance difficult. Additionally, costs associated with a detailed small-scale proof-of-principle test, which requires an extensive sampling and analysis program, cannot be extrapolated accurately to evaluate the costs of a full- 
scale deployment (i.e., reduction in sampling, analysis, and interpretation during full-scale deployment results in economy of scale). Given the site-specific nature of the ISRM technology, the cost associated with full-scale deployment is most strongly affected by aquifer and contaminant plume characteristics at the selected barrier location (barrier length required, aquifer thickness and depth, reducible Fe(III), groundwater velocity). 


\subsection{References}

Amonette JE, JE Szecsody, HT Schaef, JC Templeton, YA Gorby, and JS Fruchter. 1994. "Abiotic Reduction of Aquifer Materials by Dithionite: A Promising In-Situ Remediation Technology." Proceedings of the 33rd Hanford Symposium on Health and the Environment - In Situ Remediation: Scientific Basis for Current and Future Technologies, pp. 851-882. Pacific Northwest National Laboratory, Richland, Washington.

ASTM. 1986. "Standard practice for description and identification of soil (visual-manual procedure)." Annual Book of ASTM Standards, Volume 4.08. ASTM D 2488-84, American Society for Testing and Materials, Philadelphia.

Cantrell KJ, TL Liikala, TJ Gilmore, and GV Last. 1998. Initial Review of the Treatment Operations at the Installation Logistics Center, Fort Lewis, Washington. PNNL-11913, Pacific Northwest National Laboratory, Richland, Washington.

Ebasco (formerly Envirosphere). 1988. "Fort Lewis Logistics Center Remedial Investigation Final Report." Prepared for the U.S. Army Corps of Engineers, Seattle, by Ebasco Environmental, Bellevue, Washington.

Ebasco. 1993. "Fort Lewis Logistics Center, Lower Aquifer Groundwater Study, Final Technical Memorandum." Prepared for the U.S. Army Corps of Engineers, Seattle, by Ebasco Environmental, Bellevue, Washington.

Fruchter JS, JM Zachara, JK Fredrickson, CR Cole, JE Amonette, TO Stevens, DJ Holford, LE Eary, GD Black, and VR Vermeul. 1992. "Manipulation of Natural Subsurface Processes: Field Research and Validation." Pacific Northwest Laboratory Annual Report for 1991 to the DOE Office of Energy Research, Part 2: Environmental Sciences. PNL-8000 Pt. 2, Pacific Northwest Laboratory, Richland, Washington, pp. 88-106.

Fruchter JS, FA Spane, JK Fredrickson, CR Cole, JE Amonette, JC Templeton, TO Stevens, DJ Holford, LE Eary, BN Bjornstad, GD Black, JM Zachara, and VR Vermeul. 1994. Manipulation of Natural Subsurface Processes: Field Research and Validation. PNL-10123, Pacific Northwest National Laboratory, Richland, Washington.

Fruchter JS, JE Amonette, CR Cole, YA Gorby, MD Humphrey, JD Istok, FA Spane, JE Szecsody, SS Teel, VR Vermeul, MD Williams, and SB Yabusaki. 1996. In Situ Redox Manipulation Field Injection Test Report-Hanford 100 H Area. PNNL-1 1372, Pacific Northwest National Laboratory, Richland, Washington. 
Fruchter JS, CR. Cole, MD Williams, VR Vermeul, JE Amonette, JE Szecsody, JD Istok, and $M$ Humphrey. 2000. "Creation of a Subsurface Treatment Zone for Aqueous Chromate Contamination Using In Situ Redox Manipulation." Ground Water Monit. Remed., spring 2000.

Hantush MS. 1960. "Modification of the Theory of Leaky Aquifers." Journal of Geophysical Research, 65(11):3713-3725.

Hoopes JA and DRF Harleman. 1967. "Dispersion in radial flow from a recharge well." Journal of Geophysical Research 72(14):3595-3607.

Istok JD, JE Amonette, CR Cole, JS Fruchter, MD Humphrey, JE Szecsody, SS Teel, VR Vermeul, MD Williams, and SB Yabusaki. 1999. "In Situ Redox Manipulation by Dithionite Injection: Intermediate-Scale Laboratory Experiments." Groundwater, 37:884-889.

Last GV and TL Liikala. 1987. A field guide for well-site geologists: Cable-tool drilling. PNL-6392, Pacific Northwest Laboratory, Richland, Washington.

Logan RL, TJ Walsh, and WG Gerstel. 2000. "A revision of Quaternary stratigraphy in the southern Puget lowland, Washington." Geological Society of America Abstracts with Programs, 32(6):A-25.

Neuman SP. 1972. "Theory of Flow in Unconfined Aquifers Considering Delayed Response of the Water Table." Water Resources Research 8(4):1031-1045.

Shannon and Wilson. 1986. "Source areas, occurrence, and recommended remedial alternatives for trichloroethylene in groundwater, Fort Lewis Logistics Center, Fort Lewis, Washington." W-4487-04, Shannon and Wilson, Inc., Seattle, Washington.

Szecsody JE, JS Fruchter, DS Sklarew, and JC Evans. 2000. In Situ Redox Manipulation of Subsurface Sediments from Fort Lewis, Washington: Iron Reduction and TCE Dechlorination Mechanisms. PNNL-13178, Pacific Northwest National Laboratory, Richland, Washington.

Troost KG, JB Mahoney, DB Booth, and RK Borden. 1998. "Discriminating glacial from nonglacial deposits in Pleistocene sediments of the south-central Puget Lowland." Program with Abstracts, Association of Engineering Geologists Annual Meeting, Seattle, p. 130.

Troost KG. 2000. "The Olympia nonglacial interval in the southern Puget Lowland, Washington." Geological Society of America Abstracts with Programs, 32(6):A-72.

URS Greiner Woodward Clyde. 2000. Draft Fort Lewis Logistics Center Remedial Action Monitoring, Fourth Annual Monitoring Report. Prepared for U.S. Army Corps of Engineers, Seattle, Washington.

USACE. 1992. Phase I Well Field Design - Final Design analysis Groundwater Treatment Logistics Center, Fort Lewis, Washington. Volume I Report, U.S. Army Corps of Engineers, Seattle, Washington. 
USACE. 1993. Logistics Center Groundwater Treatment: Phase II-Geotechnical Design Analysis (90\% Design Submittal), Fort Lewis, Washington. PN 035756, U.S. Army Corps of Engineers, Seattle, Washington.

Walters $\mathrm{KL}$ and Kimmel GE. 1968. "Groundwater occurrence and stratigraphy of unconsolidated deposits, central Pierce County, Washington." Water Supply Bulletin No. 22, Washington State Department of Water Resources, Olympia, Washington.

Woodward-Clyde. 1997. "East Gate disposal yard expanded site investigation: site history and conceptual site model." Prepared for the U. S. Army Corps of Engineers by Woodward-Clyde, Seattle, Washington.

Williams MD, SB Yabusaki, CR Cole, and VR Vermeul. 1994. "In Situ Redox Manipulation Field Experiment: Design Analysis." In Situ Remediation: Scientific Basis for Current and Future Technologies, GW Gee and NR Wing, eds. Battelle Press, Columbus, Ohio.

Williams MD, VR Vermeul, JE Szecsody, JS Fruchter, and CR Cole. 1999a. 100-D Area In Situ Redox Treatability Test for Chromate-Contaminated Groundwater: FY 1998 Year-End Report. PNNL-12153, Pacific Northwest National Laboratory Richland, Washington.

Williams MD, VR Vermeul, M Oostrom, JC Evans, JS Fruchter, JD Istok, MD Humphrey, DC Lanigan, JE Szecsody, MD White, TW Wietsma, and CR Cole. 1999b. Anoxic Plume Attenuation in a Fluctuating Water Table System: Impact of 100-D Area In Situ Redox Manipulation on Downgradient Dissolved Oxygen Concentrations. PNNL-12192, Pacific Northwest National Laboratory, Richland, Washington.

Williams MD and M Oostrom. 2000. "Oxygenation of anoxic water in a fluctuating water table system." Journal of Hydrology, 230:70-85. 
Appendix A

Geochemical Reactions for Remediation of TCE 


\section{Appendix A}

\section{Geochemical Reactions for Remediation of TCE}

\section{A.1 Iron Reduction Mechanism}

The remediation technology used for Fort Lewis is based on the proven ability of reduced (ferrous) iron to abiotically degrade TCE and other organic contaminants (Roberts et al. 1996). The ISRM technology used existing iron in aquifer sediment that was chemically treated with a reductant (sodium dithionite buffered at high $\mathrm{pH}$ ) for a short time (typically 24 to 60 hours) to reduce $\mathrm{Fe}(\mathrm{III})$ oxides present in the sediment to adsorbed or structural Fe(II) phases. This reduction of aquifer sediments results in the groundwater redox conditions becoming reducing and the disappearance of dissolved oxygen in water, as shown conceptually in Figure A.1 ( 0 to 0.1 years).

a)

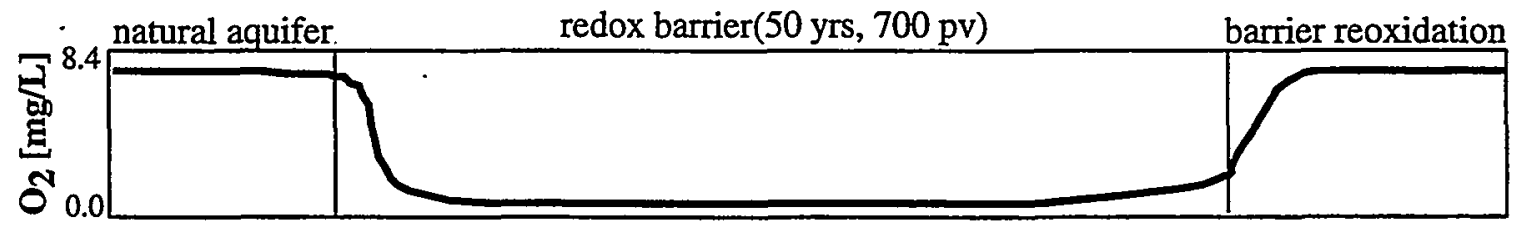

b)

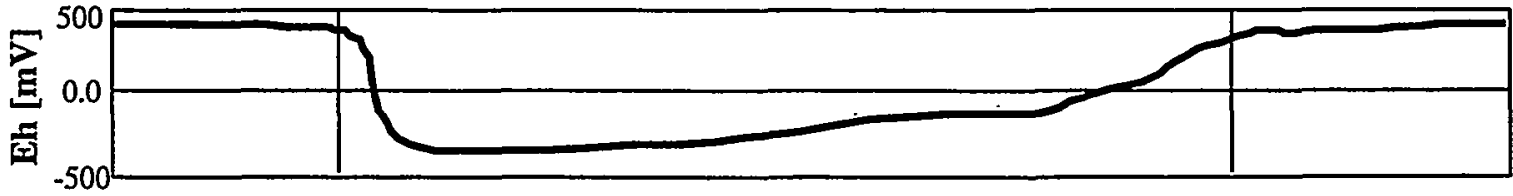

c)

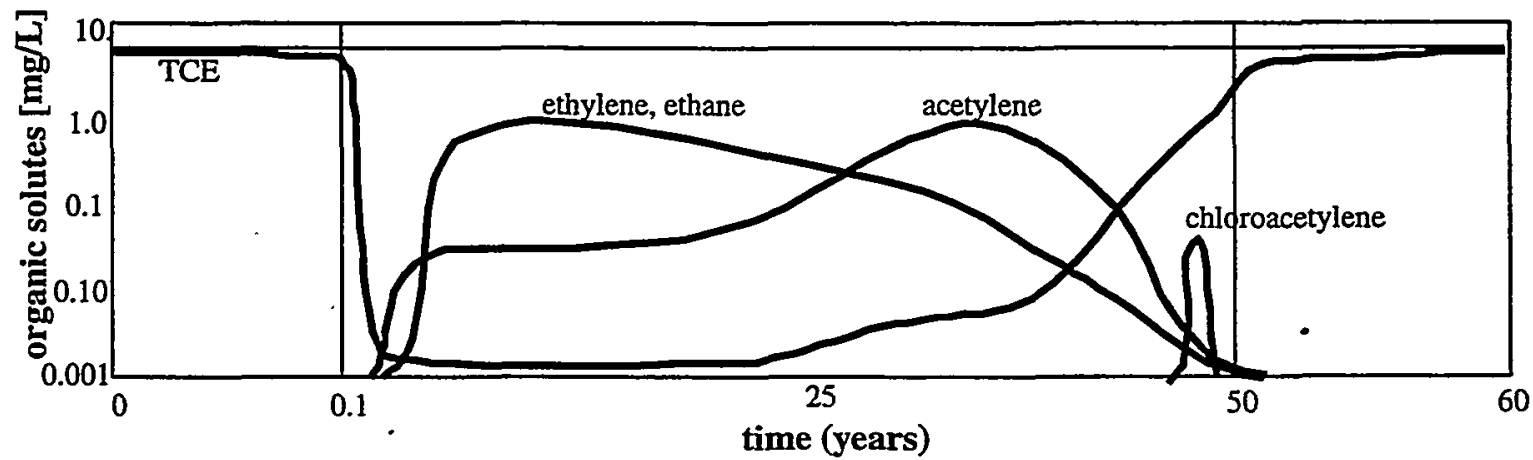

Figure A.1. Conceptual Diagram Showing the Influence of a Redox Barrier as a Function of Time On: a) Dissolved Oxygen in Water Which is the Main Barrier Oxidant, b) the Redox Conditions of Groundwater, and c) TCE and Degradation Products Resulting from Dechlorination by the Reduced Iron in the Redox Barrier 
The TCE dechlorination mechanism with reduced iron of this chemical treatment is generally the same as zero-valent permeable iron walls (shown in Figure A.lc). Zero-valent iron/mixed metal barriers also rely on the oxidation of ferrous (adsorbed or Fe(II) minerals such as green rust) (Genin et al. 1998) to ferric iron as the electron donor for remediation of chlorinated aliphatic contaminants (Balko and Tratnyek 1998; Johnson et al. 1998) or reduction of metals such as chromate (Blowes et al. 1997; Buerge and Hug 1997), and not the oxidation of $\mathrm{Fe}^{\circ}$. Although aqueous $\mathrm{Fe}(\mathrm{II})$ can reduce chromate (Eary and Rai 1988), adsorbed or structural Fe(II) on a Fe(III) oxide, clay surface, or zero-valent iron surface is necessary for dechlorination reactions. The role of the surface is not clearly understood.

The dithionite chemical treatment dissolves and reduces amorphous and some crystalline Fe(III) oxides. The reduced $\mathrm{Fe}$ (II) created by the dithionite chemical treatment appears to be present in at least two different $\mathrm{Fe}$ (II) phases: adsorbed $\mathrm{Fe}(\mathrm{II})$ and $\mathrm{Fe}(\mathrm{II})$-carbonate (siderite); adsorbed $\mathrm{Fe}$ (II) appears to be the dominant $\mathrm{Fe}(\mathrm{II})$ phase. Other, unidentified $\mathrm{Fe}(\mathrm{II})$ mineral phases may also be produced. Although more than one Fe(III) phase is likely reduced in a natural sediment, it can be useful to determine how simple a chemical model is needed to describe the observations. The reaction that describes a single phase of iron that is reduced by sodium dithionite:

$$
\left.\mathrm{S}_{2} \mathrm{O}_{4}{ }^{-2}+2 \equiv \mathrm{Fe}^{3+}+2 \mathrm{H}_{2}\right) \Longleftrightarrow 2 \equiv \mathrm{Fe}^{2+}+2\left(\mathrm{SO}_{3}{ }^{-2}\right)+4 \mathrm{H}^{+}
$$

shows that the forward rate is a function of the dithionite concentration and the square of the reducible iron concentration (rate is overall a third-order function of concentration). The aqueous Fe(II) produced has a high affinity for surfaces, so is quickly adsorbed. Therefore, Fe(II) mobility in mid- to high-pH, low ionic-strength groundwater (e.g., Fort Lewis groundwater) is extremely limited, and iron is not expected to leach from sediments during the dithionite treatment. Aqueous iron measurements in previous studies have shown $<1 \%$ iron leaching even after 600 pore volumes of groundwater passed through a sediment column. Corresponding solid iron measurements of sediments used in these columns showed $4 \%$ to $10 \%$ loss of iron. Iron mobility is somewhat higher during the actual dithionite injection as a high ionic strength solution of other cations $\left(0.06 \mathrm{M} \mathrm{Na}^{+}\right.$and $0.24 \mathrm{M} \mathrm{K}^{+}$in this case) competes for the same adsorption sites as $\mathrm{Fe}^{2+}$, causing some $\mathrm{Fe}^{2+}$ desorption. Previous experimental transport studies with dithionite injection into sediments have shown $0 \%$ to $12 \%$ iron loss after 40 pore volumes of dithionite treatment.

Experimental evidence from previous studies with Hanford sediments (Istok et al. 1999; Fruchter et al. 2000) have shown that two parallel reduction reactions are needed to describe iron reduction data (i.e., a fraction of sites are quickly reduced and a fraction more slowly reduced). This may be the result of the reduction of two or more major Fe(III) phases. Based on this hypothesis, a second reduction reaction was added with a second ferric iron phase symbolized by $\equiv \mathrm{Fe}^{3+}$ :

$$
\mathrm{S}_{2} \mathrm{O}_{4}{ }^{-2}+2 \equiv \mathrm{Fe}^{3+}+2 \mathrm{H}_{2} \mathrm{O} \Longleftrightarrow 2 \equiv \mathrm{Fe}^{2+}+2\left(\mathrm{SO}_{3}{ }^{-2}\right)+4 \mathrm{H}^{+}
$$


The total number of oxidized or reduced iron sites is the sum of sites in Reactions 1 and 2 . If the number of slowly reducing sites (Reaction 2) is small and the mass of iron far in excess of the dithionite, Reaction 1 can be reduced to a kinetic first-order reaction in which $\mathrm{Fe}^{3+}$ remains constant:

$$
\mathrm{S}_{2} \mathrm{O}_{4}^{-2} \Longleftrightarrow 2 \mathrm{Fe}^{2+}+\mathrm{SO}_{3}^{-2}
$$

Reaction 3 is not a balanced reaction, but is meant to illustrate the species upon which the reaction rate is dependent. Other studies of this chemical treatment have shown that reactions 1 and 2 can be approximated in some cases with reaction 3 with a pseudo-first order rate of $\sim 5 \mathrm{~h}$ (half-life). Another reaction occurs in the system, which describes the disproportionation of dithionite in contact with sediment:

$$
2 \mathrm{~S}_{2} \mathrm{O}_{4}{ }^{-2}+\mathrm{H}_{2} \mathrm{O} \Longleftrightarrow \mathrm{S}_{2} \mathrm{O}_{3}{ }^{-2}+2\left(\mathrm{HSO}_{3}{ }^{-}\right)
$$

that accounts for the mass loss of dithionite that cannot be used for iron reduction. Other studies have shown that this reaction has a half-life of $\sim 27$ hours (basaltic sediments). The consequence of this reaction is to limit how slowly dithionite can be reacted with (i.e., injected into) sediment in the field. If dithionite is injected too slowly, a significant amount of its mass is lost to disproportionation.

Although Fe(III) phases are the most significant phases that react with dithionite, other mineral phases present in natural sediments may also be reduced and use some of the dithionite. Previous studies have shown that some manganese reduction occurs because of the dithionite treatment of Hanford sediment, although reduced $\mathrm{Mn}(\mathrm{II}, 0)$ phases were about $3.4 \%$ of the mass of reduced iron phases.

\section{A.2 Sediment Oxidation Mechanisms}

The oxidation of the adsorbed and structural $\mathrm{Fe}(\mathrm{II})$ in the sediments of the permeable redox barrier occurs naturally by the inflow of dissolved oxygen through the barrier but can also be initiated by contaminants such as chromate, TCE, nitrate, uranium, or other reducible species that may be present. If redox equilibrium completely defined the mechanism (i.e., no effects from activation energies), and contaminants are present in equal molar concentrations, they would be reduced faster in the following order:

$$
\text { chromate }>\text { dissolved oxygen }>\text { nitrate }>\text { uranium }>\text { TCE }
$$

In relatively uncontaminated aquifers, dissolved oxygen in water is the dominant oxidant of reduced iron species because contaminants are generally present in lower molar concentrations relative to dissolved oxygen. The oxidation of reduced iron in pure mineral phases is described by the following reactions, first by dissolved oxygen and then with other contaminants. Fe(II) species that are known to exist in the dithionite-reduced sediments include adsorbed $\mathrm{Fe}(\mathrm{II})$ and siderite $\left(\mathrm{Fe}[\mathrm{II}] \mathrm{CO}_{3}\right)$ A single mole of electrons is consumed as a mole of these species is oxidized:

$$
\mathrm{Fe}^{2+} \Longleftrightarrow \mathrm{Fe}^{3+}+\mathrm{e}^{-} \quad \mathrm{Eh}=-0.77 \mathrm{v}
$$




$$
\begin{array}{ll}
\mathrm{Fe}^{2+}+3 \mathrm{H}_{2} \mathrm{O} \Longleftrightarrow \mathrm{Fe}(\mathrm{OH})_{3}(\mathrm{~s})+3 \mathrm{H}^{+}+\mathrm{e}^{-} & \mathrm{Eh}=-0.56 \mathrm{v} \\
\mathrm{FeCO}_{3}(\mathrm{~s})+3 \mathrm{H}_{2} \mathrm{O} \Longleftrightarrow \mathrm{Fe}(\mathrm{OH})_{3}(\mathrm{~s})+2 \mathrm{H}^{+}+\mathrm{HCO}_{3}^{-}+\mathrm{e}^{-} &
\end{array}
$$

The use of dissolved oxygen as an oxidant is generally divided into two electron sequences that, when combined, yield:

$$
\mathrm{O}_{2}+4 \mathrm{H}^{+}+4 \mathrm{e}^{-} \Longleftrightarrow \mathrm{H}_{2} \mathrm{O}, \quad \mathrm{Eh}=1.23 \mathrm{v}
$$

which shows that 4 moles of electrons are needed per mole of $\mathrm{O}_{2}$ consumed. The rate of this reaction (Reaction 9) has generally been observed to be first order at a fixed $\mathrm{pH}$, and the rate increases 100-fold for a unit increase in $\mathrm{pH}$. Assuming one type of surface iron (adsorbed Fe[II]) is oxidized by dissolved oxygen (Reactions 5 and 8):

$$
4 \mathrm{Fe}^{2+}+\mathrm{O}_{2}+4 \mathrm{H}^{+} \Leftrightarrow 4 \mathrm{Fe}^{3+}+2 \mathrm{H}_{2} \mathrm{O} \quad \mathrm{Eh}=-1.85 \mathrm{v}
$$

Four moles of $\mathrm{Fe}(\mathrm{II})$ are oxidized per mole of $\mathrm{O}_{2}$ consumed. At oxygen-saturated conditions $(8.4 \mathrm{mg}$ $\left.\mathrm{L}^{-1} \mathrm{O}_{2}, 1 \mathrm{~atm}, 25^{\circ} \mathrm{C}\right), 1.05 \mathrm{mmol} \mathrm{L}^{-1} \mathrm{Fe}(\mathrm{II})$ is consumed. Experimental evidence indicates that the oxygenation of $\mathrm{Fe}(\mathrm{II})$ in solutions $(\mathrm{pH}>5)$ is generally found to be first order with respect to $\mathrm{Fe}$ (II) and $\mathrm{O}_{2}$ concentration and second-order with respect to $\mathrm{OH}^{-}$. The rate of oxidation of $\mathrm{Fe}(\mathrm{II})$ in solution by oxygen at pH 8 is a few minutes (Eary and Rai 1988; Buerge and Hug 1997). In contrast, the oxidation rate (as a half-life) observed in natural sediments (surface Fe[II] thought to be adsorbed Fe[II] and $\mathrm{Fe}[\mathrm{II}] \mathrm{CO}_{3}$ ) was found to be 0.3 to $1.1 \mathrm{hr}$.

The oxidation of reduced sediment appears to be more complex than can be described with a single oxidation reaction and is likely controlled by both chemical and physical processes. Experimental evidence during iron oxidation experiments indicates that a second type of reduced iron species is present (siderite) in minor concentrations. In addition, a minor fraction of reduced iron sites (presumed to be siderite) appears to be more slowly oxidized, so a second oxidation reaction,

$$
4 \mathrm{FeCO}_{3}(\mathrm{~s})+\mathrm{O}_{2}+4 \mathrm{H}^{+} \Leftrightarrow 4 \mathrm{Fe}^{3+}+2 \mathrm{H}_{2} \mathrm{O}+4 \mathrm{CO}_{3}{ }^{2-}
$$

was considered in the reaction model used. Both of these reactions (10 and 11) show that 4 moles of Fe(II) are consumed per mole of oxygen consumed. The Fe(III) produced in reactions (10) and (11) quickly precipitates as $\mathrm{Fe}(\mathrm{OH})_{3}$. The oxidation of redox barrier in an aquifer by dissolved oxygen is shown conceptually in Figure 1.1 ( 0.1 to 50 years), in which the Eh remains negative but slowly increases over the same period of no dissolved oxygen breakthrough.

\section{A.3 TCE Degradation}

At the Fort Lewis site, the abiotic degradation of TCE and other organic contaminants is being tested using the ISRM treatment technology. In this case, the organic contaminants are electron acceptors. The 
degradation pathway of TCE by dithionite-reduced sediment has been investigated in other studies as well as in Fort Lewis sediments. Degradation pathways for most organic compounds, including TCE, are complex, involving multiple, and potentially parallel reaction steps. Of four possible abiotic degradation pathways for TCE, the two considered most common are reductive elimination and hydrogenolysis. Reductive elimination has been shown to be the major pathway in other studies using zero-valent and ferrous iron (Sivavec et al. 1996; Orth and Gillham 1996). Reductive elimination reactions include (Roberts et al. 1996)

$$
\begin{array}{ll}
\text { TCE }+2 \mathrm{e}^{-} \Leftrightarrow \text { chloroacetylene }+2 \mathrm{Cl}^{-} ; & \mathrm{Eh}=0.60 \mathrm{v} \\
\text { PCE }-+2 \mathrm{e}^{-} \Leftrightarrow \text { dichloroacetylene }+2 \mathrm{Cl}^{-} ; & \mathrm{Eh}=0.63 \mathrm{v} \\
\text { cis } ; \text { trans-DCE }+2 \mathrm{e}-\Leftrightarrow \text { acetylene }+2 \mathrm{Cl}^{-} ; & \mathrm{Eh}=0.56 \mathrm{v}
\end{array}
$$

which describes the destruction of TCE and polychloroethylene (PCE) to easily degraded (abiotically or biotically) chlorinated acetylene products. Abiotic degradation of these products by hydrogenolysis:

$$
\begin{array}{ll}
\text { dichloroacetylene }+\mathrm{H}^{+}+2 \mathrm{e}^{-} \Leftrightarrow \text { chloroacetylene }+\mathrm{Cl}^{-} ; & \mathrm{Eh}=0.56 \mathrm{v} \\
\text { chloroacetylene }+\mathrm{H}^{+}+2 \mathrm{e}^{-} \Leftrightarrow \text { acetylene }+\mathrm{Cl}^{-} ; & \mathrm{Eh}=0.50 \mathrm{v} \\
\text { acetylene }+2 \mathrm{H}^{+}+2 \mathrm{e}^{-} \Leftrightarrow \text { ethylene; } & \mathrm{Eh}=0.39 \mathrm{v}
\end{array}
$$

apparently proceeds rapidly because chlorinated acetylenes are unstable (Delavarenne and Viehe 1969). The degradation of TCE to ethylene by reductive elimination (or hydrogenolysis, as discussed below) involves the production of 6 moles of electrons or $22 \mathrm{mg} \mathrm{L}^{-1} \mathrm{TCE}$ needed to oxidize the equivalent mass of $\mathrm{Fe}(\mathrm{II})$ as water saturated with dissolved oxygen $\left(1.05 \mathrm{mmol} \mathrm{L}^{-1} \mathrm{Fe}[\mathrm{II}]\right)$. Therefore, water containing partial oxygen saturation and $\sim 1.0 \mathrm{mg} \mathrm{L}^{-1} \mathrm{TCE}$ (as likely present in the Fort Lewis aquifer with $0.3 \mathrm{mg} / \mathrm{L}$ in solution and 2 times that mass adsorbed) means that TCE has an insignificant impact on Fe(II) oxidation and remediation barrier lifetime. In the event that the reduced iron barrier is exhausted, previous laboratory studies with the Hanford $100-\mathrm{D}$ and $100-\mathrm{H}$ sediments have shown that sediment can be re-reduced with only a small (5\% to $10 \%)$ loss in capacity. Hydrogenolysis reactions include

$$
\begin{array}{ll}
\mathrm{TCE}+\mathrm{H}^{+}+2 \mathrm{e}^{-} \Leftrightarrow 1,1-\mathrm{DCE} \text { (more likely or cis-DCE }+\mathrm{Cl}^{-} ; & \mathrm{Eh}=0.51 \mathrm{v} \\
1,1-\mathrm{DCE} \text { or cis-DCE }+\mathrm{H}^{+}+2 \mathrm{e}^{-} \Leftrightarrow \text { vinyl chloride }+\mathrm{Cl}^{-} ; & \mathrm{Eh}=0.41 \mathrm{v}
\end{array}
$$

which describes the degradation of TCE involving the production then destruction of dichloroethylene (DCE) and vinyl chloride intermediates (generally more difficult to degrade). These reduction potentials are lower than reductive elimination, indicating they are less likely to occur abiotically. Activation energies and the specific electron transfer mechanism, which does involve the Fe(III) oxide surface, may also influence which reactions actually do occur. Studies of TCE degradation pathways using zero-valent iron and various Fe(II) minerals (Roberts et al. 1996; Sivavec and Horney 1995; Thornton et al. 1998) 
indicate that reductive elimination is the major pathway, with minor amounts of DCE isomers and vinyl chloride produced from the hydrogenolysis pathway. One study also indicates that the DCE isomers and vinyl chloride slowly degraded to ethylene.

The TCE reaction pathway can be used to model the observed rate of TCE degradation. Because acetylene is the main reaction product observed, the combination of reactions (6), (12), and (16) described - the major TCE degradation pathway:

$$
\mathrm{TCE}+\mathrm{H}^{+}+4 \mathrm{Fe}^{2+} \Leftrightarrow \text { acetylene }+3 \mathrm{Cl}^{-}+4 \mathrm{Fe}^{3+}
$$

A set of differential mass flux equations for (20) and (10) that describes iron oxidation by dissolved oxygen) for the 7 species can be written and simultaneously solved to define the rate of change of TCE. The mass flux equation for TCE:

$$
\text { - TCE } / \bullet t=-k_{f 20}[\text { TCE }]\left[\mathrm{H}^{+}\right]\left[\mathrm{Fe}^{2+}\right]^{4}+-\mathrm{k}_{\mathrm{b} 20}[\text { acetylene }][\mathrm{C} 1] 3\left[\mathrm{Fe}^{3+}\right]^{4}
$$

describes mass fluxes as a function of each constituent concentration to each respective stoichiometric coefficient. The set of differential equations can be numerically solved ( 55 mixed equilibrium and kinetic reactions with 71 species described in Szecsody et al. [1995, 1998a, 1998b]), but this type of detailed modeling is useful only if extensive knowledge of the reaction parameters exists. In the case of TCE degradation, not enough is known about the reaction pathways and reaction parameters to justify this approach.

Simpler models can describe the TCE degradation rate accurately under specific conditions. The equation describing the TCE degradation rate can be greatly simplified assuming no backward mass flux and that the $\mathrm{pH}$ is buffered:

$$
-\mathrm{TCE}^{\prime} / \bullet t=-\mathrm{k}_{\mathrm{f} 20}[\mathrm{TCE}]\left[\mathrm{Fe}^{2+}\right]^{4}
$$

which shows that the TCE degradation rate is a function of a rate coefficient $\left(k_{\mathrm{f} 20}^{\prime}\right)$, the TCE concentration, and the ferrous iron concentration (raised to a power $>1$ ). Therefore, as the sediment is slowly oxidized by both dissolved oxygen (reaction 10) and TCE (reaction 20), the observed overall TCE degradation rate (JTCE'/ $/ \partial$ ) will decrease. Over a small number of pore volumes, the $\mathrm{Fe}^{2+}$ concentration can be assumed constant, and the TCE degradation rate simplifies to a first-order reaction that can be integrated:

$$
\begin{aligned}
& \partial T C E ' / \partial t=-k '{ }^{2} 2[T C E] \\
& T C E=T C E_{t=0} e^{-\lambda t}
\end{aligned}
$$

Both the pseudo-first-order approach (reaction 23) and the fixed-pH approach (numerical solution to reactions 10 and 22) were used to describe the TCE degradation data in this study. As stated earlier, because the actual TCE degradation rate is a function of $\mathrm{Fe}^{2+}$ and decreases over time, the first-order halflife will appear to decrease at progressively later points in time. As the overall TCE degradation rate 
decreases, the relative concentrations of degradation products change (Figure 1.1) during flow through a redox barrier in an aquifer. In general, final degradation products (ethylene, ethane) appear when all reactions are occurring at the fastest rates, and, as reactions slow, intermediates (acetylene) and finally the initial degradation product of TCE dechlorination (chloroacetylene) appears.

\section{A.4 References}

Balko B and P Tratnyek. 1998. "Photoeffects on the Reduction of Carbon Tetrachloride by Zero-Valent Iron." J. Phys. Chem. 102(8):1459-1465.

Blowes D, C Ptacek, and J Jambor. 1997. "In Situ Remediation of Cr(VI) Contaminated Groundwater Using Permeable Reactive Walls: Laboratory Studies.” Env. Sci. Technol. 31(12):3348-3357.

Buerge IJ and SJ Hug. 1997. "Kinetics and pH Dependence of Chromium(VI) Reduction by Iron(II)." Environ. Sci. Technol. 31(5):1426-1432.

Delavarenne S and H Viehe. 1969. Chemistry of Acetylenes. Marcel Dekker, New York, pp. 651-750.

Eary L and D Rai. 1988. "Chromate Removal from Aqueous Wastes by Reduction with Ferrous Ion." Environ. Sci. and Technol. 22:972-977.

Fruchter J, C Cole, M Williams, V Vermeul, J Amonette, J Szecsody, J Istok, and M Humphrey. 2000. "Creation of a Subsurface Treatment Zone for Aqueous Chromate Contamination Using In Situ Redox Manipulation." Ground Water Monit. Remed., spring 2000.

Istok JD, JE Amonette, CR Cole, JS Fruchter, MD Humphrey, JE Szecsody, SS Teel, VR Vermeul, MD Williams, and SB Yabusaki. 1999. "In Situ Redox Manipulation by Dithionite Injection: Intermediate-Scale Laboratory Experiments." Groundwater, 37:884-889.

Johnson T, W Fish, Y Gorby, and P Tratnyek. 1998. "Degradation of Carbon Tetrachloride: Complexation Effects on the Oxide Surface." J. Cont. Hyd. 29:379-398.

Orth and Gillham. 1996.

Roberts A, L Totten, W Arnold, D Burris, and T Campbell. 1996. "Reductive Elimination of Chlorinated Ethylenes by Zero-Valent Metals." Environ. Sci. Technol. 30(8):2654-2659.

Sivavec T and D Horney. 1995. "Reductive Dechlorination of Chlorinated Ethenes by Iron Metal and Iron Sulfide Minerals." Emerging Technologies in Hazardous Waste Management VII, p. 42-45. American Chemical Society, Atlanta, Georgia.

Szecsody JE, A Chilakapati, JM Zachara, KM Salvage, and GT Yeh. 1995. Experimental and simulation results of reactive transport of Co(II/III)EDTA at variable pH." EOS, American Geophysical Union, $76(46): 221$ 
Szecsody J, A Chilikapati, J Zachara, and A Garvin. 1998a. "Influence of Iron Oxide Lens Shape on CoII/IIIEDTA Reactive Transport Through Spatially Heterogeneous Sediment." Water Res. Res. 25(10):2501-14.

Szecsody J, A Chilikapati, J Zachara, P Jardine, and A Ferrency. 1998b. "mportance of Flow and Particle-Scale Heterogeneity on CoII/IIIEDTA Reactive Transport." Hydrology 209(1-4):112-136.

Thornton E, J Szecsody, K Cantrell, C Thompson, J Evans, J Fruchter, and A Mitroshkov. 1998. "Reductive Dechlorination of TCE by Dithionite." Physical, Chemical, and Thermal Technologies for Remediation of Chlorinated and Recalcitrant Compounds, G. Wickromanayake and R Hinchee, eds., p. 335-340. 
Appendix B

\section{Analytical Methods}




\section{Appendix B}

\section{Analytical Methods}

In this appendix analytical methods and the rationale for selection are discussed. The methodologies include those employed both in the onsite laboratory as well as analytical laboratory measurements performed on samples shipped to PNNL.

Field measurement of basic groundwater chemistry parameters was made onsite using a series of inline flow probes. Parameters measured included: $\mathrm{pH}$, dissolved oxygen (DO), electrical conductivity (EC), temperature, and bromide (during tracer tests only). Grab samples were collected from the same flow stream and subjected to prompt analysis for dithionite content by flow injection analysis. Samples were also collected periodically for off-site analysis. These typically consisted of a series of samples from each well in turn for: volatile organics (VOAs), acetylene, common anions, and trace metals. VOA analyses required collection of three identical samples in $40-\mathrm{ml}$ brown- glass, septum-sealed, VOA vials each containing 100 microliters of 1:1 Ultrex hydrochloric acid as a preservative. Acetylene samples followed an identical protocol except that only two samples per well were collected for each event. Anion samples were collected in duplicate without preservative using $20-\mathrm{ml}$ polyethylene vials. Trace metals samples were collected as single aliquots using $20-\mathrm{ml}$, acid pre-cleaned polyethylene vials containing 1:1 Ultrex nitric acid as a preservative. Each trace element sample was filtered with a disposable in-line 0.45 -micron filter cartridge during filling of the plastic vial. Samples were stored at reduced temperature $\left(4^{\circ} \mathrm{C}\right.$ nominal $)$ prior to shipping, during shipping, and prior to analysis at PNNL. All VOA, acetylene, and anion samples were analyzed as promptly as possible following collection. Holding times were typically from 2 to 7 days although some were longer during the earlier phases of the project. Trace element samples were collected during all sampling events including injection and withdrawal, however, only selected samples were subjected to analysis.

\section{B.1 Geochemical Field Parameter Monitoring Probes}

A sample distribution manifold containing the electrochemical flow probes was located inside the field laboratory trailer. The probes had the following specifications:

- $\mathrm{pH}$ measurements were performed with an Oaktron WD-35615 series $\mathrm{pH}$ meter with $\mathrm{pH}$ range from 2.00 to 16.00 with a measurement accuracy of $\pm 0.05 \mathrm{pH}$ units. Multipoint $\mathrm{pH}$ calibration with commercial $\mathrm{pH}$ buffers and temperature compensation.

- Temperature measurement also made with Oaktron pH WD-35615 pH meter. Temperature range is $0.0^{\circ} \mathrm{C}$ to $100.0^{\circ} \mathrm{C}$ with and accuracy $\pm 0.5^{\circ} \mathrm{C}$. 
- Conductivity measurement made with Oaktron WD-35607 series conductivity meter with and operating range of 0.0 to $199.9 \mathrm{mS}$ and an accuracy of $\pm 0.05 \mathrm{mS}$.

- Dissolved oxygen was determined with an Orion Model 810 dissolved oxygen meter and probe. Measurement range was 0 to $20 \mathrm{ppm}$ with an accuracy of $\pm 0.1 \mathrm{ppm}$.

- For the one tracer test performed a Cole Palmer ion selective bromide electrode was added to the manifold. The electrode had an operating range of 0.4 to $79,900 \mathrm{ppm}$ with a precision of $\pm 2 \%$ of each full-scale reading.

\section{B.2 Onsite Laboratory Analyses}

The following section provides a description of the on-site laboratory equipment and analytical methods used for field analysis of geochemical parameters, dithionite concentration measured before and during the four injection series. In addition, the method used for field measurement of TCE/DCE during the initial well drilling operation is also detailed.

\section{B.2.1 Groundwater Geochemical Analyses}

In addition to the standard probe system used for routine measurements described in Section B.1, onsite laboratory instruments (microelectrodes) were also employed as a quality check for duplicate measurement of $\mathrm{pH}$, dissolved oxygen, and conductivity in all samples. This in-line electrode system was designed for much smaller volumes (0.02 in. -diameter in flow path), so that only a small amount of water was needed for accurate measurement ( 1 to $2 \mathrm{~mL}$ ). Flow through instrumentation consisted of an oxygen electrode (Microelectrodes Inc.), $\mathrm{pH}$ electrode (Fisher Scientific), and an electrical conductivity electrode (Dionex Instruments).

Laboratory electrodes were calibrated several times each day by injecting calibration standards through the in-line system. Water temperature, as measured by each instrument was also recorded for calibration purposes. Three $\mathrm{pH}$ calibration standards were used for the multipoint calibration (4.0, 7.0, and 10.0). Calibration of the laboratory instruments indicated that the $\mathrm{pH}$ electrodes were stable over the course for the field experiment. Accuracy of $\mathrm{pH}$ was $\pm 0.02 \mathrm{pH}$ units. Two electrical conductivity standards were used for calibration $(1000$ and $10,000 \mu \mathrm{S})$. Very stable behavior of the system was typical, indicating that frequent recalibration was not necessary. Accuracy of the electrical conductivity measurements was $\pm 1 \mu \mathrm{S}$. The oxygen electrode was calibrated with two solutions, oxygen saturated water $(8.2 \mathrm{mg} / \mathrm{L})$ and oxygen free water produced by dithionite treatment. Oxygen electrode recalibration did exhibit significant drift as the electrodes aged. Accuracy for dissolved oxygen was estimated to be \pm $0.1 \mathrm{mg} / \mathrm{L}$. 


\section{B.2.2 Dithionite Analyses}

Dithionite concentrations were measured on well samples and dithionite injection solutions using laboratory equipment housed in the onsite laboratory. Two separate dilution/UV absorbance systems were used to handle the sample load and provide a backup capability in the event of equipment failure. Dithionite was measured by UV absorbance at $315 \mathrm{~nm}$ with a fixed wavelength $\mathrm{UV}$ detector (HP 1050 series). A computer-controlled, automated dilution system was used to adjust sample concentration to the appropriate range for linear Beer's Law performance.

Multiple dithionite determinations were commonly made on each sample to insure accurate results. Measurements were extremely reproducible if sequential samples had similar concentrations of dithionite, but additional measurements were sometimes necessary, for example, to accurately measure a high concentration of dithionite directly after a sample of oxygenated water. Blanks, (oxygen free dilution water) were frequently injected to insure that the dilution system was operating correctly. Dithionite standards were periodically made up by weighing of reagent materials (including buffers) and immediately analyzed. For the highest dithionite concentration used, dilutions of up to $600 \mathrm{X}$ were required. Samples with lower dithionite concentrations were diluted less or not at all, giving detection limits of down to $10^{-7} \mathrm{~mol} / \mathrm{L}$ dithionite for undiluted samples. A detailed diagram of the instrumentation used for dithionite analysis is shown in Figure B-1.

\section{B.2.3 Onsite Volatile Organic Analyses}

During the initial well installation operation (RM-1A through RM-9) samples were collected from each of the boreholes at various depth intervals prior to well completion and analyzed on site for volatile organic content using a field portable GC-MS. The instrument used was a Viking SpectraTrak 620 field transportable GC-MS. The protocols used were otherwise identical to those implemented in a laboratory at PNNL (PNL-VOA4) using a more typical laboratory type instrument. PNL-VOA4 is described in more detail below under Section A.3.1. The main purpose of the in-field preliminary sample analyses was to assure that suitable levels of TCE were present at the site prior to completion of wells and continued drilling. That goal was accomplished fully by the field measurements, which were later confirmed in the laboratory using duplicate samples. It was noted during these activities that the there was a slight trend toward increasing TCE concentration with depth.

\section{B.3 Offsite Laboratory Analyses}

Analyses requiring full laboratory protocols were performed at PNNL on samples shipped back to the laboratory at the conclusion of sampling. Although all measurements were performed in-house at PNNL, a number of different laboratories and protocols were used. Two different methods were available for analysis of the key analytes of concern, trichloroethene (TCE) and cis-1, 2-dichloroethene (DCE). 


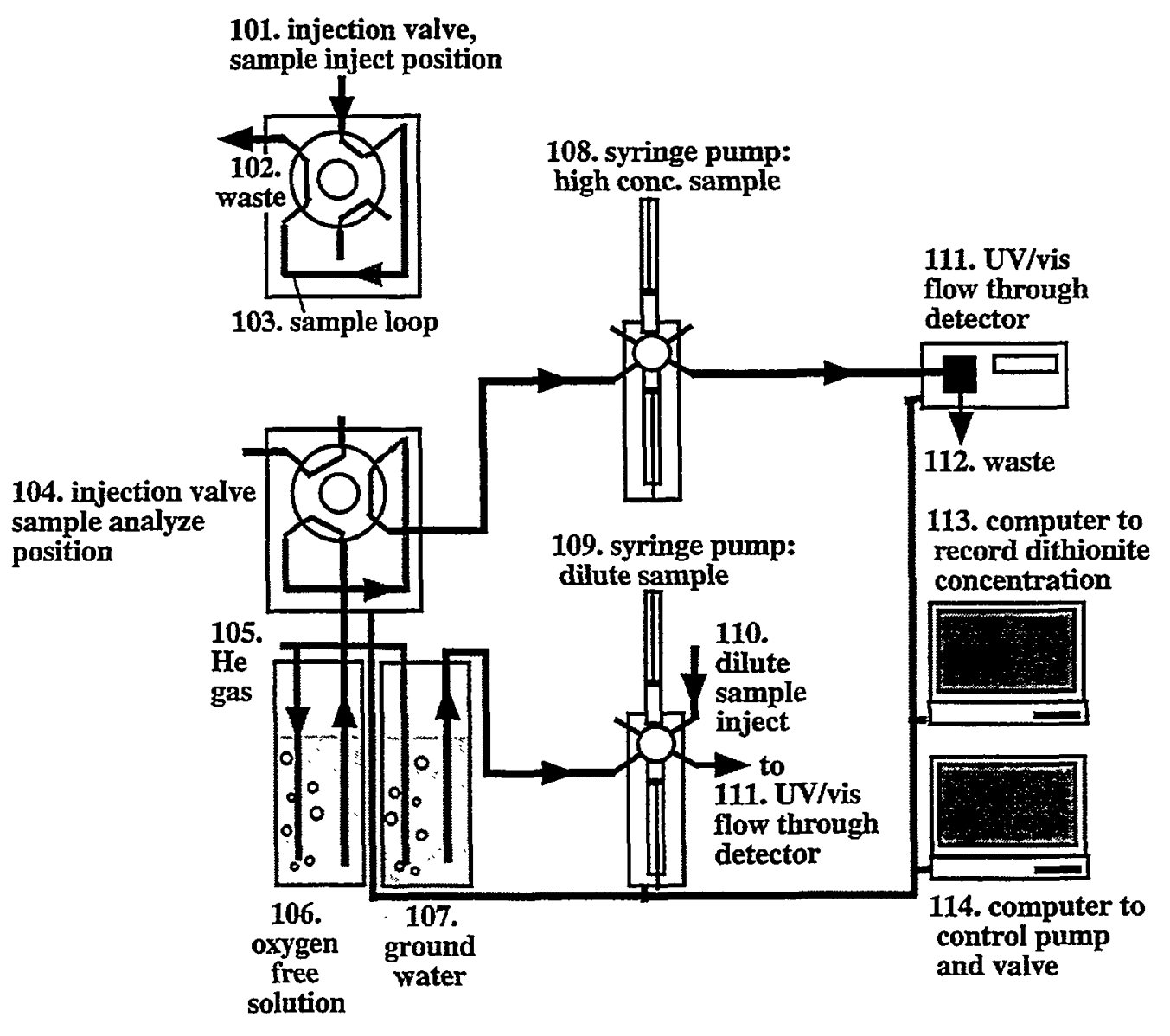

Figure B.1. Diagram of Dithionite Analysis System Developed for Automated Concentration Measurements from Manual Samples of Fluid Streams. Operations of the system are: (a) sample injection, (b) dilution (c) mixing, and (d) measurement. A sample with a high dithionite concentration is injected at the injection valve (101) into a small loop (103), where the excess is waste (102). The program that controls all fluid operations is then initiated on a computer (114), which switches the injection valve (104) and draws degassed water (106) into a syringe (108). The sample and degassed water are mixed between two syringes (108), and then injected into a UV detector (111) where the signal and baseline are recorded (113). A sample in a syringe with a low dithionite concentration is placed on the syringe pump $(109,110)$ and directly injected into the UV detector (111) with no dilution. Groundwater (107) is used for the baseline signal in this case. 


\section{B.3.1 Volatile Organic Analysis by GC/MS-Headspace Sample Introduction (PNL VOA4)}

PNL-VOA4 employs a combination of gas chromatography-mass spectrometry (GC-MS) combined with headspace sample introduction to detect, identify, and quantify a series of volatile organic constituents in groundwater or waste water samples collected in headspace-free, 40-mL VOA vials. The instrument used for laboratory analysis was a Hewlett-Packard 5971 GC-MS. Separations were achieved with a J\&W Scientific 60- meter long, DB-1 column (0.32 mm i.d., $1 \mu \mathrm{m}$ thick film). Prior to analysis, $15 \mathrm{~mL}$ aliquots of the water samples were removed from each vial, aliquots of internal standard added, and the caps replaced. The internal standard mixture contained: pentafluorobenzene, 1, 2-difluorobenzene, d5-chlorobenzene, and d4-dichlorobenzene. Each vial was then place into a block heater and heated to $95^{\circ} \mathrm{C}$ for 1 hour. An aliquot of the headspace gas was sampled with a gas-tight syringe and injected into the GC-MS which then ran through a complete temperature ramped separation program. The system was calibrated with a 4-level calibration standard spanning a concentration range of from 6.4 to $640 \mathrm{ppb}$. An $80 \mathrm{ppb}$ continuing calibration verification (CCV) standard was also used to assure the stability of the calibration. A continuing calibration blank (CCB) was injected between any standards and samples to assure that system memory effects were negligible. All standards, blanks, and samples were prepared in an identical manner. The calibration standard used was a dilution of a commercially prepared and certified multicomponent mixture supplied by Restek. A second standard supplied by Supelco was also used as a QC cross-check. Method precision was estimated from replicates to be $10 \%$ RSD. The method detection limit was approximately $0.1 \mathrm{ppb}$ for most compounds of interest including TCE and DCE. Because the method had a very wide linear dynamic range, samples were run undiluted in all cases. EnviroQuant software supplied by HP was used for quantitation of target compounds and verification of mass spectral purity. Chromatograms were also routinely inspected for the presence of additional peaks not included in the target list.

\section{B.3.2 Volatile Organic Analysis by Gas Chromatography-Purge-and Trap Sample Introduction (PNL-VOA3 or SW-846 8021)}

PNL-VOA3 was based on EPA Method 502.2 and is also very similar to EPA SW-846 Method 8021. It is a gas chromatography based method employing an OI purge and trap (P\&T) system for sample introduction, a Hewlett-Packard 5890 gas chromatograph for separations and a sequential tandem pair of detectors, an OI photoionization detector (PID) and an OI halogen selective electrolytic conductivity detector (ELCD). The OI P\&T module is equipped with a 27-position autosampler. It employs a $5 \mathrm{ml}$ sample loop and uses a three-bed trap (Tenax, silica gel, and charcoal) for sample preconcentration. The GC separation column is a 105-meter long Restek Rtx-502.2 megabore column $(0.53 \mathrm{~mm}$ i.d., $3.0 \mu \mathrm{m}$ thick film). The system was calibrated with the same standards as described under section A.3.1, however, because the dynamic range was much more constrained for this technique, the calibration ranged only from 4 to $24 \mathrm{ppb}$. Samples with higher concentrations of TCE (which was most of them) were diluted by an appropriate amount. Check standards run at the end of each batch typically agreed with the initial calibration to within $5 \%$ or better. Method replication precision was typically better than $5 \%$. The method detection limit was approximately $0.2 \mathrm{ppb}$ for both TCE and DCE. All data reported used the PID for primary quantitation with the ELCD channel used primarily as a QC check. Integration and quantitation was performed using PE Nelson Turbochrom software. 


\section{B.3.3 Acetylene Analysis by Gas Chromatography-Headspace Sample Introduction}

Acetylene, ethene, and ethane are reductive degradation products of trichloroethene. The presence of any of these species (particularly acetylene) following dithionite treatment can be taken as a priori evidence that the process has been effective in stimulating abiotic reductive dechlorination. Since standard VOA techniques do not retain acetylene quantitatively or provide optimal separations, some analytical development work was needed to find a satisfactory assay method. It was found that a GCGasPro column (J\&W Scientific) provided exceptionally good separations for the species of interest. Separation and detection was performed on an HP 5890 gas chromatograph equipped with a flame ionization detector and a 30-m-long x $0.32 \mathrm{~mm}$ i.d. GC-GasPro PLOT column. A headspace method was used for sample introduction. Twenty-ml aliquots were removed from each vial prior to analysis with a large volume syringe. An aliquot of fluorobenzene internal standard was injected through the cap. The internal standard was used as a quality control check and for approximate quantitation of TCE/DCE, however, only the acetylene data was reported to the database. Following addition of the internal standard, each vial was stirred with a vortex mixer for 30 seconds and allowed to equilibrate at room temperature for a minimum of $\mathbf{3 0}$ minutes prior to analysis. A gas tight syringe was used to remove a $1 \mathrm{ml}$ aliquot of headspace gas from each vial. The gas was injected into the $\mathrm{GC}$ through the split injector port. The injector was set to a split ratio of 5:1. A single level calibration standard purchased from Scott Specialty gases was used for quantitation of acetylene in terms of ppmv in the headspace. Final reported data was converted to $\mathrm{ppb}$ in solution using a published value of the dimensionless form of the Henry's Law constant for acetylene (0.49). The method detection limit for acetylene was approximately $0.06 \mathrm{ppb}$. Method precision typically averaged approximately $10 \%$ RSD for samples run in batches on the same day.

\section{B.3.4 Common Anions by Ion Chromatography (EPA 300.0)}

During the course of this work, three different ion chromatography methods were used in two different laboratories. All three methods followed the general guidelines of EPA Method 300.0 amended to reflect instrumentation differences. Initial work was performed according to the in-house method PNL-IC1. A Dionex 4000i ion chromatograph equipped with IonPac AS4A guard and separations columns and a membrane suppressor were used for separation and detection. Quantitation was performed with a Nelson 3000 Series Chromatographic Data System. A Waters 710 autosampler was used for sample introduction. A six-level calibration standard spanning the range of 0.1 to $100 \mathrm{ppm}$ was used for quantitation. Because of the wide dynamic range spanned, a cubic fit was used for construction of calibration curves for the species of interest: fluoride, chloride, nitrate, sulfate, and phosphate. A certified standard purchased from High Purity Standards, Inc. was used for preparation of secondary standard dilutions. A response factor for sulfite was determined by preparing a series of in-house sulfite standards and carefully determining the relative response for sulfite relative to sulfate and phosphate. Because sulfite and sulfate were not fully resolved by the separation column, samples containing sulfite required manual reintegration and recalculation of the respective concentrations of the two species. 
Partway through the project, a new ion chromatograph system was purchased. The new instrument had significantly improved separation and detection capabilities. The instrument was a Dionex DX-120 equipped with an IonPac AS9-HC column, an ASRS-Anion Self Regenerating Suppressor, and a 72 position Dionex autosampler. Operating conditions were adjusted as appropriate for that instrument but the $\mathrm{ICl}$ protocol was otherwise maintained. The AS9 column had greatly improved resolution with the sulfate and sulfite peaks separated by more than 3 minutes. Dionex PeakNet Software was used for quantitation and reporting. The method detection limits for IC1 is approximately $0.2 \mathrm{ppm}$. Replication precision is typically better than $5 \% \mathrm{RSD}$.

A number of anion samples were also run at a second PNNL lab. The method used is internally documented as PNL-AIAL-03. Protocols are taken also from EPA 300.0., however, the details of implementation are somewhat different than for PNL-IC1. The instrument used was a Dionex 4000i equipped with IonPac AS4A guard and separations columns and an Anion Self Regenerating Suppressor. All samples were injected manually into a sampling loop. Data was recorded on a strip chart recorder with quantitation taken from peak height. Calibration curves were constructed for each species using a minimum of 3 calibration levels. Up to 5 levels were used in some cases for samples spanning a wide range of concentrations. Mid-range check standards were run between groups of 5 samples and at the end. Standards were prepared in-house by weighing of high-purity reagent materials. Sulfite standards were freshly prepared for each batch. Sulfite standards were stabilized with glycerin to retard oxidation. Samples were diluted as appropriate to fall within the linear range for sulfate. The method detection limit was calculated based on the baseline noise. For sulfate, the MDL was conservatively estimated to be 0.1 ppm. Replication precision was typically better than 5\% RSD.

\section{B.3.5 Trace Metals by Inductively Coupled Plasma-Optical Emission Spectroscopy (ICP- OES; EPA SW-846 Method 6010)}

Trace metals samples were analyzed by Inductively Coupled Plasma-Optical Emission Spectroscopy (ICP-OES) according to the protocols documented as PNL-ALO 211.2. The PNNL method is derived from EPA SW-846 Method 6010 as adapted to more modern instrumentation. The instrument used was a Thermo Jarrell-Ash AP/Iris. Manual sample addition was performed in all cases. Trace element samples were run in a $2 \%$ nitric acid matrix. Injection and withdrawal samples were analyzed for total sulfur. That data was used to calculate sulfur mass balance. Total sulfur measurements were performed on unpreserved samples. Prior to analysis, the samples were treated with $1 \%$ ammonium hydroxide and $1 \%$ hydrogen peroxide and allowed to digest overnight at room temperature. Except for the sample prep and selection of analytical wavelength, analytical conditions were otherwise the same for the sulfur and trace element analyses. Single element NIST traceable solution standards purchased from Johnson-Mathey were used to produce multicomponent calibration standards. A multicomponent check standard purchased from High Purity Standards, Inc. was used for independent calibration verification. All data analysis was performed by the Thermospec software. Detection limits were based on three standard deviations of the baseline for each element. Detection limits varied widely for different elements with the most favorable cases ranging down to the low ppb levels. Precision for replicate analysis was typically $5 \%$ RSD or less but occasionally was higher for samples with high levels of total dissolved solids. 


\section{B.3.6 Trace Metals by Inductively Coupled Plasma-Mass Spectrometry (ICP-MS); EPA SW-846 Methods 6020}

In order to verify that the dithionite treatment had not adversely impacted aquifer water quality, a more capable analytical method, ICP-MS, was used for post-test analysis of trace metals samples. The method was used after it was concluded that the more routine ICP-OES method (6010) was not adequately sensitive for a number of key elements (particularly arsenic) with respect to Primary Drinking Water Limits. The method used was internally documented as PNL-ALO-280. It follows the protocols set forth in EPA SW-846 Method 6020. The laboratory has been certified by the State of Washington for performance of this test. The instrument used was a VG PlasmaQuad PQ2+ STE. The instrument was equipped with both ultrasonic and Meinhard type nebulizers. Samples were introduced into the system manually through a peristaltic pump. Indium and thallium spikes were added to each sample to determine if plasma suppression was present associated with elevated dissolved solids. Samples exhibiting suppression effects were diluted as appropriate. The instrument was calibrated with a mixture of NIST traceable commercial standards at four calibration levels. An independent calibration verification standard obtained from a second vendor was used as a QC check. Matrix spikes were also used as QC checks. Method detection limits varied with individual elements and as a function of dilution. Under the most favorable circumstances (i.e. no dilution) detection limits of as low as $0.001 \mathrm{ppb}$ were typical for many elements. Replication precision was typically less than 1\% RSD for clean samples and generally better than $5 \%$ for samples with high dissolved solids content. 


\section{Appendix C}

\section{Hydrogeologic Setting, Well Construction Summaries, and As-Built Diagrams}




\section{Appendix C}

\section{Hydrogeologic Setting. Well Construction Summaries, and As-Built Diagrams}

\section{C.1 Regional Geologic Setting}

The study area (Figure 1.1) lies approximately 10 miles south of Tacoma, within the southern part of the Puget Lowland physiographic province of western Washington. The Puget Lowland is a north south trending structural trough bounded to the east by the Cascade Mountains and to the west by the Olympic Mountains. The trough is filled with a thick sequence of mostly unconsolidated, Quaternary-age, sedimentary deposits. For the last 1 to 2 million years, the Puget Lowland has predominantly been under the influence of the repeated advance and retreat of Puget Lobe of the Cordilleran Ice Sheet, which as recently as 16,000 years ago filled the Puget Lowland with hundreds to thousands of feet of glacial ice (Porter and Swanson 1998). The last major glaciation deposited the Vashon Drift, which includes those deposits laid down during the Vashon Stage of the Fraser Glaciation.

Previous investigations (Griffin et al. 1962; Ebasco 1988; 1993; USACE 1993; Woodward-Clyde 1997) reveal the stratigraphy to be diverse, erratic, and complex. In general, the geology can be characterized as a stacked braided stream system incised into tills and or fluvial/lacustrine (nonglacial) sediments. The complexity and variability inherent of the glacial environment is demonstrated geomorphically by the wide variety of glacial deposits and land forms left behind during the last deglaciation (Figure C.1).

\section{C.1.1 Late-Pleistocene (Vashon) Glaciation in the Puget Lowland}

Late Pleistocene glaciation in the Puget Lowland left behind two types of deposits: glacial outwash and till. Sheets of glacial outwash, composed predominantly of reworked gravel and sand, were deposited by proglacial streams with the advance and retreat of the Puget Lobe and occasionally by outburst floods from ice-dammed lakes in the region. Till, on the other hand, is an ice-contact type of deposit. Two types of glacial till are represented in the subsurface, lodgement till and ablation till. Lodgement till, locally known as "hardpan," is very dense, unstratified, compact gravel in a matrix of clay, silt, and sand deposited at the base of the glacier and subsequently overridden by the glacier. Ablation till is a loose, heterogeneous mixture of soil types similar to lodgement till but derived from melting glacial ice (Ebasco 1988). 


\section{Geomorphic Map}

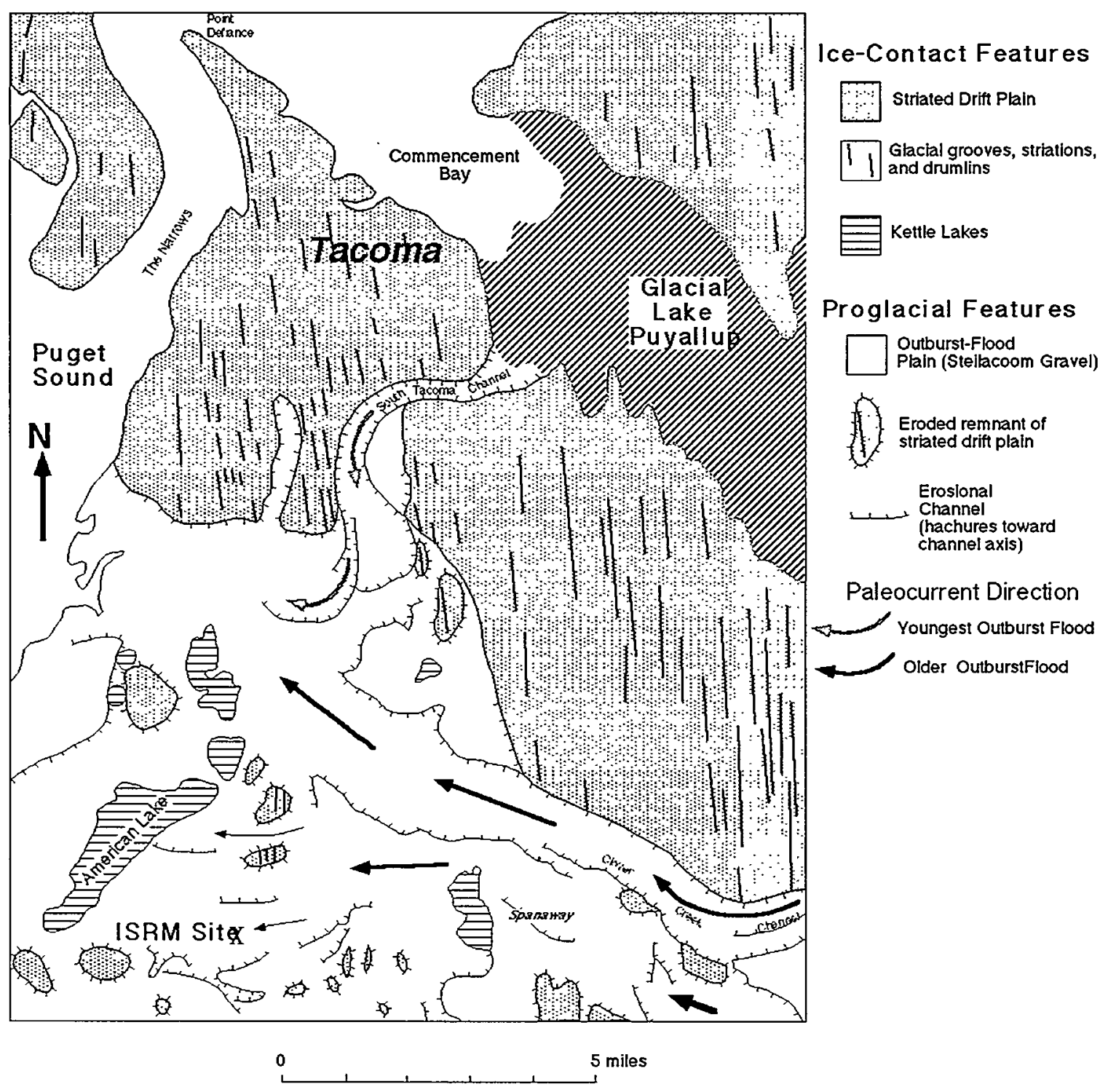

Figure C.1. Geomorphic Map of a Portion of the Puget Lowland

The region is situated on a broad, poorly drained, upland plain of glacial drift exhibiting low to moderate relief (Walters and Kimmel 1968). This upland plain is subdivided into two geomorphic units, a striated drift plain and an outburst-flood plain (Figure C.1). The youngest strata within the striated drift plain, which underlies most of the city of Tacoma, consists of glacially sculpted till. This last-glacial till was deposited and overridden by several hundreds of feet or more of glacial ice. At the surface are many subparallel grooves and drumlin-like ice-contact features aligned parallel to the direction of ice movement 
(north to south) during the last retreat of the ice sheet; the longest ridges being 2 to 3 miles in length. The sediments within these ridges are heterogeneous and complex, and some of the deposits appear to be nonglacial (Laprade 2000).

As the ice retreated northward for the last time around 16,000 years ago, a large ice-dammed lake (Glacial Lake Puyallup) formed in the present Puyallup River valley east of Tacoma. The lake filled until it overtopped drainage divides along its western margin. The lake drained suddenly, at least four different times, as indicated by the number of distinct erosional channels that were created when successively lower outlets from the ice-dammed lake were exposed by the northward receding ice margin. The last two flood channels to drain glacial Lake Puyallup (Clover Creek channel and South Tacoma channel) are shown in Figure C.1; evidence for two other older flood events from glacial Lake Puyallup (Kirby/Muck Creek and Ohop) lie off the map to the southeast (Walters and Kimmel 1968).

Floodwaters from each of the channels drained westward eroding into the older striated drift plain. In Figure C. 1 it is apparent how floodwaters coming down the Clover Creek channel were initially confined to a relatively narrow channel but eventually fanned out near Spanaway, seven miles east of Fort Lewis, creating a huge, gently west-sloping, outburst-flood plain. From Spanaway westward is a network of braided flood channels that blanketed the area with outburst flood gravel (i.e., the Steilacoom Gravel). Subsequent to the outburst flood down Clover Creek the ice continued its retreat northward. Lake Puyallup filled one last time and ultimately overtopped another divide, creating a new outlet referred to as the South Tacoma channel. This last flood event locally incised into the older outburst-flood deposits previously deposited via the Clover Creek channel (Figure C.1).

The last glacial ice-contact feature to form was kettle lakes, which began to develop when large blocks of ice stagnated after becoming separated from the main ice mass as it retreated northward. The stagnant ice blocks were subsequently buried or partially buried beneath an aggrading blanket of outwash sand and gravel, from meltwater streams as well as outburst floods from glacial Lake Puyallup. The stagnant ice did not melt completely until after Glacial Lake Puyallup drained for the last time. The ice blocks melted slowly, forming isolated sinkholes that later filled with groundwater. The kettles and depressions range in size from $50 \mathrm{ft}$ to 2 miles. A string of kettle lakes formed north and west of Fort Lewis. American Lake is one of these.

Due to the retreat and melting of the glaciers, sea level has risen about $300 \mathrm{ft}$ since the last ice age. This rise in sea level flooded all the lowland areas formerly occupied with ice, created the waterways of Puget Sound, and raised groundwater levels. Only minor modification of the land surface has occurred in the last 15,000 years via surface streams and organic depression fillings (Ebasco 1988).

\section{C.1.2 Regional Stratigraphy}

The generalized stratigraphy for the southern Puget Lowland is presented in Figure C.2. 
Generalized StratigraphySouth-Central Puget Lowland

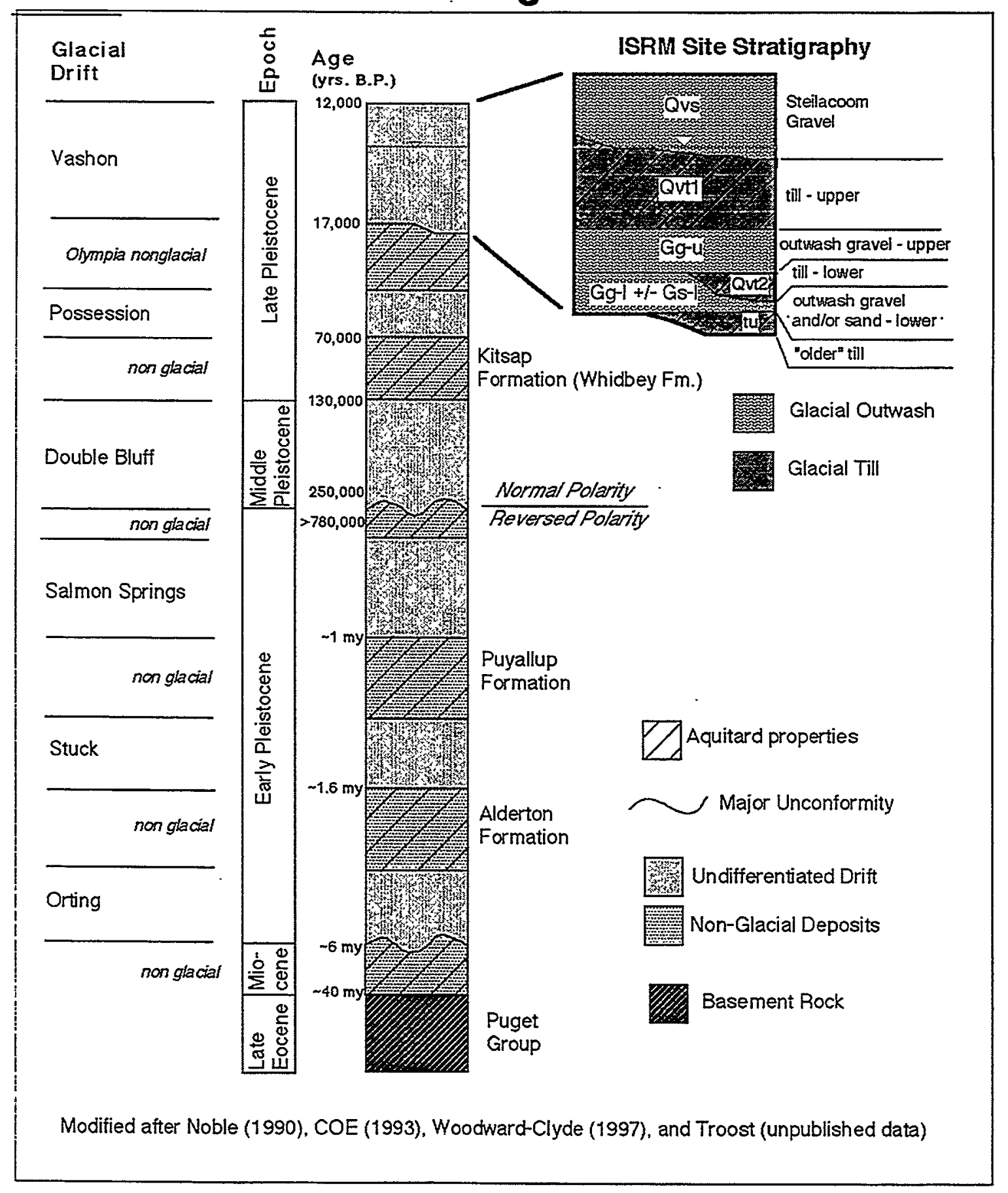

Ft.Lewis.strat

Figure C.2. Generalized Stratigraphy of the South-Central Puget Lowland 
Based on the oxygen-isotope record preserved in deep marine cores, seven major interglacial-glacial cycles have occurred in the last 620,000 years, although many more glacial-interglacial cycles may extend back 2.5 million years into the earliest Pleistocene (Morrison 1991). Earlier reports (Ebasco 1988, 1993) presented evidence for only four glacial-interglacial cycles. From youngest to oldest, these include the Vashon, pre-Vashon-post-Kitsap, Salmon Springs, and Stuck glacial-drift sequences. More recently, Troost et al. (1998) recognized two other glacial-drift sequences (Possession and Double Bluff) within the pre-Vashon-post-Kitsap sequence reported earlier. An older, early Pleistocene glaciation, which deposited the Orting Drift, is also reported in the literature (Walters and Kimmel 1968; Shannon and Wilson 1986). Thus it appears that within the published geologic record there is evidence for at least six major glacial-interglacial cycles within the Puget Lowland. The youngest, pre-Vashon non-glacial unit, which was deposited about 17,000 years B.P., is referred to as the Olympia nonglacial interval. Older nonglacial units, from youngest to oldest, include the Kitsap, Puyallup, and Alderton Formations, respectively.

The region is underlain by $2000 \mathrm{ft}$ or more of unconsolidated Pleistocene sediments (Hall and Othberg 1974) that consist of alternating layers of glacial, and non-glacial deposits from at least six periods of glacial advance and retreat. Deposits of coarse-grained glacial drift (till and outwash) are often separated by finer-grained, non-glacial fluvial, lacustrine, and marsh deposits (Walters and Kimmel 1968). The predominant surficial deposit in the area is a mantle of outwash gravel (Steilacoom Gravel); these gravels were deposited in braided channels that formed just downstream of glacial Lake Puyallup (Figure C.1), as the lake rapidly drained at the end of the last ice age. The Steilacoom Gravel is considered to be part of the Vashon Drift and is composed of consistently coarse gravel in a sand matrix. The relative consistency of grain size and singular source (outburst floods from Lake Puyallup) serve to differentiate the Steilacoom Gravel from other members of the underlying Vashon Drift (WoodwardClyde 1997).

Below the Steilacoom Gravel is a sequence of glacial sediments associated with the last advance and retreat of the ice sheet during the late Pleistocene (13,000-17,000 years B.P.); these deposits belong to the Vashon Drift (Figure C.2). Multiple till layers separated by layers of glacial outwash suggest that there were at least two, and perhaps more, secondary advances of the Puget Lobe in the area during this time. Lying stratigraphically below the Vashon Drift are several units of undetermined age (Ebasco 1993). The upper unit is an undifferentiated till layer consisting of lodgment till, glaciomarine drift, glaciolacustrine deposits, and lesser amounts of ablation till. Underlying the undifferentiated till is a unit of nonglacial alluvial deposits (Olympia nonglacial interval); the Olympia Beds vary from silt to sandy gravel and contain organic debris (Troost 2000). Another sequence of glacial drift (Possession Drift) may be present atop the laterally extensive Whidbey Formation (Borden and Troost 1995) formerly referred to as the Kitsap Formation.

The silts, fine sands, and peat of the Whidbey Formation, equivalent to the Qpv unit of Logan et al. (2000), represent non-glacial (fluvial and lacustrine) deposits laid down during the last major interglacial period, which lasted from about 70,000-130,000 years B.P. (Morrison 1991). The Whidbey Formation is important because it forms the shallowest, regionally extensive aquitard between the unconfined aquifer 
(i.e., Vashon Drift) and deeper groundwater systems (Noble 1990; Borden and Troost 1995). The maximum thickness of the Kitsap is not known, but it has been inferred to be up to $150 \mathrm{ft}$ thick in central Pierce County (Walters and Kimmel 1968).

Another pre-Vashon glacial sequence (Double Bluff) may exist between the Kitsap Formation and the underlying Salmon Springs Drift (Troost et al. 1998). The Salmon Springs Drift has a reversed magnetopolarity and therefore assigned to the Matuyama Polarity Epoch (Mankinen and Dalrymple 1979), dated at $>780,000$ years B.P. (Morrison 1991). Below the Salmon Springs Drift may be at least two other episodes of nonglacial (Puyallup Formation, Alderton Formation) and glacial (Stuck Drift, Orting Drift) deposition (Figure C.2). Underlying the Orting Drift are unconsolidated and undifferentiated lacustrine and volcaniclastic deposits of Miocene age (Walters and Kimmel 1968). The predominance of volcanic material within these sediments differentiates them from the Pleistocene deposits.

\section{C.2 References}

Borden RK and KG Troost. 1995. "Hydrogeologic implications of refined central Pierce County stratigraphy." Abstracts from the 1st Symposium on the Hydrogeology of Washington State, Washington State Department of Ecology, Olympia, p. 117-118.

Ebasco. 1988 Fort Lewis Logistics Center, Remedial investigation final report. Ebasco Environmental, prepared for the U.S. Army Corps of Engineers, Seattle.

Ebasco. 1993. "Fort Lewis Logistics Center, Lower aquifer groundwater study, final technical memorandum." Prepared for the U.S. Army Corps of Engineers, Seattle, by Ebasco Environmental, Bellevue, Washington.

Griffin WC, JE Sceva, HA Swenson, and MJ Mundorff. 1962. "Water resources of the Tacoma Area, Washington." U.S. Geological Survey, Water Supply Paper.

Hall JB and KL Othberg. 1974. "Thickness of unconsolidated sediments, Puget Lowland, Washington." Geologic Map GM-12, Washington Department of Natural Resources, Olympia.

Laprade WT. 2000. "Revelations in Seattle ridges." Geological Society of America Abstracts with Programs, 32(6):A-23.

Logan RL, TJ Walsh, and WG Gerstel. 2000. "A revision of Quaternary stratigraphy in the southern Puget Lowland, Washington." Geological Society of America Abstracts with Programs, 32(6):A-25.

Mankinen EA and GB Dalrymple. 1979. "Revised geomagnetic polarity time scale for the interval 0-5 m.y.B.P.” Journal of Geophysical Research, 84(B2):615-626.

Morrison RB ed. 1991. "Quaternary Nonglacial Geology: Conterminous U.S." Decade of North American Geology. Geological Society of America, Vol. K-2, p. 1-12. 
Noble JB. 1990. "Proposed revision of nomenclature for the Pleistocene stratigraphy of coastal Pierce County, Washington." Open-File Report 90-4, Washington Department of Natural Resources, Olympia.

Porter SC and TW Swanson. 1998. "Radiocarbon age constraints on rates of advance and retreat of the Puget Lobe of the Cordilleran Ice Sheet during the last glaciation." Quaternary Research, v. 50, pp. 205213.

Shannon and Wilson. 1986. Source areas, occurrence, and recommended remedial alternatives for trichloroethylene in groundwater, Fort lewis Logistics Center, Fort Lewis, Washington. W-4487-04, Shannon and Wilson, Inc., Seattle.

Troost KG, JB Mahoney, DB Booth, and RK Borden. 1998. "Discriminating glacial from nonglacial deposits in Pleistocene sediments of the south-central Puget Lowland." Program with Abstracts, Association of Engineering Geologists Annual Meeting, Seattle, p. 130.

Troost KG. 2000. "The Olympia nonglacial interval in the southern Puget Lowland, Washington." Geological Society of America Abstracts with Programs, 32(6):A-72.

USACE. 1993. Logistics Center groundwater treatment: Phase II, 90\% design submittal, Fort Lewis, Washington. PN 035756, U.S. Army Corps of Engineers, Seattle.

Walters KL and GE Kimmel. 1968. "Groundwater occurrence and stratigraphy of unconsolidated deposits, central Pierce County, Washington." Water Supply Bulletin No. 22, State of Wahington Department of Water Resources, Olympia.

Woodward-Clyde. 1997. "East Gate disposal yard expanded site investigation: Site history and conceptual site model." Prepared for the U.S. Army Corps of Engineers, Seattle. 



\section{C.3 Well Completion Summaries}





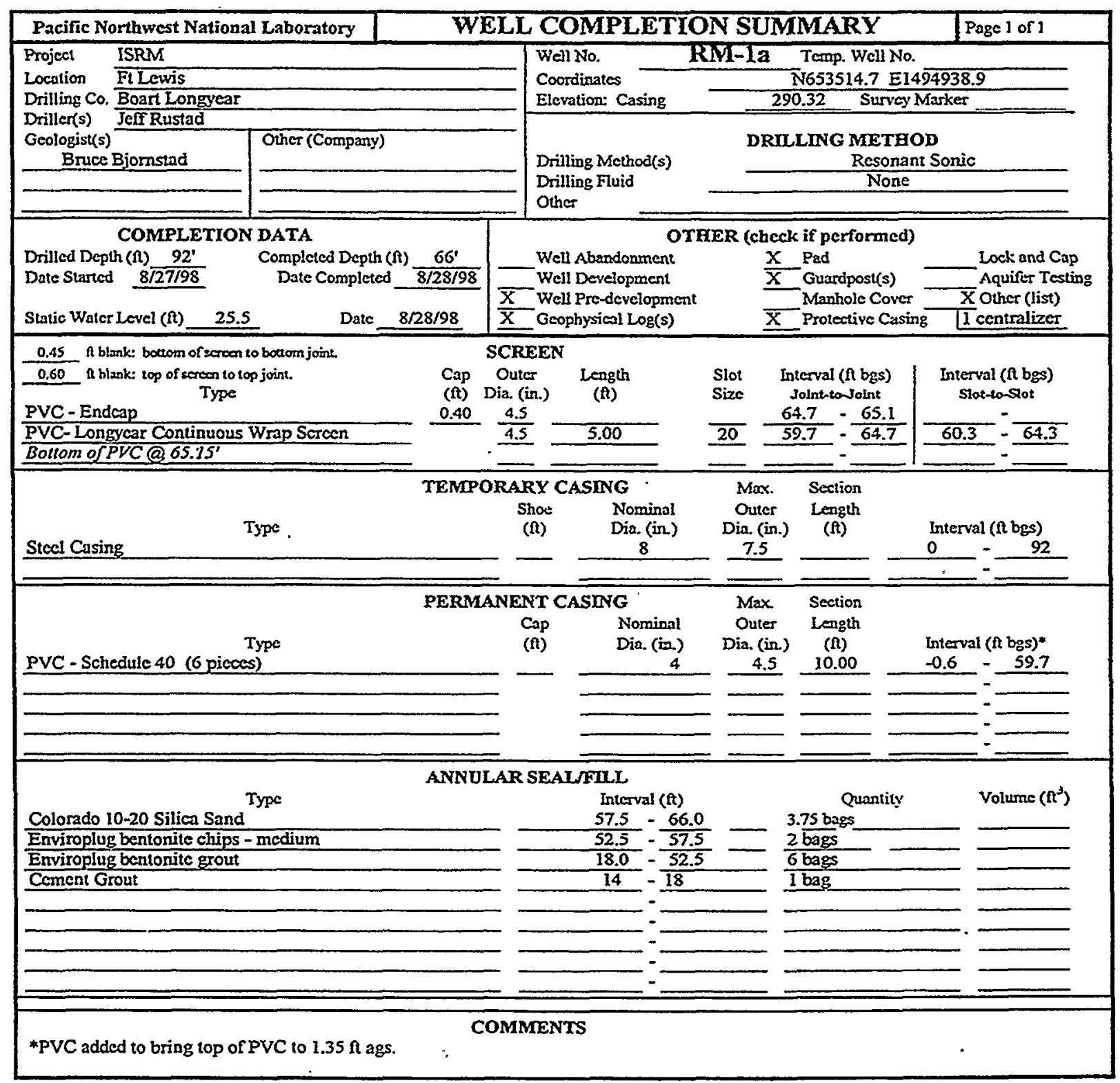

C. 11 


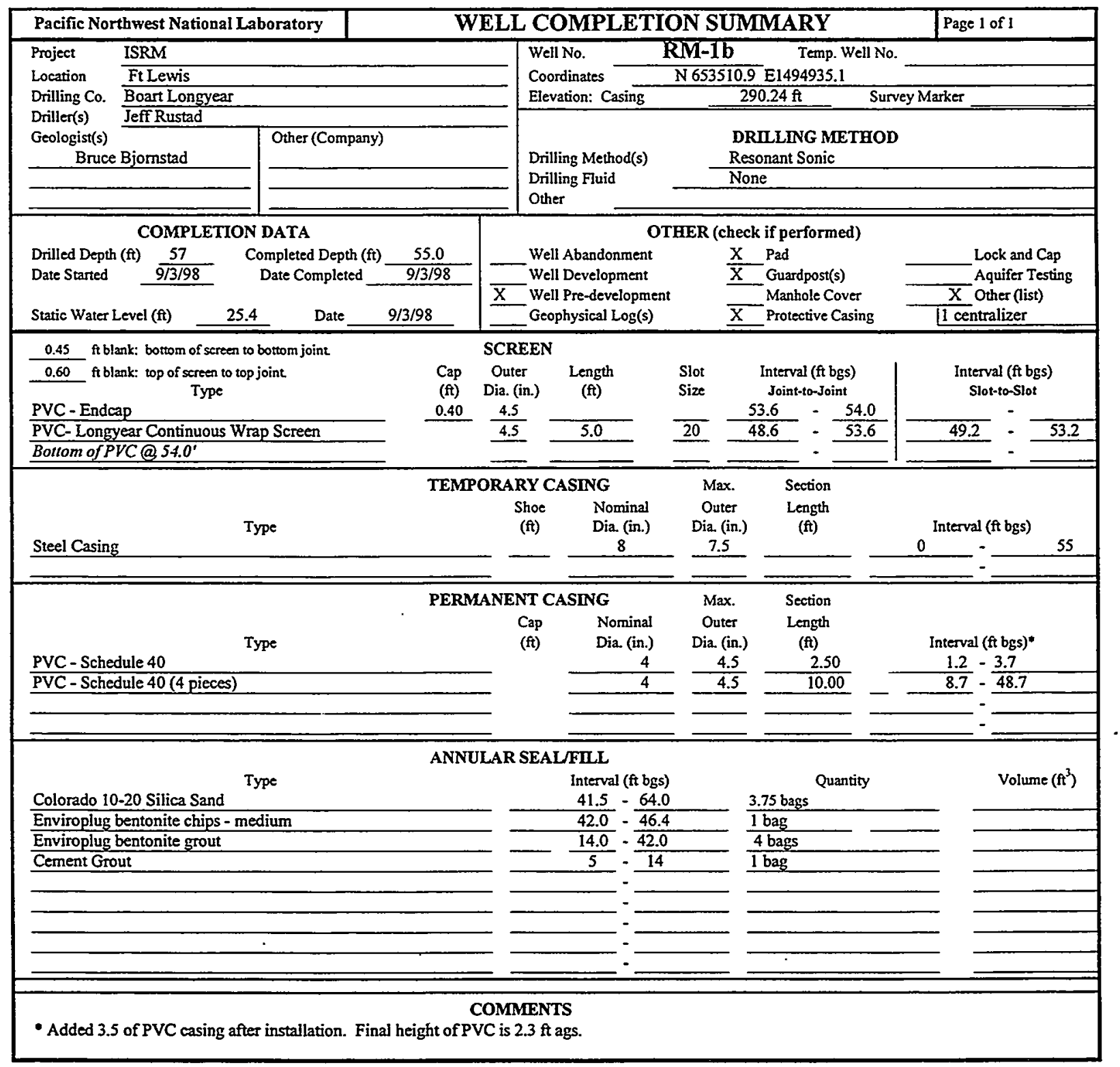

C. 12 


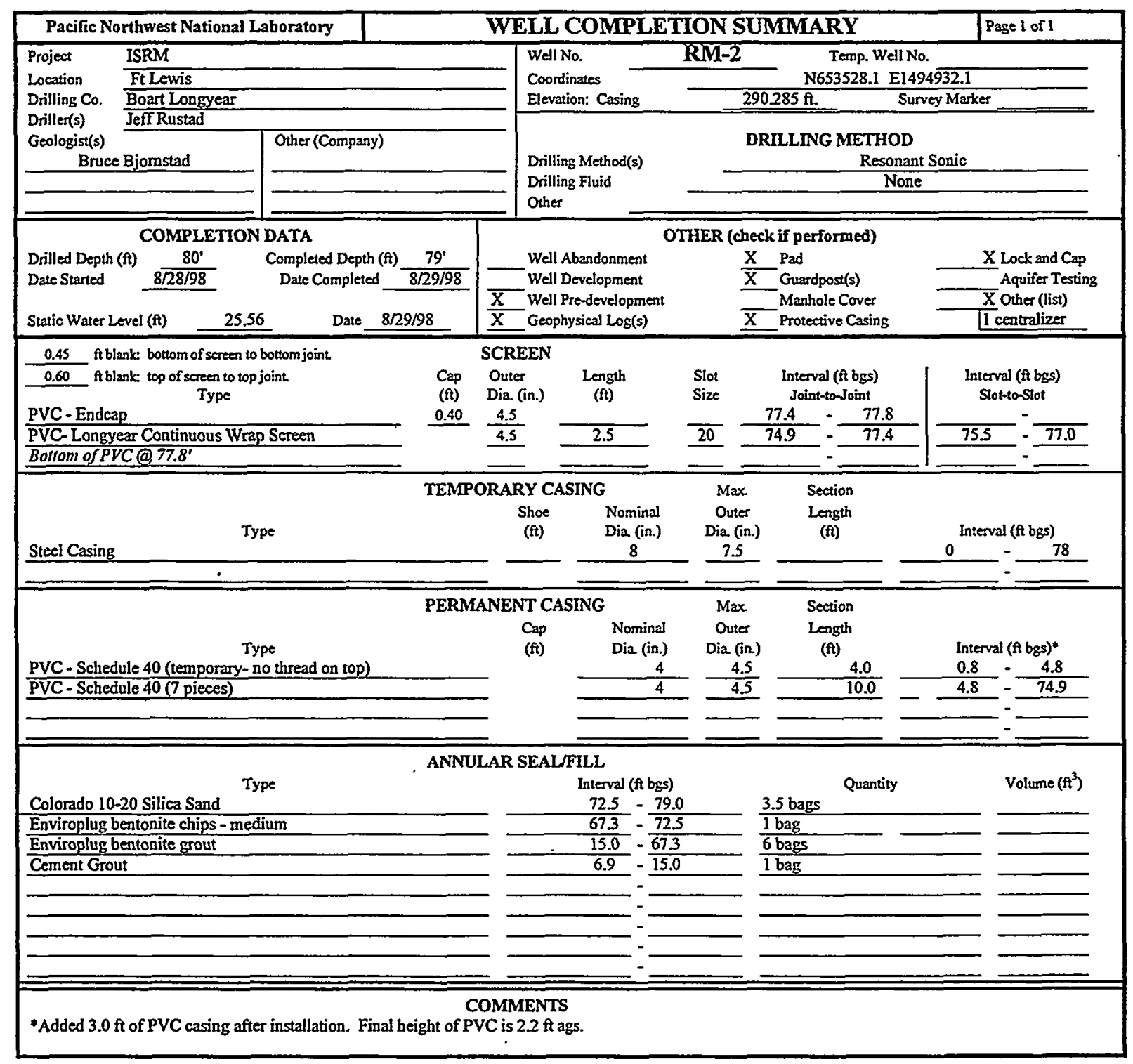

C. 13 


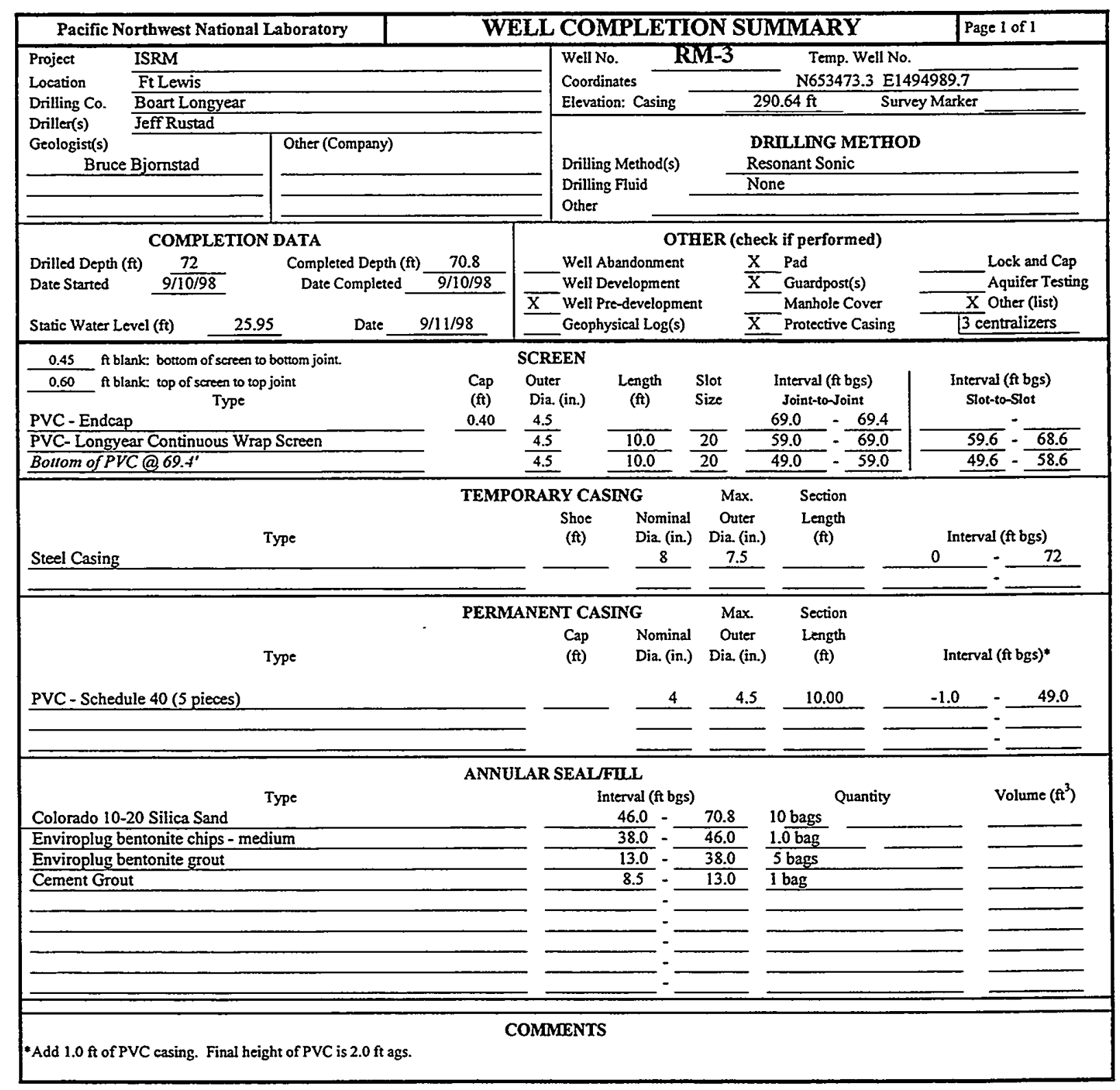




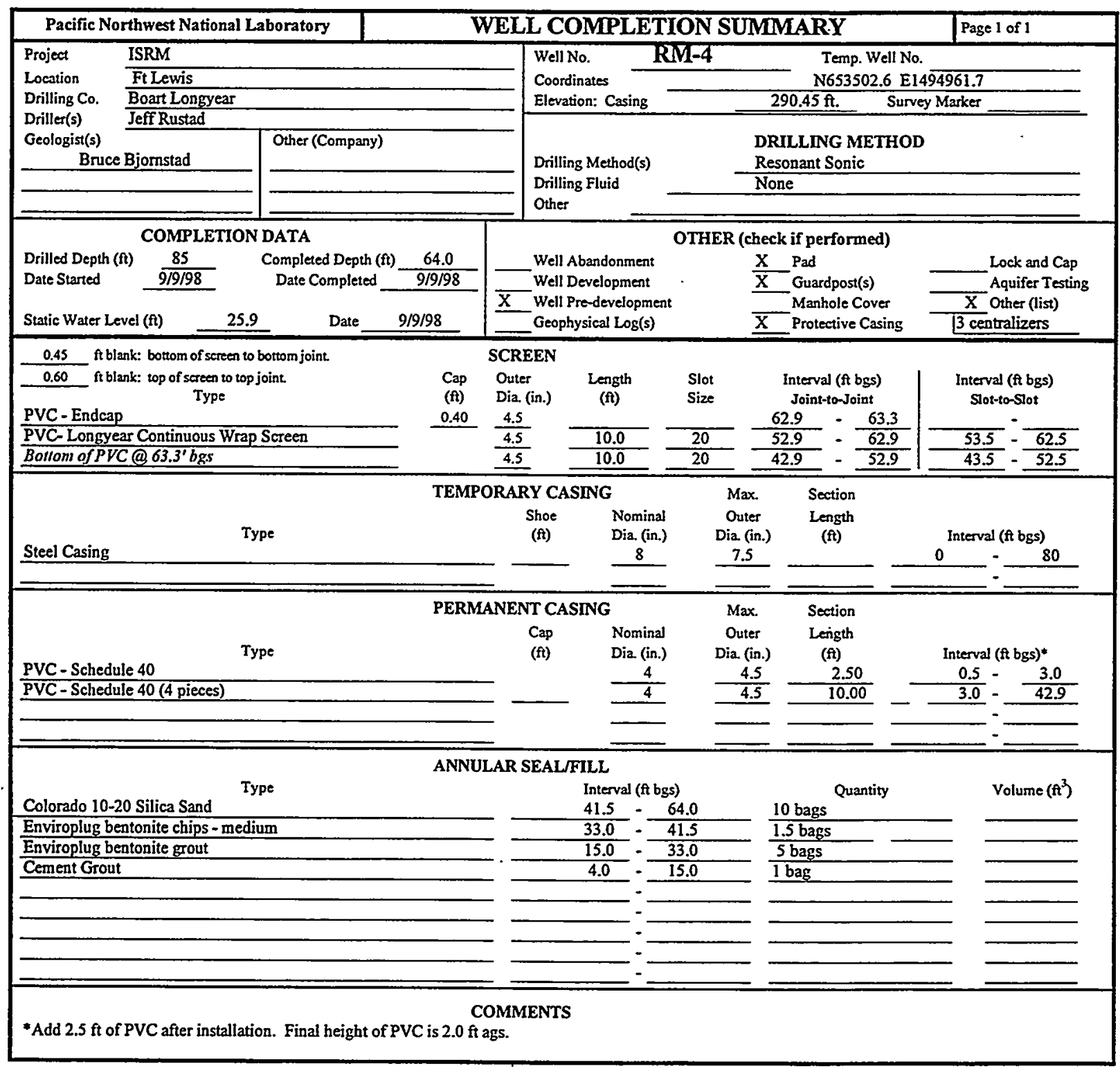




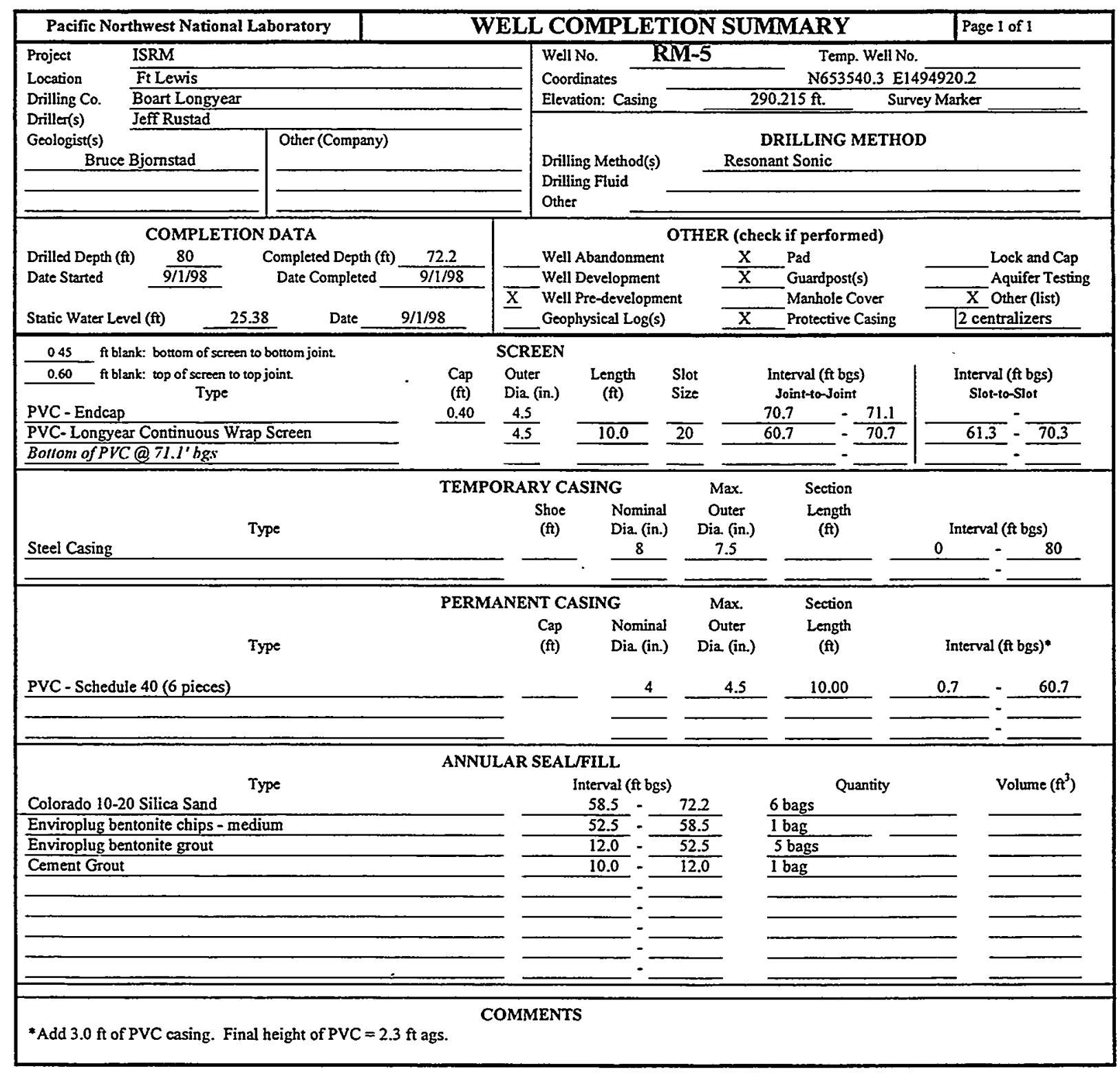




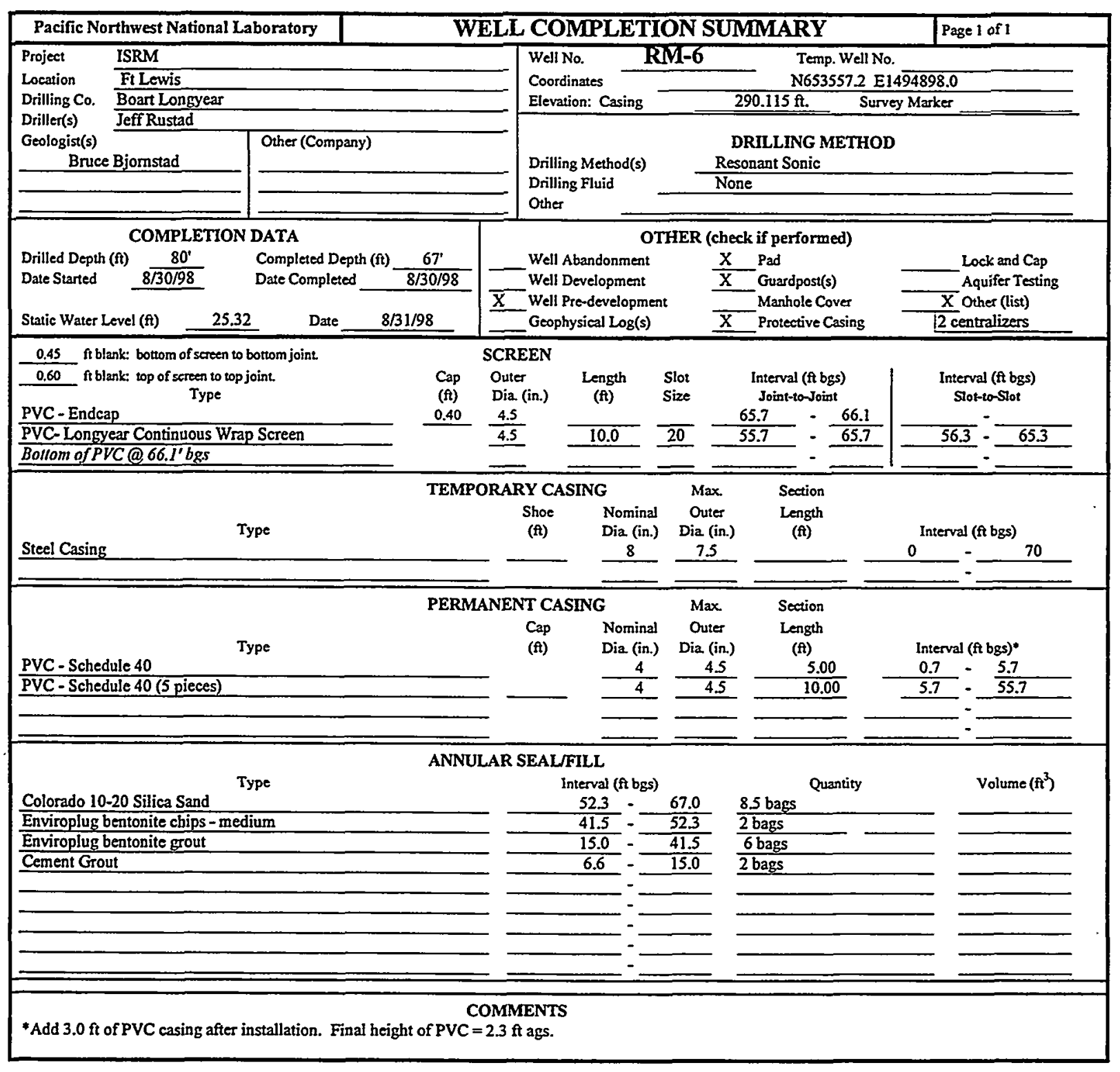




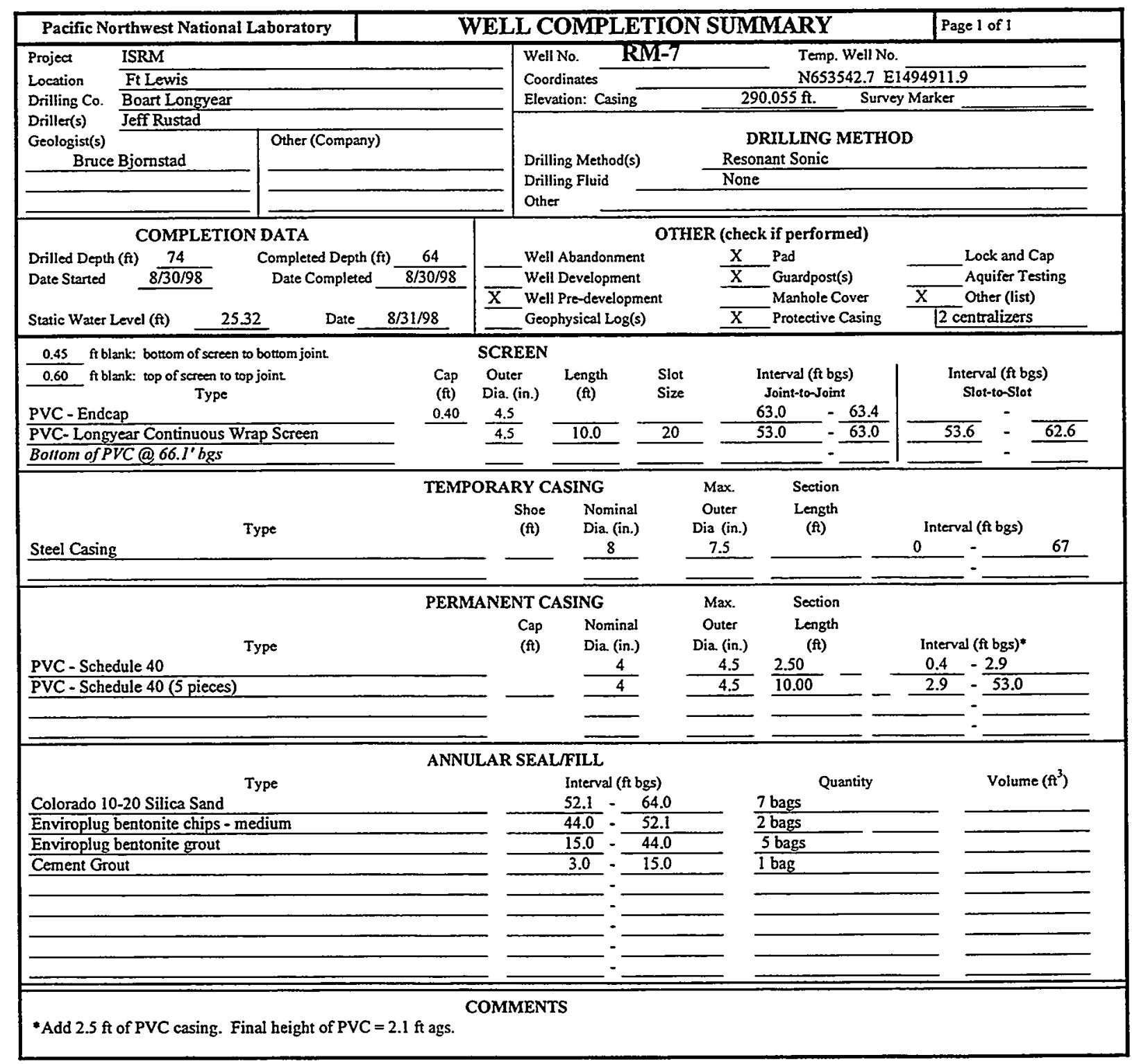




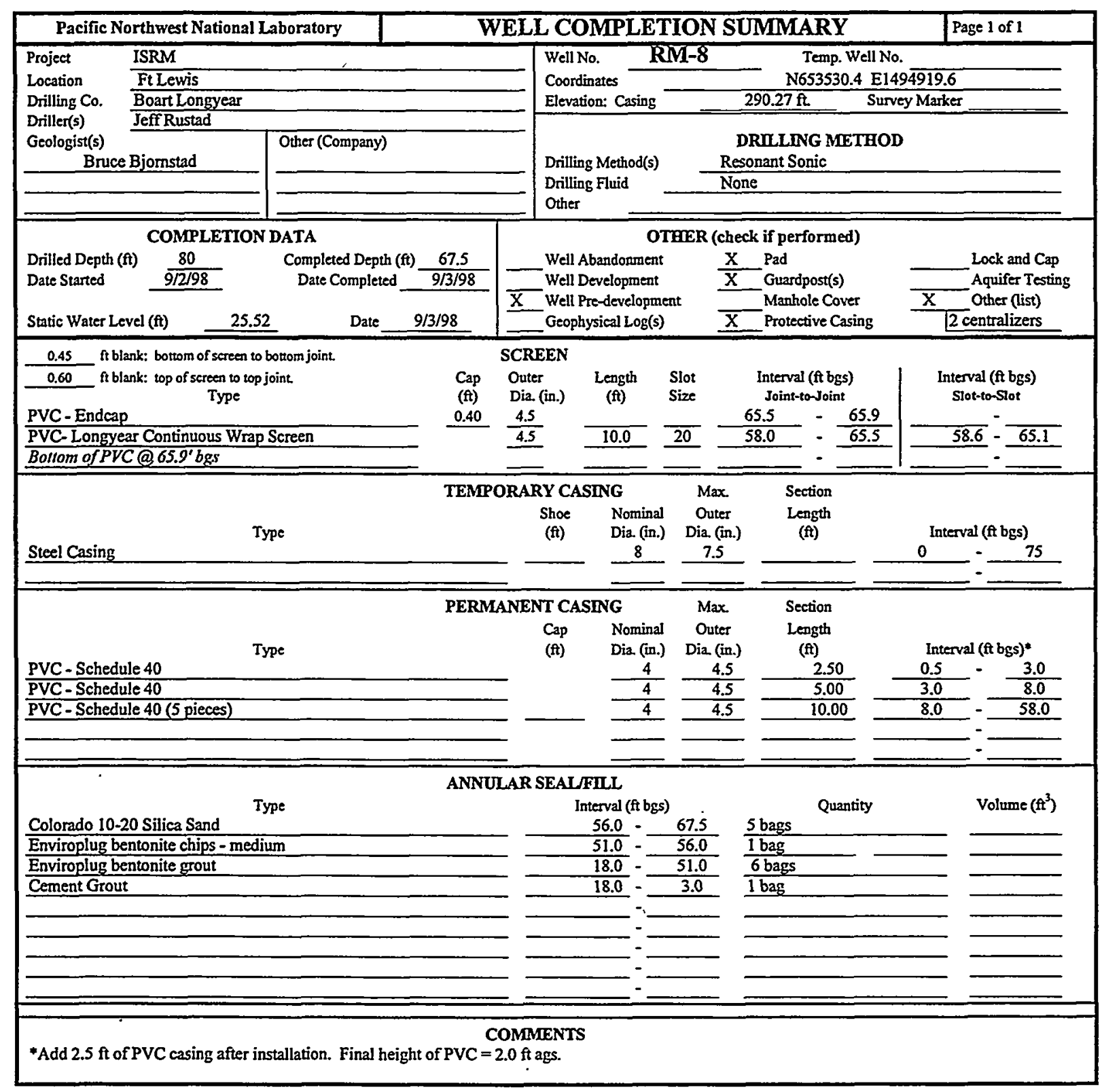




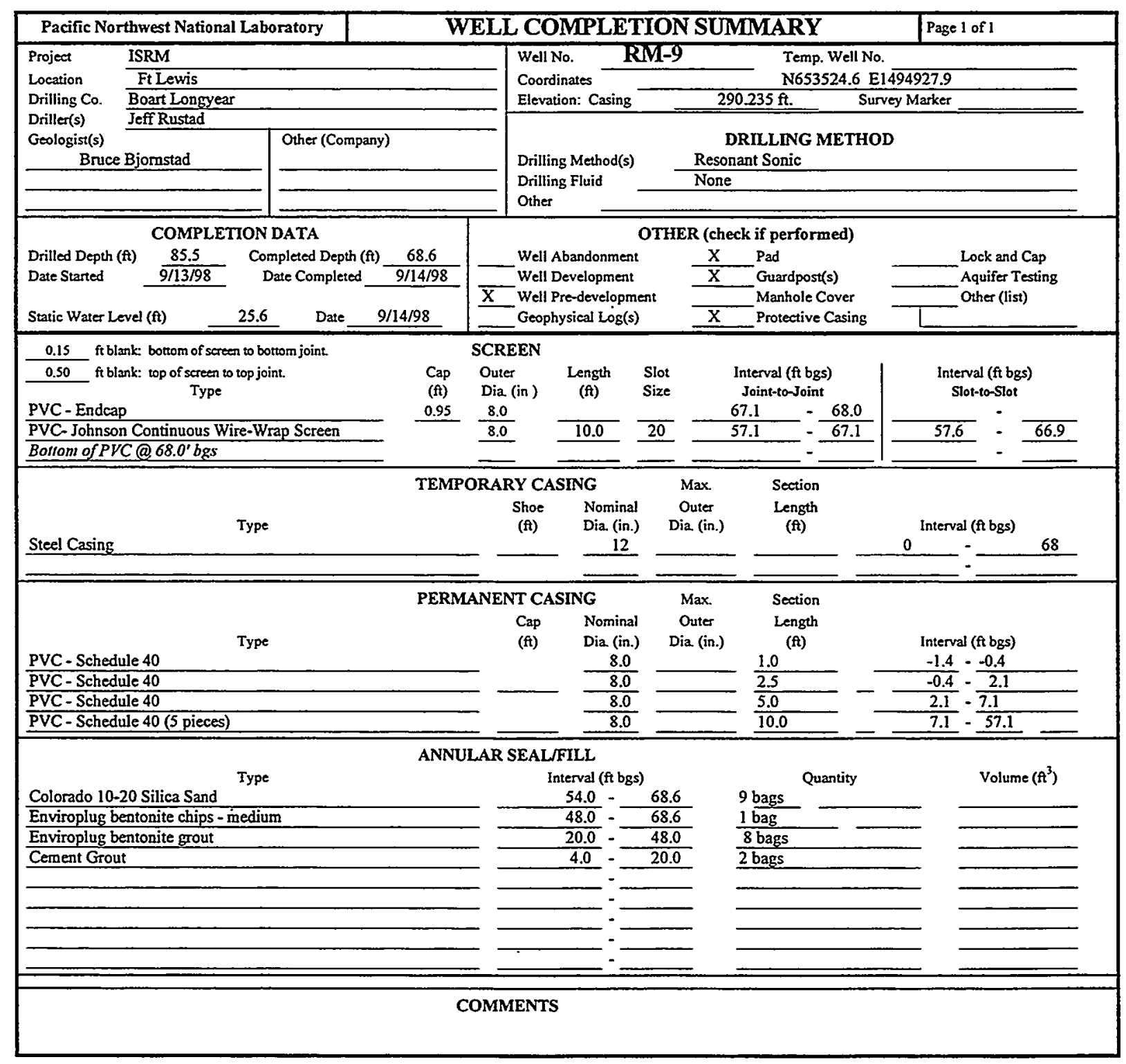




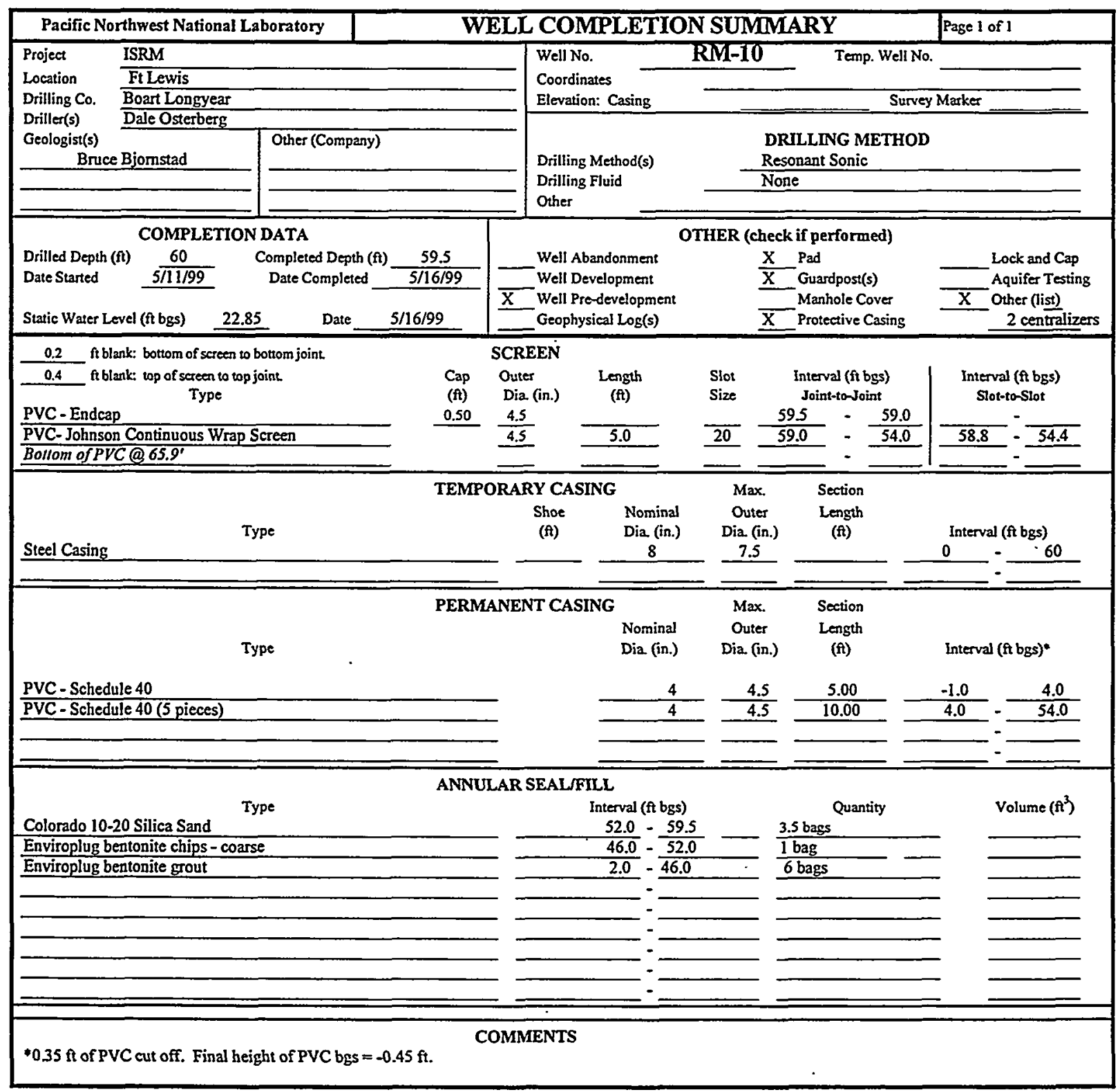




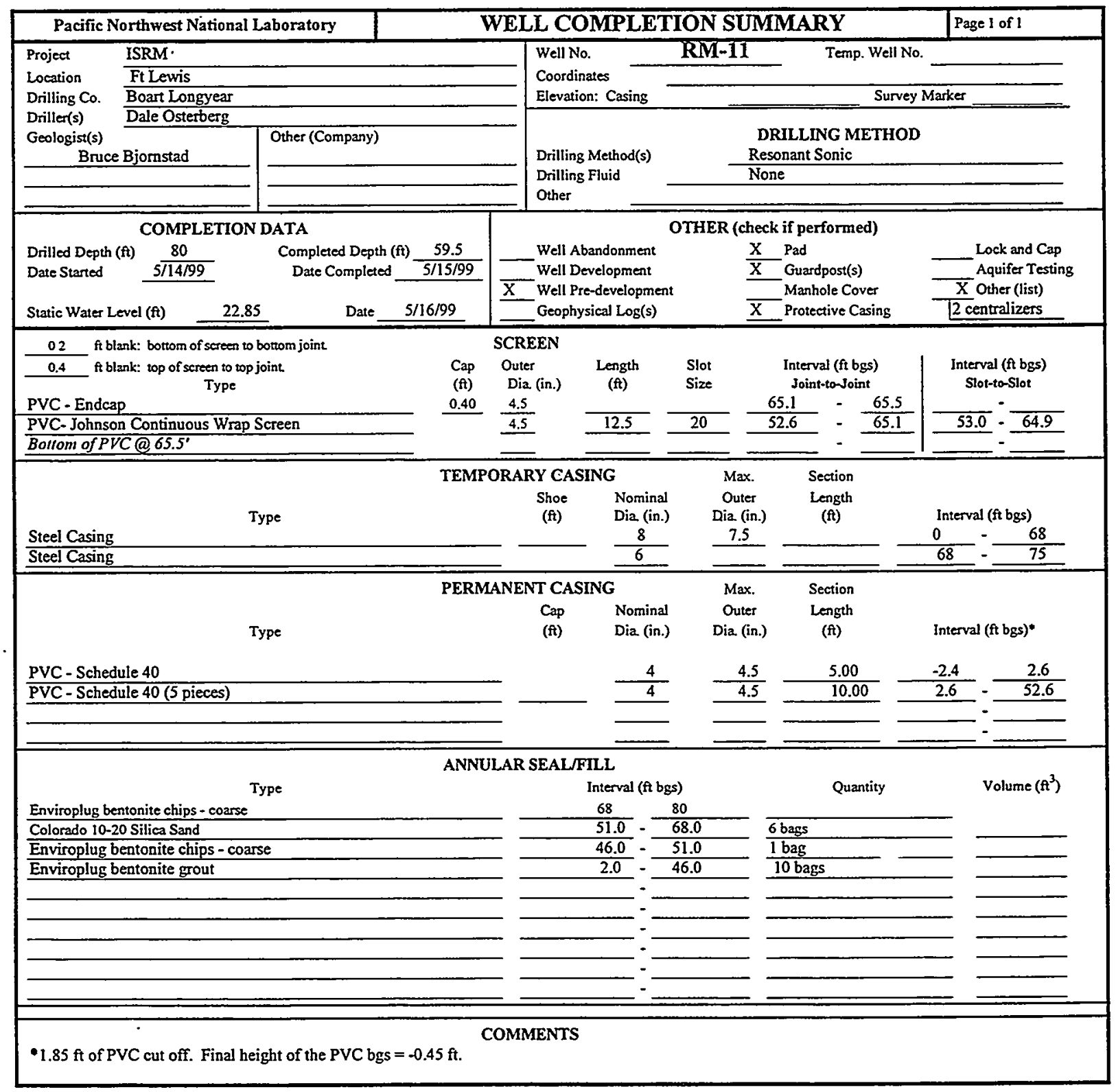




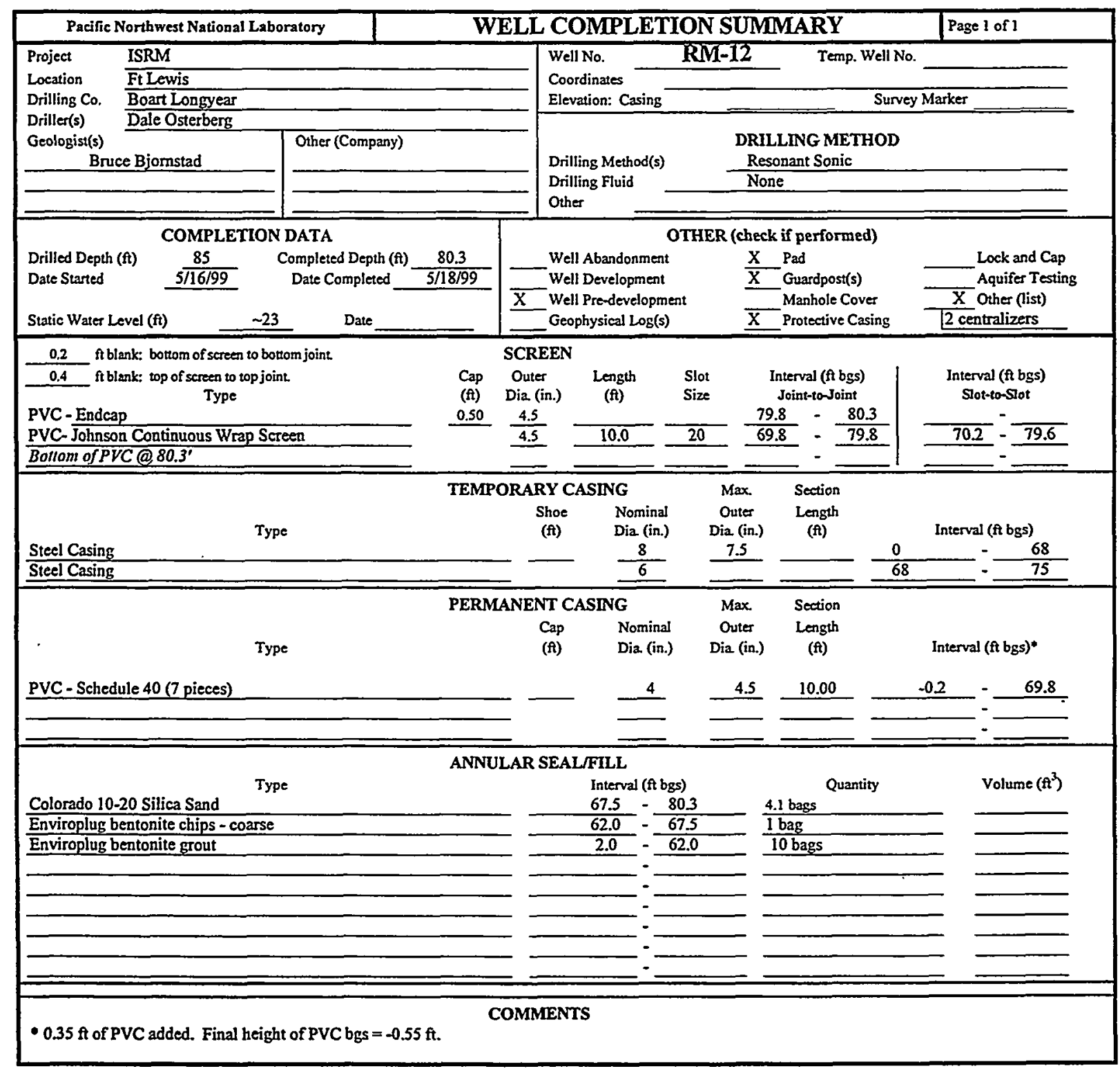




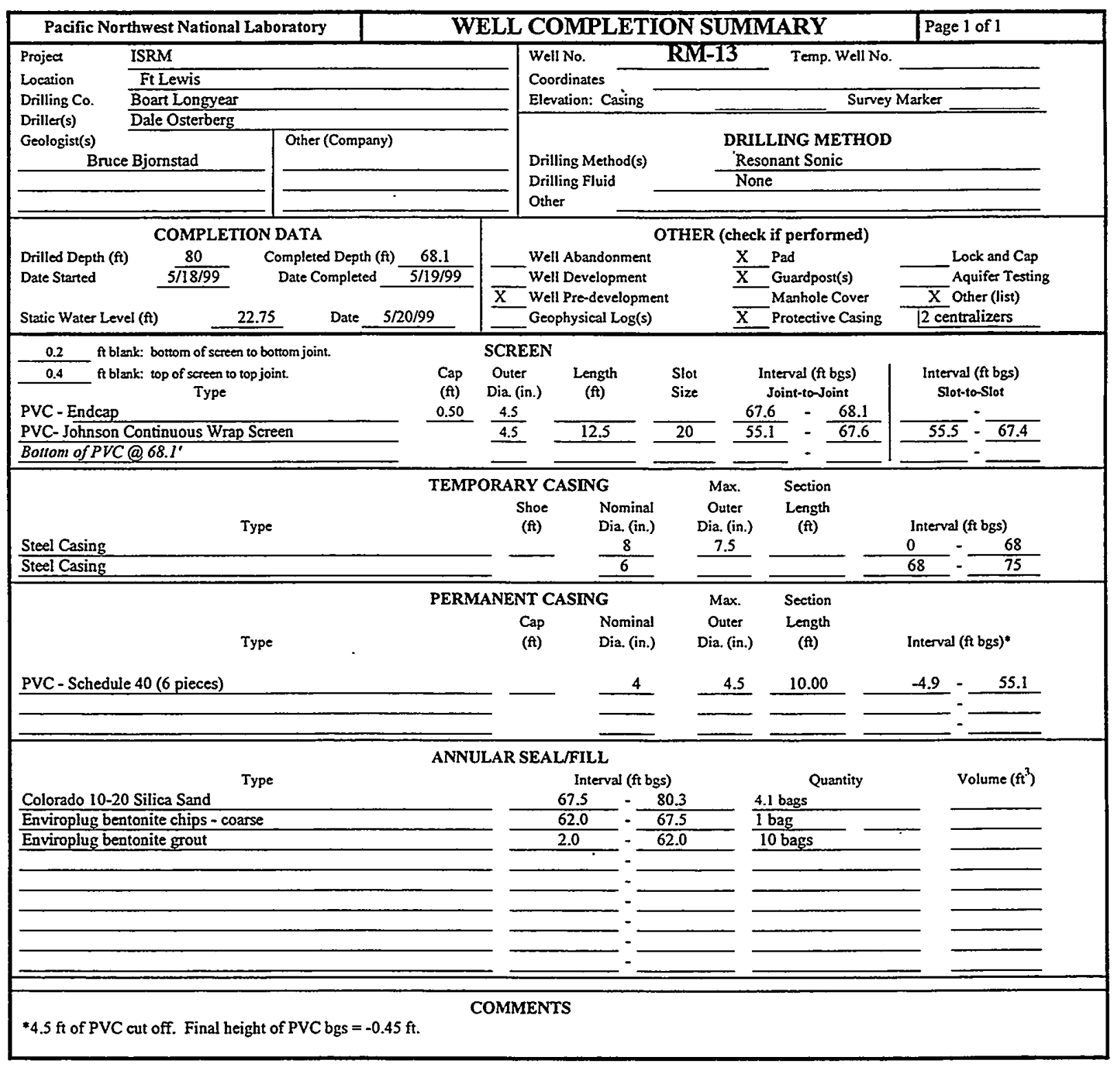




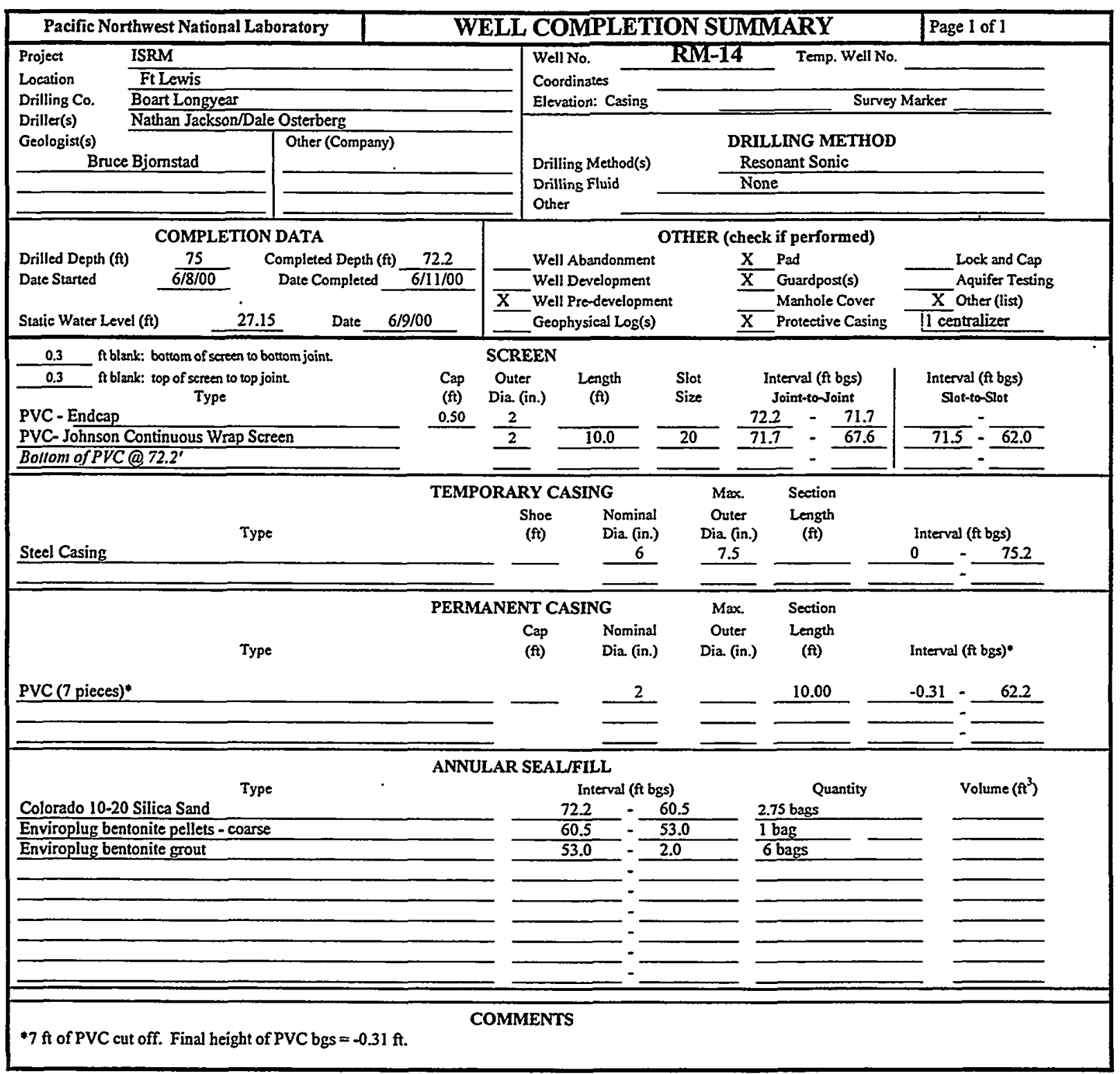




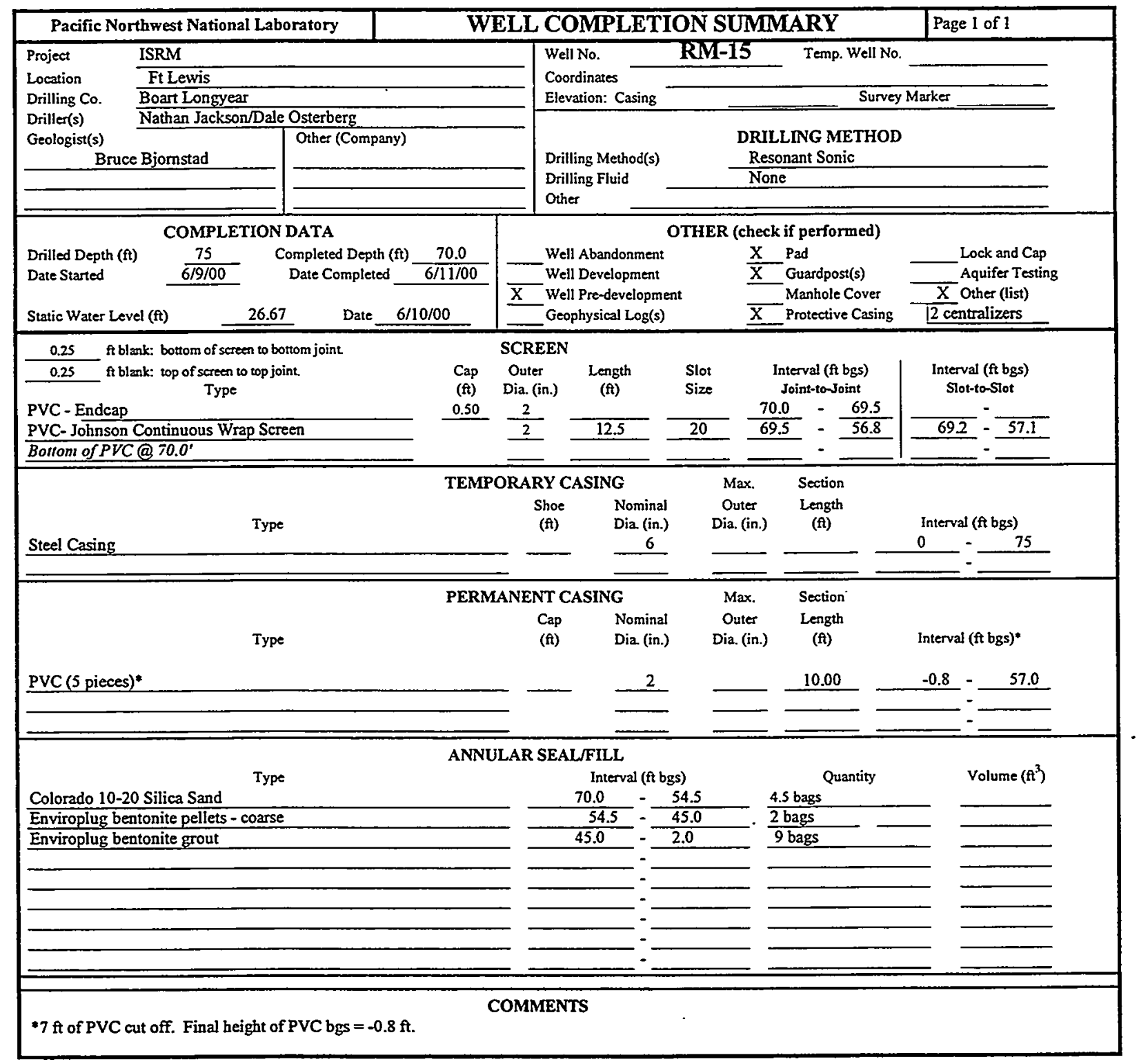




\section{C.4 As-Built Diagrams}

C. 27 

Hole abandoned - no well installation
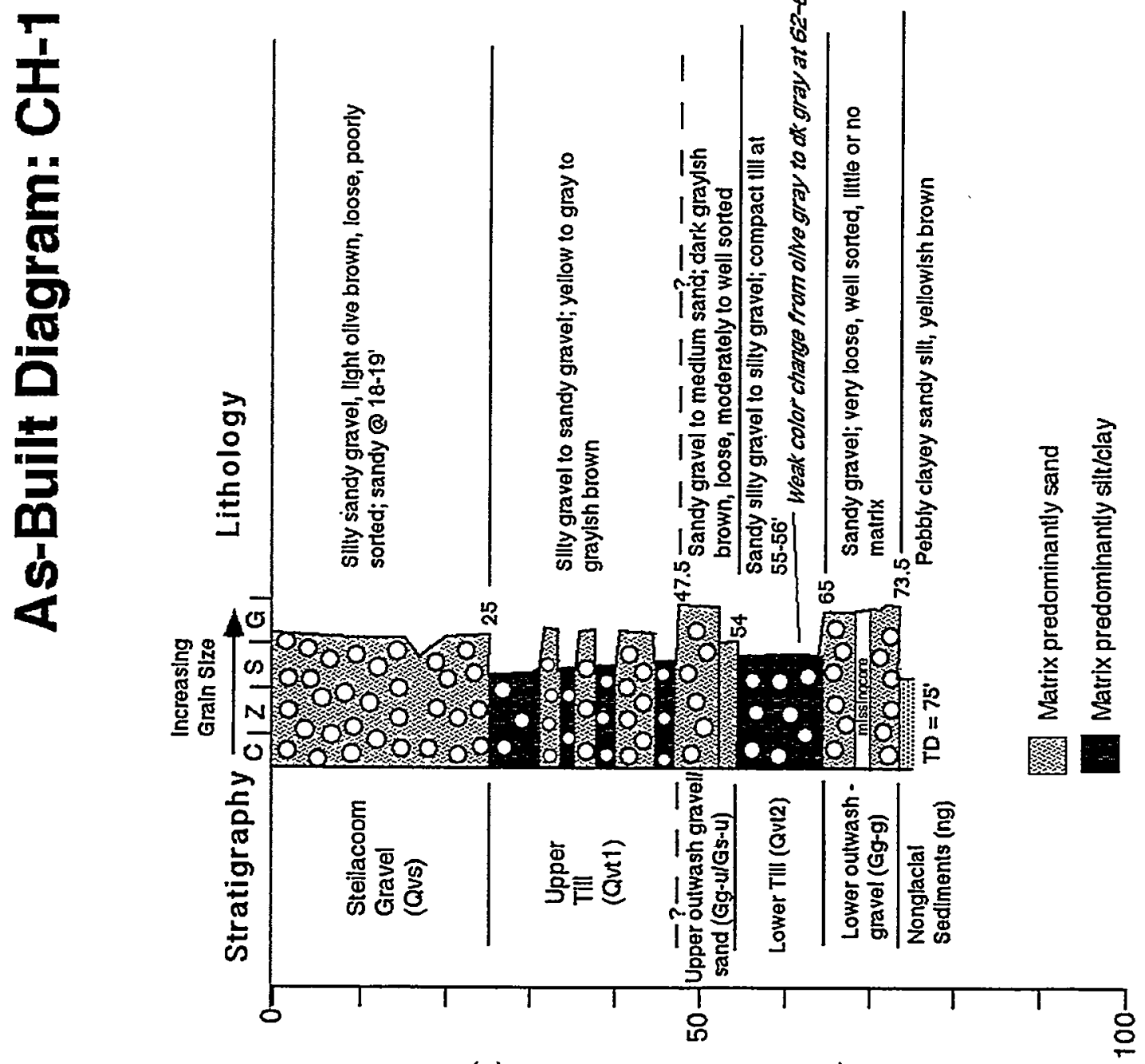

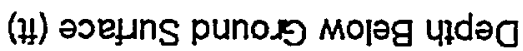




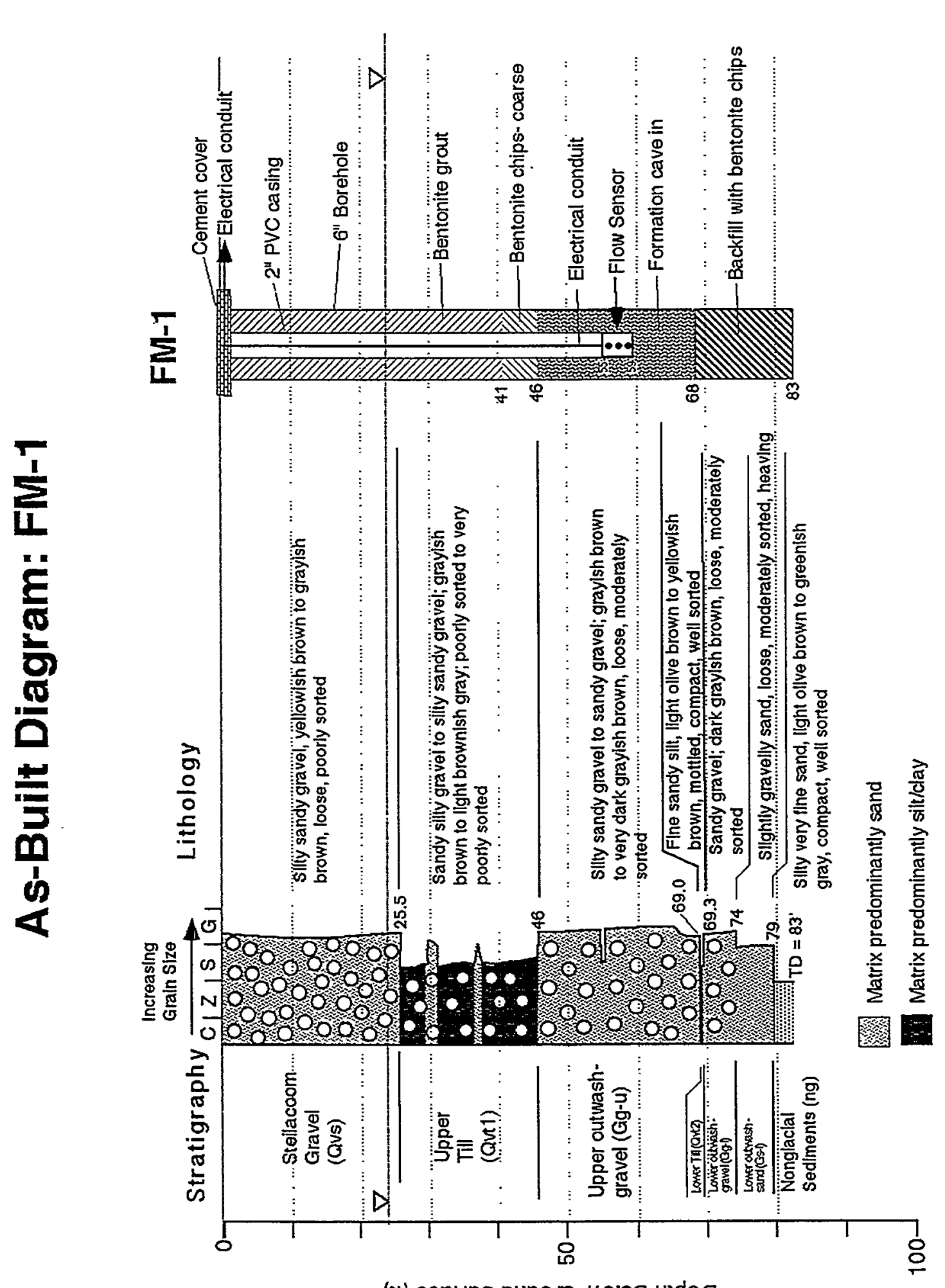

(H) әэeuns punox Mo|əg पldəa 


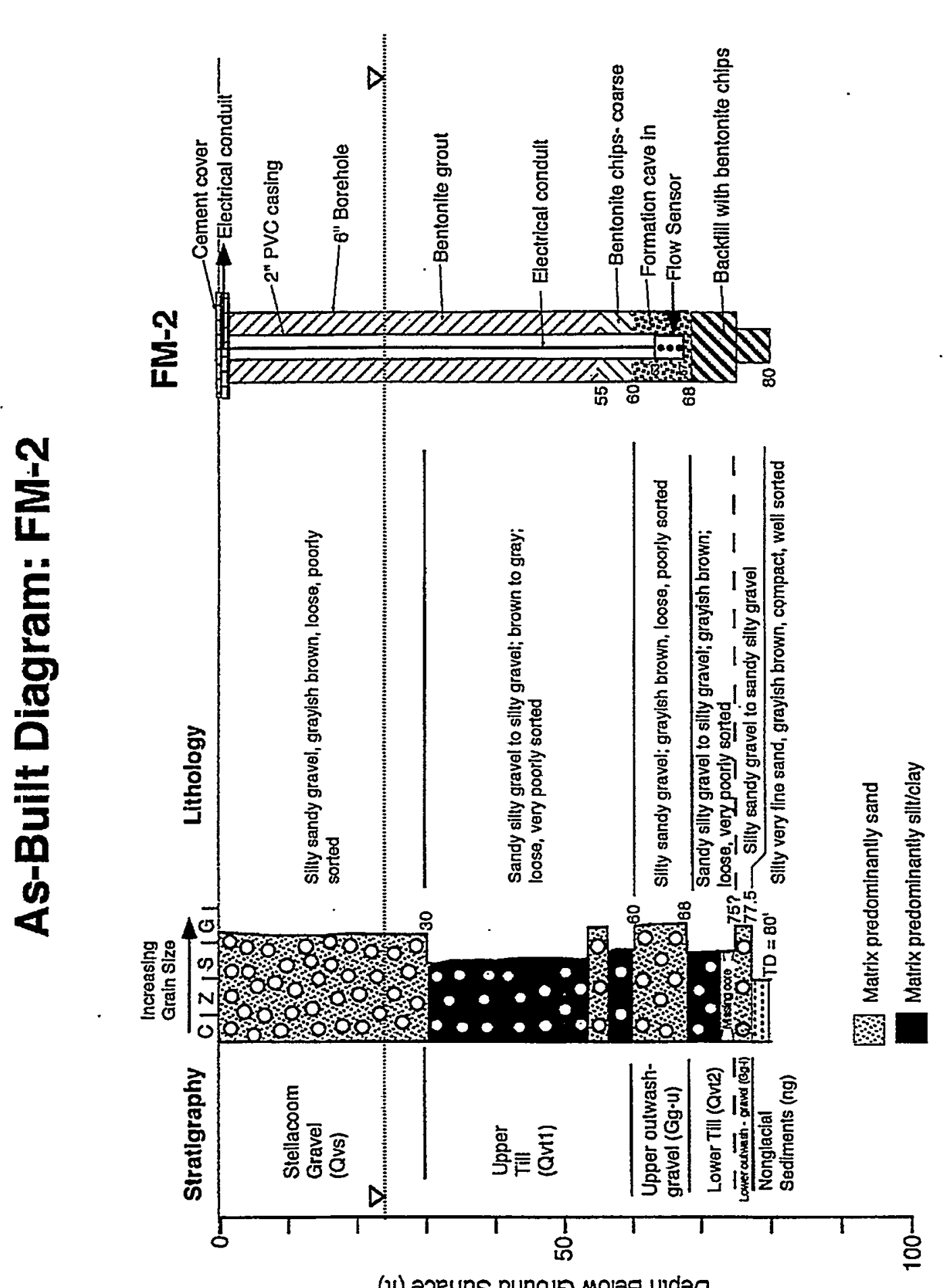

(H) aseuns punojo mopeg yidaO 


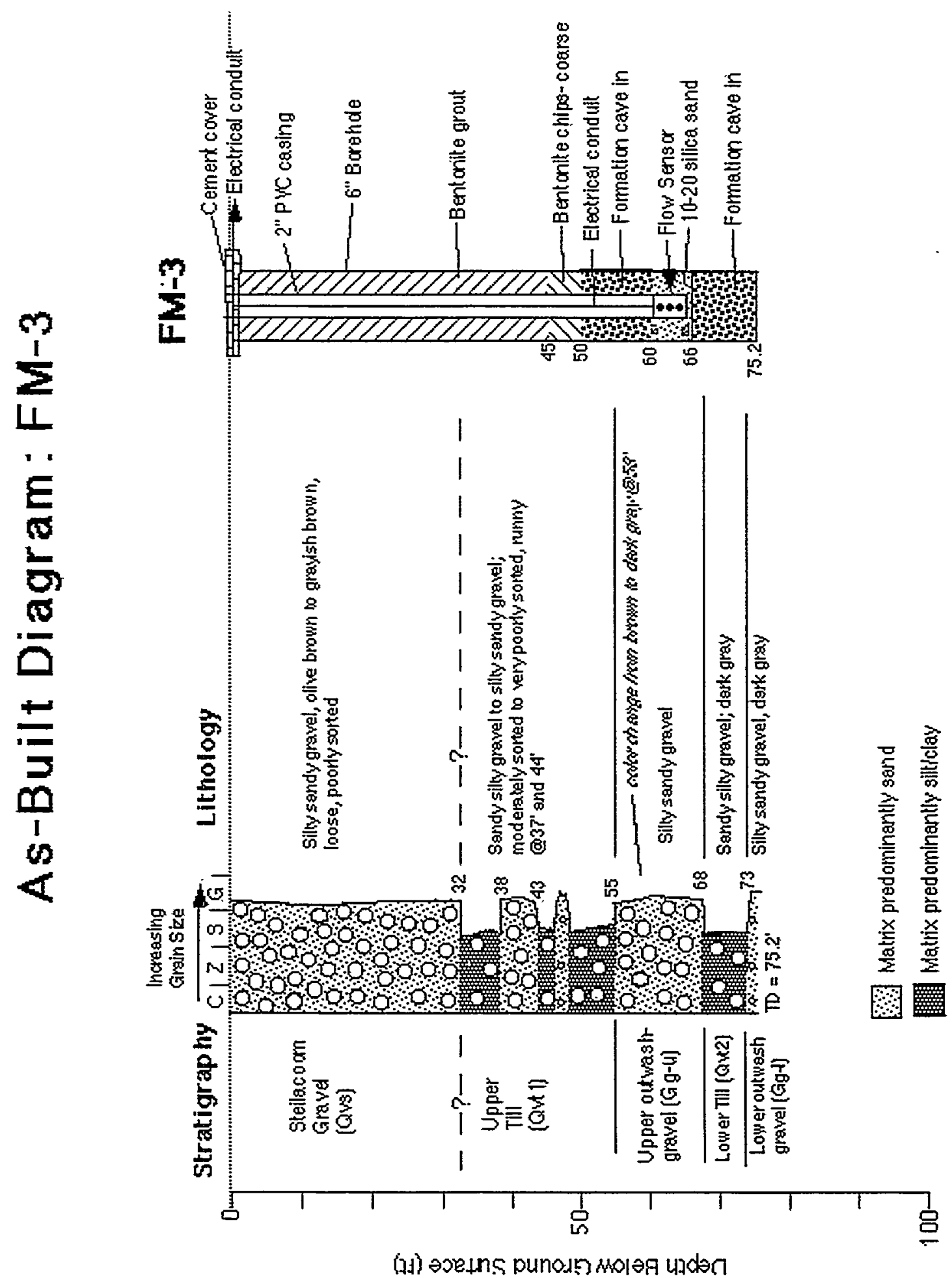




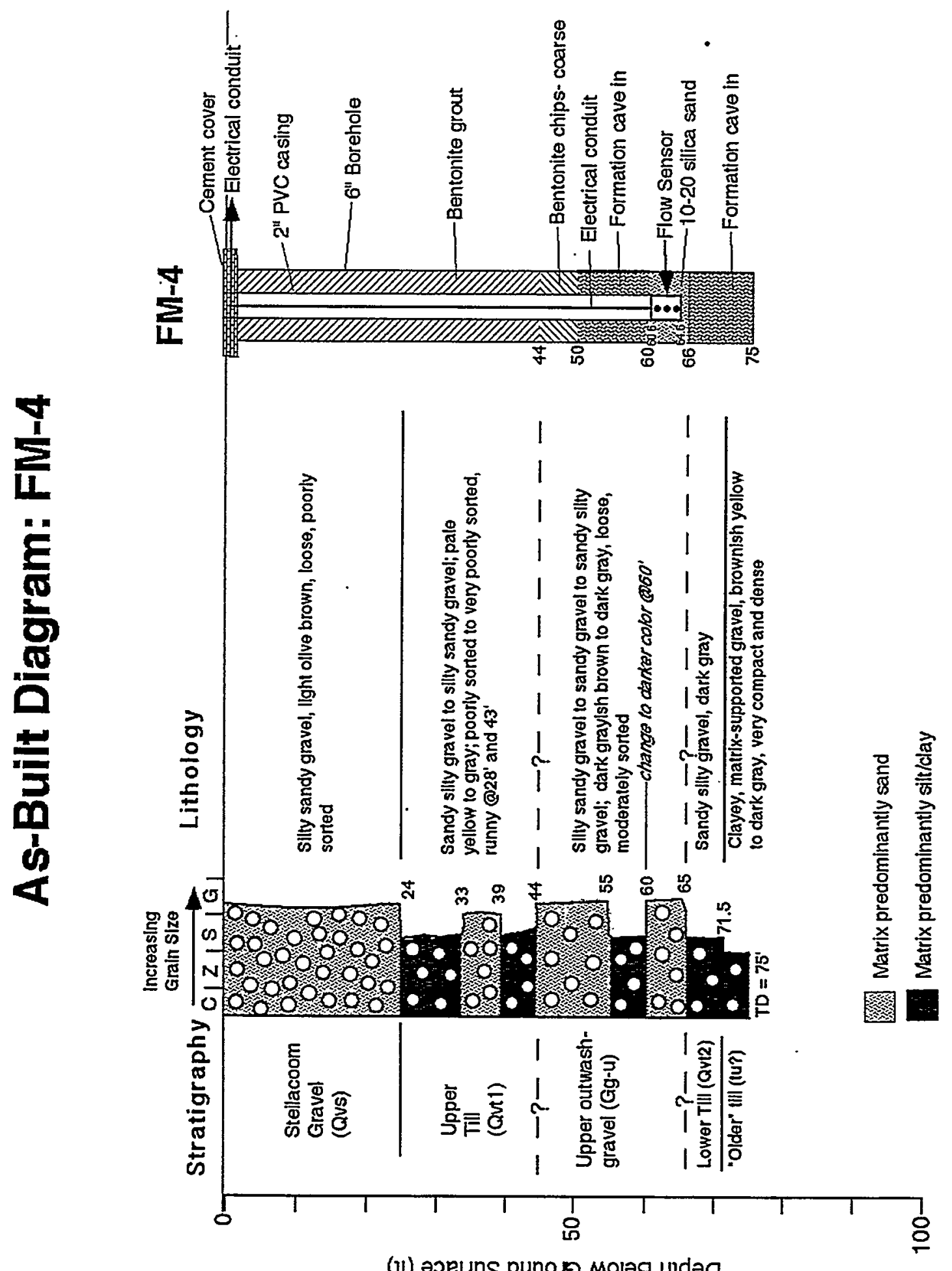

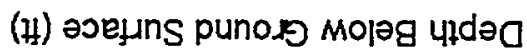




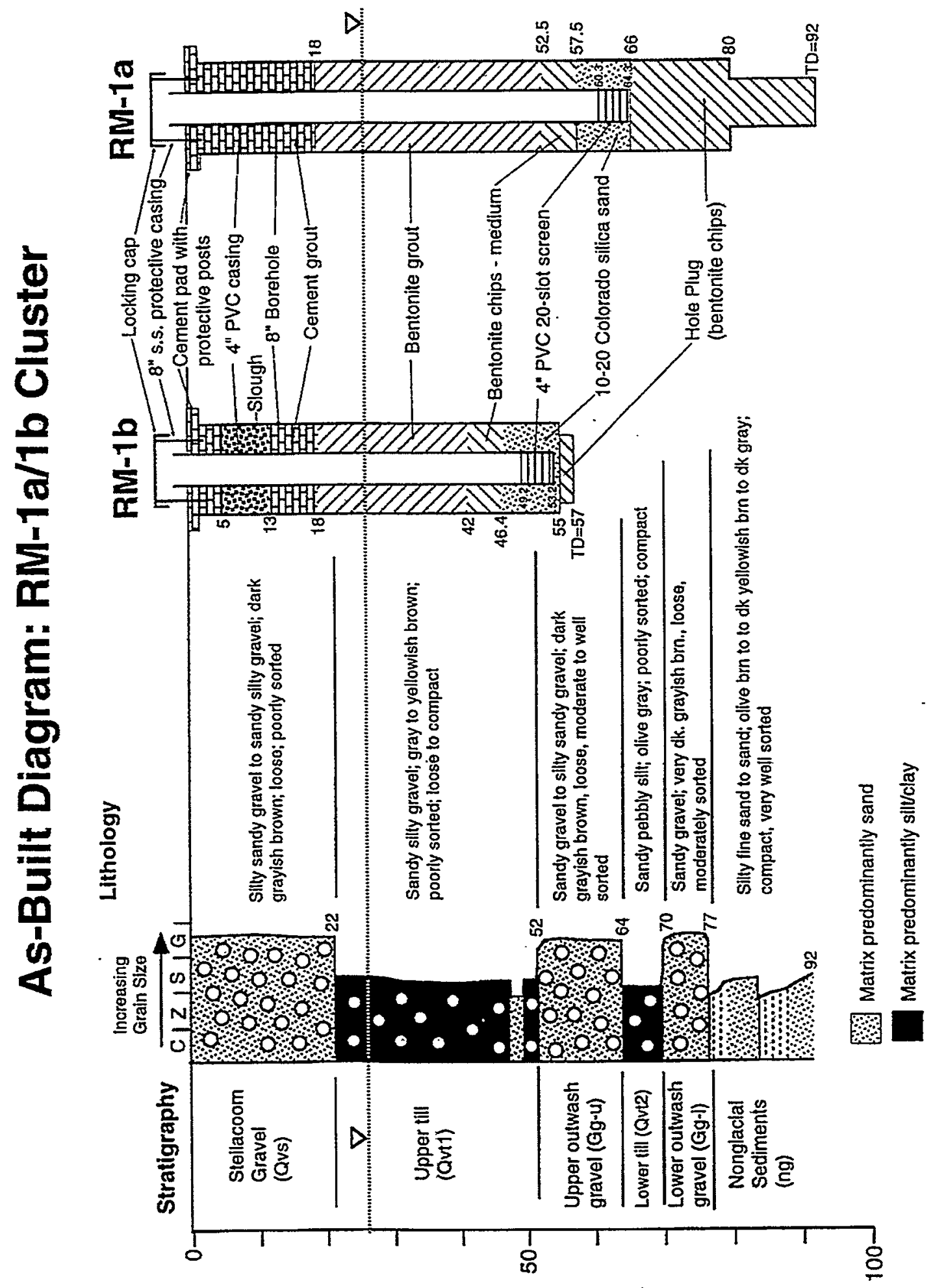

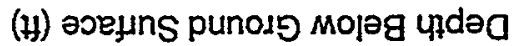




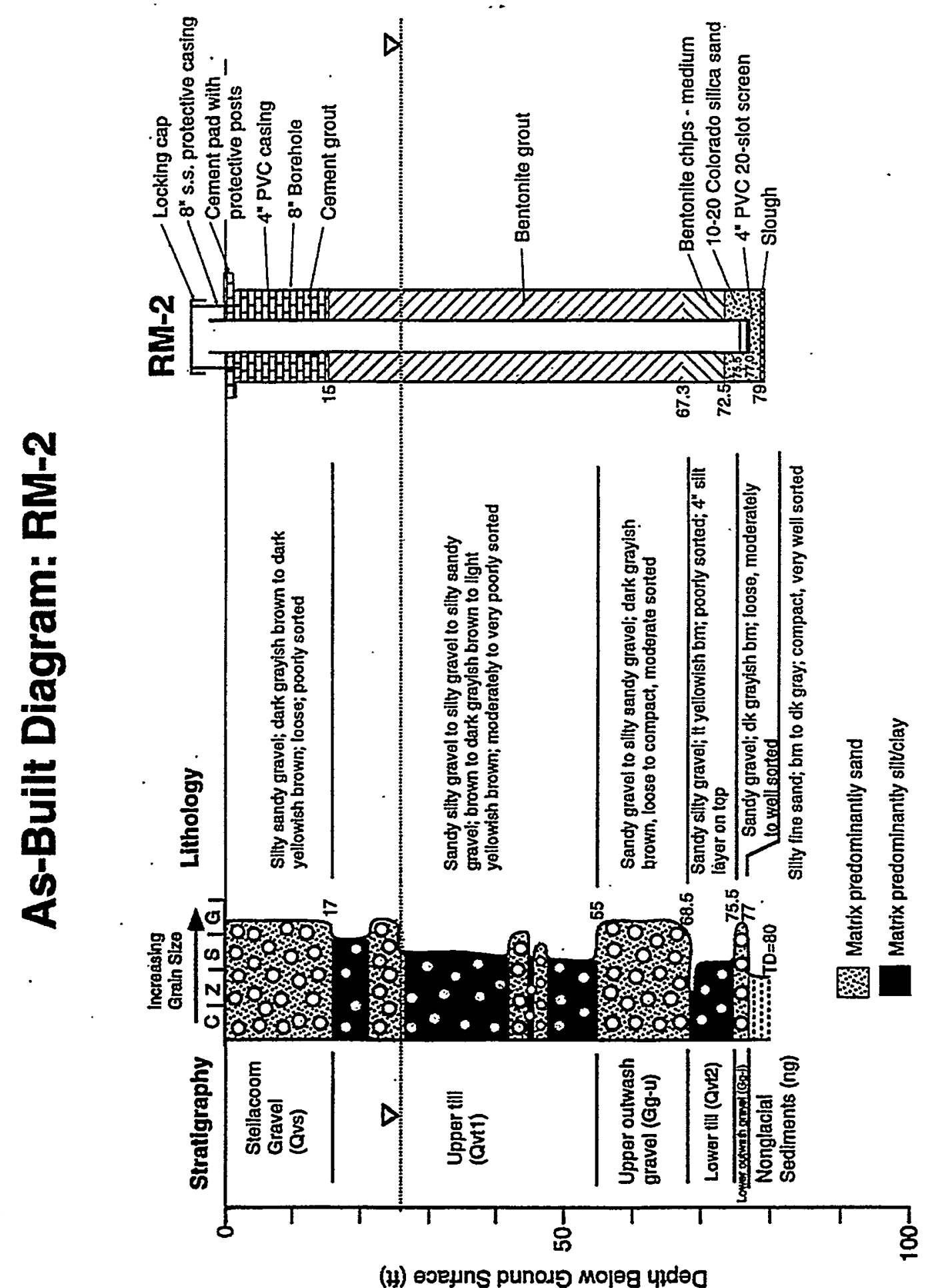




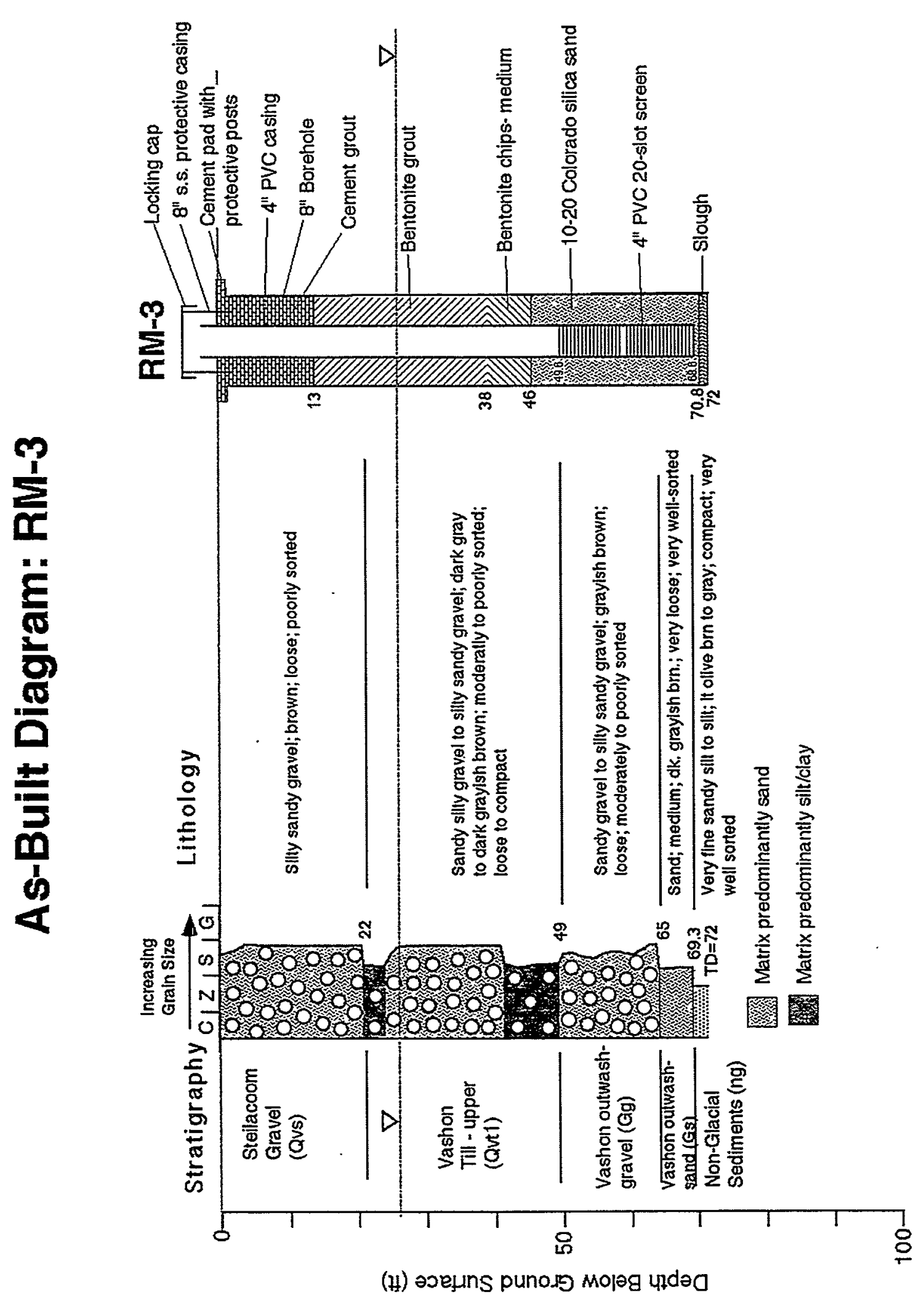

C. 36 


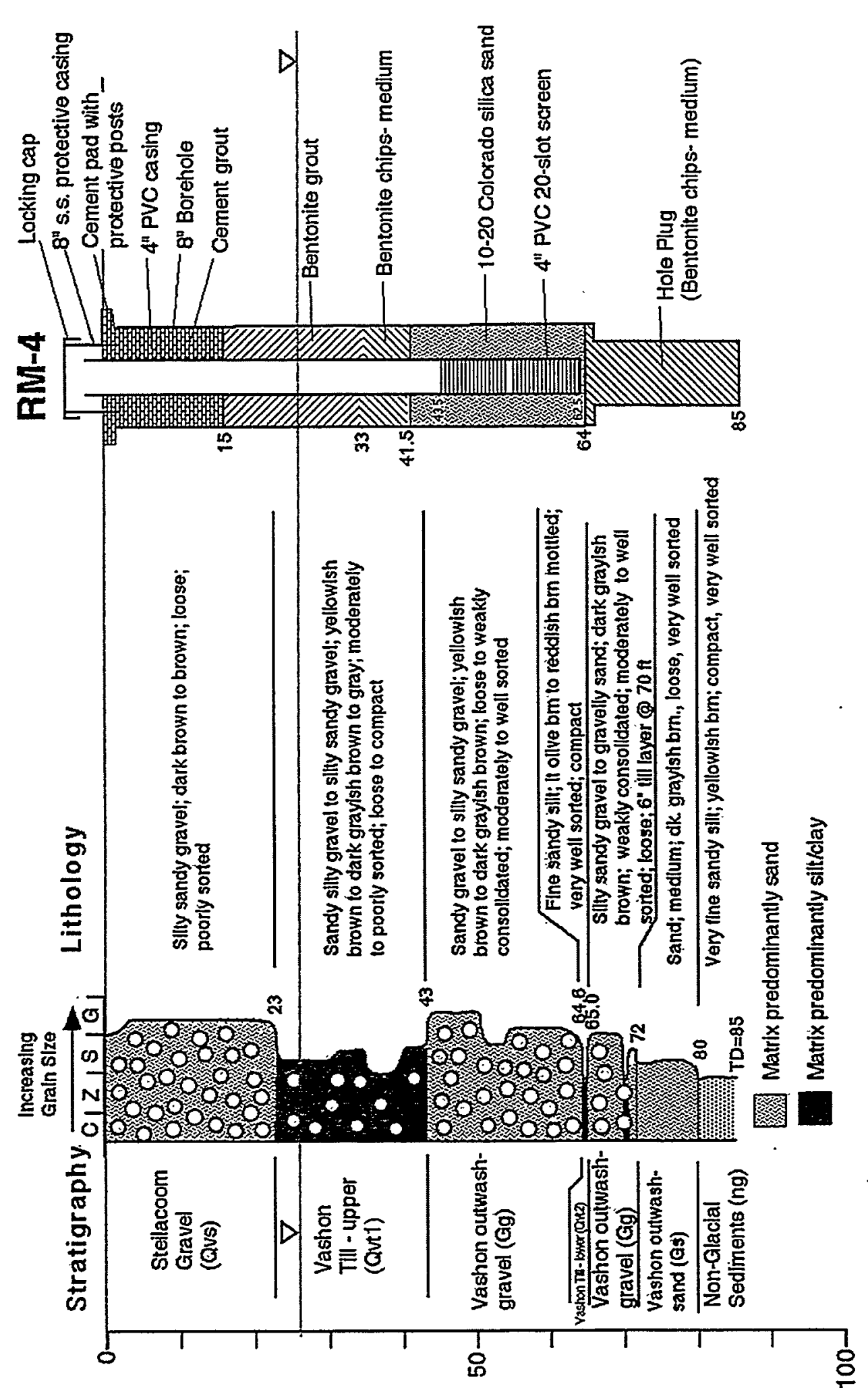

(H) əJeนns punoฌ MO|әg पldəO 


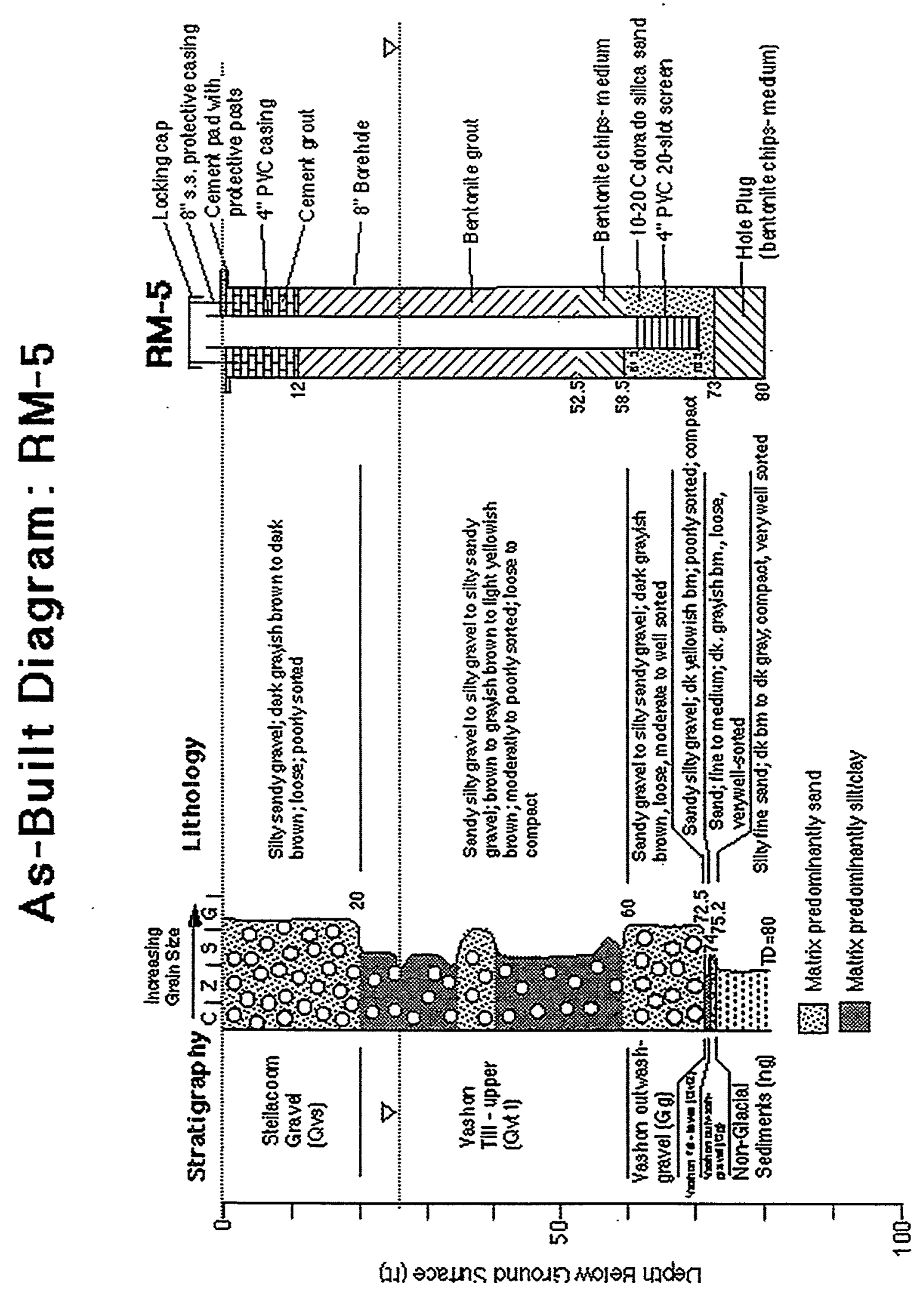

C. 38 


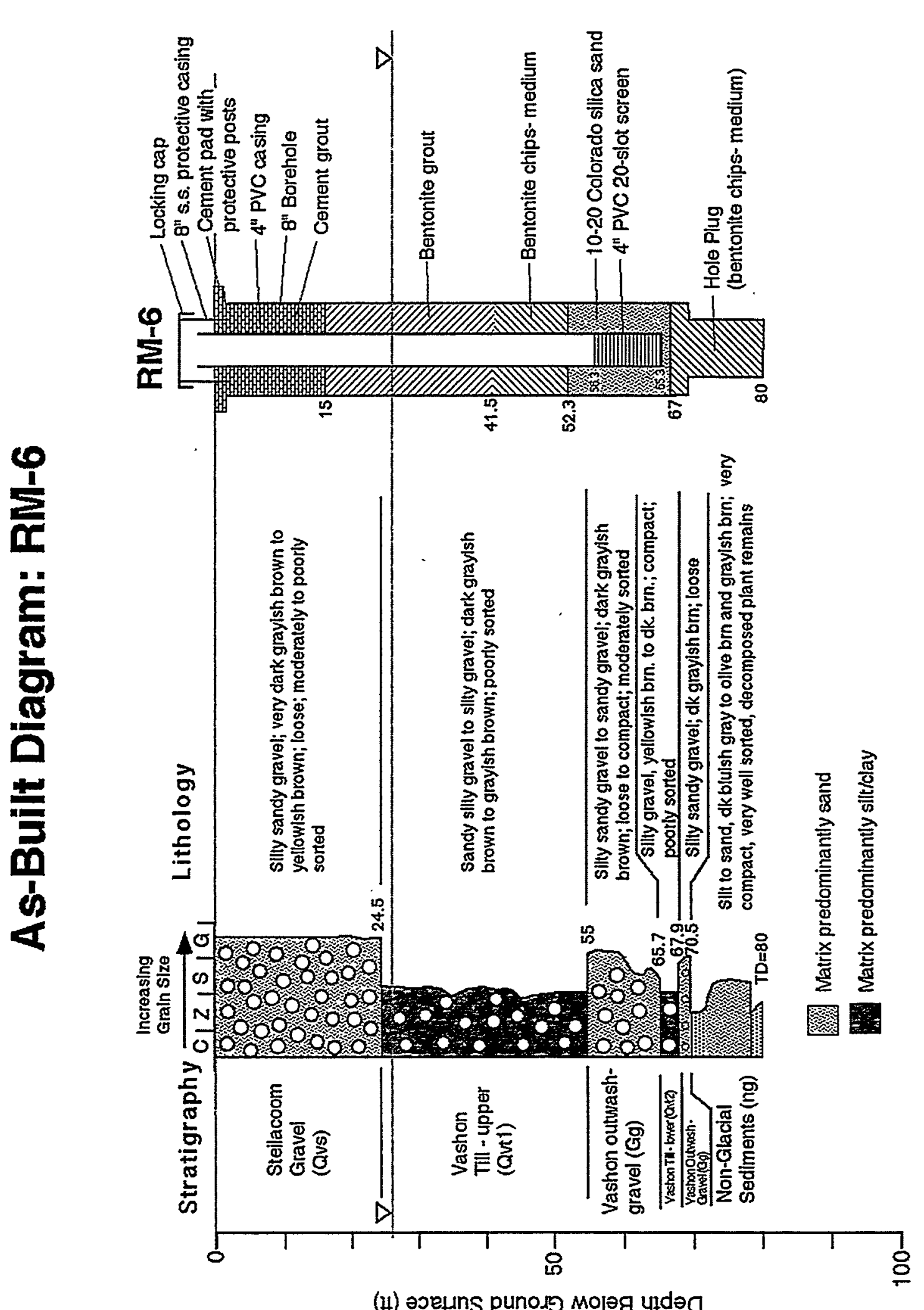

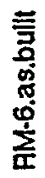

(H) ววeนuns punoฌ Moləg undəa 


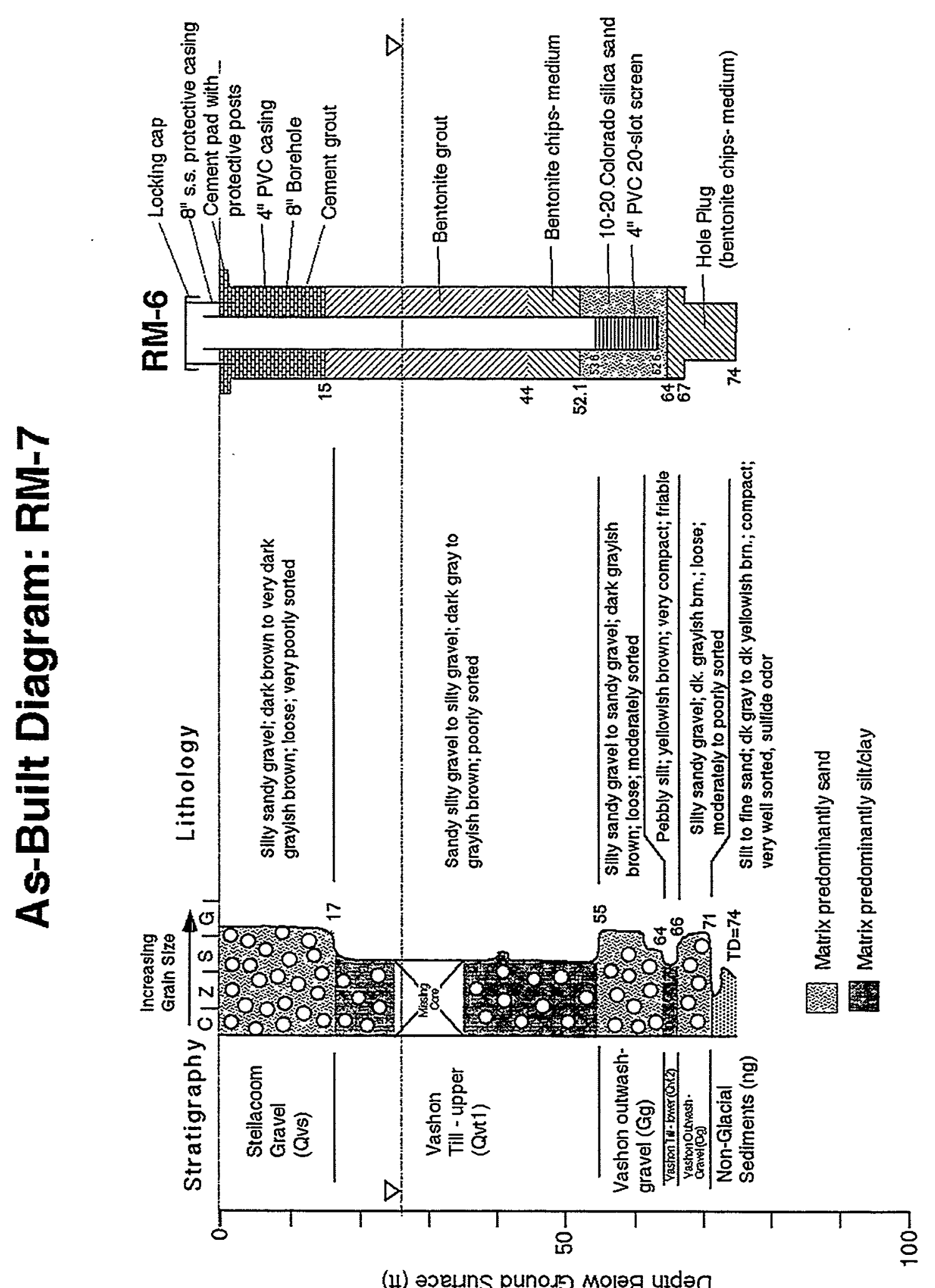

C. 40 


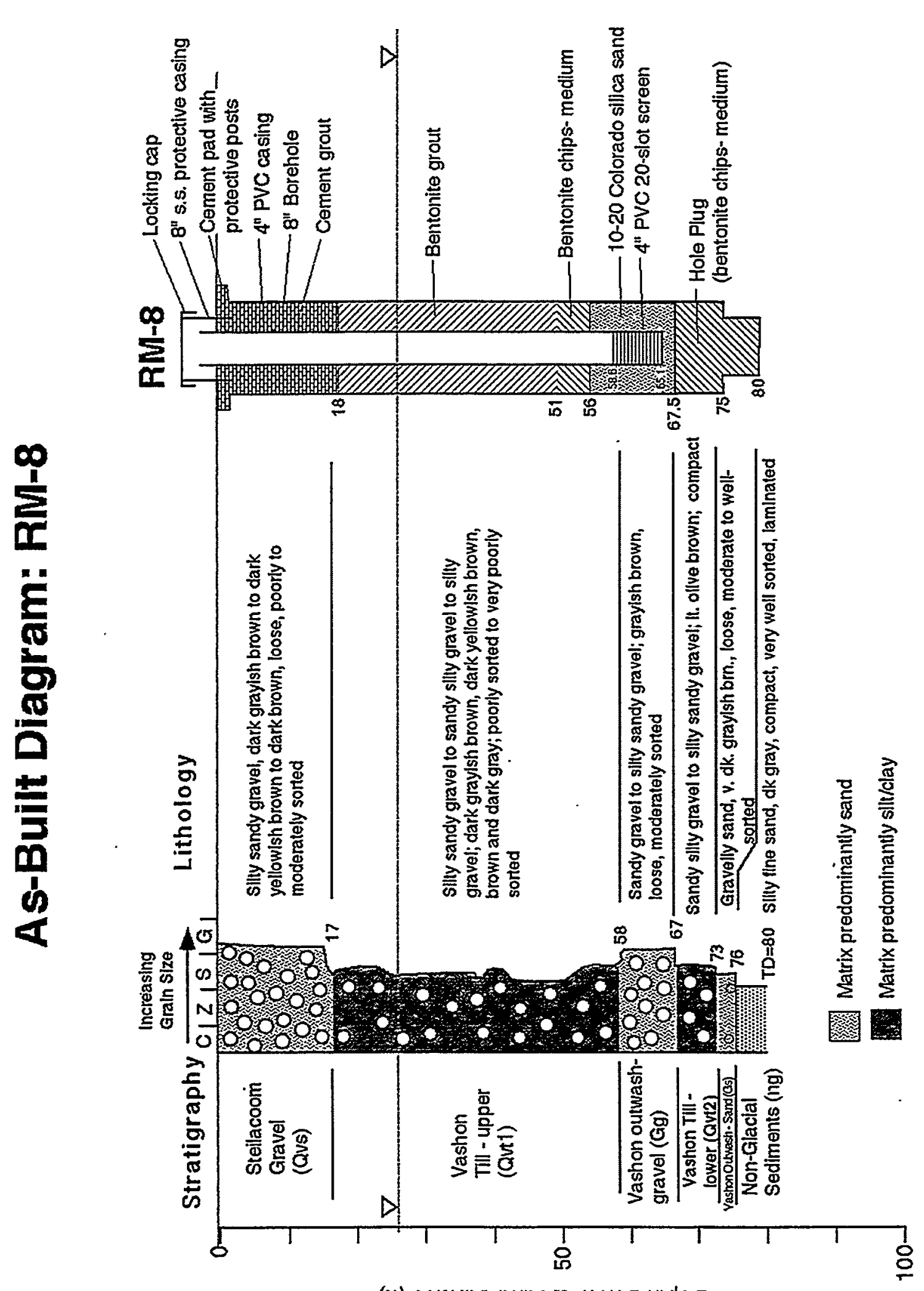

(4) əoefuns punos moləg undəa 


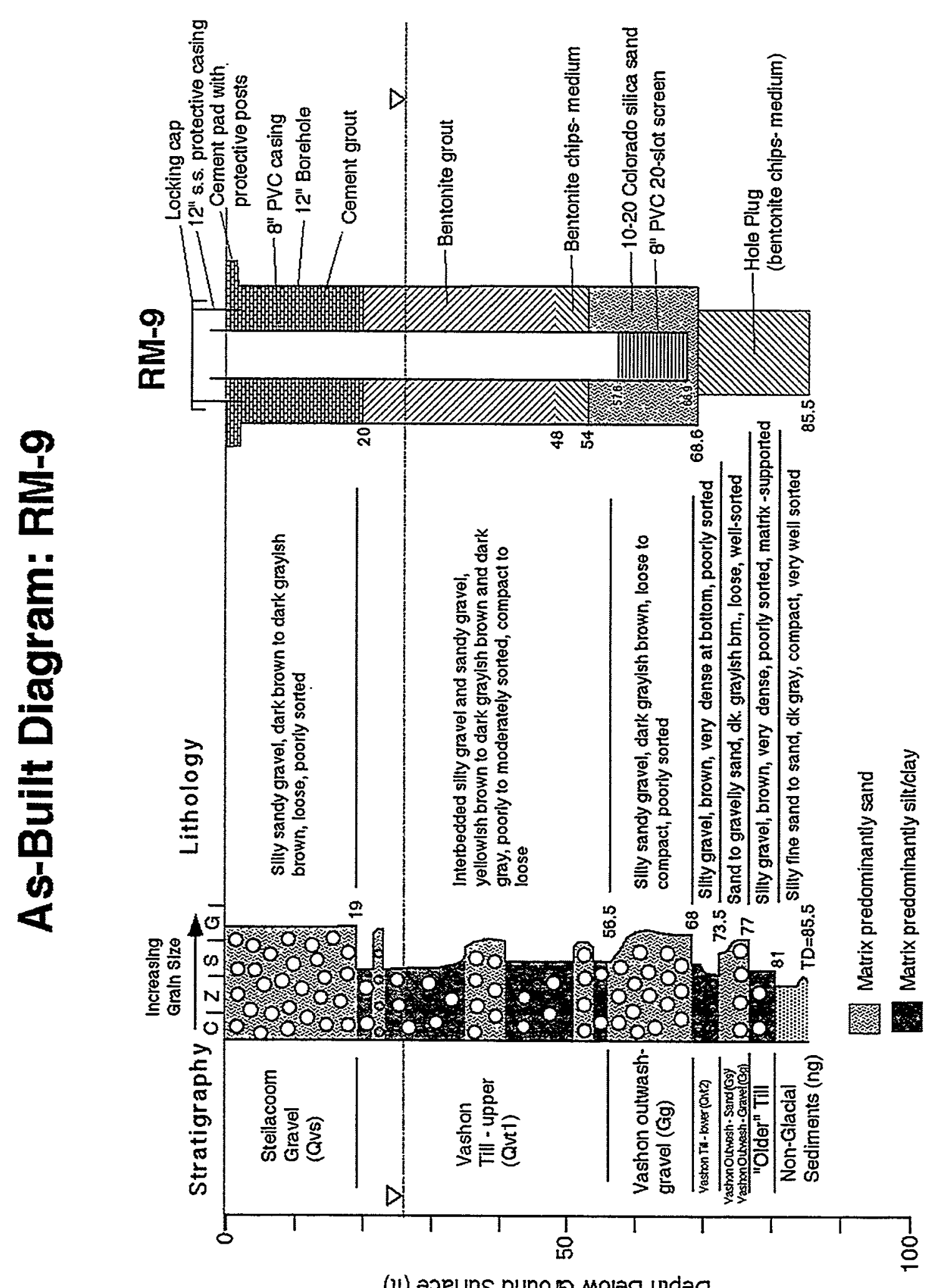

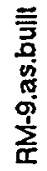

(4) aseuns puno® Moləg undəo 


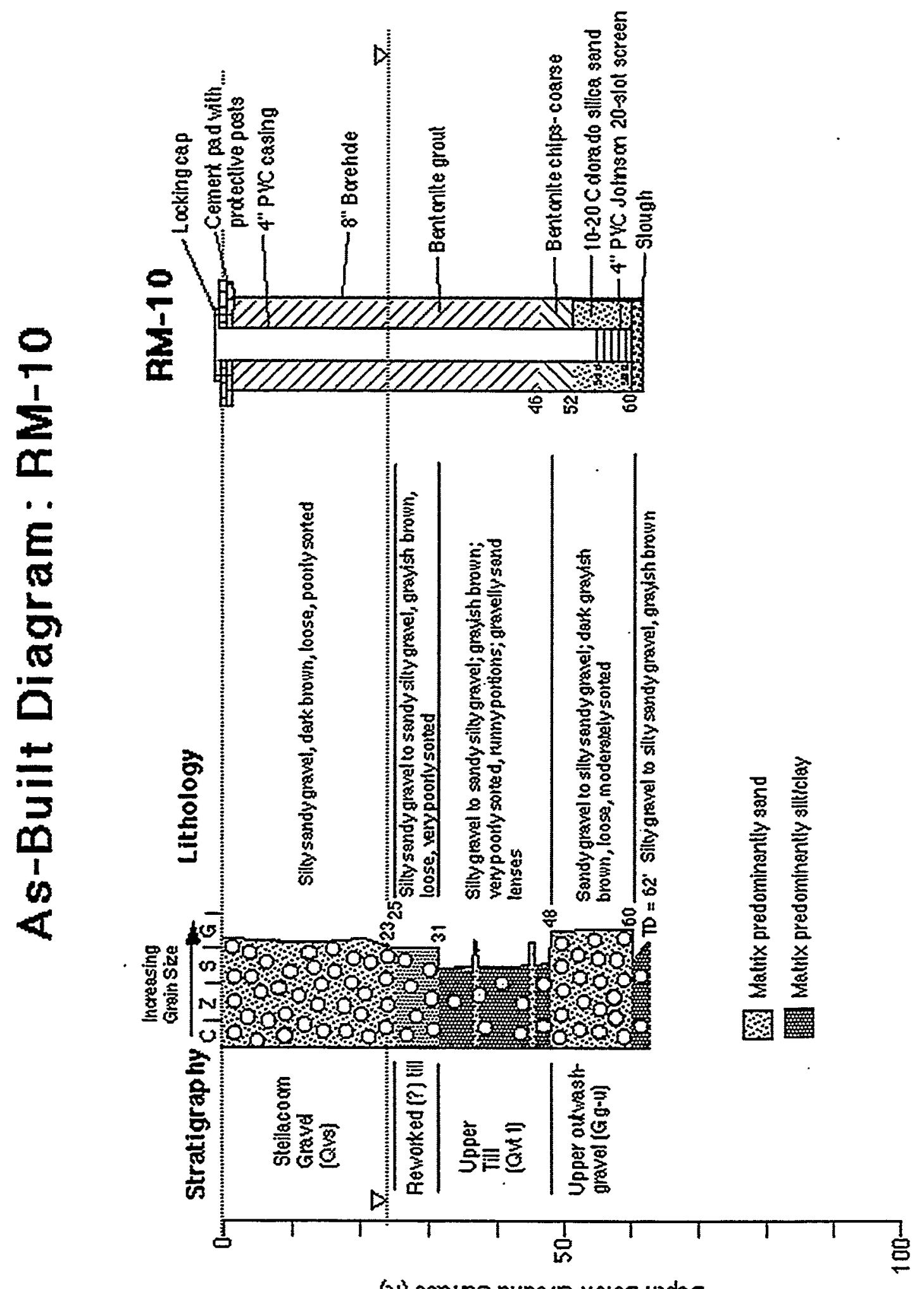

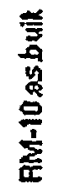

(a) əxeins punoxs soləa uldan 


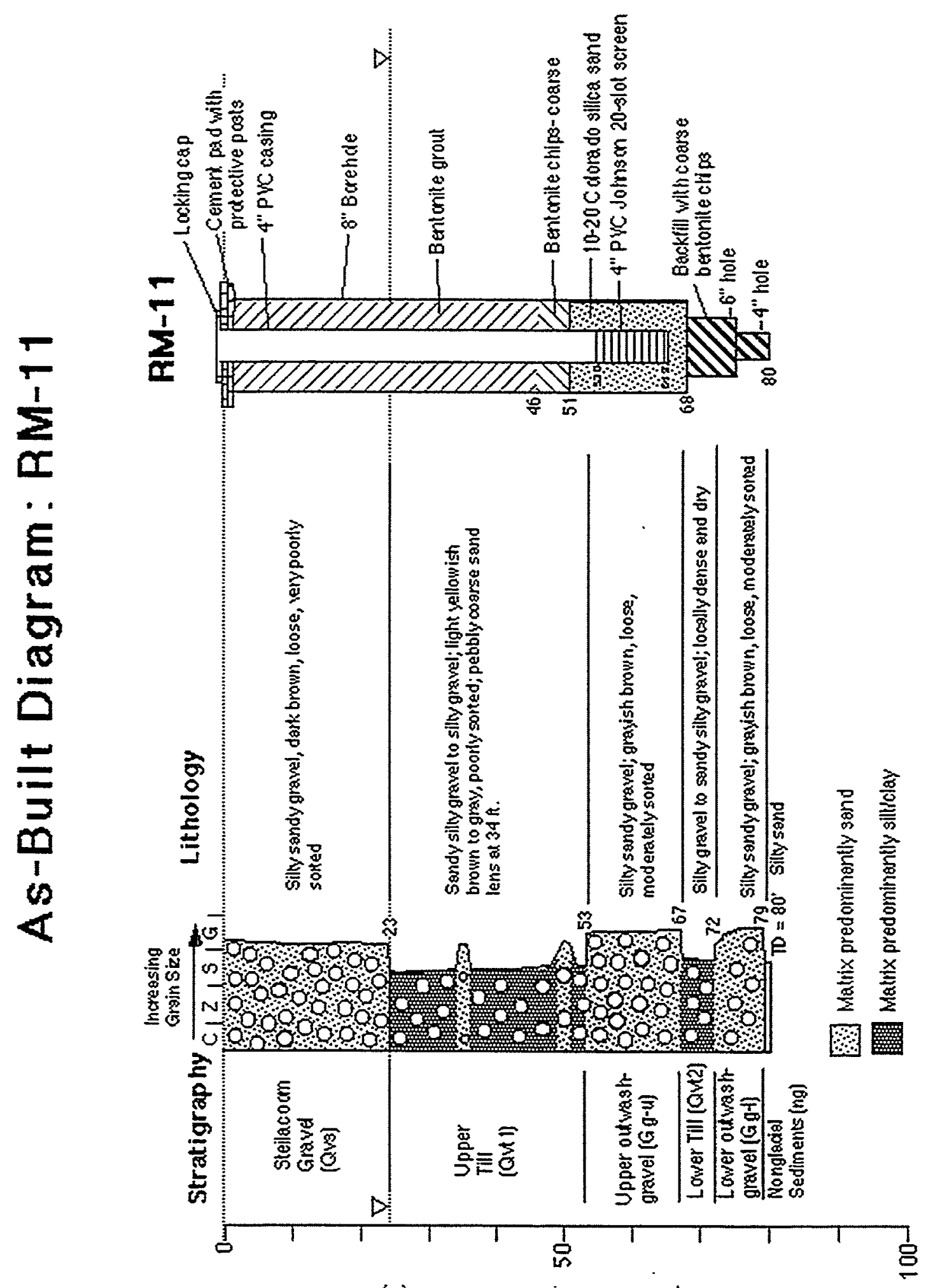

(ด) วre Ins punoin Moləa undan 


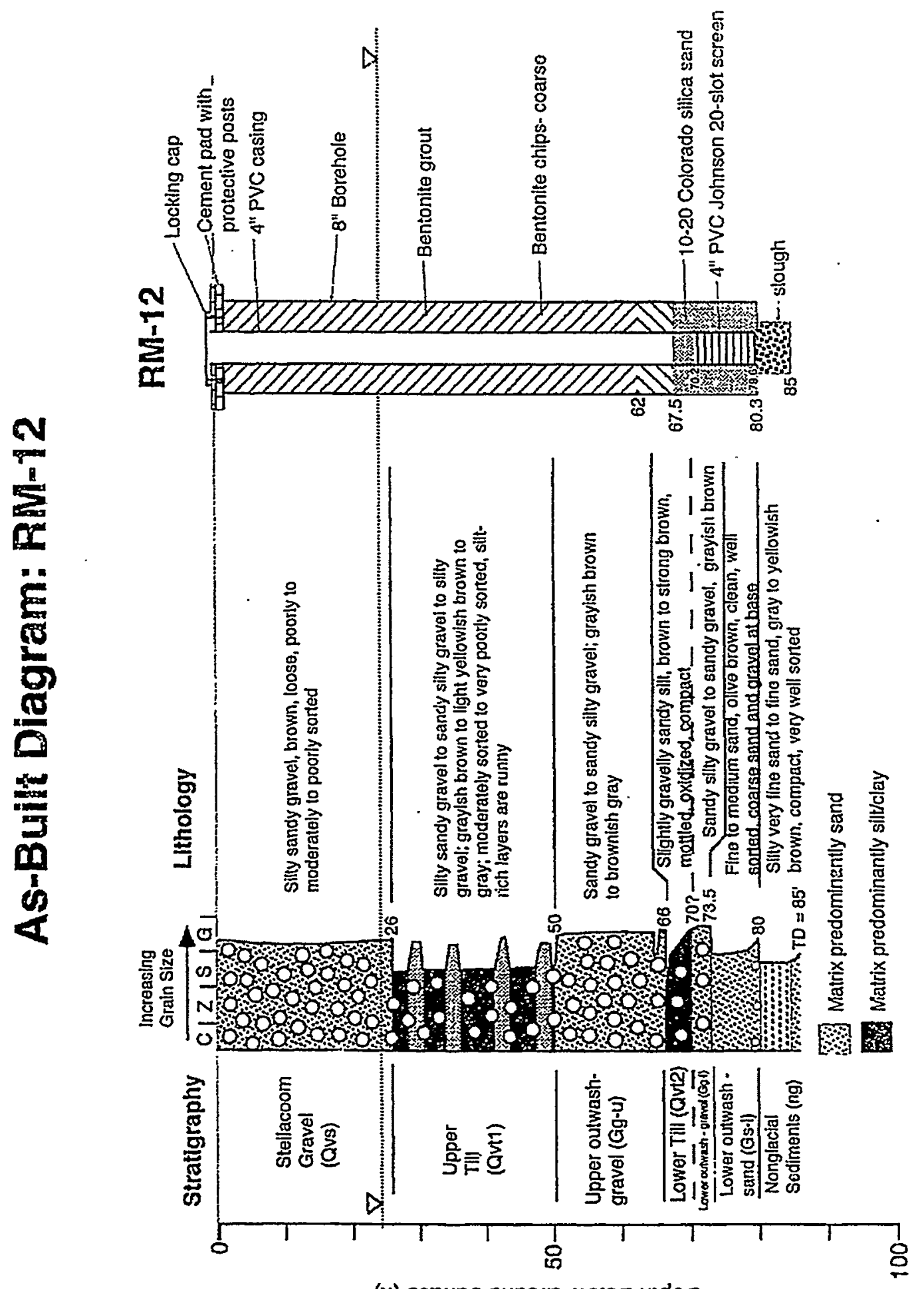

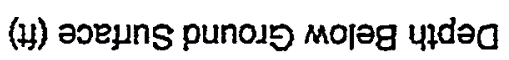




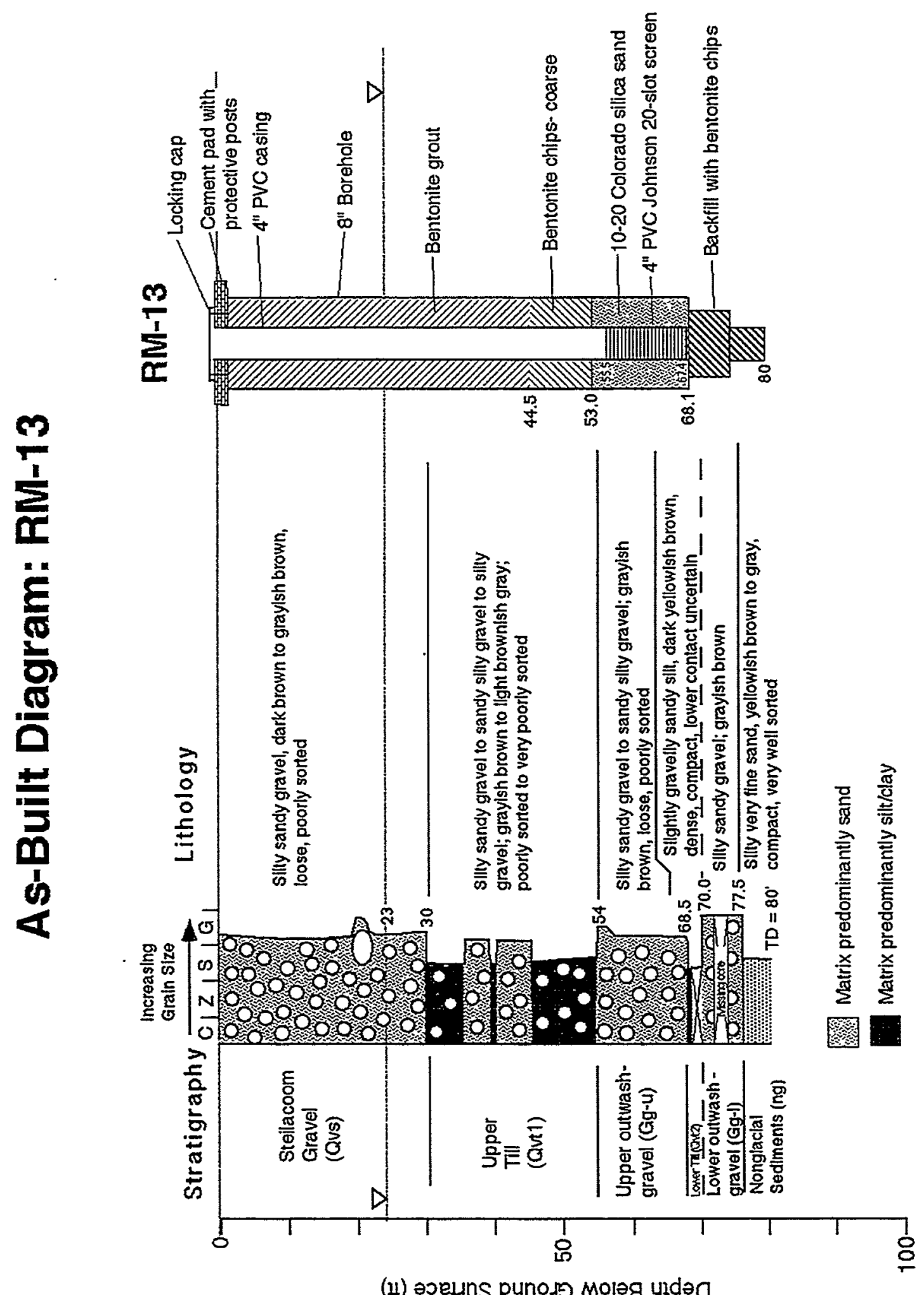

C. 46 


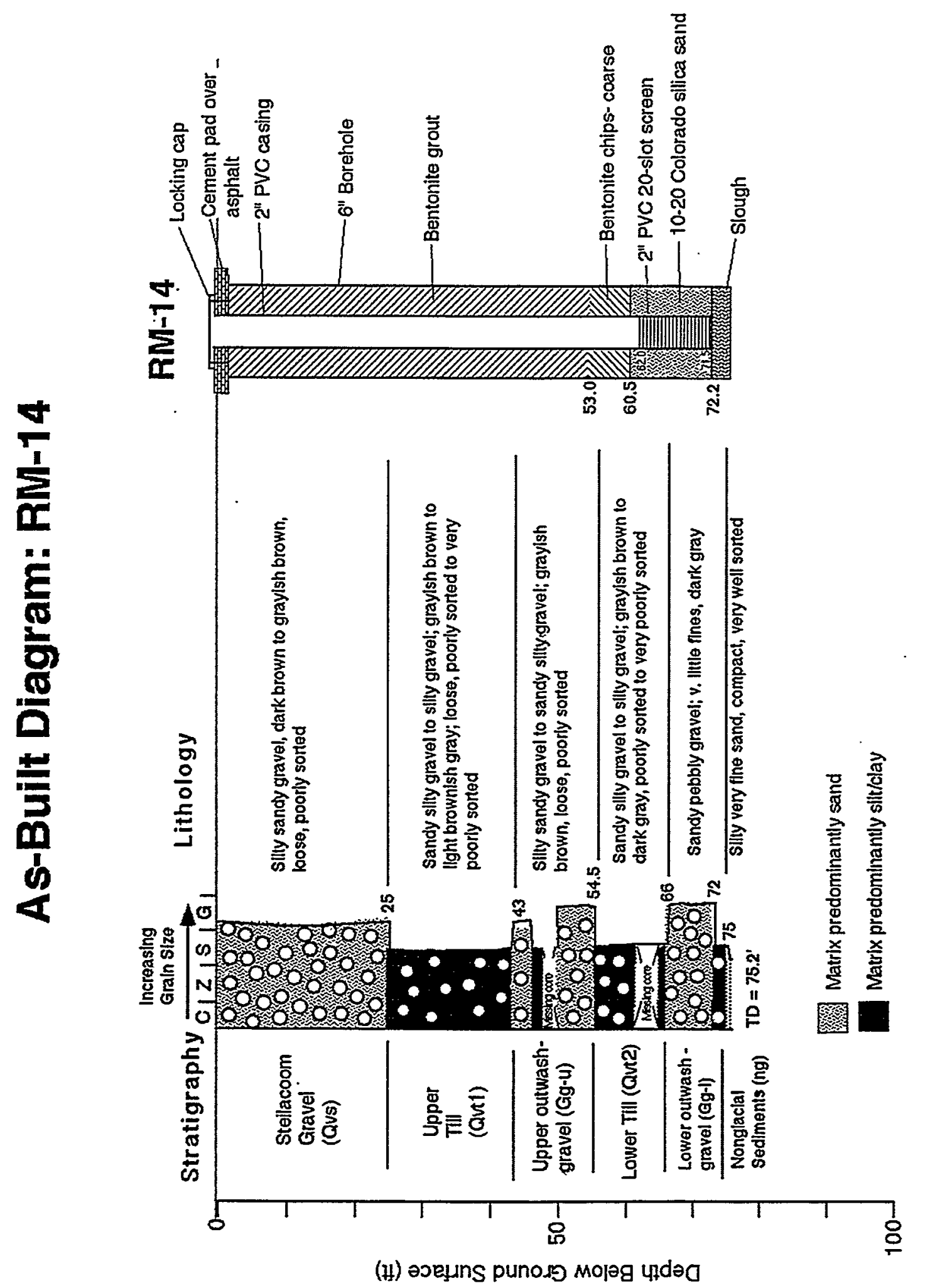




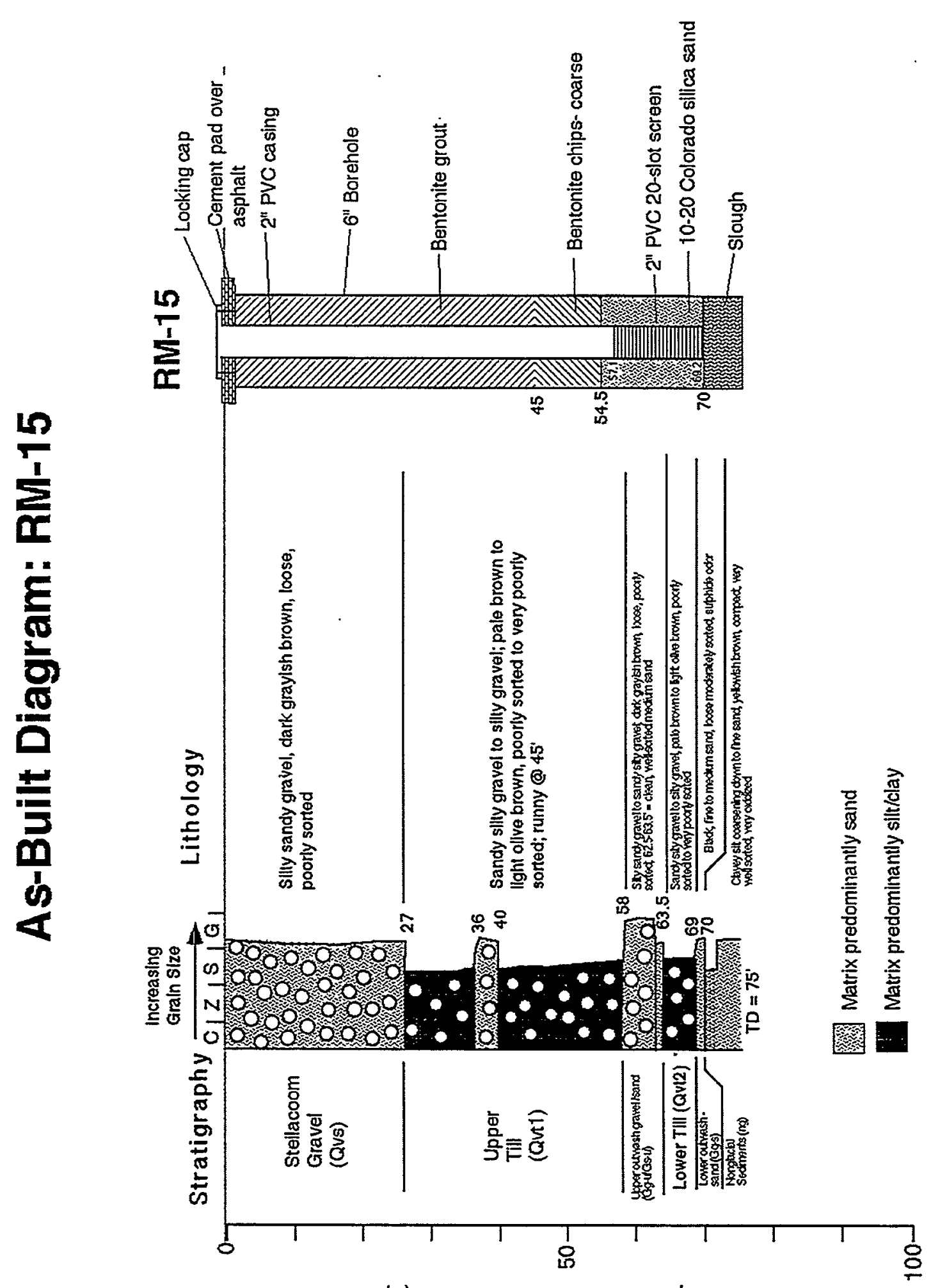

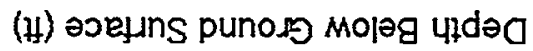




\section{Appendix D}

\section{Hydraulic Testing Results}




\section{Appendix D}

\section{Analytical Methods}

\section{Contents}

- Baseline Hydraulic Test Analyses

- Post-Injection Hydraulic Test Analyses

- Observed Pressure Responses in the Injection Well (RM-9) Throughout the Various Phases of Testing

- Observed Pressure Responses in the Injection Well (RM-9) Throughout the Various Phases of Testing

- Observed Pressure Responses in Monitoring Well RM-5 Throughout the Various Phases of Testing

- Observed Pressure Responses in the Monitoring Well RM-7 Throughout the Various Phases of Testing

- Observed Pressure Responses in the Monitoring Well RM-8 Throughout the Various Phases of Testing

D.1 
Baseline Hydraulic Test Analyses

D.3 
Pacific Northwest

National Laboratory

Operated by Battelle for the

US Department of Energy
Pumping test analysis

HANTUSH's method

Leaky aquifer, no aquitard storage
Date: $4 / 13 / 00$

Page 1

Project: Fort Lewis ISRM POPT

Evaluated by: VRV

Pumping Test No. Constant-Rate Discharge Test

Test conducted on: $9 / 20 / 98$

RM-5 Drawdown Data

Discharge 80.30 U.S.gal/min

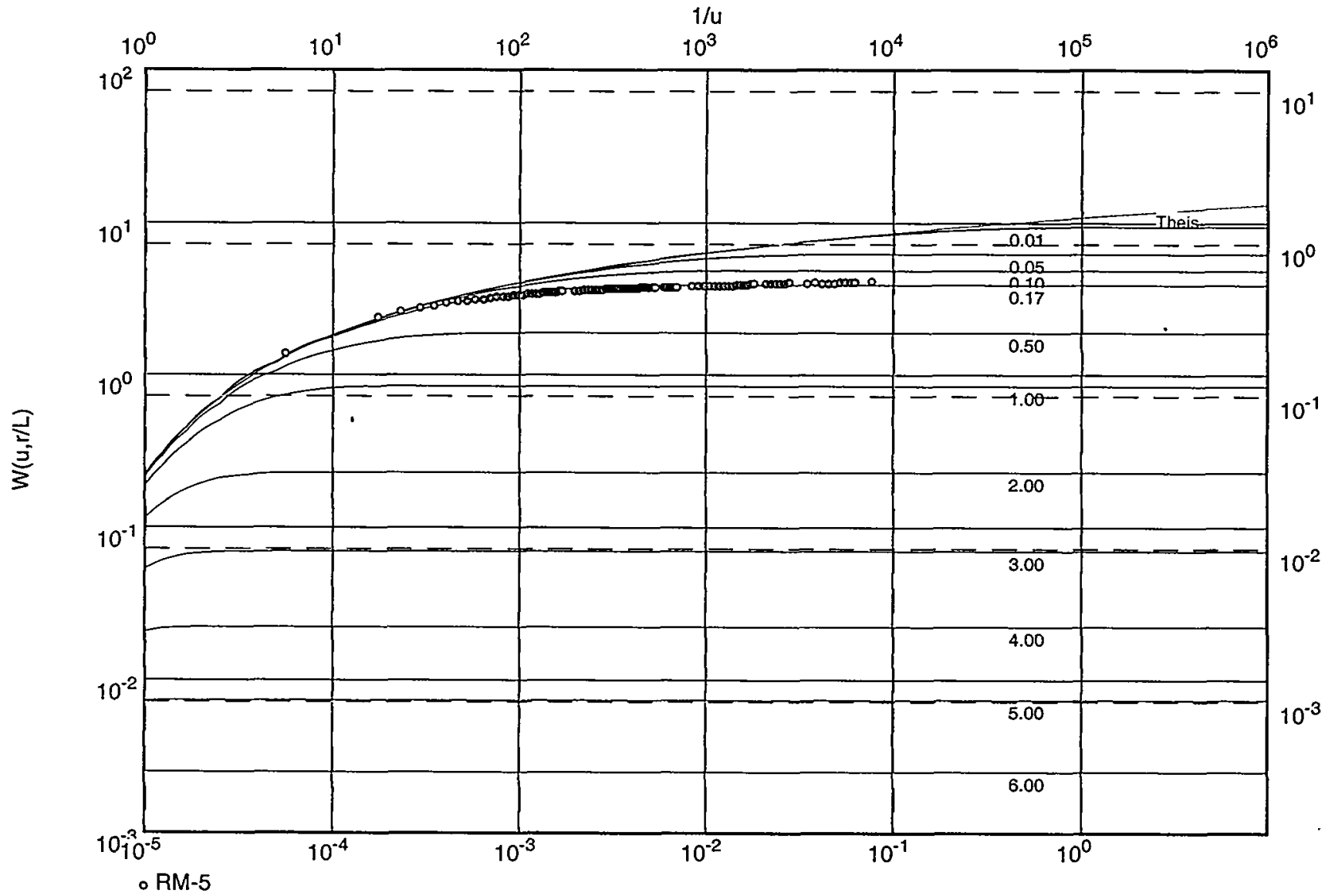

Transmissivity $\left[\mathrm{ft}^{2} / \mathrm{d}\right]: 8.90 \times 10^{3}$

Hydraulic conductivity [ft/d]: $7.42 \times 10^{2}$

Aquifer thickness [ft]: 12.00

Storativity: $1.14 \times 10^{-3}$

Hydraulic resistance (c) [d]: $1.18 \times 10^{0}$ 
Pacific Northwest

National Laboratory

Operated by Battelle for the

US Department of Energy
Pumping test analysis

HANTUSH's method

Leaky aquifer, no aquitard storage
Date: $4 / 13 / 00$

Page 1

Project: Fort Lewis ISRM POPT

Evaluated by: VRV

Pumping Test No. Constant-Rate Discharge Test

Test conducted on: 9/20/98

RM-5 Pressure Recovery Data

Discharge 80.30 U.S.gal/min

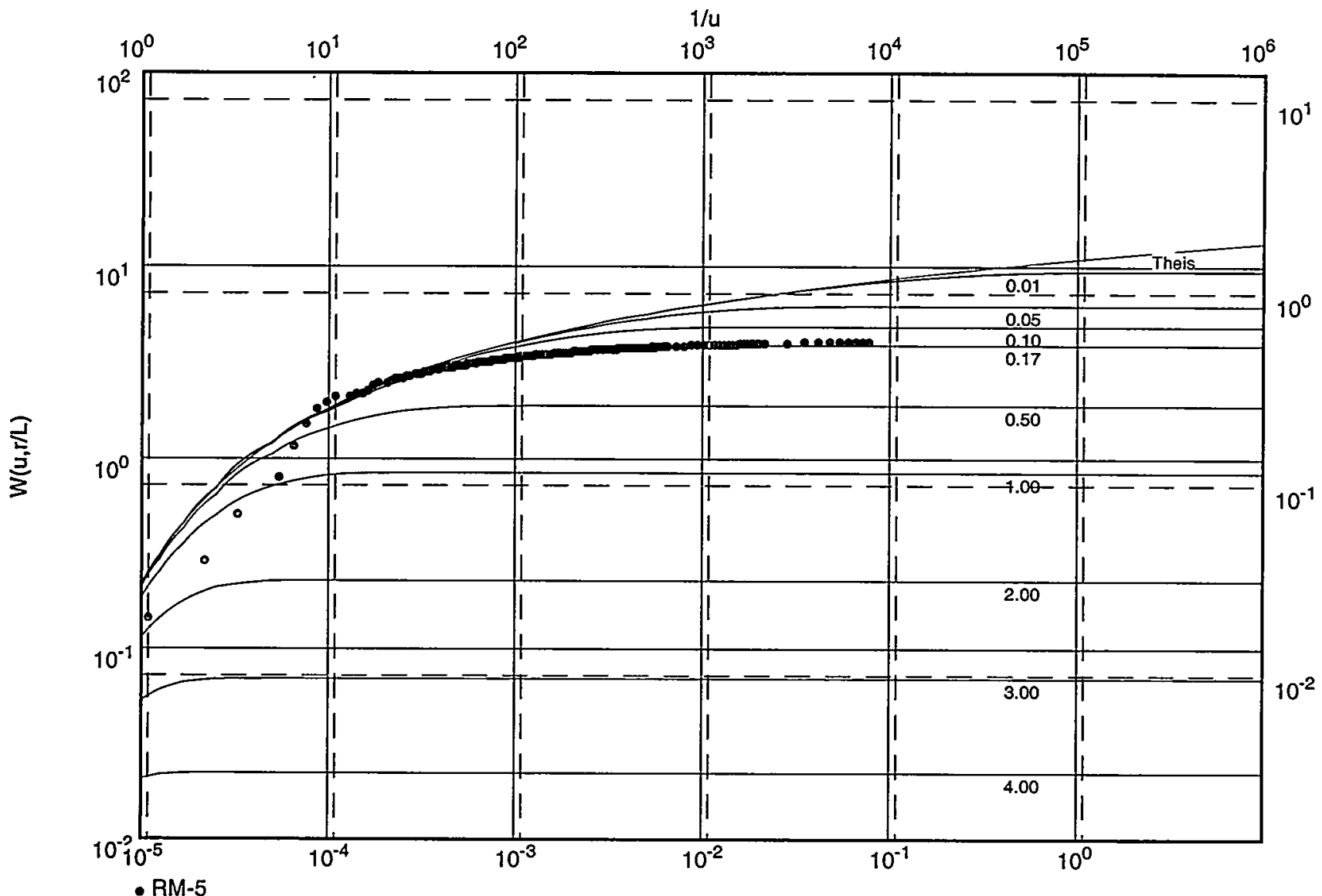

Transmissivity $\left[\mathrm{ft}^{2} / \mathrm{d}\right]: 8.90 \times 10^{3}$

Hydraulic conductivity [ft/d]: $7.42 \times 10^{2}$

Aquifer thickness [ft]: 12.00

Storativity: $1.06 \times 10^{-3}$

Hydraulic resistance (c) [d]: $1.19 \times 10^{0}$ 
Pacific Northwest

National Laboratory

Operated by Battelle for the

US Department of Energy
Pumping test analysis

HANTUSH's method

Leaky aquifer, no aquitard storage

\begin{tabular}{|l|l|}
\hline Pumping Test No. Constant-Rate Discharge Test & Test conducted on: 9/20/98 \\
\hline RM-7 Drawdown Data & \\
\hline Discharge 80.30 U.S.gal/min &
\end{tabular}

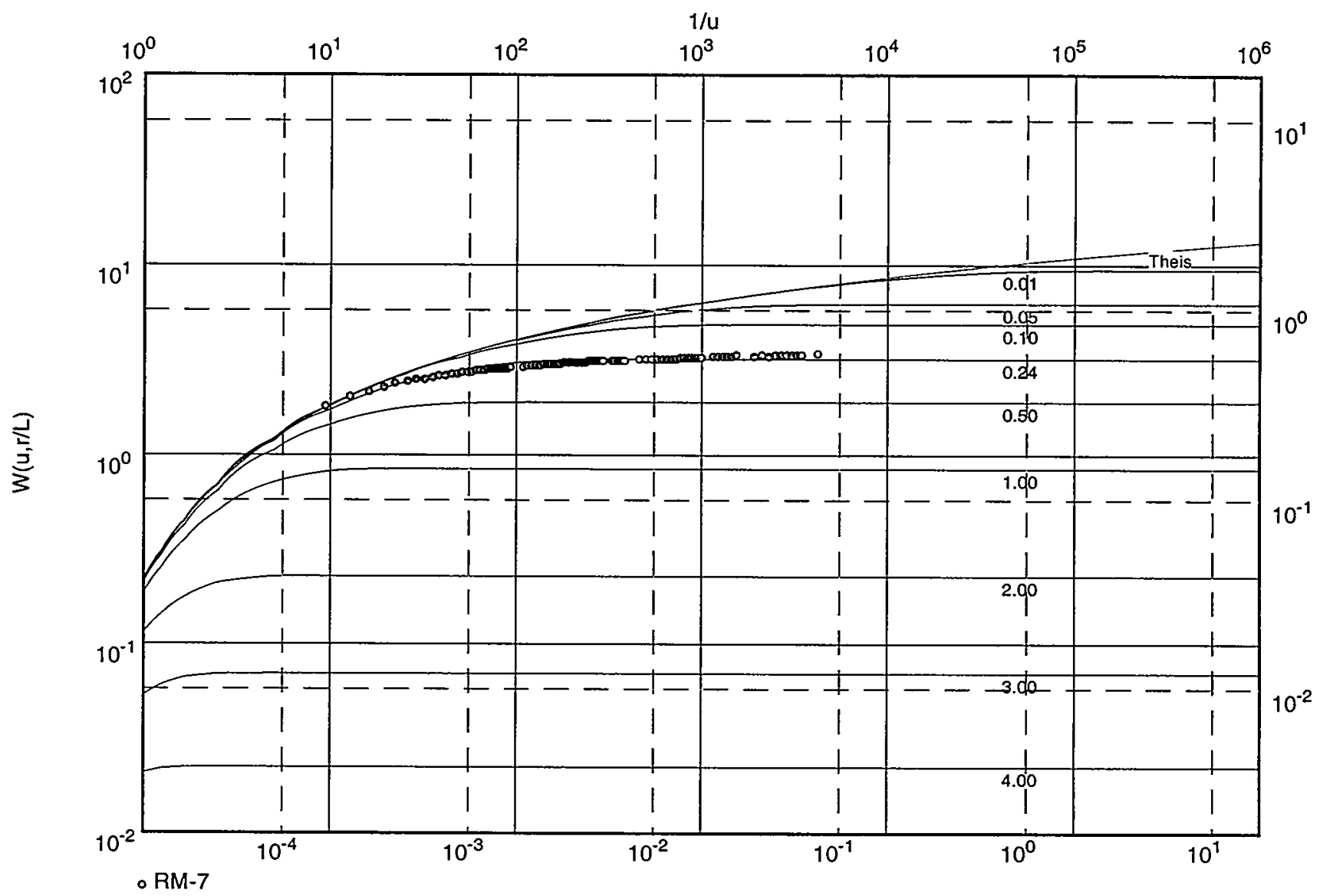

Transmissivity [ft²/d]: $7.10 \times 10^{3}$

Hydraulic conductivity [ft/d]: $5.92 \times 10^{2}$

Aquifer thickness [ft]: 12.00

Storativity: $8.73 \times 10^{-4}$

Hydraulic resistance (c) [d]: $1.43 \times 10^{0}$ 
Pacific Northwest

National Laboratory

Operated by Battelle for the

US Department of Energy
Pumping test analysis

HANTUSH's method

Leaky aquifer, no aquitard storage

Evaluated by: VRV

\begin{tabular}{|l|l}
\hline Pumping Test No. Constant-Rate Discharge Test & Test conducted on: 9/20/98 \\
\hline RM-7 Pressure Recovery Data & \\
\hline Discharge 80.30 U.S.gal/min & \\
\hline
\end{tabular}

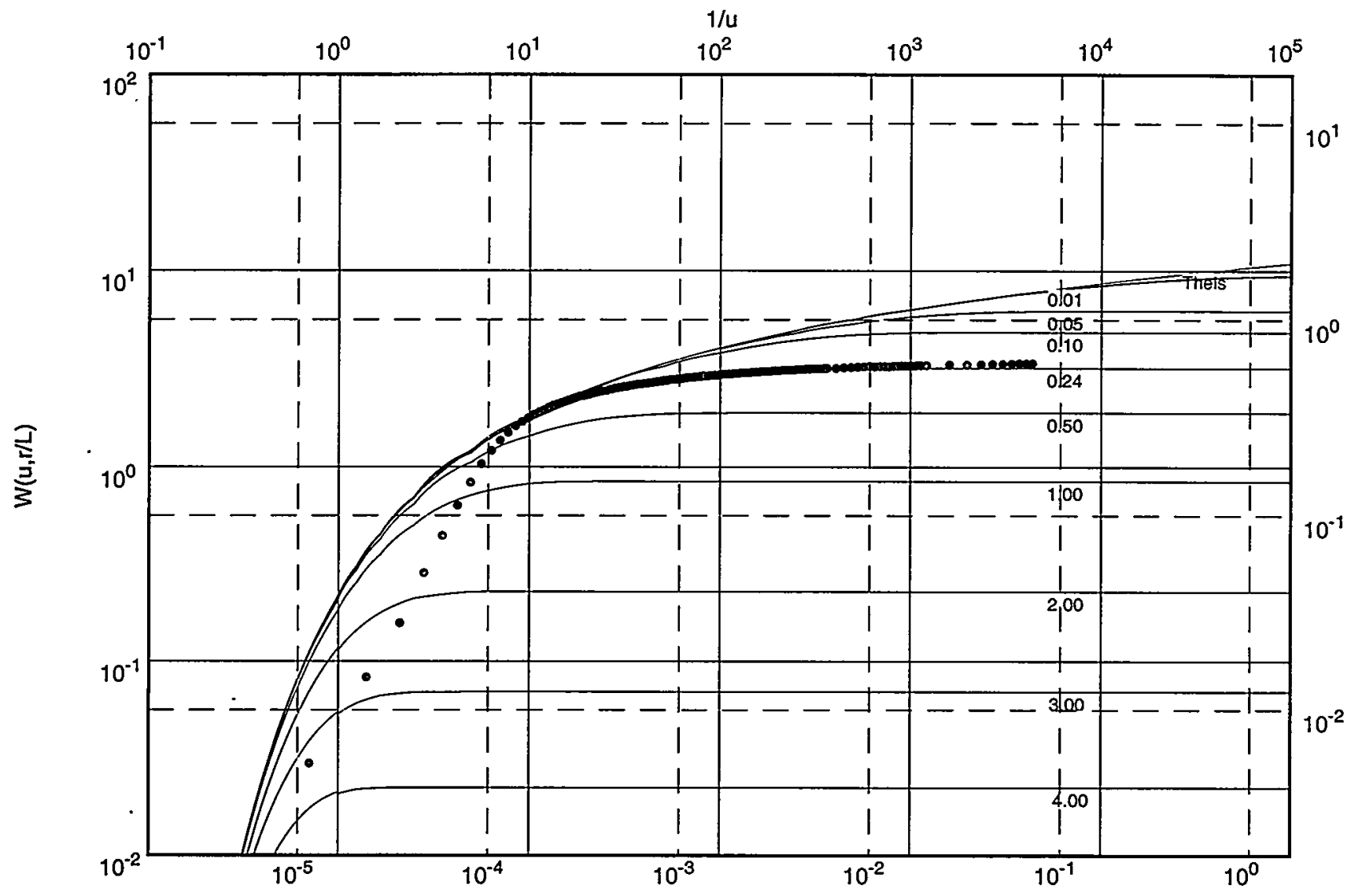

- RM-7

Transmissivity [ft²/d]: $6.90 \times 10^{3}$

Hydraulic conductivity [ft/d]: $5.75 \times 10^{2}$

Aquifer thickness [ft]: 12.00

Storativity: $7.71 \times 10^{-4}$

Hydraulic resistance (c) [d]: $1.47 \times 10^{\circ}$ 
Pacific Northwest

National Laboratory

Operated by Battelle for the

US Department of Energy
Pumping test analysis

HANTUSH's method

Leaky aquifer, no aquitard storage
Date: $4 / 13 / 00$

Page 1

Project: Fort Lewis ISRM POPT

Evaluated by: VRV

Pumping Test No. Constant-Rate Discharge Test

Test conducted on: 9/20/98

RM-8 Drawdown Data

Discharge 80.30 U.S.gal/min

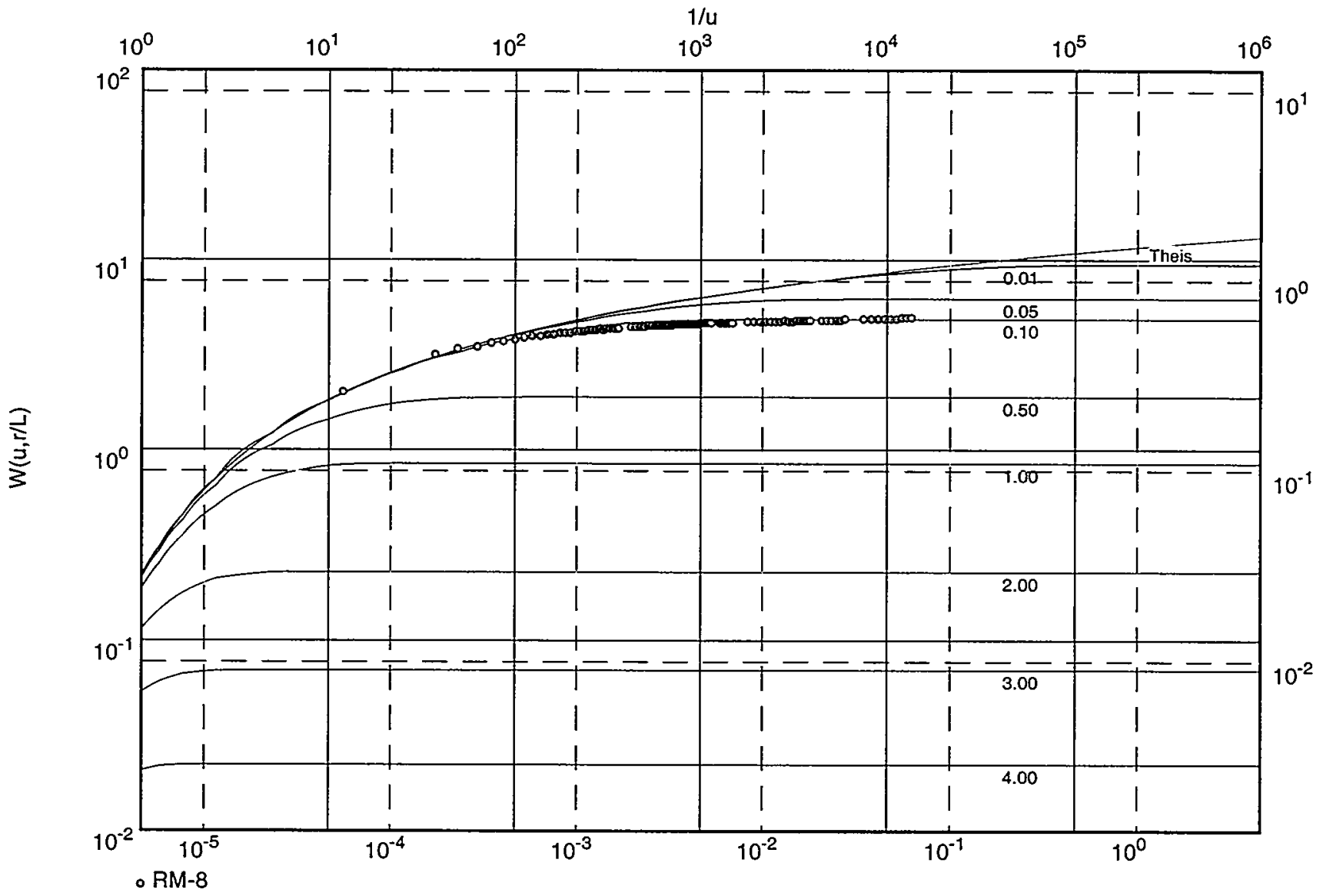

Transmissivity $\left[\mathrm{ft}^{2} / \mathrm{d}\right]: 9.50 \times 10^{3}$

Hydraulic conductivity [tt/d]: $7.91 \times 10^{2}$

Aquifer thickness [ft]: 12.00

Storativity: $1.69 \times 10^{-3}$

Hydraulic resistance (c) [d]: $1.09 \times 10^{0}$ 
Pacific Northwest

National Laboratory

Operated by Battelle for the

US Department of Energy
Pumping test analysis

HANTUSH's method

Leaky aquifer, no aquitard storage

Pumping Test No. Constant-Rate Discharge Test Test conducted on: 9/20/98

RM-8 Pressure Recovery Data

Discharge 80.30 U.S.gal/min

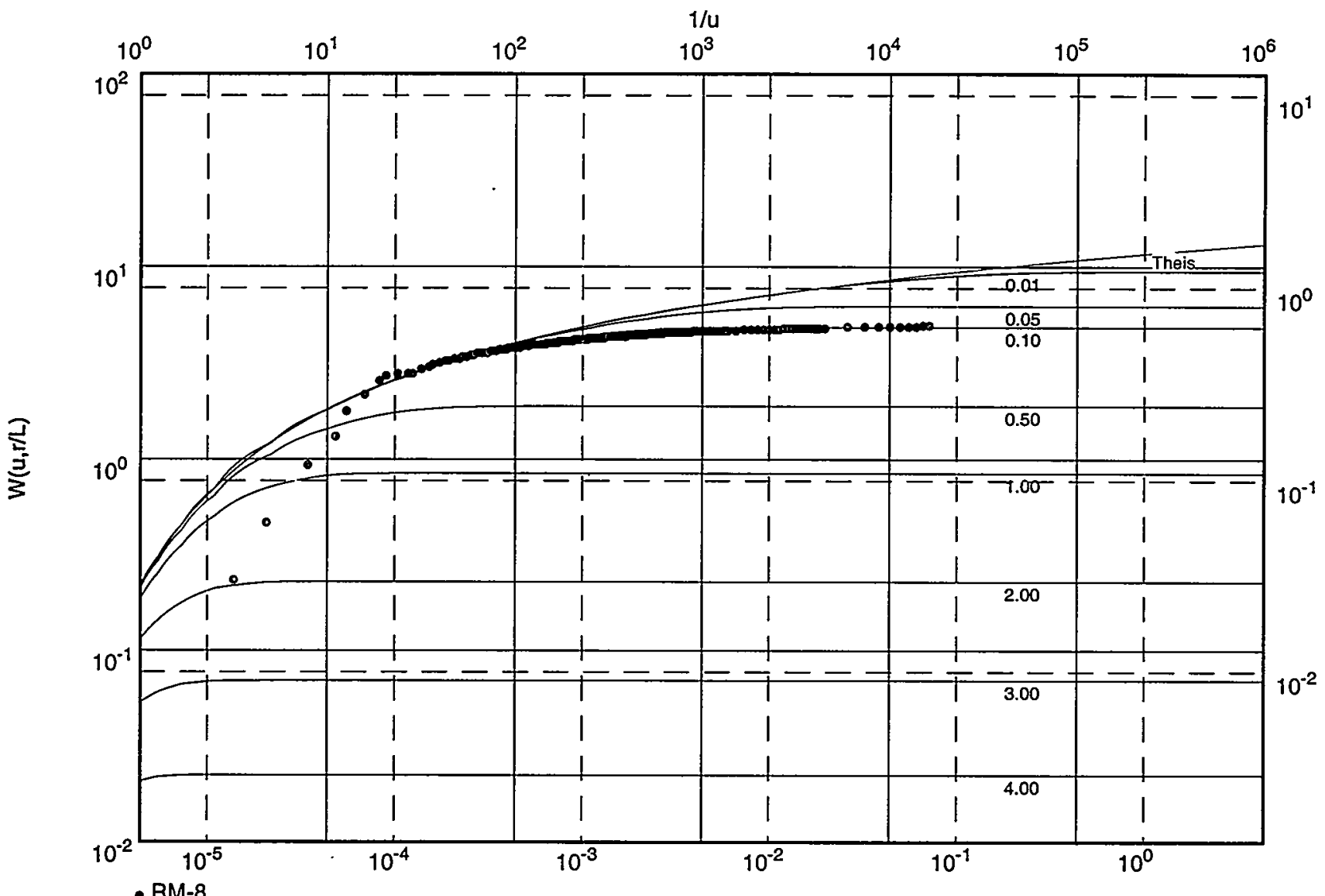

Transmissivity $[\mathrm{ft} / \mathrm{d}]: 9.50 \times 10^{3}$

Hydraulic conductivity [ft/d]: $7.91 \times 10^{2}$

Aquifer thickness [ft]: 12.00

Storativity: $1.59 \times 10^{-3}$

Hydraulic resistance (c) [d]: $1.09 \times 10^{0}$ 


\begin{tabular}{|c|c|c|c|c|}
\hline \multirow{3}{*}{$\begin{array}{l}\text { Pacific Northwest } \\
\text { National Laboratory } \\
\text { Operated by Battelle for the } \\
\text { US Department of Energy }\end{array}$} & \multirow{3}{*}{\multicolumn{2}{|c|}{$\begin{array}{l}\text { Pumping test analysis } \\
\text { HANTUSH's method } \\
\text { Leaky aquifer, no aquitard storage }\end{array}$}} & Date: $4 / 13 / 00$ & Page 1 \\
\hline & & & \multicolumn{2}{|c|}{ Project: Fort Lewis ISRM POPT } \\
\hline & & & \multicolumn{2}{|c|}{ Evaluated by: VRV } \\
\hline \multicolumn{2}{|c|}{ Pumping Test No. Constant-Rate Discharge Test } & \multicolumn{3}{|c|}{ Test conducted on: $9 / 20 / 98$} \\
\hline \multicolumn{5}{|l|}{ RM-1a Drawdown Data } \\
\hline \multicolumn{2}{|c|}{ Discharge 80.30 U.S.gal/min } & & & \\
\hline
\end{tabular}

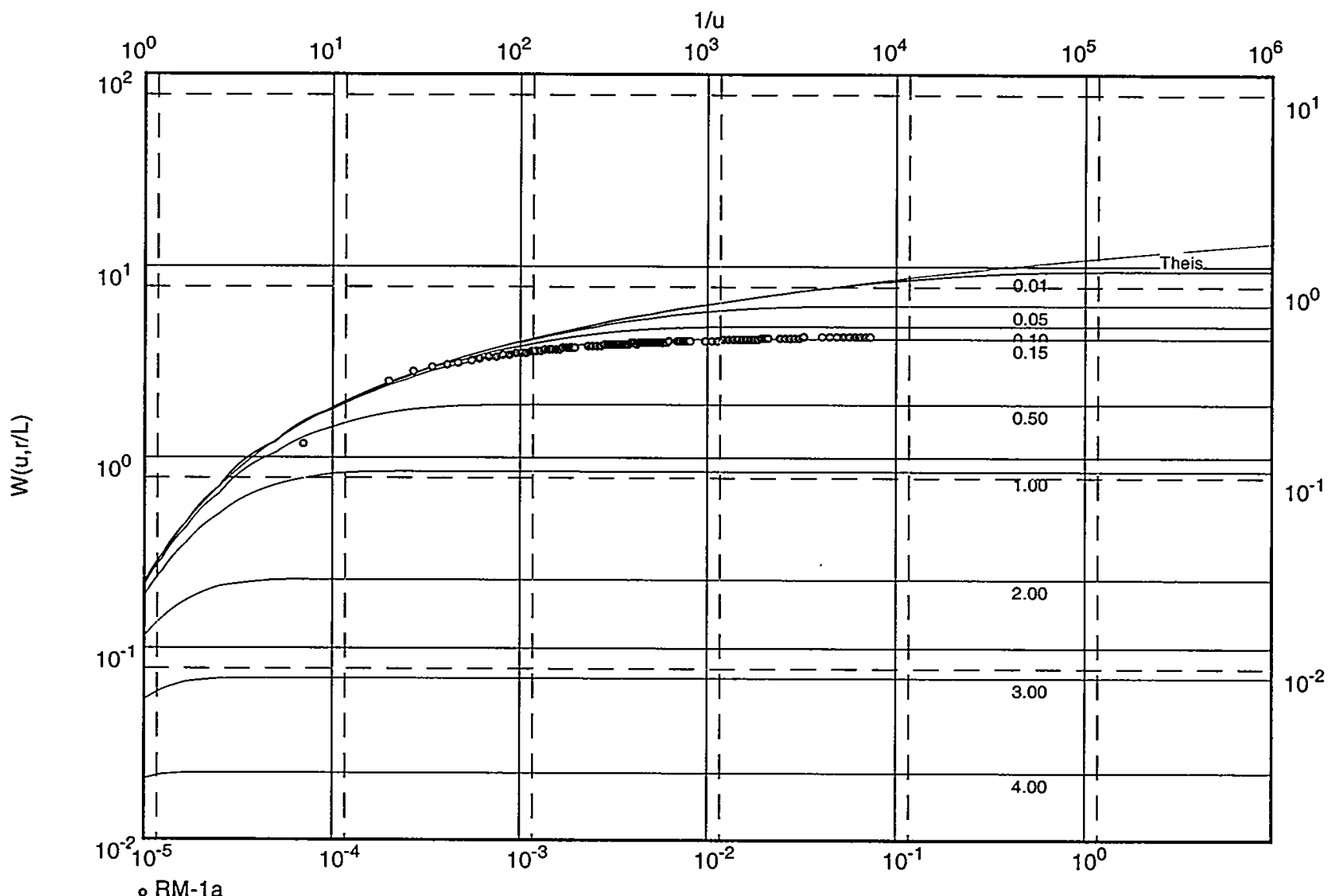

Transmissivity $[\mathrm{ft} 2 / \mathrm{d}]: 9.60 \times 10^{3}$

Hydraulic conductivity [ft/d]: $8.00 \times 10^{2}$

Aquifer thickness [ft]: 12.00

Storativity: $1.49 \times 10^{-3}$

Hydraulic resistance (c) [d]: $1.01 \times 10^{0}$ 
Pacific Northwest

National Laboratory

Operated by Battelle for the

US Department of Energy
Pumping test analysis

HANTUSH's method

Leaky aquifer, no aquitard storage

Project: Fort Lewis ISRM POPT

Evaluated by: VRV

Pumping Test No. Constant-Rate Discharge Test

Test conducted on: $9 / 20 / 98$

RM-1a Pressure Recovery Data

Discharge 80.30 U.S.gal/min

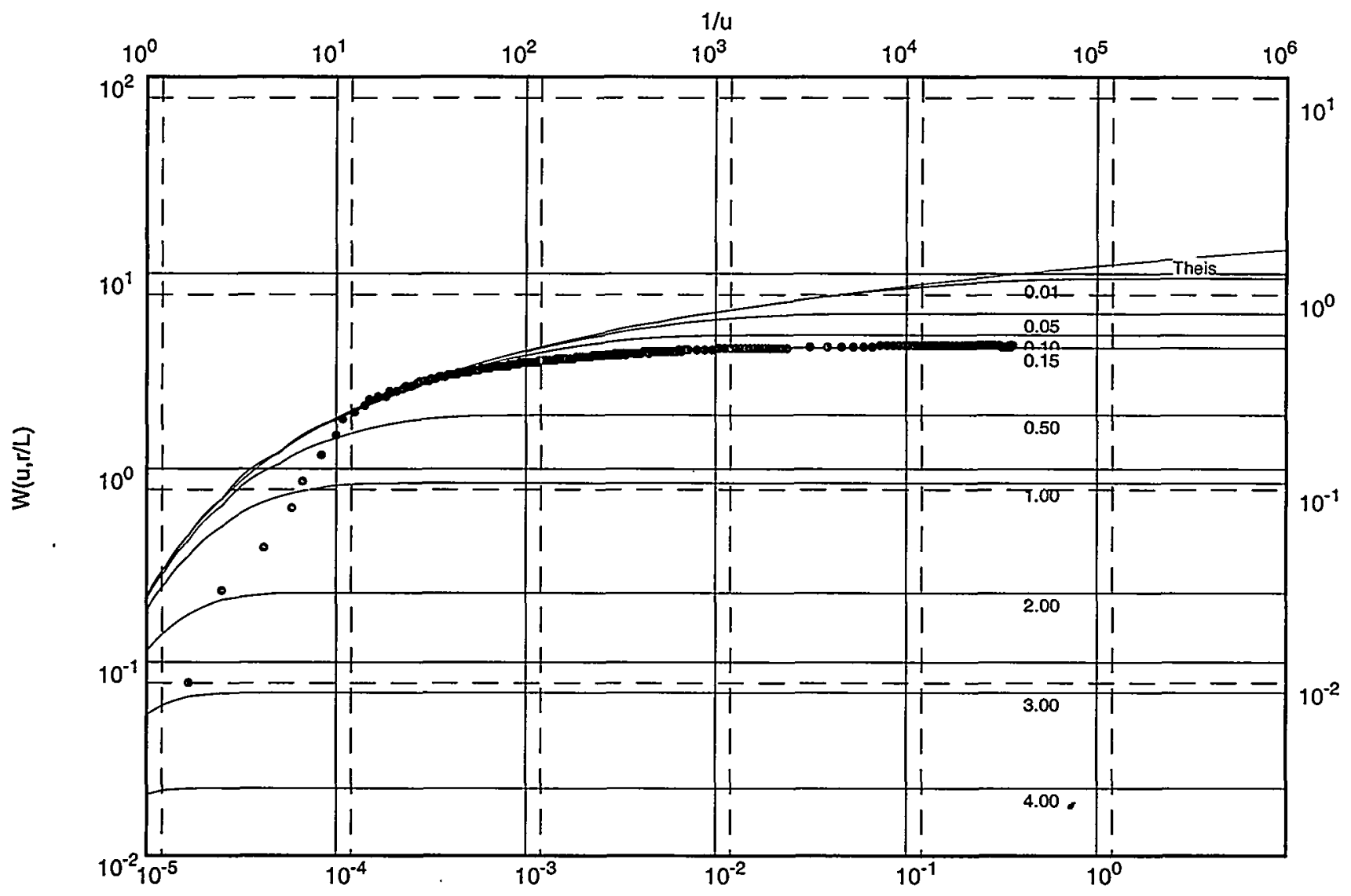

- $\mathrm{RM}-1 \mathrm{a}$

Transmissivity [ft/2/d]: $9.60 \times 10^{3}$

Hydraulic conductivity [ft/d]: $8.00 \times 10^{2}$

Aquifer thickness [ft]: 12.00

Storativity: $1.45 \times 10^{-3}$

Hydraulic resistance (c) [d]: $1.01 \times 10^{0}$ 


\section{Post-Injection Hydraulic Test Analyses}

D.13 
Pacific Northwest

National Laboratory

Operated by Battelle for the

US Department of Energy
Pumping test analysis

HANTUSH's method

Leaky aquifer, no aquitard storage
Date: $4 / 13 / 00$

Page 1

Project: Fort Lewis ISRM POPT

Evaluated by: VRV

Pumping Test No. Withdrawal Phase of Dithionite Inj \#4

Test conducted on: $3 / 27 / 00$

RM-5 Pressure Recovery Data

Discharge 109.80 U.S.gal/min

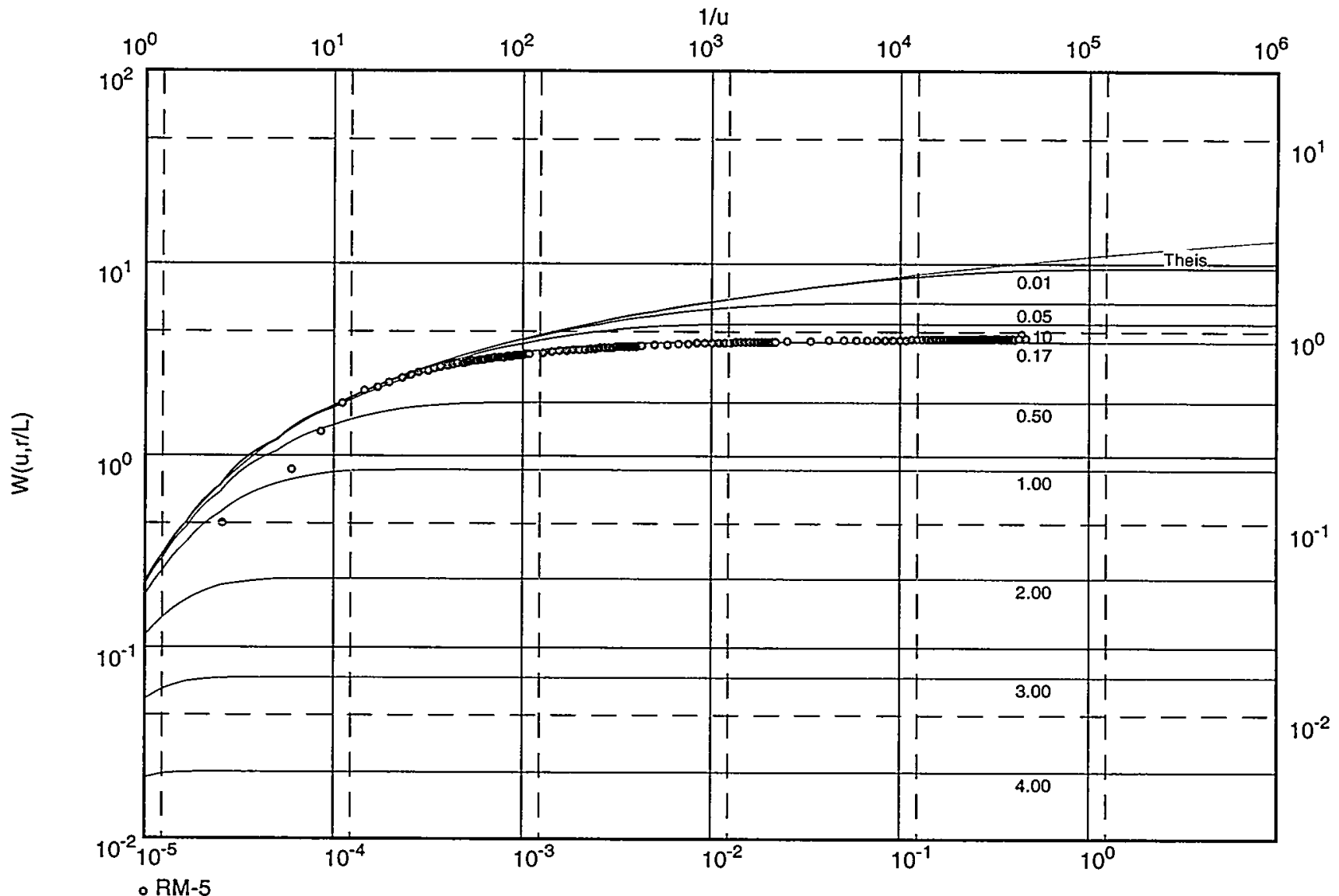

Transmissivity [ft2/d]: $7.45 \times 10^{3}$

Hydraulic conductivity [ft/d]: $6.20 \times 10^{2}$

Aquifer thickness [ft]: 12.00

Storativity: $7.87 \times 10^{-4}$

Hydraulic resistance (c) [d]: $1.42 \times 10^{\circ}$ 
Pacific Northwest

National Laboratory

Operated by Battelle for the

US Department of Energy
Pumping test analysis

HANTUSH's method

Leaky aquifer, no aquitard storage

Project: Fort Lewis ISRM POPT

Evaluated by: VRV

Pumping Test No. Withdrawal Phase of Dithionite Inj \#4

Test conducted on: $3 / 27 / 00$

RM-7 Pressure Recovery Data

Discharge 109.80 U.S.gal/min

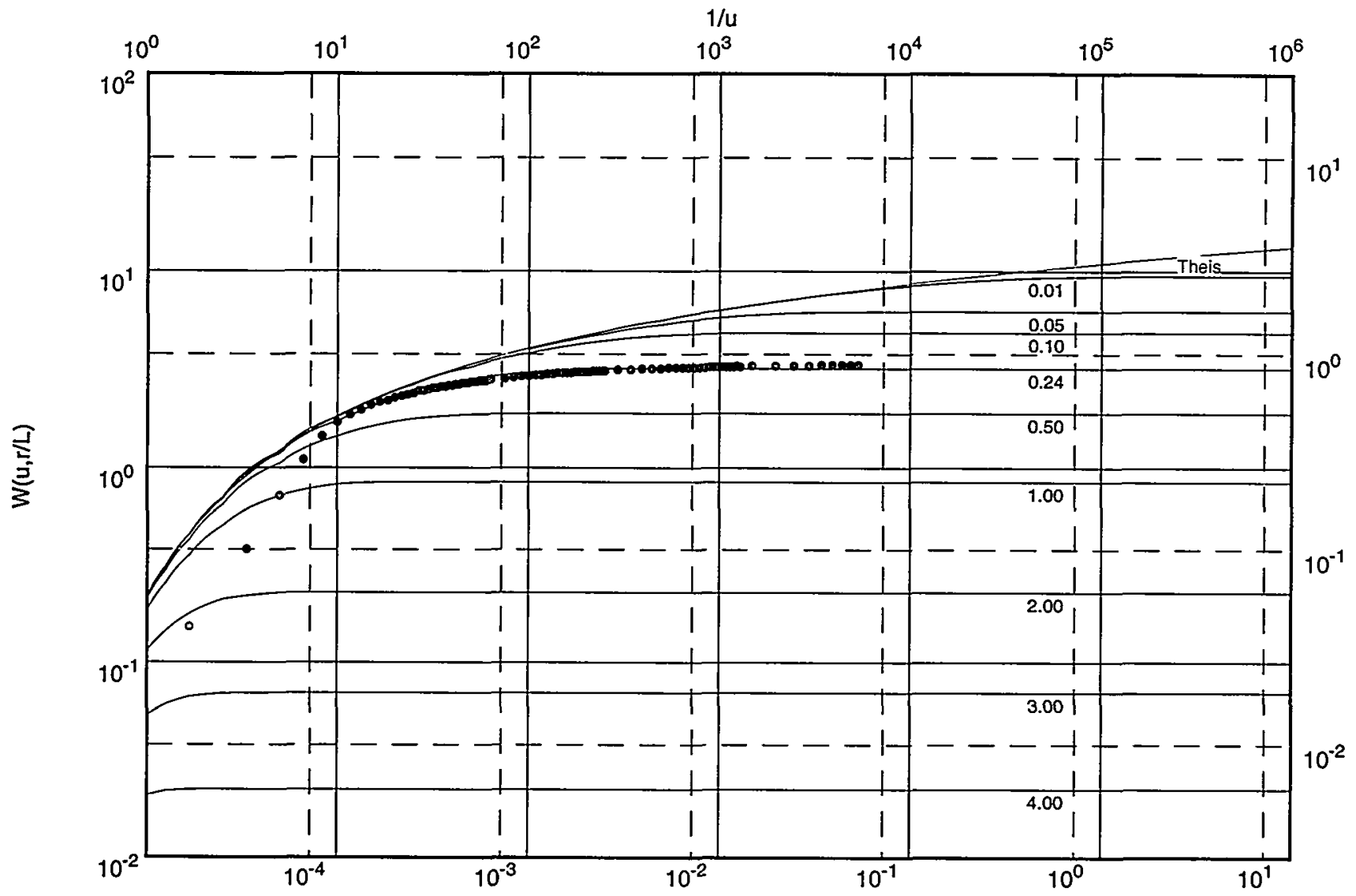

- $\mathrm{RM}-7$

Transmissivity [ft2/d]: $6.35 \times 10^{3}$

Hydraulic conductivity [ft/d]: $5.29 \times 10^{2}$

Aquifer thickness [ft]: 12.00

Storativity: $6.00 \times 10^{-4}$

Hydraulic resistance (c) [d]: $1.59 \times 10^{\circ}$

D. 15 


\begin{tabular}{|c|c|c|c|c|}
\hline \multirow{3}{*}{$\begin{array}{l}\text { Pacific Northwest } \\
\text { National Laboratory } \\
\text { Operated by Battelle for the } \\
\text { US Department of Energy }\end{array}$} & \multirow{3}{*}{\multicolumn{2}{|c|}{$\begin{array}{l}\text { Pumping test analysis } \\
\text { HANTUSH's method } \\
\text { Leaky aquifer, no aquitard storage }\end{array}$}} & Date: $4 / 13 / 00$ & Page 1 \\
\hline & & & \multicolumn{2}{|c|}{ Project: Fort Lewis ISRM POPT } \\
\hline & & & \multicolumn{2}{|c|}{ Evaluated by: VRV } \\
\hline \multicolumn{2}{|c|}{ Pumping Test No. Withdrawal Phase of Dithionite Inj \#4 } & \multicolumn{3}{|c|}{ Test conducted on: $3 / 27 / 00$} \\
\hline \multicolumn{2}{|l|}{ RM-8 Pressure Recovery Data } & & & \\
\hline Discharge 109.80 U.S.gal $/ \mathrm{min}$ & & & & \\
\hline
\end{tabular}

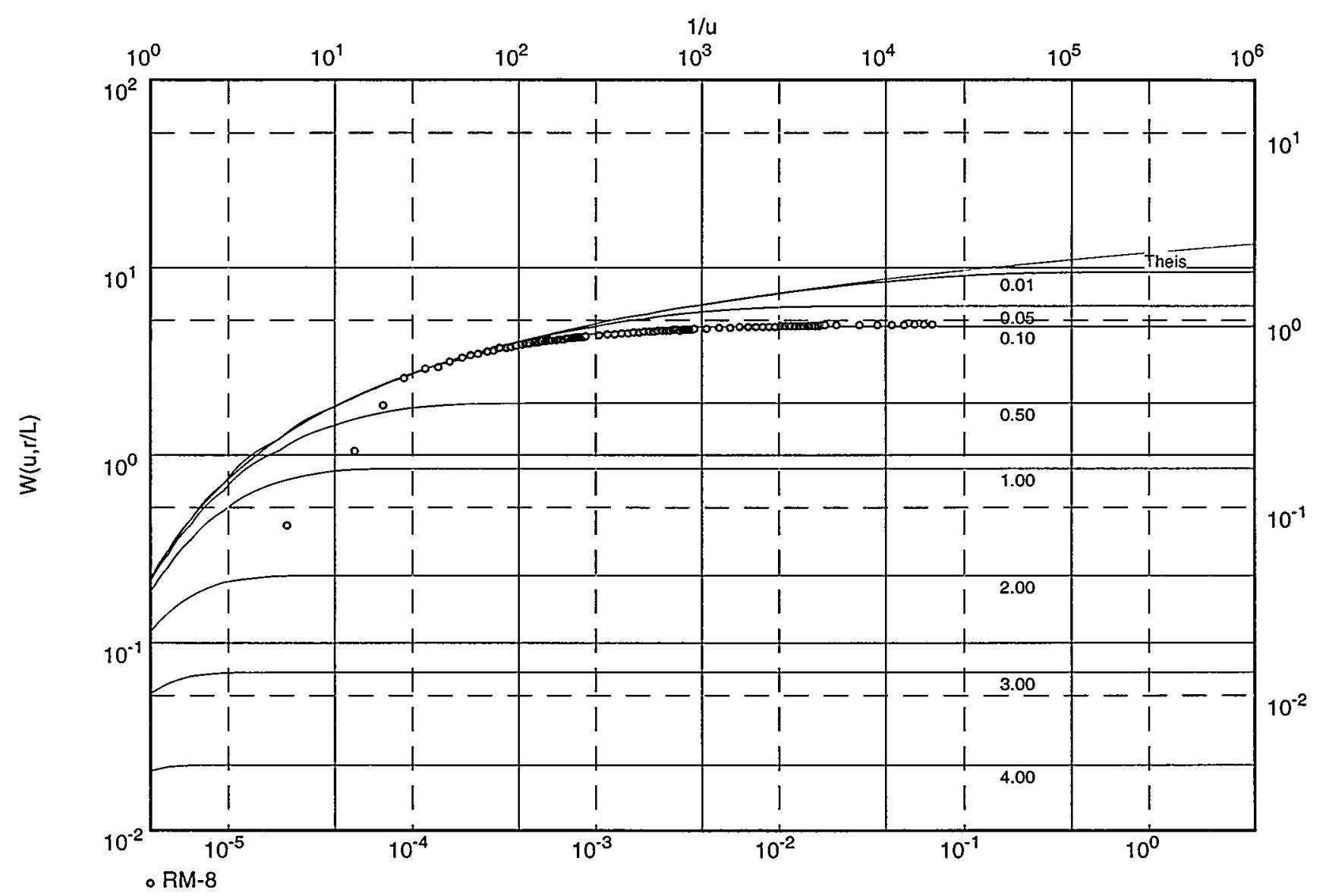

Transmissivity $\left[\mathrm{ft}^{2} / \mathrm{d}\right]: 8.80 \times 10^{3}$

Hydraulic conductivity [ft/d]: $7.33 \times 10^{2}$

Aquifer thickness [ft]: 12.00

Storativity: $1.28 \times 10^{-3}$

Hydraulic resistance (c) [d]: $1.18 \times 10^{\circ}$ 
Observed Pressure Responses in the Injection Well (RM-9) Throughout the Various Phases of Testing 


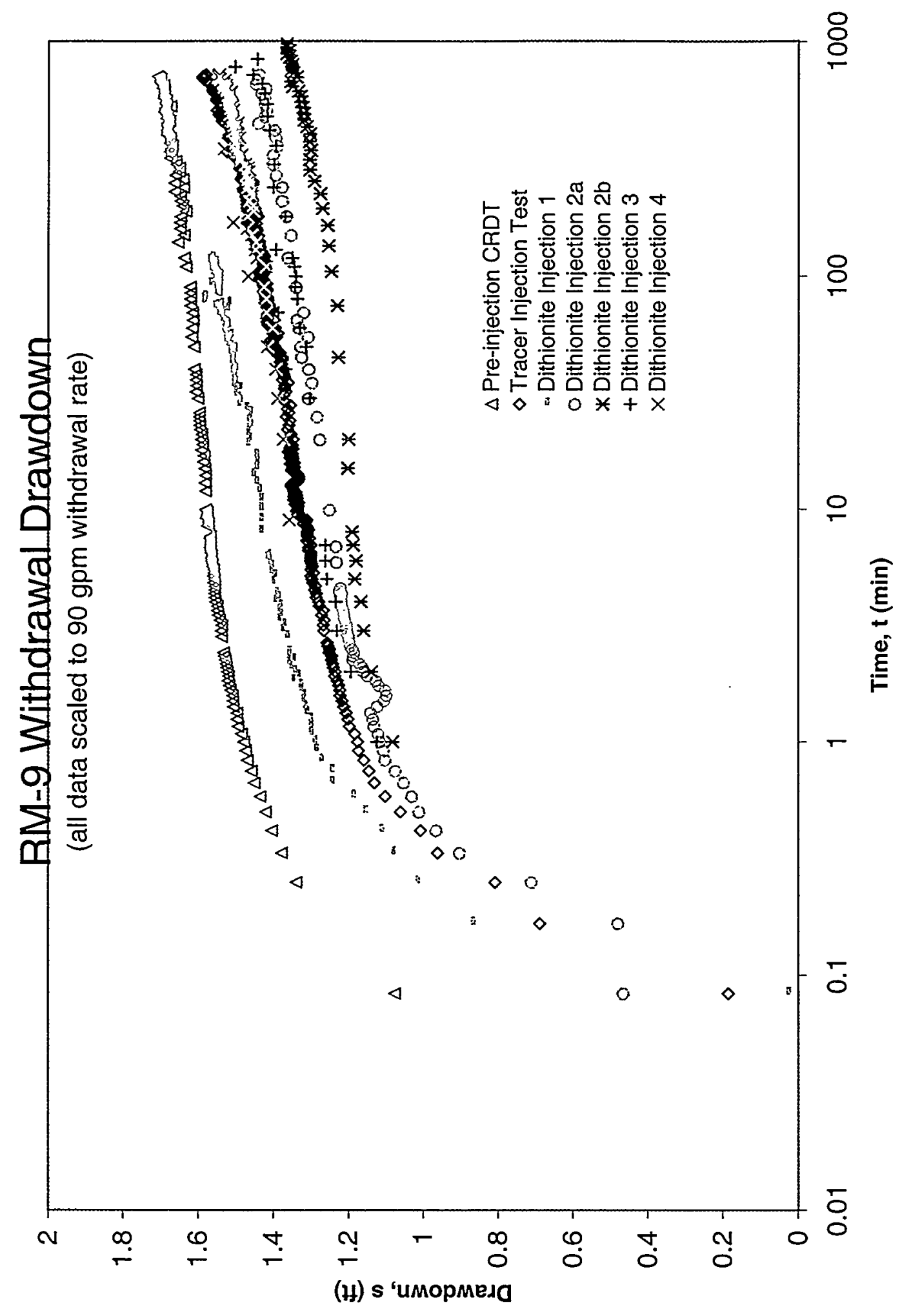

D. 18 


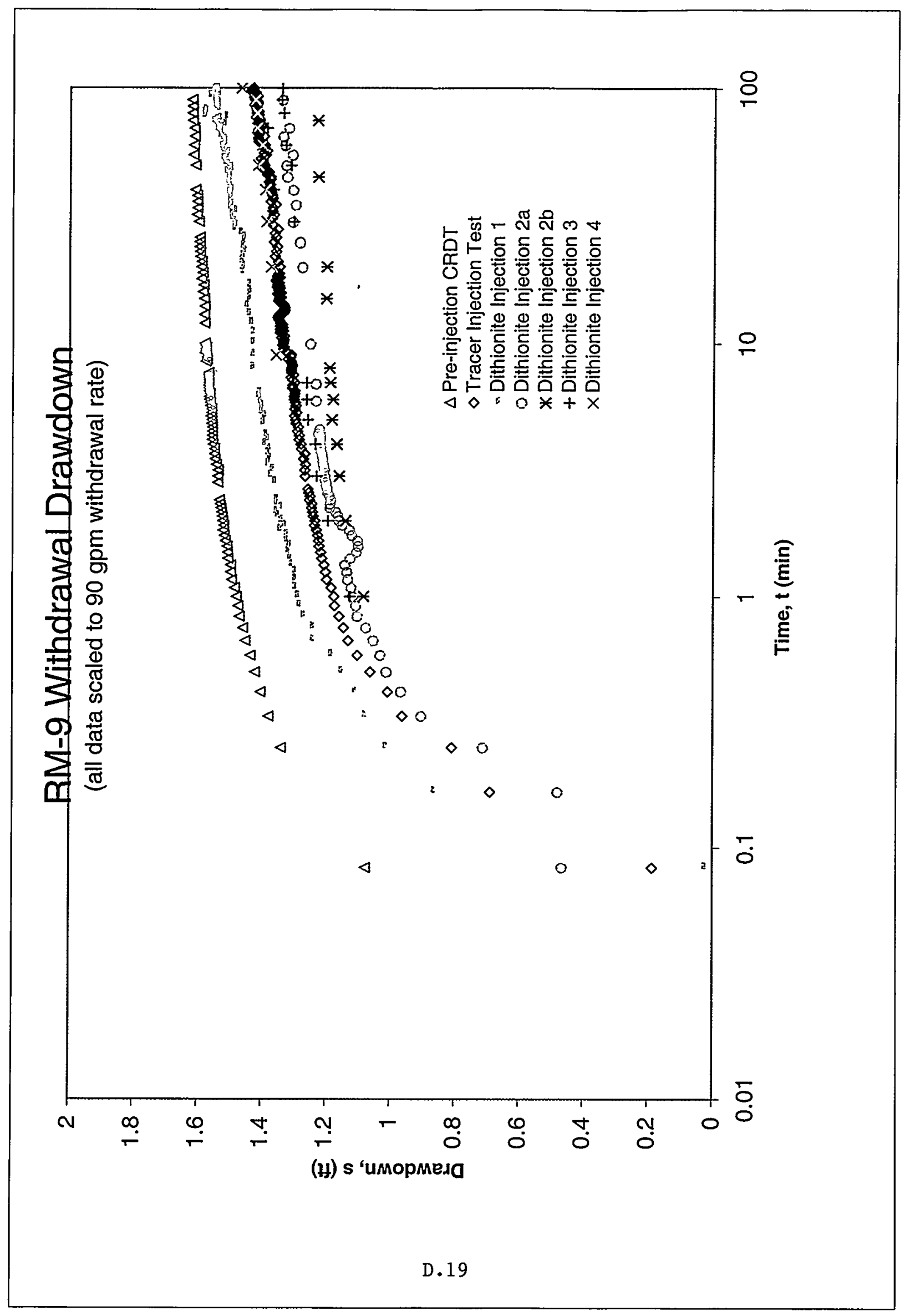




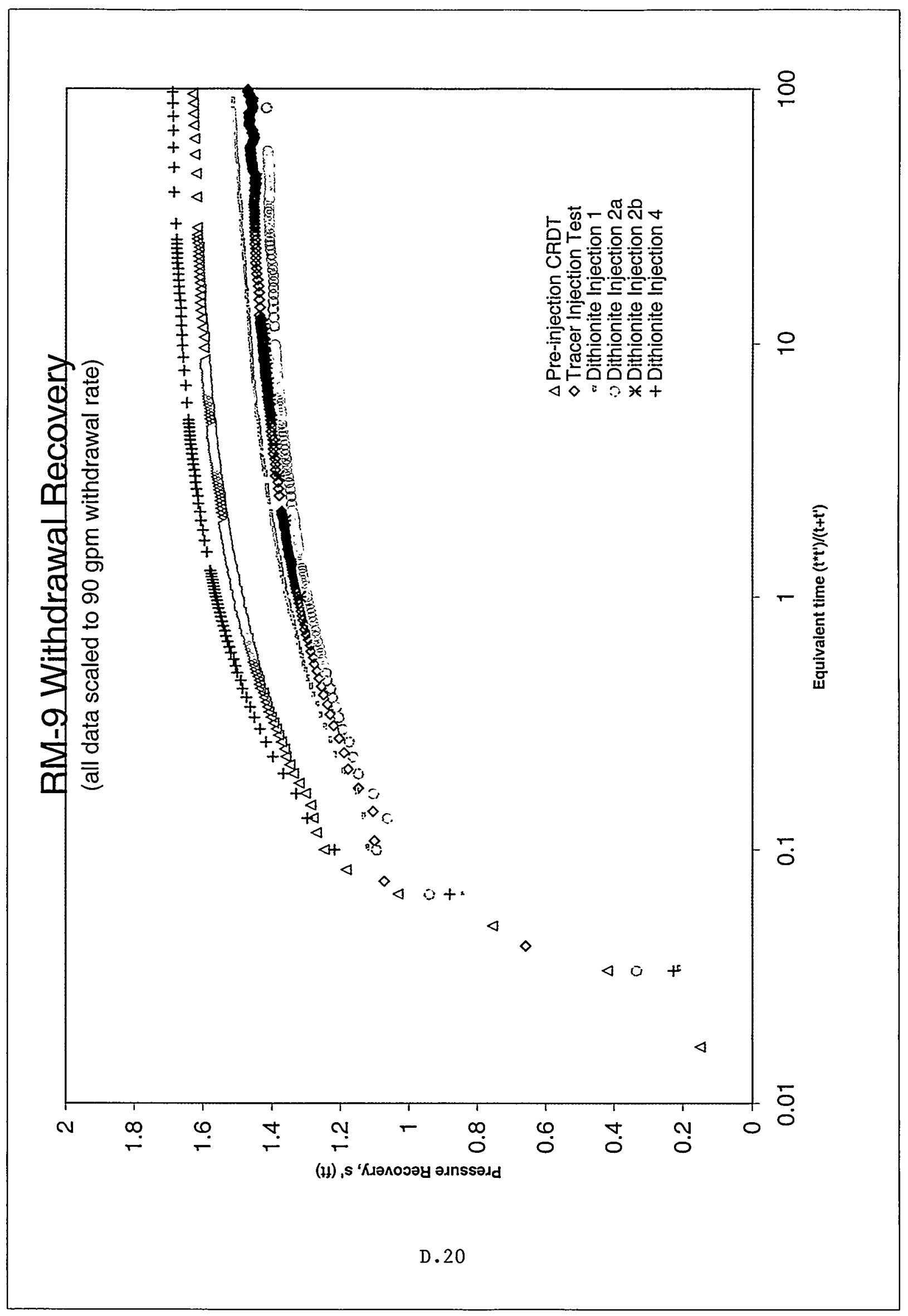


Observed Pressure Responses in Monitoring Well RM-5 Throughout the Various Phases of Testing 


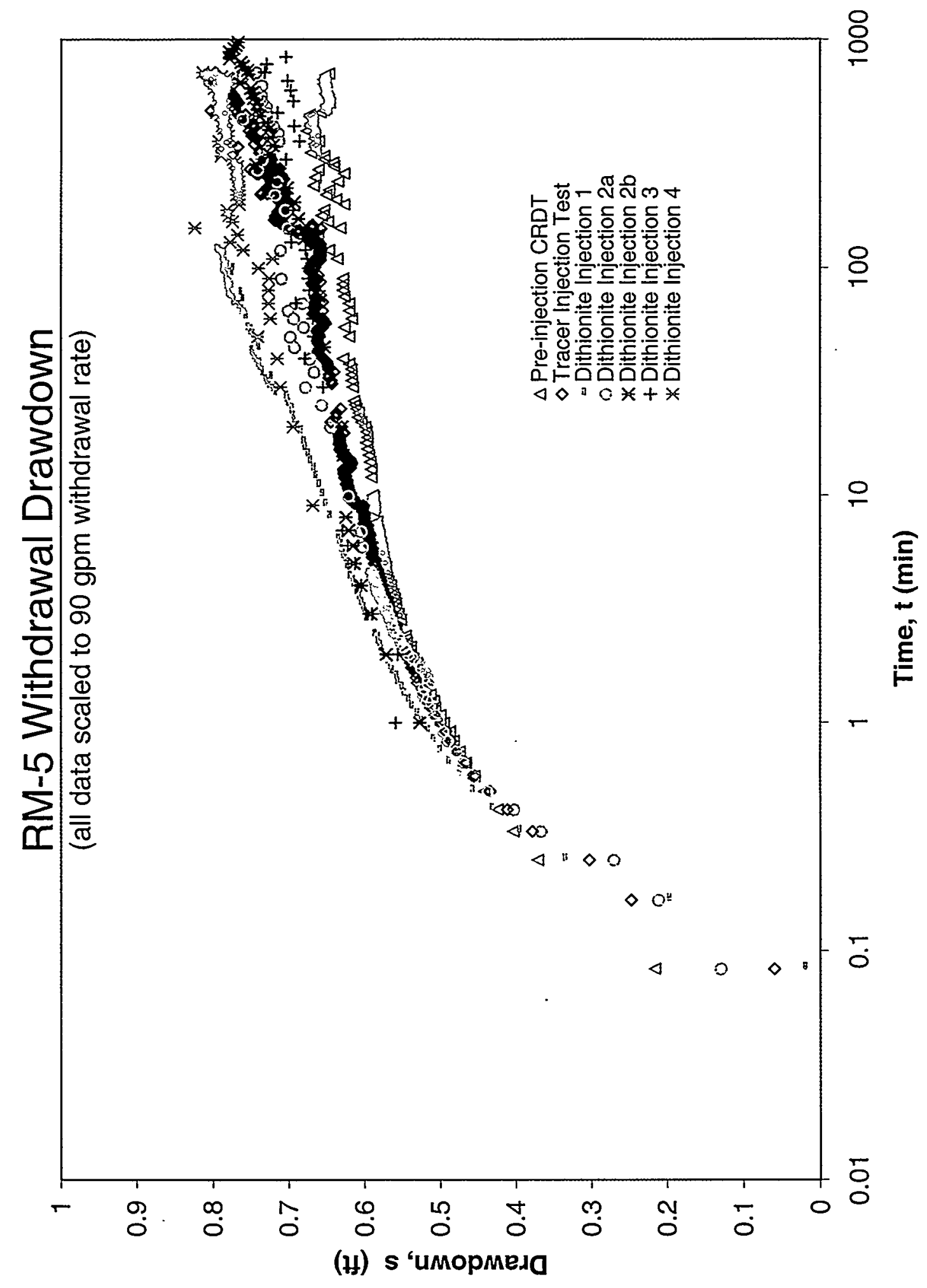

D. 22 


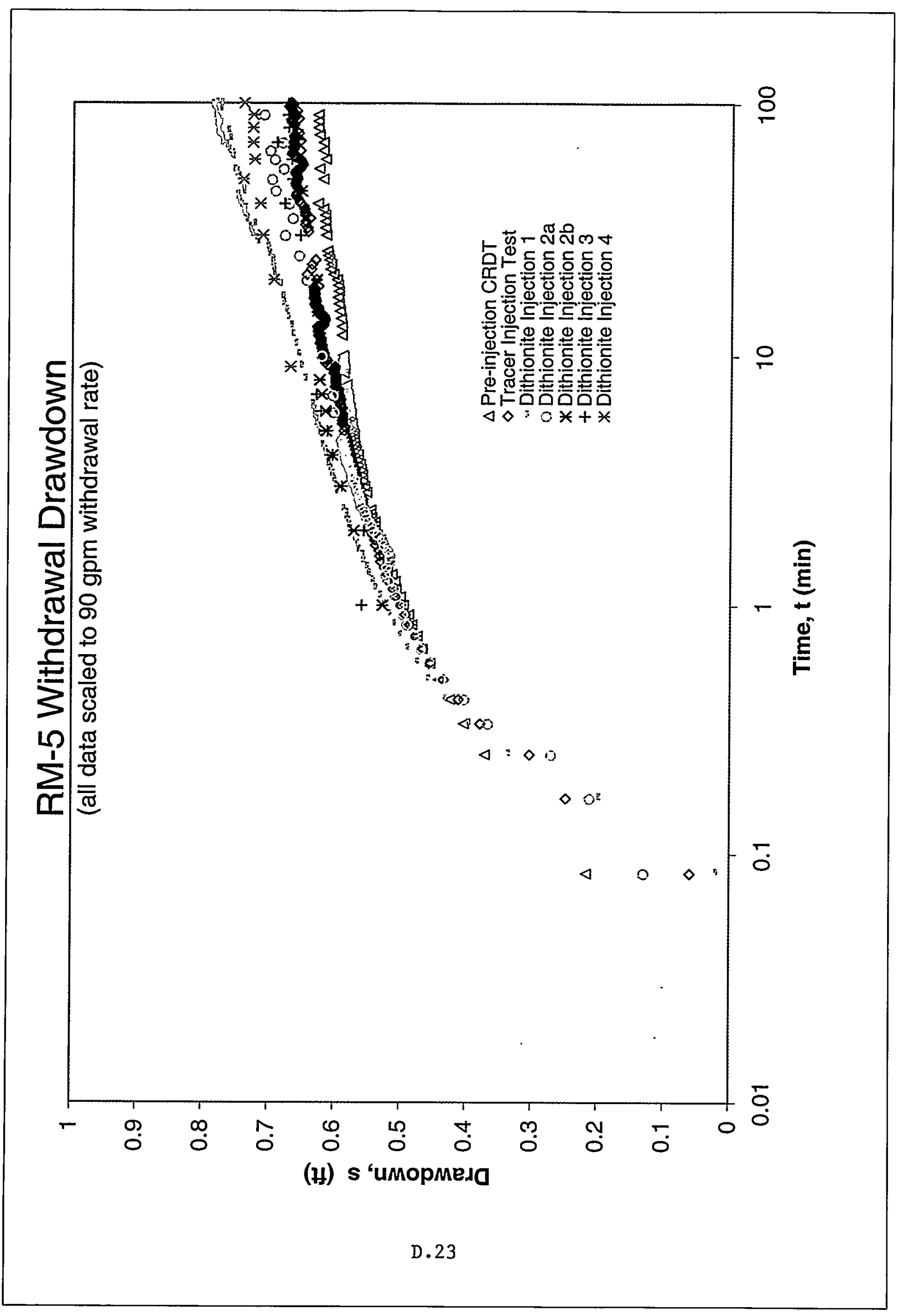




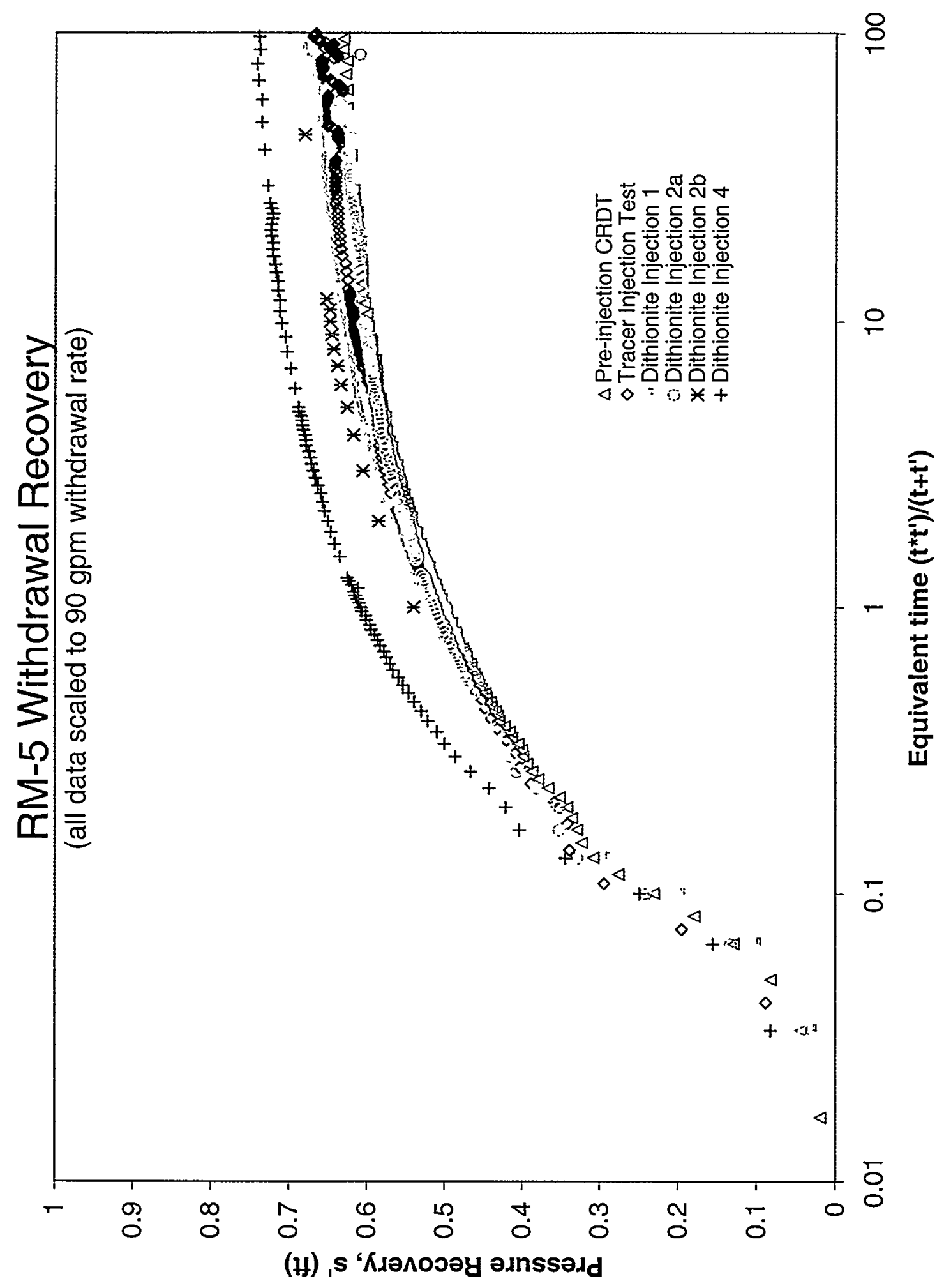

D. 24 


\section{Observed Pressure Responses in Monitoring Well RM-7 Throughout the Various Phases of Testing}




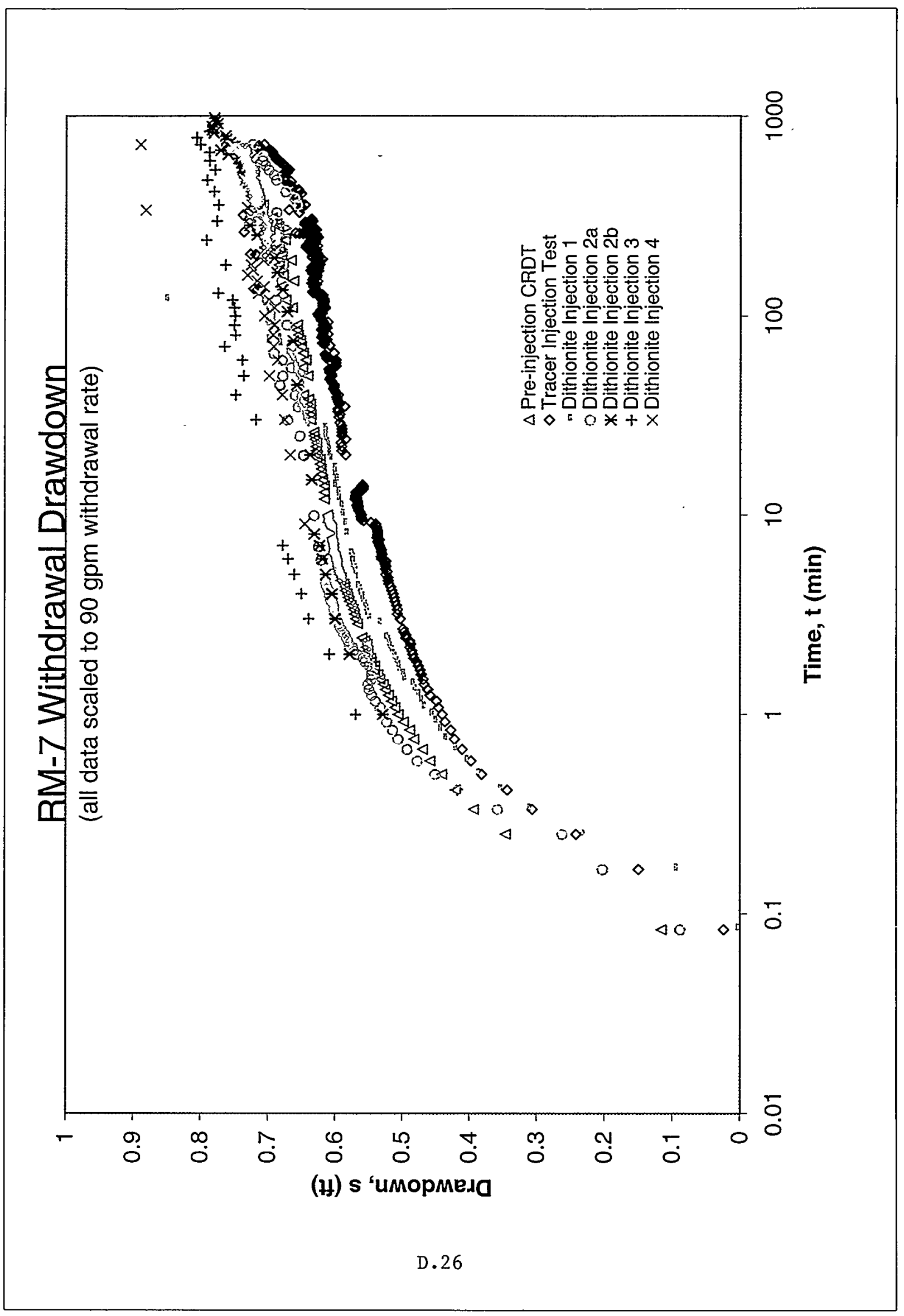




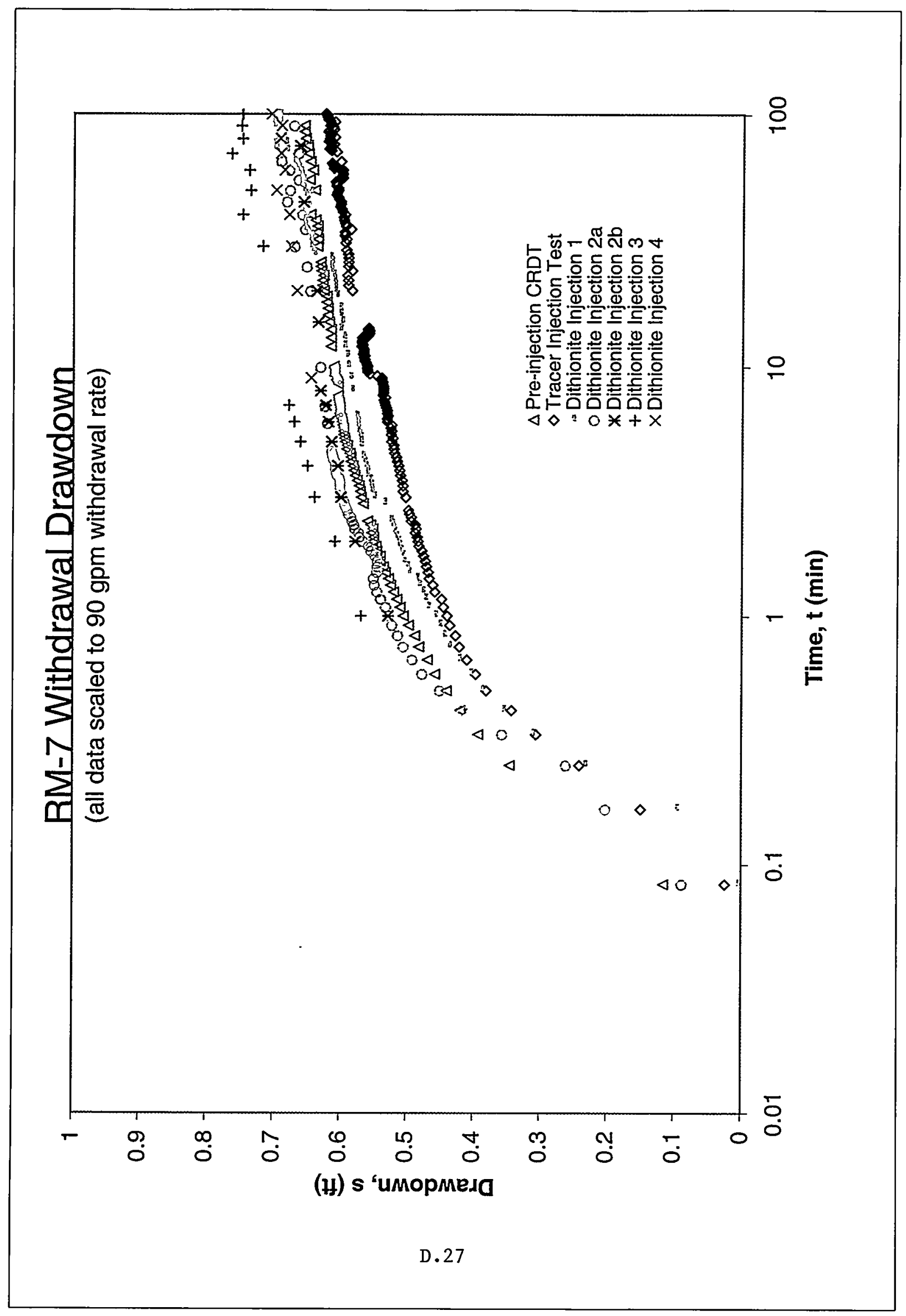




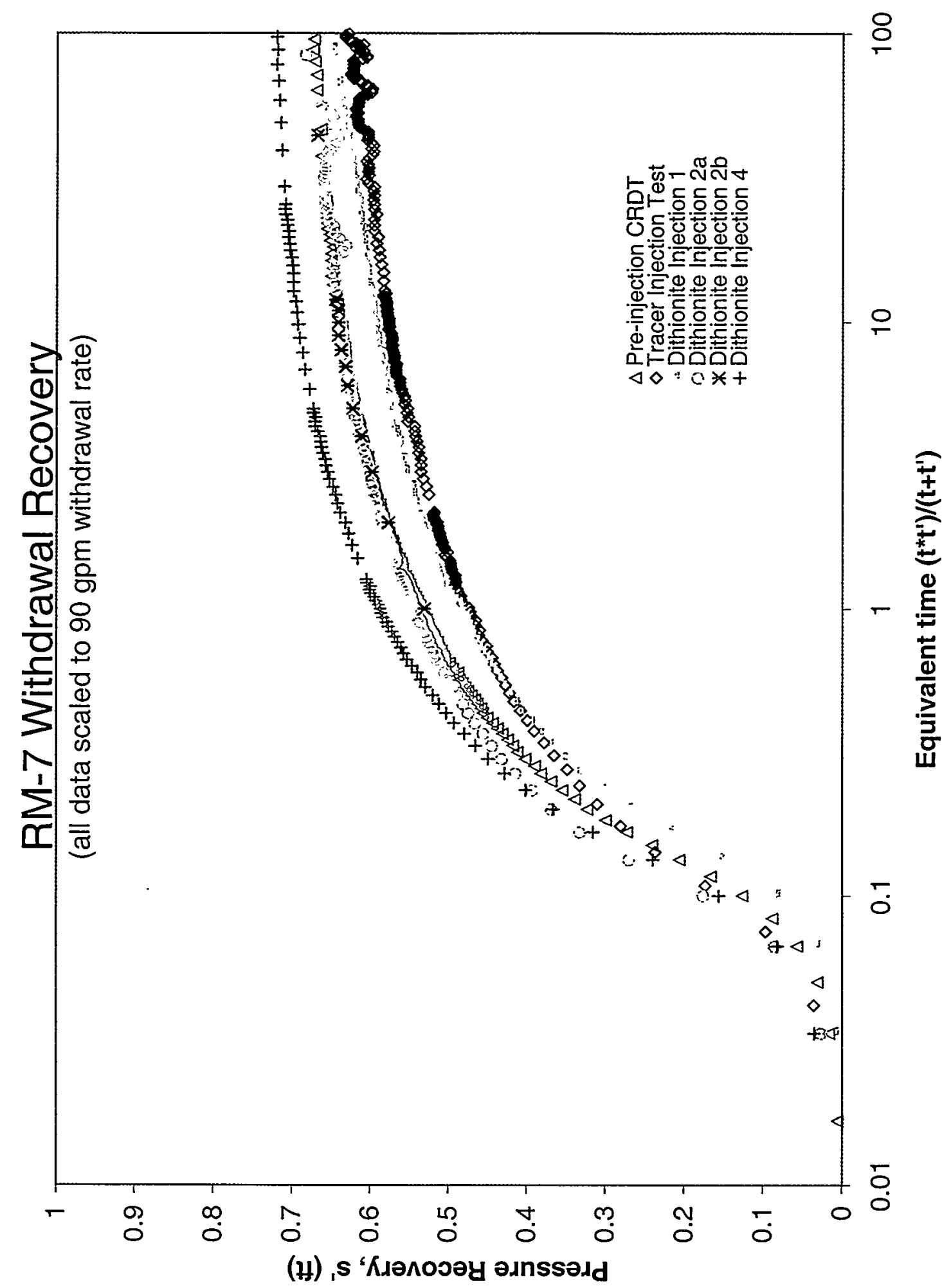

D. 28 
Observed Pressure Responses in Monitoring Well RM-8+ Throughout the Various Phases of Testing 


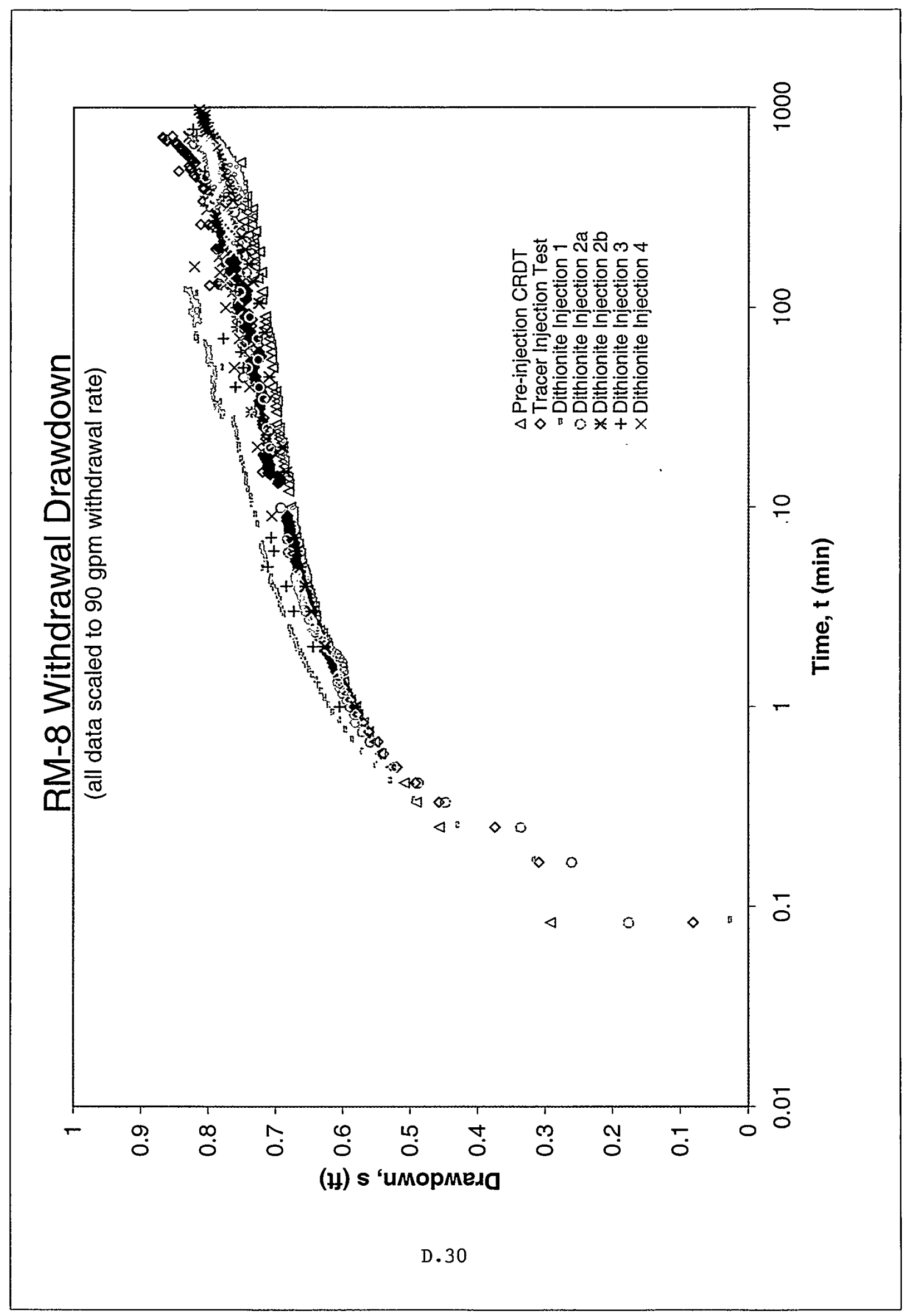




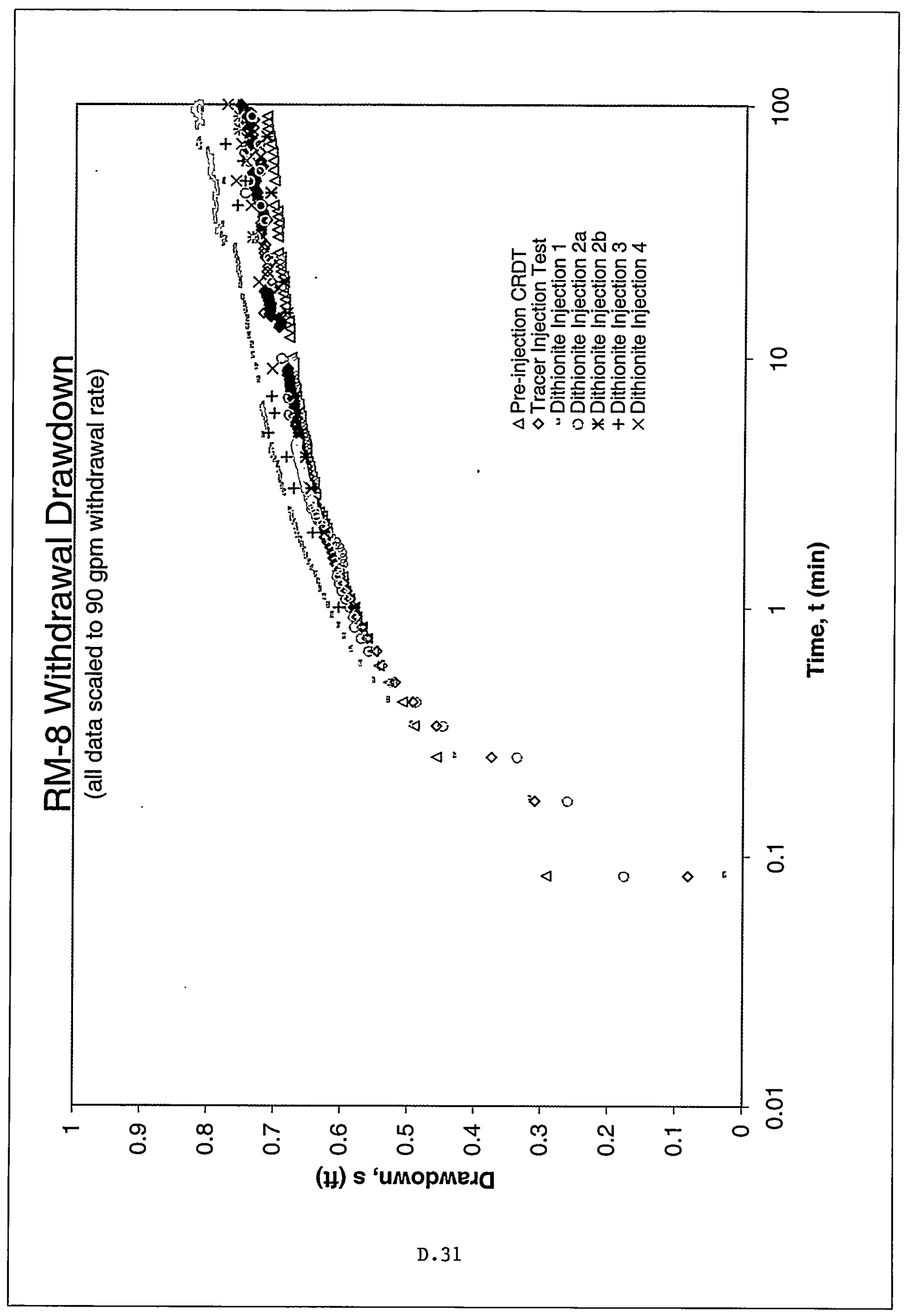




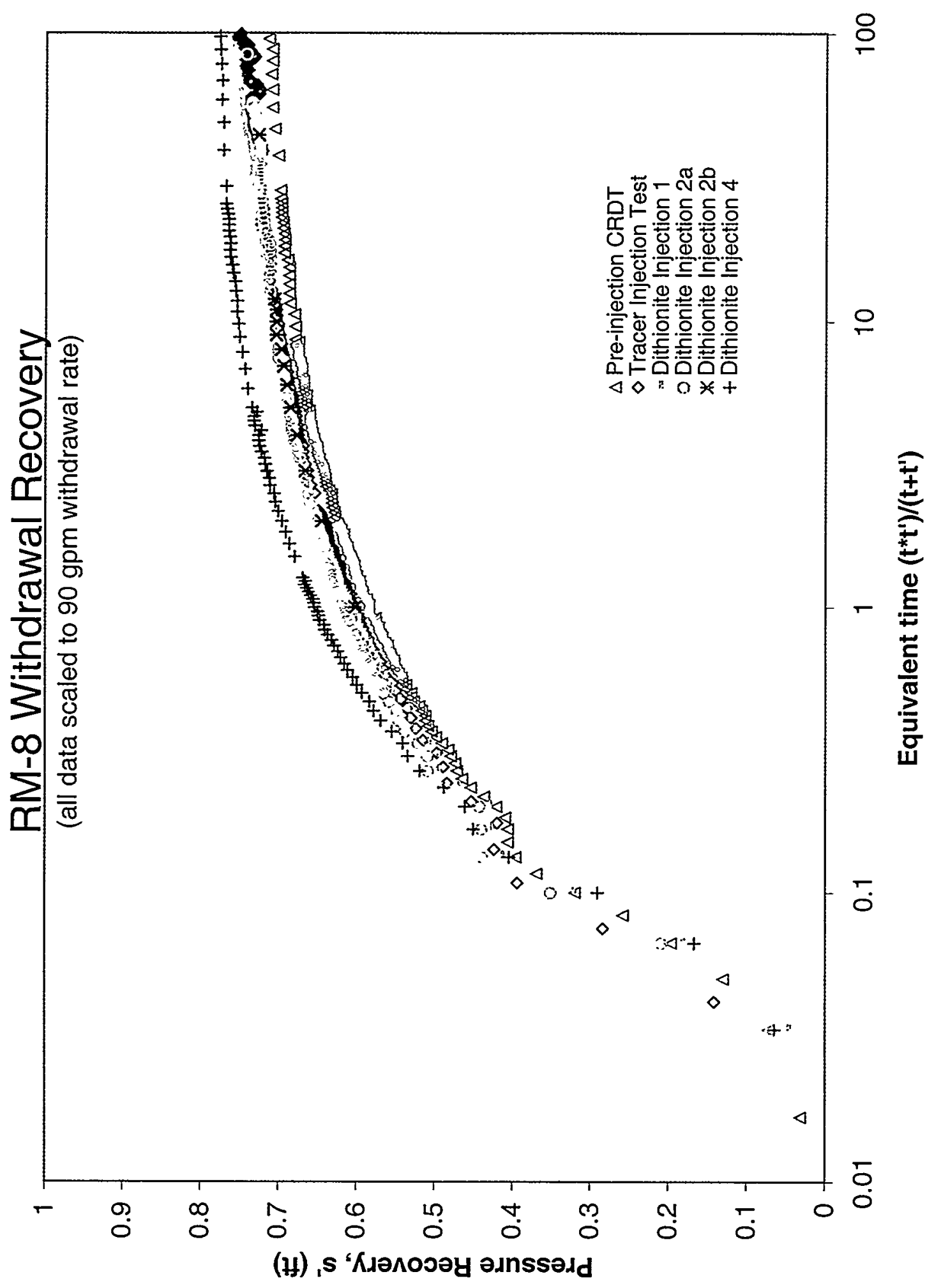

D. 32 
Appendix E

\section{Baseline Chemistry}




\section{Appendix E}

\section{Baseline Chemistry}

The following tables provide a more comprehensive listing of analytical data collected prior to the start of injections. Only the data considered to be the most reliable is listed here. Species analyzed include TCE and DCE (Table E.1), Acetylene (Table E.2), Common Anions (Table E.3), Water Chemistry Parameters (EC, pH, and DO; Table E.4), and Trace Metals (Table E.5).

Table E.1. TCE and DCE Measured by Purge-and-Trap-Gas Chromatography

\begin{tabular}{|c|c|c|c|c|c|c|c|}
\hline $\begin{array}{c}\text { Sample } \\
\text { ID }\end{array}$ & Well & Collection Daté & $\begin{array}{l}\text { TCE } \\
\text { PPB }\end{array}$ & , & $\begin{array}{c}\text { cis-1,2DCE } \\
\text { PŔ }\end{array}$ & & 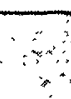 \\
\hline FL20 & RM-1A & $09 / 30 / 98$ & 121 & D & 8.9 & $\mathbf{J}$ & D \\
\hline FL34 & $\mathrm{RM}-1 \mathrm{~A}$ & $10 / 12 / 98$ & 134 & $D$ & 10.7 & $\mathrm{~J}$ & D \\
\hline FL21 & RM-1B & 09/30/98 & 106 & $\mathrm{D}$ & 9.2 & $\mathrm{~J}$ & D \\
\hline FL35 & RM-1B & $10 / 12 / 98$ & 142 & $\mathrm{D}$ & 15.3 & J & D \\
\hline FL22 & $\mathrm{RM}-2$ & 09/30/98 & 133 & $\mathrm{D}$ & 15.6 & $J$ & D \\
\hline FL36 & RM-2 & $10 / 12 / 98$ & 153 & $\mathrm{D}$ & 19.1 & $\mathrm{~J}$ & $\mathrm{D}$ \\
\hline FL23 & RM-3 & $09 / 30 / 98$ & 117 & $\mathrm{D}$ & 8.9 & $\mathrm{~J}$ & D \\
\hline FL31 & $\mathrm{RM}-3$ & $09 / 30 / 98$ & 123 & $\mathrm{D}$ & 10.0 & J & $\mathrm{D}$ \\
\hline FL37 & RM-3 & $10 / 12 / 98$ & 139 & $\mathrm{D}$ & 14.0 & $J$ & $\mathrm{D}$ \\
\hline FL24 & $\mathrm{RM}-4$ & $09 / 30 / 98$ & 124 & $D$ & 10.4 & $\mathrm{~J}$ & D \\
\hline FL38 & $\mathrm{RM}-4$ & $10 / 12 / 98$ & 142 & $D$ & 13.3 & J & $\mathrm{D}$ \\
\hline FL25 & RM-5 & $09 / 30 / 98$ & 148 & $\mathrm{D}$ & 14.4 & $\mathrm{~J}$ & $\mathrm{D}$ \\
\hline FL39 & RM-5 & $10 / 12 / 98$ & 159 & $\mathrm{D}$ & 15.5 & $\mathrm{~J}$ & D \\
\hline FL26 & RM-6 & $09 / 30 / 98$ & 103 & $\mathrm{D}$ & 15.3 & $\mathrm{~J}$ & $\mathrm{D}$ \\
\hline FL40 & RM-6 & $10 / 12 / 98$ & 155 & $\mathrm{D}$ & 23.1 & $\mathrm{~J}$ & $\mathrm{D}$ \\
\hline FL41 & RM-6 & $10 / 12 / 98$ & 159 & $\mathrm{D}$ & 26.3 & $\mathrm{~J}$ & $\mathrm{D}$ \\
\hline FL27 & RM-7 & $09 / 30 / 98$ & 144 & $\mathrm{D}$ & 12.9 & $\mathrm{~J}$ & $\mathrm{D}$ \\
\hline FL42 & RM-7 & $10 / 12 / 98$ & 165 & $\mathrm{D}$ & 19.2 & $J$ & $\mathrm{D}$ \\
\hline FL43 & RM-7 & $10 / 12 / 98$ & 177 & $\mathrm{D}$ & 17.2 & $\mathbf{J}$ & $\mathrm{D}$ \\
\hline FL28 & RM-8 & $09 / 30 / 98$ & 141 & $\mathrm{D}$ & 11.4 & $\mathbf{J}$ & $\mathrm{D}$ \\
\hline FL44 & RM-8 & $10 / 12 / 98$ & 164 & $D$ & 17.0 & $\mathrm{~J}$ & $\mathrm{D}$ \\
\hline FL29 & RM-9 & $09 / 30 / 98$ & 122 & $\mathrm{D}$ & 9.4 & $\mathbf{J}$ & $\mathrm{D}$ \\
\hline FL30 & RM-9 & $09 / 30 / 98$ & 126 & $D$ & 10.2 & $\mathbf{J}$ & $\mathrm{D}$ \\
\hline
\end{tabular}

E.1 
Table E.2. Acetylene Measurements by Headspace-Gas Analysis

\begin{tabular}{|c|c|c|c|}
\hline $\begin{array}{l}\text { Samplét } \\
\mathbf{m}\end{array}$ & $\begin{array}{l}\text { Well } \\
\therefore\end{array}$ & ollectiondoate & $\begin{array}{l}\text { Acetyene } \\
\text { Pp }\end{array}$ \\
\hline FL20 & RM-1A & $09 / 30 / 98$ & $<0.06$ \\
\hline FL21 & RM-1B & $09 / 30 / 98$ & $<0.06$ \\
\hline FL22 & RM-2 & $09 / 30 / 98$ & $<0.06$ \\
\hline FL23 & RM-3 & $09 / 30 / 98$ & $<0.06$ \\
\hline FL24 & RM-4 & $09 / 30 / 98$ & $<0.06$ \\
\hline FL25 & RM-5 & 09/30/98 & $<0.06$ \\
\hline FL26 & RM-6 & $09 / 30 / 98$ & $<0.06$ \\
\hline FL27 & RM-7 & $09 / 30 / 98$ & $<0.06$ \\
\hline FL28 & RM-8 & $09 / 30 / 98$ & $<0.06$ \\
\hline FL30 & RM-9 & 09/30/98 & $<0.06$ \\
\hline
\end{tabular}


Table E.3. Common Anions by Ion Chromatography

\begin{tabular}{|c|c|c|c|c|c|c|c|}
\hline \multirow{2}{*}{$\begin{array}{c}\text { Sample } \\
\text { ID } \\
\text { FL20 }\end{array}$} & \multirow{2}{*}{ 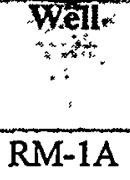 } & \multirow{2}{*}{$\begin{array}{c}\text { Collections } \\
\text { Date } \\
\text { ons }\end{array}$} & \multicolumn{2}{|c|}{ 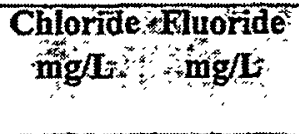 } & \multirow{2}{*}{$\begin{array}{l}\text { Nitrate } \\
\text { mgick } \\
\text { (asNO } \\
1.73)\end{array}$} & \multicolumn{2}{|c|}{$\begin{array}{c}\text { Phosphatensifife } \\
\text { mg/L }\end{array}$} \\
\hline & & & 2.40 & $<0.2$ & & $<0.2$ & 6.87 \\
\hline FL34 & RM-1A & $10 / 12 / 98$ & 2.35 & $<0.2$ & 1.75 & $<0.2$ & 6.22 \\
\hline FL47 & RM-1A & $11 / 09 / 98$ & 2.47 & $<0.2$ & 2.56 & $<0.2$ & 6.45 \\
\hline FL21 & RM-1B & $09 / 30 / 98$ & 3.05 & $<0.2$ & 1.86 & 0.43 & 18.7 \\
\hline FL35 & RM-1B & $10 / 12 / 98$ & 2.99 & $<0.2$ & 1.77 & 0.58 & 20.5 \\
\hline FL52 & RM-1B & $11 / 09 / 98$ & 3.03 & $<0.2$ & 2.07 & 0.33 & 17.8 \\
\hline FL22 & $\mathrm{RM}-2$ & $09 / 30 / 98$ & 2.42 & $<0.2$ & 1.93 & $<0.2$ & 8.10 \\
\hline FL36 & RM-2 & $10 / 12 / 98$ & 2.38 & $<0.2$ & 2.55 & $<0.2$ & 7.07 \\
\hline FL54 & RM-2 & $11 / 09 / 98$ & 2.57 & $<0.2$ & 2.13 & $<0.2$ & 6.71 \\
\hline FL23 & $\mathrm{RM}-3$ & $09 / 30 / 98$ & 2.38 & $<0.2$ & 2.21 & $<0.2$ & 5.87 \\
\hline FL31 & $\mathrm{RM}-3$ & 09/30/98 & 2.30 & $<0.2$ & 2.14 & $<0.2$ & 5.84 \\
\hline FL37 & RM-3 & $10 / 12 / 98$ & 2.36 & $<0.2$ & 2.17 & $<0.2$ & 5.72 \\
\hline FL55 & RM-3 & $11 / 09 / 98$ & 2.53 & $<0.2$ & 2.20 & $<0.2$ & 6.12 \\
\hline FL56 & $\mathrm{RM}-3$ & $11 / 09 / 98$ & 2.51 & $<0.2$ & 2.16 & $<0.2$ & 6.04 \\
\hline FL24 & $\mathrm{RM}-4$ & $09 / 30 / 98$ & 2.43 & $<0.2$ & 2.01 & $<0.2$ & 5.98 \\
\hline FL38 & $\mathrm{RM}-4$ & $10 / 12 / 98$ & 2.36 & $<0.2$ & 1.88 & $<0.2$ & 5.92 \\
\hline FL48 & RM-4 & $11 / 09 / 98$ & 2.44 & $<0.2$ & 2.05 & $<0.2$ & 6.02 \\
\hline FL25 & RM-5 & $09 / 30 / 98$ & 2.50 & $<0.2$ & 1.76 & $<0.2$ & 6.57 \\
\hline FL39 & RM-5 & $10 / 12 / 98$ & 2.63 & $<0.2$ & 2.55 & $<0.2$ & 6.42 \\
\hline FL57 & RM-5 & $11 / 09 / 98$ & 2.67 & $<0.2$ & 2.30 & $<0.2$ & 6.93 \\
\hline FL26 & RM-6 & $09 / 30 / 98$ & 2.62 & $<0.2$ & 0.82 & $<0.2$ & 11.2 \\
\hline FL40 & RM-6 & $10 / 12 / 98$ & 2.59 & $<0.2$ & 2.27 & $<0.2$ & 6.97 \\
\hline FL41 & RM-6 & $10 / 12 / 98$ & 2.47 & $<0.2$ & 2.23 & $<0.2$ & 6.93 \\
\hline FL50 & RM-6 & $11 / 09 / 98$ & 2.53 & $<0.2$ & 1.41 & $<0.2$ & 8.79 \\
\hline FL27 & RM-7 & $09 / 30 / 98$ & 2.48 & $<0.2$ & 2.42 & $<0.2$ & 6.46 \\
\hline FL42 & RM-7 & $10 / 12 / 98$ & 2.44 & $<0.2$ & 2.51 & $<0.2$ & 6.31 \\
\hline FL43 & $\mathrm{RM}-7$ & $10 / 12 / 98$ & 2.44 & $<0.2$ & 2.48 & $<0.2$ & 6.25 \\
\hline FL49 & $\mathrm{RM}-7$ & $11 / 09 / 98$ & 2.52 & $<0.2$ & 2.56 & $<0.2$ & 6.53 \\
\hline FL28 & RM-8 & $09 / 30 / 98$ & 2.45 & $<0.2$ & 2.58 & $<0.2$ & 6.33 \\
\hline FL44 & RM-8 & $10 / 12 / 98$ & 2.44 & $<0.2$ & 2.56 & $<0.2$ & 6.10 \\
\hline FL51 & RM-8 & $11 / 09 / 98$ & 2.50 & $<0.2$ & 2.52 & $<0.2$ & 6.43 \\
\hline FL29 & RM-9 & $09 / 30 / 98$ & 2.51 & $<0.2$ & 2.52 & $<0.2$ & 6.14 \\
\hline FL30 & RM-9 & $09 / 30 / 98$ & 2.36 & $<0.2$ & 2.49 & $<0.2$ & 6.07 \\
\hline FL58 & RM-9 & $11 / 09 / 98$ & 2.66 & $<0.2$ & 2.49 & $<0.2$ & 6.27 \\
\hline FL59 & RM-9 & $11 / 09 / 98$ & 2.60 & $<0.2$ & 2.47 & $<0.2$ & 6.27 \\
\hline
\end{tabular}


Table E.4. Water Chemistry Parameters

\begin{tabular}{|c|c|c|c|c|c|}
\hline $\begin{array}{c}\text { Samplée } \\
\mathbf{m} \\
\end{array}$ & Well & $\begin{array}{l}\text { Collection } \\
\text { Date }\end{array}$ & $\begin{array}{c}\text { Efectrical } \\
\text { Conductivity } \\
\text { MicroSiemens }\end{array}$ & $\begin{array}{l}\text { Dissolyed } \\
\text { oxygen } \\
\text { ppin }\end{array}$ & $\mathbf{p} \mathbf{H}$ \\
\hline FL20 & RM-1A & $09 / 30 / 98$ & $159^{\circ}$ & 4.5 & 6.53 \\
\hline FL34 & $R M-1 A$ & $10 / 12 / 98$ & 120 & 4.1 & 6.74 \\
\hline FL47 & RM-1A & $11 / 09 / 98$ & 115 & 4.3 & 6.76 \\
\hline FL21 & $\mathrm{RM}-1 \mathrm{~B}$ & $09 / 30 / 98$ & 189 & 4.0 & 6.81 \\
\hline FL35 & $R M-1 B$ & $10 / 12 / 98$ & 172 & 1.6 & 7.06 \\
\hline FL52 & $\mathrm{RM}-1 \mathrm{~B}$ & $11 / 09 / 98$ & 128 & 3.2 & 6.94 \\
\hline FL22 & $\mathrm{RM}-2$ & $09 / 30 / 98$ & 169 & 5.0 & 6.69 \\
\hline FL36 & $\mathrm{RM}-2$ & $10 / 12 / 98$ & 125 & 4.3 & 6.88 \\
\hline FL54 & $\mathrm{RM}-2$ & $11 / 09 / 98$ & 106 & 4.7 & 6.89 \\
\hline FL23 & RM-3 & $09 / 30 / 98$ & 161 & 8.0 & 6.33 \\
\hline FL37 & RM-3 & $10 / 12 / 98$ & 117 & 6.3 & 6.75 \\
\hline FL55 & RM-3 & $11 / 09 / 98$ & 99 & 6.0 & 6.76 \\
\hline FL24 & $\mathrm{RM}-4$ & $09 / 30 / 98$ & 154 & 5.6 & 6.63 \\
\hline FL38 & $\mathrm{RM}-4$ & $10 / 12 / 98$ & 117 & 5.0 & 6.75 \\
\hline FL48 & $\mathrm{RM}-4$ & $11 / 09 / 98$ & 107 & 5.1 & 6.76 \\
\hline FL25 & RM-5 & $09 / 30 / 98$ & 160 & 4.5 & 6.78 \\
\hline FL39 & RM-5 & $10 / 12 / 98$ & 128 & 3.8 & 6.93 \\
\hline FL57 & $R M-5$ & $11 / 09 / 98$ & 109 & 4.5 & 6.91 \\
\hline FL26 & $\mathrm{RM}-6$ & $09 / 30 / 98$ & 174 & 1.0 & 7.23 \\
\hline FLA0 & RM-6 & $10 / 12 / 98$ & 133 & 4.0 & 7.11 \\
\hline FL41 & $R M-6$ & $10 / 12 / 98$ & 135 & 3.7 & 7.14 \\
\hline FL50 & $R M-6$ & $11 / 09 / 98$ & 129 & 1.4 & 7.14 \\
\hline FL27 & $\mathrm{RM}-7$ & $09 / 30 / 98$ & 151 & 6.7 & 6.69 \\
\hline FL42 & RM-7 & $10 / 12 / 98$ & 125 & 5.5 & 6.86 \\
\hline FL43 & RM-7 & $10 / 12 / 98$ & 124 & 5.5 & 6.86 \\
\hline FL 49 & RM-7 & $11 / 09 / 98$ & 113 & 5.3 & 6.87 \\
\hline FL28 & RM-8 & $09 / 30 / 98$ & 148 & 7.2 & 6.60 \\
\hline FL44 & RM-8 & $10 / 12 / 98$ & 121 & 5.9 & 6.86 \\
\hline FL51 & $\mathrm{RM}-8$ & $11 / 09 / 98$ & 114 & 5.7 & 6.82 \\
\hline FL29 & RM-9 & $09 / 30 / 98$ & 146 & 6.9 & 6.61 \\
\hline FL45 & RM-9 & $10 / 12 / 98$ & 121 & 5.9 & 6.78 \\
\hline FL58 & RM-9 & $11 / 09 / 98$ & 102 & 5.6 & 6.80 \\
\hline
\end{tabular}


Table E.5a. Trace Metals by Inductively Coupled Argon Plasma-Optical Emission Spectroscopy (Al, As, $\mathrm{Ba}, \mathrm{Be}, \mathrm{B}$, and $\mathrm{Cd}$ )

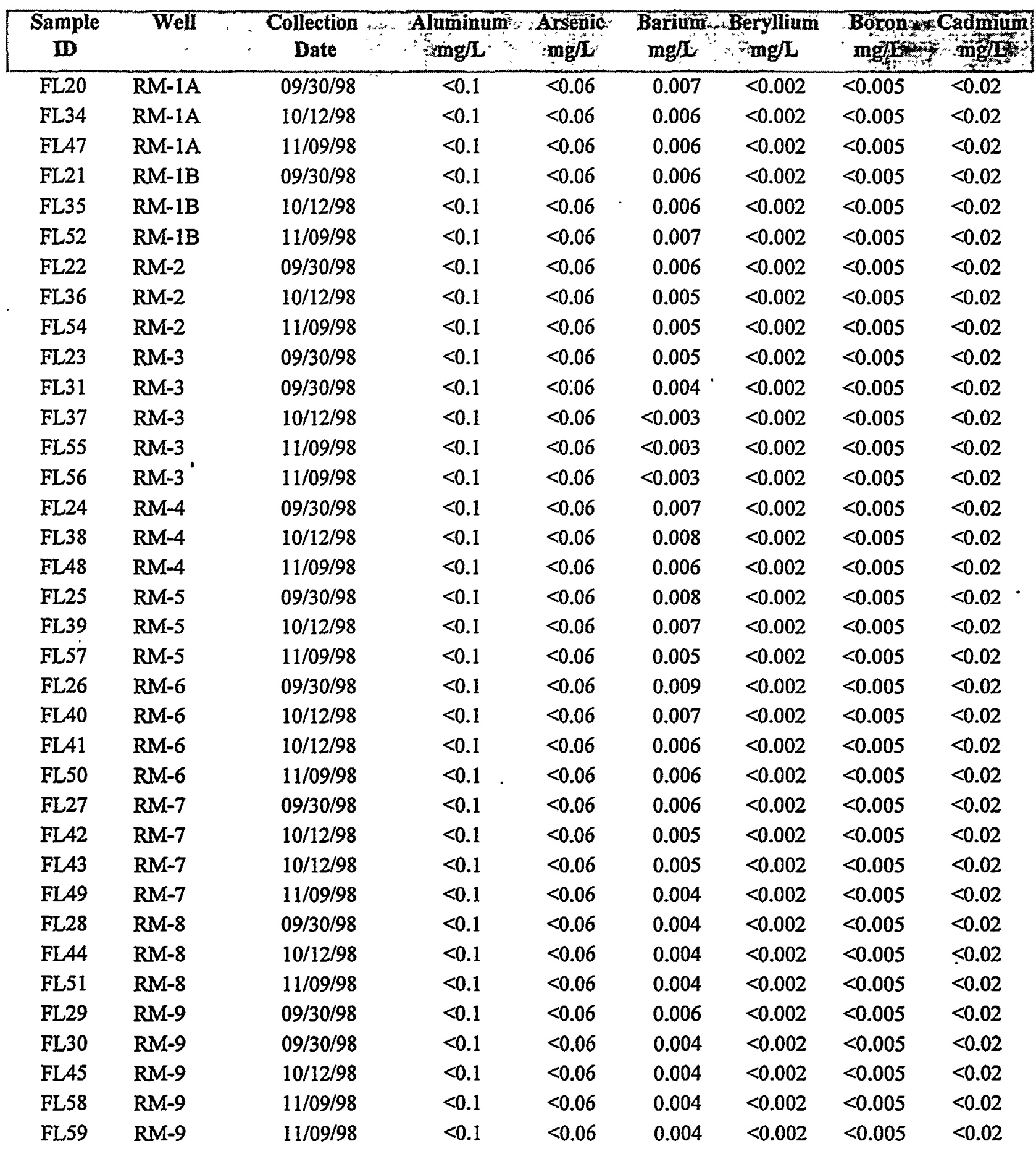


Table E.5b. Trace Metals by Inductively Coupled Argon Plasma-Optical Emission Spectroscopy ( $\mathrm{Ca}, \mathrm{Cr}, \mathrm{Co}, \mathrm{Cu}, \mathrm{Fe}$, and $\mathrm{Pb}$ )

\begin{tabular}{|c|c|c|c|c|c|c|c|c|}
\hline $\begin{array}{l}\text { Sample } \\
\text { ID }\end{array}$ & Well & $\begin{array}{l}\text { Collection } \\
\text { Oate }\end{array}$ & $\begin{array}{l}\text { Galcium } \\
\text { mg } \mathbf{L}\end{array}$ & $\begin{array}{l}\text { Chromium } \\
\text { migh }\end{array}$ & $\begin{array}{l}\text { Cobale } \\
\text { mgit }\end{array}$ & $\begin{array}{l}\text { Copper } \\
\text { mg } \mathrm{L}\end{array}$ & $\begin{array}{l}\text { Trom } \\
\text { mgit }\end{array}$ & $\begin{array}{l}\text { Legats } \\
\text { mg }\end{array}$ \\
\hline FL20 & $\mathrm{RM}-1 \mathrm{~A}$ & $09 / 30 / 98$ & 13 & $<0.04$ & $<0.004$ & $<0.006$ & 0.02 & $<0.06$ \\
\hline FL34 & RM-1A & $10 / 12 / 98$ & 14 & $<0.04$ & $<0.004$ & $<0.006$ & $<0.005$ & $<0.06$ \\
\hline FL47 & $\mathrm{RM}-1 \mathrm{~A}$ & $11 / 09 / 98$ & 14 & $<0.04$ & $<0.004$ & $<0.006$ & 0.01 & $<0.06$ \\
\hline FL21 & RM-1B & 09/30/98 & 15 & $<0.04$ & $<0.004$ & $<0.006$ & 0.03 & $<0.06$ \\
\hline FL35 & $\mathrm{RM}-1 \mathrm{~B}$ & $10 / 12 / 98$ & 15 & $<0.04$ & $<0.004$ & $<0.006$ & 0.044 & $<0.06$ \\
\hline FL52 & RM-1B & $11 / 09 / 98$ & 15 & $<0.04$ & $<0.004$ & $<0.006$ & 0.026 & $<0.06$ \\
\hline FL22 & $\mathrm{RM}-2$ & $09 / 30 / 98$ & 14 & $<0.04$ & $<0.004$ & $<0.006$ & $<0.005$ & $<0.06$ \\
\hline FL36 & $\mathrm{RM}-2$ & $10 / 12 / 98$ & 14 & $<0.04$ & $<0.004$ & $<0.006$ & 0.016 & $<0.06$ \\
\hline FL54 & $\mathrm{RM}-2$ & $11 / 09 / 98$ & 14 & $<0.04$ & $<0.004$ & $<0.006$ & 0.01 & $<0.06$ \\
\hline FL23 & RM-3 & $09 / 30 / 98$ & 13 & $<0.04$ & $<0.004$ & $<0.006$ & $<0.005$ & $<0.06$ \\
\hline FL31 & RM-3 & $09 / 30 / 98$ & 13 & $<0.04$ & $<0.004$ & $<0.006$ & $<0.005$ & $<0.06$ \\
\hline FL37 & $\mathrm{RM}-3$ & $10 / 12 / 98$ & 14 & $<0.04$ & $<0.004$ & $<0.006$ & $<0.005$ & $<0.06$ \\
\hline FL55 & RM-3 & $11 / 09 / 98$ & 13 & $<0.04$ & $<0.004$ & $<0.006$ & $<0.005$ & $<0.06$ \\
\hline FL56 & RM-3 & $11 / 09 / 98$ & 13 & $<0.04$ & $<0.004$ & $<0.006$ & $<0.005$ & $<0.06$ \\
\hline FL24 & $\mathrm{RM}-4$ & $09 / 30 / 98$ & 13 & $<0.04$ & $<0.004$ & $<0.006$ & 0.019 & $<0.06$ \\
\hline FL38 & $\mathrm{RM}-4$ & $10 / 12 / 98$ & 14 & $<0.04$ & $<0.004$ & $<0.006$ & 0.012 & $<0.06$ \\
\hline FL48 & $\mathrm{RM}-4$ & $11 / 09 / 98$ & 13 & $<0.04$ & $<0.004$ & $<0.006$ & 0.02 & $<0.06$ \\
\hline FL25 & RM-5 & $09 / 30 / 98$ & 14 & $<0.04$ & $<0.004$ & $<0.006$ & 0.015 & $<0.06$ \\
\hline FL39 & $\mathrm{RM}-5$ & $10 / 12 / 98$ & 15 & $<0.04$ & $<0.004$ & $<0.006$ & 0.01 & $<0.06$ \\
\hline FL57 & RM-5 & $11 / 09 / 98$ & 14 & $<0.04$ & $<0.004$ & $<0.006$ & 0.008 & $<0.06$ \\
\hline FL26 & $R M-6$ & $09 / 30 / 98$ & 15 & $<0.04$ & $<0.004$ & $<0.006$ & 0.018 & $<0.06$ \\
\hline FL40 & RM-6 & $10 / 12 / 98$ & 15 & $<0.04$ & $<0.004$ & $<0.006$ & $<0.005$ & $<0.06$ \\
\hline FL41 & RM-6 & $10 / 12 / 98$ & 15 & $<0.04$ & $<0.004$ & $<0.006$ & $<0.005$ & $<0.06$ \\
\hline FL50 & RM-6 & $11 / 09 / 98$ & 14 & $<0.04$ & $<0.004$ & $<0.006$ & 0.021 & $<0.06$ \\
\hline FL27 & RM-7 & $09 / 30 / 98$ & 14 & $<0.04$ & $<0.004$ & $<0.006$ & 0.013 & $<0.06$ \\
\hline FL42 & RM-7 & $10 / 12 / 98$ & 14 & $<0.04$ & $<0.004$ & $<0.006$ & $<0.005$ & $<0.06$ \\
\hline FL43 & RM-7 & $10 / 12 / 98$ & 14 & $<0.04$ & $<0.004$ & $<0.006$ & $<0.005$ & $<0.06$ \\
\hline FL49 & RM-7 & $11 / 09 / 98$ & 13 & $<0.04$ & $<0.004$ & $<0.006$ & 0.018 & $<0.06$ \\
\hline FL28 & RM-8 & $09 / 30 / 98$ & 14 & $<0.04$ & $<0.004$ & 0.091 & 0.011 & $<0.06$ \\
\hline FL44 & RM-8 & $10 / 12 / 98$ & 14 & $<0.04$ & $<0.004$ & $<0.006$ & $<0.005$ & $<0.06$ \\
\hline FL51 & RM-8 & $11 / 09 / 98$ & 14 & $<0.04$ & $<0.004$ & $<0.006$ & $<0.005$ & $<0.06$ \\
\hline FL29 & RM-9 & $09 / 30 / 98$ & 15 & $<0.04$ & $<0.004$ & $<0.006$ & 0.014 & $<0.06$ \\
\hline FL30 & RM-9 & $09 / 30 / 98$ & 14 & $<0.04$ & $<0.004$ & 0.019 & 0.019 & $<0.06$ \\
\hline FL45 & RM-9 & $10 / 12 / 98$ & 14 & $<0.04$ & $<0.004$ & $<0.006$ & 0.01 & $<0.06$ \\
\hline FL58 & RM-9 & $11 / 09 / 98$ & 13 & $<0.04$ & $<0.004$ & $<0.006$ & 0.011 & $<0.06$ \\
\hline FL59 & RM-9 & $11 / 09 / 98$ & 13 & $<0.04$ & $<0.004$ & $<0.006$ & 0.010 & $<0.06$ \\
\hline
\end{tabular}


Table E.5c. Trace Metals by Inductively Coupled Argon Plasma-Optical Emission Spectroscopy (Li, Mg, Mn, $\mathrm{Mo}, \mathrm{Ni}$, and $\mathrm{K}$ )) .

\begin{tabular}{|c|c|c|c|c|c|c|c|c|}
\hline $\begin{array}{l}\text { Sample } \\
\text { ID }\end{array}$ & Well & $\begin{array}{c}\text { Collection } \\
\text { Date"." }\end{array}$ & $\begin{array}{c}\text { Lithium } \\
\text { mgu }\end{array}$ & 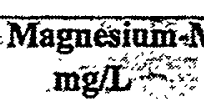 & $\begin{array}{c}\text { Tanganesezx } \\
\text { mg/L }\end{array}$ & $\begin{array}{l}\text { Jybdenum } \\
\text { mig/l. }\end{array}$ & $\begin{array}{l}\text { Nickeldi } \\
\text { mgit }\end{array}$ & tásion \\
\hline FL20 & $\overline{R M}-1 \mathrm{~A}$ & $09 / 30 / 98$ & $<0.02$ & 5.8 & 0.063 & $<0.06$ & $<0.01$ & 1.8 \\
\hline FL34 & RM-1A & $10 / 12 / 98$ & $<0.02$ & 5.9 & $<0.004$ & $<0.06$ & $<0.01$ & 1.8 \\
\hline FLA7 & RM-1A & $11 / 09 / 98$ & $<0.02$ & 5.9 & 0.013 & $<0.06$ & $<0.01$ & 2.2 \\
\hline FL21 & RM-1B & $09 / 30 / 98$ & $<0.02$ & 6.9 & 0.22 & $<0.06$ & $<0.01$ & 2.0 \\
\hline FL35 & RM-1B & $10 / 12 / 98$ & $<0.02$ & 6.7 & 0.18 & $<0.06$ & $<0.01$ & 2.1 \\
\hline FL52 & RM-1B & $11 / 09 / 98$ & $<0.02$ & 6.7 & 0.043 & $<0.06$ & $<0.01$ & 2.1 \\
\hline FL22 & RM-2 & $09 / 30 / 98$ & $<0.02$ & 6.1 & 0.022 & $<0.06$ & $<0.01$ & 1.8 \\
\hline FL36 & RM-2 & $10 / 12 / 98$ & $<0.02$ & 6.2 & 0.009 & $<0.06$ & $<0.01$ & 1.8 \\
\hline FL54 & RM-2 & $11 / 09 / 98$ & $<0.02$ & 6.1 & $<0.004$ & $<0.06$ & 0.017 & 2.2 \\
\hline FL23 & RM-3 & $09 / 30 / 98$ & $<0.02$ & 5.9 & 0.039 & $<0.06$ & $<0.01$ & 1.6 \\
\hline FL31 & RM-3 & $09 / 30 / 98$ & $<0.02$ & 5.8 & 0.04 & $<0.06$ & $<0.01$ & 1.6 \\
\hline FL37 & RM-3 & $10 / 12 / 98$ & $<0.02$ & 6.0 & 0.022 & $<0.06$ & $<0.01$ & 1.7 \\
\hline FL55 & RM-3 & $11 / 09 / 98$ & $<0.02$ & 5.9 & $<0.004$ & $<0.06$ & 0.018 & 1.7 \\
\hline FL56 & RM-3 & $11 / 09 / 98$ & $<0.02$ & 5.7 & $<0.004$ & $<0.06$ & 0.016 & 1.6 \\
\hline FL24 & RM-4 & $09 / 30 / 98$ & $<0.02$ & 5.7 & 0.09 & $<0.06$ & $<0.01$ & 1.7 \\
\hline FL38 & RM-4 & $10 / 12 / 98$ & $<0.02$ & 6.0 & 0.047 & $<0.06$ & $<0.01$ & 1.8 \\
\hline FL48 & $\mathrm{RM}-4$ & $11 / 09 / 98$ & $<0.02$ & 5.8 & 0.011 & $<0.06$ & $<0.01$ & 1.8 \\
\hline FL25 & RM-5 & 09/30/98 & $<0.02$ & 6.2 & 0.14 & $<0.06$ & $<0.01$ & 1.8 \\
\hline FL39 & $\mathrm{RM}-5$ & $10 / 12 / 98$ & $<0.02$ & 6.7 & 0.042 & $<0.06$ & $<0.01$ & 1.9 \\
\hline FL57 & $\mathrm{RM}-5$ & $11 / 09 / 98$ & $<0.02$ & 6.5 & $<0.004$ & $<0.06$ & 0.017 & 1.9 \\
\hline FL26 & RM-6 & $09 / 30 / 98$ & $<0.02$ & 6.7 & 0.22 & $<0.06$ & $<0.01$ & 2.1 \\
\hline FLA0 & RM-6 & $10 / 12 / 98$ & $<0.02$ & 6.8 & 0.13 & $<0.06$ & $<0.01$ & 2.0 \\
\hline FL4I & RM-6 & $10 / 12 / 98$ & $<0.02$ & 6.9 & 0.13 & $<0.06$ & $<0.01$ & 2.0 \\
\hline FL50 & RM-6 & $11 / 09 / 98$ & $<0.02$ & 6.6 & 0.034 & $<0.06$ & $<0.01$ & 2.0 \\
\hline FL27 & RM-7 & $09 / 30 / 98$ & $<0.02$ & 6.5 & 0.036 & $<0.06$ & $<0.01$ & 1.7 \\
\hline FL42 & RM-7 & $10 / 12 / 98$ & $<0.02$ & 6.5 & 0.011 & $<0.06$ & $<0.01$ & 1.7 \\
\hline FL43 & RM-7 & $10 / 12 / 98$ & $<0.02$ & 6.5 & 0.011 & $<0.06$ & $<0.01$ & 1.8 \\
\hline FL49 & RM-7 & $11 / 09 / 98$ & $<0.02$ & 6.2 & 0.024 & $<0.06$ & $<0.01$ & 1.7 \\
\hline FL28 & RM-8 & $09 / 30 / 98$ & $<0.02$ & 6.1 & 0.013 & $<0.06$ & $<0.01$ & 1.7 \\
\hline FL44 & RM-8 & $10 / 12 / 98$ & $<0.02$ & 6.3 & 0.006 & $<0.06$ & $<0.01$ & 1.7 \\
\hline FL51 & RM-8 & $11 / 09 / 98$ & $<0.02$ & 6.1 & $<0.004$ & $<0.06$ & $<0.01$ & 2.2 \\
\hline FL29 & RM-9 & $09 / 30 / 98$ & $<0.02$ & 6.2 & 0.036 & $<0.06$ & $<0.01$ & 1.6 \\
\hline FL30 & RM-9 & $09 / 30 / 98$ & $<0.02$ & 5.9 & 0.013 & $<0.06$ & $<0.01$ & 1.6 \\
\hline FL45 & RM-9 & $10 / 12 / 98$ & $<0.02$ & 6.3 & 0.008 & $<0.06$ & $<0.01$ & 1.7 \\
\hline FL58 & RM-9 & $11 / 09 / 98$ & $<0.02$ & 6.1 & $<0.004$ & $<0.06$ & 0.11 & 2.2 \\
\hline FL59 & RM-9 & $11 / 09 / 98$ & $<0.02$ & 6.0 & $<0.004$ & $<0.06$ & 0.27 & 2.2 \\
\hline
\end{tabular}


Table E.5d. Trace Metals by Inductively Coupled Argon Plasma-Optical Emission Spectroscopy ( $\mathrm{Se}, \mathrm{Si}, \mathrm{Ag}, \mathrm{Na}, \mathrm{Sr}, \mathrm{V}$, and $\mathrm{Zn}$ )

\begin{tabular}{|c|c|c|c|c|c|c|c|c|c|}
\hline $\begin{array}{c}\text { Sample } \\
\mathbf{I}\end{array}$ & $\begin{array}{l}\text { Well } \\
\vdots \\
\vdots\end{array}$ & $\begin{array}{l}\text { Collection } \\
\text { Date }\end{array}$ & mgn & $\begin{array}{l}\text { Silicon } \\
\text { ing }\end{array}$ & $\begin{array}{l}\text { Silver } \\
\text { mg/L. }\end{array}$ & $\begin{array}{l}\text { Sodium } \\
\text { mgth }\end{array}$ & $\begin{array}{l}\text { trontinm } \\
\text { mitity }\end{array}$ & $\begin{array}{l}\text { anadiuma } \\
\text { mg/L }\end{array}$ & ming \\
\hline FL20 & RM-1A & $09 / 30 / 98$ & $<0.06$ & 11 & $<0.02$ & 6.6 & 0.073 & $<0.02$ & $<0.006$ \\
\hline FL34 & $R M-1 A$ & $10 / 12 / 98$ & $<0.06$ & 0.2 & $<0.02$ & 6.4 & 0.072 & $<0.02$ & $<0.006$ \\
\hline FL47 & RM-1A & $11 / 09 / 98$ & $<0.06$ & 11 & $<0.02$ & 6.2 & 0.074 & $<0.02$ & $<0.006$ \\
\hline FL21 & RM-1B & $09 / 30 / 98$ & $<0.06$ & 11 & $<0.02$ & 12.0 & 0.083 & $<0.02$ & $<0.006$ \\
\hline FL35 & RM-1B & $10 / 12 / 98$ & $<0.06$ & 11 & $<0.02$ & 12.0 & 0.082 & $<0.02$ & $<0.006$ \\
\hline FL52 & RM-1B & $11 / 09 / 98$ & $<0.06$ & 11 & $<0.02$ & 11.0 & 0.081 & $<0.02$ & $<0.006$ \\
\hline FL22 & $R M-2$ & $09 / 30 / 98$ & $<0.06$ & 12 & $<0.02$ & 7.1 & 0.074 & $<0.02$ & $<0.006$ \\
\hline FL36 & RM-2 & $10 / 12 / 98$ & $<0.06$ & 11 & $<0.02$ & 6.9 & 0.074 & $<0.02$ & $<0.006$ \\
\hline FL54 & RM-2 & $11 / 09 / 98$ & $<0.06$ & 12 & $<0.02$ & 6.3 & 0.074 & $<0.02$ & $<0.006$ \\
\hline FL23 & RM-3 & $09 / 30 / 98$ & $<0.06$ & 12 & $<0.02$ & 5.5 & 0.070 & $<0.02$ & $<0.006$ \\
\hline FL31 & $R M-3$ & $09 / 30 / 98$ & $<0.06$ & 12 & $<0.02$ & 5.5 & 0.070 & $<0.02$ & $<0.006$ \\
\hline FL37 & RM-3 & $10 / 12 / 98$ & $<0.06$ & 12 & $<0.02$ & 5.5 & 0.070 & $<0.02$ & $<0.006$ \\
\hline FL55 & RM-3 & $11 / 09 / 98$ & $<0.06$ & 12 & $<0.02$ & 5.7 & 0.071 & $<0.02$ & $<0.006$ \\
\hline FL56 & $\mathrm{RM}-3$ & $11 / 09 / 98$ & $<0.06$ & 12 & $<0.02$ & 5.4 & 0.068 & $<0.02$ & $<0.006$ \\
\hline FL24 & $\mathrm{RM}-4$ & $09 / 30 / 98$ & $<0.06$ & 12 & $<0.02$ & 5.9 & 0.070 & $<0.02$ & $<0.006$ \\
\hline FL38 & RM-4 & $10 / 12 / 98$ & $<0.06$ & 12 & $<0.02$ & 6.1 & 0.073 & $<0.02$ & $<0.006$ \\
\hline FL48 & $R M-4$ & $11 / 09 / 98$ & $<0.06$ & 12 & $<0.02$ & 5.8 & 0.071 & $<0.02$ & $<0.006$ \\
\hline FL25 & RM-5 & $09 / 30 / 98$ & $<0.06$ & 12 & $<0.02$ & 6.4 & 0.074 & $<0.02$ & $<0.006$ \\
\hline FL39 & RM-5 & $10 / 12 / 98$ & $<0.06$ & 12 & $<0.02$ & 6.5 & 0.078 & $<0.02$ & $<0.006$ \\
\hline FL57 & RM-5 & $11 / 09 / 98$ & $<0.06$ & 12 & $<0.02$ & 6.3 & 0.076 & $<0.02$ & $<0.006$ \\
\hline FL26 & RM-6 & $09 / 30 / 98$ & $<0.06$ & 10 & $<0.02$ & 9.6 & 0.080 & $<0.02$ & $<0.006$ \\
\hline FL40 & $R M-6$ & $10 / 12 / 98$ & $<0.06$ & 1.0 & $<0.02$ & 7.2 & 0.078 & $<0.02$ & $<0.006$ \\
\hline FL41 & RM-6 & $10 / 12 / 98$ & $<0.06$ & 12 & $<0.02$ & 7.2 & 0.078 & $<0.02$ & $<0.006$ \\
\hline FL:50 & $\mathrm{RM}-6$ & $11 / 09 / 98$ & $<0.06$ & 11 & $<0.02$ & 7.7 & 0.078 & $<0.02$ & $<0.006$ \\
\hline FL27 & RM-7 & $09 / 30 / 98$ & $<0.06$ & 12.5 & $<0.02$ & 6.2 & 0.076 & $<0.02$ & $<0.006$ \\
\hline FL42 & $\mathrm{RM}-7$ & $10 / 12 / 98$ & $<0.06$ & 12 & $<0.02$ & 6.0 & 0.074 & $<0.02$ & $<0.006$ \\
\hline FL43 & RM-7 & $10 / 12 / 98$ & $<0.06$ & 12 & $<0.02$ & 6.1 . & 0.075 & $<0.02$ & $<0.006$ \\
\hline FL49 & RM-7 & $11 / 09 / 98$ & $<0.06$ & 12 & $<0.02$ & 5.8 & 0.072 & $<0.02$ & $<0.006$ \\
\hline FL28 & RM-8 & $09 / 30 / 98$ & $<0.06$ & 12 & $<0.02$ & 5.8 & 0.072 & $<0.02$ & 0.041 \\
\hline FL44 & RM-8 & $10 / 12 / 98$ & $<0.06$ & 12 & $<0.02$ & 5.9 & 0.073 & $<0.02$ & $<0.006$ \\
\hline FL51 & RM-8 & $11 / 09 / 98$ & $<0.06$ & 12 & $<0.02$ & 5.8 & 0.073 & $<0.02$ & $<0.006$ \\
\hline FL29 & RM-9 & $09 / 30 / 98$ & $<0.06$ & 12 & $<0.02$ & 5.7 & 0.076 & $<0.02$ & $<0.006$ \\
\hline FL30 & RM-9 & $09 / 30 / 98$ & $<0.06$ & 12 & $<0.02$ & 5.6 & 0.072 & $<0.02$ & $<0.006$ \\
\hline FL45 & RM-9 & $10 / 12 / 98$ & $<0.06$ & 12 & $<0.02$ & 5.8 & 0.074 & $<0.02$ & $<0.006$ \\
\hline FL58 & RM-9 & $11 / 09 / 98$ & $<0.06$ & 12 & $<0.02$ & 5.8 & 0.071 & $<0.02$ & $<0.006$ \\
\hline FL59 & RM-9 & $11 / 09 / 98$ & $<0.06$ & 12 & $<0.02$ & 5.7 & 0.069 & $<0.02$ & $<0.006$ \\
\hline
\end{tabular}


Appendix F

Tracer Test 


\section{Appendix F}

\section{Tracer Test}

This appendix contains the bromide breakthrough curves (BTC) for all the wells at the Fort Lewis ISRM proof-of-principle test site during the Bromide Tracer Test. The bromide data shown are from samples collected during the test and measured in the laboratory using ion chromatography. The bromide tracer test is discussed in Section 6. Table 6.1 contains a summary of the test operation.

Bromide Tracer Test Breakthrough Curves

F.1 

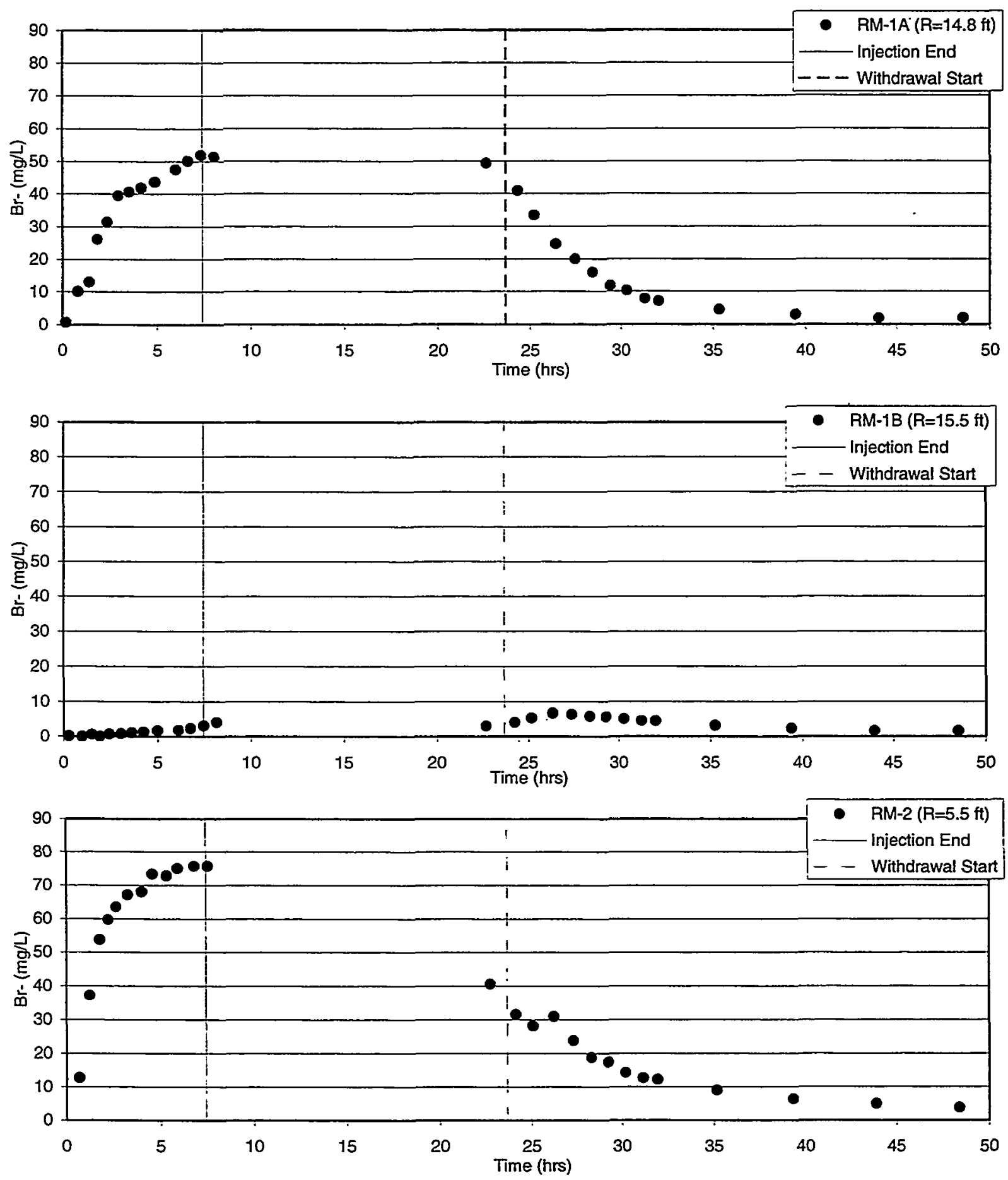

Bromide Tracer Test Breakthrough Curves

F.2 

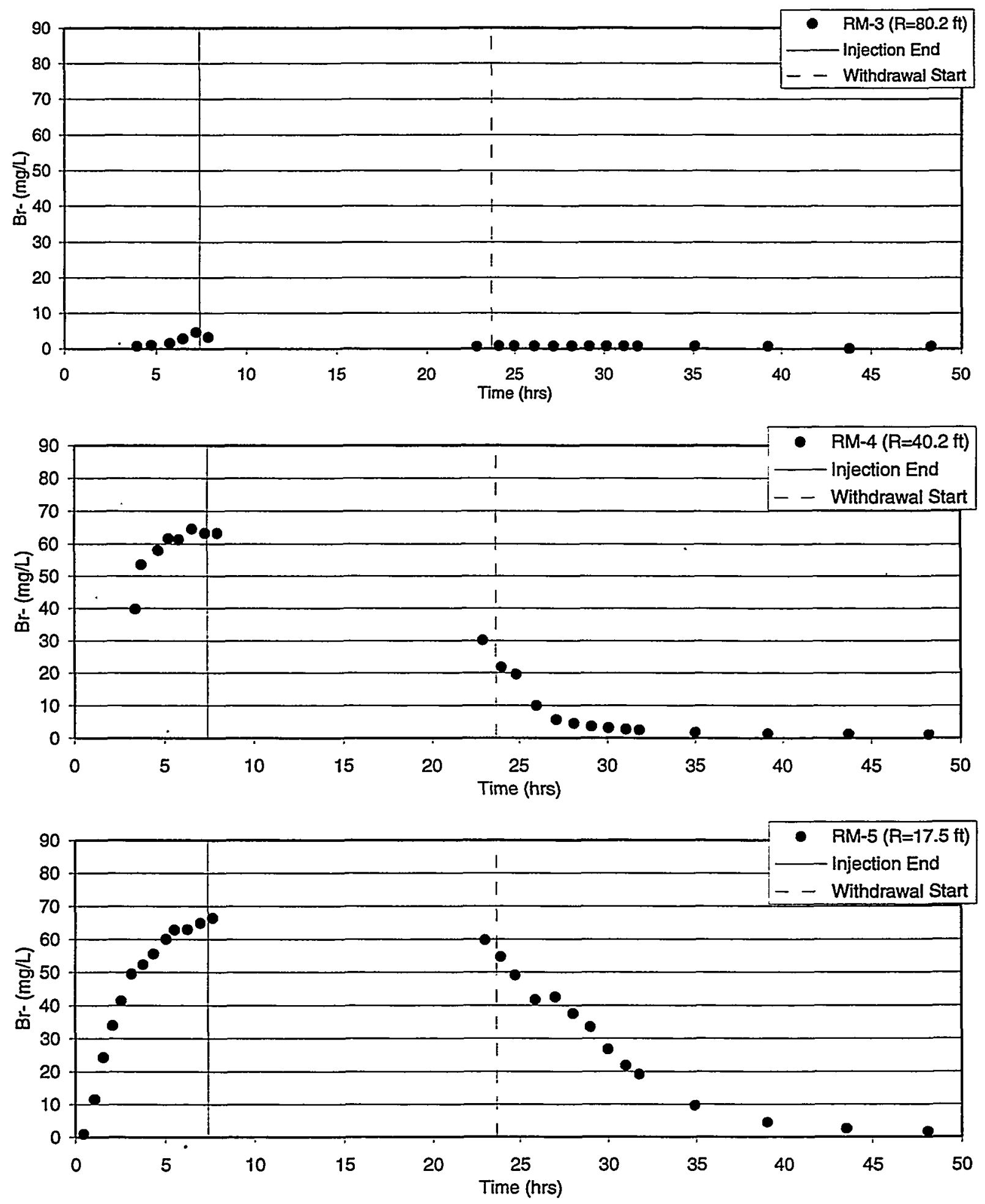

Bromide Tracer Test Breakthrough Curves

F.3 

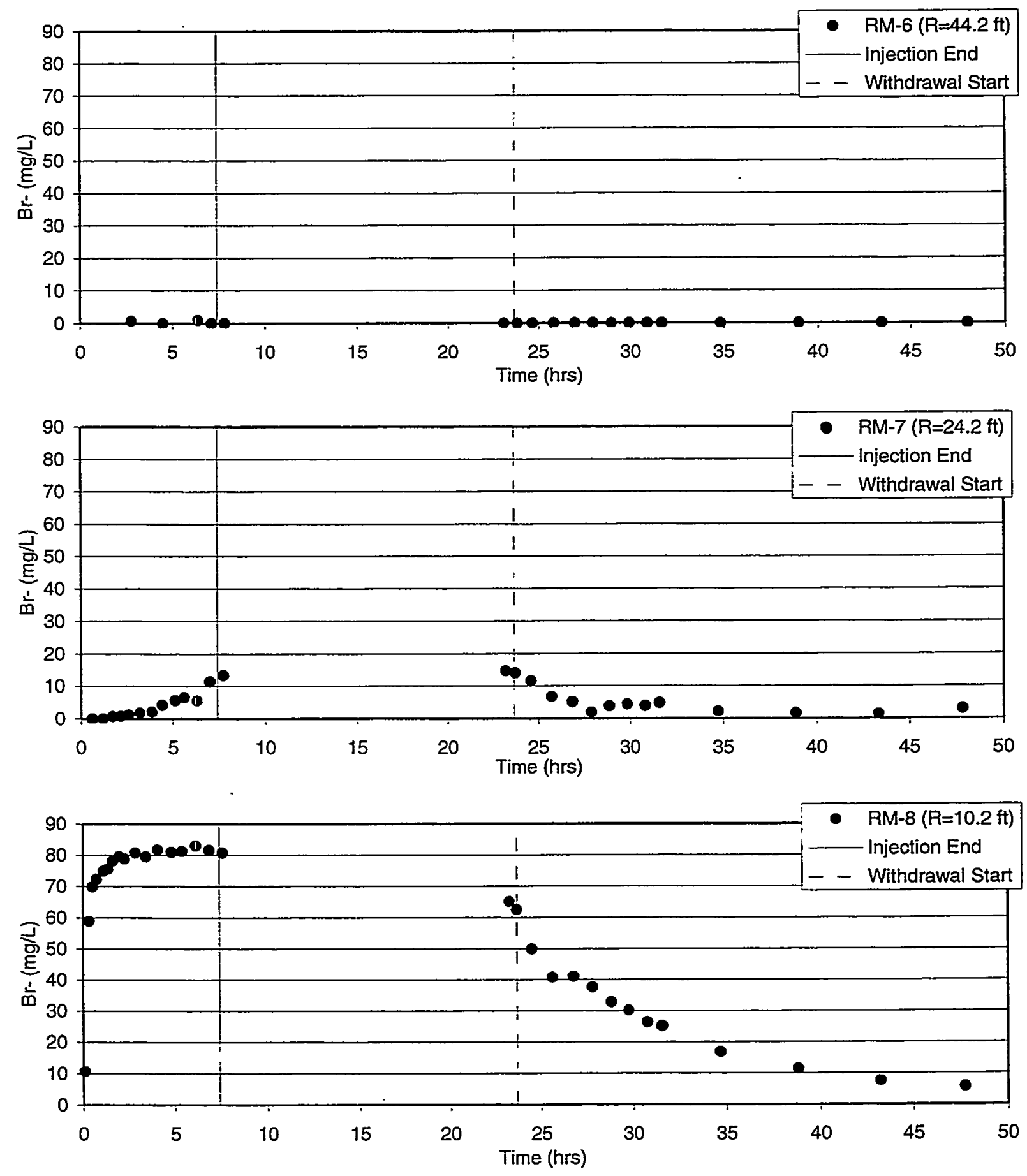

Bromide Tracer Test Breakthrough Curves

F.4 


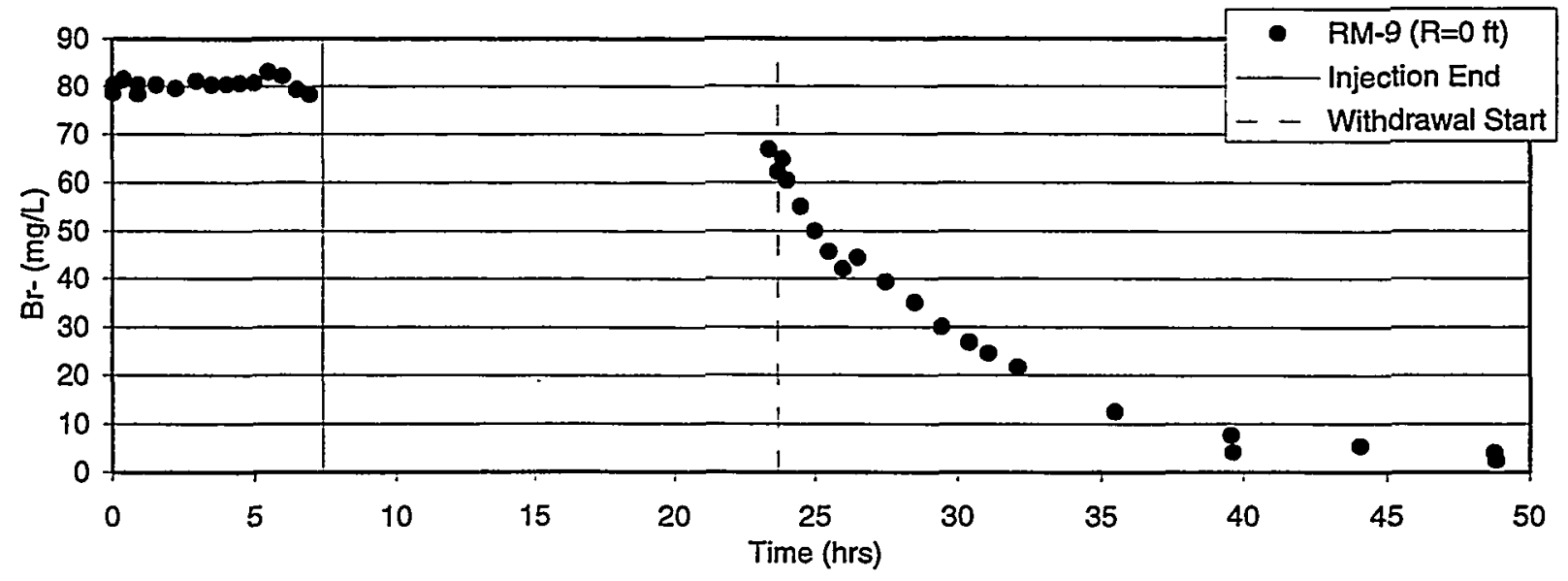

Bromide Tracer Test Breakthrough Curves

F.5 
Appendix G

\section{Dithionite Injection/Withdrawal}




\section{Appendix G}

\section{Dithionite Injection/Withdrawal}

This appendix contains dithionite and electrical conductivity (EC) measurements for all wells at the Fort Lewis proof-of-principle test site during dithionite Injection/Withdrawal Tests 1, 2a, 2b, 3, and 4. These data are from samples collected and analyzed at the ISRM site. These tests are discussed in Section 6. Table 6.1 contains a summary of the test operations. 

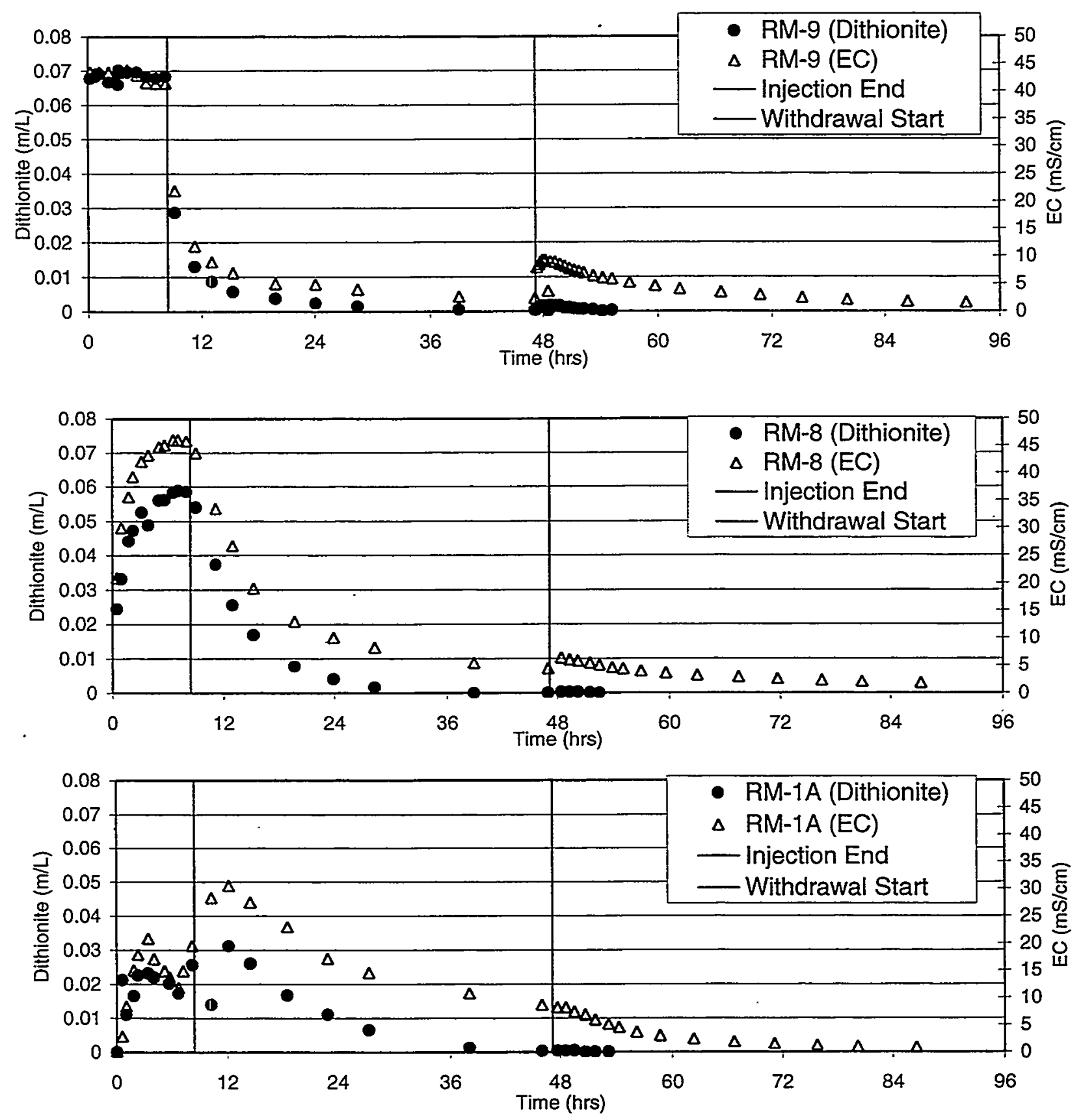

Dithionite Injection/Withdrawal \#1

G.2 

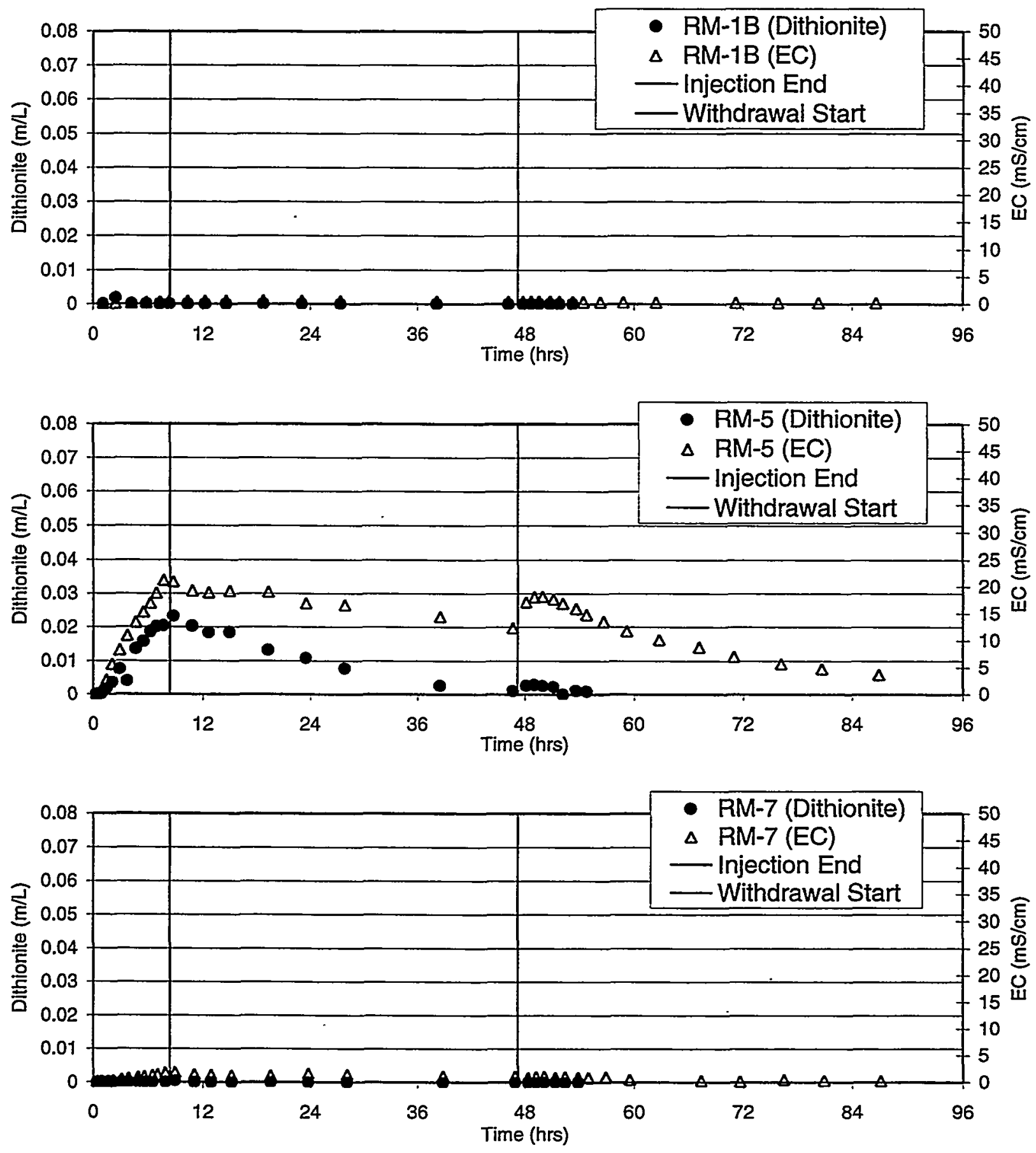

Dithionite Injection/Withdrawal \#1

G.3 

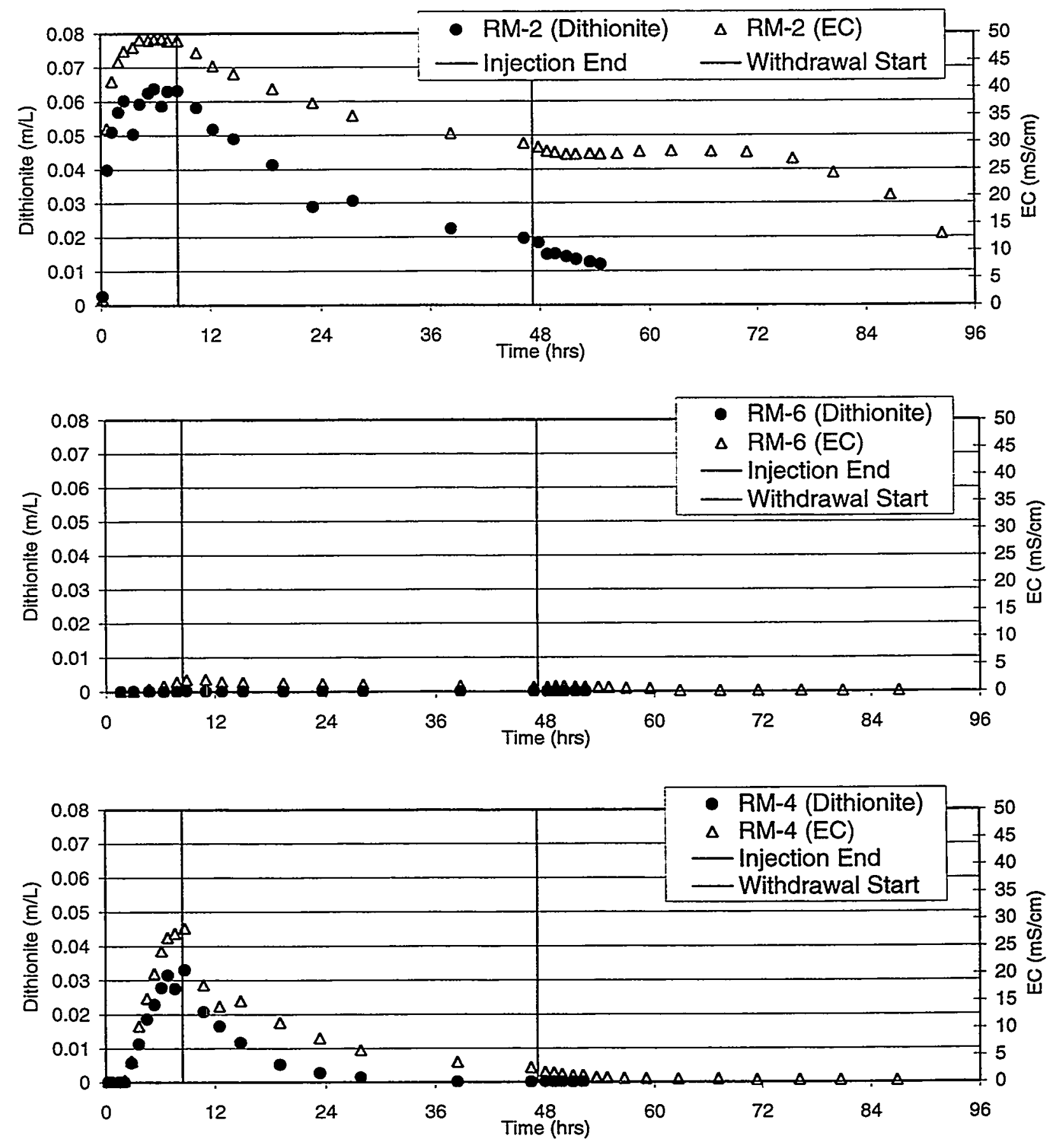

Dithionite Injection/Withdrawal \#1

G.4 


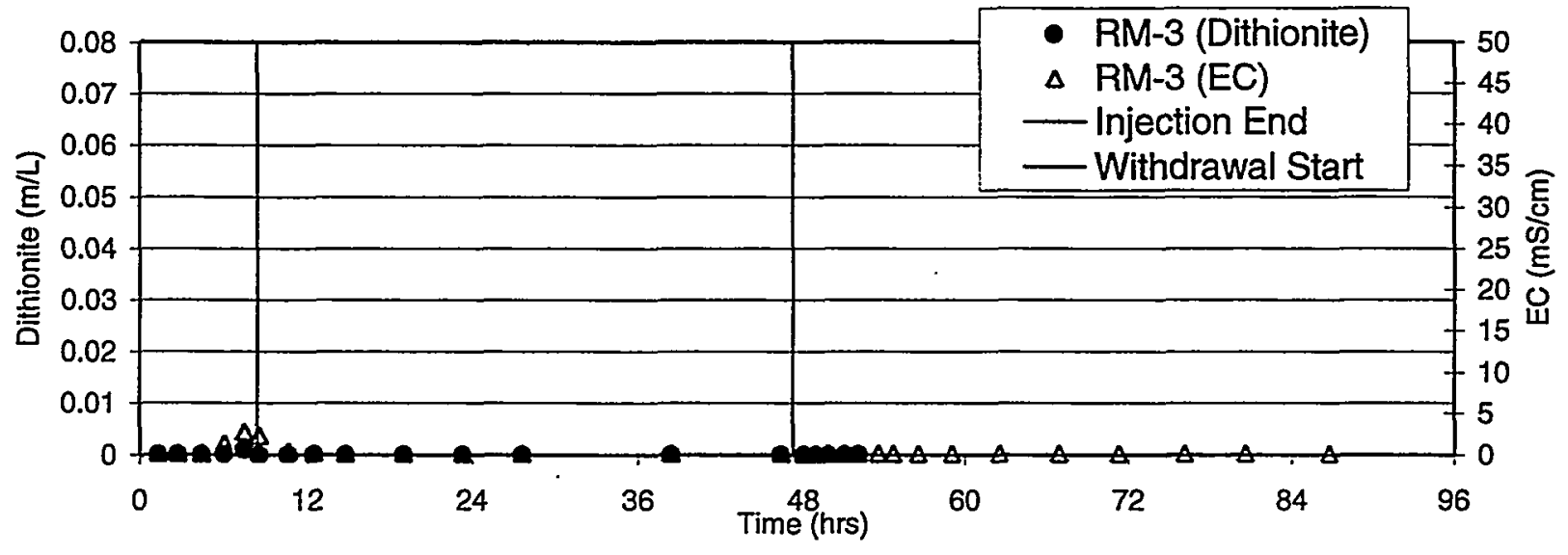

Dithionite Injection/Withdrawal \#1

G.5 

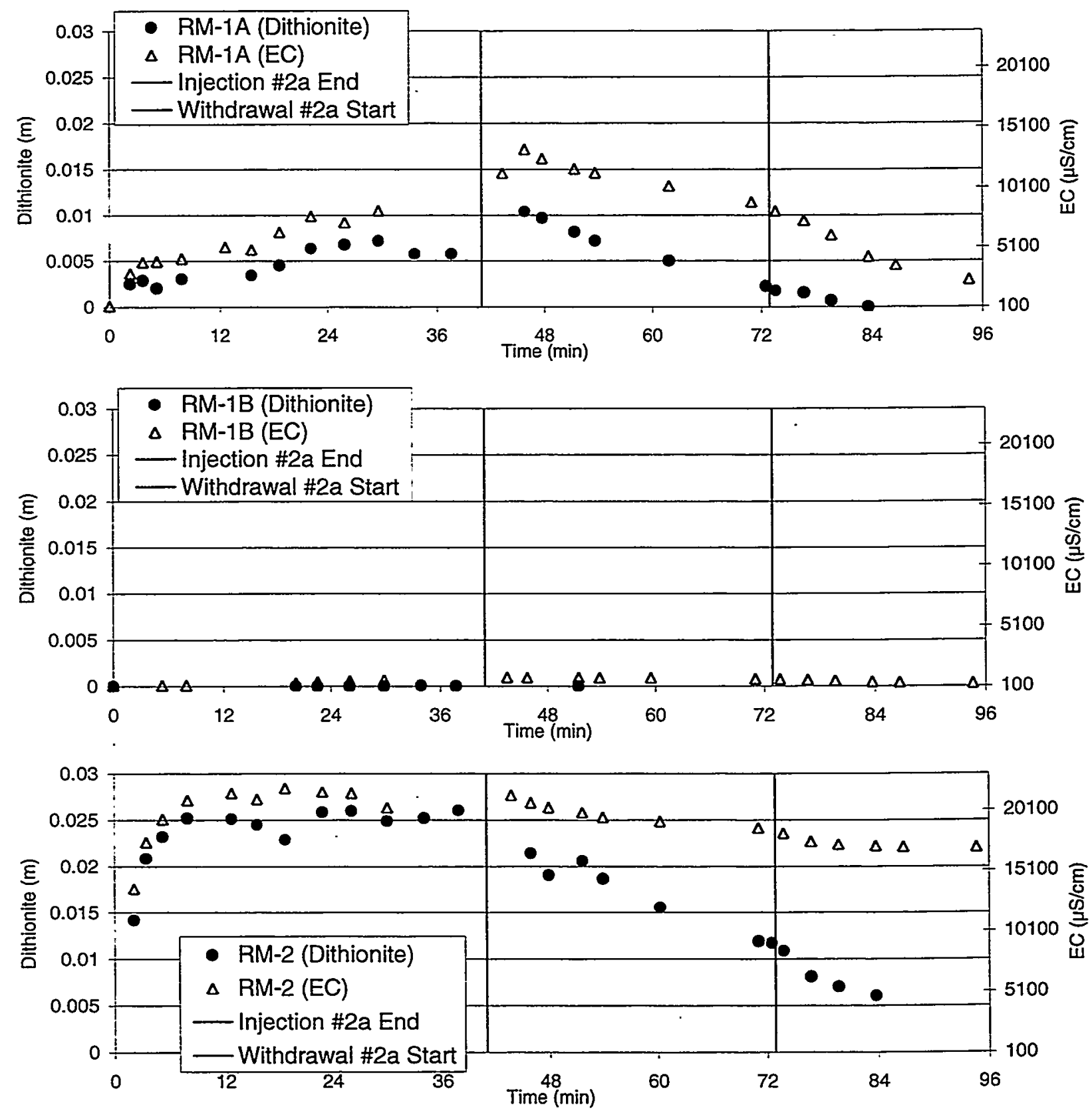

Dithionite Injection/Withdrawal $\# 2 a$

G.6 

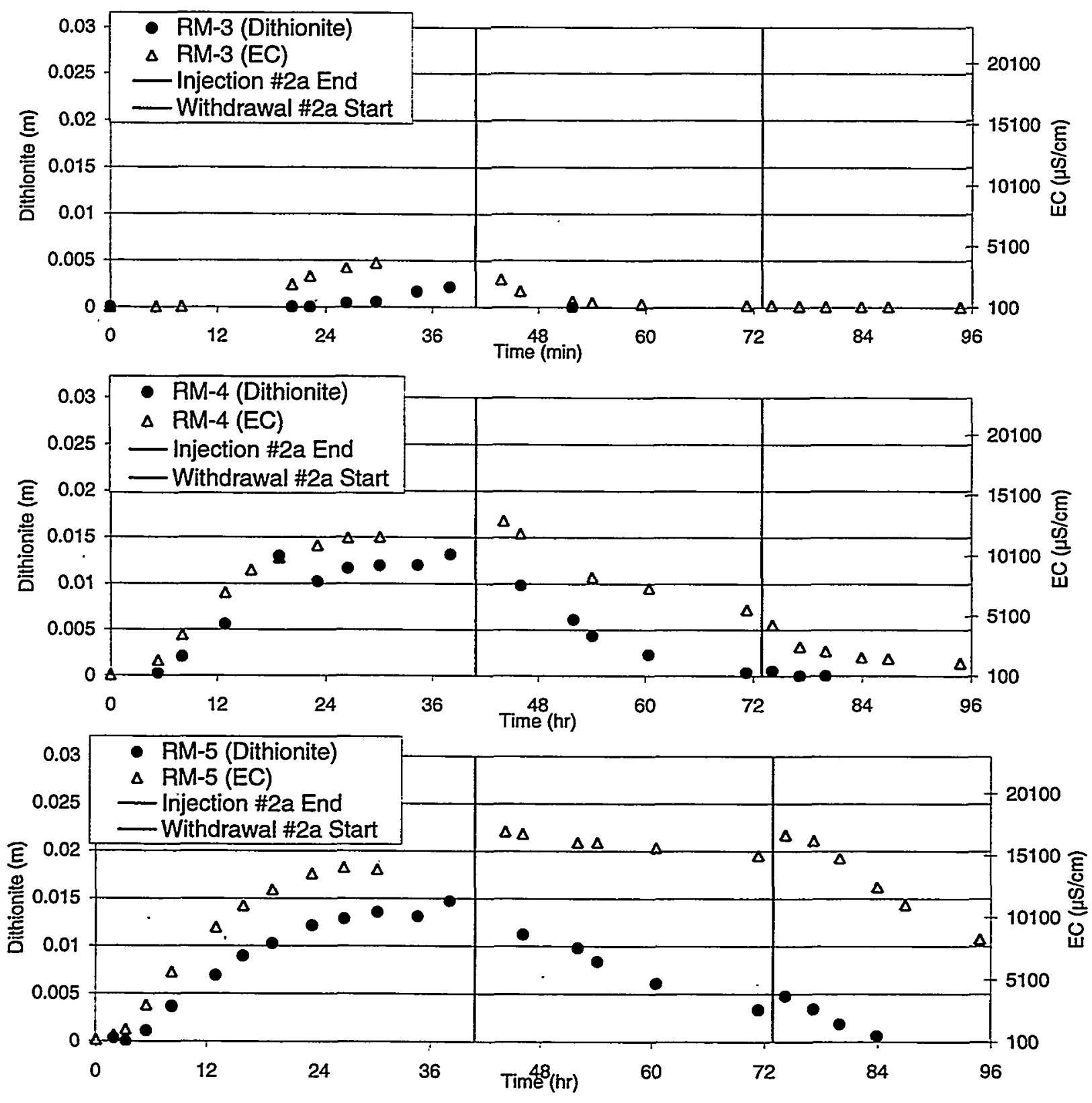

Dithionite Injection/Withdrawal \#2a

G.7 

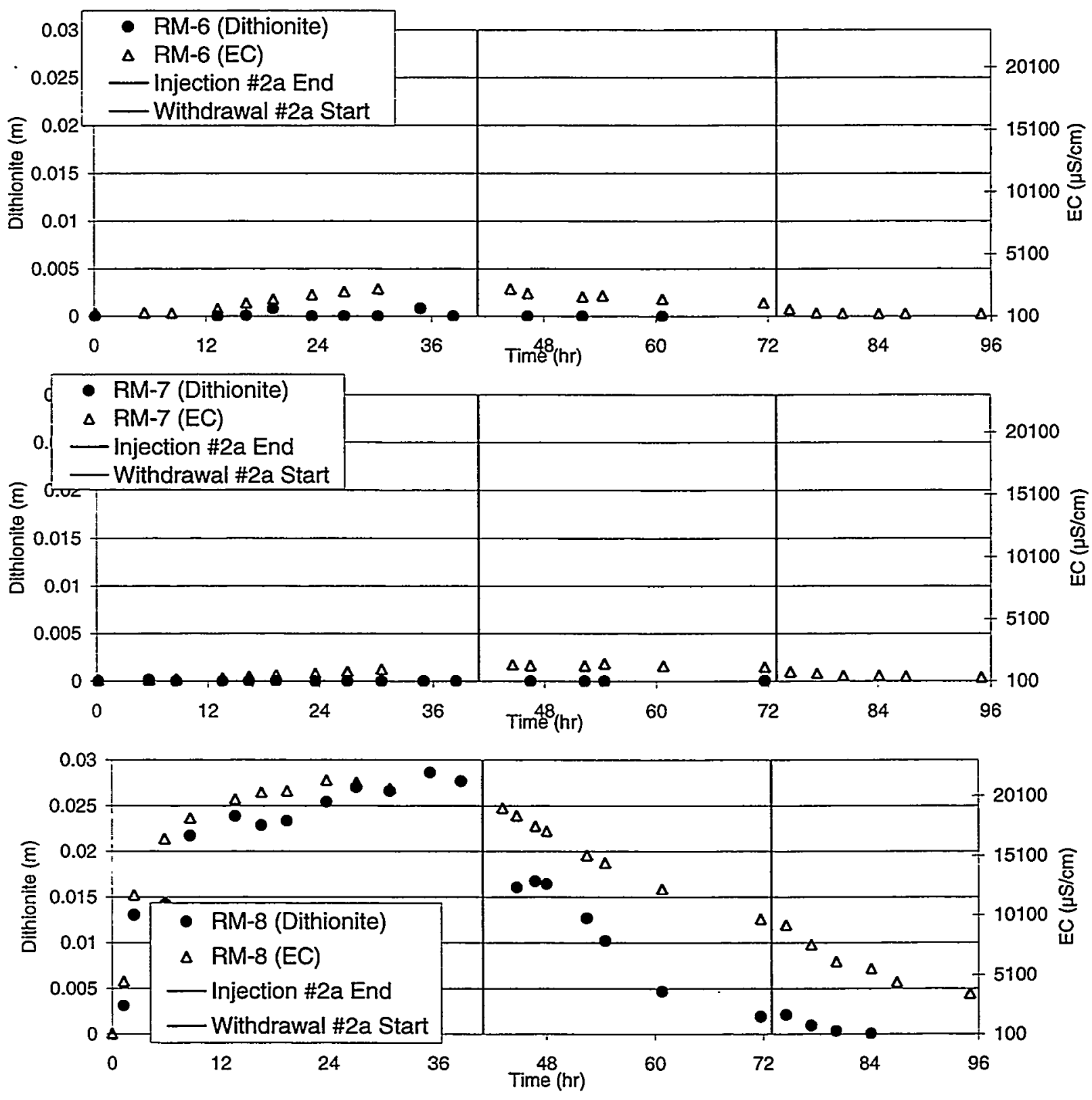

Dithionite Injection/Withdrawal \#2a

G.8 

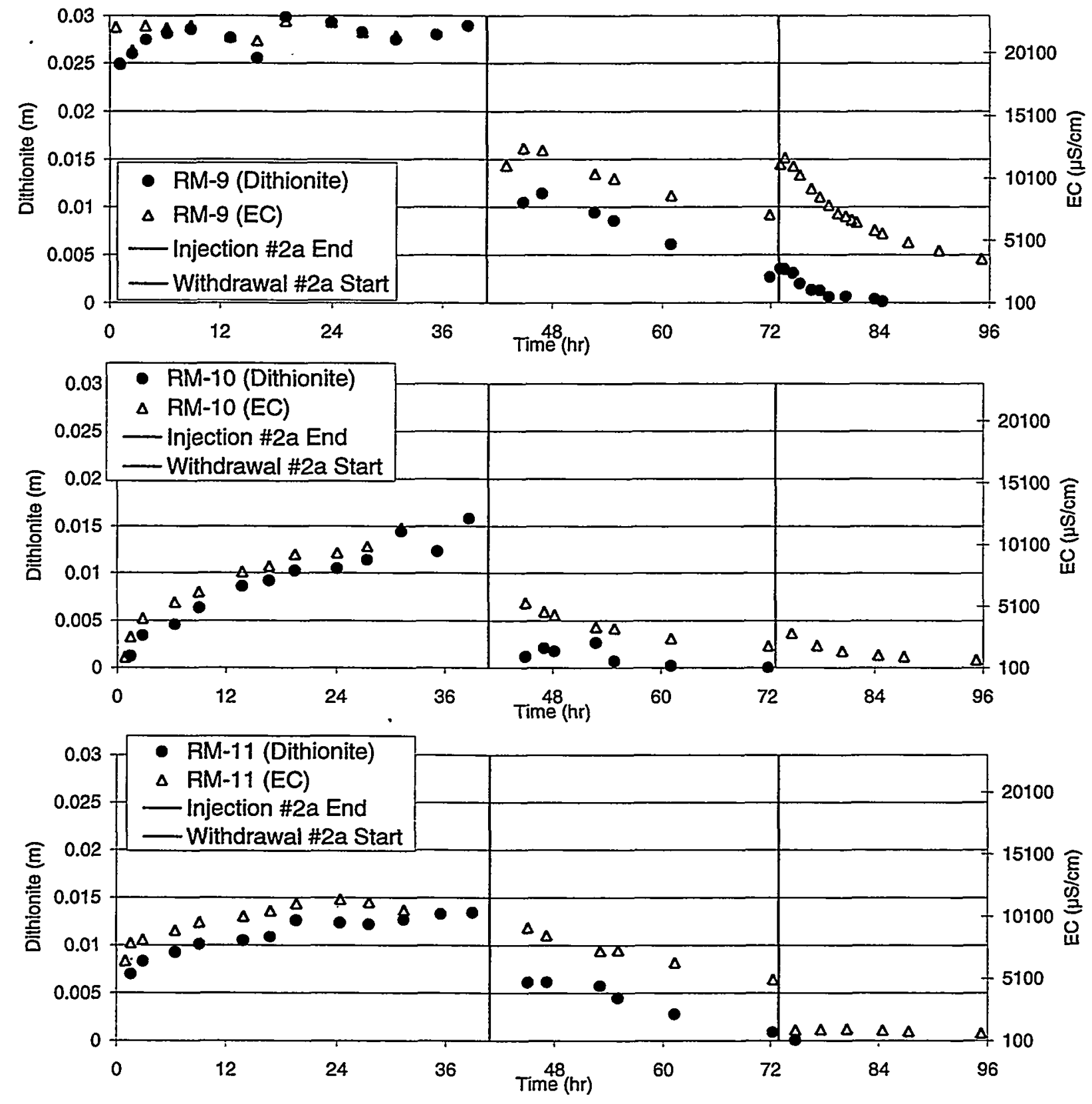

Dithionite Injection/Withdrawal \#2a

G.9 

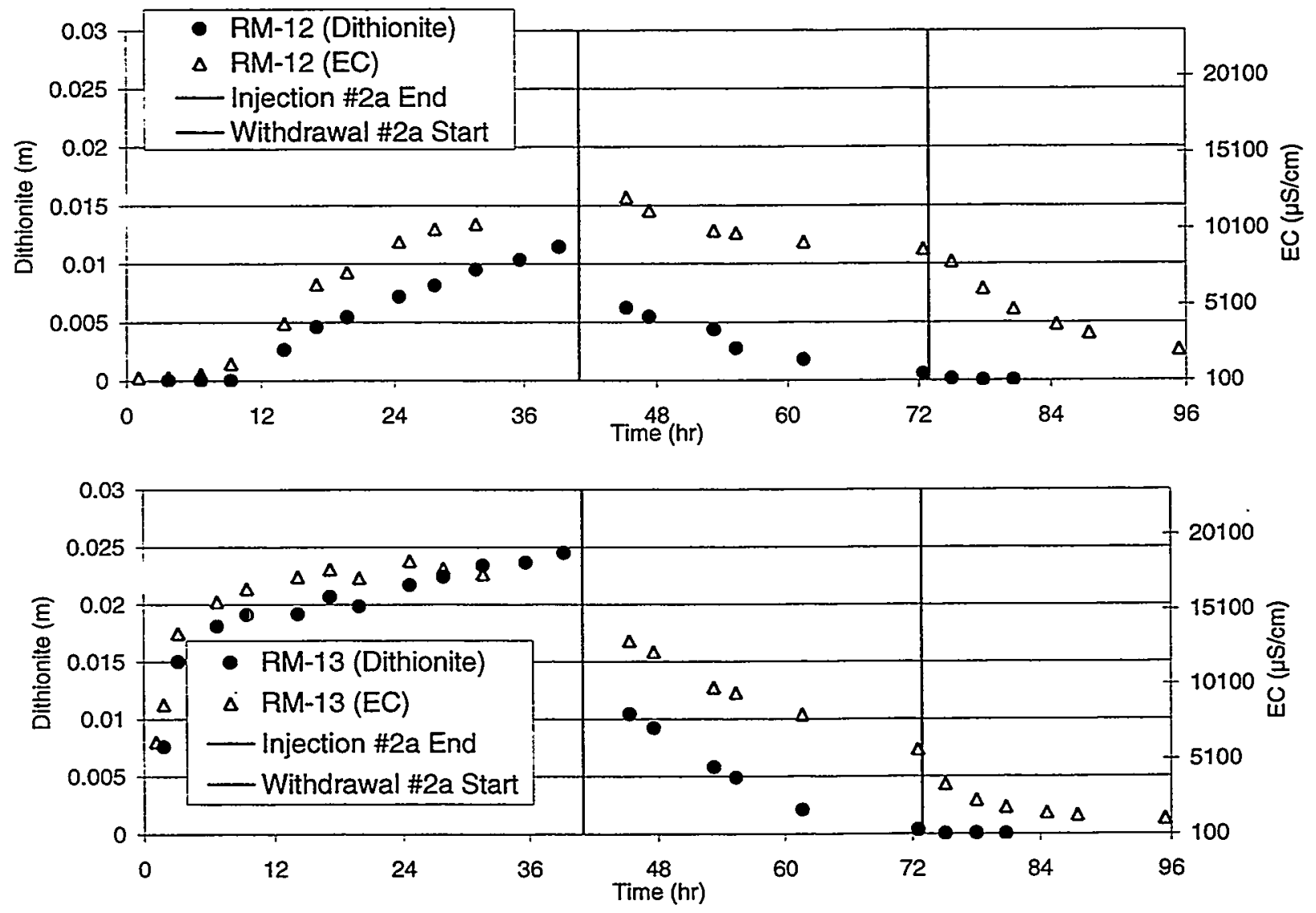

Dithionite Injection/Withdrawal \#2a

G.10 

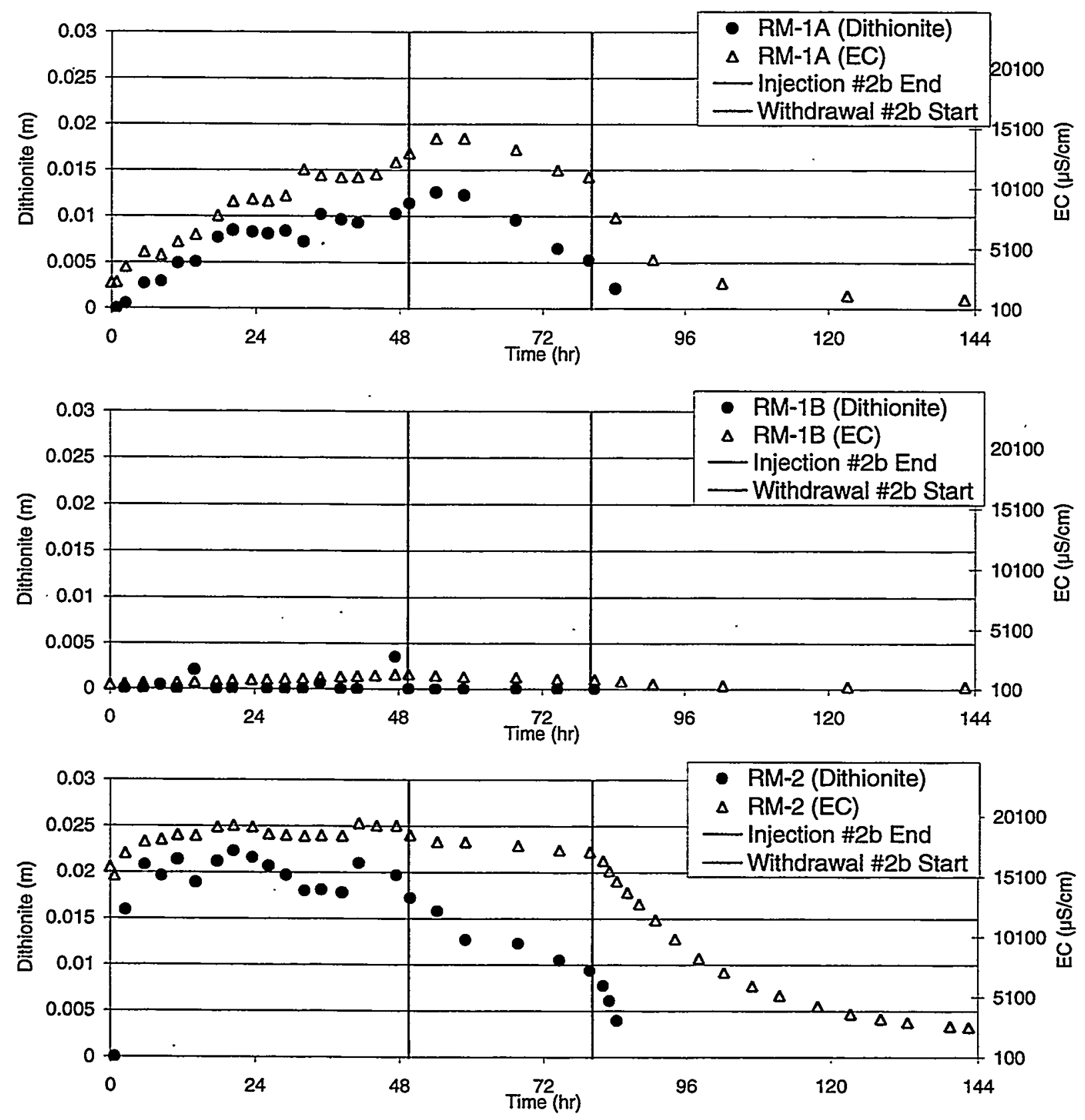

Dithionite Injection/Withdrawal \#2b

G.11 

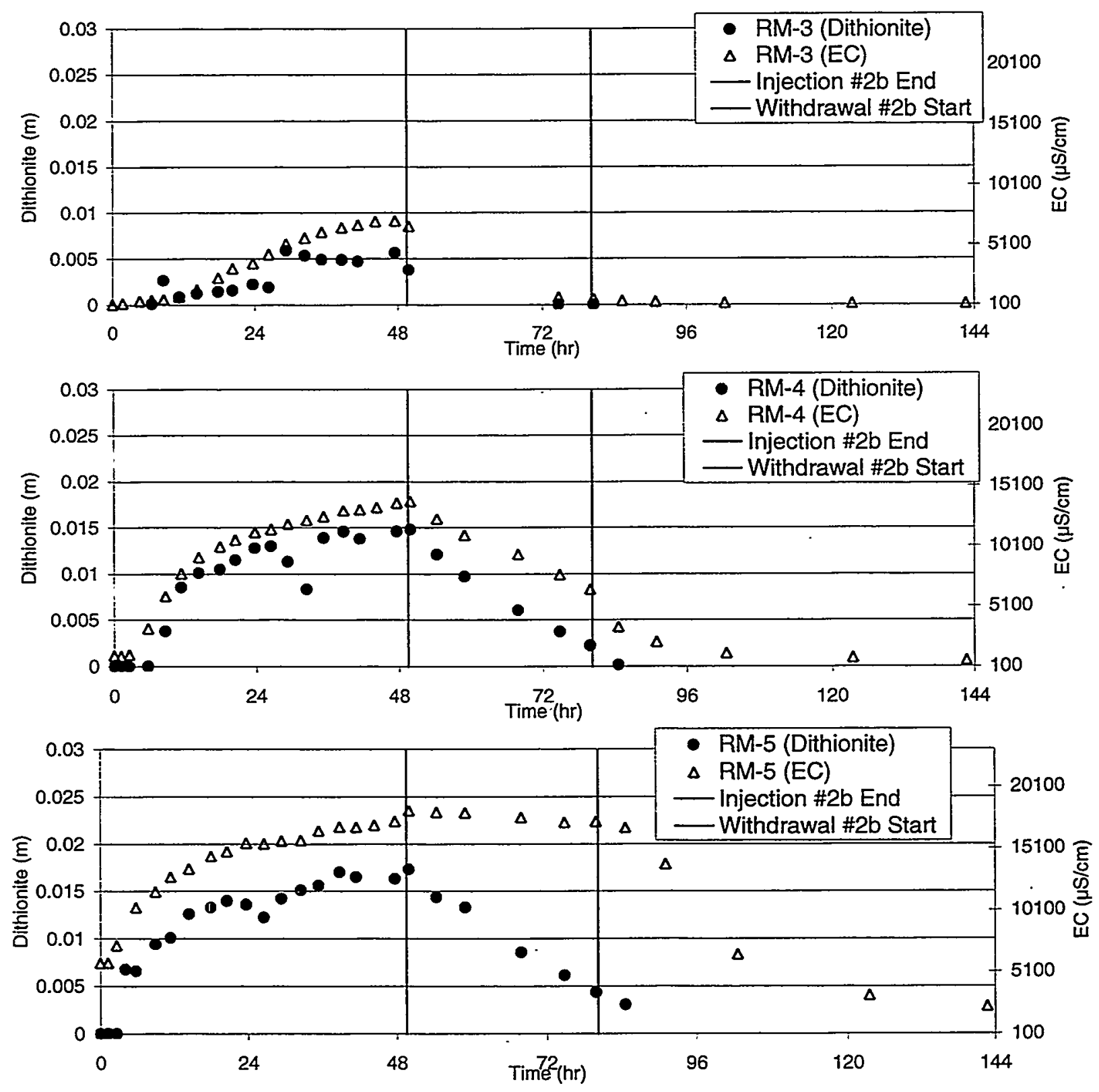

Dithionite Injection/Withdrawal \#2b

G.12 

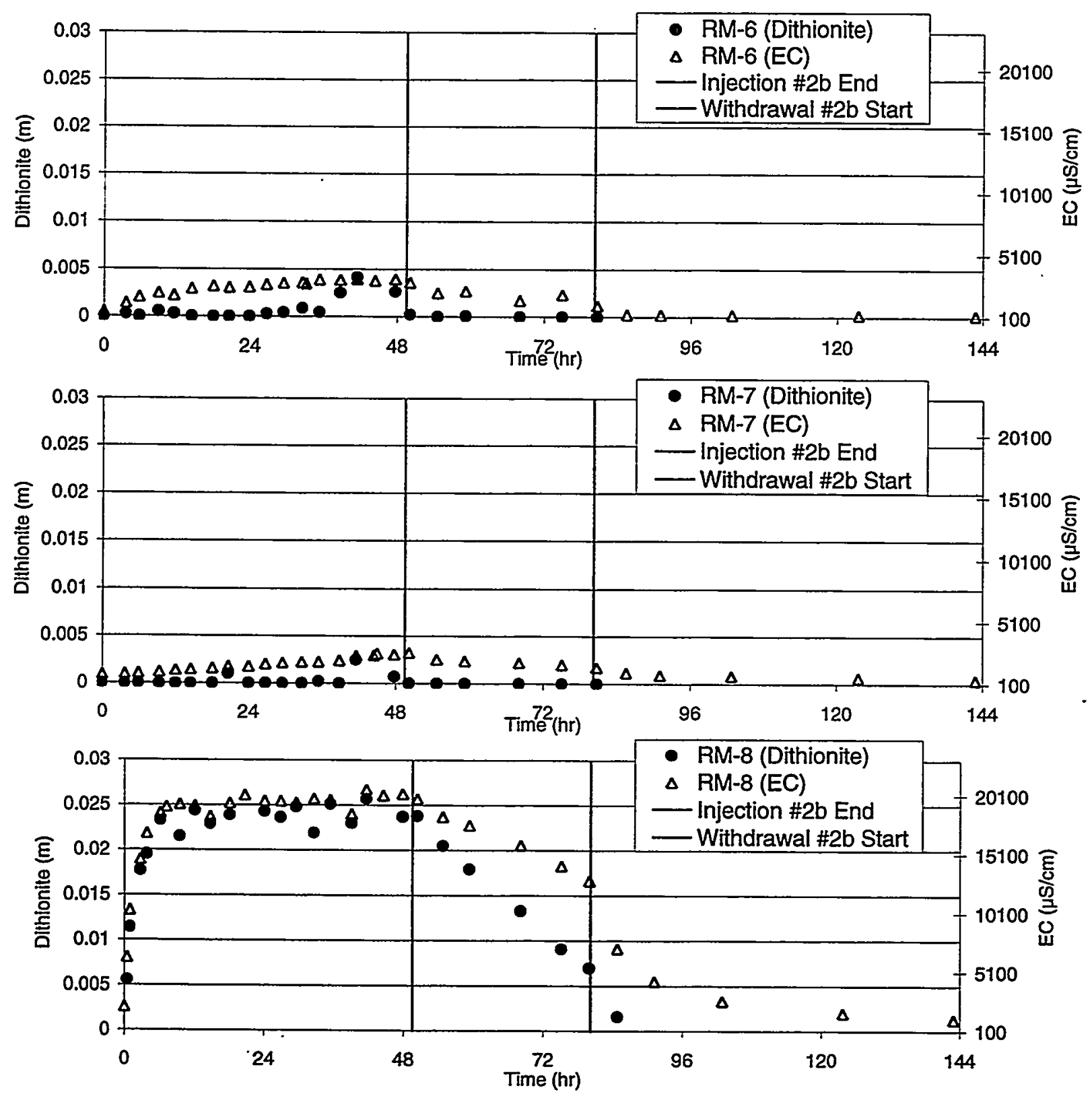

Dithionite Injection/Withdrawal $\# 2 \mathrm{~b}$

G.13 

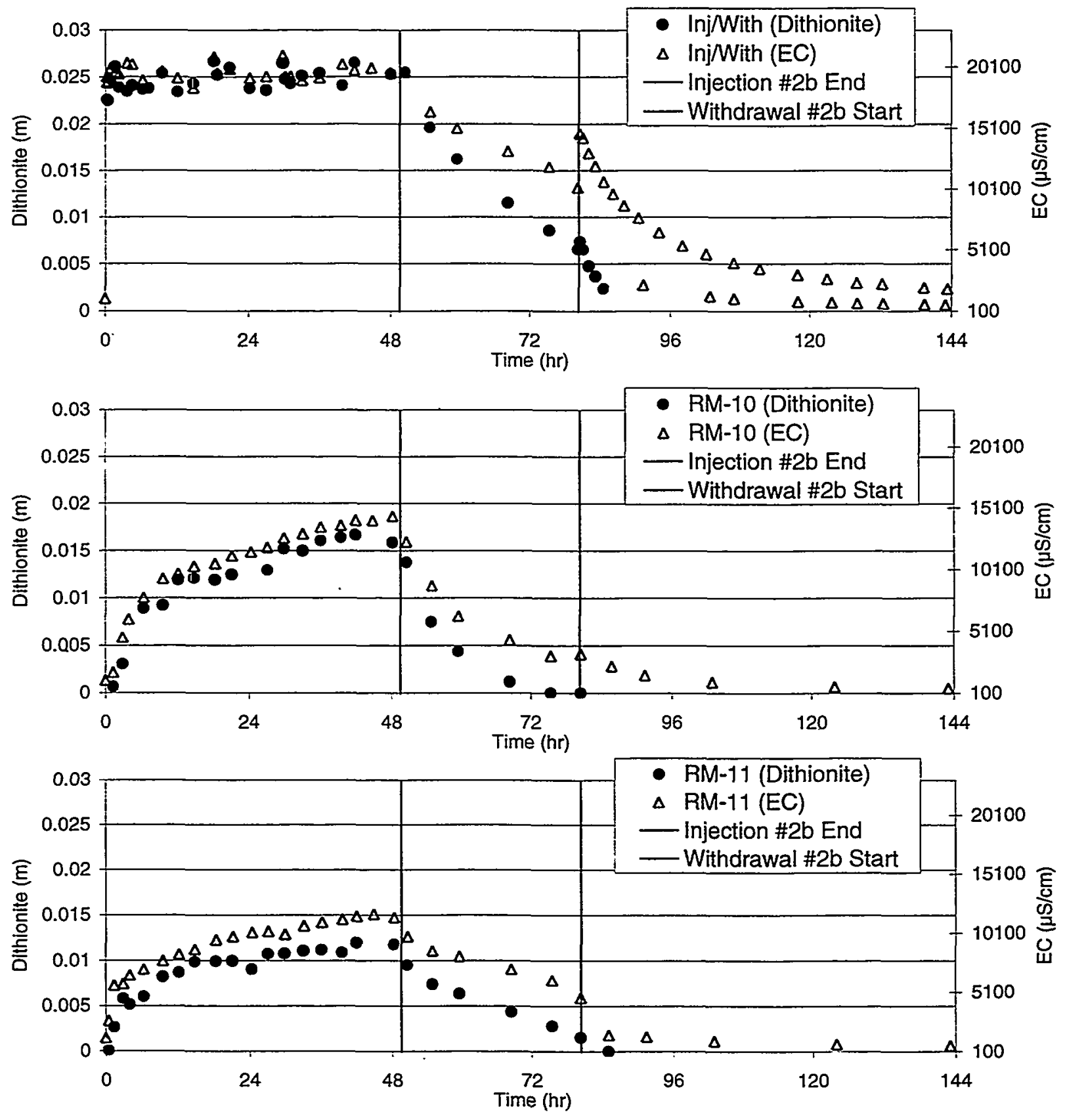

Dithionite Injection/Withdrawal \#2b

$$
\text { G.14 }
$$



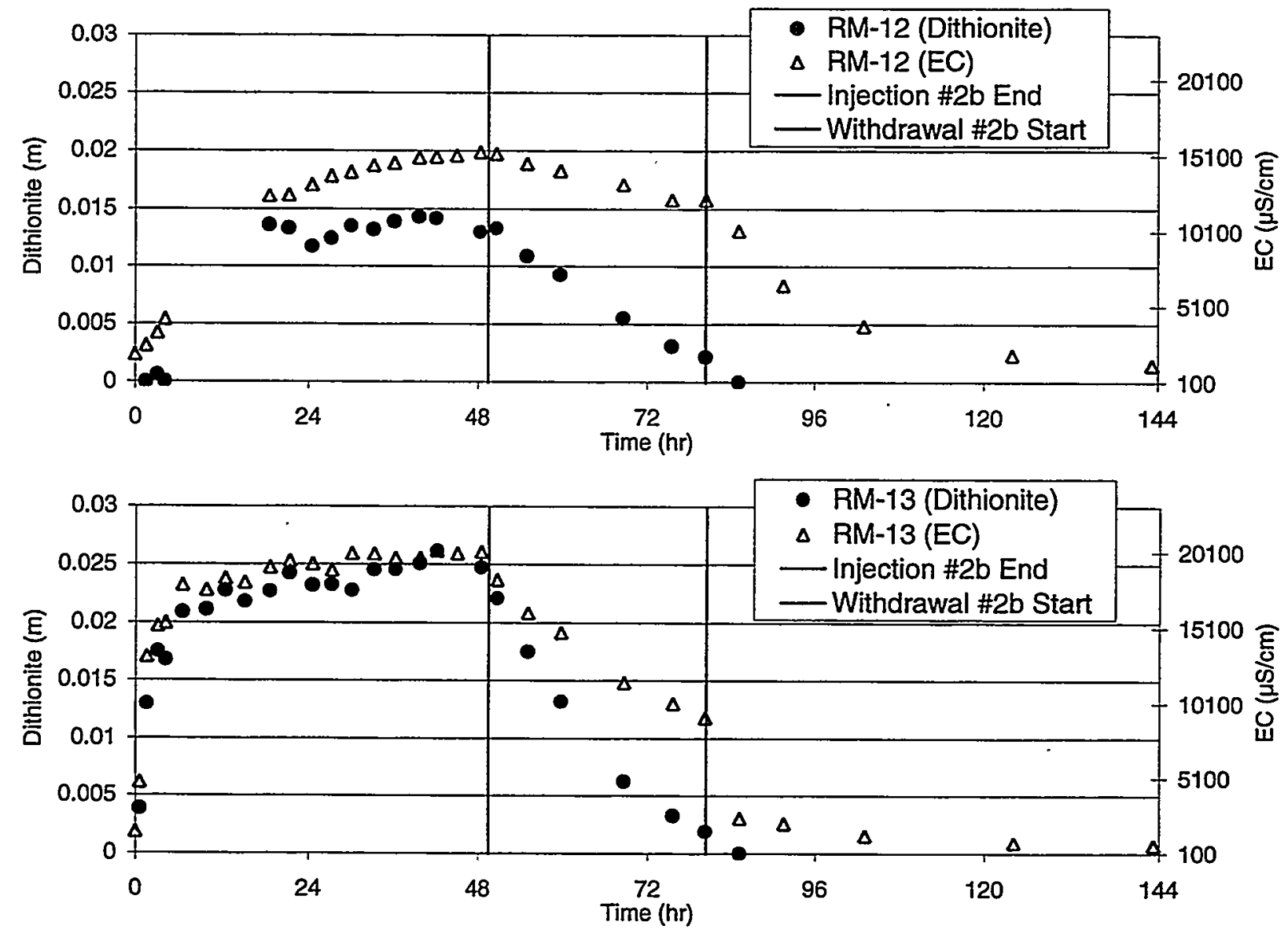

Dithionite Injection/Withdrawal \#2b

G.15 

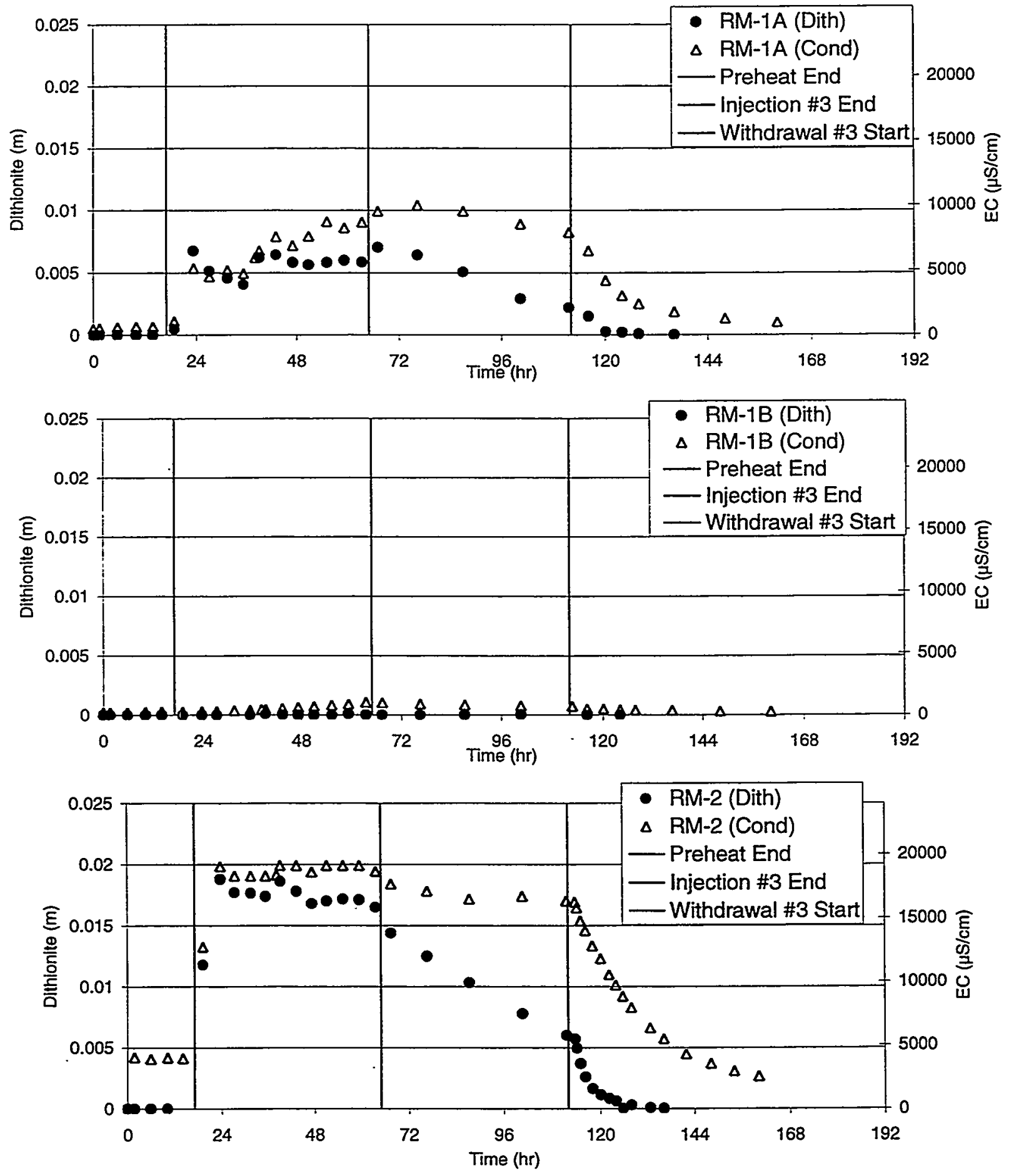

Dithionite Injection/Withdrawal \#3

G.16 

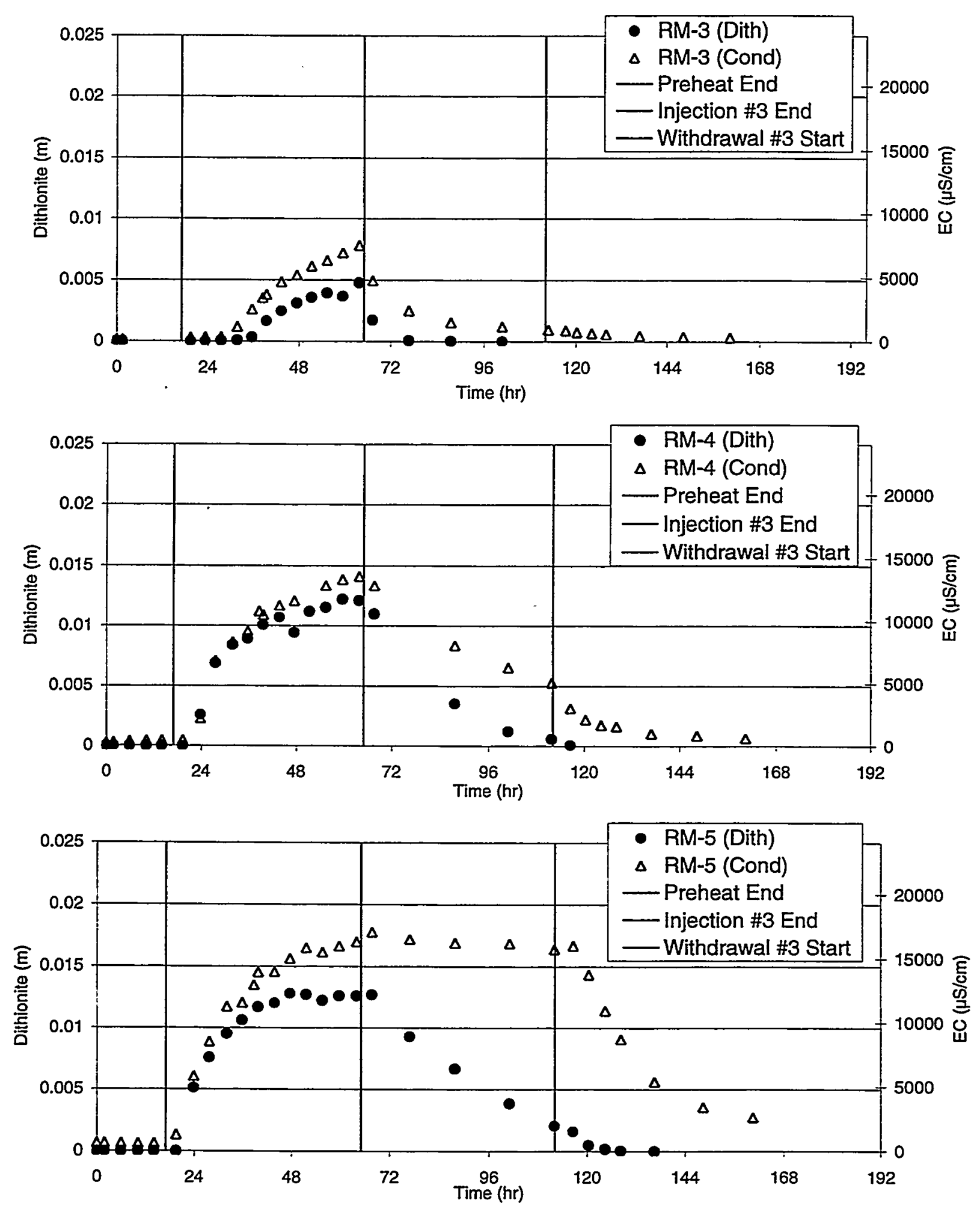

Dithionite Injection/Withdrawal \#3

G.17 

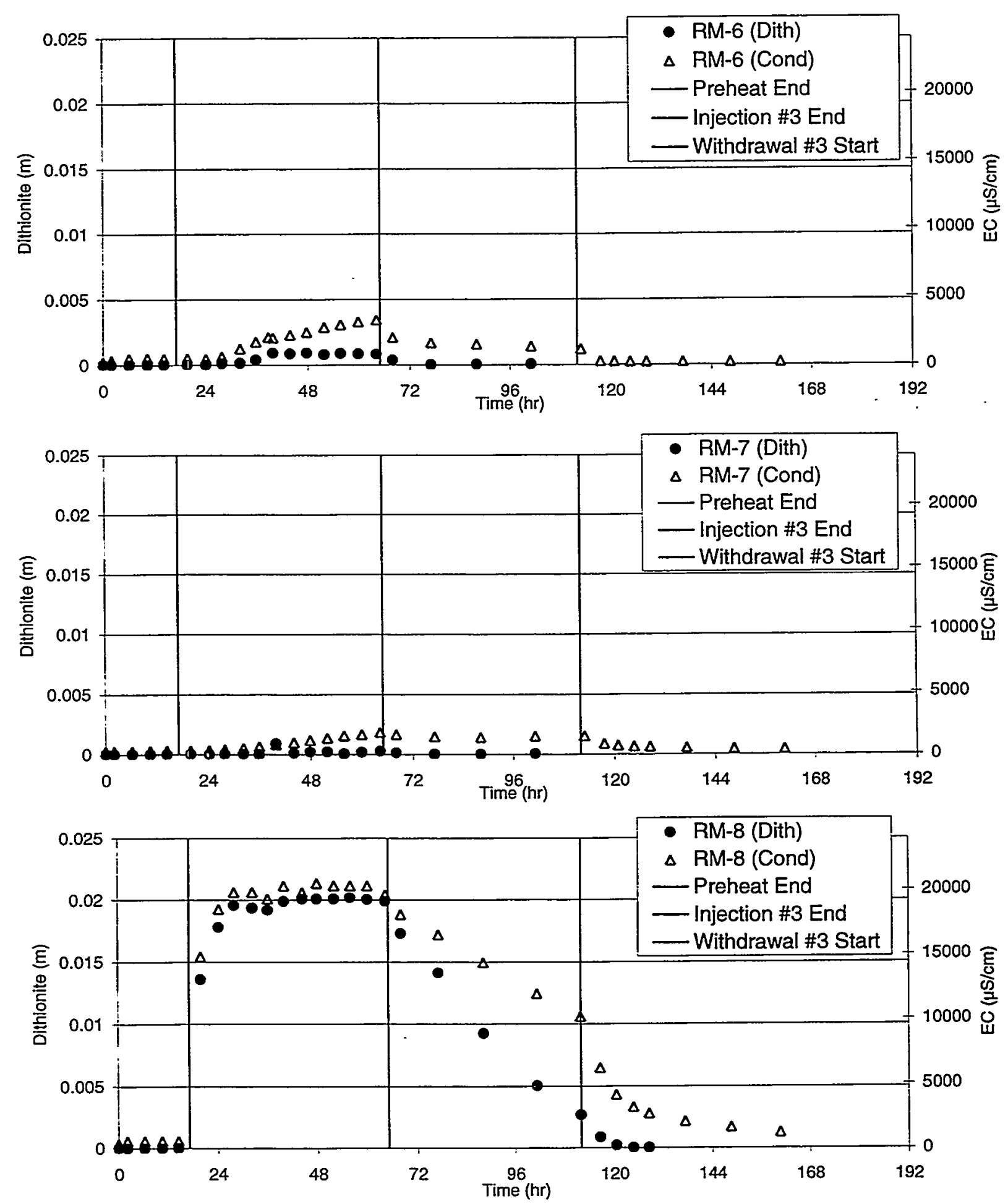

Dithionite Injection/Withdrawal \#3

G.18 

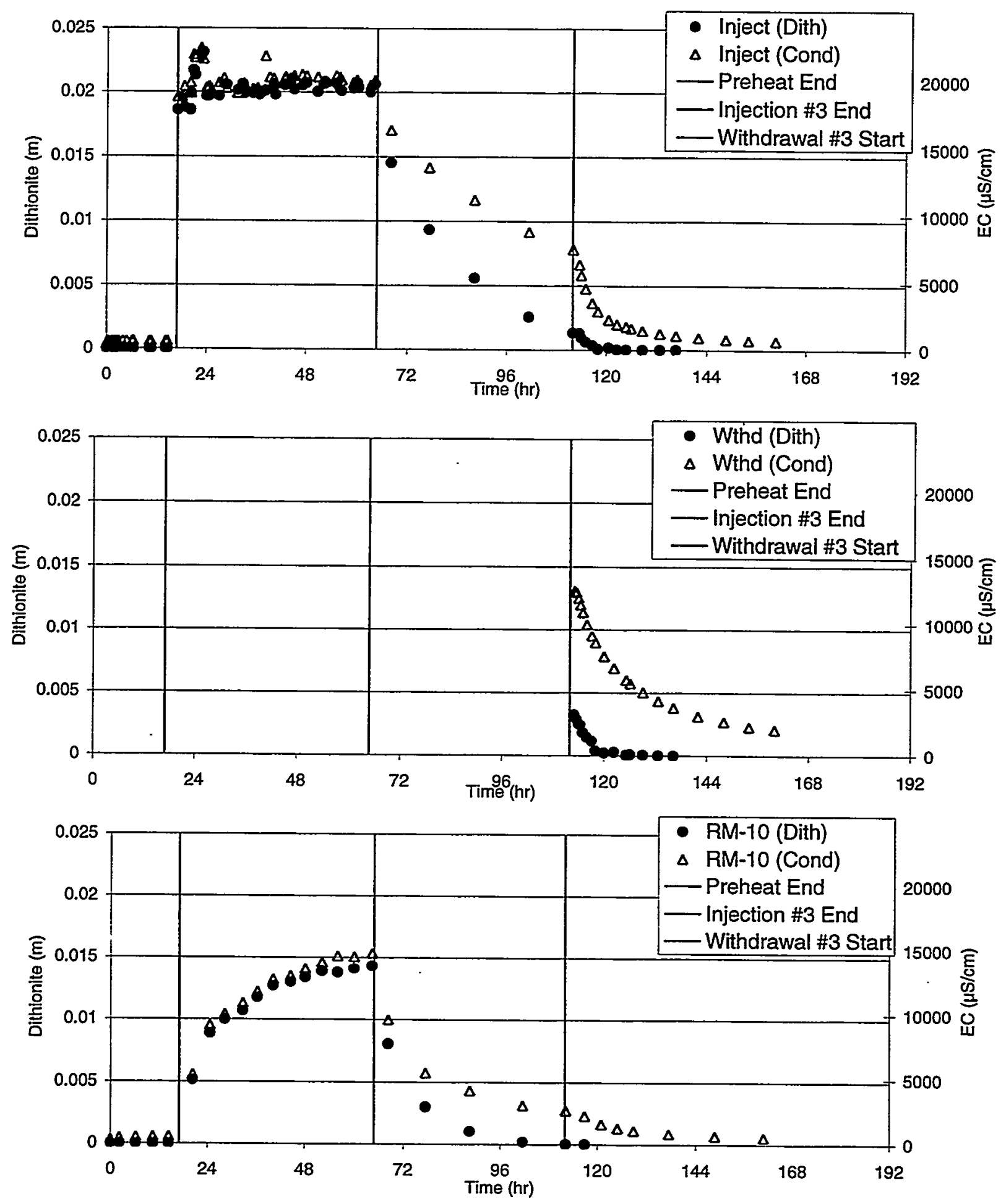

Dithionite Injection/Withdrawal \#3

G.19 

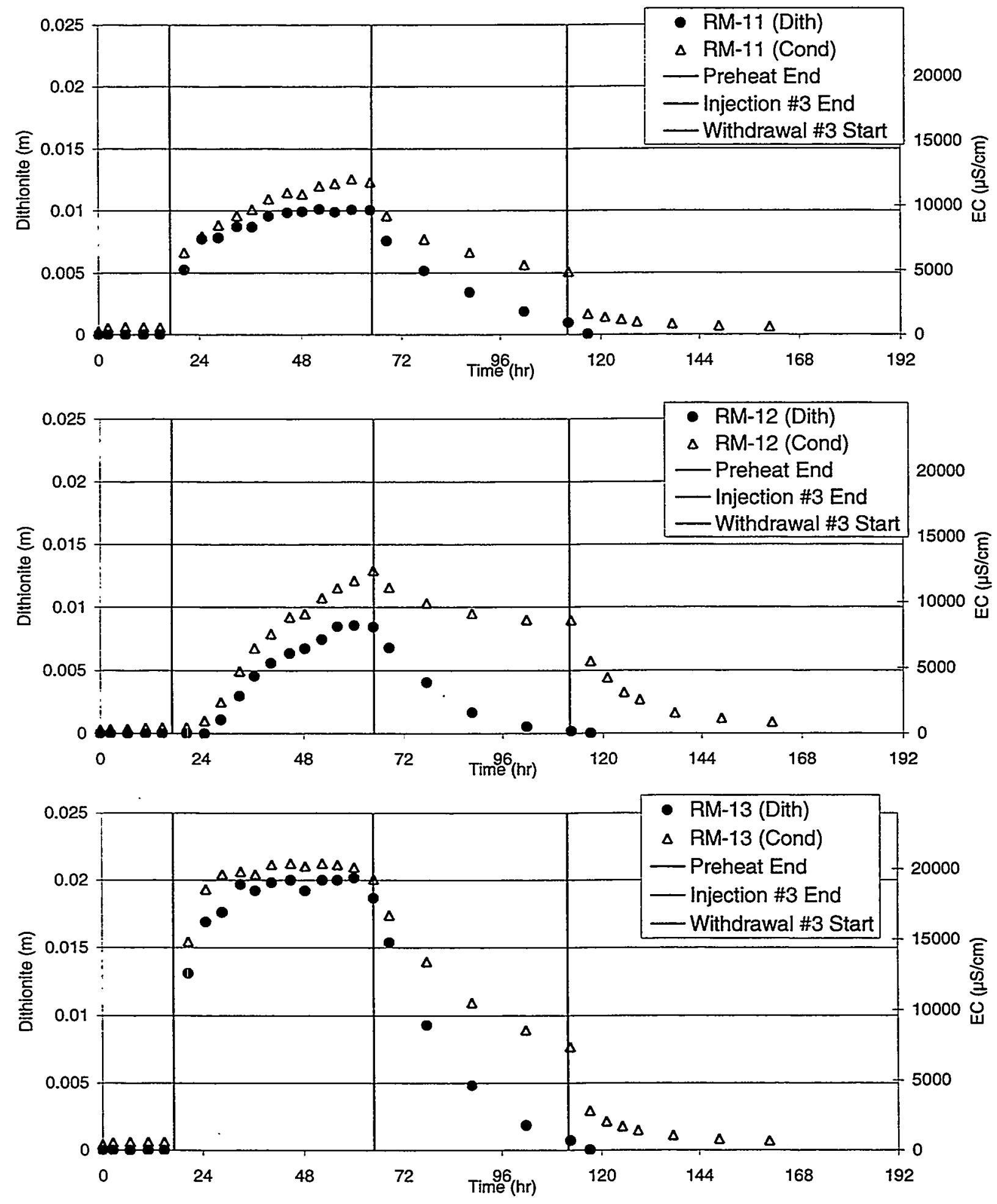

Dithionite Injection/Withdrawal \#3

G.20 

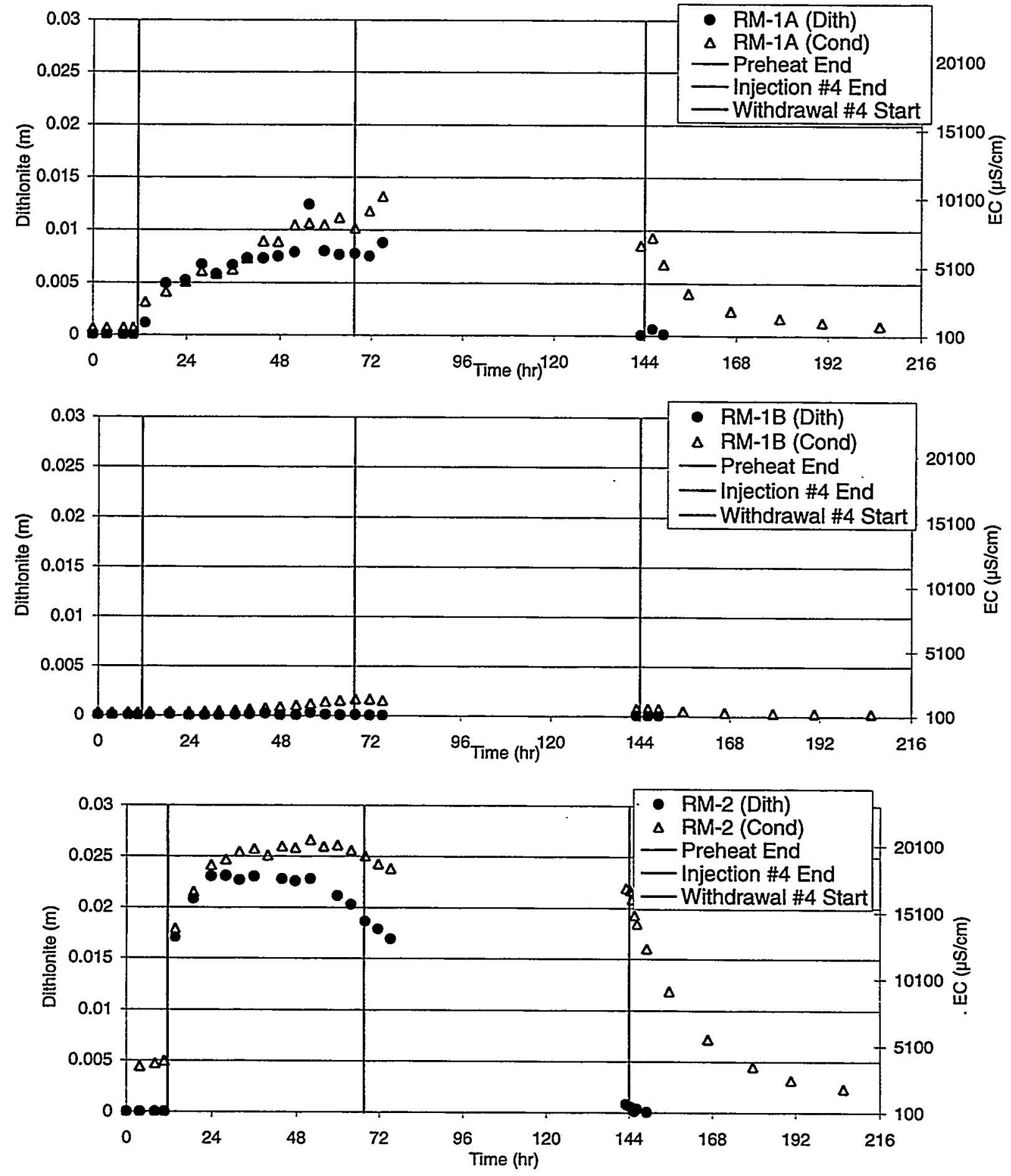

Dithionite Injection/Withdrawal \#4

G.21 

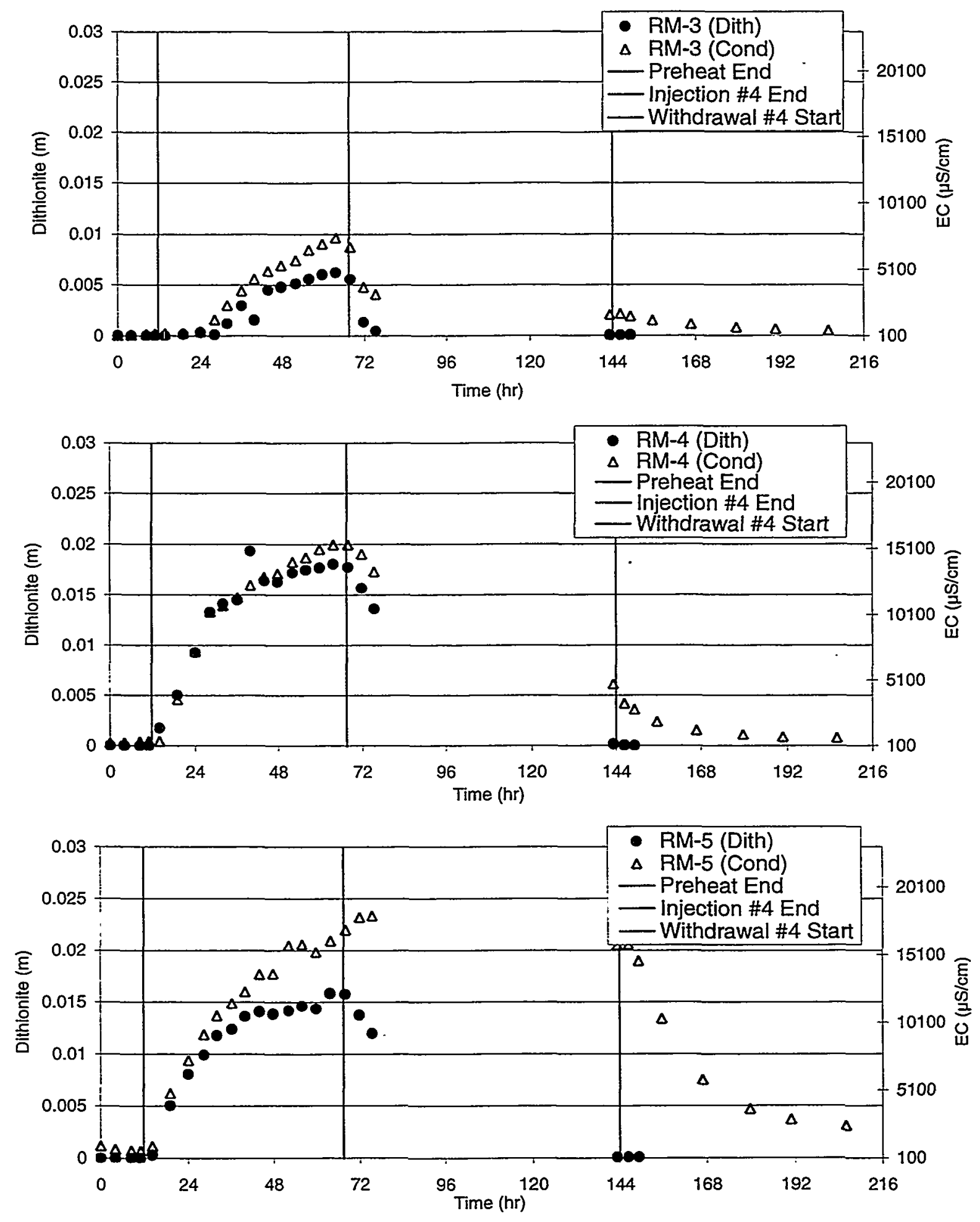

Dithionite Injection/Withdrawal \#4

G.22 

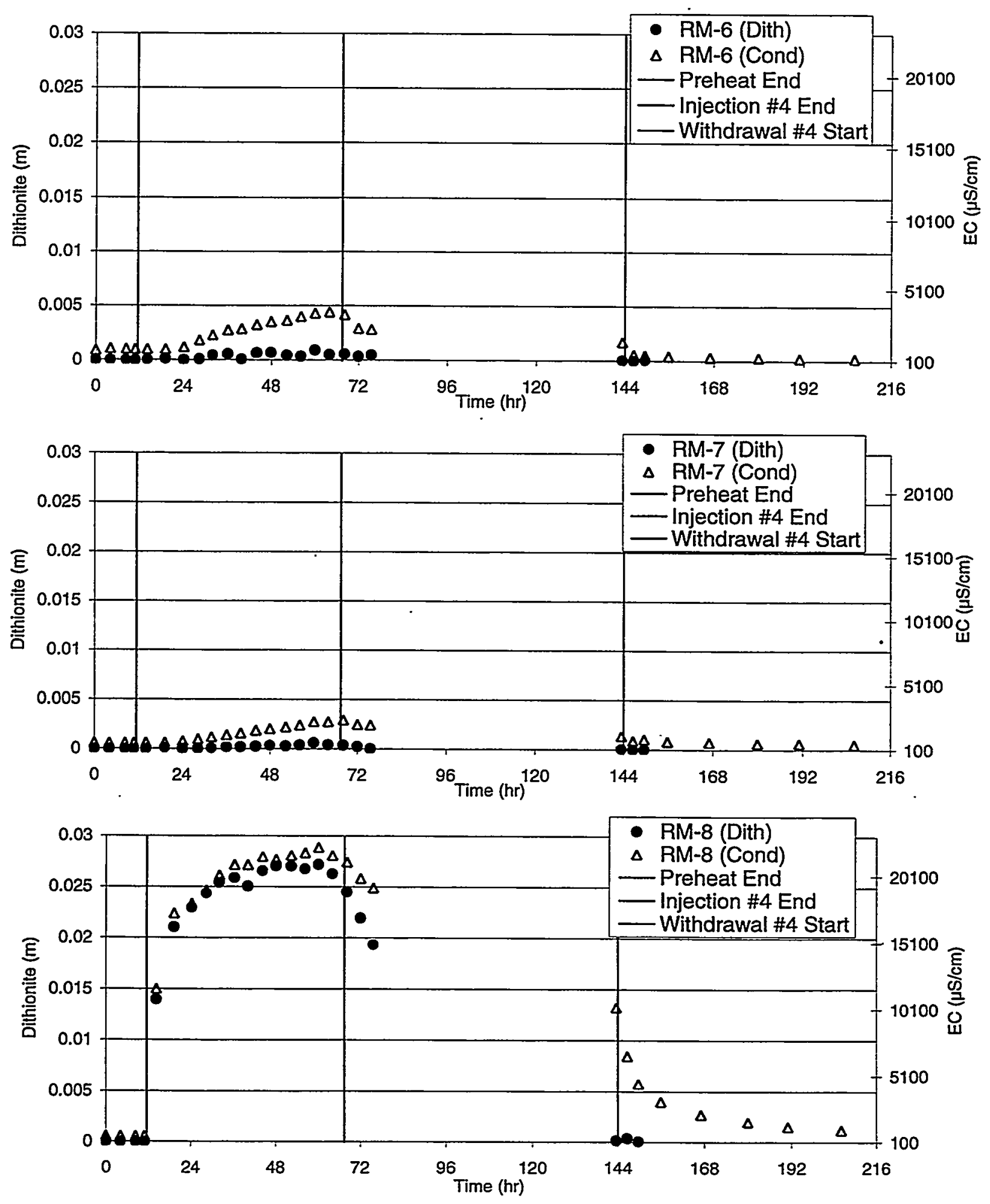

Dithionite Injection/Withdrawal \#4

G.23 

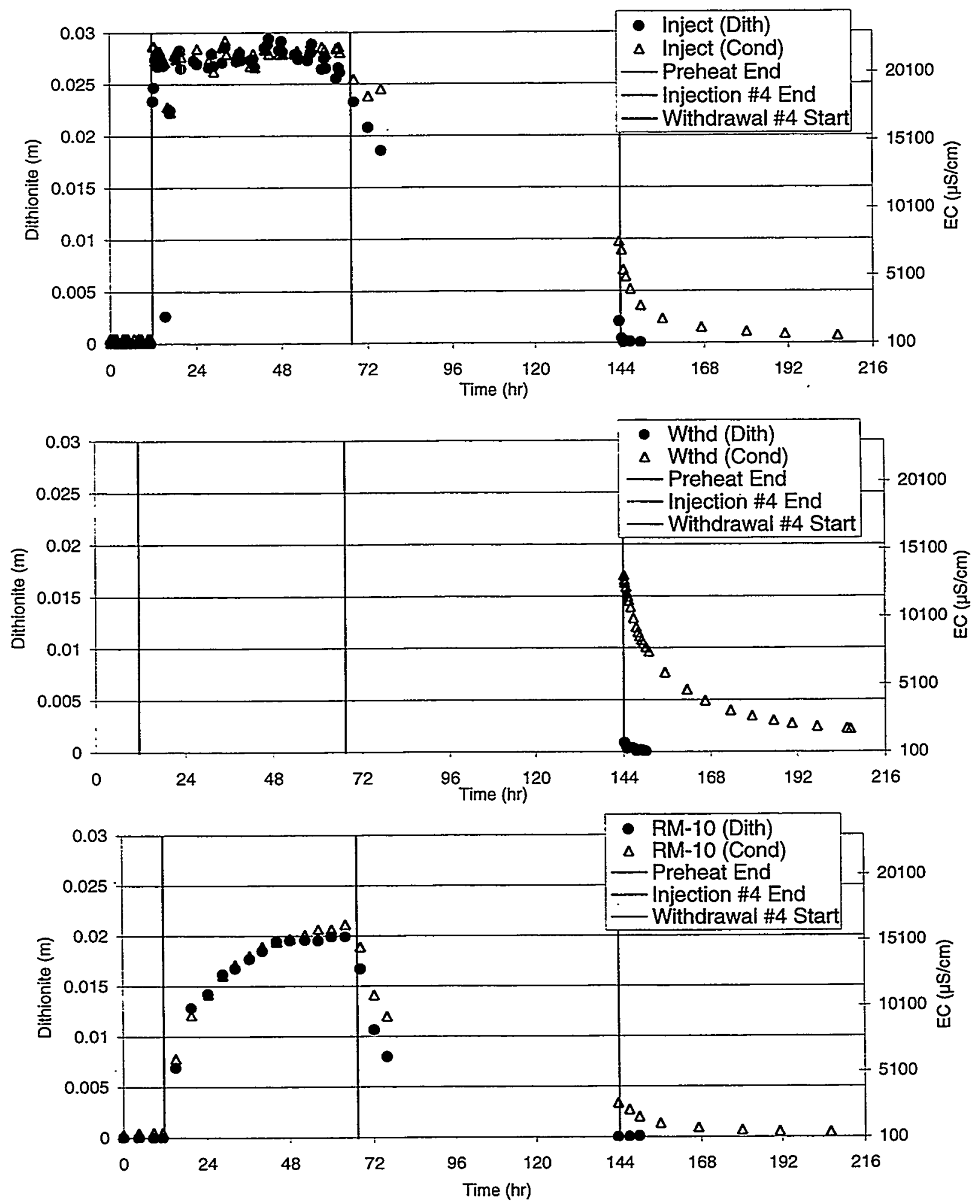

Dithionite Injection/Withdrawal \#4 

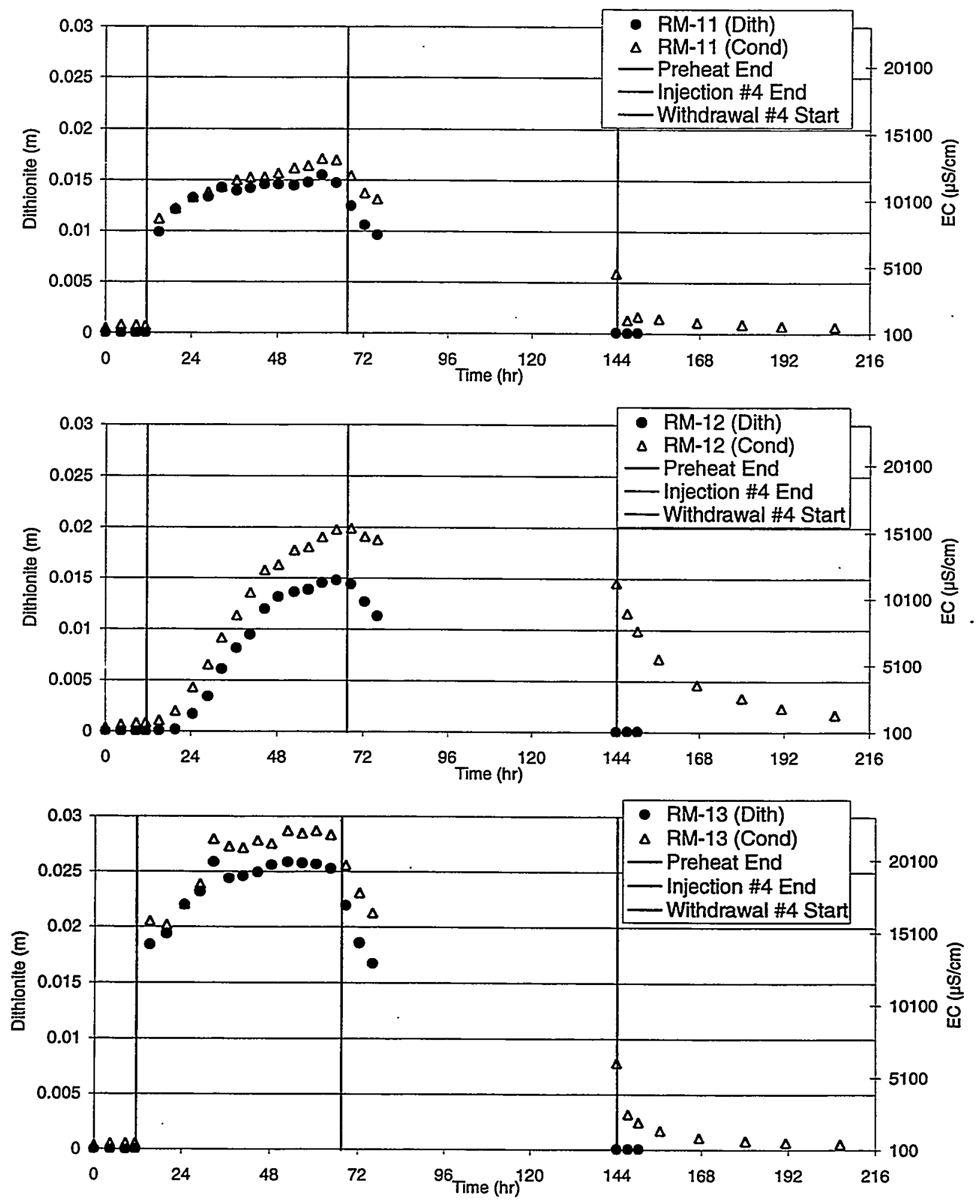

Dithionite Injection/Withdrawal \#4

G.25 


\section{Appendix H}

\section{Post-Injection Performance Assessment Monitoring}




\section{Appendix H}

\section{Post-Injection Performance Assessment Monitoring}

The following tables provide a more comprehensive listing of analytical data collected following each injection. Only the data considered the most reliable are listed here. Species analyzed include TCE and DCE (Table H.1), Acetylene (Table H.2), Common Anions (Table H.3), Water Chemistry Parameters (EC, pH, DO, and Eh; Table H.4), Trace Metals by ICP-OES (Table H.5), and Trace Metals by ICP-MS (Table H.6). An additional group of elements was also measured by ICP-MS on the FL1200 series samples. Results for many species were typically non-detects at an MOL of at least $0.0001 \mathrm{mg} / \mathrm{L}$ or better. Because data for those elements provided little useful additional information, detailed tabulations were not prepared for this report. Table H.7 contains a list of the elements surveyed together with estimated non-detect levels. 
Table H.1a. TCE and DCE Measured by Purge-and-Trap Gas Chromatography (RM-1A, RM-1B, and RM-2)

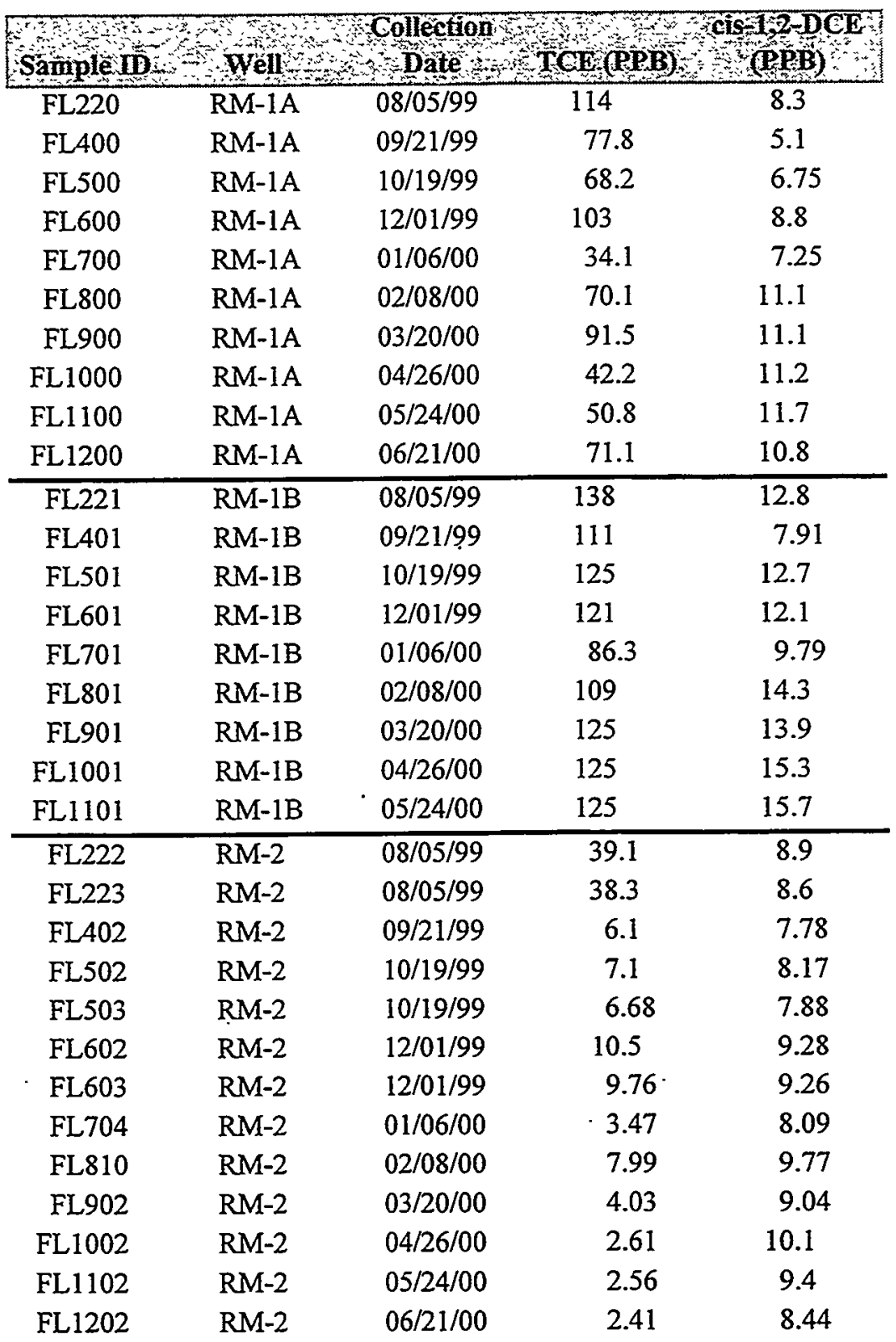


Table H.1b. TCE and DCE Measured by Purge-and-Trap-Gas Chromatography (RM-3, RM-4, and RM-5)

\begin{tabular}{|c|c|c|c|c|}
\hline Sample: & Weil & $\begin{array}{l}\text { Collection } \\
\text { Datete }\end{array}$ & TCE (PPB) & $\begin{array}{r}5=1,2, D C \\
(\mathbf{P P B})\end{array}$ \\
\hline FL224 & RM-3 & $08 / 05 / 99$ & 127 & 9.2 \\
\hline FL404 & RM-3 & $09 / 21 / 99$ & 139 & 6.71 \\
\hline FL504 & $R M-3$ & $10 / 19 / 99$ & 110 & 7.51 \\
\hline FL604 & $R M-3$ & $12 / 01 / 99$ & 134 & 10.1 \\
\hline FL703 & RM-3 & $01 / 06 / 00$ & 107 & 8.39 \\
\hline FL702 & RM-3 & $01 / 06 / 00$ & 120 & 9.99 \\
\hline FL802 & RM-3 & $02 / 08 / 00$ & 138 & 12 \\
\hline FL803 & RM-3 & $02 / 08 / 00$ & 136 & 11.9 \\
\hline FL903 & RM-3 & $03 / 20 / 00$ & 141 & 11.3 \\
\hline FL904 & RM-3 & $03 / 20 / 00$ & 147 & 11.3 \\
\hline FL1003 & RM-3 & $04 / 26 / 00$ & 113 & 10.1 \\
\hline FL1004 & RM-3 & $04 / 26 / 00$ & 108 & 10.2 \\
\hline FL1 103 & RM-3 & $05 / 24 / 00$ & 135 & 10.6 \\
\hline FL1104 & RM-3 & $05 / 24 / 00$ & 141 & 10.5 \\
\hline FL1203 & RM-3 & $06 / 21 / 00$ & 142 & 9.96 \\
\hline FL1204 & RM-3 & $06 / 21 / 00$ & 149 & 10.3 \\
\hline FL225 & $\overline{\mathrm{RM}-4}$ & $08 / 05 / 99$ & 130 & 8.6 \\
\hline FLA05 & $\mathrm{RM}-4$ & $09 / 21 / 99$ & 110 & 5.33 \\
\hline FL505 & RM-4 & $10 / 19 / 99$ & 116 & 8.57 \\
\hline FL605 & RM-4 & $12 / 01 / 99$ & 120 & 9.22 \\
\hline FL705 & RM-4 & $01 / 06 / 00$ & 85.1 & 8.12 \\
\hline FL804 & RM-4 & $02 / 08 / 00$ & 114 & 11.3 \\
\hline FL905 & RM-4 & $03 / 20 / 00$ & 120 & 11.1 \\
\hline FL1005 & RM-4 & $04 / 26 / 00$ & 90.8 & 11.5 \\
\hline FL1105 & $\mathrm{RM}-4$ & $05 / 24 / 00$ & 93.4 & 10.7 \\
\hline FL1205 & $\mathrm{RM}-4$ & $06 / 21 / 00$ & 122 & 10.8 \\
\hline FL226 & $\overline{\mathrm{RM}-5}$ & $08 / 05 / 99$ & 99.5 & 9.2 \\
\hline FL406 & RM-5 & $09 / 21 / 99$ & 29.2 & 12.5 \\
\hline FL506 & RM-5 & $10 / 19 / 99$ & 36.7 & 9.15 \\
\hline FL606 & RM-5 & $12 / 01 / 99$ & 56.4 & 9.77 \\
\hline FL714 & RM-5 & $01 / 06 / 00$ & 13.2 & 10.2 \\
\hline FL714 & RM-5 & $01 / 06 / 00$ & 13.2 & 10.1 \\
\hline FL813 & RM-5 & $02 / 08 / 00$ & 24.4 & 10.9 \\
\hline FL814 & RM-5 & $02 / 08 / 00$ & 22.9 & 11.2 \\
\hline FL906 & RM-5 & $03 / 20 / 00$ & 22.2 & 11.8 \\
\hline FL907 & RM-5 & $03 / 20 / 00$ & 25.6 & 12.1 \\
\hline FL1006 & RM-5 & $04 / 26 / 00$ & 5.55 & 10.9 \\
\hline FL1007 & RM-5 & $04 / 26 / 00$ & 5.29 & 10.8 \\
\hline FL1106 & RM-5 & $05 / 24 / 00$ & 7.73 & 9.73 \\
\hline FL1107 & RM-5 & $05 / 24 / 00$ & 6.69 & 9.63 \\
\hline FL1206 & RM-5 & $06 / 21 / 00$ & 10.4 & 10.5 \\
\hline FL1207 & RM-5 & $06 / 21 / 00$ & 13.3 & 10.8 \\
\hline
\end{tabular}


Table H.1c. TCE and DCE Measured by Purge-and-Trap Gas Chromatography (RM-6, RM-7, and RM-8)

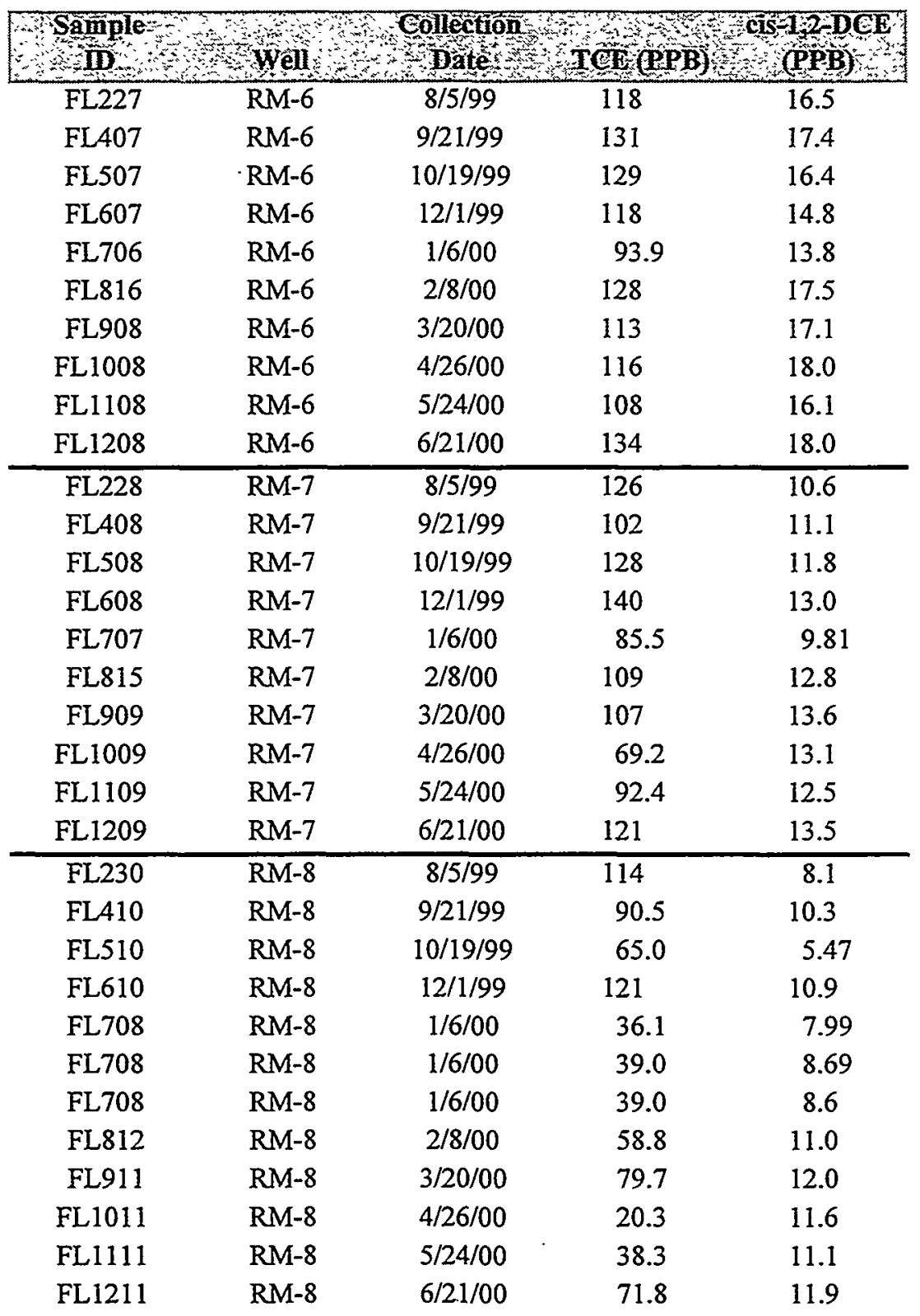

H.4 
Table H.1d. TCE and DCE Measured by Purge-and-Trap Gas Chromatography (RM-9, RM-10, and RM-11)

\begin{tabular}{|c|c|c|c|c|}
\hline Sampled & Well & $\begin{array}{c}\text { Collection } \\
\text { Daté }\end{array}$ & TCE (PeB) & $\begin{array}{l}\text { Cis-1,2DCE } \\
\text { PPB }\end{array}$ \\
\hline FL231 & RM-9 & $8 / 5 / 99$ & 121 & 8.7 \\
\hline FL411 & RM-9 & $9 / 21 / 99$ & 107 & 10.2 \\
\hline FL403 & RM-9 & 9/21/99 & 103 & 7 \\
\hline FL511 & RM-9 & $10 / 19 / 99$ & 106 & 9.37 \\
\hline FL611 & RM-9 & $12 / 1 / 99$ & 117 & 9.92 \\
\hline FL710 & RM-9 & $1 / 6 / 00$ & 46.1 & 7.99 \\
\hline FL710 & RM-9 & $1 / 6 / 00$ & 22.9 & 3.3 \\
\hline FL710 & RM-9 & $1 / 6 / 00$ & 49.1 & 7.57 \\
\hline FL808 & RM-9 & $2 / 8 / 00$ & 90.9 & 11 \\
\hline FL912 & RM-9 & $3 / 20 / 00$ & 96.1 & 11.8 \\
\hline FL1012 & RM-9 & $4 / 26 / 00$ & 28.9 & 11.2 \\
\hline FL1112 & RM-9 & $5 / 24 / 00$ & 56.7 & 11.3 \\
\hline FL1212 & RM-9 & $6 / 21 / 00$ & 98.2 & 11.3 \\
\hline FL232 & $\mathrm{RM}-10$ & $8 / 5 / 99$ & 123 & 9.8 \\
\hline FL412 & RM-10 & 9/21/99 & 108 & 9.89 \\
\hline FL512 & RM-10 & $10 / 19 / 99$ & 95.4 & 8.51 \\
\hline FL612 & RM-10 & $12 / 1 / 99$ & 123 & 10.3 \\
\hline FL613 & RM-10 & $12 / 1 / 99$ & 126 & 10.5 \\
\hline FL711 & RM-10 & $1 / 6 / 00$ & 55.8 & 6.34 \\
\hline FL805 & RM-10 & $2 / 8 / 00$ & 97.8 & 11.3 \\
\hline FL913 & RM-10 & $3 / 20 / 00$ & 123 & 12.4 \\
\hline FL1013 & $R M-10$ & $4 / 26 / 00$ & 62 & 12.5 \\
\hline FL1113 & RM-10 & $5 / 24 / 00$ & 62 & 11.1 \\
\hline FL1213 & RM-10 & $6 / 21 / 00$ & 101 & 11.6 \\
\hline FL233 & RM-11 & $8 / 5 / 99$ & 114 & 9.7 \\
\hline FL234 & $\mathrm{RM}-11$ & $8 / 5 / 99$ & 124 & 10.1 \\
\hline FLA13 & RM-11 & $9 / 21 / 99$ & 106 & 10.4 \\
\hline FL414 & RM-11 & $9 / 21 / 99$ & 108 & 10.6 \\
\hline FL513 & RM-11 & $10 / 19 / 99$ & 105 & 10 \\
\hline FL514 & RM-11 & $10 / 19 / 99$ & 99.4 & 9.23 \\
\hline FL614 & RM-11 & 12/1/99 & 131 & 11.9 \\
\hline FL712 & RM-11 & $1 / 6 / 00$ & 64.4 & 7.43 \\
\hline FL712 & RM-11 & $1 / 6 / 00$ & 64.4 & 7.43 \\
\hline FL807 & RM-11 & $2 / 8 / 00$ & 82.6 & 12.1 \\
\hline FL914 & RM-11 & $3 / 20 / 00$ & 105 & 12.5 \\
\hline FL1014 & RM-11 & $4 / 26 / 00$ & 67.6 & 12.5 \\
\hline FL1114 & RM-11 & $5 / 24 / 00$ & 76.9 & 11.9 \\
\hline FL1214 & RM-1 1 & $6 / 21 / 00$ & 115 & 12.6 \\
\hline
\end{tabular}


Table H.1e. TCE and DCE Measured by Purge-and-Trap Gas Chromatography (RM-12, RM-13, RM-14, and RM-15)

\begin{tabular}{|c|c|c|c|c|}
\hline $\begin{array}{l}\text { W } \\
\text { Sample }\end{array}$ & Whis & $\begin{array}{l}\text { Collection } \\
\text { Date }\end{array}$ & 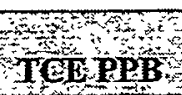 & $\begin{array}{r}\text { CIS 1 2NDCE } \\
\text { PPB }\end{array}$ \\
\hline FL235 & $\mathrm{RM}-12$ & $8 / 5 / 99$ & 148 & 16.2 \\
\hline FL415 & $R M-12$ & $9 / 21 / 99$ & 108 & 13.6 \\
\hline FL515 & RM-12 & $10 / 19 / 99$ & 75.6 & 6.69 \\
\hline FL615 & RM-12 & $12 / 1 / 99$ & 152 & 14.4 \\
\hline FL715 & RM-12 & $1 / 6 / 00$ & 82.7 & 9.08 \\
\hline FL806 & RM-12 & $2 / 8 / 00$ & 142 & 17.9 \\
\hline FL915 & $\mathrm{RM}-12$ & $3 / 20 / 00$ & 159 & 19.5 \\
\hline FL1015 & RM-12 & $4 / 26 / 00$ & 79.8 & 17.1 \\
\hline FL1115 & RM-12 & $5 / 24 / 00$ & 118 & 17.2 \\
\hline$\overline{\text { FL236 }}$ & RM-13 & $8 / 5 / 99$ & 120 & 9 \\
\hline FL416 & RM-13 & $9 / 21 / 99$ & 35.7 & 4.3 \\
\hline FL516 & $\mathrm{RM}-13$ & $10 / 19 / 99$ & 96.6 & 9.57 \\
\hline FL616 & $\mathrm{RM}-13$ & $12 / 1 / 99$ & 112 & 9.94 \\
\hline FL716 & $R M-13$ & $1 / 6 / 00$ & 42.9 & 6.57 \\
\hline FL811 & RM-13 & $2 / 8 / 00$ & 91.3 & 11.3 \\
\hline FL916 & RM-13 & $3 / 20 / 00$ & 108 & 12.7 \\
\hline FL1016 & RM-13 & $4 / 26 / 00$ & 46.7 & 14.1 \\
\hline FL1116 & $R M-13$ & $5 / 24 / 00$ & 43.6 & 11.6 \\
\hline FL1216 & RM-13 & $6 / 21 / 00$ & 68 & 12.5 \\
\hline FL1215 & RM-14 & $6 / 21 / 00$ & 18 & 10.5 \\
\hline FL1201 & RM-15 & $6 / 21 / 00$ & 34.4 & 10.9 \\
\hline
\end{tabular}

H. 6 
Table H.2a. Acetylene Measurements by Headspace Gas Analysis (RM-1A, RM-1B, and RM-2)

\begin{tabular}{cccc}
\hline SampleT & Weil & Collection Date & Acetylene PPB \\
\hline FL100 & RM-1A & $12 / 1 / 98$ & 0.86 \\
FL120 & RM-1A & $12 / 15 / 98$ & 0.38 \\
FL140 & RM-1A & $1 / 5 / 99$ & 0.08 \\
FL400 & RM-1A & $9 / 21 / 99$ & 0.22 \\
FL500 & RM-1A & $10 / 19 / 99$ & 0.20 \\
FL600 & RM-1A & $12 / 1 / 99$ & 0.15 \\
FL700 & RM-1A & $1 / 6 / 00$ & 1.64 \\
FL800 & RM-1A & $2 / 8 / 00$ & 0.55 \\
FL900 & RM-1A & $3 / 20 / 00$ & 0.47 \\
FL1000 & RM-1A & $4 / 26 / 00$ & 2.48 \\
FL1100 & RM-1A & $5 / 24 / 00$ & 0.89 \\
FL1200 & RM-1A & $6 / 21 / 00$ & 0.54 \\
\hline FL101 & RM-1B & $12 / 1 / 98$ & $0.12 \mathrm{~J}$ \\
FL121 & RM-1B & $12 / 15 / 98$ & $0.12 \mathrm{~J}$ \\
FL141 & RM-1B & $1 / 5 / 99$ & 0.22 \\
FL401 & RM-1B & $9 / 21 / 99$ & $<0.06$ \\
FL501 & RM-1B & $10 / 19 / 99$ & $<0.05$ \\
FL601 & RM-1B & $12 / 1 / 99$ & $<0.05$ \\
FL701 & RM-1B & $1 / 6 / 00$ & $<0.06$ \\
FL801 & RM-1B & $2 / 8 / 00$ & $<0.06$ \\
FL901 & RM-1B & $3 / 20 / 00$ & $<0.06$ \\
FL1001 & RM-1B & $4 / 26 / 00$ & $<0.06$ \\
FL1101 & RM-1B & $5 / 24 / 00$ & $<0.06$ \\
\hline FL102 & RM-2 & $12 / 1 / 98$ & 4.14 \\
FL122 & RM-2 & $12 / 15 / 98$ & 2.62 \\
FL142 & RM-2 & $1 / 5 / 99$ & 2.89 \\
FL402 & RM-2 & $9 / 21 / 99$ & 4.75 \\
FL502 & RM-2 & $10 / 19 / 99$ & 5.48 \\
FL503 & RM-2 & $10 / 19 / 99$ & 5.48 \\
FL602 & RM-2 & $12 / 1 / 99$ & 1.19 \\
FL603 & RM-2 & $12 / 1 / 99$ & 1.45 \\
FL704 & RM-2 & $1 / 6 / 00$ & 5.96 \\
FL810 & RM-2 & $2 / 8 / 00$ & 1.85 \\
FL902 & RM-2 & $3 / 20 / 00$ & 0.74 \\
FL1002 & RM-2 & $4 / 26 / 00$ & 7.56 \\
FL1102 & RM-2 & $5 / 24 / 00$ & 5.94 \\
FL1202 & RM-2 & $6 / 21 / 00$ & 5.57 \\
& & & \\
\hline
\end{tabular}


Table H.2b. Acetylene Measurements by Headspace Gas Analysis (RM-3, and RM-4)

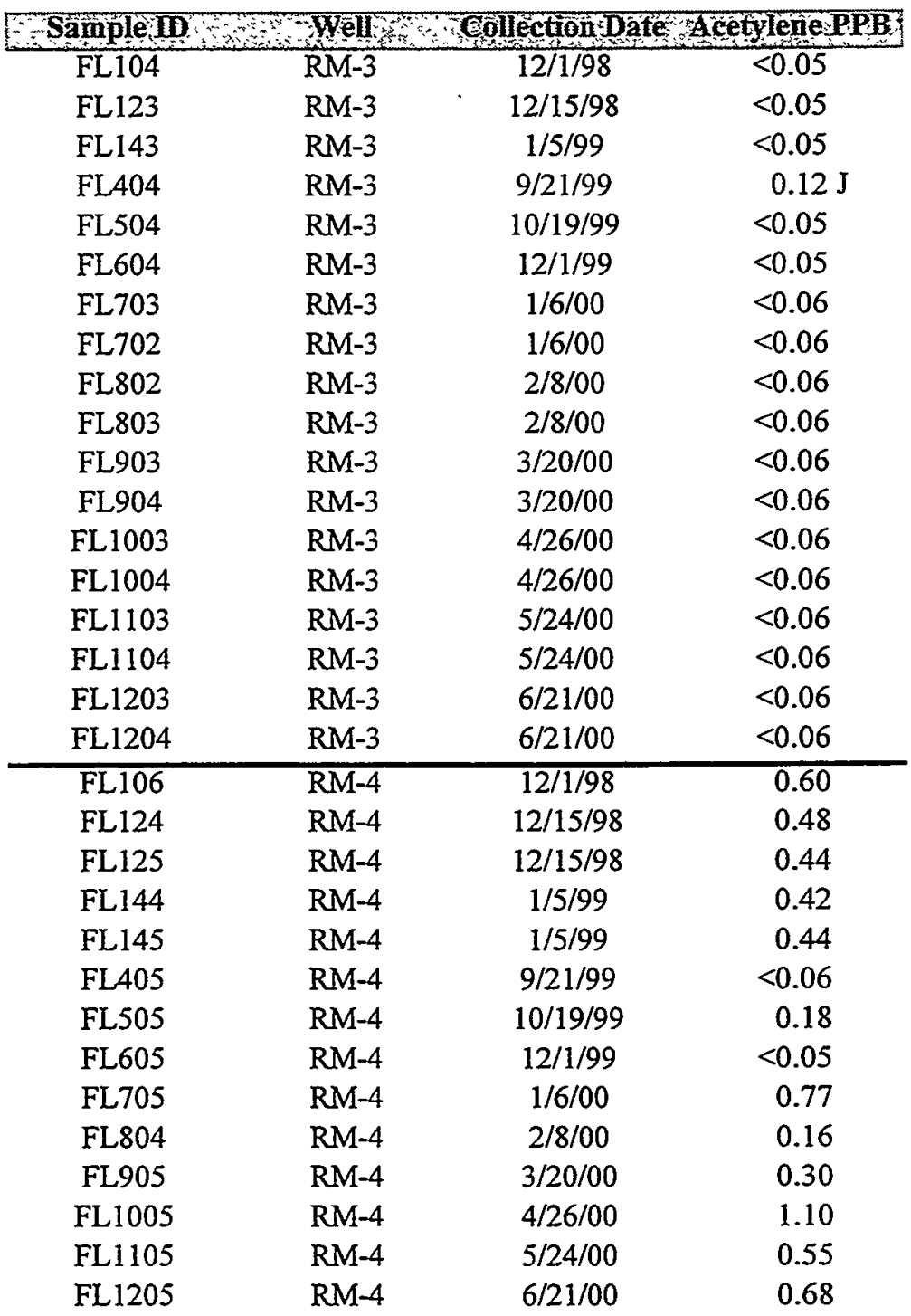


Table H.2c. Acetylene Measurements by Headspace Gas Analysis (RM-5, and RM-6)

\begin{tabular}{cccc}
\hline Sample ID & Well & Collection: & Acetylene.PPB \\
\hline FL107 & RM-5 & $12 / 1 / 98$ & 3.14 \\
FL126 & RM-5 & $12 / 15 / 98$ & 2.14 \\
FL146 & RM-5 & $1 / 5 / 99$ & 1.48 \\
FL406 & RM-5 & $9 / 21 / 99$ & 4.15 \\
FL506 & RM-5 & $10 / 19 / 99$ & 4.17 \\
FL606 & RM-5 & $12 / 1 / 99$ & 4.13 \\
FL713 & RM-5 & $1 / 6 / 00$ & 7.99 \\
FL714 & RM-5 & $1 / 6 / 00$ & 8.41 \\
FL813 & RM-5 & $2 / 8 / 00$ & 2.66 \\
FL814 & RM-5 & $2 / 8 / 00$ & 2.91 \\
FL906 & RM-5 & $3 / 20 / 00$ & 0.65 \\
FL907 & RM-5 & $3 / 20 / 00$ & 0.75 \\
FL1006 & RM-5 & $4 / 26 / 00$ & 7.48 \\
FL1007 & RM-5 & $4 / 26 / 00$ & 7.36 \\
FL1106 & RM-5 & $5 / 24 / 00$ & 4.20 \\
FL1107 & RM-5 & $5 / 24 / 00$ & 4.34 \\
FL1206 & RM-5 & $6 / 21 / 00$ & 4.88 \\
FL1207 & RM-5 & $6 / 21 / 00$ & 3.98 \\
\hline FL108 & RM-6 & $12 / 1 / 98$ & 0.40 \\
FL127 & RM-6 & $12 / 15 / 98$ & 0.44 \\
FL128 & RM-6 & $12 / 15 / 98$ & 0.48 \\
FL148 & RM-6 & $1 / 5 / 99$ & $<0.06$ \\
FL407 & RM-6 & $9 / 21 / 99$ & $<0.06$ \\
FL507 & RM-6 & $10 / 19 / 99$ & $<0.05$ \\
FL607 & RM-6 & $12 / 1 / 99$ & $<0.05$ \\
FL706 & RM-6 & $1 / 6 / 00$ & $<0.06$ \\
FL816 & RM-6 & $2 / 8 / 00$ & $<0.06$ \\
FL908 & RM-6 & $3 / 20 / 00$ & $<0.06$ \\
FL1008 & RM-6 & $4 / 26 / 00$ & $<0.06$ \\
FL1108 & RM-6 & $5 / 24 / 00$ & $<0.06$ \\
FL1208 & RM-6 & $6 / 21 / 00$ & $<0.06$ \\
& & & \\
\hline
\end{tabular}


Table H.2d. Acetylene Measurements by Headspace Gas Analysis (RM-7, RM-8, and RM-9)

\begin{tabular}{cccc}
\hline Sample D & RMel & Collection Date & AcetylerepPB \\
\hline FL110 & RM-7 & $12 / 1 / 98$ & 0.20 \\
FL129 & RM-7 & $12 / 15 / 98$ & 0.14 \\
FL149 & RM-7 & $1 / 5 / 99$ & 0.17 \\
FL150 & RM-7 & $1 / 5 / 99$ & 0.19 \\
FL408 & RM-7 & $9 / 21 / 99$ & $<0.06$ \\
FL508 & RM-7 & $10 / 19 / 99$ & $<0.05$ \\
FL608 & RM-7 & $12 / 1 / 99$ & $<0.05$ \\
FL707 & RM-7 & $1 / 6 / 00$ & $<0.06$ \\
FL815 & RM-7 & $2 / 8 / 00$ & $<0.06$ \\
FL909 & RM-7 & $3 / 20 / 00$ & $<0.06$ \\
FL1009 & RM-7 & $4 / 26 / 00$ & $<0.06$ \\
FL1109 & RM-7 & $5 / 24 / 00$ & $<0.06$ \\
FL1209 & RM-7 & $6 / 21 / 00$ & $<0.06$ \\
\hline FL111 & RM-8 & $12 / 1 / 98$ & 0.62 \\
FL130 & RM-8 & $12 / 15 / 98$ & 0.24 \\
FL151 & RM-8 & $1 / 5 / 99$ & 0.39 \\
FL410 & RM-8 & $9 / 21 / 99$ & 0.40 \\
FL510 & RM-8 & $10 / 19 / 99$ & 0.43 \\
FL610 & RM-8 & $12 / 1 / 99$ & 0.29 \\
FL708 & RM-8 & $1 / 6 / 00$ & 4.18 \\
FL812 & RM-8 & $2 / 8 / 00$ & 0.63 \\
FL911 & RM-8 & $3 / 20 / 00$ & 0.93 \\
FL1011 & RM-8 & $4 / 26 / 00$ & 4.55 \\
FL1111 & RM-8 & $5 / 24 / 00$ & 2.65 \\
FL1211 & RM-8 & $6 / 21 / 00$ & 3.27 \\
\hline FL112 & RM-9 & $12 / 1 / 98$ & 0.38 \\
FL132 & RM-9 & $12 / 15 / 98$ & 0.26 \\
FL152 & RM-9 & $1 / 5 / 99$ & 0.26 \\
FL411 & RM-9 & $9 / 21 / 99$ & 0.08 \\
FL403 & RM-9 & $9 / 21 / 99$ & 0.08 \\
FL511 & RM-9 & $10 / 19 / 99$ & 0.15 \\
FL611 & RM-9 & $12 / 1 / 99$ & 0.22 \\
FL710 & RM-9 & $1 / 6 / 00$ & 2.59 \\
FL808 & RM-9 & $2 / 8 / 00$ & 0.43 \\
FL912 & RM-9 & $3 / 20 / 00$ & 0.62 \\
FL1012 & RM-9 & $4 / 26 / 00$ & 5.44 \\
FL1112 & RM-9 & $5 / 24 / 00$ & 2.62 \\
FL1212 & RM-9 & $6 / 21 / 00$ & 1.72 \\
& & & \\
\hline
\end{tabular}


Table H.2d. Acetylene Measurements by Headspace Gas Analysis (RM-10, RM-11, and RM-12)

\begin{tabular}{cccc}
\hline Sainplem & Well & Collection Date & Acetylene PPB \\
\hline FL412 & RM-10 & $9 / 21 / 99$ & $<0.06$ \\
FL512 & RM-10 & $10 / 19 / 99$ & $<0.05$ \\
FL612 & RM-10 & $12 / 1 / 99$ & $<0.05$ \\
FL613 & RM-10 & $12 / 1 / 99$ & $<0.05$ \\
FL711 & RM-10 & $1 / 6 / 00$ & 1.00 \\
FL805 & RM-10 & $2 / 8 / 00$ & 0.36 \\
FL913 & RM-10 & $3 / 20 / 00$ & 0.46 \\
FL1013 & RM-10 & $4 / 26 / 00$ & 1.06 \\
FL1113 & RM-10 & $5 / 24 / 00$ & 0.59 \\
FL1213 & RM-10 & $6 / 21 / 00$ & 0.99 \\
\hline FL413 & RM-11 & $9 / 21 / 99$ & $<0.06$ \\
FL414 & RM-11 & $9 / 21 / 99$ & $0.08 \mathrm{~J}$ \\
FL513 & RM-11 & $10 / 19 / 99$ & $<0.05$ \\
FL514 & RM-11 & $10 / 19 / 99$ & $<0.06$ \\
FL614 & RM-11 & $12 / 1 / 99$ & $<0.05$ \\
FL712 & RM-11 & $1 / 6 / 00$ & 0.93 \\
FL807 & RM-11 & $2 / 8 / 00$ & 0.13 \\
FL914 & RM-11 & $3 / 20 / 00$ & 0.20 \\
FL1014 & RM-11 & $4 / 26 / 00$ & 0.99 \\
FL1114 & RM-11 & $5 / 24 / 00$ & 0.50 \\
FL1214 & RM-11 & $6 / 21 / 00$ & 0.68 \\
\hline FL415 & RM-12 & $9 / 21 / 99$ & 0.17 \\
FL515 & RM-12 & $10 / 19 / 99$ & $<0.06$ \\
FL615 & RM-12 & $12 / 1 / 99$ & $<0.05$ \\
FL715 & RM-12 & $1 / 6 / 00$ & 0.52 \\
FL806 & RM-12 & $2 / 8 / 00$ & 0.35 \\
FL915 & RM-12 & $3 / 20 / 00$ & 0.22 \\
FL1015 & RM-12 & $4 / 26 / 00$ & 2.03 \\
FL1115 & RM-12 & $5 / 24 / 00$ & 0.91 \\
& & & \\
\hline
\end{tabular}

H.11 
Table H.2e. Acetylene Measurements by Headspace Gas Analysis (RM-13, RM-14, and RM-15)

\begin{tabular}{cccc}
\hline Sample:D & Well & Collectiondate AcetylegepPB \\
\hline FL416 & RM-13 & $9 / 21 / 99$ & 0.64 \\
FL516 & RM-13 & $10 / 19 / 99$ & 0.32 \\
FL616 & RM-13 & $12 / 1 / 99$ & 0.26 \\
FL716 & RM-13 & $1 / 6 / 00$ & 1.89 \\
FL811 & RM-13 & $2 / 8 / 00$ & 0.92 \\
FL916 & RM-13 & $3 / 20 / 00$ & 0.92 \\
FL1016 & RM-13 & $4 / 26 / 00$ & 2.30 \\
FL1116 & RM-13 & $5 / 24 / 00$ & 1.31 \\
FL1216 & RM-13 & $6 / 21 / 00$ & 3.25 \\
FL1215 & RM-14 & $6 / 21 / 00$ & 3.15 \\
\hline FL1201 & RM-15 & $6 / 21 / 00$ & 1.76
\end{tabular}

H.12 
Table H.3a. Common Anions by Ion Chromatography (Wells RM-1A and RM-1B)

\begin{tabular}{|c|c|c|c|c|c|c|c|c|c|}
\hline Sample ID & Well & $\begin{array}{l}\text { Collection } \\
\text { Date }\end{array}$ & $\begin{array}{l}\text { Chlorid } \\
\text { mogt }\end{array}$ & $\begin{array}{l}\text { Fluoride } \\
\text { mgle }\end{array}$ & $\begin{array}{l}\text { Tfrateng } \\
\text { (aśno }\end{array}$ & $\begin{array}{l}\text { Phosphat } \\
\text { inght }\end{array}$ & $\begin{array}{l}\text { Sulfate } \\
\text { tigh }\end{array}$ & $\begin{array}{l}\text { Sulfite } \\
\text { mgfte }\end{array}$ & $\begin{array}{l}\text { losulate } \\
\text { ngth }\end{array}$ \\
\hline FL100 & RM-1A & $12 / 1 / 98$ & 4.40 & $<2$ & $<2$ & $<2$ & 133 & 2.47 & \\
\hline FL120 & RM-1A & $12 / 15 / 98$ & 4.26 & $<2$ & $<2$ & $<2$ & 105 & 13.1 & \\
\hline FL140 & RM-1A & $1 / 5 / 99$ & 3.48 & $<0.2$ & $<0.2$ & $<0.2$ & 81.8 & $<0.3$ & \\
\hline FL160 & RM-1A & 2/9/99 & 3.71 & $<2$ & $<2$ & $<2$ & 81.6 & & \\
\hline FL200 & RM-1A & $4 / 1 / 99$ & 2.21 & $<2$ & $<2$ & $<2$ & 63.6 & $<3$ & \\
\hline FL220 & RM- $1 A$ & $8 / 5 / 99$ & 2.24 & 0.35 & $<0.2$ & $<0.2$ & 46.0 & & \\
\hline FL340 & $\mathrm{RM}-1 \mathrm{~A}$ & $8 / 22 / 99$ & 23.1 & $<20$ & $<20$ & $<20$ & 161 & $<20$ & \\
\hline FL400 & RM-1A & 9/21/99 & 3.19 & $<2$ & $<2$ & $<2$ & 220 & $<3$ & \\
\hline FL400 & RM-1A & 9/21/99 & & & & & 239 & $<1$ & \\
\hline FL500 & RM-1A & $10 / 19 / 99$ & & & & & 164 & 1.20 & $<0.1$ \\
\hline FL600 & RM-1A & $12 / 1 / 99$ & & & & & 107 & $<1$ & $<0.1$ \\
\hline FL600 & $R M-1 A$ & $12 / 1 / 99$ & 2.01 & 0.17 & $<2$ & $<2$ & 115 & & \\
\hline FL700 & RM-1A & $1 / 6 / 00$ & & & & & 300 & 0.40 & \\
\hline FL800 & $R M-1 A$ & $2 / 8 / 00$ & & & & & 190 & $<3$ & \\
\hline FL900 & RM-1A & $3 / 20 / 00$ & & & & & 130 & $<3$ & \\
\hline FL1 100 & RM-1A & $5 / 24 / 00$ & & & & & 307 & & \\
\hline FL1200 & RM-1A & $6 / 21 / 00$ & & & & & 156 & & \\
\hline FL101 & RM-1B & $12 / 1 / 98$ & 3.34 & 0.28 & 1.89 & $<0.2$ & 44.6 & $<3$ & \\
\hline FL121 & RM-1B & $12 / 15 / 98$ & 3.01 & 0.26 & 1.62 & $0.3 \mathrm{~J}$ & 41.7 & $<3$ & \\
\hline FL141 & RM-1B & $1 / 5 / 99$ & 3.67 & $<2$ & $<2$ & $<2$ & 31.8 & $<3$ & \\
\hline FL161 & RM-1B & $2 / 9 / 99$ & 4.30 & $<2$ & $<2$ & $<2$ & 22.8 & & \\
\hline FL202 & RM-1B & $4 / 1 / 99$ & 2.42 & $<2$ & $<2$ & $<2$ & 14.9 & $<3$ & \\
\hline FL201 & $R M-1 B$ & $4 / 1 / 99$ & 2.70 & $<2$ & $<2$ & $<2$ & 16.7 & $<3$ & \\
\hline FL221 & RM-1B & $8 / 5 / 99$ & 2.46 & 0.24 & 1.15 & $<0.2$ & 20.7 & & \\
\hline FL341 & RM-1B & $8 / 22 / 99$ & 42.3 & $<20$ & $<20$ & $<20$ & 73.3 & $<20$ & \\
\hline FL401 & $\mathrm{RM}-1 \mathrm{~B}$ & 9/21/99 & 3.17 & $<2$ & $<2$ & $<2$ & 65.3 & $<3$ & \\
\hline FL501 & RM-1B & $10 / 19 / 99$ & & & & & 56.0 & $<0.4$ & $<0.1$ \\
\hline FL601 & RM-1B & $12 / 1 / 99$ & 2.69 & $0.08 \mathrm{~J}$ & 1.38 & $<2$ & 30.0 & $0.16 \mathrm{~J}$ & $<2$ \\
\hline FL601 & RM-1B & $12 / 1 / 99$ & & & & & 31.0 & $<0.8$ & \\
\hline FL701 & RM-1B & $1 / 6 / 00$ & & & & & 120 & 8.00 & \\
\hline FL801 & RM-1B & $2 / 8 / 00$ & & & & & 95.0 & $<1.4$ & \\
\hline FL901 & $R M-1 B$ & $3 / 20 / 00$ & & & & & 57.0 & $<1.3$ & \\
\hline FL1 101 & $R M-1 B$ & $5 / 24 / 00$ & & & & & 136 & & \\
\hline
\end{tabular}

H.13 
Table H.3b. Common Anions by Ion Chromatography (Well RM-2)

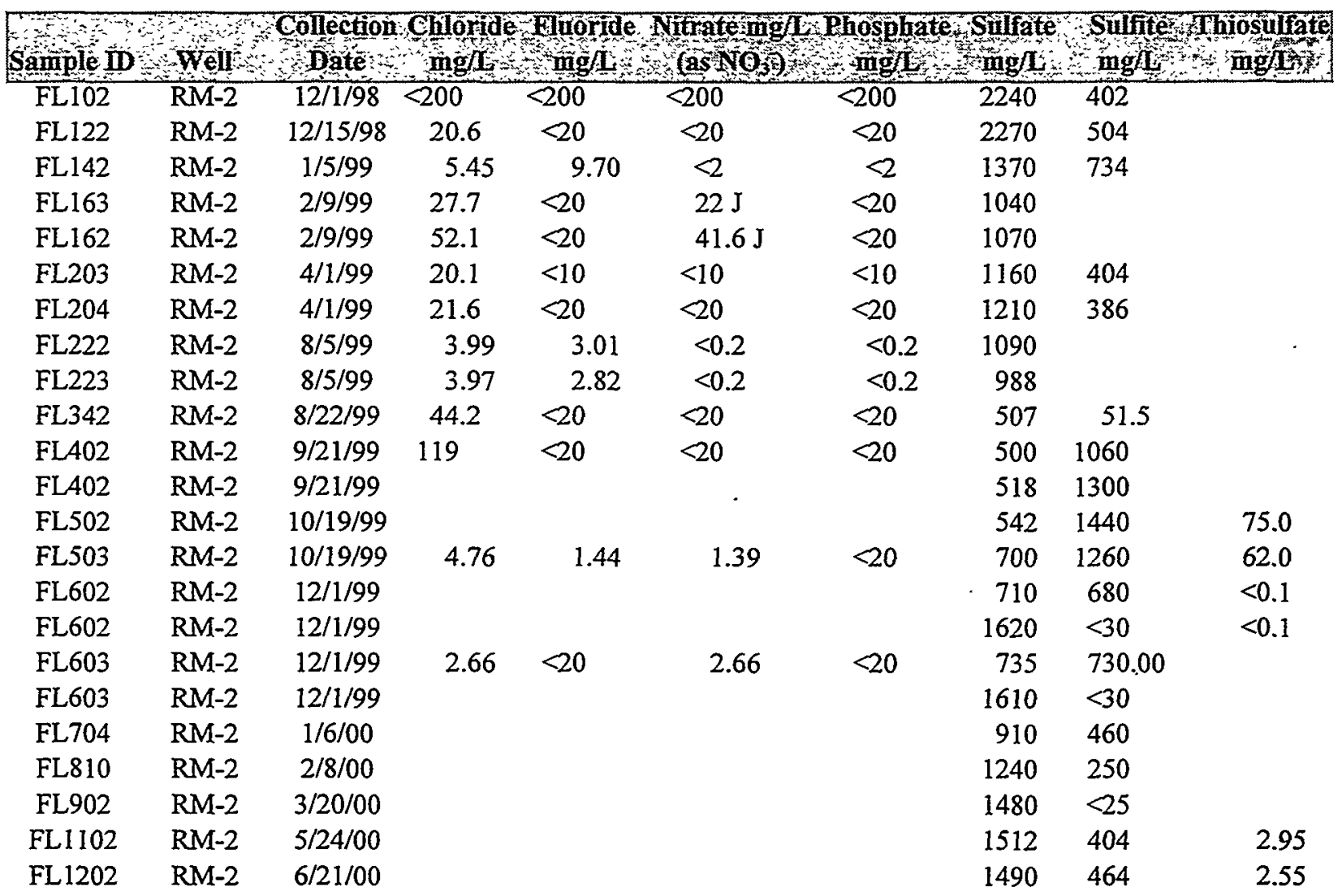


Table H.3c. Common Anions by Ion Chromatography (Wells RM-3 and RM-4)

\begin{tabular}{|c|c|c|c|c|c|c|c|c|c|}
\hline Sámple i & Wêll & $\begin{array}{l}\text { Collection } \\
\text { Date }\end{array}$ & $\begin{array}{l}\text { Chlorid } \\
\text { mg/L }\end{array}$ & $\begin{array}{l}\text { Eliforide } \\
\text { mig/ }\end{array}$ & $\begin{array}{l}\text { itrateng } \\
\text { (asNo }\end{array}$ & $\begin{array}{l}\text { Plosplat } \\
\text { mogl }\end{array}$ & osulfate & $\begin{array}{l}\text { Sulfite } \\
\text { mgds }\end{array}$ & 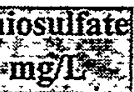 \\
\hline FL104 & RM-3 & $12 / 1 / 98$ & 2.70 & $<0.2$ & 2.20 & $<0.2$ & 6.18 & $<3$ & \\
\hline FL105 & RM-3 & $12 / 1 / 98$ & 2.64 & $<0.2$ & 2.19 & $<0.2$ & 6.21 & $<3$ & \\
\hline FL123 & RM-3 & $12 / 15 / 98$ & 2.48 & $<0.2$ & 1.97 & $<0.2$ & 7.36 & $<3$ & \\
\hline FL143 & RM-3 & $1 / 5 / 99$ & 3.63 & $<2$ & $<2$. & $<2$ & 6.83 & $<3$ & \\
\hline FLI64 & RM-3 & $2 / 9 / 99$ & 3.88 & $<2$ & $<2$ & $<2$ & 7.29 & & \\
\hline FL205 & RM-3 & $4 / 1 / 99$ & 3.02 & $<2$ & $<2$ & $<2$ & 7.60 & $<3$ & \\
\hline FL224 & $\mathrm{RM}-3$ & $8 / 5 / 99$ & 2.28 & $<0.2$ & 2.54 & $<0.2$ & 6.53 & & \\
\hline FL343 & RM-3 & $8 / 22 / 99$ & 2.23 & 0.21 & 2.18 & $<0.2$ & 18.7 & $<0.2$ & \\
\hline FL404 & $\mathrm{RM}-3$ & 9/21/99 & 3.13 & $<2$ & $<2$ & $<2$ & 6.58 & $<3$ & \\
\hline FL504 & RM-3 & $10 / 19 / 99$ & & & & & 6.10 & $<0.2$ & $<0.1$ \\
\hline FL604 & RM-3 & 12/1/99 & 2.64 & 0.03 & 1.90 & $<2$ & 5.80 & $<0.3$ & $<0.1$ \\
\hline FL604 & RM-3 & $12 / 1 / 99$ & & & & & 5.85 & $<3$ & \\
\hline FL703 & RM-3 & $1 / 6 / 00$ & & & & & 18.0 & 0.20 & \\
\hline FL702 & $\mathrm{RM}-3$ & $1 / 6 / 00$ & & & & & 18.0 & 0.20 & \\
\hline FL802 & RM-3 & $2 / 8 / 00$ & & & & & 8.10 & $<0.14$ & \\
\hline FL803 & $R M-3$ & $2 / 8 / 00$ & & & & & 7.90 & $<0.14$ & \\
\hline FL903 & RM-3 & $3 / 20 / 00$ & & & & & 7.10 & $<0.3$ & \\
\hline FL904 & RM-3 & $3 / 20 / 00$ & & & & & 7.10 & $<0.3$ & \\
\hline FL1103 & $R M-3$ & $5 / 24 / 00$ & & & & & 19.0 & & \\
\hline FL1 104 & RM-3 & $5 / 24 / 00$ & & & & & 18.9 & & \\
\hline FL1203 & RM-3 & $6 / 21 / 00$ & & & & & 6.60 & & \\
\hline FL1204 & $\mathrm{RM}-3$ & $6 / 21 / 00$ & & & & & 6.59 & & \\
\hline FL106 & $\mathrm{RM}-4$ & $12 / 1 / 98$ & 3.43 & $<1$ & 1.57 & $<1$ & 43.5 & $<3$ & \\
\hline FL124 & RM-4 & $12 / 15 / 98$ & 2.53 & 0.29 & 1.64 & 0.20 & 36.9 & $<3$ & \\
\hline FL125 & $\mathrm{RM}-4$ & $12 / 15 / 98$ & 2.53 & 0.30 & 1.64 & $<0.2$ & 36.9 & $<3$ & \\
\hline FL144 & $R M-4$ & $1 / 5 / 99$ & 3.61 & $<2$ & $<2$ & $<2$ & 25.3 & $<3$ & \\
\hline FL145 & $\mathrm{RM}-4$ & $1 / 5 / 99$ & 3.87 & $<2$ & $<2$ & $<2$ & 28.1 & $<3$ & \\
\hline FL166 & $\mathrm{RM}-4$ & $2 / 9 / 99$ & 4.59 & $<2$ & $<2$ & $<2$ & 36.5 & & \\
\hline FL206 & $R M-4$ & $4 / 1 / 99$ & 3.17 & $<2$ & $<2$ & $<2$ & 15.6 & $<3$ & \\
\hline FL225 & $\mathrm{RM}-4$ & $8 / 5 / 99$ & 2.29 & $<0.2$ & 1.59 & $<0.2$ & 7.59 & & \\
\hline FL344 & $\mathrm{RM}-4$ & $8 / 22 / 99$ & 42.4 & $<20$ & $<20$ & $<20$ & 103 & $<20$ & \\
\hline FL405 & RM-4 & 9/21/99 & 3.53 & $<2$ & $<2$ & $<2$ & 57.0 & $<3$ & \\
\hline FL505 & RM-4 & $10 / 19 / 99$ & & & & & 34.0 & $<0.5$ & $<0.1$ \\
\hline FL605 & $\mathrm{RM}-4$ & $12 / 1 / 99$ & 2.81 & 0.06 & 0.35 & 0.09 & 26.0 & $<0.3$ & $<0.1$ \\
\hline FL605 & RM-4 & $12 / 1 / 99$ & & & & & 26.3 & $<30$ & \\
\hline FL705 & $\mathrm{RM}-4$ & $1 / 6 / 00$ & & & & & 130 & $<2$ & \\
\hline FL804 & $\mathrm{RM}-4$ & $2 / 8 / 00$ & & & & & 61.0 & $<0.7$ & \\
\hline FL905 & RM-4 & $3 / 20 / 00$ & & & & & 36.0 & $<1$ & \\
\hline FL1 105 & RM-4 & $5 / 24 / 00$ & & & & & 82.5 & & \\
\hline FL1205 & $R M-4$ & $6 / 21 / 00$ & & & & & 27.3 & & \\
\hline
\end{tabular}

H.15 
Table H.3d. Common Anions by Ion Chromatography (Well RM-5)

\begin{tabular}{|c|c|c|c|c|c|c|c|c|c|}
\hline $\begin{array}{l}\text { S } \\
\text { Sampleuto }\end{array}$ & $\begin{array}{l}\text { Wel } \\
\text { Wul }\end{array}$ & $\begin{array}{l}\text { Collection } \\
\text { Date }\end{array}$ & 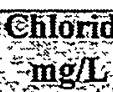 & $\begin{array}{l}\text { Flíorids } \\
\text { mgtL }\end{array}$ & $\begin{array}{l}\text { Nitratemg } \\
\mathrm{u} \text { (aso }\end{array}$ & $\begin{array}{l}\text { Enhosphat } \\
\text { ngf }\end{array}$ & $\begin{array}{l}\text { Sulfate } \\
\text { nugt }\end{array}$ & Sulfite & $\begin{array}{l}\text { hiosulfate } \\
\text { mgth }\end{array}$ \\
\hline FL107 & RM-5 & $12 / 1 / 98$ & 5.81 & $<2$ & $<2$ & $<2$ & 281 & 12.5 & \\
\hline FL126 & RM-5 & $12 / 15 / 98$ & 4.07 & $<2$ & $<2$ & $<2$ & 183 & 33.3 & \\
\hline FLI46 & RM-5 & $1 / 5 / 99$ & 4.26 & $<2$ & $<2$ & $<2$ & 144 & 26.4 & \\
\hline FL176 & RM-5 & $2 / 9 / 99$ & 5.93 & $<2$ & $<2$ & $<2$ & 140 & & \\
\hline FL177 & RM-5 & $2 / 9 / 99$ & 5.76 & $<2$ & $<2$ & $<2$ & 150 & & \\
\hline FL178 & RM-5 & 2/9/99 & 5.89 & $<2$ & $<2$ & $<2$ & 159 & & \\
\hline FL171 & RM-5 & 2/9/99 & 4.84 & $<2$ & $<2$ & $<2$ & 158 & & \\
\hline FL208 & RM-5 & $4 / 1 / 99$ & 3.10 & $<2$ & $<2$ & $<2$ & 91.3 & $<3$ & \\
\hline FL226 & RM-5 & $8 / 5 / 99$ & 2.32 & 0.54 & $<0.2$ & $<0.2$ & 139 & & \\
\hline FL345 & RM-5 & $8 / 22 / 99$ & 22.7 & $<20$ & $<20$ & $<20$ & 326 & 100 & \\
\hline FL406 & RM-5 & $9 / 21 / 99$ & 3.60 & $<2$ & $<2$ & $<2$ & 278 & 120 & \\
\hline FL406 & RM-5 & $9 / 21 / 99$ & & & & & 420 & & \\
\hline FL506 & RM-5 & $10 / 19 / 99$ & & & & & 280 & $<3$ & $<0.1$ \\
\hline FL606 & RM-5 & $12 / 1 / 99$ & 2.81 & 0.26 & 0.35 & 0.72 & 157 & $<1$ & $<0.1$ \\
\hline FL606 & RM-5 & $12 / 1 / 99$ & & & & & 159 & $<3$ & \\
\hline FL713 & RM-5 & $1 / 6 / 00$ & & & & & 480 & 8.00 & \\
\hline FL714 & RM-5 & $1 / 6 / 00$ & & & & & 490 & 8.00 & \\
\hline FL813 & RM-5 & $2 / 8 / 00$ & & & & & 400 & $<6$ & \\
\hline FL814 & RM-5 & $2 / 8 / 00$ & & & & & 400 & $<6$ & \\
\hline FL906 & RM-5 & $3 / 20 / 00$ & & & & & 275 & $<5$ & \\
\hline FL907 & RM-5 & $3 / 20 / 00$ & & & & & 280 & $<5$ & \\
\hline FL1 106 & RM-5 & $5 / 24 / 00$ & & & & & 525 & 5.40 & \\
\hline FL1 107 & RM-5 & $5 / 24 / 00$ & & & & & 530 & 4.92 & \\
\hline FL1206 & RM-5 & $6 / 21 / 00$ & & & & & 332 & 12.10 & \\
\hline FL1207 & RM-5 & $6 / 21 / 00$ & & & & & 330 & & \\
\hline
\end{tabular}

H.16 
Table H.3e. Common Anions by Ion Chromatography (Well RM-6 and RM-7)

\begin{tabular}{|c|c|c|c|c|c|c|c|c|c|}
\hline Sămple & Wello & $\begin{array}{l}\text { Gollection } \\
\text { pate }\end{array}$ & $\begin{array}{l}\text { Chlorid } \\
\text { ungul }\end{array}$ & 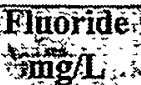 & tratengis & hosphat & 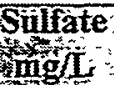 & $\begin{array}{l}\text { Sulfite } \\
\text { ingut }\end{array}$ & iosulfate \\
\hline FL108 & RM-6 & $12 / 1 / 98$ & 4.54 & $<2$ & $<2$ & $<2$ & 89.8 & 3.34 & \\
\hline FL109 & RM-6 & $12 / 1 / 98$ & 4.74 & $<2$ & $<2$ & $<2$ & 90.1 & 2.90 & \\
\hline FL127 & RM-6 & $12 / 15 / 98$ & 4.24 & $<2$ & $<2$ & $<2$ & 79.0 & 5.78 & \\
\hline FL148 & RM-6 & 1/5/99 & 4.03 & $<2$ & $<2$ & $<2$ & 98.8 & 13.40 & \\
\hline FL168 & RM-6 & $2 / 9 / 99$ & 4.46 & $<2$ & $<2$ & $<2$ & 141 & & \\
\hline FL167 & RM-6 & 2/9/99 & 4.95 & $<2$ & $<2$ & $<2$ & 150 & & \\
\hline FL209 & RM-6 & $4 / 1 / 99$ & 3.63 & $<2$ & $<2$ & $<2$ & 126 & $<2$ & \\
\hline FL227 & RM-6 & $8 / 5 / 99$ & 2.37 & 0.39 & $<0.2$ & $<0.2$ & 56.3 & & \\
\hline FL227 & RM-6 & $8 / 5 / 99$ & & & & & 57.7 & & \\
\hline FL346 & RM-6 & $8 / 22 / 99$ & 2.28 & 0.28 & 1.18 & $<0.2$ & 43.7 & $<0.2$ & \\
\hline FL407 & RM-6 & $9 / 21 / 99$ & 3.57 & $<2$ & $<2$ & $<2$ & 93.1 & $<3$ & \\
\hline FL507 & RM-6 & $10 / 19 / 99$ & & & & & 60.0 & $<1$ & $<0.1$ \\
\hline FL607 & RM-6 & $12 / 1 / 99$ & 2.69 & 0.05 & 0.95 & 0.04 & 34.5 & $<0.5$ & $<0.1$ \\
\hline FL607 & RM-6 & $12 / 1 / 99$ & & & & & 36.7 & $<30$ & \\
\hline FL706 & RM-6 & $1 / 6 / 00$ & & & & & 250 & $<3$ & \\
\hline FL816 & RM-6 & $2 / 8 / 00$ & & & & & 270 & $<3$ & \\
\hline FL908 & RM-6 & $3 / 20 / 00$ & & & & & 270 & $<5$ & \\
\hline FL1108 & RM-6 & $5 / 24 / 00$ & & & & & 311 & & \\
\hline FL1208 & RM-6 & $6 / 21 / 00$ & & & & & 184 & & \\
\hline FL110 & RM-7 & $12 / 1 / 98$ & 4.50 & $<2$ & $<2$ & $<2$ & 104 & $<2$ & \\
\hline FL129. & RM-7 & $12 / 15 / 98$ & 4.32 & $<2$ & $<2$ & $<2$ & 77.3 & 6.37 & \\
\hline FL149 & RM-7 & $1 / 5 / 99$ & 3.59 & $<0.2$ & $<0.2$ & $<0.2$ & 73.6 & 6.64 & \\
\hline FL150 & RM-7 & $1 / 5 / 99$ & 3.95 & $<2$ & $<2$ & $<2$ & 75.6 & 7.16 & \\
\hline FL179 & RM-7 & 2/9/99 & 6.34 & $<2$ & $<2$ & $<2$ & 91.8 & & \\
\hline FL180 & RM-7 & 2/9/99 & 6.28 & $<2$ & $<2$ & $<2$ & 88.7 & & \\
\hline FL181 & RM-7 & 2/9/99 & 6.70 & $<2$ & $<2$ & $<2$ & 117 & & \\
\hline FL172 & RM-7 & 2/9/99 & 5.92 & $<2$ & $<2$ & $<2$ & 96.6 & & \\
\hline FL210 & $\mathrm{RM}-7$ & $4 / 1 / 99$ & 3.40 & $<2$ & $<2$ & $<2$ & 73.0 & $<3$ & \\
\hline FL228 & RM-7 & $8 / 5 / 99$ & 2.37 & 0.33 & $<0.2$ & $<0.2$ & 30.8 & & \\
\hline FL347 & RM-7 & $8 / 22 / 99$ & 41.2 & $<20$ & $<20$ & $<20$ & 137 & $<20$ & \\
\hline FL408 & RM-7 & 9/21/99 & 3.62 & $<2$ & $<2$ & $<2$ & 121 & $<3$ & \\
\hline FL508 & RM-7 & $10 / 19 / 99$ & & & & & 60.0 & $<1$ & $<0.1$ \\
\hline FL608 & $\mathrm{RM}-7$ & $12 / 1 / 99$ & 2.79 & 0.06 & 0.34 & $<2$ & 31.9 & $<0.5$ & $<0.1$ \\
\hline FL608 & $\mathrm{RM}-7$ & $12 / 1 / 99$ & & & & & 32.0 & $<30$ & \\
\hline FL707 & RM-7 & $1 / 6 / 00$ & & & & & 230 & $<3$ & \\
\hline FL815 & RM-7 & $2 / 8 / 00$ & & & & & 180 & $<3$ & \\
\hline FL909 & RM-7 & $3 / 20 / 00$ & & & & & 160 & $<3$ & \\
\hline FL1 109 & $\mathrm{RM}-7$ & $5 / 24 / 00$ & & & & & 259 & & \\
\hline FL1209 & RM-7 & $6 / 21 / 00$ & & & & & 123 & & \\
\hline
\end{tabular}


Table H.3f. Common Anions by Ion Chromatography (Well RM-8)

\begin{tabular}{|c|c|c|c|c|c|c|c|c|c|}
\hline Sample ID & Well & $\begin{array}{l}\text { Collection } \\
\text { Date }\end{array}$ & Chlorid & $\begin{array}{l}\text { Fluorid } \\
\text { mgth }\end{array}$ & $\begin{array}{l}\text { Nitrateng } \\
\text { (as No }\end{array}$ & Phospha & Sulfat & $\begin{array}{l}\text { Sulfife } \\
\text { ngfI }\end{array}$ & $\begin{array}{l}\text { Thiosulatite } \\
\text { mofd }\end{array}$ \\
\hline FL111 & RM-8 & $12 / 1 / 98$ & 4.43 & $<2$ & $<2$ & $<2$ & 117 & $<30$ & \\
\hline FL130 & RM-8 & $12 / 15 / 98$ & 4.61 & $<2$ & $<2$ & $<2$ & 96.6 & 7.00 & \\
\hline FL151 & RM-8 & $1 / 5 / 99$ & 3.80 & $<2$ & $<2$ & $<2$ & 65.3 & 3.12 & \\
\hline FL173 & RM-8 & 2/8/99 & 5.11 & $<2$ & $<2$ & $<2$ & 65.1 & & \\
\hline FL174 & RM-8 & $2 / 8 / 99$ & 5.32 & $<2$ & $<2$ & $<2$ & 74.1 & & \\
\hline FL175 & RM-8 & 2/8/99 & 5.52 & $<2$ & $<2$ & $<2$ & 88.6 & & \\
\hline FL169 & RM-8 & 2/9/99 & 4.45 & $<2$ & $<2$ & $<2$ & 70.7 & & \\
\hline FL211 & RM-8 & 4/9/99 & 3.61 & $<2$ & $<2$ & $<2$ & 45.8 & $<3$ & \\
\hline FL230 & RM-8 & $8 / 5 / 99$ & 2.30 & 0.40 & $<0.2$ & 0.23 & 37.0 & & \\
\hline FL348 & RM-8 & $8 / 22 / 99$ & 43.9 & $<20$ & $<20$ & $<20$ & 254 & $<20$ & \\
\hline FL410 & RM-8 & $9 / 21 / 99$ & 3.90 & $<2$ & $<2$ & $<2$ & 96.5 & 10.10 & \\
\hline FL510 & RM-8 & 10/19/99 & & & & & 81.0 & $<0.2$ & $<0.1$ \\
\hline FL610 & RM-8 & $12 / 1 / 99$ & 2.68 & 0.04 & $<2$ & 0.67 & 64.9 & $<0.5$ & $<0.1$ \\
\hline FL610 & RM-8 & $12 / 1 / 99$ & & & & & 67.0 & $<30$ & \\
\hline FL708 & RM-8 & $1 / 6 / 00$ & & & & & 340 & $<3$ & \\
\hline FL812 & RM-8 & $2 / 8 / 00$ & & & & & 210 & $<1$ & \\
\hline FL911 & RM-8 & $3 / 20 / 00$ & & & & & 120 & $<3$ & \\
\hline FL1111 & RM-8 & $5 / 24 / 00$ & & & & & 262 & & \\
\hline FL1211 & RM-8 & $6 / 21 / 00$ & & & & & 97.8 & & \\
\hline
\end{tabular}


Table H.3g. Common Anions by Ion Chromatography (Well RM-9 and RM-10)

\begin{tabular}{|c|c|c|c|c|c|c|c|c|c|}
\hline Samplè Io & Well & $\begin{array}{c}\text { Collection } \\
\text { Date }\end{array}$ & $\begin{array}{l}\text { Chloride } \\
\text { migh }\end{array}$ & $\begin{array}{l}\text { Fluorid } \\
\text { mght }\end{array}$ & $\begin{array}{l}\text { Nitrate ging } \\
\text { (asto } 3\end{array}$ & $\begin{array}{l}\text { Phosphate } \\
\text { mghts }\end{array}$ & $\begin{array}{l}\text { Sulfate } \\
\text { ing } / \mathrm{L}\end{array}$ & $\begin{array}{l}\text { Sulfite } \\
\text { mginf }\end{array}$ & iosiluate \\
\hline FL112 & RM-9 & $12 / 1 / 98$ & 5.15 & $<2$ & $<2$ & $<2$ & 82.2 & $<30$ & \\
\hline FL133 & RM-9 & $12 / 15 / 98$ & 4.88 & $<2$ & 2.05 & $<2$ & 71.8 & $<30$ & \\
\hline FL132 & RM-9 & $12 / 15 / 98$ & 4.71 & $<2$ & $<2$ & $<2$ & 79.9 & $<30$ & \\
\hline - FL135 & RM-9 & $12 / 15 / 98$ & 5.32 & $<2$ & $<2$ & $<2$ & 82.2 & $<30$ & \\
\hline FL136 & RM-9 & $12 / 15 / 98$ & 2.68 & 0.54 & 1.25 & $<0.2$ & 102 & $<30$ & \\
\hline FL134 & RM-9 & $12 / 15 / 98$ & 5.00 & $<2$ & $<2$ & $<2$ & 79.4 & $<30$ & \\
\hline FL137 & RM-9 & $12 / 15 / 98$ & 5.08 & $<2$ & $<2$ & $<2$ & 91.6 & $<30$ & \\
\hline FL152 & RM-9 & $1 / 5 / 99$ & 3.85 & $<2$ & $<2$ & $<2$ & 44.1 & $<30$ & \\
\hline FL170 & RM-9 & 2/9/99 & 4.27 & $<2$ & $<2$ & $<2$ & 68.1 & & \\
\hline FL212 & RM-9 & $4 / 1 / 99$ & 4.11 & $<2$ & $<2$ & $<2$ & 52.3 & $<3$ & \\
\hline FL231 & RM-9 & $8 / 5 / 99$ & 2.25 & 0.30 & 0.39 & $<0.2$ & 19.1 & & \\
\hline FL349 & RM-9 & $8 / 22 / 99$ & 42.5 & $<20$ & $<20$ & $<20$ & 130 & $<20$ & \\
\hline FLA11 & RM-9 & 9/21/99 & 3.95 & $<2$ & $<2$ & $<2$ & 59.3 & 2.91 & \\
\hline FL403 & RM-9 & 9/21/99 & 3.00 & $<2$ & $<2$ & $<2$ & 67.9 & $<3$ & \\
\hline FLA03 & RM-9 & $9 / 21 / 99$ & & & & & 68.0 & $<1$ & \\
\hline FL511 & RM-9 & $10 / 19 / 99$ & & & & & 48.0 & $<0.5$ & $<0.12$ \\
\hline FL611 & RM-9 & $12 / 1 / 99$ & 2.61 & 0.06 & 2.61 & 0.67 & 51.0 & $<30$ & $<0.1$ \\
\hline FL611 & RM-9 & $12 / 1 / 99$ & & & & & 51.4 & & \\
\hline FL710 & RM-9 & $1 / 6 / 00$ & & & & & 245 & 0.20 & \\
\hline FL808 & RM-9 & $2 / 8 / 00$ & & & & & 140 & $<1$ & \\
\hline FL912 & RM-9 & $3 / 20 / 00$ & & & & & 88.0 & $<3$ & \\
\hline FL1112 & RM-9 & $5 / 24 / 00$ & & & & & 181 & & \\
\hline FL1212 & RM-9 & $6 / 21 / 00$ & & & & & 71.7 & & \\
\hline FL232 & $\overline{R M-10}$ & $8 / 5 / 99$ & 2.27 & 0.29 & 0.31 & $<0.2$ & 19.1 & & \\
\hline FL350 & RM-10 & $8 / 22 / 99$ & 28.2 & $<20$ & $<20$ & $<20$ & 101 & $<20$ & \\
\hline FL412 & RM-10 & $9 / 21 / 99$ & 4.01 & $<2$ & $<2$ & $<2$ & 78.1 & $<3$ & \\
\hline FL512 & RM-10 & $10 / 19 / 99$ & & & & & 54.0 & $<1$ & $<0.1$ \\
\hline FL612 & RM-10 & $12 / 1 / 99$ & 2.64 & 0.05 & $<2$ & 0.63 & 48.0 & $<0.5$ & $<0.1$ \\
\hline FL613 & RM-10 & $12 / 1 / 99$ & 2.64 & 0.05 & $<2$ & 0.70 & 48.0 & $<0.5$ & $<0.1$ \\
\hline FL613 & $\mathrm{RM}-10$ & $12 / 1 / 99$ & & & & & 48.3 & $<30$ & \\
\hline FL612 & RM-10 & $12 / 1 / 99$ & & & & & 48.4 & $<30$ & \\
\hline FL711 & RM-10 & $1 / 6 / 00$ & & & & & 180 & $<3$ & . \\
\hline FL805 & RM-10 & $2 / 8 / 00$ & & & & & 79.0 & $<0.7$ & \\
\hline FL913 & RM-10 & $3 / 20 / 00$ & & . & & & 52.0 & $<1$ & \\
\hline FL1113 & RM-10 & $5 / 24 / 00$ & & & & & 146 & & \\
\hline FL1213 & RM-10 & $6 / 21 / 00$ & & & & & 68.1 & & \\
\hline
\end{tabular}


Table H.3h. Common Anions by Ion Chromatography

(Wells RM-11, RM-12, RM-13, RM-14, and RM-15)

\begin{tabular}{|c|c|c|c|c|c|c|c|c|c|}
\hline \multirow{3}{*}{$\frac{\text { Sample }}{\text { FL233 }}$} & \multirow{3}{*}{$\frac{\text { Well }}{\text { RM-11 }}$} & \multirow{2}{*}{\multicolumn{2}{|c|}{$\begin{array}{l}\text { Collection Chloride } \\
\text { Date ng } \mathrm{m}\end{array}$}} & \multirow{2}{*}{$\begin{array}{l}\text { Fluoride } \\
\text { mgl }\end{array}$} & \multirow{3}{*}{ 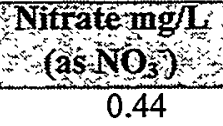 } & \multirow{2}{*}{$\begin{array}{l}\text { Phosphat } \\
\text { nog th- }\end{array}$} & \multirow{2}{*}{ Suifate } & \multicolumn{2}{|c|}{ Sulfite Thiosulfafe } \\
\hline & & & & & & & & $m g t$ & $\mathbf{m g} \mathrm{I}$ \\
\hline & & $8 / 5 / 99$ & 2.32 & 0.24 & & $<0.2$ & 25.1 & & \\
\hline FL234 & RM-11 & $8 / 5 / 99$ & 2.31 & 0.24 & 0.42 & $<0.2$ & 25.4 & & \\
\hline FL351 & RM-11 & $8 / 22 / 99$ & 43.4 & $<20$ & $<20$ & $<20$ & 153 & $<20$ & \\
\hline FL413 & RM-11 & $9 / 21 / 99$ & 4.07 & $<2$ & $<2$ & $<2$ & 91.2 & 4.23 & \\
\hline FL414 & RM-11 & $9 / 21 / 99$ & 4.02 & $<2$ & $<2$ & $<2$ & 98.9 & $<3$ & \\
\hline FL513 & RM-11 & $10 / 19 / 99$ & & & & & 68.0 & $<1$ & $<0.1$ \\
\hline FL514 & RM-11 & $10 / 19 / 99$ & & & & & 66.0 & $<1$ & $<0.1$ \\
\hline FL614 & RM-11 & $12 / 1 / 99$ & 2.75 & 0.05 & 0.48 & 0.65 & 43.0 & $<0.5$ & $<0.1$ \\
\hline FL614 & RM-11 & $12 / 1 / 99$ & & & & & 44.3 & $<30$ & \\
\hline FL712 & $\mathrm{RM}-11$ & $1 / 6 / 00$ & & & & & 210 & $<2$ & \\
\hline FL807 & RM-11 & $2 / 8 / 00$ & & & & & 130 & $<1$ & \\
\hline FL914 & RM-11 & $3 / 20 / 00$ & & & & & 110 & $<3$ & \\
\hline FL1114 & RM-11 & $5 / 24 / 00$ & & & & & 174 & & \\
\hline FL1214 & $\mathrm{RM}-11$ & $6 / 21 / 00$ & & & & & 123 & & \\
\hline FL235 & $\mathrm{RM}-12$ & $8 / 5 / 99$ & 2.50 & 0.33 & 1.79 & $<0.2$ & 50.7 & & \\
\hline FL352 & RM-12 & $8 / 22 / 99$ & 46.0 & $<20$ & $<20$ & $<20$ & 296 & $<20$ & \\
\hline FL415 & RM-12 & 9/21/99 & $4.5 \mathrm{I}$ & $<2$ & $<2$ & $<2$ & 144 & 22.4 & \\
\hline FL515 & RM-12 & $10 / 19 / 99$ & & & & & 103 & $<1$ & $<0.1$ \\
\hline FL615 & $\mathrm{RM}-12$ & $12 / 1 / 99$ & 2.71 & 0.04 & 0.02 & 0.64 & 49.0 & $<1$ & $<0.1$ \\
\hline FL615 & $\mathrm{RM}-12$ & $12 / 1 / 99$ & & & & & 58.4 & $<30$ & \\
\hline FL715 & $\mathrm{RM}-12$ & $1 / 6 / 00$ & & & & & 180 & 8.00 & \\
\hline FL806 & $\mathrm{RM}-12$ & $2 / 8 / 00$ & & & & & 100 & $<1$ & \\
\hline FL915 & RM-12 & $3 / 20 / 00$ & & & & & 74.0 & $<3$ & \\
\hline FL1115 & $\mathrm{RM}-12$ & $5 / 24 / 00$ & & & & & 210 & & \\
\hline FL236 & $\overline{R M}-13$ & $8 / 5 / 99$ & 2.30 & 0.21 & $<0.2$ & $<0.2$ & 21.3 & & \\
\hline FL353 & RM-13 & $8 / 22 / 99$ & 45.6 & $<20$ & $<20$ & $<20$ & 156 & $<20$ & \\
\hline FL416 & RM-13 & $9 / 21 / 99$ & 3.99 & $<2$ & $<2$ & $<2$ & 136 & 9.51 & \\
\hline FL416 & RM-13 & $9 / 21 / 99$ & & & & & 150 & & \\
\hline FL516 & RM-13 & $10 / 19 / 99$ & & & & & 94.0 & $<1$ & \\
\hline FL616 & $R M-13$ & $12 / 1 / 99$ & 2.69 & 0.05 & 0.02 & 0.72 & 72.0 & $<1$ & \\
\hline FL616 & RM-13 & $12 / 1 / 99$ & & & & & 85.0 & $<30$ & \\
\hline FL716 & RM-13 & $1 / 6 / 00$ & & & & & 190 & 4.00 & \\
\hline FL811 & RM-13 & $2 / 8 / 00$ & & & & & 96.0 & $<1$ & \\
\hline FL916 & RM-13 & $3 / 20 / 00$ & & & & & 58.0 & $<1$ & \\
\hline FL1116 & RM-13 & $5 / 24 / 00$ & & & & & 167 & & \\
\hline FL1216 & $\mathrm{RM}-13$ & $6 / 21 / 00$ & & & & & 96.6 & & \\
\hline FL1215 & $\mathrm{RM}-14$ & $6 / 21 / 00$ & & & & & 289 & & \\
\hline FL1201 & $\mathrm{RM}-15$ & $6 / 21 / 00$ & & & & & 308 & 1.20 & \\
\hline
\end{tabular}


Table H.4a. Water Chemistry Parameters (Wells RM-la and RM-1B)

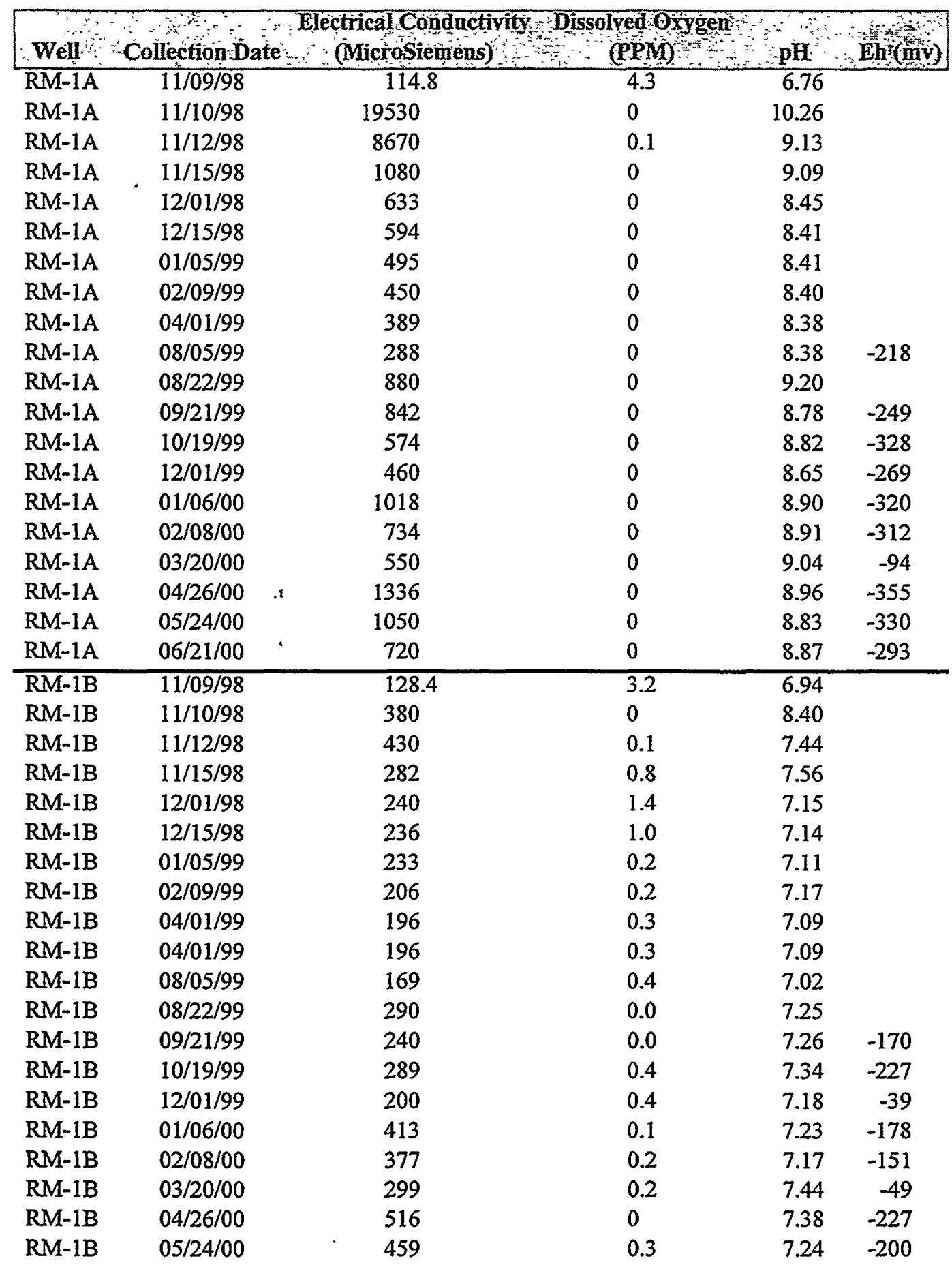


Table H.4b. Water Chemistry Parameters (Well RM-2)

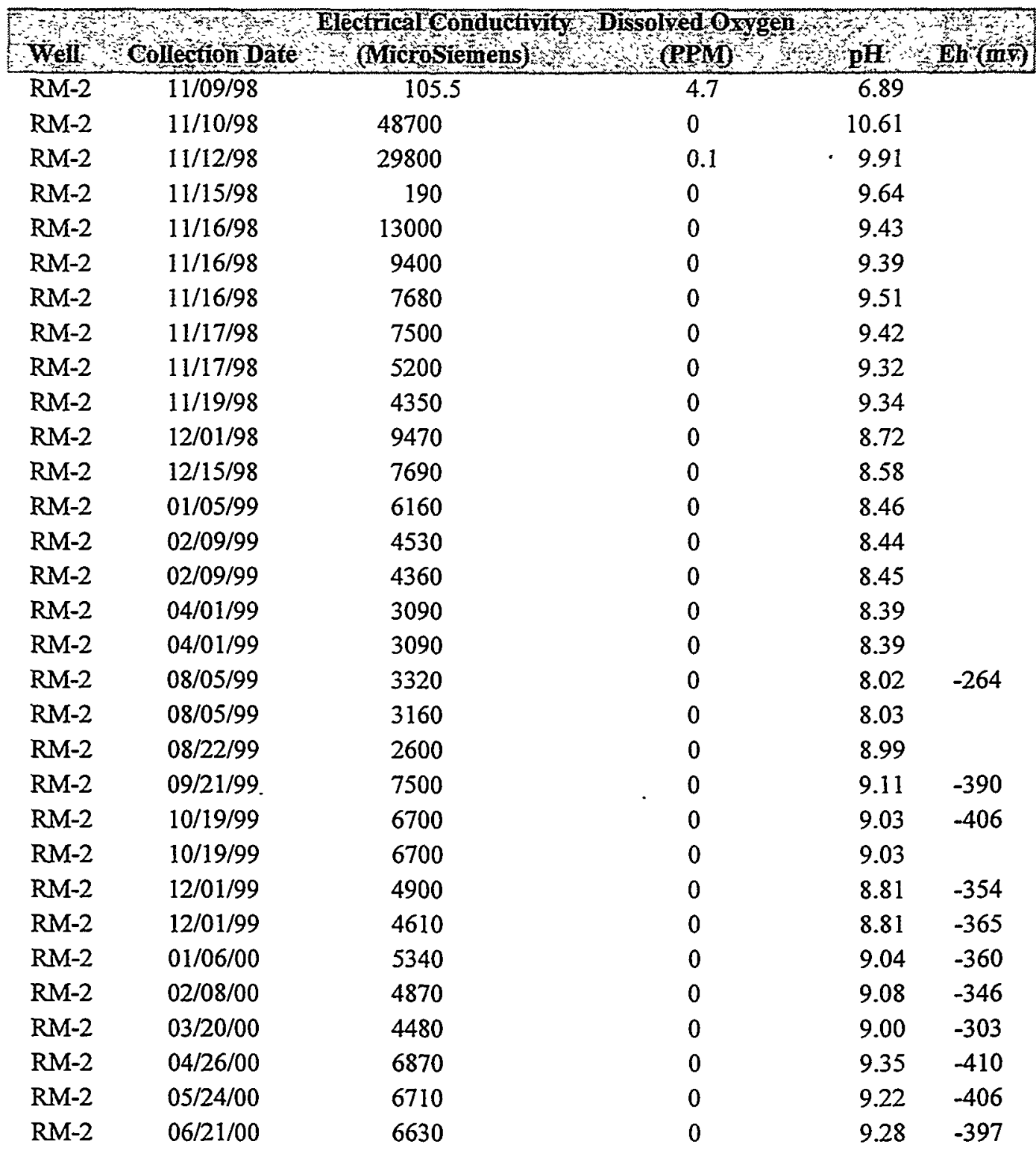


Table H.4c. Water Chemistry Parameters (Well RM-3)

\begin{tabular}{|c|c|c|c|c|c|}
\hline Wêli & Collectioni Date & $\begin{array}{l}\text { ElectricalCondinctivy } \\
\text { (Microsiemens) }\end{array}$ & $\begin{array}{l}\text { dyed oxyget } \\
(\overline{\mathrm{P} P M})\end{array}$ & pH & Eh (miv) \\
\hline $\mathrm{RM}-3$ & $11 / 09 / 98$ & 98.5 & 6.0 & 6.76 & \\
\hline$R M-3$ & $11 / 10 / 98$ & 2320 & 0.0 & 8.85 & \\
\hline RM-3 & $11 / 12 / 98$ & 128 & 6.7 & 7.27 & \\
\hline RM-3 & $11 / 15 / 98$ & 125 & 7.0 & 7.32 & \\
\hline RM-3 & $12 / 01 / 98$ & 122.8 & 6.8 & 6.80 & \\
\hline RM-3 & $12 / 01 / 98$ & 122.9 & 6.8 & 6.79 & \\
\hline RM-3 & $12 / 15 / 98$ & 122 & 6.5 & 6.83 & \\
\hline RM-3 & $01 / 05 / 99$ & 124 & 5.7 & 6.76 & \\
\hline RM-3 & $02 / 09 / 99$ & 126.7 & 4.1 & 6.95 & \\
\hline $\mathrm{RM}-3$ & $04 / 01 / 99$ & 131.6 & 4.6 & 6.75 & \\
\hline $\mathrm{RM}-3$ & $08 / 05 / 99$ & 109 & 7.2 & 6.89 & -30 \\
\hline $\mathrm{RM}-3$ & $08 / 22 / 99$ & 170 & 4.0 & 7.25 & \\
\hline$R M-3$ & $09 / 21 / 99$ & 114 & 6.5 & 6.86 & 74 \\
\hline $\mathrm{RM}-3$ & $10 / 19 / 99$ & 107.5 & 6.3 & 6.92 & 49.6 \\
\hline RM-3 & $12 / 01 / 99$ & 112 & 6.3 & 6.86 & 107 \\
\hline $\mathrm{RM}-3$ & $01 / 06 / 00$ & 165.3 & 1.4 & 7.07 & 15 \\
\hline RM-3 & $01 / 06 / 00$ & 163.5 & 1.5 & 7.06 & -13.4 \\
\hline RM-3 & $02 / 08 / 00$ & 132.9 & 4.2 & 7.00 & -20 \\
\hline$R M-3$ & $02 / 08 / 00$ & 131 & & 8.91 & 10 \\
\hline $\mathrm{RM}-3$ & $03 / 20 / 00$ & 123.2 & 4.3 & 6.98 & 73 \\
\hline RM-3 & $03 / 20 / 00$ & 122.3 & 4.3 & 6.99 & 111 \\
\hline RM-3 & $04 / 26 / 00$ & 281 & 0 & 7.95 & -347 \\
\hline RM-3 & $04 / 26 / 00$ & 287 & 0 & 7.89 & -347 \\
\hline RM-3 & $05 / 24 / 00$ & 167 & 1.0 & 7.33 & -33 \\
\hline RM-3 & $05 / 24 / 00$ & 167 & 2.1 & 7.30 & -33 \\
\hline$R M-3$ & $06 / 21 / 00$ & 130.8 & 6.2 & 7.09 & 32 \\
\hline RM-3 & $06 / 21 / 00$ & 130.4 & 5.5 & 7.26 & 32 \\
\hline
\end{tabular}


Table H.4d. Water Chemistry Parameters (Well RM-4)

\begin{tabular}{|c|c|c|c|c|c|}
\hline \multicolumn{6}{|c|}{ 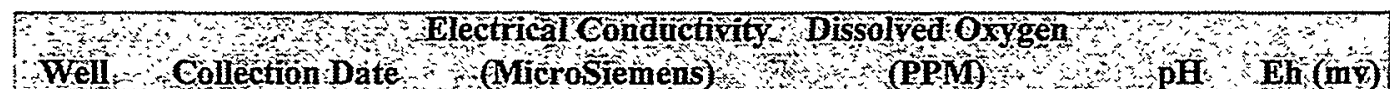 } \\
\hline RM-4 & $11 / 09 / 98$ & 106.7 & 5.1 & 6.76 & \\
\hline$R M-4$ & $11 / 10 / 98$ & 28300 & 0 & 10.35 & \\
\hline$R M-4$ & $11 / 12 / 98$ & 2690 & 0.1 & 9.03 & \\
\hline $\mathrm{RM}-4$ & $11 / 15 / 98$ & 470 & 0 & 9.10 & \\
\hline RM-4 & $12 / 01 / 98$ & 303 & 0 & 8.43 & \\
\hline $\mathrm{RM}-4$ & $12 / 15 / 98$ & 268 & 0 & 8.45 & \\
\hline$R M-4$ & $12 / 15 / 98$ & 268 & 0 & 8.45 & \\
\hline$R M-4$ & $01 / 05 / 99$ & 261 & 0 & 8.35 & \\
\hline RM-4 & $02 / 09 / 99$ & 248 & 0 & 8.22 & \\
\hline $\mathrm{RM}-4$ & $04 / 01 / 99$ & 208 & 0.4 & 7.85 & \\
\hline $\mathrm{RM}-4$ & $08 / 05 / 99$ & 148 & 3.8 & 7.32 & -105 \\
\hline$R M-4$ & $08 / 22 / 99$ & 600 & 0 & 8.93 & \\
\hline $\mathrm{RM}-4$ & $09 / 21 / 99$ & 360 & 0 & 8.62 & -240 \\
\hline $\mathrm{RM}-4$ & $10 / 19 / 99$ & 266 & 0 & 8.70 & -275 \\
\hline $\mathrm{RM}-4$ & $12 / 01 / 99$ & 239 & 0 & 8.58 & -262 \\
\hline RM-4 & $01 / 06 / 00$ & 528 & 0 & 8.74 & -315 \\
\hline$R M-4$ & $02 / 08 / 00$ & 344 & 0.1 & 8.91 & -284 \\
\hline $\mathrm{RM}-4$ & $03 / 20 / 00$ & 286 & 0.2 & 8.95 & -247 \\
\hline RM-4 & $04 / 26 / 00$ & 737 & 0 & 8.83 & -331 \\
\hline $\mathrm{RM}-4$ & $05 / 24 / 00$ & 422 & 0 & 8.90 & -310 \\
\hline $\mathrm{RM}-4$ & $06 / 21 / 00$ & 280 & 0 & 8.88 & -287 \\
\hline
\end{tabular}


Table H.4e. Water Chemistry Parameters (Well RM-5)

\begin{tabular}{|c|c|c|c|c|c|}
\hline Wêll & Collection Date & $\begin{array}{l}\text { Electrical Conductiv } \\
\text { (Microsiemeñs) }\end{array}$ & $\begin{array}{r}\text { Dissolved } \\
\text { (PPM) }\end{array}$ & $\mathbf{p} \mathbf{\mathbf { H }}$ & Eh (iniv) \\
\hline RM-5 & $11 / 09 / 98$ & 108.5 & 4.5 & 6.91 & \\
\hline RM-5 & $11 / 10 / 98$ & 20900 & 0 & 10.11 & \\
\hline RM-5 & $11 / 12 / 98$ & 12370 & 0.1 & 9.05 & \\
\hline RM-5 & $11 / 15 / 98$ & 2200 & 0 & 9.10 & \\
\hline RM-5 & $12 / 01 / 98$ & 1119 & 0 & 8.46 & \\
\hline RM-5 & $12 / 15 / 98$ & 925 & 0 & 8.44 & \\
\hline RM-5 & $01 / 05 / 99$ & 777 & 0 & 8.40 & \\
\hline RM-5 & 02/09/99 & 559 & 0 & 8.47 & \\
\hline RM-5 & 02/09/99 & 591 & 0 & 8.49 & \\
\hline RM-5 & 02/09/99 & 609 & 0 & 8.51 & \\
\hline RM-5 & 02/09/99 & 631 & 0 & 8.56 & \\
\hline RM-5 & 04/01/99 & 471 & 0 & 8.54 & \\
\hline RM-5 & $08 / 05 / 99$ & 498 & 0 & 8.35 & -232 \\
\hline RM-5 & 08/22/99 & 2300 & 0 & 9.11 & \\
\hline RM-5 & $09 / 21 / 99$ & 1600 & 0 & 9.13 & -360 \\
\hline RM-5 & $10 / 19 / 99$ & 992 & 0 & 9.11 & -322 \\
\hline $\mathrm{RM}-5$ & $12 / 01 / 99$ & 631 & 0 & 9.06 & -312 \\
\hline RM-5 & $01 / 06 / 00$ & 1729 & 0 & 9.22 & -335 \\
\hline RM-5 & 01/06/00 & 1786 & 0 & 9.24 & -325 \\
\hline RM-5 & $02 / 08 / 00$ & 1270 & 0 & 9.17 & -307 \\
\hline RM-5 & $03 / 20 / 00$ & 991 & 0 & 9.30 & -270 \\
\hline RM-5 & $03 / 20 / 00$ & 1045 & 0 & 9.32 & -275 \\
\hline RM-5 & $04 / 26 / 00$ & 2050 & 0 & 9.32 & -385 \\
\hline RM-5 & 04/26/00 & 2150 & 0 & 9.31 & -387 \\
\hline RM-5 & $05 / 24 / 00$ & 1700 & 0 & 9.37 & -368 \\
\hline RM-5 & $05 / 24 / 00$ & 1790 & 0 & 9.30 & -390 \\
\hline RM-5 & $06 / 21 / 00$ & 1352 & 0 & 9.48 & -358 \\
\hline RM-5 & $06 / 21 / 00$ & 1508 & 0 & 9.48 & -376 \\
\hline
\end{tabular}


Table H.4f. Water Chemistry Parameters (Well RM-6)

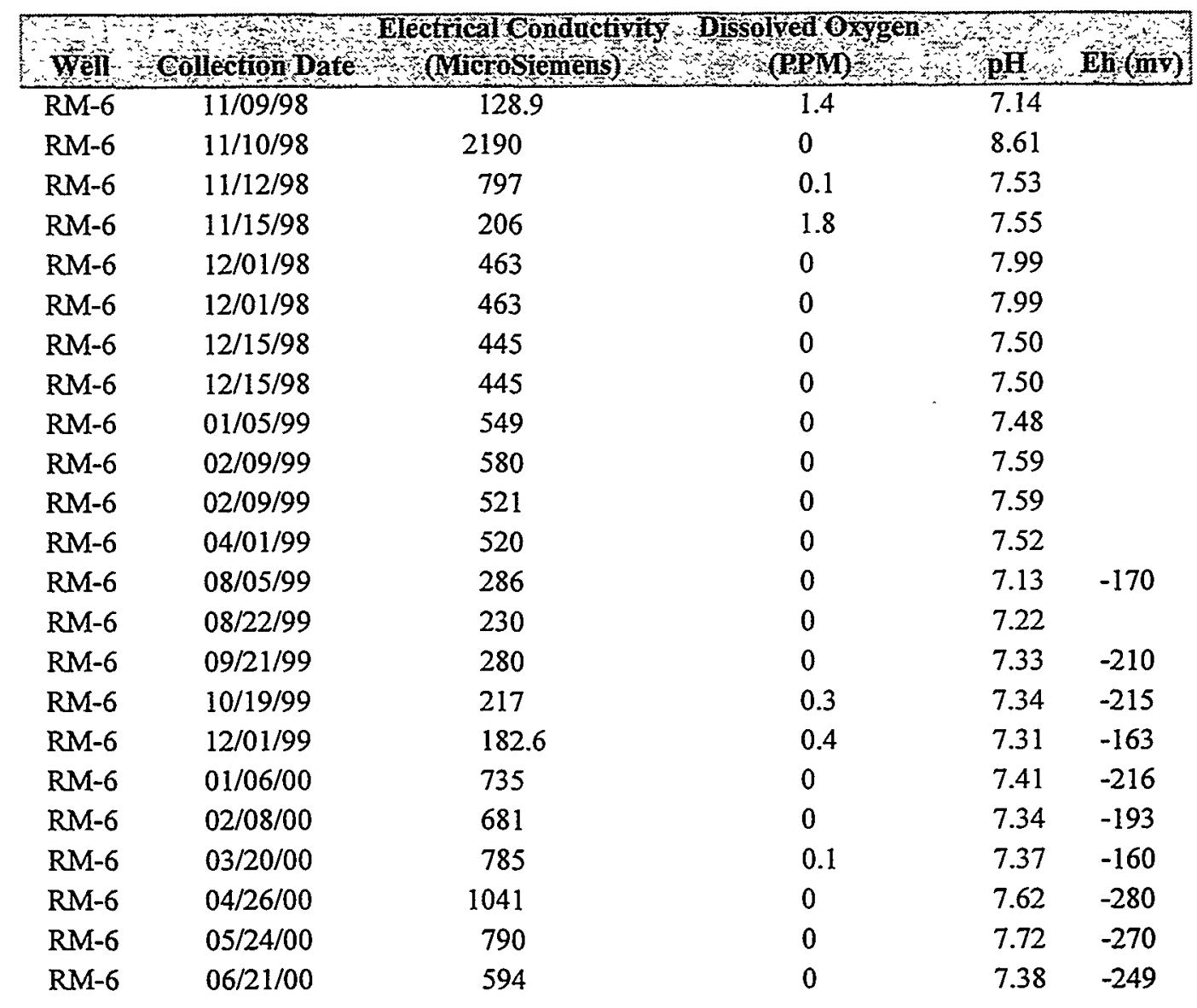


Table H.4g. Water Chemistry Parameters (Well RM-7)

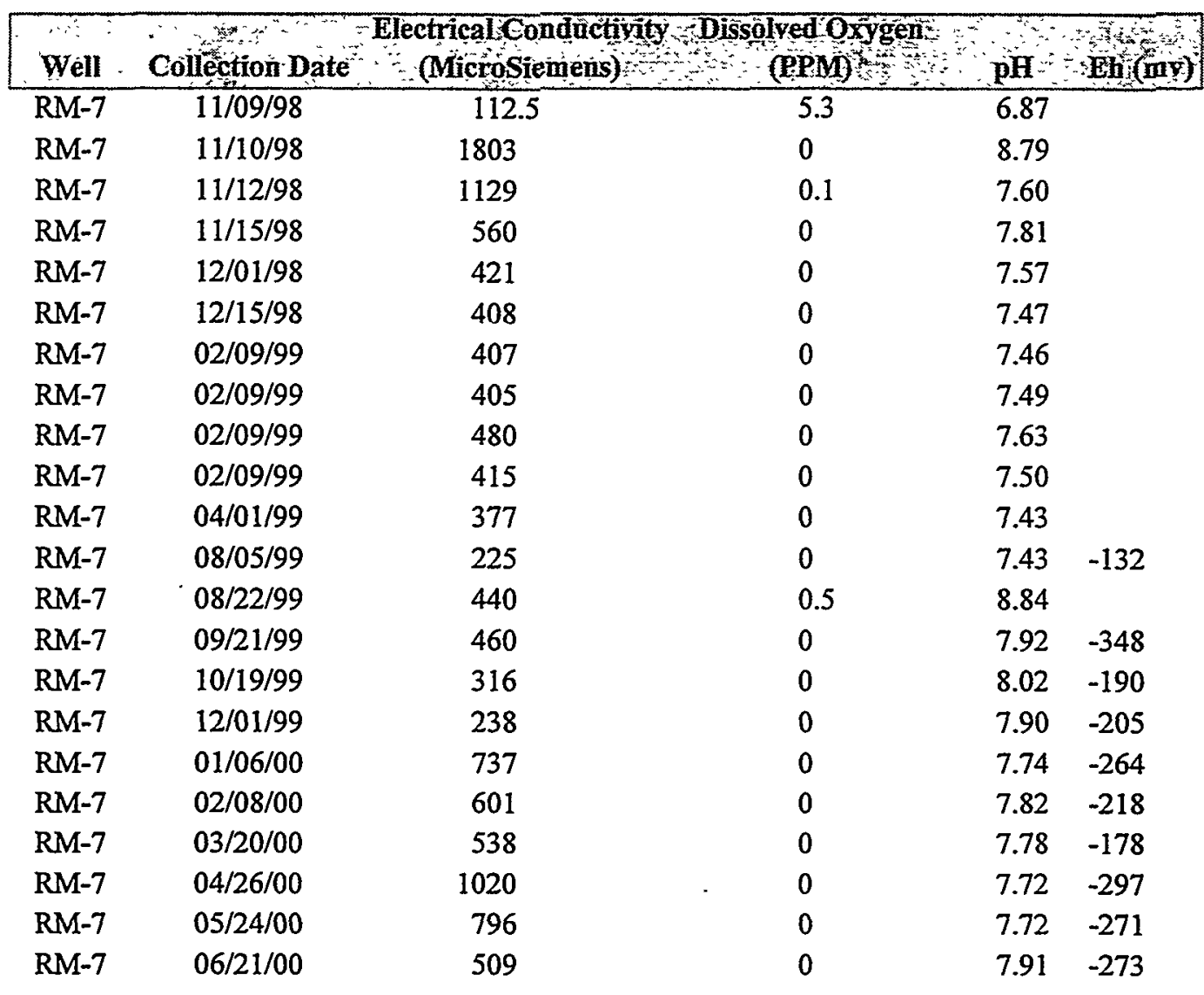


Table H.4h. Water Chemistry Parameters (Well RM-8)

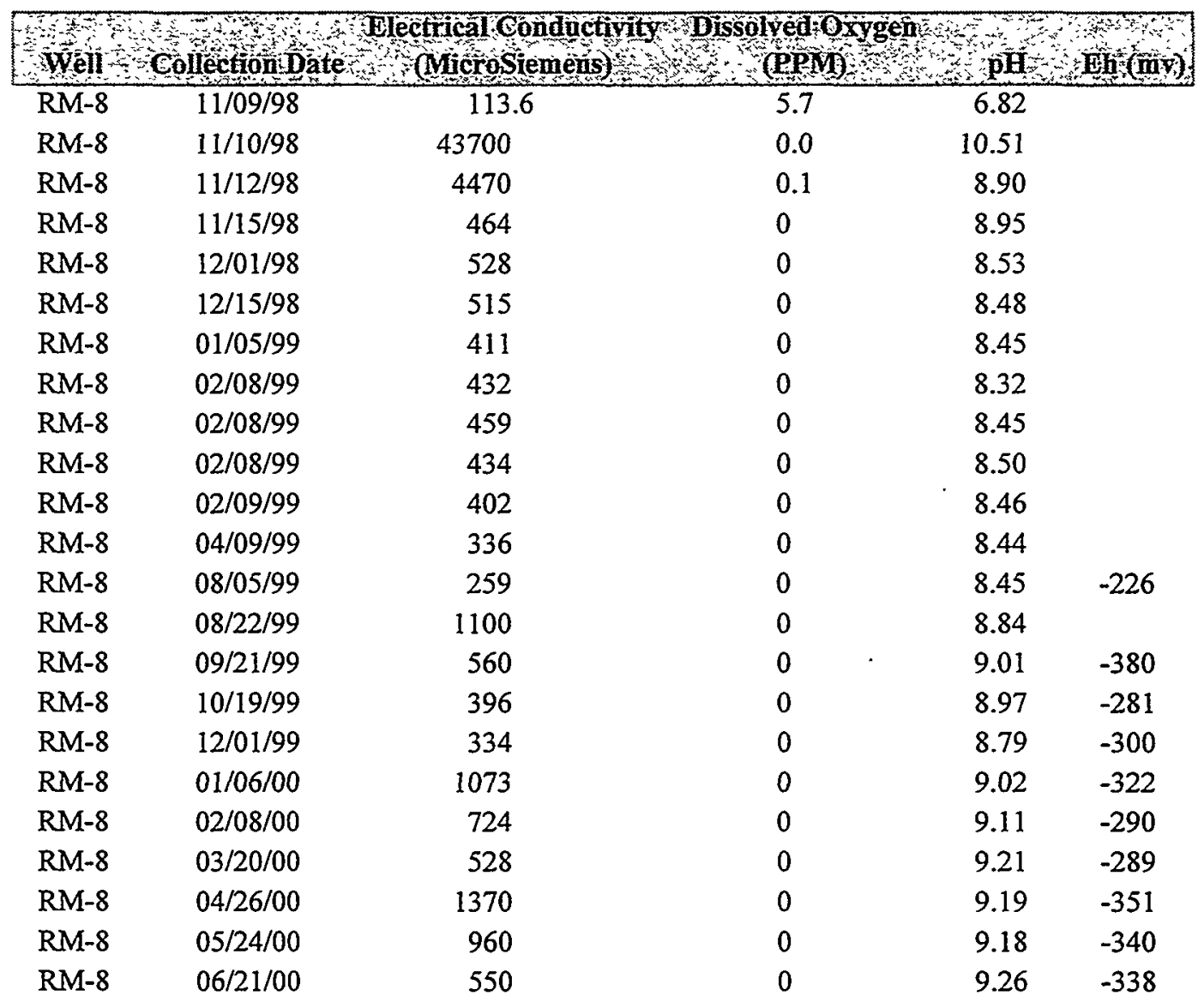


Table H.4i. Water Chemistry Parameters (Well RM-9)

\begin{tabular}{|c|c|c|c|c|c|}
\hline Well & Collection Date & $\begin{array}{l}\text { Electrical Conductivity } \\
\text { (Microsiemens) }\end{array}$ & $\begin{array}{l}\text { Pissolvedoxygen } \\
\text { (PPM) }\end{array}$ & $\mathbf{p H}$ & Eht(niv) \\
\hline $\mathrm{RM}-9$ & $11 / 09 / 98$ & 102.2 & 5.6 & 6.80 & \\
\hline RM-9 & $11 / 10 / 98$ & 24300 & 0 & 10.52 & \\
\hline RM-9 & $11 / 12 / 98$ & 1760 & 0.1 & 9.28 & \\
\hline RM-9 & $11 / 15 / 98$ & 562 & 0 & 8.76 & \\
\hline RM-9 & $12 / 01 / 98$ & 411 & 0 & 8.55 & \\
\hline RM-9 & $12 / 15 / 98$ & 395 & 0 & 8.50 & \\
\hline RM-9 & $12 / 15 / 98$ & 429 & 0 & 8.52 & \\
\hline RM-9 & $12 / 15 / 98$ & 440 & 0 & 8.53 & \\
\hline RM-9 & $12 / 15 / 98$ & 454 & 0 & 8.52 & \\
\hline RM-9 & $12 / 15 / 98$ & 411 & 0 & 8.50 & \\
\hline RM-9 & $12 / 15 / 98$ & 466 & 0 & 8.52 & \\
\hline RM-9 & $01 / 05 / 99$ & 340 & 0 & 8.45 & \\
\hline RM-9 & 02/09/99 & 395 & 0 & 8.43 & \\
\hline RM-9 & 04/01/99 & 332 & 0 & 8.39 & \\
\hline RM-9 & 08/05/99 & 219 & 0.2 & 8.16 & -154 \\
\hline RM-9 & $08 / 22 / 99$ & 600 & 0 & 8.76 & \\
\hline RM-9 & $09 / 21 / 99$ & 369 & 0 & 8.88 & -286 \\
\hline RM-9 & $10 / 19 / 99$ & 296 & 0 & 8.81 & -293 \\
\hline RM-9 & $12 / 01 / 99$ & 292 & 0 & 8.70 & -254 \\
\hline RM-9 & $01 / 06 / 00$ & 839 & 0 & 9.06 & -321 \\
\hline RM-9 & $02 / 08 / 00$ & 556 & 0 & 9.05 & -319 \\
\hline RM-9 & $03 / 20 / 00$ & 438 & 0 & 9.15 & -299 \\
\hline RM-9 & $04 / 26 / 00$ & 1121 & 0 & 9.22 & -361 \\
\hline RM-9 & $05 / 24 / 00$ & 715 & 0 & 9.10 & -348 \\
\hline RM-9 & $06 / 21 / 00$ & 408 & 0 & 9.09 & -347 \\
\hline
\end{tabular}


Table H.4j. Water Chemistry Parameters (Wells RM-10, RM-11, and RM-12)

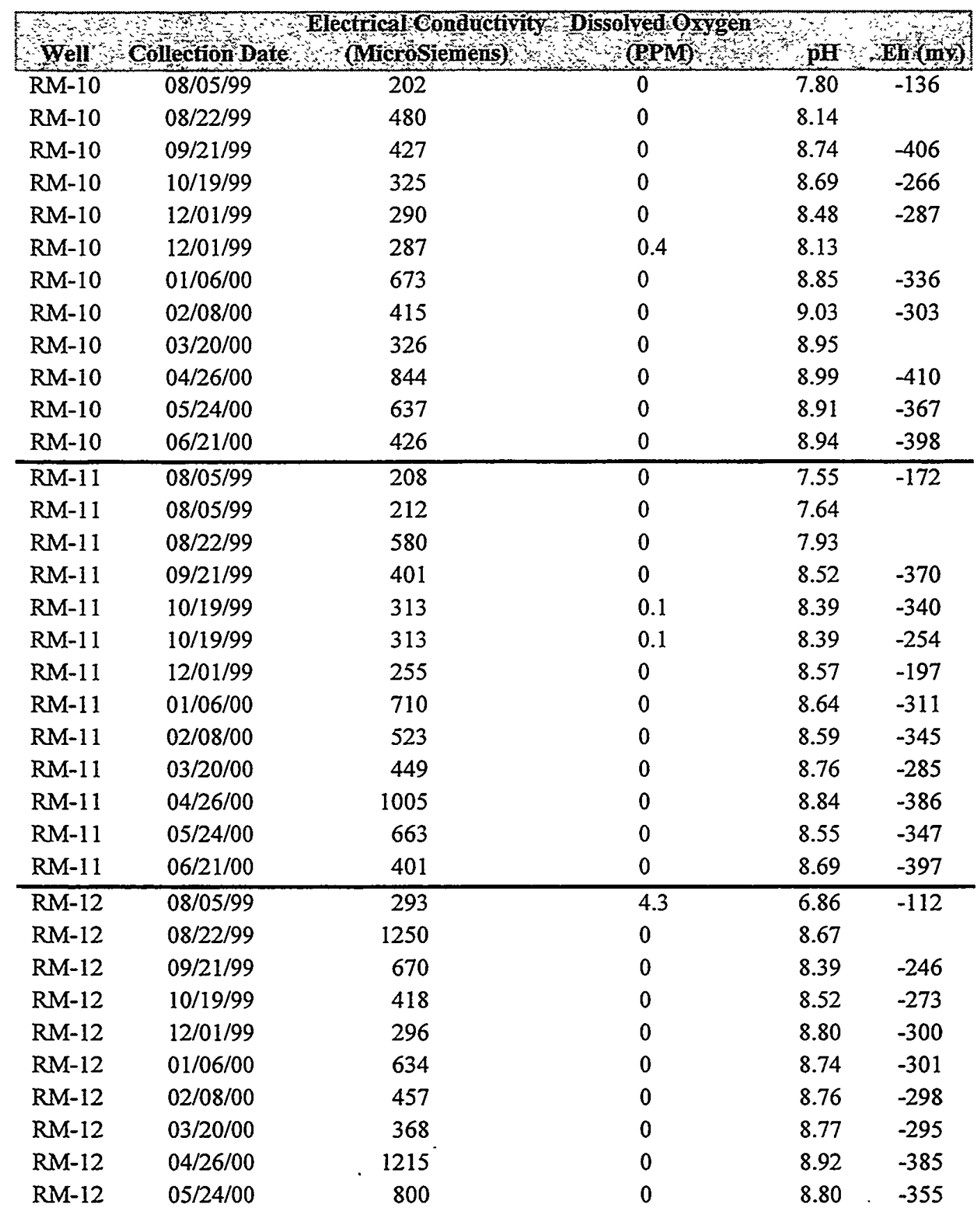


Table H.4k. Water Chemistry Parameters (Wells RM-13, RM-14, and RM-15)

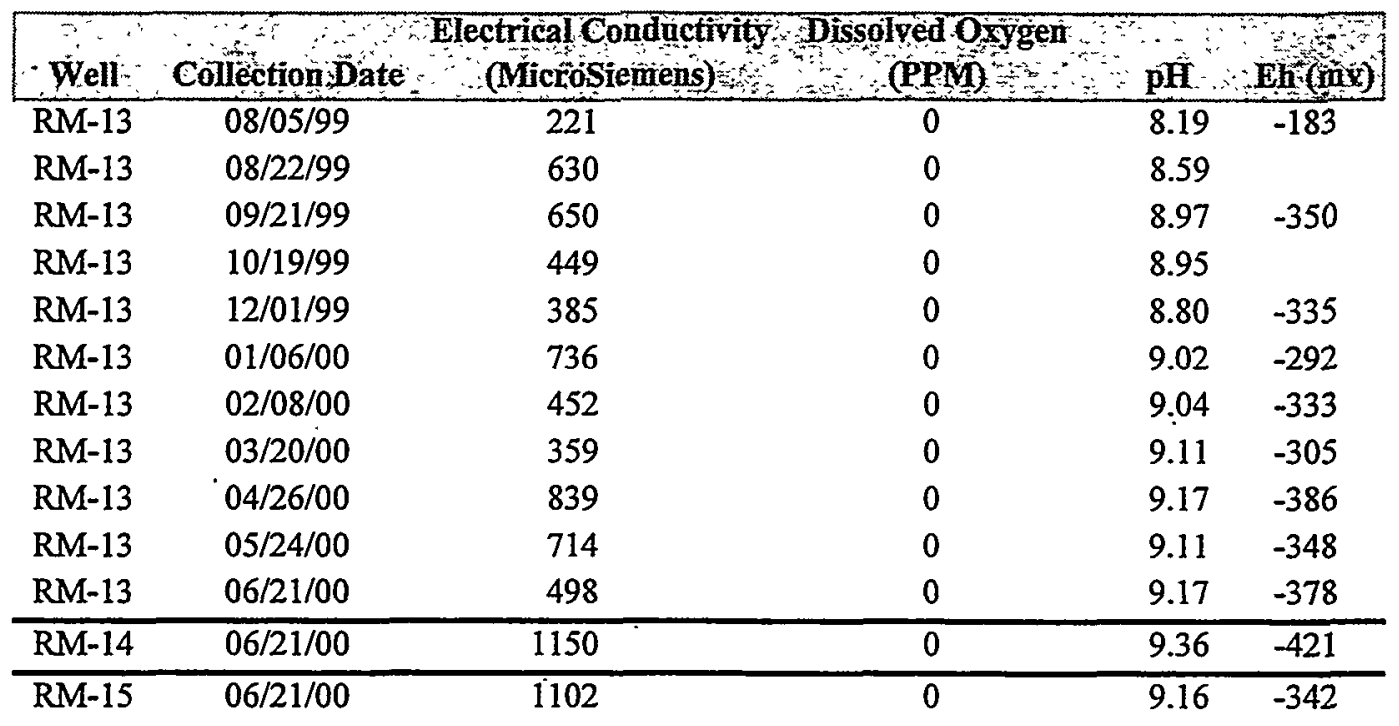

Table H.5a. Trace Metals by Inductively Coupled Argon Plasma-Optical Emission Spectroscopy ( $\mathrm{Al}, \mathrm{As}, \mathrm{Ba}, \mathrm{Be}, \mathrm{B}$, and $\mathrm{Cd}$ )

\begin{tabular}{|c|c|c|c|c|c|c|c|c|c|}
\hline & 21 & \multicolumn{3}{|c|}{ Collection Aluminum Antimony } & \multirow{2}{*}{$\begin{array}{l}\text { Arsenic } \\
\text { magt: }\end{array}$} & \multicolumn{2}{|c|}{ Bárinm Berallium } & \multicolumn{2}{|c|}{ Boron f Gadnium } \\
\hline Sample $\mathbf{m}$ & 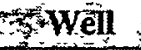 & Date & mgll & ing & & mgd & mgll & mgd & $\mathrm{mgI}$ \\
\hline FL100 & $\mathrm{RM}-1 \mathrm{~A}$ & 1-Dec-98 & $<0.1$ & $\mathrm{NA}$ & $<0.06$ & $<0.005$ & $<0.002$ & $<0.005$ & $<0.02$ \\
\hline FL101 & RM-1B & 1-Dec-98 & $<0.1$ & NA & $<0.06$ & 0.014 & $<0.002$ & $<0.005$ & $<0.02$ \\
\hline FL102 & RM-2 & 1-Dec-98 & $<0.1$ & NA & $<0.06$ & 0.016 & $<0.002$ & $<0.005$ & $<0.02$ \\
\hline FL104 & RM-3 & 1-Dec-98 & $<0.1$ & NA & $<0.06$ & $<0.005$ & $<0.002$ & $<0.005$ & $<0.02$ \\
\hline FL105 & RM-3 & 1-Dec-98 & $<0.1$ & NA & $<0.06$ & $<0.005$ & $<0.002$ & $<0.005$ & $<0.02$ \\
\hline FL106 & RM-4 & 1-Dec-98 & $<0.1$ & NA & $<0.06$ & 0.007 & $<0.002$ & $<0.005$ & $<0.02$ \\
\hline FL, 107 & RM-5 & 1-Dec-98 & $<0.1$ & NA & $<0.06$ & 0.026 & $<0.002$ & $<0.005$ & $<0.02$ \\
\hline FL108 & RM-6 & 1-Dec-98 & $<0.1$ & NA & $<0.06$ & 0.057 & $<0.002$ & 0.009 & $<0.02$ \\
\hline FL109 & RM-6 & 1-Dec-98 & $<0.1$ & NA & $<0.06$ & 0.057 & $<0.002$ & 0.009 & $<0.02$ \\
\hline FL1 10 & RM-7 & 1-Dec-98 & $<0.1$ & NA & $<0.06$ & 0.021 & $<0.002$ & 0.009 & $<0.02$ \\
\hline FL111 & RM-8 & 1-Dec-98 & $<0.1$ & NA & $<0.06$ & 0.006 & $<0.002$ & 0.007 & $<0.02$ \\
\hline FL1 12 & RM-9 & 1-Dec-98 & $<0.1$ & NA & $<0.06$ & $<0.005$ & $<0.002$ & 0.008 & $<0.02$ \\
\hline
\end{tabular}


Table H.5b. Trace Metals by Inductively Coupled Argon Plasma-Optical Emission Spectroscopy $(\mathrm{Ca}, \mathrm{Cr}, \mathrm{Co}, \mathrm{Cu}, \mathrm{Fe}$, and $\mathrm{Pb}$ )

\begin{tabular}{|c|c|c|c|c|c|c|c|c|c|}
\hline $\begin{array}{l}\text { Sấplét } \\
\text { Sand }\end{array}$ & 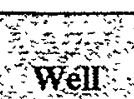 & $\begin{array}{l}\text { Eollection } \\
\text { Date }\end{array}$ & $\begin{array}{l}\text { alcum } \\
\text { mgit }\end{array}$ & $\begin{array}{l}\text { Chromiu } \\
\text { nog/t }\end{array}$ & $\begin{array}{l}\text { Cobalt } \\
\text { mgtet }\end{array}$ & $\begin{array}{l}\text { Coppe } \\
\text { mgrt }\end{array}$ & Hon mg & $\begin{array}{l}\text { Lead } \\
\text { mgtht }\end{array}$ & within \\
\hline FL100 & $\mathrm{RM}-1 \mathrm{~A}$ & $1-\operatorname{Dec}-98$ & 24 & $<0.04$ & $<0.004$ & $<0.006$ & 0.15 & $<0.06$ & $<0.02$ \\
\hline FL101 & RM-1B & 1-Dec-98 & 22 & $<0.04$ & $<0.004$ & $<0.006$ & 0.03 & $<0.06$ & $<0.02$ \\
\hline FL102 & $\mathrm{RM}-2$ & 1-Dec-98 & 36 & $<0.04$ & $<0.004$ & 0.22 & 2.40 & $<0.06$ & $<0.02$ \\
\hline FL104 & RM-3 & 1-Dec-98 & 12 & $<0.04$ & $<0.004$ & $<0.006$ & 0.01 & $<0.06$ & $<0.02$ \\
\hline FL105 & RM-3 & 1-Dec-98 & 13 & $<0.04$ & $<0.004$ & $<0.006$ & 0.02 & $<0.06$ & $<0.02$ \\
\hline FL106 & $\mathrm{RM}-4$ & 1-Dec-98 & 13 & $<0.04$ & $<0.004$ & $<0.006$ & 0.05 & $<0.06$ & $<0.02$ \\
\hline FL107 & RM-5 & 1-Dec-98 & 34 & $<0.04$ & $<0.004$ & $<0.006$ & 0.30 & $<0.06$ & $<0.02$ \\
\hline FL108 & RM-6 & 1-Dec-98 & 39 & $<0.04$ & $<0.004$ & $<0.006$ & 0.02 & $<0.06$ & $<0.02$ \\
\hline FL109 & $\mathrm{RM}-6$ & 1-Dec-98 & 39 & $<0.04$ & $<0.004$ & 0.16 & 0.04 & $<0.06$ & $<0.02$ \\
\hline FL1 10 & RM-7 & 1-Dec-98 & 26 & $<0.04$ & $<0.004$ & 0.014 & 0.01 & $<0.06$ & $<0.02$ \\
\hline FL111 & RM-8 & 1-Dec-98 & 24 & $<0.04$ & $<0.004$ & $<0.006$ & 0.07 & $<0.06$ & $<0.02$ \\
\hline FL112 & RM-9 & 1-Dec-98 & 20 & $<0.04$ & $<0.004$ & $<0.006$ & 0.05 & $<0.06$ & $<0.02$ \\
\hline
\end{tabular}

Table H.5c. Trace Metals by Inductively Coupled Argon Plasma-Optical Emission Spectroscopy ( $\mathrm{Li}, \mathrm{Mg}, \mathrm{Mn}, \mathrm{Mo}, \mathrm{Ni}$, and $\mathrm{K}$ )

\begin{tabular}{|c|c|c|c|c|c|c|c|c|}
\hline $\begin{array}{l}\text { Sâple } \\
\text { Sâno }\end{array}$ & Well & 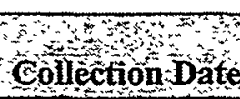 & $\begin{array}{l}\text { Magnesium } \\
\text { mgdis }\end{array}$ & $\begin{array}{l}\text { Tangan } \\
\mathrm{ng} \mathrm{m}\end{array}$ & Mercar & $\begin{array}{l}\text { Molybdenum } \\
\text { ngtt }\end{array}$ & $\begin{array}{l}\text { Nickel } \\
\text { mgd }\end{array}$ & Cotassium \\
\hline FL100 & $\mathrm{RM}-1 \mathrm{~A}$ & 1-Dec-98 & 14 & 0.15 & NA & $<0.06$ & $<0.01$ & 109 \\
\hline FL101 & RM-1B & 1-Dec-98 & 10 & 0.29 & NA & $<0.06$ & $<0.01$ & 7.9 \\
\hline FL102 & RM-2 & 1-Dec-98 & 150 & 0.19 & NA & $<0.06$ & 0.1 & 2,600 \\
\hline FL104 & RM-3 & 1-Dec-98 & 5.7 & 0.016 & NA & $<0.06$ & $<0.01$ & 2.1 \\
\hline FL105 & RM-3 & 1-Dec-98 & 6.1 & 0.017 & NA & $<0.06$ & $<0.01$ & 2.1 \\
\hline FL106 & $R M-4$ & 1-Dec-98 & 8 & 0.28 & NA & $<0.06$ & $<0.01$ & 51 \\
\hline FL107 & RM-5 & 1-Dec-98 & 23 & 0.31 & NA & $<0.06$ & $<0.01$ & 205 \\
\hline FL108 & RM-6 & 1-Dec-98 & 20 & 0.58 & NA & $<0.06$ & 0.02 & 28 \\
\hline FL109 & RM-6 & 1-Dec-98 & 19 & 0.53 & NA & $<0.06$ & 0.021 & 29 \\
\hline FL1 10 & RM-7 & 1-Dec-98 & 18 & 0.84 & NA & $<0.06$ & 0.022 & 23 \\
\hline FL111 & RM-8 & 1-Dec-98 & 12 & 0.14 & NA & $<0.06$ & $<0.01$ & 90 \\
\hline FL1 12 & RM-9 & 1-Dec-98 & 9.4 & 0.18 & NA & $<0.06$ & $<0.01$ & 72 \\
\hline
\end{tabular}


Table H.5d. Trace Metals by Inductively Coupled Argon Plasma-Optical Emission Spectroscopy ( $\mathrm{Se}, \mathrm{Si}, \mathrm{Ag}, \mathrm{Na}, \mathrm{Sr}, \mathrm{V}$, and $\mathrm{Zn}$ )

\begin{tabular}{|c|c|c|c|c|c|c|c|c|c|c|}
\hline $\begin{array}{c}\text { Sample } \\
\mathbf{D}^{-}\end{array}$ & $\begin{array}{r}x \\
\text { Wêll }\end{array}$ & $\begin{array}{l}\text { Collection } \\
\text { Date }\end{array}$ & $\begin{array}{l}\text { Rubidiu } \\
\text { m mill }\end{array}$ & 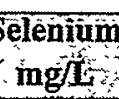 & $\begin{array}{l}\text { Silicon } \\
\text { mgtL }\end{array}$ & $\begin{array}{l}\text { Silver } \\
\text { nigh }\end{array}$ & $\begin{array}{c}\text { Sodnum } \\
\text { moftit }\end{array}$ & $\begin{array}{l}\text { Strontiúm } \\
\text { pagl }\end{array}$ & $\begin{array}{l}\text { Thall } \\
\text { mang } \\
\text { mung }\end{array}$ & $\begin{array}{l}\text { Tinis } \\
\text { moto }\end{array}$ \\
\hline FL100 & $\mathrm{RM}-1 \mathrm{~A}$ & $1-\mathrm{Dec}-98$ & $\mathrm{NA}$ & $<0.06$ & 5.7 & $<0.02$ & 41 & 0.35 & NA & NA \\
\hline FL101 & $R M-1 B$ & 1-Dec-98 & NA & $<0.06$ & 10 & $<0.02$ & 15 & 0.14 & NA & NA \\
\hline FL102 & RM-2 & 1-Dec-98 & NA & $<0.06$ & 2.8 & $<0.02$ & 970 & 0.66 & NA & NA \\
\hline FL104 & RM-3 & 1-Dec-98 & NA & $<0.06$ & 12 & $<0.02$ & 5.9 & 0.066 & NA & NA \\
\hline FL105 & RM-3 & 1-Dec-98 & NA & $<0.06$ & 12 & $<0.02$ & 5.9 & 0.072 & NA & NA \\
\hline FL106 & RM-4 & 1-Dec-98 & NA & $<0.06$ & 8.8 & $<0.02$ & 14 & 0.13 & NA & $\mathrm{NA}$ \\
\hline FL107 & RM-5 & 1-Dec-98 & NA & $<0.06$ & 13 & $<0.02$ & 95 & 0.36 & $\mathrm{NA}$ & NA \\
\hline FL108 & RM-6 & 1-Dec-98 & $N A$ & $<0.06$ & 10 & $<0.02$ & 37 & 0.28 & NA & NA \\
\hline FL109 & RM-6 & 1-Dec-98 & $\mathrm{NA}$ & $<0.06$ & 9.4 & $<0.02$ & 37 & 0.28 & $\mathrm{NA}$ & $\mathrm{NA}$ \\
\hline FL1 10 & RM-7 & 1-Dec-98 & NA & $<0.06$ & 4.3 & $<0.02$ & 23 & 0.25 & NA & NA \\
\hline FL111 & RM-8 & 1-Dec-98 & NA & $<0.06$ & 2.7 & $<0.02$ & 32 & 0.15 & NA & $\mathrm{NA}$ \\
\hline FL112 & RM-9 & 1-Dec-98 & NA & $<0.06$ & 6.3 & $<0.02$ & 22 & 0.12 & NA & NA \\
\hline
\end{tabular}

Table H.5e. Trace Metals by Inductively Coupled Argon Plasma-Optical Emission Spectroscopy $(\mathrm{Ti}, \mathrm{W}, \mathrm{U}, \mathrm{V}$, and $\mathrm{Zn}$ )

\begin{tabular}{|c|c|c|c|c|c|c|c|}
\hline \multirow{2}{*}{\multicolumn{2}{|c|}{ Sample ID }} & Collection & Ritaniüi & \multicolumn{3}{|c|}{ Tungsten Uraniūind Yanadium } & \multirow{2}{*}{ ZincingA } \\
\hline & & Date: & $\mathrm{mg}$ & mgl & ing & mg & \\
\hline FL100 & RM-1A & 1-Dec-98 & NA & $\mathrm{NA}$ & NA & $<0.02$ & 0.010 \\
\hline FL101 & RM-1B & 1-Dec-98 & NA & NA & NA & $<0.02$ & 0.013 \\
\hline FL102 & RM-2 & 1-Dec-98 & NA & NA & NA & $<0.02$ & 0.027 \\
\hline FL104 & RM-3 & 1-Dec-98 & NA & NA & NA & $<0.02$ & 0.011 \\
\hline FL105 & RM-3 & 1-Dec-98 & NA & NA & NA & $<0.02$ & 0.010 \\
\hline FL106 & $\mathrm{RM}-4$ & 1-Dec-98 & NA & NA & NA & $<0.02$ & 0.008 \\
\hline FL107 & RM-5 & 1-Dec-98 & NA & $\mathrm{NA}$ & NA & $<0.02$ & 0.009 \\
\hline FL108 & RM-6 & 1-Dec-98 & NA & NA & NA & $<0.02$ & 0.009 \\
\hline FL109 & RM-6 & 1-Dec-98 & NA & NA & NA & $<0.02$ & 0.013 \\
\hline FL110 & RM-7 & 1-Dec-98 & NA & NA & NA & $<0.02$ & 0.006 \\
\hline FL111 & RM-8 & 1-Dec-98 & NA & NA & NA & $<0.02$ & 0.007 \\
\hline FL112 & RM-9 & 1-Dec-98 & NA & NA & NA & $<0.02$ & 0.007 \\
\hline
\end{tabular}


Table H.6a. Trace Metals by Inductively Coupled Argon Plasma-Mass Spectrometry (Al, $\mathrm{Sb}, \mathrm{As}, \mathrm{Ba}, \mathrm{Be}, \mathrm{B}$, and $\mathrm{Cd}$ )

\begin{tabular}{|c|c|c|c|c|c|c|c|c|c|}
\hline Sample $\mathbf{W}$ & Wen & $\begin{array}{l}\text { Eolecton } \\
\text { ODate }\end{array}$ & mot & mg & $\begin{array}{l}\text { Arsemc } \\
\text { mogty }\end{array}$ & mg & mgat & $\begin{array}{l}\text { Boron } \\
\text { ng:L }\end{array}$ & Cadmum \\
\hline FL500 & $\mathrm{RM}-1 \mathrm{~A}$ & $19-$ Oct-99 & $<0.06$ & 0.00089 & $<0.005$ & 0.0072 & $\mathrm{NA}$ & $\mathrm{NA}$ & $<0.003$ \\
\hline FL501 & RM-1B & $19-$ Oct-99 & $<0.06$ & 0.00066 & $<0.005$ & 0.0164 & NA & NA & $<0.003$ \\
\hline FL502 & RM-2 & $19-$ Oct- 99 & $<0.06$ & 0.00552 & $<0.005$ & 0.1250 & NA & NA & $<0.003$ \\
\hline FL503 & RM-2 & $19-$ Oct-99 & $<0.06$ & 0.00244 & $<0.005$ & 0.1280 & NA & NA & $<0.003$ \\
\hline FL504 & RM-3 & 19 -Oct-99 & $<0.06$ & 0.00070 & $<0.005$ & 0.0056 & NA & NA & $<0.003$ \\
\hline FL505 & RM-4 & $19-$ Oct-99 & $<0.06$ & 0.00091 & $<0.005$ & 0.0034 & NA & NA & $<0.003$ \\
\hline FL506 & RM-5 & $19-$ Oct-99 & $<0.06$ & 0.00125 & $<0.005$ & 0.0105 & NA & NA & $<0.003$ \\
\hline FL507 & RM-6 & $19-$ Oct-99 & $<0.06$ & 0.00080 & $<0.005$ & 0.0269 & NA & NA & $<0.003$ \\
\hline FL508 & RM-7 & $19-$ Oct-99 & $<0.06$ & 0.00083 & $<0.005$ & 0.0108 & NA & NA & $<0.003$ \\
\hline FL510 & RM-8 & $19-$ Oct-99 & $<0.06$ & 0.00099 & $<0.005$ & 0.0021 & NA & NA & $<0.003$ \\
\hline FL511 & RM-9 & $19-$ Oct-99 & $<0.06$ & 0.00109 & $<0.005$ & 0.0017 & NA & NA & $<0.003$ \\
\hline FL512 & RM-10 & $19-$ Oct-99 & $<0.06$ & 0.00098 & $<0.005$ & 0.0061 & NA & NA & 0.0060 \\
\hline FL513 & RM-11 & $19-$ Oct-99 & $<0.06$ & 0.00093 & $<0.005$ & 0.0159 & NA & NA & $<0.003$ \\
\hline FL514 & RM-11 & 19-Oct-99 & $<0.06$ & 0.00086 & $<0.005$ & 0.0165 & $\mathrm{NA}$ & NA & $<0.003$ \\
\hline FL515 & RM-12 & 19-Oct-99 & $<0.06$ & 0.00094 & $<0.005$ & 0.0051 & NA & NA & $<0.003$ \\
\hline FL516 & RM-13 & $19-$ Oct-99 & $<0.06$ & 0.00103 & $<0.005$ & 0.0026 & NA & NA & $<0.003$ \\
\hline FL1200H & $\overline{R M-1 A}$ & 21-Jun-00 & 0.036 & 0.0013 & 0.0025 & $\overline{0.006}$ & $<0.0002$ & $\overline{\mathrm{NA}}$ & 0.00005 \\
\hline FL1202H & $\mathrm{RM}-2$ & 21-Jun-00 & 0.030 & 0.0020 & 0.0018 & 0.187 & $<0.0002$ & NA & 0.00007 \\
\hline FL1203K & RM-3 & 21-Jun-00 & 0.029 & 0.0010 & 0.0012 & 0.008 & $<0.0002$ & NA & 0.00002 \\
\hline FL1204H & RM-3 & 21-Jun-00 & 0.030 & 0.0012 & 0.0012 & 0.008 & $<0.0002$ & NA & $<0.00002$ \\
\hline FL1205H & RM-4 & 21-Jun-00 & 0.030 & 0.0011 & 0.0016 & 0.002 & $<0.0002$ & NA & 0.00003 \\
\hline FL1206K & RM-5 & 21-Jun-00 & 0.058 & 0.0015 & 0.0016 & 0.009 & $<0.0002$ & NA & 0.00002 \\
\hline FL1207H & RM-5 & 21-Jun-00 & 0.078 & 0.0014 & 0.0016 & 0.010 & $<0.0002$ & NA & 0.00006 \\
\hline FL1208H & RM-6 & 21-Jun-00 & 0.026 & 0.0011 & 0.0022 & 0.043 & $<0.0002$ & NA & 0.00018 \\
\hline FL1209H & RM-7 & 21-Jun-00 & 0.079 & 0.0011 & 0.0039 & 0.024 & $<0.0002$ & NA & 0.00027 \\
\hline FL1209H Rep & RM-7 & 21-Jun-00 & 0.075 & 0.0012 & 0.0039 & 0.025 & $<0.0002$ & $\mathrm{NA}$ & 0.00026 \\
\hline FL1211H & RM-8 & 21-Jun-00 & 0.039 & 0.0010 & 0.0006 & 0.002 & $<0.0002$ & $\mathrm{NA}$ & $<0.00002$ \\
\hline FL1212H & RM-9 & 21-Jun-00 & 0.026 & 0.0010 & 0.0006 & 0.001 & $<0.0002$ & $\mathrm{NA}$ & $<0.00002$ \\
\hline FL1213H & RM-10 & 21-Jun-00 & 0.034 & 0.0013 & 0.0016 & 0.003 & $<0.0002$ & NA & 0.00002 \\
\hline FL1214H & RM-11 & 21-Jun-00 & 0.028 & 0.0009 & 0.0017 & 0.014 & $<0.0002$ & NA & 0.00004 \\
\hline FL1216H & RM-13 & 21-Jun-00 & 0.031 & 0.0010 & 0.0005 & 0.002 & $<0.0002$ & $\mathrm{NA}$ & 0.00004 \\
\hline FL1215H & RM-14 & 21-Jun-00 & 0.073 & 0.0015 & 0.0014 & 0.025 & $<0.0002$ & $\mathrm{NA}$ & 0.00005 \\
\hline FL1201H & RM-15 & 21-Jun-00 & 0.056 & 0.0014 & 0.0020 & 0.029 & $<0.0002$ & NA & 0.00019 \\
\hline
\end{tabular}


Table H.6b. Trace Metals by Inductively Coupled Argon Plasma-Mass Spectrometry $(\mathrm{Ca}, \mathrm{Cr}, \mathrm{Co}, \mathrm{Cu}, \mathrm{Fe}, \mathrm{Pb}$, and $\mathrm{Li}$ )

\begin{tabular}{|c|c|c|c|c|c|c|c|c|c|}
\hline Sample W & Well & $\begin{array}{l}\text { Collection } \\
\text { Date }\end{array}$ & $\begin{array}{l}\text { alchum* } \\
\text { mghL }\end{array}$ & $\begin{array}{l}\text { hromium } \\
\text { mg/Lth }\end{array}$ & $\begin{array}{l}\text { Cobalt } \\
\text { mgite }\end{array}$ & $\begin{array}{l}\text { Copper } \\
\text { mgdi }\end{array}$ & Tron* & $\begin{array}{l}\text { Lead } \\
\text { mgits }\end{array}$ & Tithinim \\
\hline FL500 & RM-1A & 19-Oct-99 & 20 & $<0.0015$ & $\mathrm{NA}$ & $<0.005$ & 0.07 & $<0.009$ & $\mathrm{NA}$ \\
\hline FL501 & RM-1B & 19-Oct-99 & 20 & $<0.0015$ & $\mathrm{NA}$ & $<0.005$ & 0.04 & $<0.009$ & NA \\
\hline FL502 & $\mathrm{RM}-2$ & $19-$ Oct-99 & 20 & $<0.0015$ & NA & $<0.005$ & 1.40 & $<0.009$ & NA \\
\hline FL503 & RM-2 & 19-Oct-99 & 13 & $<0.0015$ & NA & $<0.005$ & 1.40 & $<0.009$ & NA \\
\hline FL504 & RM-3 & 19-Oct-99 & 11 & $<0.0015$ & $\mathrm{NA}$ & $<0.005$ & 0.02 & $<0.009$ & $\mathrm{NA}$ \\
\hline FL505 & $\mathrm{RM}-4$ & 19-Oct-99 & 12 & $<0.0015$ & $\mathrm{NA}$ & $<0.005$ & 0.05 & $<0.009$ & $\mathrm{NA}$ \\
\hline FL506 & RM-5 & 19-Oct-99 & 11 & $<0.0015$ & NA & $<0.005$ & 0.11 & $<0.009$ & NA \\
\hline FL507 & RM-6 & 19-Oct-99 & 24 & $<0.0015$ & $\mathrm{NA}$ & $<0.005$ & 0.08 & $<0.009$ & NA \\
\hline FL508 & RM-7 & $19-$ Oct-99 & 23 & $<0.0015$ & $\mathrm{NA}$ & $<0.005$ & 0.01 & $<0.009$ & NA \\
\hline FL510 & RM-8 & $19-$ Oct-99 & 10 & $<0.0015$ & $\mathrm{NA}$ & $<0.005$ & 0.05 & $<0.009$ & NA \\
\hline FL511 & RM-9 & 19-Oct-99 & 10 & $<0.0015$ & NA & $<0.005$ & 0.04 & $<0.009$ & NA \\
\hline FL512 & RM-10 & 19-Oct-99 & 13 & $<0.0015$ & NA & $<0.005$ & 0.03 & $<0.009$ & NA \\
\hline FL513 & RM-1 1 & 19-Oct-99 & 17 & $<0.0015$ & $\mathrm{NA}$ & $<0.005$ & 0.06 & $<0.009$ & $\mathrm{NA}$ \\
\hline FL514 & RM-11 & $19-$ Oct-99 & 14 & $<0.0015$ & $\mathrm{NA}$ & $<0.005$ & 0.06 & $<0.009$ & NA \\
\hline FL515 & RM-12 & 19-Oct-99 & 20 & $<0.0015$ & $\mathrm{NA}$ & $<0.005$ & 0.09 & $<0.009$ & NA \\
\hline FL516 & RM-13 & 19-Oct-99 & 12 & $<0.0015$ & $\mathrm{NA}$ & $<0.005$ & 0.05 & $<0.009$ & NA \\
\hline FL1200H & $\overline{R M-1 A}$ & 21-Jun-00 & 16 & 0.001 & $\overline{0.045}$ & 0.0029 & 0.31 & $<0.009$ & $<0.002$ \\
\hline FL1202H & $\mathrm{RM}-2$ & 21-Jun-00 & 7 & 0.001 & 0.060 & 0.0044 & 1.59 & $0.00044 \mathrm{~B}$ & 0.003 \\
\hline FL1203K & RM-3 & 21-Jun-00 & 17 & $<0.001$ & 0.001 & 0.0026 & 0.15 & $0.00019 \mathrm{~B}$ & 0.002 \\
\hline FL1204H & RM-3 & 21-Jun-00 & 17 & 0.001 & 0.001 & 0.0020 & 0.08 & $0.00020 \mathrm{~B}$ & $0.002^{\circ}$ \\
\hline FL1205H & RM-4 & 21-Jun-00 & 12 & $<0.001$ & 0.010 & 0.0010 & 0.08 & $0.00017 \mathrm{~B}$ & $<0.002$ \\
\hline FL1206K & RM-5 & 21-Jun-00 & 9 & $<0.001$ & 0.050 & 0.0012 & 0.28 & $0.00044 B$ & $<0.002$ \\
\hline FL1207H & RM-5 & 21-Jun-00 & 9 & $<0.001$ & 0.051 & 0.0028 & 0.40 & $0.00024 \mathrm{~B}$ & $<0.002$ \\
\hline FL1208H & RM-6 & 21-Jun-00 & 78 & $<0.001$ & 0.057 & 0.0123 & 0.40 & $0.00034 \mathrm{~B}$ & 0.003 \\
\hline FL1209H & RM-7 & 21-Jun-00 & 51 & $<0.001$ & 0.055 & 0.0063 & 0.22 & $<0.0002$ & 0.002 \\
\hline FL1209H Rep & RM-7 & 21-Jun-00 & 51 & $<0.001$ & 0.055 & 0.0062 & 0.22 & $0.00094 \mathrm{~B}$ & 0.003 \\
\hline FL1211H & RM-8 & 21-Jun-00 & 7 & $<0.001$ & 0.020 & 0.0010 & 0.14 & $0.00094 \mathrm{~B}$ & $<0.002$ \\
\hline FL1212H & RM-9 & 21-Jun-00 & 8 & $<0.001$ & 0.014 & 0.0009 & 0.06 & $0.00020 \mathrm{~B}$ & $<0.002$ \\
\hline FL1213H & RM-10 & 21-Jun-00 & 13 & $<0.001$ & 0.021 & 0.0011 & 0.07 & $0.00017 \mathrm{~B}$ & $<0.002$ \\
\hline FL1214H & RM-11 & 21-Jun-00 & 25 & $<0.001$ & 0.026 & 0.0014 & 0.14 & $0.00023 B$ & 0.002 \\
\hline FL1216H & RM-13 & 21-Jun-00 & 8 & $<0.001$ & 0.016 & 0.0010 & 0.07 & $0.00018 \mathrm{~B}$ & $<0.002$ \\
\hline FL1215H & RM-14 & 21-Jun-00 & 8 & $<0.001$ & 0.038 & 0.0014 & 0.20 & $0.00020 \mathrm{~B}$ & $<0.002$ \\
\hline FL1201H & RM-15 & 21-Jun-00 & 27 & 0.002 & 0.056 & 0.0047 & 0.38 & $0.00038 \mathrm{~B}$ & 0.002 \\
\hline
\end{tabular}

- 500 Series samples measured by ICP-OES for calcium and iron. 
Table H.6c. Trace Metals by Inductively Coupled Argon Plasma-Mass Spectrometry ( $\mathrm{Mg}, \mathrm{Mn}, \mathrm{Hg}, \mathrm{Mo}, \mathrm{Ni}$, and $\mathrm{K}$ )

\begin{tabular}{|c|c|c|c|c|c|c|c|c|}
\hline Sample: & Whell & Collectron & Magnestu & mgant & mgic & mogon & Nickets & ofassiam \\
\hline FL500 & $\mathrm{RM}-1 \mathrm{~A}$ & $19-$ Oct-99 & 8.6 & 0.13 & $\mathrm{NA}$ & 0.0012 & $<0.01$ & 100 \\
\hline FL501 & RM-1B & 19-Oct-99 & 8.9 & 0.53 & NA & 0.0012 & $<0.01$ & 14 \\
\hline FL502 & $\mathrm{RM}-2$ & 19-Oct-99 & 44 & 0.11 & NA & 0.022 & 0.28 & 1,520 \\
\hline FL503 & RM-2 & 19-Oct-99 & 44 & 0.11 & NA & 0.022 & 0.26 & 1,550 \\
\hline FL504 & RM-3 & 19-Oct-99 & 4.2 & 0.049 & NA & 0.00037 & $<0.01$ & 2.7 \\
\hline FL505 & $\mathrm{RM}-4$ & 19-Oct-99 & 4.1 & 0.17 & NA & 0.00037 & $<0.01$ & 44 \\
\hline FL506 & RM-5 & 19-Oct-99 & 5.5 & 0.14 & NA & 0.0023 & $<0.01$ & 210 \\
\hline FL507 & $\mathrm{RM}-6$ & 19-Oct-99 & 9.1 & 0.40 & NA & 0.0018 & $<0.01$ & 18 \\
\hline FL508 & RM-7 & 19-Oct-99 & 8.3 & 0.81 & NA & 0.00054 & $<0.01$ & 24 \\
\hline FL510 & RM-8 & 19-Oct-99 & 4.3 & 0.041 & NA & 0.00039 & $<0.01$ & 84 \\
\hline FL511 & RM-9 & 19-Oct-99 & 4.2 & 0.085 & NA & 0.00039 & $<0.01$ & 58 \\
\hline FL512 & RM-10 & 19-Oct-99 & 4.8 & 0.20 & NA & 0.00038 & $<0.01$ & 53 \\
\hline FL513 & RM-11 & 19-Oct-99 & 6.6 & 0.38 & NA & 0.00054 & $<0.01$ & 45 \\
\hline FL514 & RM-11 & 19-Oct-99 & 6.6 & 0.37 & NA & 0.00057 & $<0.01$ & 45 \\
\hline FL515 & RM-12 & 19-Oct-99 & 7.4 & 0.35 & NA & 0.00096 & $<0.01$ & 45 \\
\hline FL516 & RM-13 & 19-Oct-99 & 4.1 & 0.084 & NA & 0.00048 & $<0.01$ & 84 \\
\hline FL1200H & RM-1A & 21-Jun-00 & 9.8 & 0.11 & $<0.0003$ & 0.0062 & 0.021 & NA \\
\hline FL1202H & $\mathrm{RM}-2$ & 21-Jun-00 & 26.9 & 0.15 & $<0.0003$ & 0.0111 & 0.230 & NA \\
\hline FL1203K & RM-3 & 21-Jun-00 & 6.8 & 0.12 & $<0.0003$ & 0.0006 & 0.004 & NA \\
\hline FL1204H & RM-3 & 21-Jun-00 & 7.0 & 0.12 & $<0.0003$ & 0.0005 & 0.003 & NA \\
\hline FL1205H & $R M-4$ & 21-Jun-00 & 4.8 & 0.071 & $<0.0003$ & 0.0008 & 0.005 & NA \\
\hline FL1206K & RM-5 & 21-Jun-00 & 6.2 & 0.074 & $<0.0003$ & 0.0036 & 0.024 & NA \\
\hline FL1207H & RM-5 & 21-Jun-00 & 6.5 & 0.079 & $<0.0003$ & 0.0036 & 0.028 & NA \\
\hline FL1208H & RM-6 & $21-J u n-00$ & 32.0 & 0.83 & $<0.0003$ & 0.0034 & 0.029 & NA \\
\hline FL1209H & RM-7 & 21-Jun-00 & 18.3 & 1.35 & $<0.0003$ & 0.0020 & 0.023 & NA \\
\hline FL1209H Rep & RM-7 & 21-Jun-00 & 17.5 & 1.34 & $<0.0003$ & 0.0020 & 0.025 & NA \\
\hline FL1211H & RM-8 & 21-Jun-00 & 4.1 & 0.030 & $<0.0003$ & 0.0014 & 0.011 & NA \\
\hline FL1212H & RM-9 & 21-Jun-00 & 4.5 & 0.030 & $<0.0003$ & 0.0010 & 0.007 & NA \\
\hline FL1213H & RM-10 & 21-Jun-00 & 6.4 & 0.102 & $<0.0003$ & 0.0012 & 0.010 & NA \\
\hline FL1214H & RM-11 & 21-Jun-00 & 11.1 & 0.66 & $<0.0003$ & 0.0013 & 0.012 & NA \\
\hline FL1216H & RM-13 & 21-Jun-00 & 4.2 & 0.043 & $<0.0003$ & 0.0014 & 0.009 & NA \\
\hline FL1215H & RM-14 & 21-Jun-00 & 5.7 & 0.13 & $<0.0003$ & 0.0080 & 0.024 & NA \\
\hline FL1201H & RM-15 & 21-Jun-00 & 13.7 & 0.55 & $<0.0003$ & 0.0054 & 0.027 & NA \\
\hline
\end{tabular}

- 500 Series samples measured from magnesium and potassium by ICP-OES. 
Table H.6d. Trace Metals by Inductively Coupled Argon Plasma-Mass Spectrometry (Rb, Se, Si, Ag, Na, Sr, Tl, and Sn)

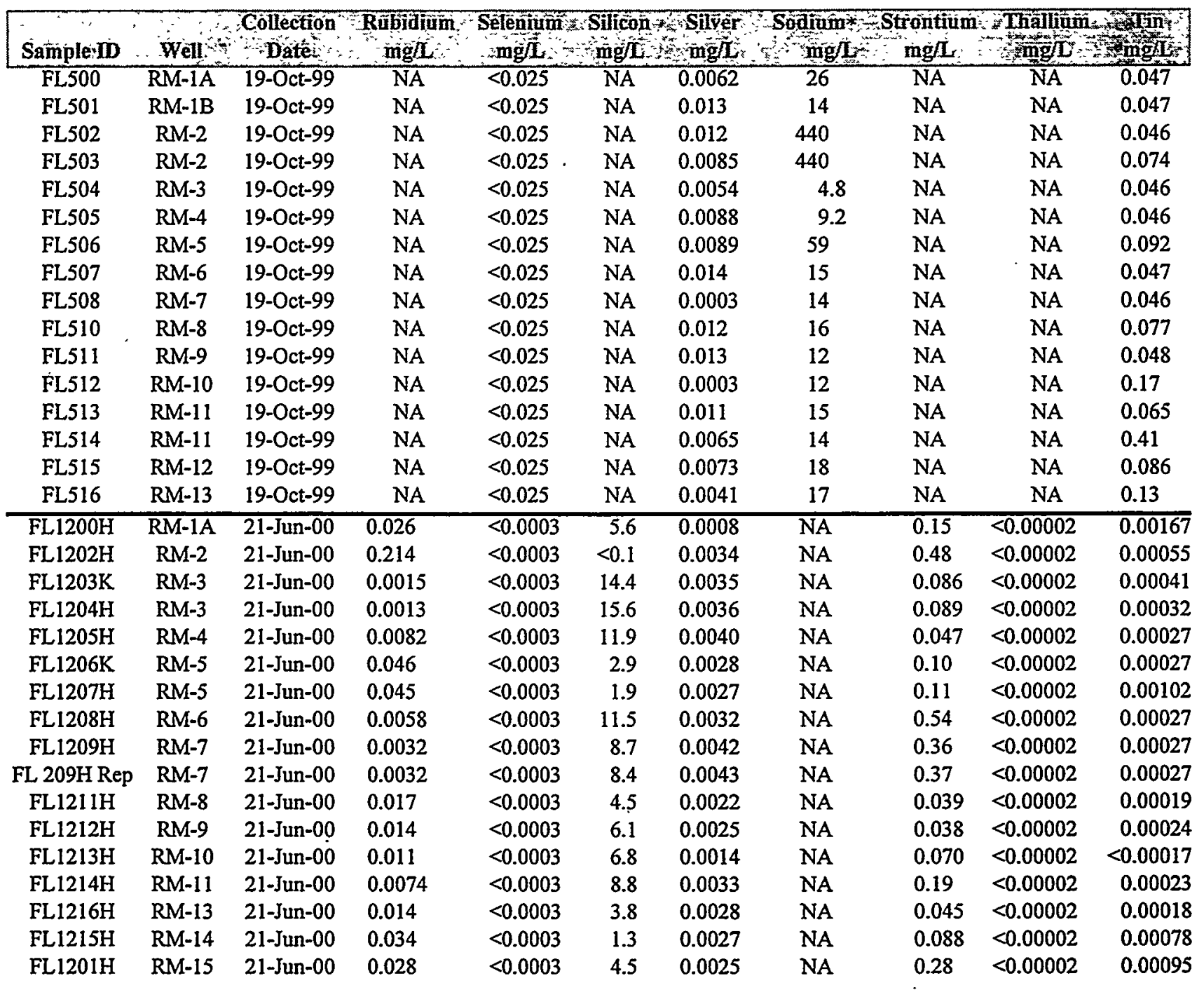

- Measured by ICP-OES. 
Table H.6e. Trace Metals by Inductively Coupled Argon Plasma-Mass Spectrometry (Ti, W, U, V, and $\mathrm{Zn}$ )

\begin{tabular}{|c|c|c|c|c|c|c|c|}
\hline Sampiêu & Weil & Date & 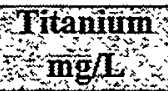 & mg & mgd & ingls & ancong \\
\hline FL500 & $\mathrm{RM}-1 \mathrm{~A}$ & $19-$ Oct-99 & $\mathrm{NA}$ & $\mathrm{NA}$ & 0.00012 & $\mathrm{NA}$ & $<0.05$ \\
\hline FL501 & RM-1B & 19-Oct-99 & NA & NA & 0.00016 & NA & $<0.05$ \\
\hline FL502 & RM-2 & 19-Oct-99 & NA & NA & $<0.00008$ & NA & $<0.05$ \\
\hline FL503 & RM-2 & 19-Oct-99 & NA & NA & $<0.00008$ & NA & $<0.05$ \\
\hline FL504 & RM-3 & 19-Oct-99 & NA & NA & $<0.00008$ & $\mathrm{NA}$ & $<0.05$ \\
\hline FL505 & RM-4 & 19-Oct-99 & NA & NA & $<0.00008$ & NA & $<0.05$ \\
\hline FL506 & RM-5 & 19-Oct-99 & NA & NA & 0.00015 & NA & $<0.05$ \\
\hline FL507 & RM-6 & 19-Oct-99 & NA & NA & 0.00014 & NA & $<0.05$ \\
\hline FL508 & RM-7 & 19-Oct-99 & NA & NA & 0.00055 & NA & $<0.05$ \\
\hline FL510 & RM-8 & 19-Oct-99 & NA & $\mathrm{NA}$ & $<0.00008$ & NA & $<0.05$ \\
\hline FL511 & RM-9 & 19-Oct-99 & NA & NA & $<0.00008$ & NA & $<0.05$ \\
\hline FL5 12 & RM-10 & 19-Oct-99 & NA & NA & $<0.00008$ & NA & $<0.05$ \\
\hline FL513 & RM-11 & 19-Oct-99 & NA & NA & 0.00014 & NA & $<0.05$ \\
\hline FL514 & $\mathrm{RM}-1 \mathrm{I}$ & 19-Oct-99 & NA & NA & 0.000125 & NA & $<0.05$ \\
\hline FL515 & RM-12 & 19-Oct-99 & NA & NA & $<0.00008$ & NA & $<0.05$ \\
\hline FL516 & RM-13 & 19-Oct-99 & NA & NA & $<0.00008$ & NA & $<0.05$ \\
\hline FL1200H & RM-1A & 21-Jun-00 & 0.00298 & 0.0055 & 0.00024 & 0.00034 & 0.0066 \\
\hline FL1202H & RM-2 & 21-Jun-00 & $<0.0002$ & 0.0135 & 0.000014 & 0.00038 & 0.012 \\
\hline FL1203K & RM-3 & 21-Jun-00 & $<0.0002$ & 0.0017 & 0.00018 & 0.0022 & 0.0023 \\
\hline FL1204H & RM-3 & 21-Jun-00 & $<0.0002$ & 0.0014 & 0.00017 & 0.0025 & 0.0049 \\
\hline FL1205H & $\mathrm{RM}-4$ & 21-Jun-00 & $<0.0002$ & 0.0016 & $<0.000002$ & $<0.00008$ & 0.0047 \\
\hline FL1206K & RM-5 & 21-Jun-00 & $<0.0002$ & 0.0027 & 0.000014 & $<0.00008$ & 0.0053 \\
\hline FL1207H & RM-5 & 21-Jun-00 & $<0.0002$ & 0.0026 & 0.000011 & $<0.00008$ & 0.028 \\
\hline FL1208H & RM-6 & 21-Jun-00 & $<0.0002$ & 0.0014 & 0.0010 & 0.00053 & 0.0074 \\
\hline FL1209H & $\mathrm{RM}-7$ & 21-Jun-00 & $<0.0002$ & 0.0012 & 0.00042 & $<0.00008$ & 0.038 \\
\hline FL 209H Rep & RM-7 & 21-Jun-00 & $<0.0002$ & 0.0011 & 0.00041 & $<0.00008$ & 0.038 \\
\hline FL1211H & RM-8 & 21-Jun-00 & $<0.0002$ & 0.0007 & $<0.000002$ & $<0.00008$ & 0.0020 \\
\hline FL1212H & RM-9 & 21-Jun-00 & $<0.0002$ & 0.0005 & $<0.000002$ & $<0.00008$ & -0.0008 \\
\hline FL1213H & RM-10 & 21-Jun-00 & $<0.0002$ & 0.0005 & $<0.000002$ & $<0.00008$ & 0.0021 \\
\hline FL1214H & RM-11 & 21-Jun-00 & $<0.0002$ & 0.0004 & 0.000070 & $<0.00008$ & 0.0060 \\
\hline FL1216H & RM-13 & 21-Jun-00 & $<0.0002$ & 0.0007 & $<0.000002$ & $<0.00008$ & 0.0065 \\
\hline FL1215H & RM-14 & 21-Jun-00 & $<0.0002$ & 0.0038 & 0.000017 & $<0.00008$ & 0.012 \\
\hline FL1201H & RM-15 & 21-Jun-00 & $<0.0002$ & 0.0044 & 0.00024 & 0.00074 & 0.011 \\
\hline
\end{tabular}


Table H.7. Elements Surveyed by Inductively Coupled Argon Plasma-Mass Spectrometry on FL1200 Series Samples for which all Results were Non-Detects

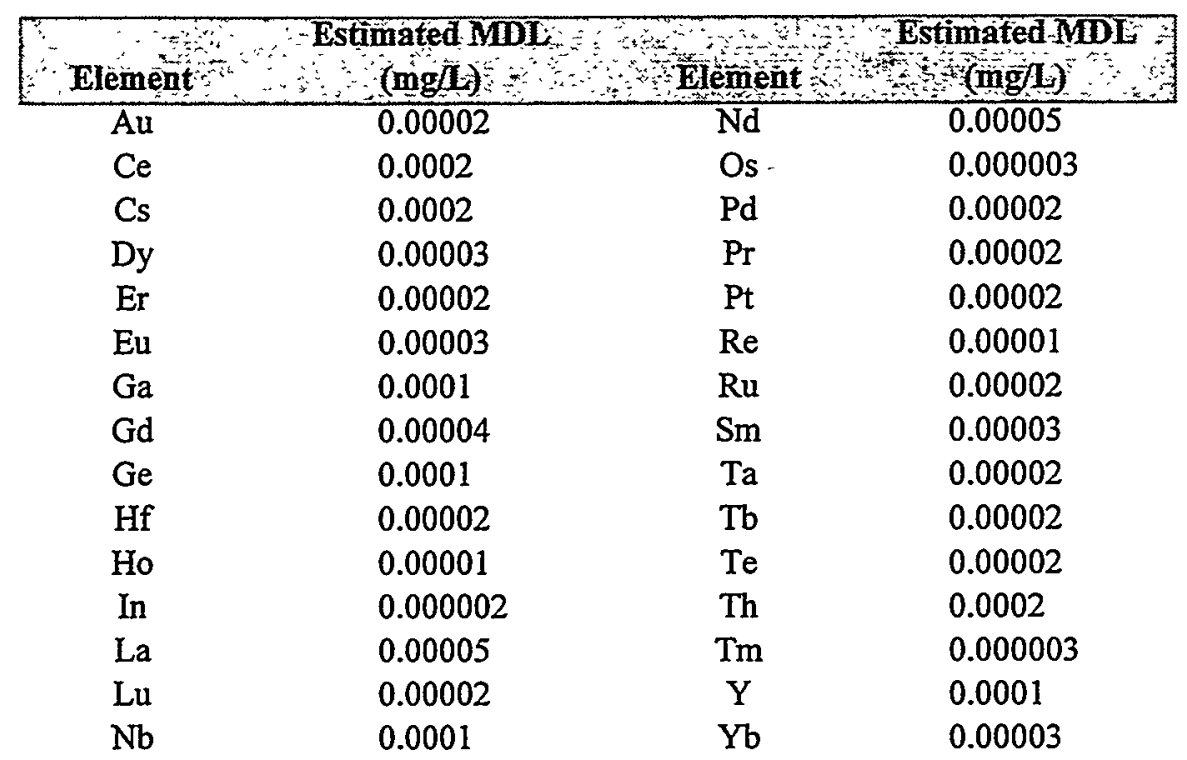




\section{Appendix I}

ISRM Sediment Sample Inventory 

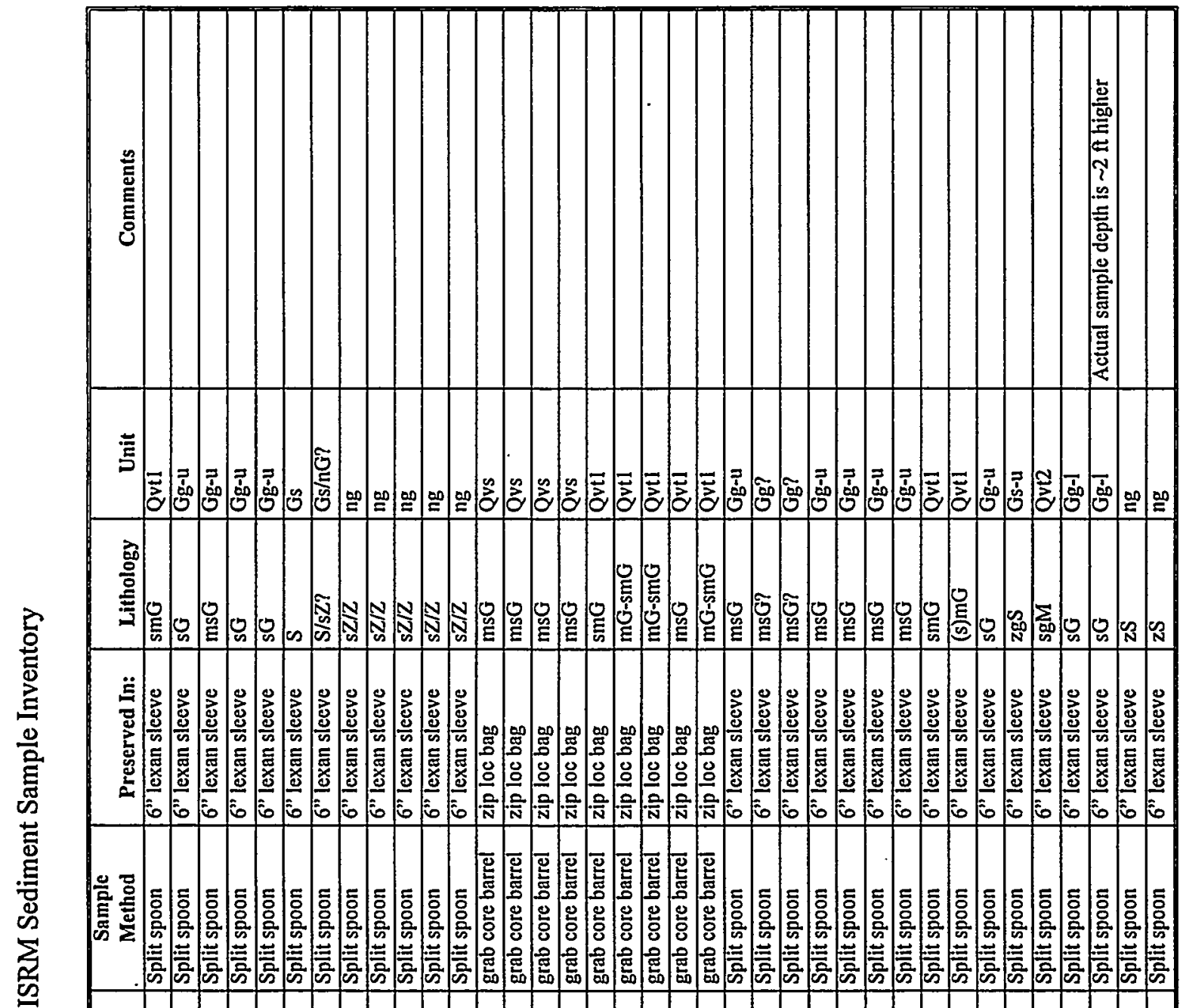

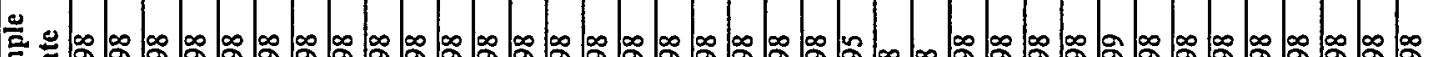
它

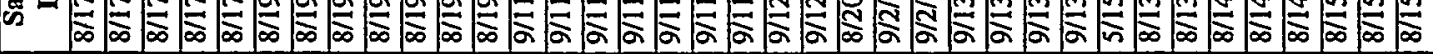

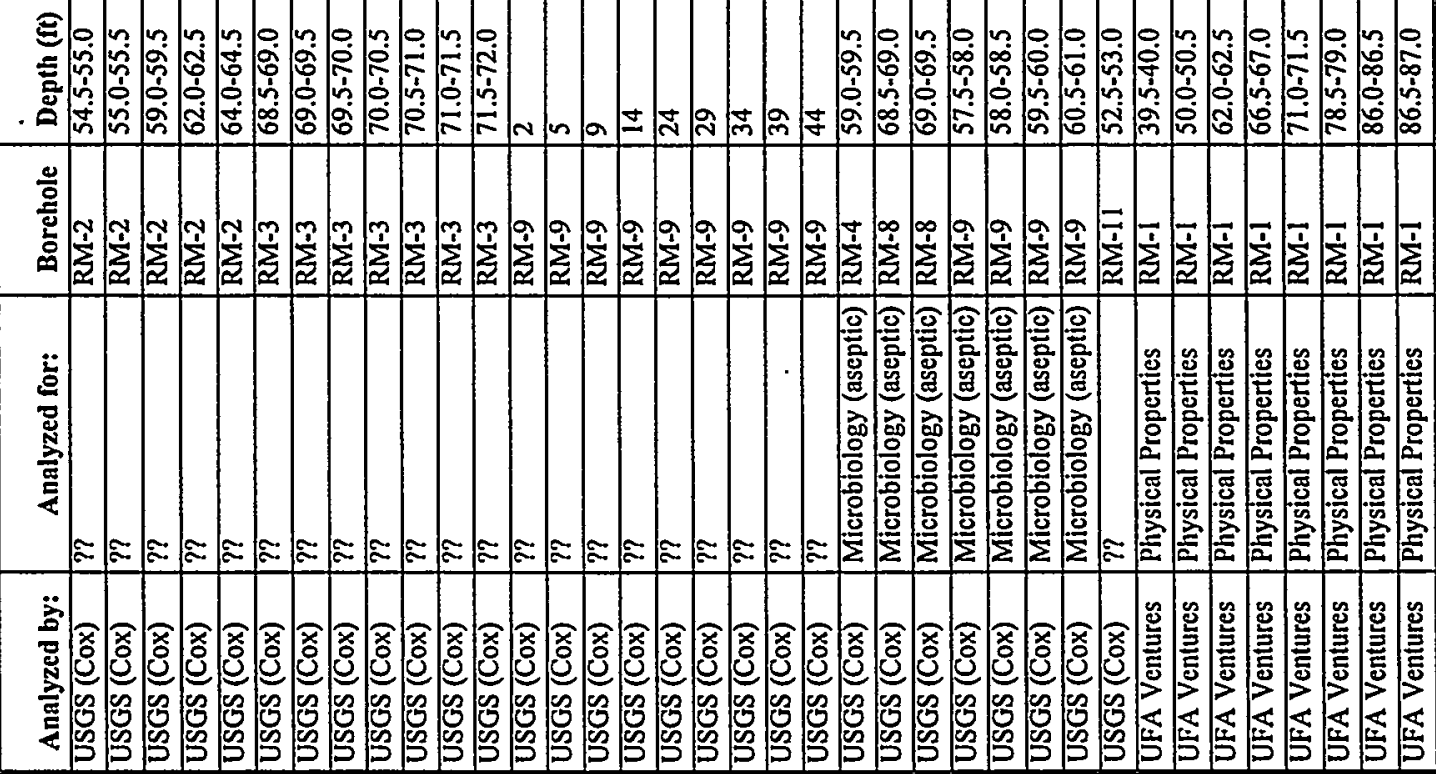




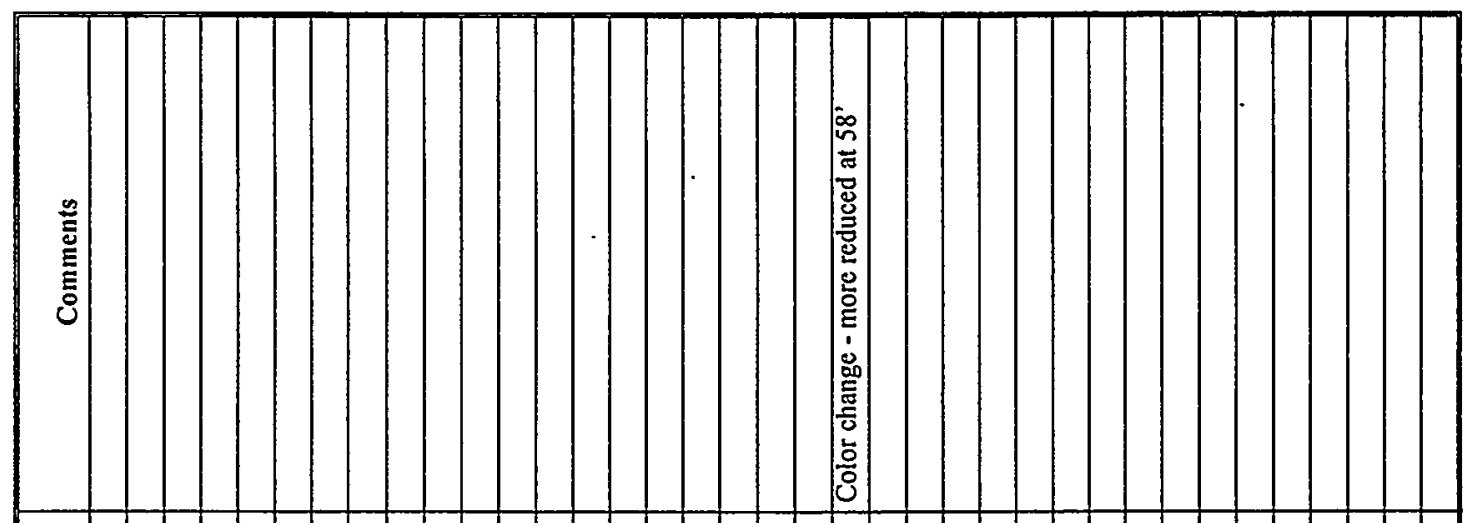

咅

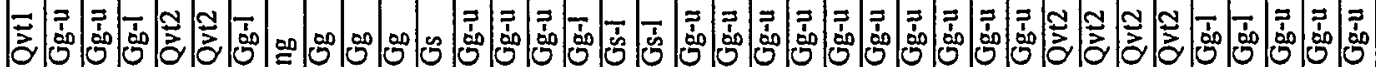

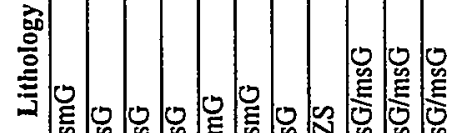

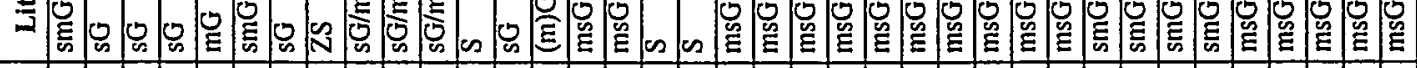

吾空

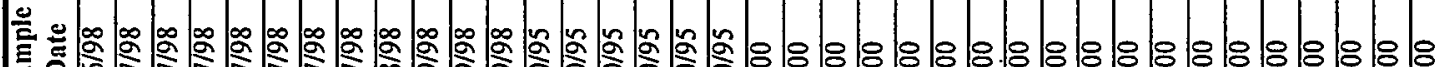

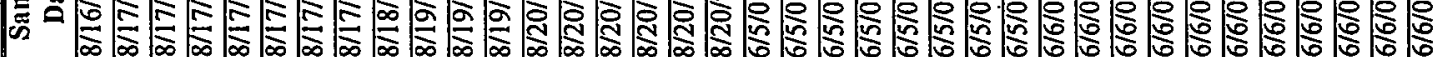

Elomem

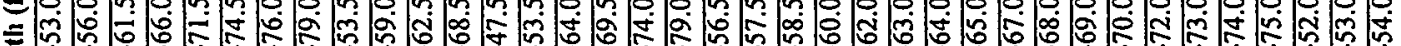

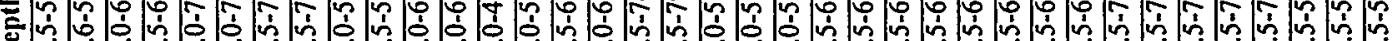

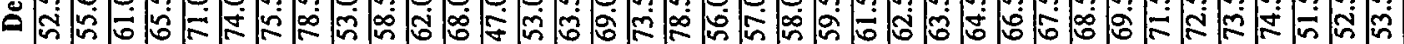

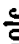

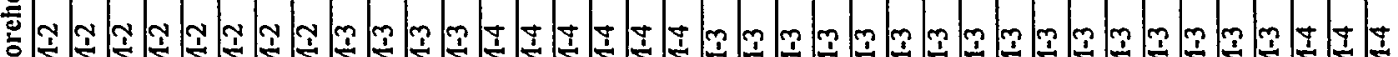

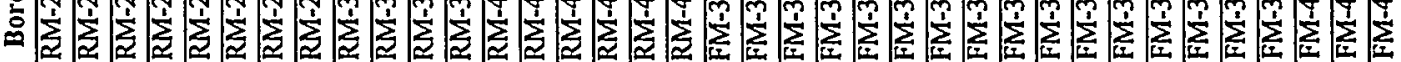

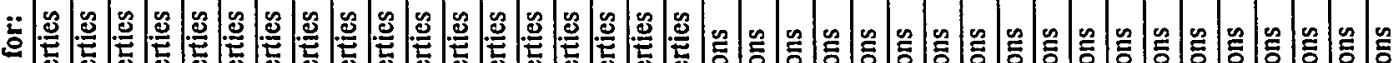

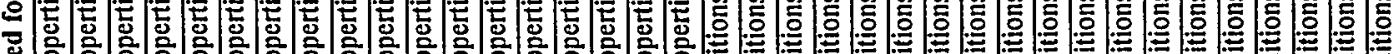

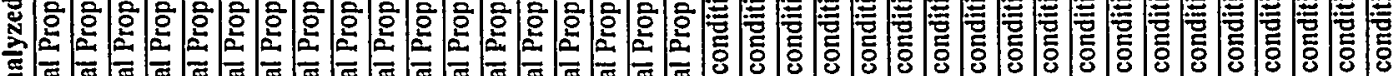

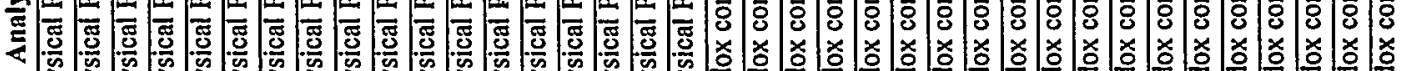

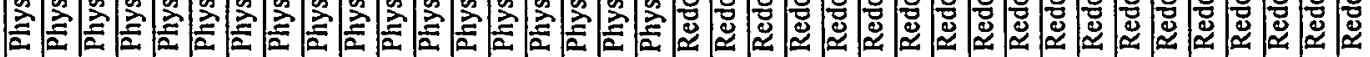

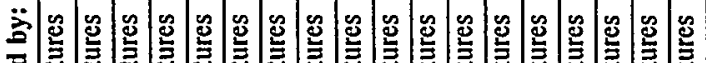

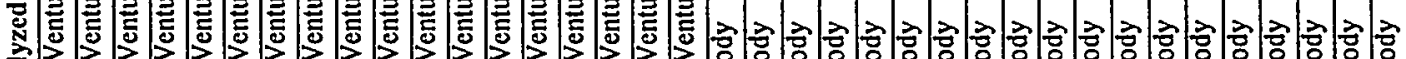

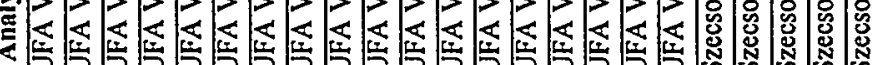




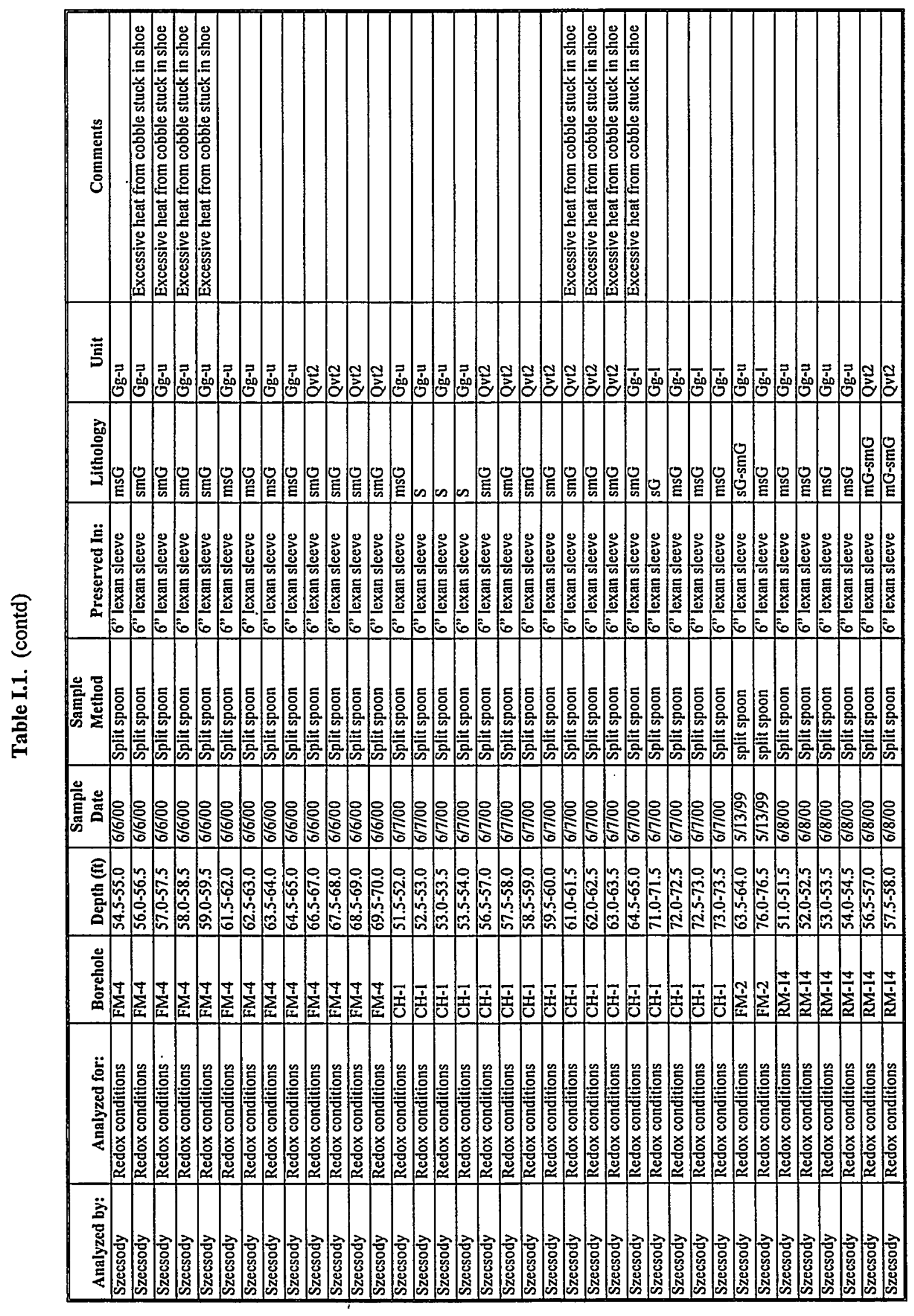




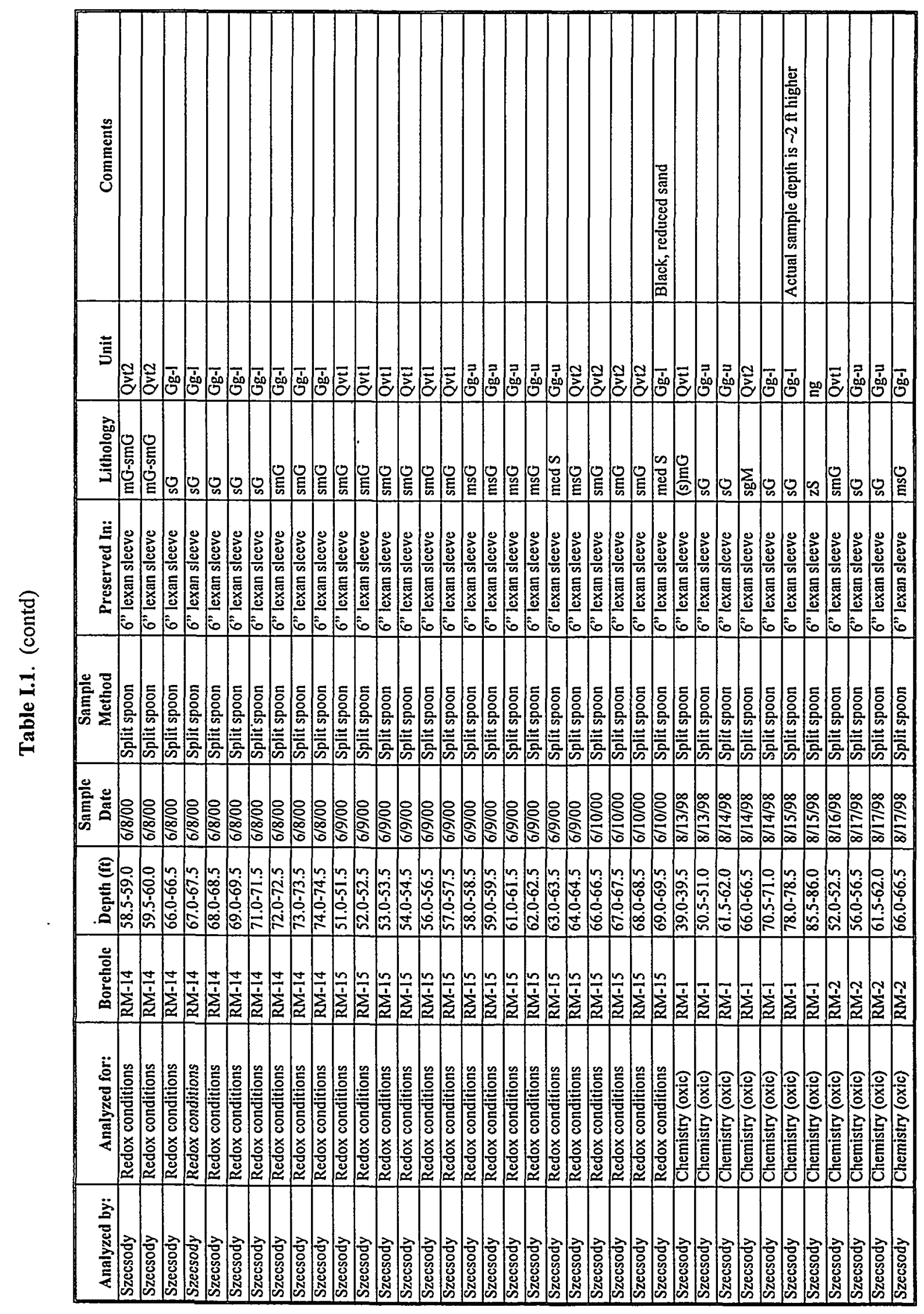




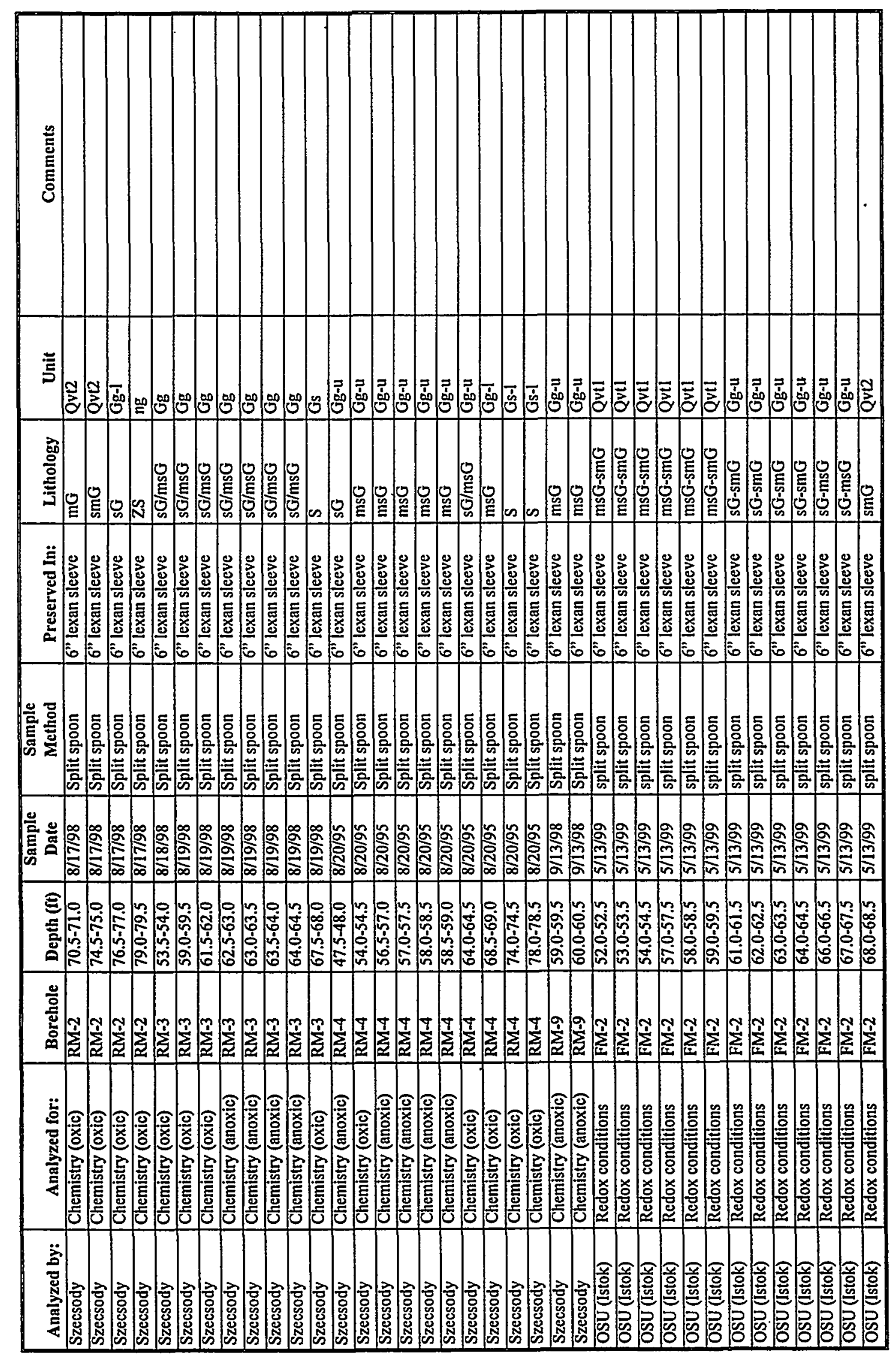




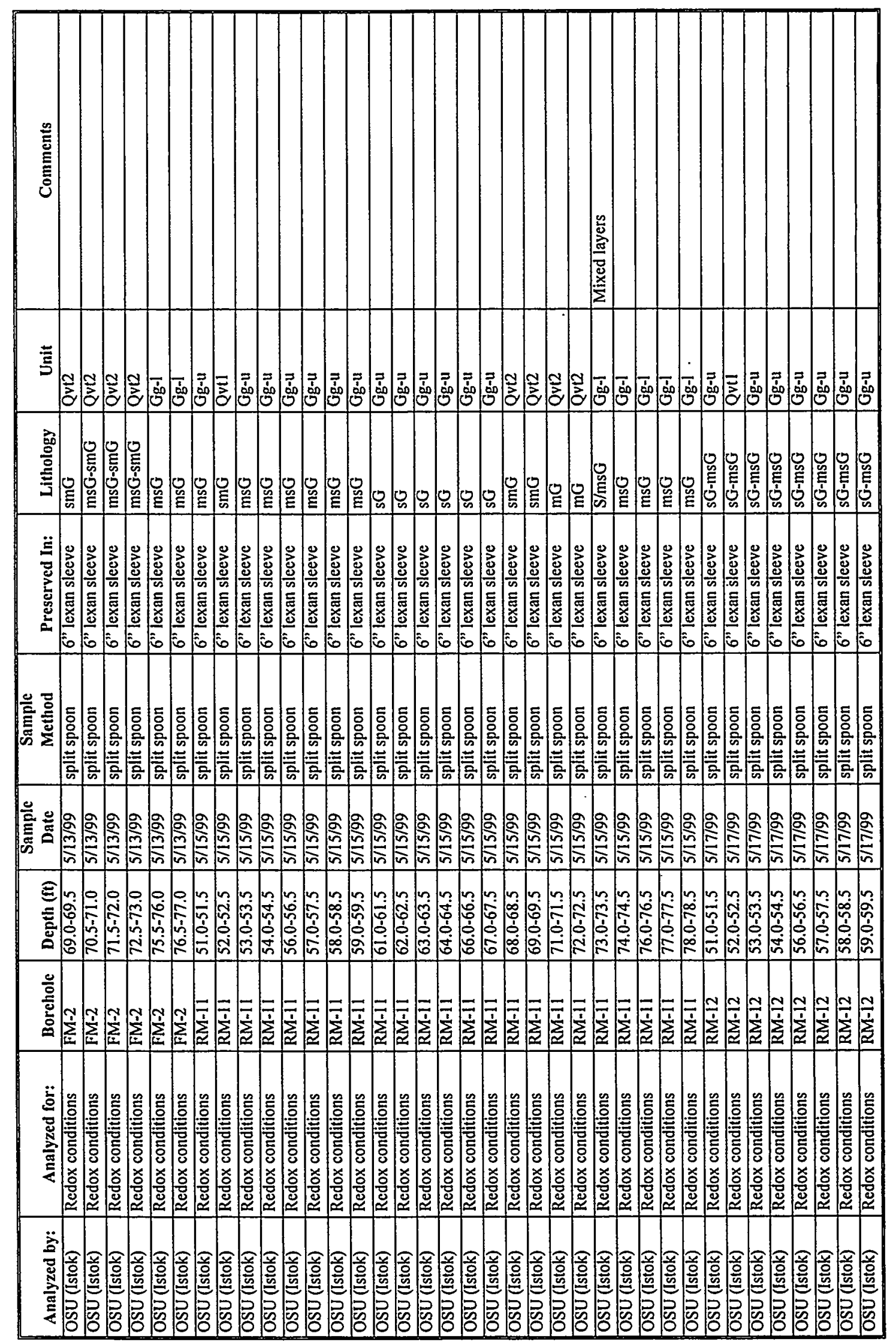




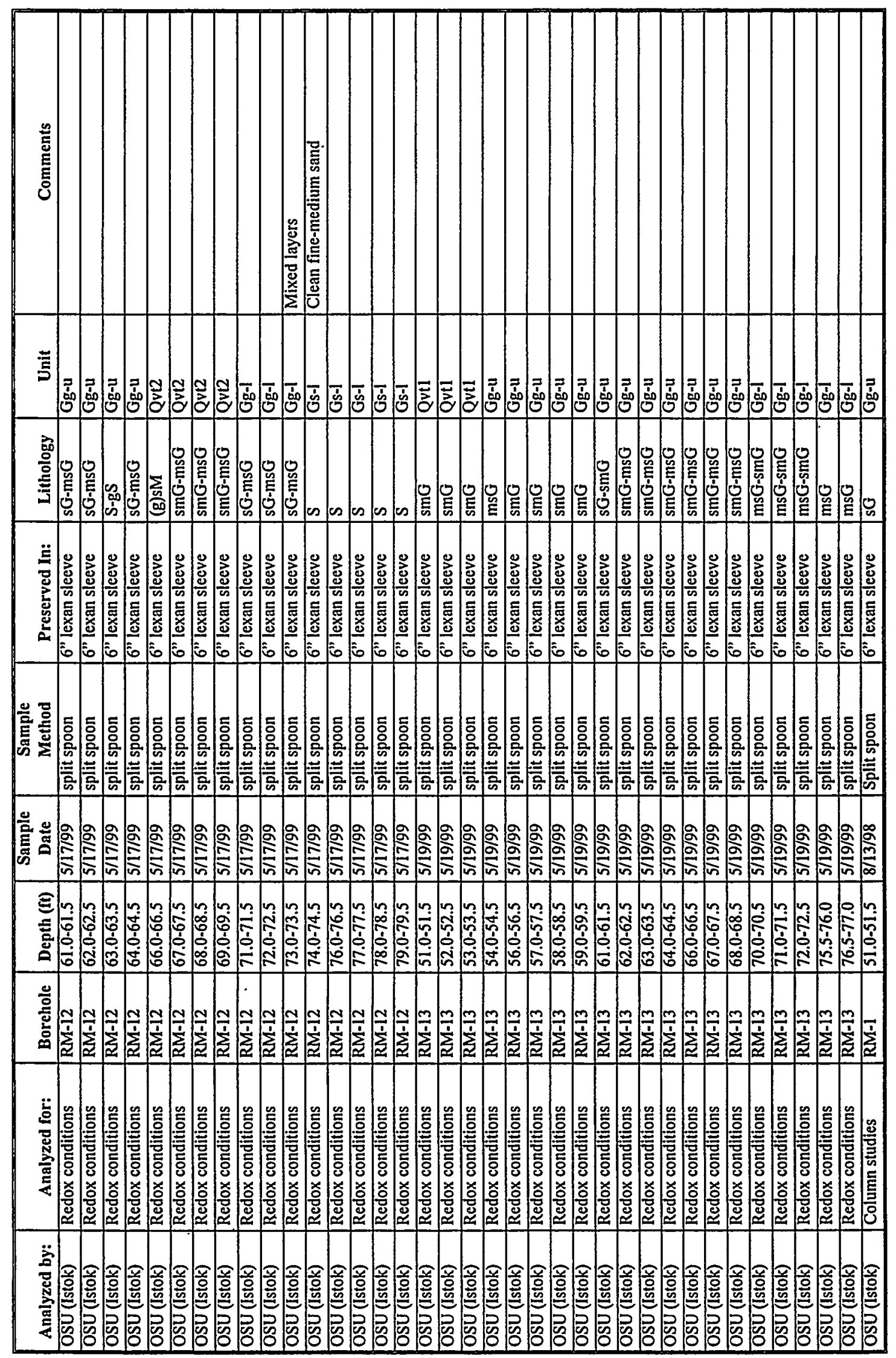




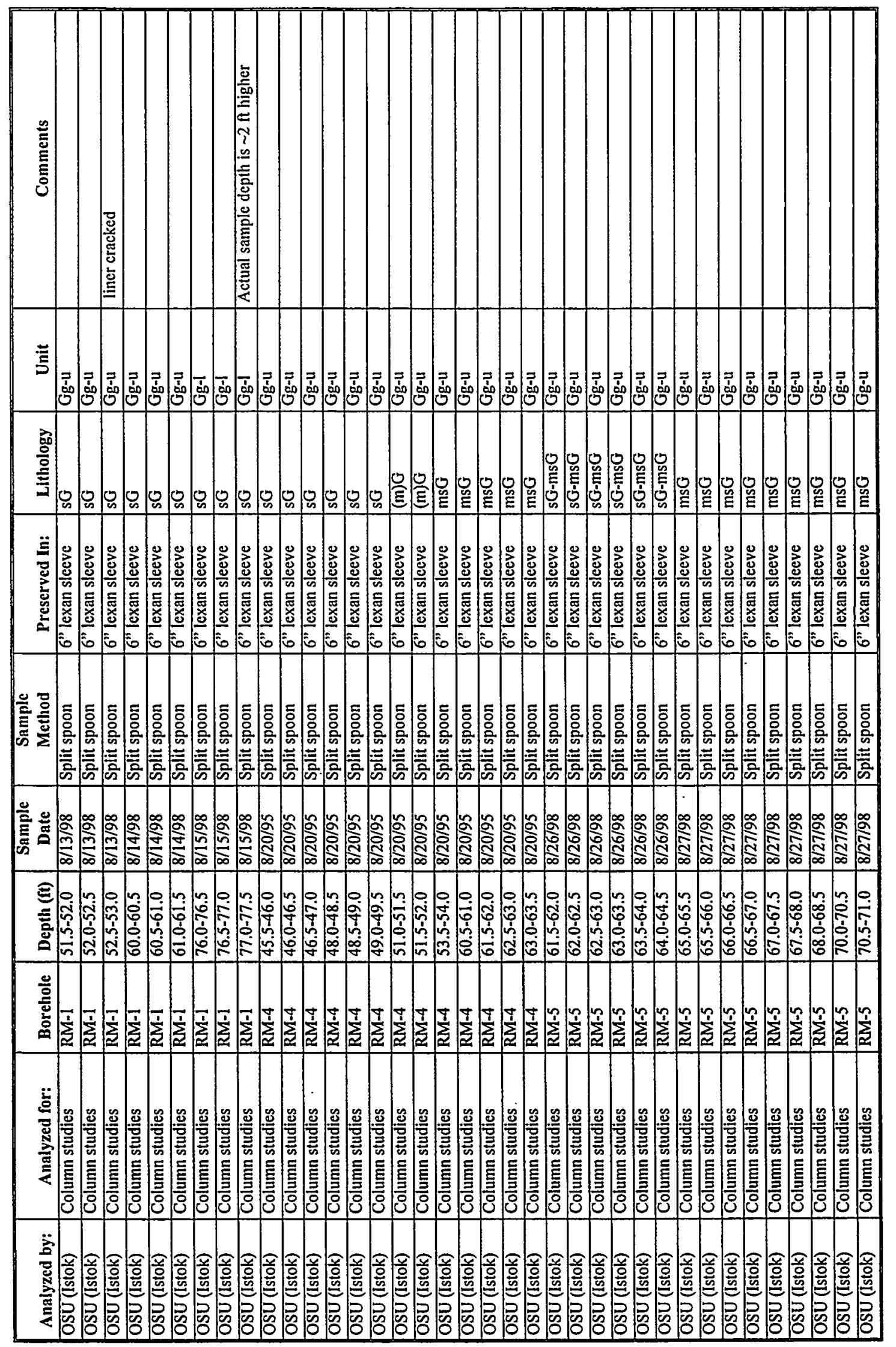




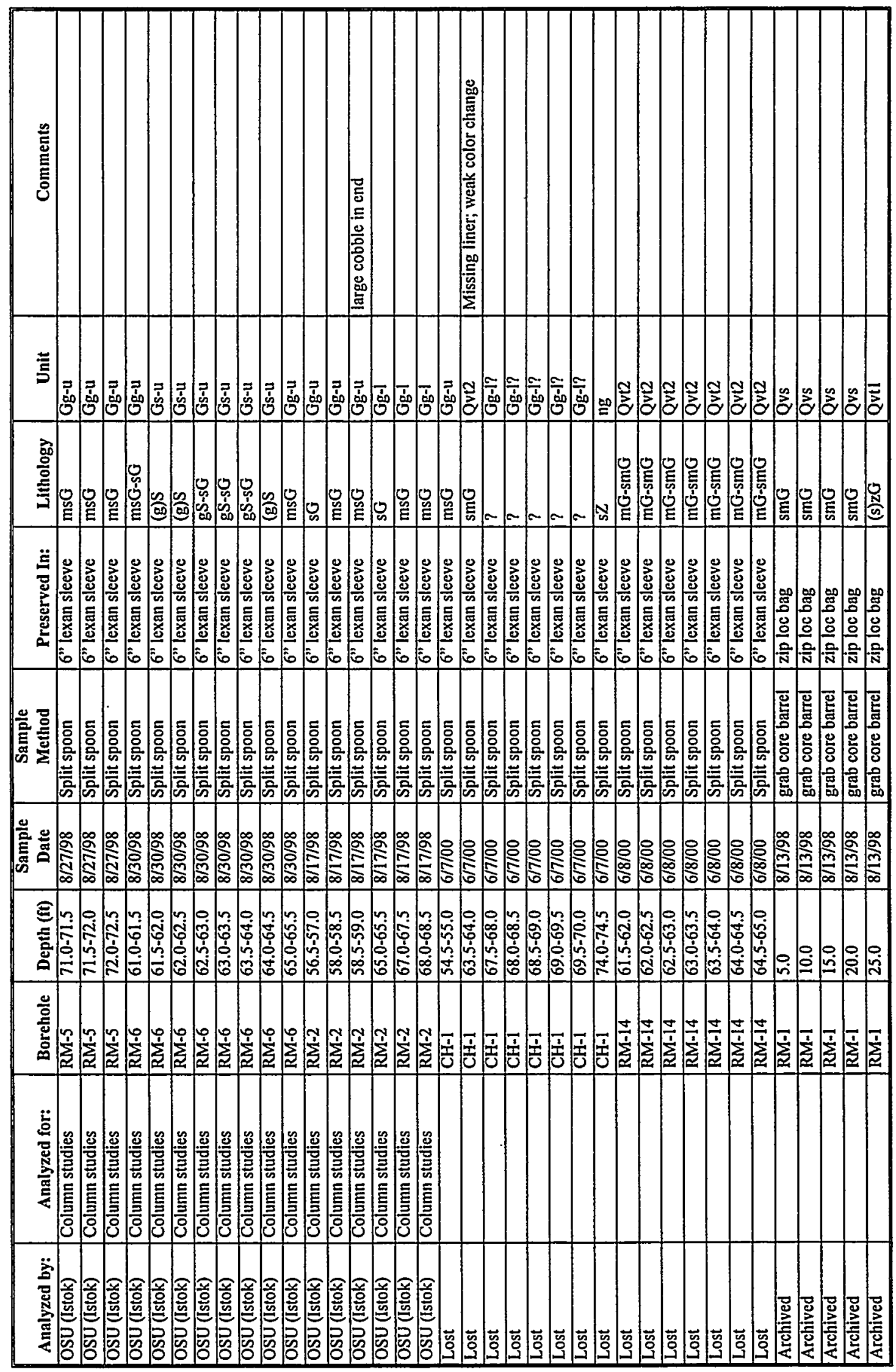




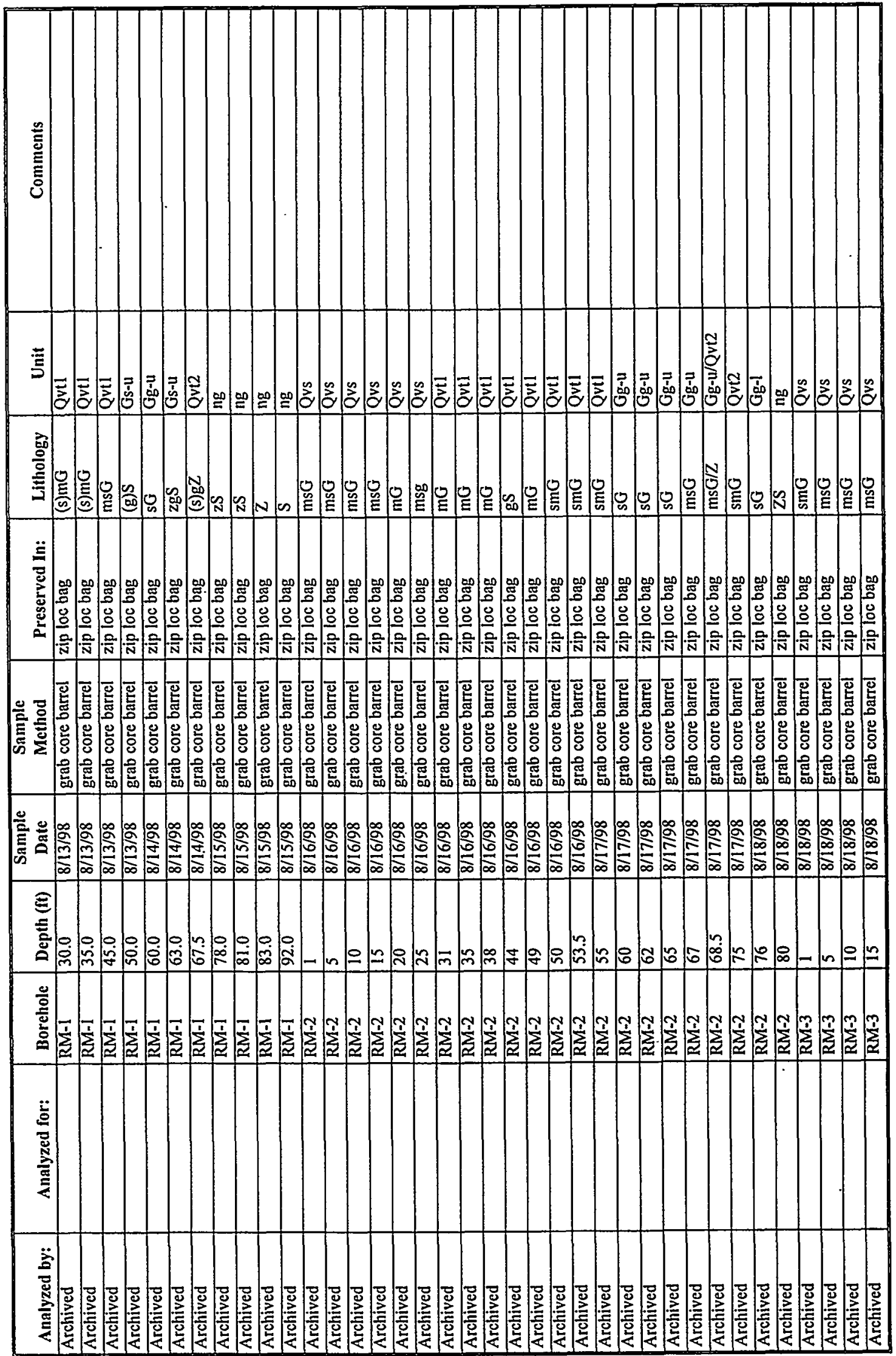




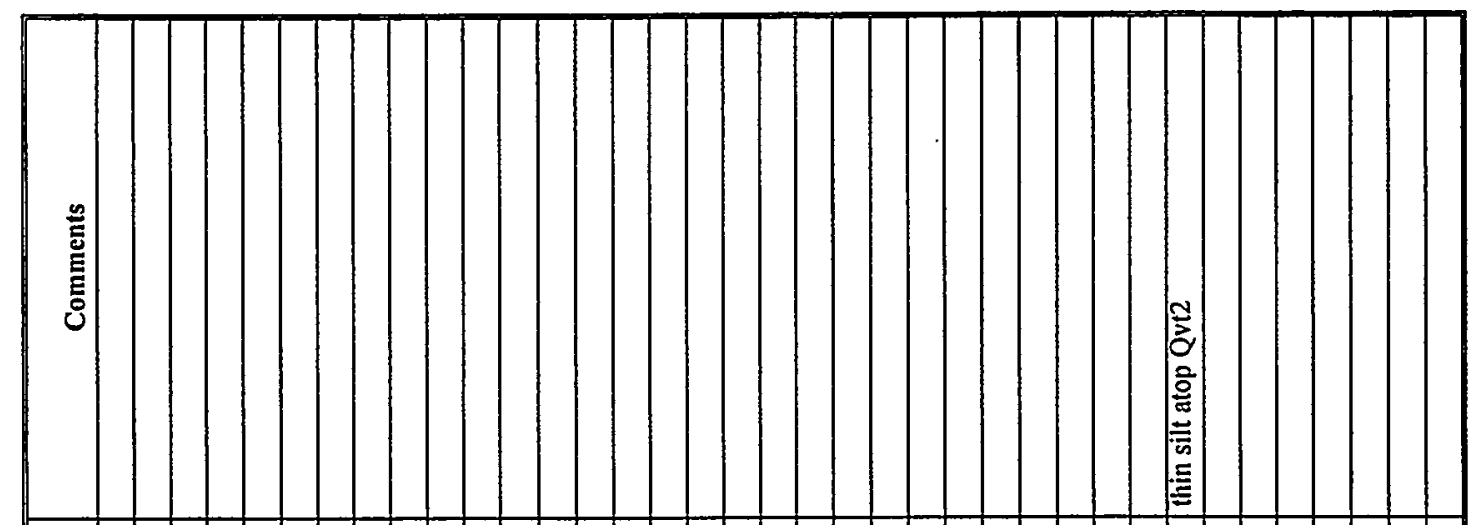

$\overline{\overline{5}}$

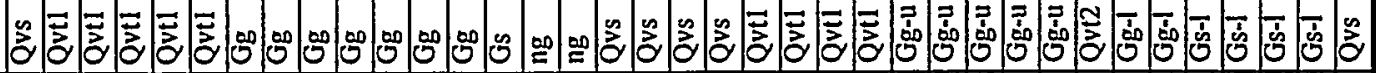

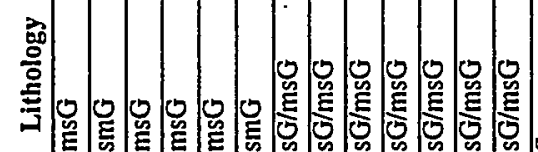

$\ddot{3}$

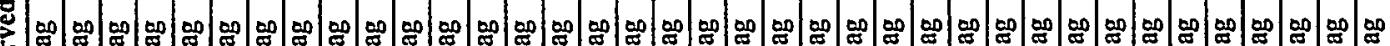

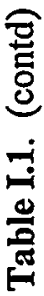

(5)

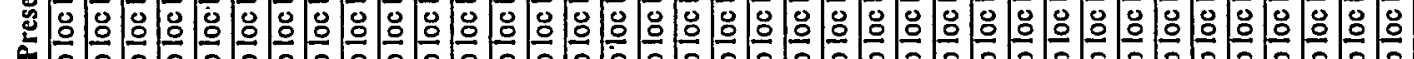

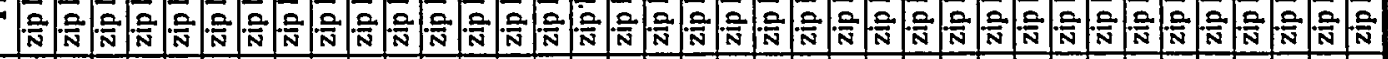

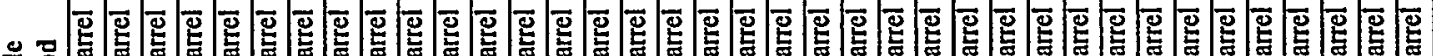
等

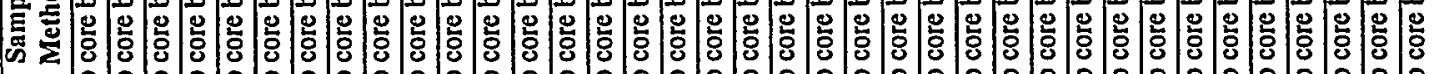

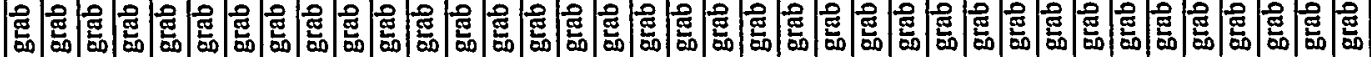

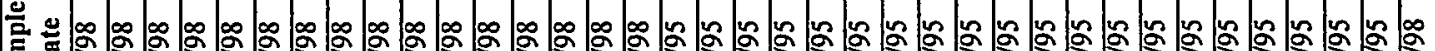

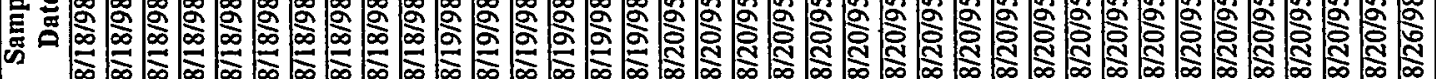

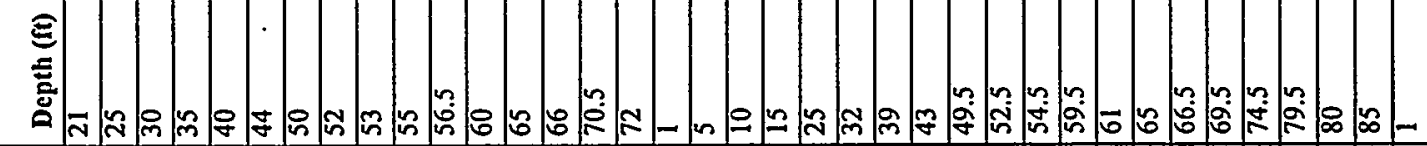
$\div$

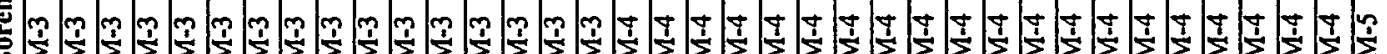

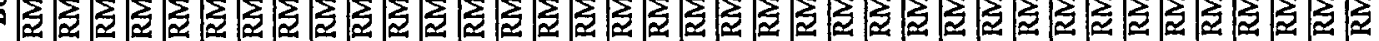

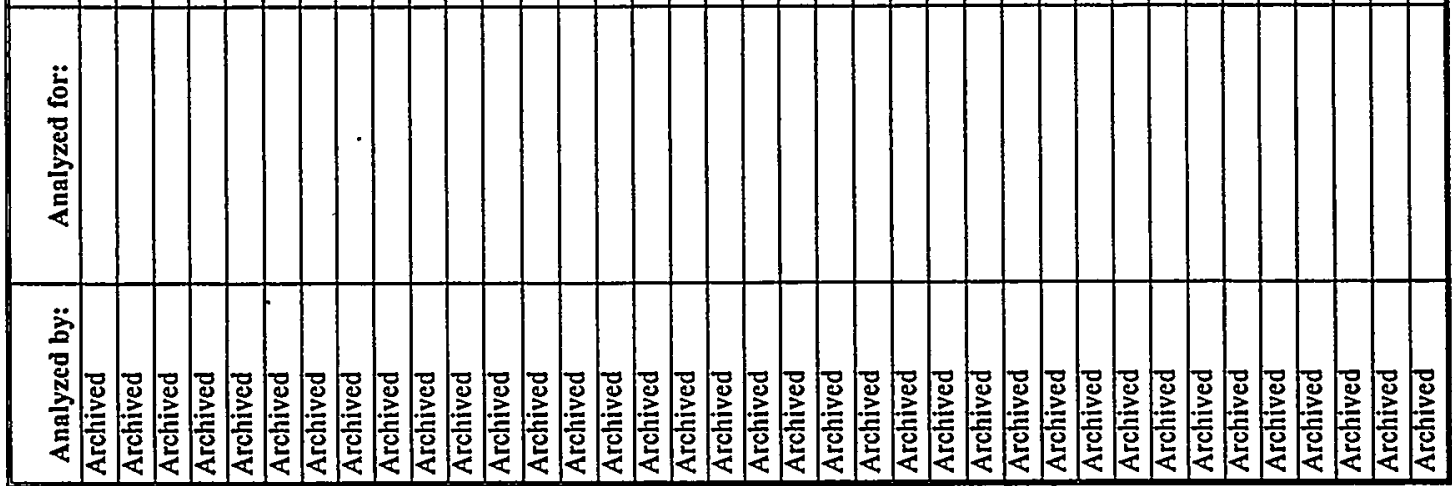




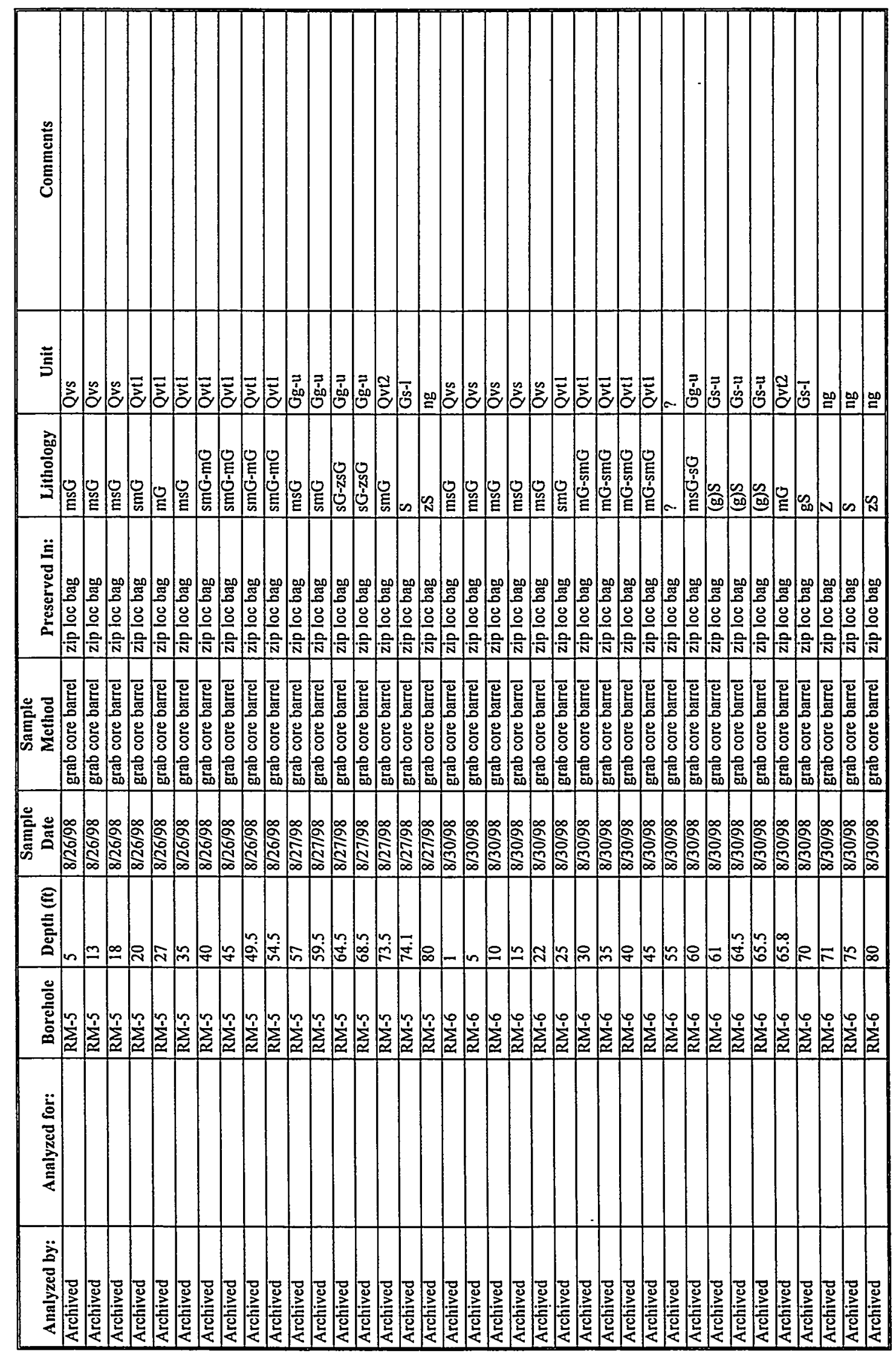

I. 12 


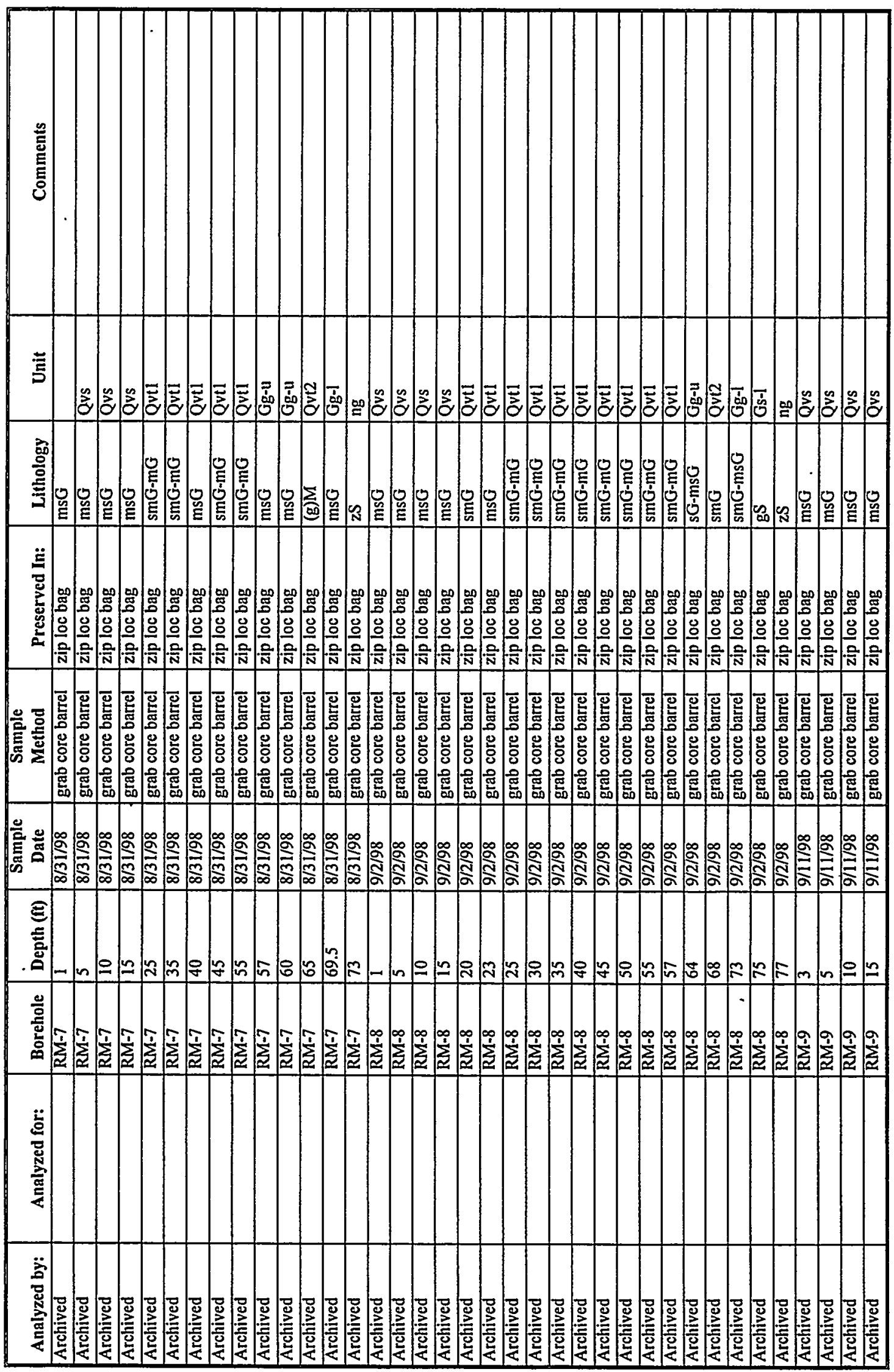




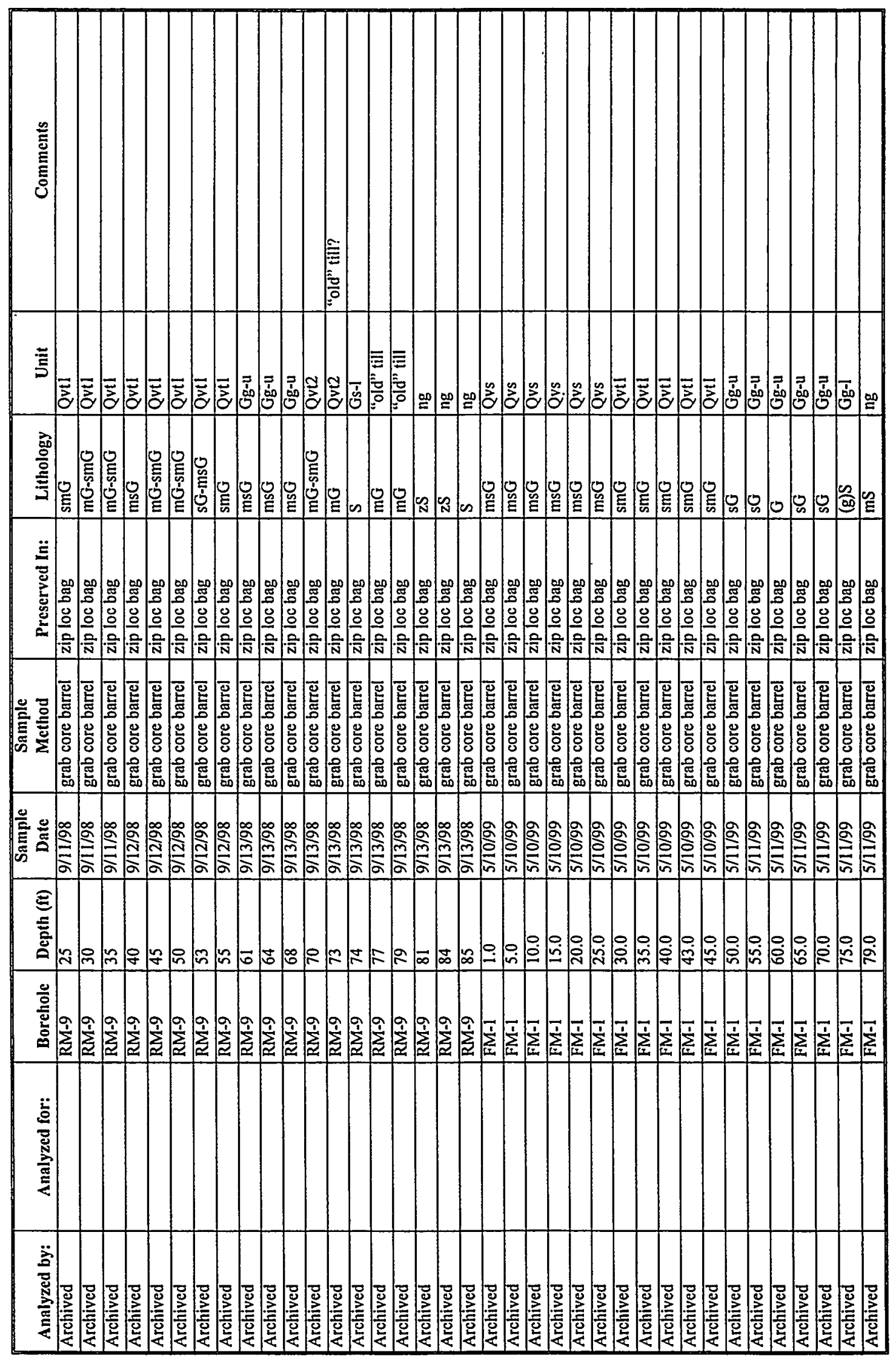

I. 14 


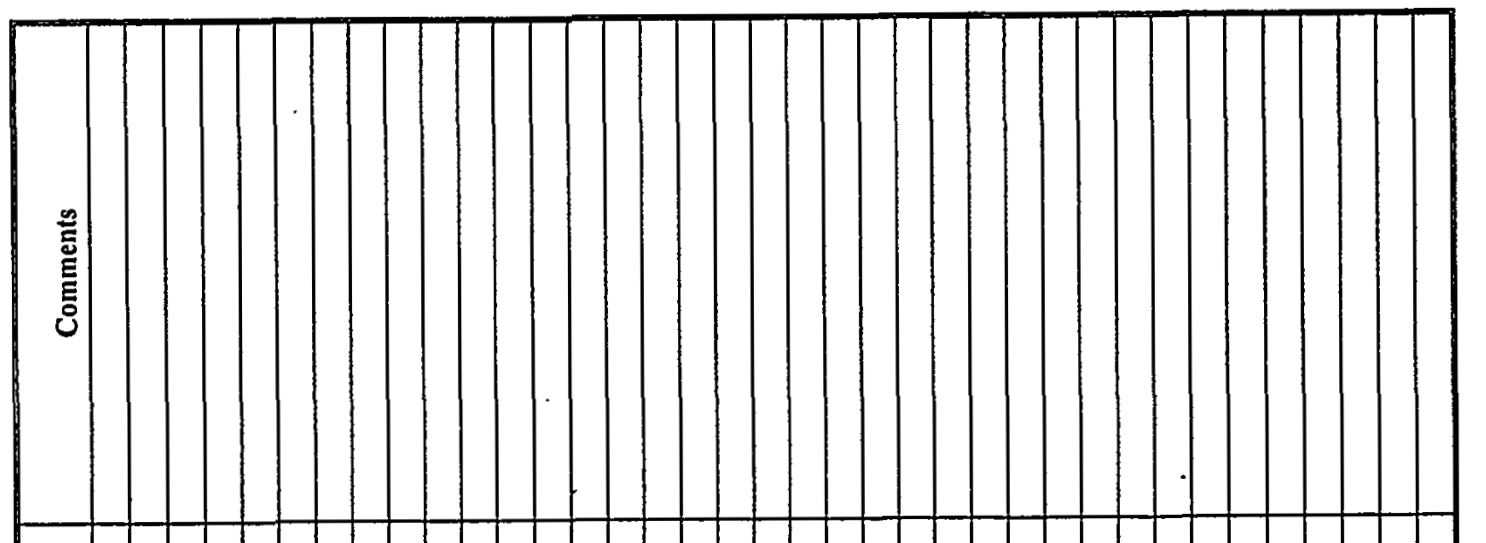

六

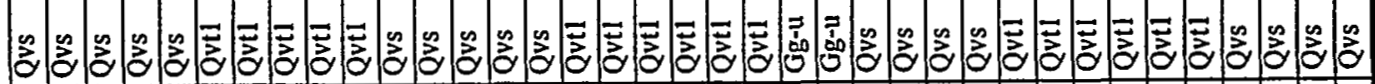
害

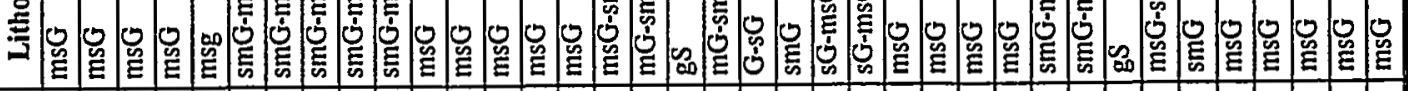
实

(5)

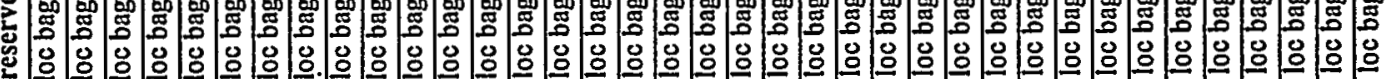

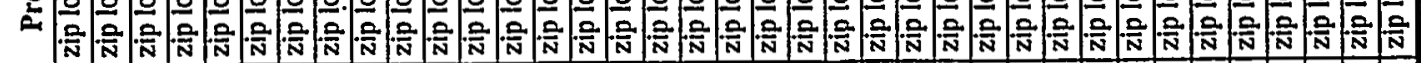

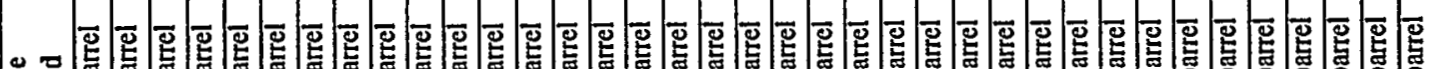

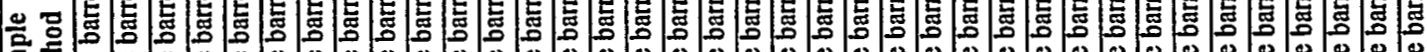
校

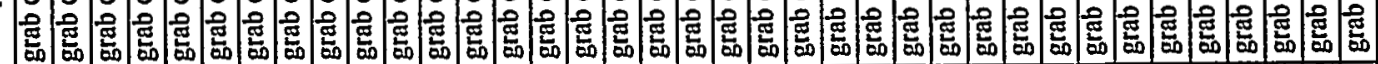

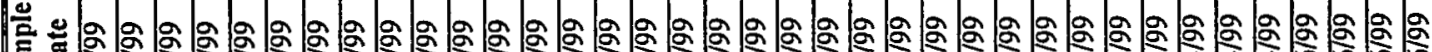

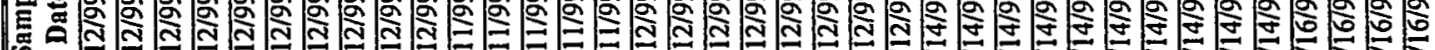

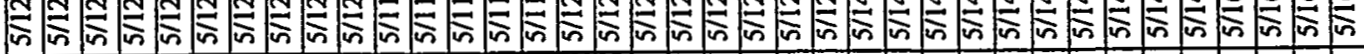

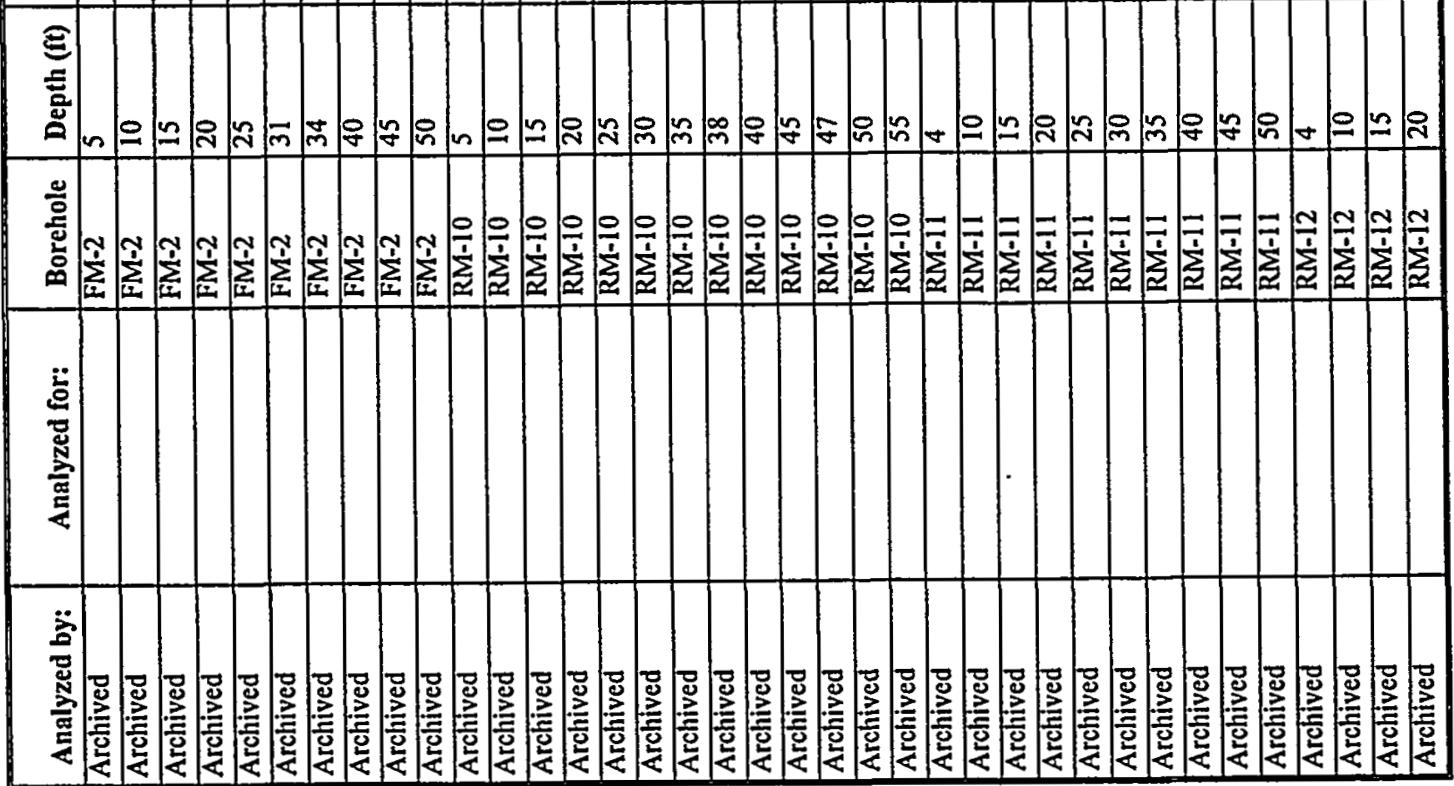




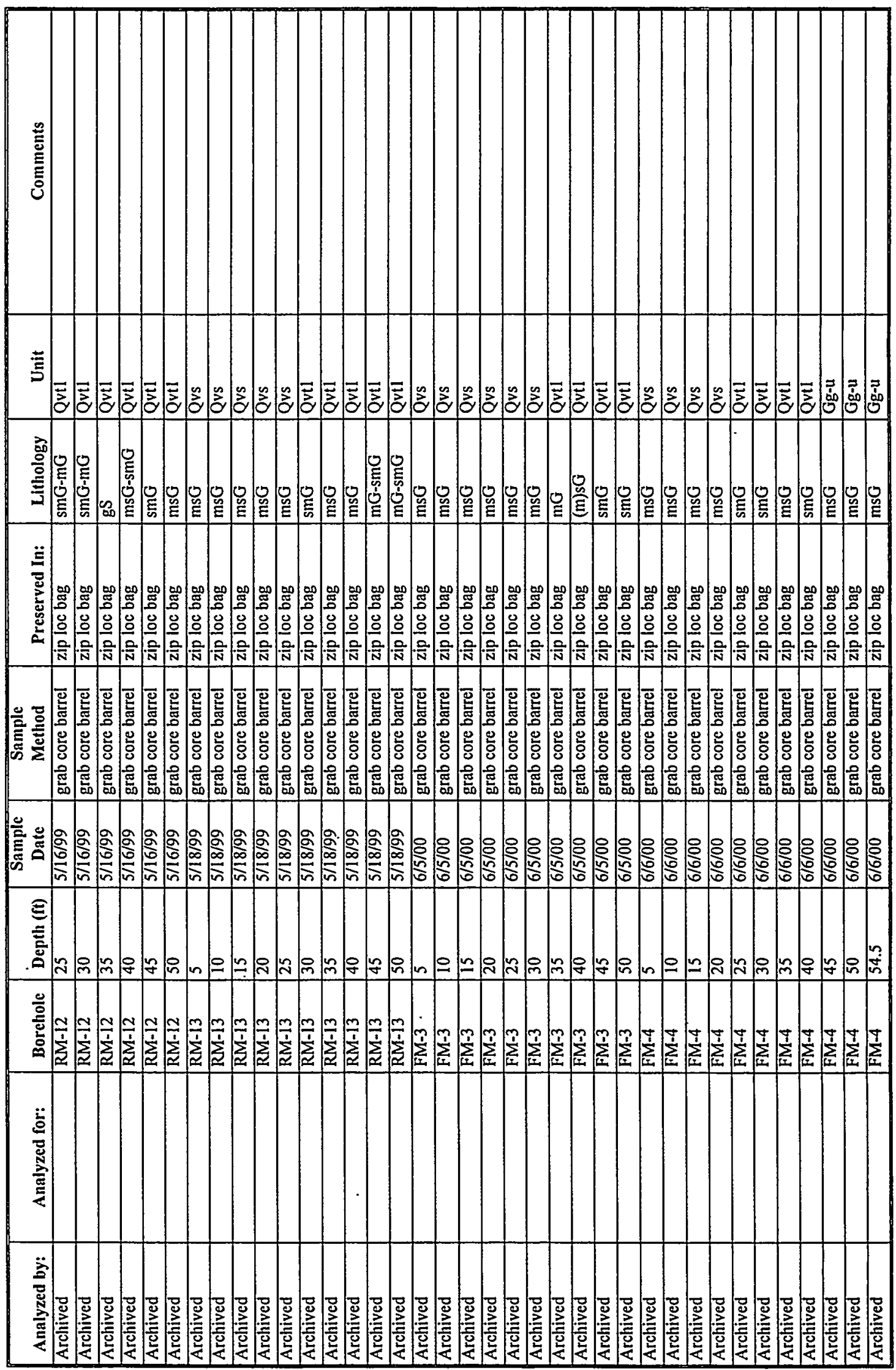




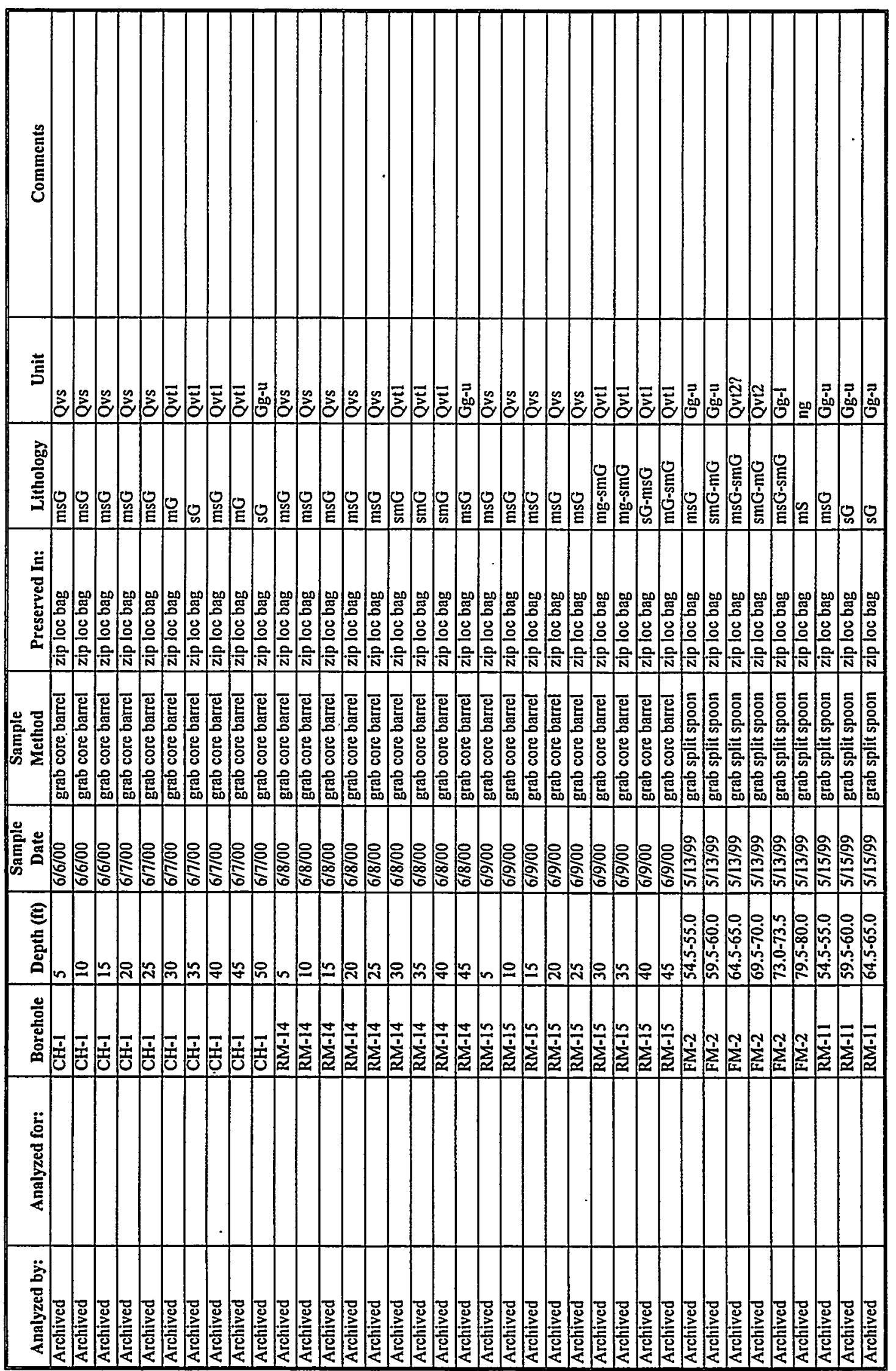




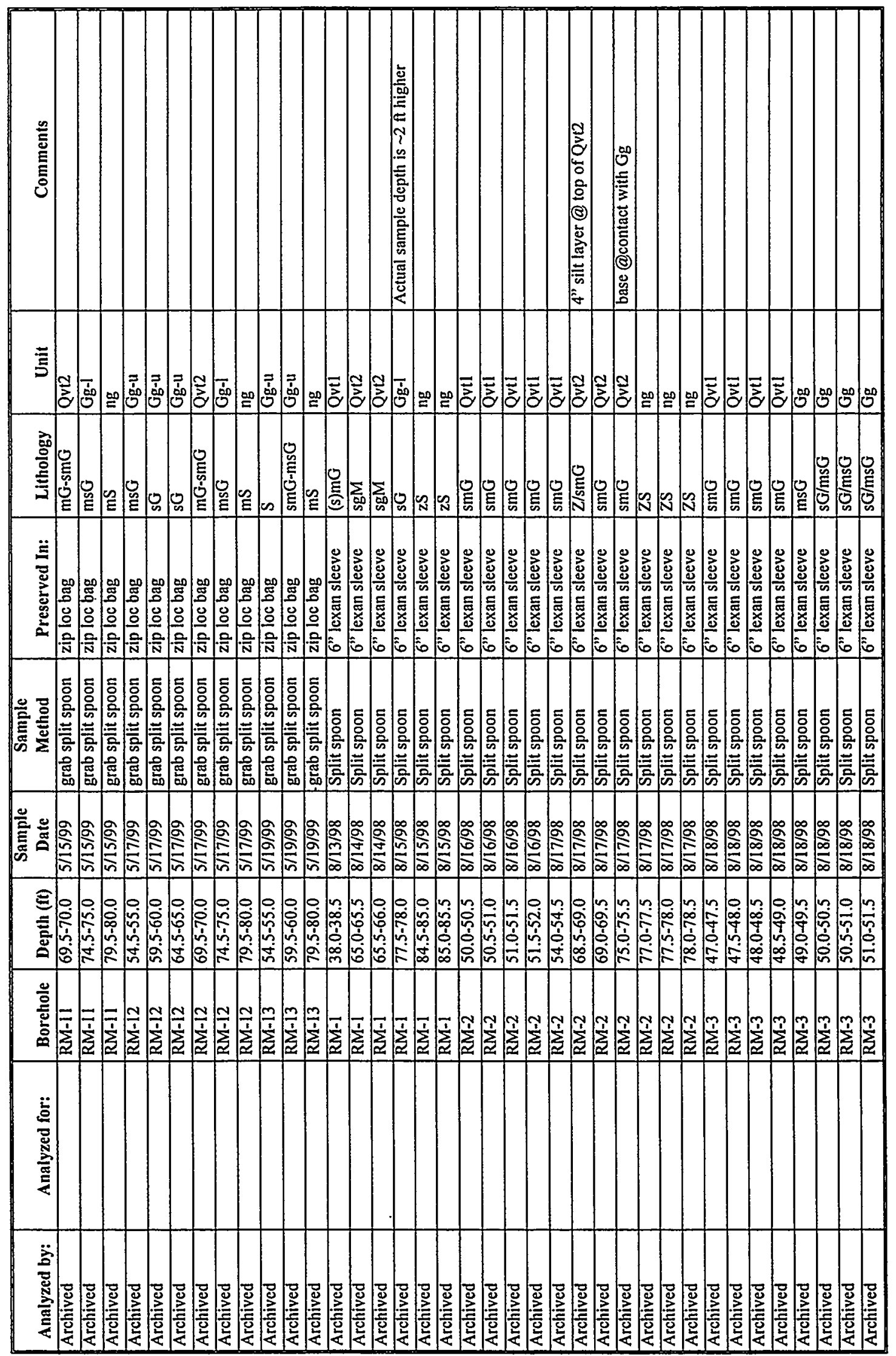




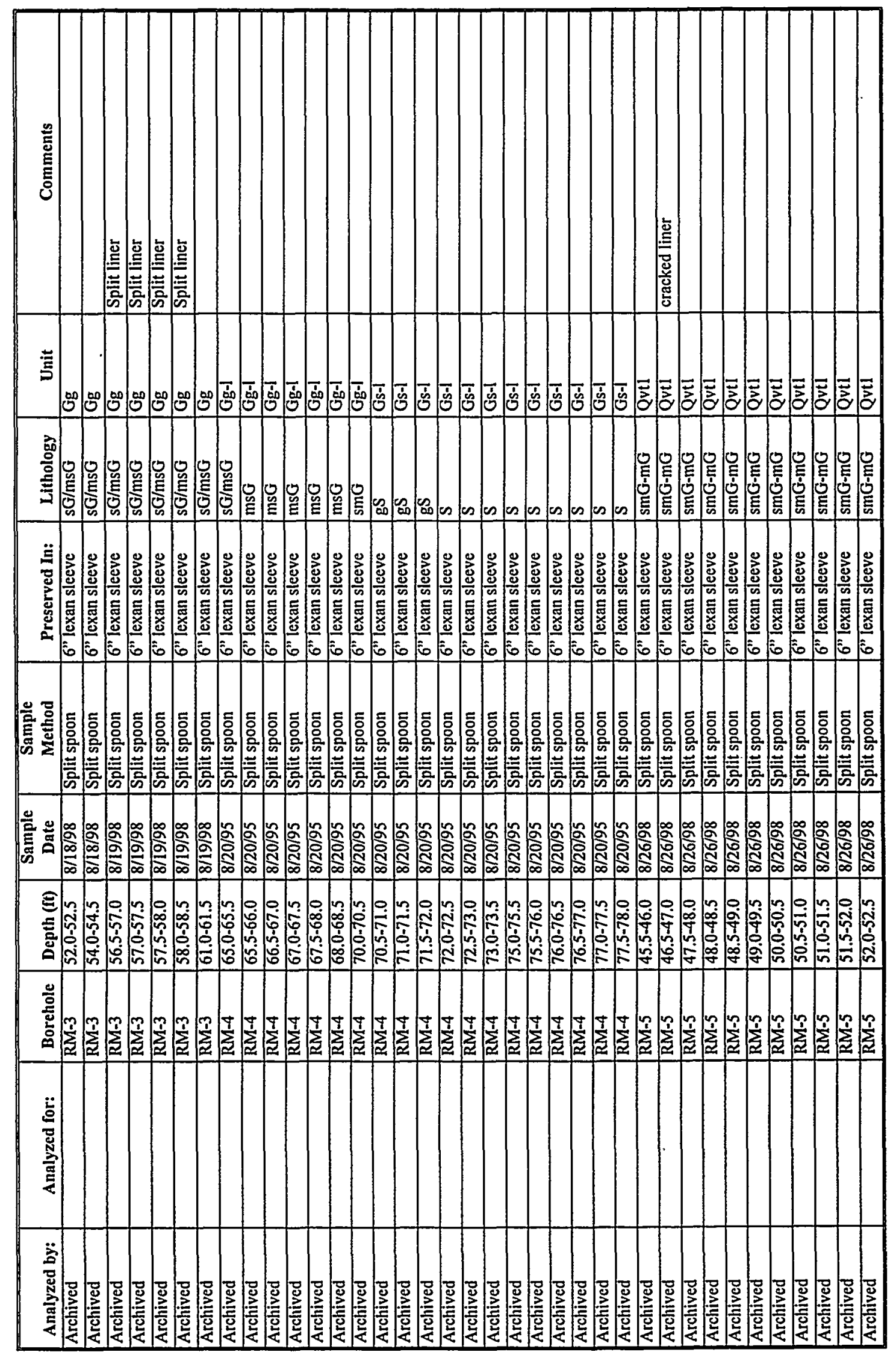




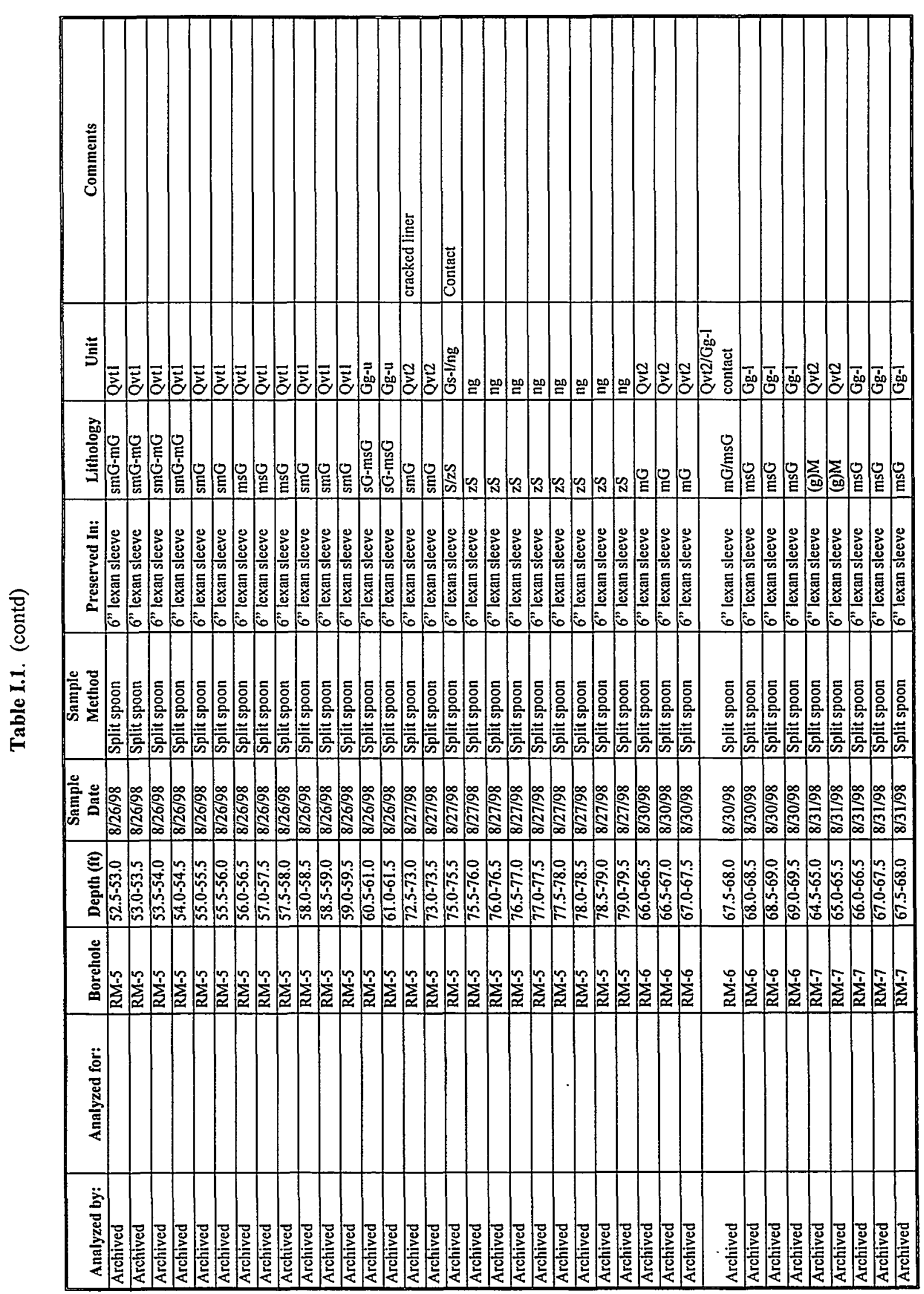




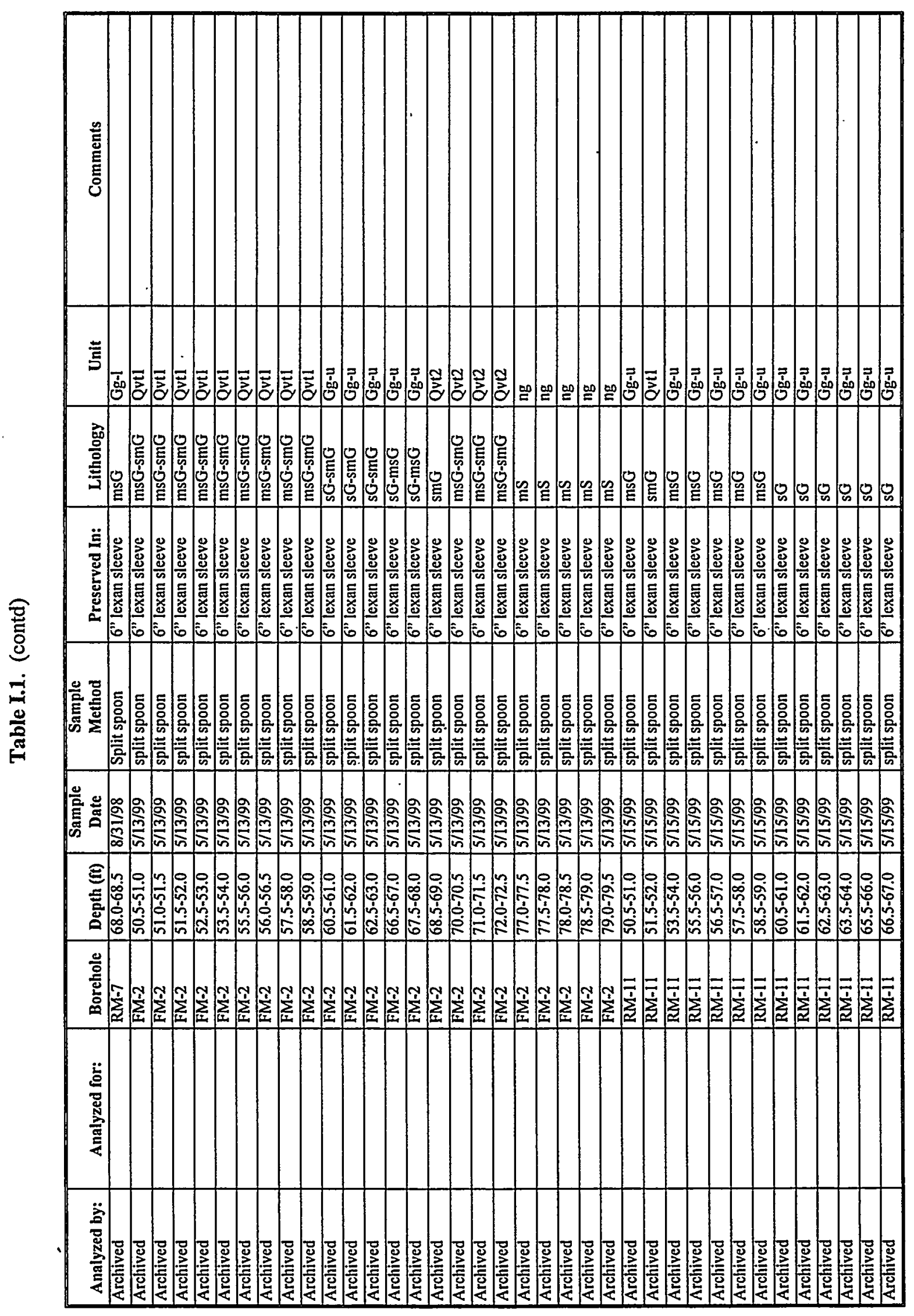




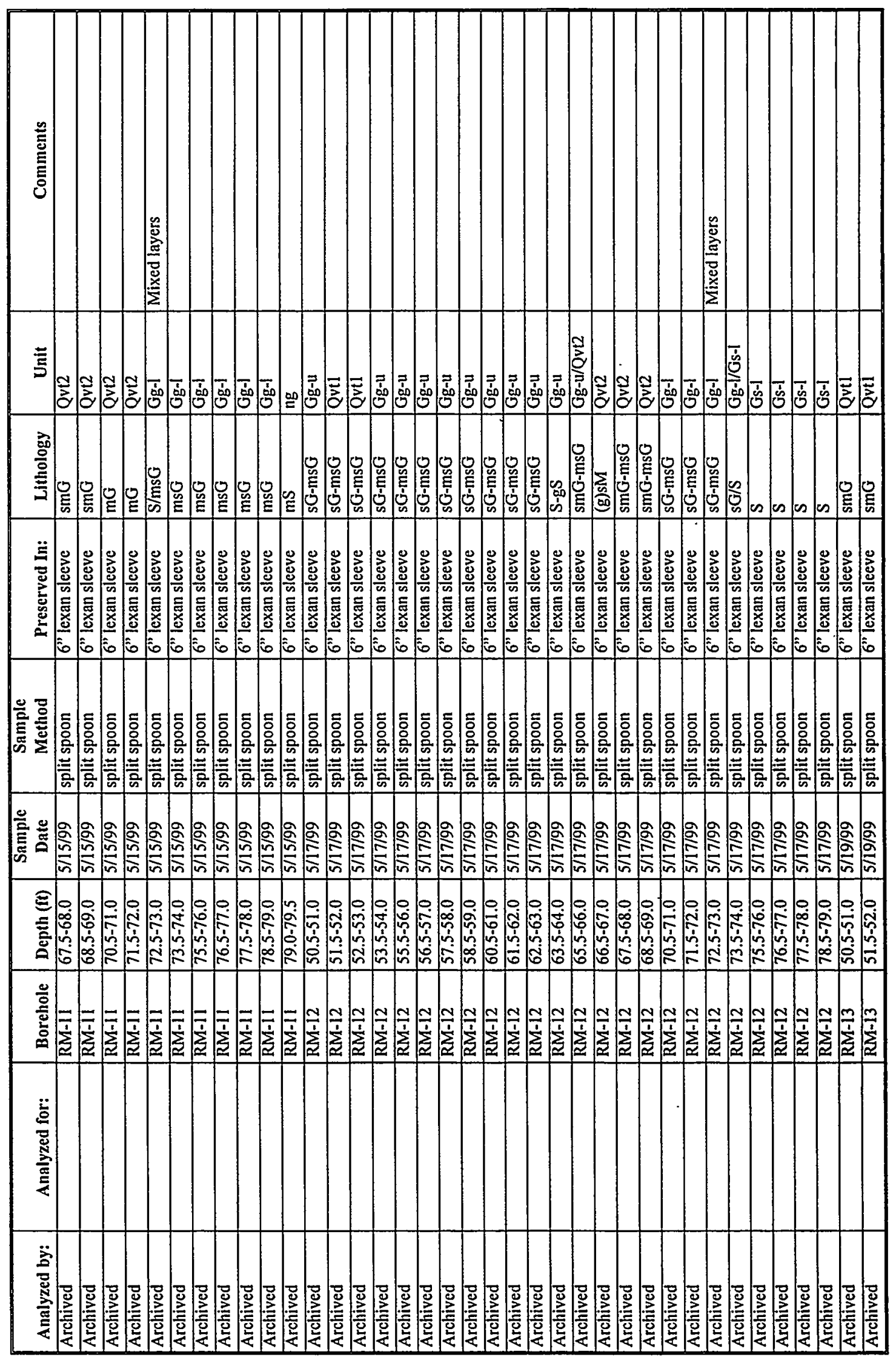




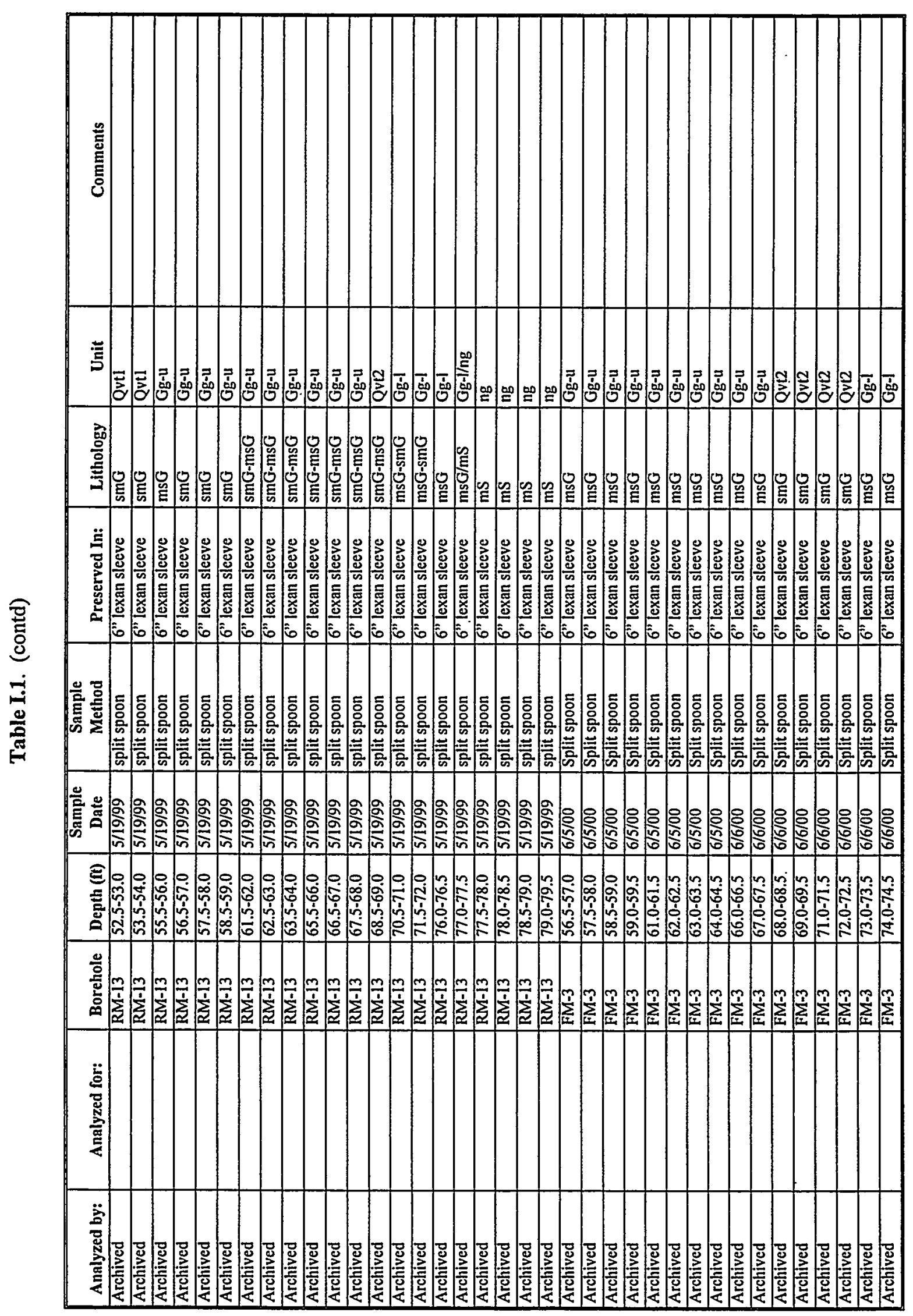




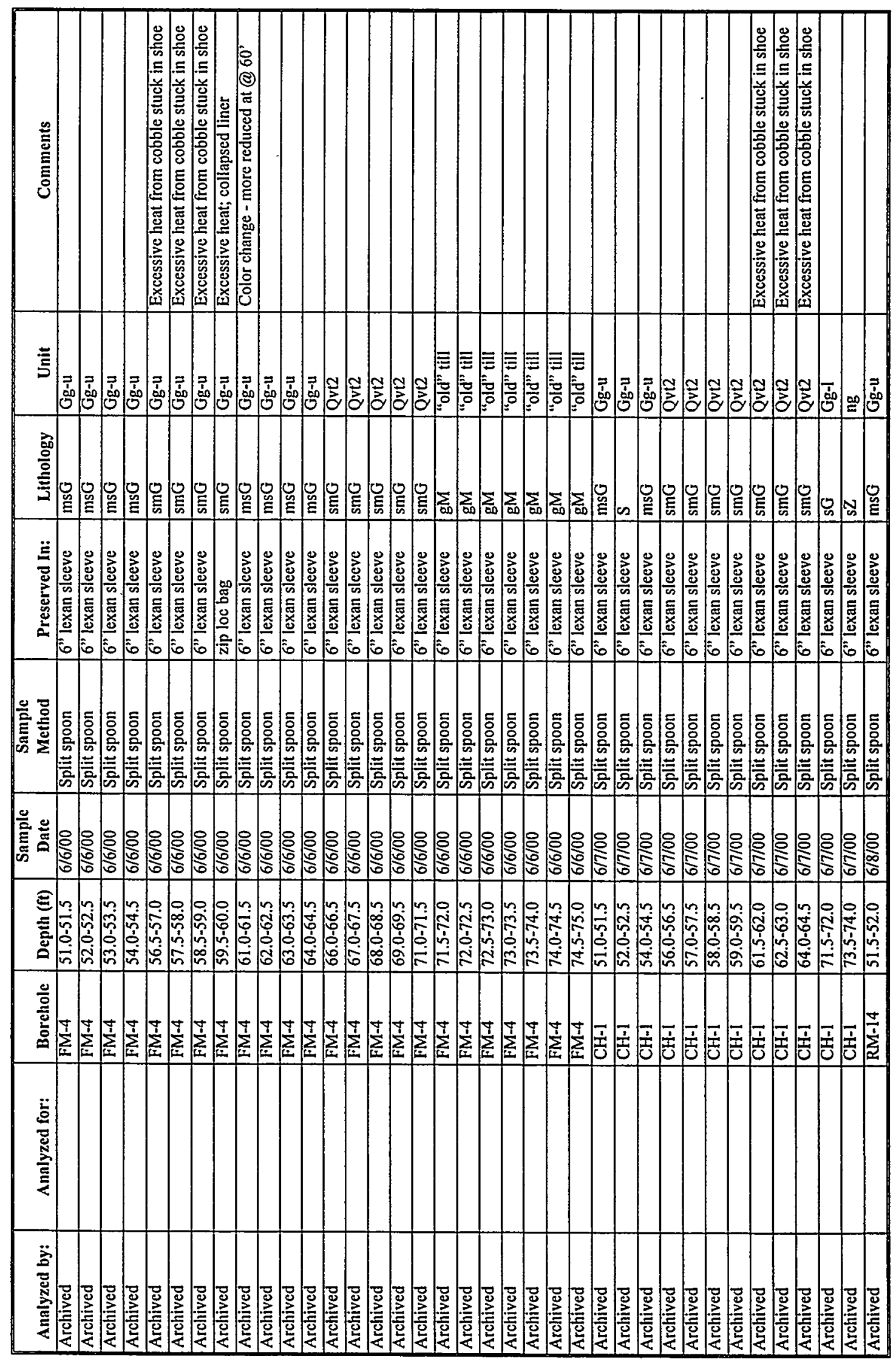




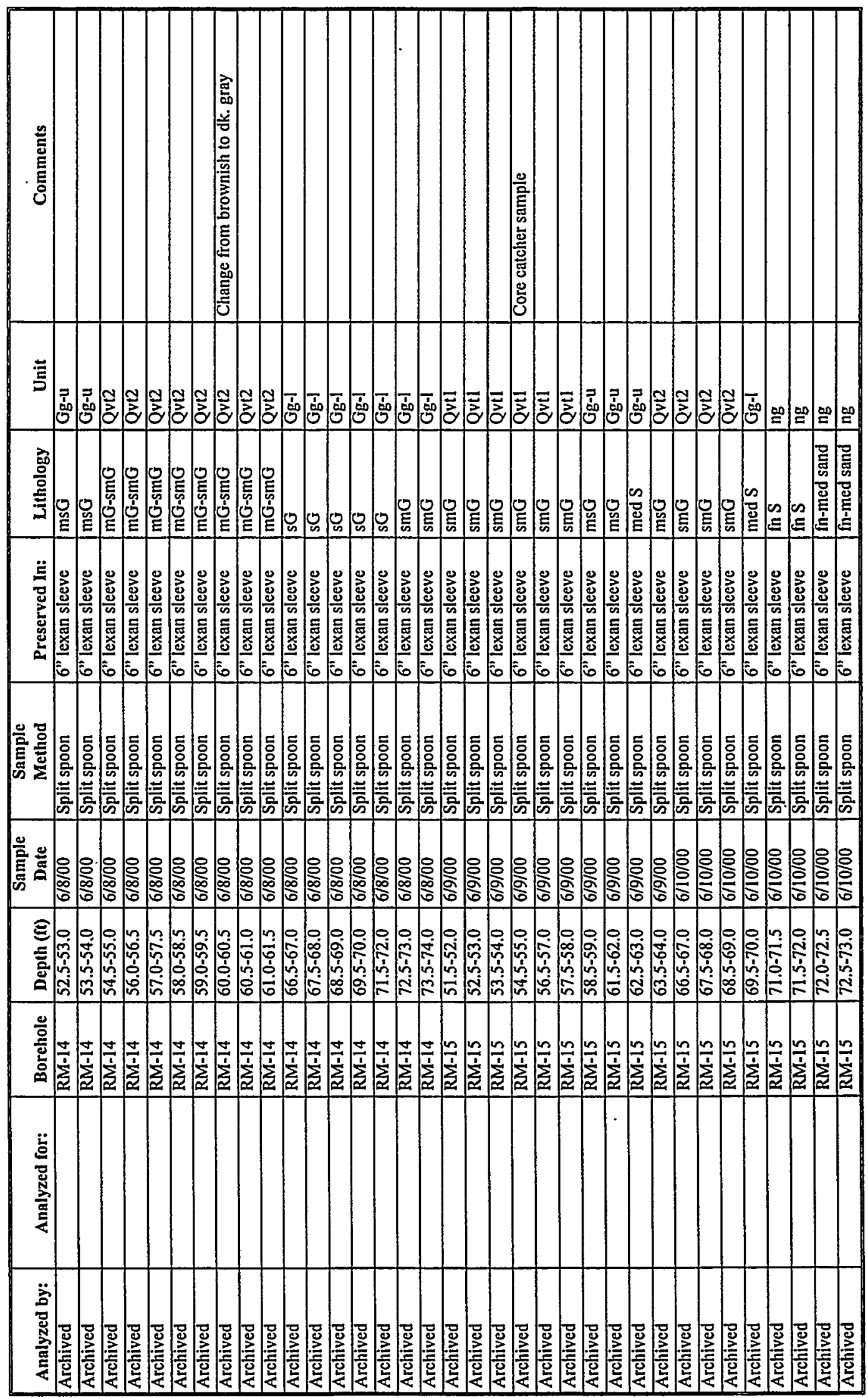




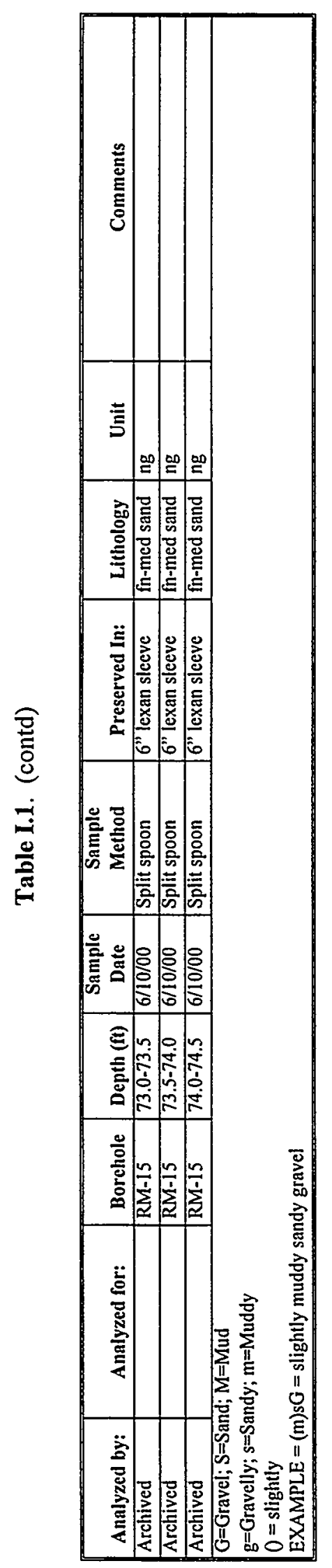




\section{Distribution}

No. of

Copies

OFFSITE

Marcia Knadle

Region 10

OEA-095

1200 Sixth Avenue

Seattle, WA 98101

U.S. Army Corps of Engineers

Seattle District

Attn: Bill Goss

Kira Lynch

Richard Smith

P.O. Box 3755

Seattle, WA 98124

U.S. EPA

Bob Keivit (2)

Washington Operations Office

300 Desmond Drive SE, Suite 102

Lacey, WA 98503

U.S. Geological Survey

Water Resources Division

Attn: Sue Kahle

Steve Cox

Rick Dinicola

1201 Pạcific Avenue, Suite 600

Tacoma, WA 98402

Rich Wilson

Public Works

Attn: AFZH-PWE MS-17

Building 2012, Room 321

Fort Lewis, WA 98433-9500
No. of

Copies

ONSITE

42 Pacific Northwest National Laboratory

B. N. Bjornstad

K6-81

J. G. Bush

K6-96

J. L. Devary

$\mathrm{K} 6-96$

J. C. Evans

K6-96

J. S. Fruchter

$\mathrm{K} 6-96$

T. L. Liikala (20)

K6-96

K. B. Olsen

K6-96

D. S. Sklarew

K6-96

R. M. Smith

$\mathrm{K} 6-96$

J. E. Szecsody

K3-61

V. R. Vermeul (5)

K6-96

M. D. Williams

K9-36

Information Release Office (7)

K1-06

Distr.1 